\title{
Statistical Summaries of Surface-Water Hydrologic Data Collected in the Suwannee River Water Management District, Florida, 1906-93
}

By M.A. Franklin, G.L. Giese, and P.R. Mixson

U.S. GEOLOGICAL SURVEY

Open-File Report 94-709-W

Prepared in cooperation with the SUWANNEE RIVER WATER MANAGEMENT DISTRICT

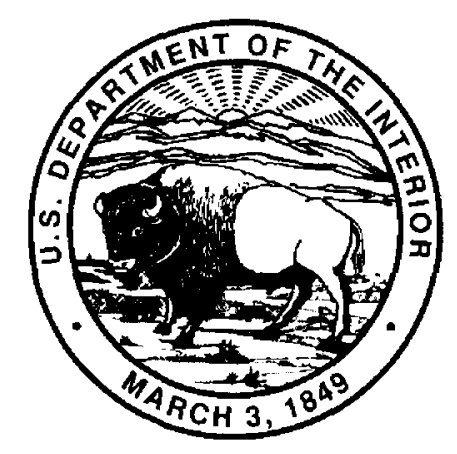




\title{
U.S. DEPARTMENT OF THE INTERIOR BRUCE BABBITT, Secretary
}

\author{
U.S. GEOLOGICAL SURVEY \\ Gordon P. Eaton, Director
}

Any use of trade, product, or firm names in this publication is for descriptive purposes only and does not imply endorsement by the U.S. Geological Survey.

For additional information write to:

Copies of this report can be purchased from:

District Chief

U.S. Geological Survey

Suite 3015

227 North Bronough Street

Tallahassee, Florida 32301
U.S. Geological Survey

Earth Science Information Center

Open-File Reports Section

P.O. Box 25286, MS 517

Denver, CO 80225-0425 


\section{CONTENTS}

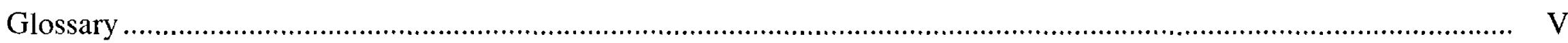

Stream and Lake Stations for Which Statistics are Included in This Report ........................................................... VII

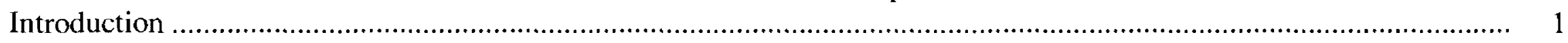

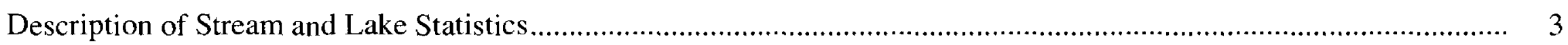

Graphs Showing Maximum, Minimum, and Mean of Monthly Mean Stream Elevations .................................... 4

Duration Curves of Daily Mean Stream Elevations .................................................................................

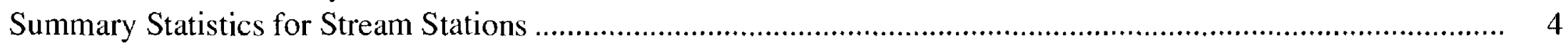

Graphs Showing Maximum, Minimum, and Mean of the Monthly Mean Stream Discharges............................... 5

Duration Curves of Daily Mean Stream Discharges ................................................................................ 5

Tables Showing Maximum, Minimum, and Mean of the Mean Monthly Stream Elevations and Discharges ........... 6

Tables Showing Duration of Daily Mean Stream Elevations and Discharges .................................................. 6

Tables Showing Lowest and Highest Mean Stream Elevations and Discharges for Various

Consecutive-Day Periods ..................................................................................................... 6

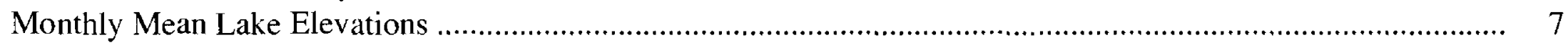

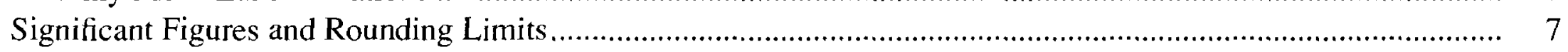

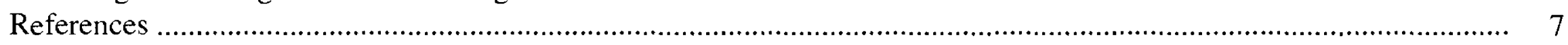

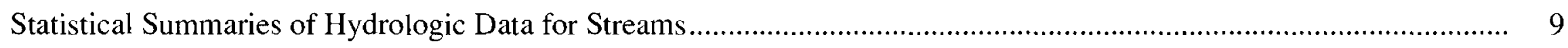

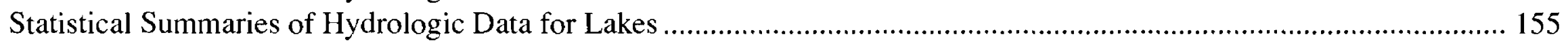

\section{FIGURES}

1. Map showing location of Suwannee River Water Management District and location of stream and lake gaging stations. 
CONVERSION FACTORS, VERTICAL DATUM, AND ABBREVIATIONS

\begin{tabular}{|c|c|c|}
\hline Multiply & By & To obtain \\
\hline \multicolumn{3}{|c|}{ Length } \\
\hline inch (in.) & 25.4 & millimeter (mm) \\
\hline foot $(f t)$ & 0.3048 & meter $(\mathrm{m})$ \\
\hline mile (mi) & 1.609 & kilometer (km) \\
\hline \multicolumn{3}{|c|}{ Area } \\
\hline acre & 4,047 & square meter $\left(\mathrm{m}^{2}\right)$ \\
\hline acre & 0.4047 & square hectometer $\left(\mathrm{hm}^{2}\right)$ \\
\hline acre & 0.004047 & square kilometer $\left(\mathrm{km}^{2}\right)$ \\
\hline square mile $\left(\mathrm{mi}^{2}\right)$ & 2.590 & square kilometer $\left(\mathrm{km}^{2}\right)$ \\
\hline \multicolumn{3}{|c|}{ Volume } \\
\hline gallon (gal) & 3.785 & liter (L) \\
\hline gallon (gal) & 0.003785 & cubic meter $\left(\mathrm{m}^{3}\right)$ \\
\hline gallon (gal) & 3.785 & cubic decimeter $\left(\mathrm{dm}^{3}\right)$ \\
\hline million gallons (Mgal) & 3,785 & cubic meter $\left(\mathrm{m}^{3}\right)$ \\
\hline cubic foot $\left(\mathrm{ft}^{3}\right)$ & 28.32 & cubic decimeter $\left(\mathrm{dm}^{3}\right)$ \\
\hline cubic foot $\left(\mathrm{ft}^{3}\right)$ & 0.028317 & cubic meter $\left(\mathrm{m}^{3}\right)$ \\
\hline acre-foot (acre-ft) & 1,233 & cubic meter $\left(\mathrm{m}^{3}\right)$ \\
\hline acre-foot (acre-ft) & 0.001233 & cubic hectometer $\left(\mathrm{hm}^{3}\right)$ \\
\hline \multicolumn{3}{|c|}{ Flow } \\
\hline cubic foot per second $\left(\mathrm{ft}^{3} / \mathrm{s}\right)$ & 0.02832 & cubic meter per second $\left(\mathrm{m}^{3} / \mathrm{s}\right)$ \\
\hline gallon per minute (gal/min) & 0.06309 & liter per second $(\mathrm{L} / \mathrm{s})$ \\
\hline million gallons per day $(\mathrm{Mgal} / \mathrm{d})$ & 0.04381 & cubic meter per second $\left(\mathrm{m}^{3} / \mathrm{s}\right)$ \\
\hline \multicolumn{3}{|c|}{ Mass } \\
\hline ton, short & 0.9072 & megagram (Mg) \\
\hline
\end{tabular}

Sea level: In this report "sea level" or "mean sea level" refers to the National Geodetic Vertical Datum of 1929--a geodetic datum derived from a general adjustment of the firstorder level nets of the United States and Canada, formerly called Sea Level Datum of 1929. 


\section{GLOSSARY}

Terms related to streamflow, water-quality, and other hydrologic data, as used in this report, are defined below. Refer to the chart for converting English units to International System (SI) Units on page IV of this report.

Acre-foot (ac-ft) is the quantity of water required to cover 1 acre to a depth of 1 foot and is equivalent to 43,560 cubic feet or about 326,000 gallons or 1,233 cubic meters.

Annual 7-day minimum is the lowest mean discharge for 7 consecutive days for a calender year or a water year. Note that most low-flow frequency analyses of annual 7-day minimum flows use a climatic year (April 1 - March 31). The date shown in the summary statistics table is the initial date of the 7-day period. (This value should not be confused with the 7-day 10-year low-flow statistic.)

Contents is the volume of water in a reservoir or lake. Unless otherwise indicated, volume is computed on the basis of a level pool and does not include bank storage.

Control designates a feature downstream from the gage that determines the stagedischarge relation at the gage. This feature may be a natural constriction of the channel, an artificial structure, or a uniform cross section over a long reach of the channel.

Control structure as used in this report is a structure on a stream or canal that is used to regulate the flow or stage of the stream or to prevent the intrusion of salt water.

Cubic feet per second $\left(\mathrm{ft}^{3} / \mathrm{s}\right)$ is the rate of discharge representing a volume of 1 cubic foot passing a given point during 1 second and is equivalent to 7.48 gallons per second or 448.8 gallons per minute or 0.02832 cubic meters per second.

Cubic-feet-per-second day (cfs-day) is the volume of water represented by a flow of 1 cubic foot per second for 24 hours. It is equivalent to 86,400 cubic feet, approximately 1.9835 acre-feet, about 646,000 gallons, or 2,445 cubic meters.

Cubic feet per second per square mile ( $\mathrm{cfsm}$ ) is the number of cubic feet of water flowing per second divided by the drainage area in squarae miles.

Discharge is the volume of water (or more broadly, volume of fluid plus suspended sediment) that passes a given point within a given period of time.

Drainage area of a stream at a specified location is that area, measured in a horizontal plane, enclosed by a topographic divide from which direct surface runoff from precipitation normally drains by gravity into the stream above the specified point. Figures of drainage area given herein include all closed basins, or noncontributing areas, within the area unless otherwise specified.

Drainage basin is a part of the surface of the earth that is occupied by a drainage system, which consists of a surface stream or a body of impounded surface water together with all tributary surface streams and bodies of impounded surface water.

Elevation, in this report, refers to the distance above or below the National Geodetic Vertical Datum of 1929 (NGVD of 1929).

Gage height (G.H.) is the water-surface elevation referred to some arbitrary gage datum. Gage height is often used interchangeably with the more general term "stage," although gage height is more appropriate when used with a reading on a gage.

Gaging station is a particular site on a stream, canal, lake, or reservoir where systematic observations of hydrologic data are obtained. 
Hydrologic unit is a geographic area representing part or all of a surface drainage basin or distinct hydrologic feature as delineated by the Office of Water Data Coordination on the State Hydrologic Unit Maps; each hydrologic unit is identified by an eight-digit number.

Instantaneous discharge is the discharge at a particular instant of time.

Mean discharge is the arithmetic mean of individual daily mean discharges during a specific period.

National Geodetic Vertical Datum of 1929 (NGVD of 1929) is a geodetic datum derived from a general adjustment of the first order level nets of both the United States and Canada. It was formerly called "Sea Level Datum of 1929" or "mean sea level" in this series of reports. Although the datum was derived from the average sea level over a period of many years at 26 tide stations along the Atlantic, Gulf of Mexico, and Pacific Coasts, it does not necessarily represent local mean sea level at any particular place.

Partial-record station is a particular site where limited streamflow and/or water-quality data are collected systematically over a period of years for use in hydrologic analyses.

Recurrence interval is the average time interval between occurrences of a hydrological event of a given or greater magnitude, usually expressed in years. May also be called return period.

Runoff in inches (in.) shows the depth to which the drainage area would be covered if all the runoff for a given time period were uniformly distributed.

Stage-discharge relation is the relation between gage height (stage) and volume of water per unit of time (discharge) flowing in a channel.

Streamflow is the discharge that occurs in a natural channel. Although the term "discharge" can be applied to the flow of a canal, the word "streamflow" uniquely describes the discharge in a surface stream course. The term "streamflow" is more general than "runoff" as streamflow may be applied to discharge whether or not it is affected by diversion or regulation.

Surface area of a lake is that area outlined on the most recent USGS topographic map as the boundary of the lake and measured by a planimeter in acres. In localities not covered by topographic maps, the areas are computed from the best maps available at the time planimetered. All areas shown are those for the stage when the planimetered map was made.

Water year in USGS reports dealing with surface-water supply is the I2-month period October 1 through September 30. The water year is designated by the calendar year in which it ends and which includes 9 of the 12 months. Thus, the year ending September 30,1993 , is called the " 1993 water year."

7-day 10-year low flow ( $7 \mathrm{Q10}$ ) is the discharge at the 10-year recurrence interval taken from a frequency curve of annual values of the lowest mean discharge for 7 consecutive days (the 7-day low flow). The average runoff is also known as basin yield. 


\section{STREAM AND LAKE STATIONS FOR WHICH STATISTICS ARE INCLUDED IN THIS REPORT \\ STREAMS}

Station

number

\section{WACCASASSA RIVER BASIN}

Waccasassa River:

Cow Creek:

Tenmile Creek at Lebanon Station, FL

02314200

\section{SUWANNEE RIVER BASIN}

Suwannee River:

Rocky Creek near Belmont, F

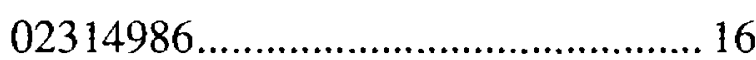

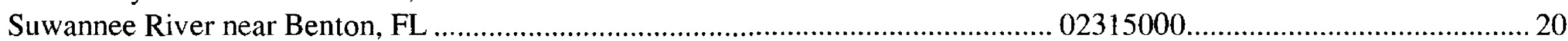

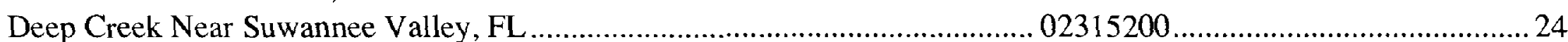

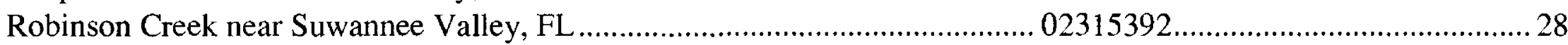

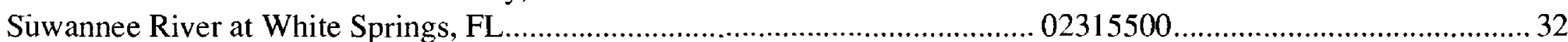

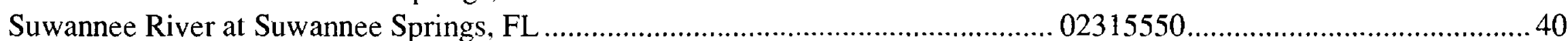

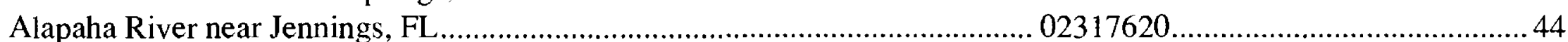

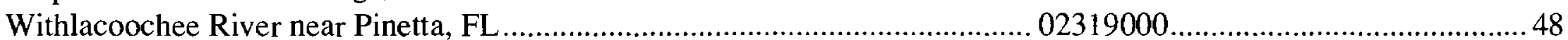

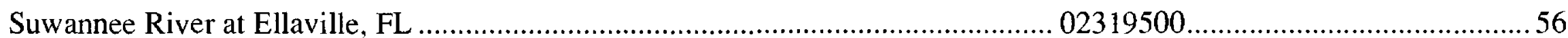

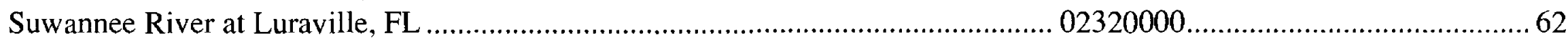

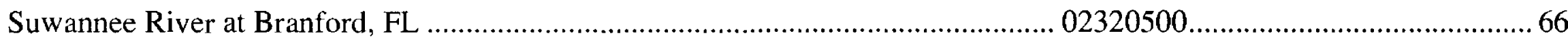

Santa Fe River:

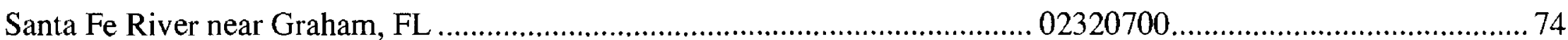

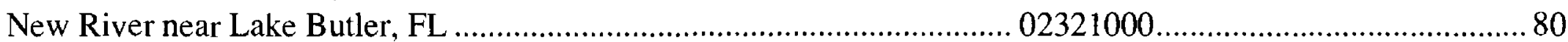

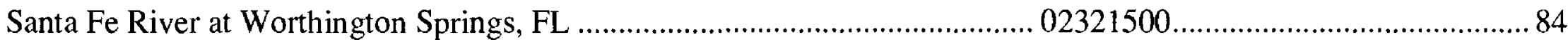

Olustee Creek:

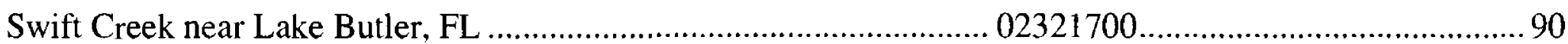

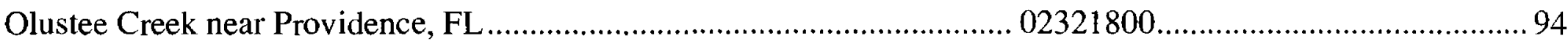

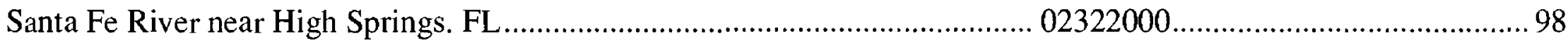

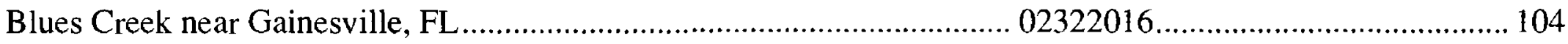

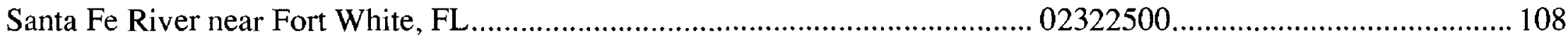

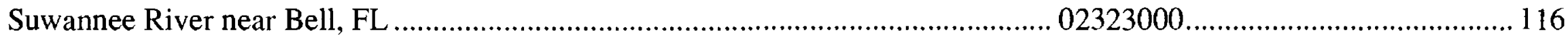

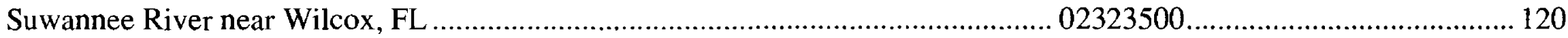

\section{STEINHATCHEE RIVER BASIN}

Steinhatchee River:

Steinhatchee River near Cross City, FL

02324000

126

\section{FENHOLLOWAY RIVER BASIN}

Fenholloway River:

Fenholloway River near Foley, FL

02324400

ECONFINA RIVER BASIN

Econfina River:

Econfina River near Perry, FL

02326000 138

AUCILLA RIVER BASIN

Aucilla River:

Aucilla River at Lamont, FL

02326500

Aucilla River near Scanlon, FL

02326512 


\section{LAKES}

\section{ST. MARYS RIVER BASIN}

\section{St. Marys River:}

Middle Prong St. Marys River:

Big Gum Swamp:

Ocean Pond at Olustee, FL

South Prong St. Marys River:

Palestine Lake near Olustee, FL

WACCASASSA RIVER BASIN

Waccasassa River:

Chunky Pond near Bronson, FL

02313510

\section{SUWANNEE RIVER BASIN}

Suwannee River:

Withlachoochee River:

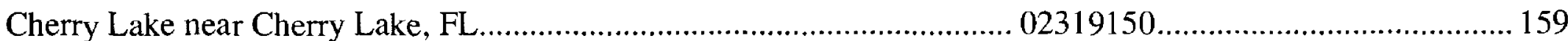

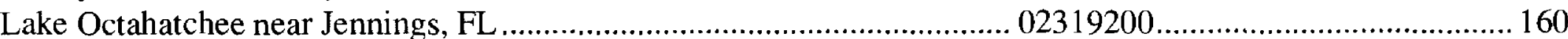

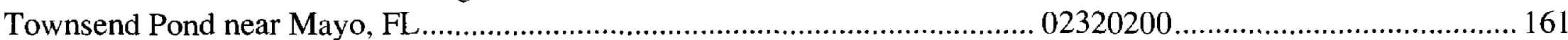

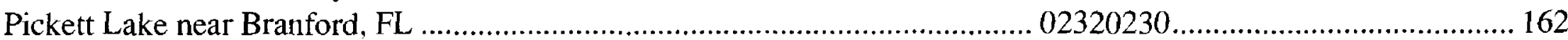

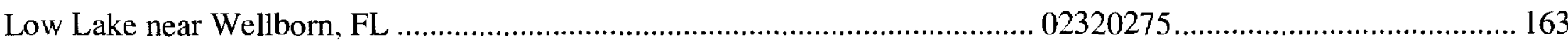

Santa Fe River:

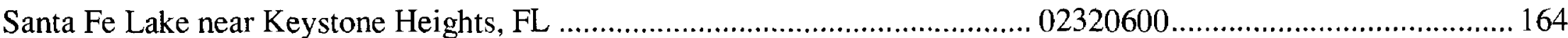

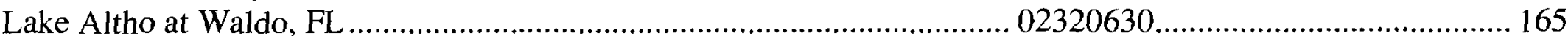

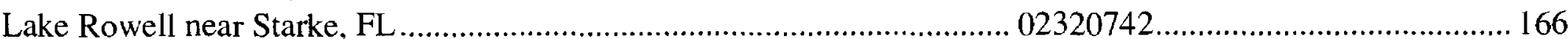

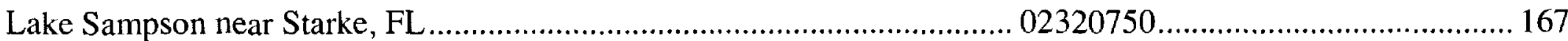

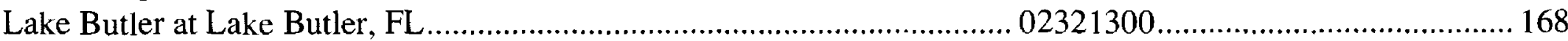

Cow Creek:

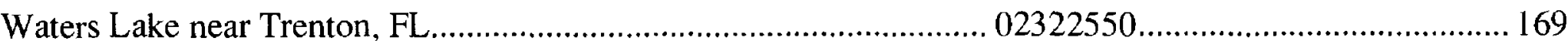

Ichetucknee River:

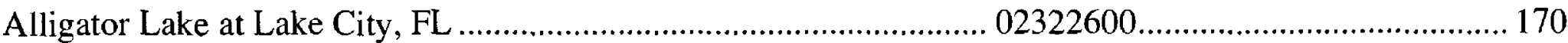

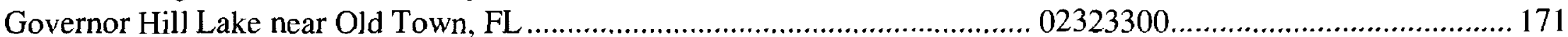

\section{ECONFINA RIVER BASIN}

Econfina River:

Sampala Lake near Greenville, FL

02325700

Andrews Lake near Shady Grove, FL

02325820 


\title{
Statistical Summaries of Surface-Water Hydrologic Data Collected in the Suwannee River Water Management District, Florida, 1906-93
}

\author{
By M.A. Franklin, G.L. Giese, and P.R. Mixson
}

\section{INTRODUCTION}

Since 1906, hydrologic data have been collected systematically on streams and lakes in the Suwannee River Water Management District (SRWMD) by the U.S. Geological Survey (USGS), the SRWMD, and other agencies. Records of stream discharge and stream and lake stage in the SRWMD (fig. 1), collected largely in cooperation with the SRWMD since 1975, have been published for many years in the USGS annual report series "Water Resources Data for Florida."

Streamflow and stream and lake elevation statistics are given in this report for sites shown in figure 1. All 27 daily streamflow stations shown are (or were) operated by the USGS in cooperation with the SRWMD; all 18 lake stage stations shown are now (1994) operated by the SRWMD, but were operated until the late 1970's by the USGS in cooperation with the SRWMD. All unregulated stream stations which have three or more years of continuous record are included in this report. All unregulated lake stations having at least three years of intermittent lake elevation readings are also included. There are many other sites in the SRWMD for which shorter records or miscellaneous measurements are available but are not included because statistical summaries for such stations require interpretive analysis beyond the scope of this report.

Basic hydrologic data have long been recognized as fundamental to the analysis of magnitude and frequency of floods, availability of water supplies, potential for reservoir storage, and permitting of waste discharges. Also, in recent times, scientists and water managers have become more aware of the complex relations between flows and water levels and the terrestrial and aquatic plant and animal life in wetland ecosystems. In 1994, the SRWMD and the USGS began a long-term program of cooperative studies designed to better understand minimum and maximum flow and water levels needed to manage the surface and ground water resources of the District and maintain or improve the various ecosystems therein. Information presented in this report, together with frequency analysis of station data, flow regionalization, studies of the relation of salinity to flow in the lower Suwannee River, definition of ground-water surface-water interactions, surface- and ground-water quality studies, and studies of interaction between surface-water bodies and wetlands, will provide the basis for the SRWMD to establish minimum flow and level requirements for streams and lakes in the SRWMD area.

This report is a necessary first step in the longterm program of study because it contains basic stream discharge and stream and lake elevation statistics, most of which are not contained in the annual report "Water Resources Data for Florida." These statistics, most of which were generated using a U.S. Geological Survey computer program, ADAPS, Automatic Data Processing System, characterize normal flows and levels and departures from normal due to floods and droughts or seasonal climatic variations. Specifically, the report presents for the period of record of each stream or lake gaging station, as appropriate:

- Minimum, maximum, and mean of monthly mean stream elevations and discharges in graphical and tabular form.

- For streams, annual mean discharge, highest and lowest annual mean discharge, highest and lowest daily mean discharge, minimum annual seven-day mean discharge, instantaneous peak discharge and elevation, instantaneous minimum discharge, and basin yield. 


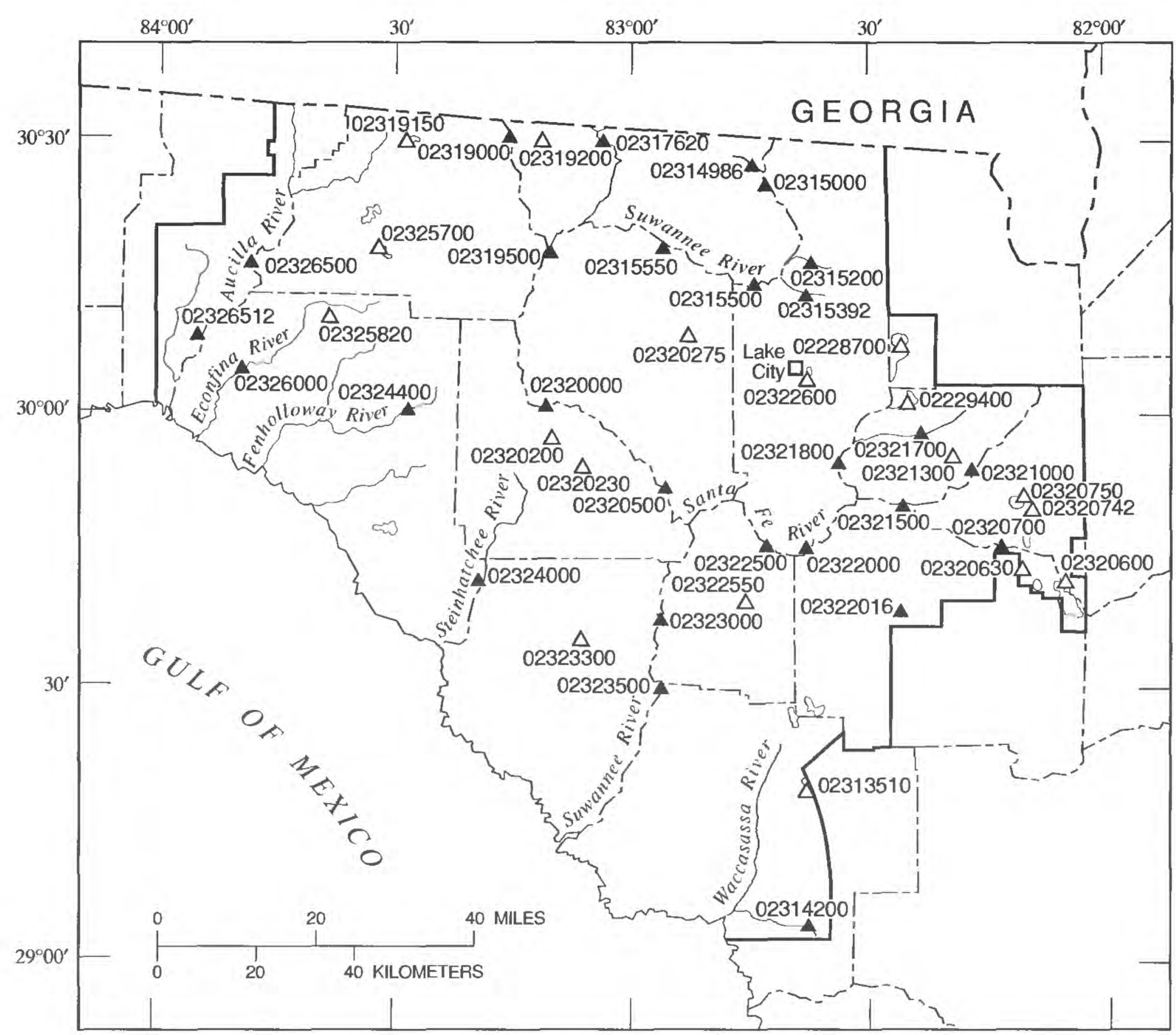

EXPLANATION

\begin{tabular}{|c|c|}
\hline & $\begin{array}{l}\text { SUWANNEE RIVER WATER } \\
\text { MANAGEMENT DISTRICT } \\
\text { BOUNDARY }\end{array}$ \\
\hline 02314200 & $\begin{array}{l}\text { STREAM GAGING } \\
\text { STATION-And number }\end{array}$ \\
\hline$\underset{\triangle}{02313510}$ & $\begin{array}{l}\text { LAKE GAGING } \\
\text { STATION-And number }\end{array}$ \\
\hline
\end{tabular}

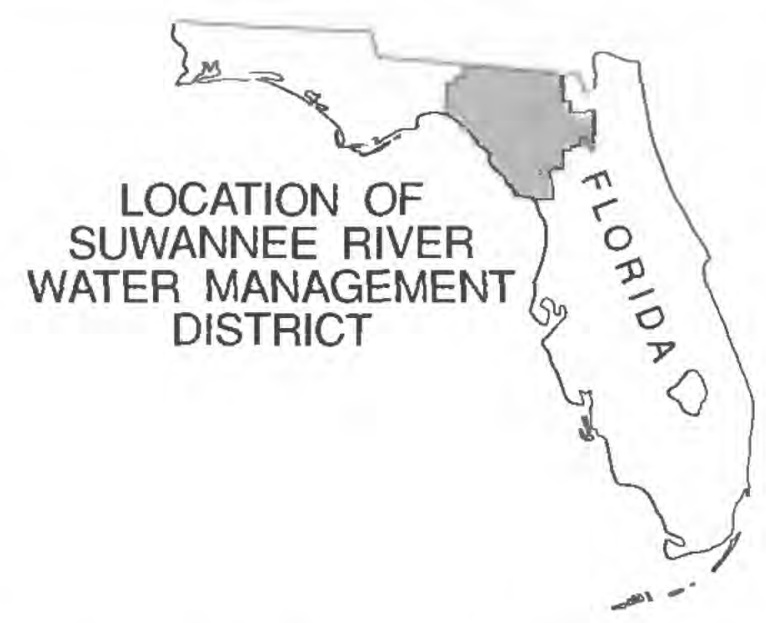

Figure 1. Map showing location of Suwannee River Water Management district and location of stream and lake gaging stations.

- Duration of annual daily mean stream elevation and discharge. Duration of daily values are shown in both graphical and tabular form.

- Duration of daily mean stream elevations and discharge, by months, in tables.

- Minimum and maximum 1-,3-,7-, 14-,30-, 60-,
90-,120-, and 183-consecutive day stream elevations and discharges (with rankings) for each year of record.

- Mean monthly lake elevations and statistics by month, including number of monthly values, mean, variance, standard deviation, skewness, and coefficient of variation. 
For convenience, a GLOSSARY of commonly used terms related to the collection and reporting of surface-water elevations and discharge is included before the Introduction section of this report.

The authors wish to acknowledge Natalie Rackley, formerly of the U.S. Geological Survey, and T.W. Grubbs, U.S. Geological Survey, for their computer assistance in the compilation of station records, and Jim Tomberlin, U.S. Geological Survey, for the mapping of stream and lake gaging stations.

\section{DESCRIPTION OF STREAM AND LAKE STATISTICS}

The following sections describe the summary statistics presented in the various graphs and tables given for each station later in this report. A running head for each station contains the name of the major basin in the SRWMD within which the station is located, the station name, and a unique downstream order identification number for the station. Streamflow stations are listed first, then lake elevation stations, each in downstream order.

The downstream ordering system has been in effect since October 1, 1950, for USGS reports. The order of listing of hydrologic-station records in this report is in a downstream direction along the main stream. All stations on a tributary entering upstream from a mainstream station are listed before that station. A station on a tributary that enters between two mainstream stations is listed between them. A similar order is followed in listing stations on first rank, second rank, and other ranks of tributaries. The rank of any tributary with respect to the stream to which it is immediately tributary is indicated by an indention in the "List of Stations..." in the front of this report. Each indention represents one rank. This downstream order and system of indention indicates which stations are on tributaries between any two stations and the rank of the tributaries. Gaps in the series of numbers allow for new stations that may be established; hence, the numbers are not consecutive.

The complete number for each station, such as 02314200 , which is left of the station name, includes the two-digit Part number " 02 " plus the 6 to 13 digit downstream-order number " 314200 ". The part number refers to an area whose boundaries coincide with natural drainage lines; for example, Part " 02 " is the South Atlantic Slope and eastern Gulf of Mexico basin.
Immediately following the heading is the station manuscript which provides, under various subheadings the following information, as appropriate, for each station:

LOCATION.--Information on locations is obtained from the most accurate maps available. Coordinates of each station are given in terms of latitude and longitude and also in terms of township, range, and quadrant.

DRAINAGE AREA.--Drainage areas are measured using the most accurate maps available. Because the type of maps varies from one drainage basin to another, the accuracy of drainage areas likewise varies. Drainage areas are updated as revised maps become available.

PERIOD OF RECORD.--This indicates the period for which records have been collected for the station or for an equivalent station. An equivalent station is one that was in operation at a time when the present station was not in operation. Also, the location of the equivalent station was such that flow can reasonably be considered equivalent to flow at the present station.

REVISED RECORDS.--Published records. because of new information, occasionally are incorrect, and revisions are printed in later reports. Listed under this heading are all the reports in which revisions have been published for the station and the water years to which the revisions apply. If a revision did not include daily, monthly, or annual figures of discharge, that fact is noted after the year dates as follows: "(M)" means that only the instantaneous maximum discharge was revised; "( $\mathrm{m})$ " that only the instantaneous minimum was revised; and "(P)" that only peak discharges were revised. If the drainage area has been revised, the report in which the most recently revised figure was first published is cited.

GAGE.--The type of gage in current use, the datum of the current gage referred to National Geodetic Vertical Datum of 1929 (NGVD of 1929; see glossary), and a condensed history of the types, locations, and datums of previous gages are given under this heading. The terms "NGVD of 1929" and "mean sea level" (MSL) are used interchangeably in this report.

REMARKS.--This paragraph is used to present information relative to the accuracy of the records, to special methods of computation, and to conditions that affect natural flow at the station. In addition, information may be presented pertaining to average 
discharge data for the period of record; to extremes data for the period of record; and, possibly, to other pertinent items. For reservoir stations, information is given on the dam forming the reservoir, the capacity, outlet works and spillway, and purpose and use of the reservoir. Regarding accuracy of the records, "excellent" means that about 95 percent of daily discharges are thought to be within 5 percent of their true values, "good" within 10 percent, and "fair" within 15 percent. Records that do not meet these criteria are rated poor.

AVERAGE DISCHARGE.-- The average discharge for the indicated water years. A water year in USGS reports dealing with surface-water supply is the 12-month period October 1 through September 30 . The water year is designated by the calendar year in which it ends and which includes 9 of the 12 months. Thus, the year ending September 301994 , is called the "1994 water year." The water year was so chosen because major floods seldom occur during September and October; thus it is unlikely that statistics on a flood (for example, flood volumes) will be distorted by ending the year at this time.

COOPERATION.--Records provided by a cooperating organization or obtained for the Geological Survey by a cooperating organization are identified here.

\section{EXTREMES FOR PERIOD OF RECORD.--}

For lake stations, the extremes of record are shown here. For stream stations, extremes are shown in a table outside the station manuscript.

\section{EXTREMES OUTSIDE PERIOD OF}

RECORD.--Included here is information concerning major floods or unusually low flows that occurred outside the stated period of record. The information may or may not have been obtained by the USGS.

\section{Graphs Showing Maximum, Minimum, and Mean of Monthly Mean Stream Elevations}

These graphs present summary statistics for monthly mean stream elevations for the period of record. Elevation, in this report, refers to the distance above or below the NGVD of 1929, otherwise referred to as mean sea level. These monthly statistics were derived from daily-mean values of continuouslyrecorded gage heights, which were converted to elevations by tying arbitrary gage datums to NGVD of 1929. The graphs show the maximum, minimum, and mean of the monthly mean stream elevations for the period of record for each month. This type of graph is used to show both the seasonal variations in elevations and extremes which have been encountered during the period of record. The relation of current conditions to long-term conditions is evident from such a record, and may be of importance where stream levels affect local aquatic and terrestrial plant and animal life, and in water supply.

\section{Duration Curves of Daily Mean Stream Elevations}

These curves show the percentage of time indicated values of daily mean stream elevation were equaled or exceeded for the period of record. For example, if an elevation of $22 \mathrm{ft}$ above mean sea level corresponds to a value of 30 for the percent of time the indicated value is equaled or exceeded, this means that a daily mean elevation of $22 \mathrm{ft}$ was equaled or exceeded 30 percent of all the days of record. Flow (and elevation) duration curves are often used to obtain a general idea of flow and storage characteristics of a basin. Searcy $(1959$, p. 22$)$ notes:

"A curve with a steep slope throughout denotes a highly variable stream whose flow is largely from direct runoff, whereas a curve with a flat slope reveals the presence of surface- or ground-water storage, which tends to equalize the flow. The slope of the lower end of the duration curve shows the characteristics of the perennial storage in the drainage basin; a flat slope at the lower end indicates a large amount of storage, and a steep slope indicates a negligible amount. Streams whose high flows come largely from snowmelt tend to have a flat slope at the upper end. The same is true for streams with large floodplain storage or those that drain swamp areas."

An elevation duration curve may also be used to estimate the percentage of time a floodplain is flooded or an area normally inundated (such as a spawning bed) is dry. The elevation duration curve can also be used to estimate the percentage of time a navigation channel is at or above a specified depth. An important limitation of duration curves is that no information is provided as to whether the daily elevation or flow values are consecutive or widely scattered in time. This information may be of importance in many applications.

\section{Summary Statistics for Stream Stations}

These tables present various summary statistics and extremes by water year for each stream station as follows: 
ANNUAL MEAN.--The arithmetic mean of the individual daily mean discharges for the year noted or for the designated period. At some stations, the yearly mean discharge is adjusted for reservoir storage or diversion. The adjusted figures are identified by a symbol and corresponding footnotes.

HIGHEST ANNUAL MEAN.--The maximum annual mean discharge occurring for the designated period.

LOWEST ANNUAL MEAN.--The minimum annual mean discharge occurring for the designated period.

HIGHEST DAILY MEAN.--The maximum daily mean discharge for the year or for the designated period.

LOWEST DAILY MEAN.--The minimum daily mean discharge for the designated period.

ANNUAL 7-DAY MINIMUM.--The lowest mean discharge for 7 consecutive days for a calendar year or a water year. Most low-flow frequency analyses of annual 7-day minimum flows use a climatic year (April 1-March 31). The date shown in the summary statistics table is the initial date of the 7day period. (This value should not be confused with the 7-day 10-year low-flow statistic.)

INSTANTANEOUS PEAK FLOW.--The maximum instantaneous discharge occurring for the designated period. Secondary instantaneous peak discharges above a selected base discharge are stored in USGS District computer files for stations meeting certain criteria. Those discharge values may be obtained by writing to the USGS District Office in Florida. (See address on page preceding "CONTENTS"..)

INSTANTANEOUS PEAK STAGE.--The maximum instantaneous stage occurring for the water year or for the designated period. If the dates of occurrence for the instantaneous peak flow and instantaneous peak stage differ, the REMARKS paragraph in the manuscript or a footnote is used to provide further information.

INSTANTANEOUS LOW FLOW.--The minimum instantaneous discharge occurring for the designated period.

ANNUAL RUNOFF.--Indicates the total quantity of water in runoff for a drainage area for the year. The value shown in inches indicates the depth to which the drainage area would be covered if all the runoff for a specified period were uniformly distributed. The value shown in cubic feet per second per square mile (CFSM) is the average number of cubic feet of water flowing per second from each square mile of area drained, assuming the runoff is distributed uniformly in time and area.

\section{Graphs Showing Maximum, Minimum, and Mean of the Monthly Mean Stream Discharges}

The graphs for stream discharge are similar to the graphs for stream elevation, and roughly follow the same trends as stream elevation. That is, months of high and low mean stream elevations will tend to coincide with months of high and low mean stream discharge. However, elevations and discharges are not linearly related due to the curvilinear nature of the relation between elevation and discharge. Whereas knowledge of stream elevations is critical where flooding and drying are issues, knowledge of discharge is critical in issues involving instream flow requirements, water supply, and point discharge permits. These type of discharge graphs are also used to compare with current conditions, especially in tracking the progress of droughts. They are more useful than the elevation graphs in this regard because the discharge at a station is an integrator of flow conditions throughout the drainage basin upstream from the station, whereas the value of stream elevation is much more local to the station.

\section{Duration Curves of Daily Mean Stream Discharges}

These curves show the percentage of time that indicated values of daily mean stream discharge were equaled or exceeded for the period of record. For example, if a discharge of 22 cubic feet per second corresponds to a value of 30 for the percent of time the indicated value is equaled or exceeded, this means that a daily mean discharge of 22 cubic feet per second was equaled or exceeded 30 percent of all the days of record. As in the case of graphs showing maximum, minimum, and mean of the monthly mean elevations and discharges, duration curves of daily mean discharge can follow the same patterns as those for elevation. The same caveats apply regarding the curvilinear relation between discharge and elevation and the greater usefulness of discharge over elevation when evaluating conditions in the entire drainage basin. Comments made in the section on duration of stream elevation regarding the relation of shape of the duration curve to basin storage characteristics and flow variability are even more applicable to discharge than to elevation. 


\section{Tables Showing Maximum, Minimum, and Mean of the Mean Monthly Stream Elevations and Discharges}

These tables list the same information previously shown in graphical form. The data are repeated in tabular form for convenience in reading numerical values. An explanation of the usefulness of these statistics is presented in the previous section.

\section{Tables Showing Duration of Daily Mean Stream Elevations and Discharges}

These tables contain information similar to that contained in the duration curves of daily mean stream elevation and discharge. Information in the column headed ANNUAL is identical to that shown before and it contains duration of elevation or discharge based on all the days of every year of record. These data are presented in tabular form for convenience in reading particular values. The columns of monthly data contain duration values based on all the days of a particular month for the period of record. Because stream elevation and discharge exhibit seasonality, it is often more meaningful to consider duration of elevation or discharge by month. During a typically wet month, a daily discharge could be exceeded only a small percentage of the time, for example, 20 percent of the days, considering all the days of all the months of record. However, considering only all the days of all the Julys of record, it may be exceeded just 50 percent of the days.

The variability of elevation and discharge on a monthly basis is much less than on an annual basis. An explanation of the usefulness of duration data is presented in the previous section.

\section{Tables Showing Lowest and Highest Mean Stream Elevations and Discharges for Various Consecutive-Day Periods}

These tables show the lowest and highest mean stream elevations and discharges for various consecutive-day periods and rankings for each climatic year of record (in the case of lowest values) and each water year (in the case of highest values). A climatic year begins April 1 and ends March 31. For example, the 1993 climatic year begins April 1, 1993, and ends March 31, 1994. The climatic year is used because lowest stream elevations and discharges typically occur in months other than March and April in much of the United States (including Florida). The use of the climatic year for lowest consecutive-day values lessens the chance that a lowest consecutiveday period will be interrupted by the end of the annual computation period. The term water year is as previously defined. The water year was used as the annual computation period for maximum values because highest elevations and flows seldom occur in September and October. This minimizes the possibility that a yearly highest consecutive-day period will be interrupted by the end of the annual computation period.

The identification of climatic years in the tables of lowest mean discharge is not made directly. Instead, it is made in terms of the water years in which the climatic year nests. For example, the lowest 7 consecutive-day elevation occurring in the water- year range designated 1976-1977 includes the period of time April 1976 through March 1977.

This report does not assign frequency of occurrence to the annual consecutive-day elevations and discharges. This procedure would involve some interpretive analysis which is beyond the scope of this report. However, the rankings for the annual lowest and highest values for the period of record give some indication of the rarity of the extreme values. The highest annual value of a particular consecutive-day elevation or discharge for the period of record would receive a rank of 1 , the next highest, a rank of 2 , and so forth. Similarly, the lowest annual value of elevation or discharge for the period of record would receive a rank of 1, the next lowest a rank of 2, and so forth.

Consecutive-day lowest flows have been used in some states as criteria for waste-discharge applications. For example, the North Carolina Department of Environment, Health, and Natural Resources (DEHNR) utilizes the low flow 7Q10, which is the annual minimum 7-consecutive day discharge which would have a 10 percent probability of occurring in any one year (Giese and Mason, 1993). The North Carolina DEHNR also uses the low flow $7 \mathrm{Q} 2$, the annual minimum consecutive-day discharge which has a 50 percent probability of occurring in any one year, in draft-storage-frequency analysis for water-supply reservoirs (Arteaga and Hubbard, 1975).

Highest consecutive-day discharges are descriptive of flood volumes and have implications in the design of reservoirs for storage of flood waters. Highest consecutive-day elevations are indicative of length of time of local flood inundation and may be of consequence to terrestrial and aquatic plant and animal species. 
Consecutive-day periods of longer than 90 days may lack meaning for many applications and should be used with circumspection. For example, a 183 consecutive-day highest discharge may actually include many days of average or even below-average discharge.

For some stations, several years of lowest and highest consecutive-day mean elevations will not be printed, while lowest and highest consecutive-day discharges will be printed for those same years because ADAPS discards the year if there is any missing record. In the annual processing of hydrographic records, missing days of daily discharge are estimated, but missing days of elevation are not; hence, the discrepancy.

\section{Monthly Mean Lake Elevations}

Tables showing monthly normal lake elevations for each month of record are given for each lake station. Although the normal monthly means are in fact mean values of the readings for each month, for most months only a single reading was taken from a nonrecording gage. Hence, the term normal was used rather than mean to avoid implying that many (or continuous) observations were available for each month. However, lake elevations usually change so slowly that a single monthly reading is sufficient in most situations to adequately characterize lake elevations. Standard statistics which characterize the distribution of normal lake elevations for each month are also given for each lake station, including number of monthly values, mean of the monthly values (Mean), variance (Var), standard deviation (Std), skewness (Slew), and coefficient of variation (Cvar). Duration of daily elevation and consecutive-day elevation data were not available because readings were only taken once a month in most cases; therefore, these analysis were omitted.

Information on lake elevations is important for water supply, recreation, evaluation of the potential for flooding, and has implications for the health of the lake ecosystem.

\section{Significant Figures and Rounding Limits}

Many of the values shown in the tables at the back of this report are computer generated by ADAPS and are unrounded. Nevertheless, the following significant figure and rounding criteria should be applied in actual use.

These criteria are based not on the accuracy of the values, but solely on their magnitude.
For daily discharge:

\begin{tabular}{|c|c|c|}
\hline $\begin{array}{l}\text { Range of discharge } \\
\text { (cubic feet per second) }\end{array}$ & $\begin{array}{l}\text { Significant } \\
\text { figures }\end{array}$ & $\begin{array}{l}\text { Rounding } \\
\text { limits }\end{array}$ \\
\hline$<0.10$ & 1 & hundredths \\
\hline $0.10-0.99$ & 2 & hundredths \\
\hline $1.0-9.9$ & 2 & tenths \\
\hline-99 & 2 & units \\
\hline$\geq 100$ & 3 & variable \\
\hline
\end{tabular}

For monthly and yearly means and average discharge:

\begin{tabular}{|c|c|c|c|}
\hline \multicolumn{2}{|c|}{$\begin{array}{l}\text { Range of discharge } \\
\text { (cubic feet per second) }\end{array}$} & $\begin{array}{c}\text { Significant } \\
\text { figures }\end{array}$ & $\begin{array}{l}\text { Rounding } \\
\text { limits }\end{array}$ \\
\hline \multicolumn{2}{|l|}{$<0.01$} & 1 & thousandths \\
\hline 0.010 & - $\quad 0.099$ & 2 & thousandths \\
\hline 0.1 .0 & $\begin{array}{l}-\quad 0.99 \\
\end{array}$ & 2 & hundredths \\
\hline 1.00 & $\begin{array}{l}-\quad 9.99 \\
\end{array}$ & 3 & hundredths \\
\hline 10.0 & - 99.9 & 3 & tenths \\
\hline \multirow[t]{2}{*}{100} & -999 & 3 & units \\
\hline & $\geq 1,000$ & 4 & variable \\
\hline
\end{tabular}

\section{REFERENCES}

Arteaga, F.E., and Hubbard, E.F., 1974, Evaluation of reservoir sites in North Carolina: U.S. Geological Survey Water Resources Investigations Report 46-74, $66 \mathrm{p}$.

Giese, G.L., and Mason, Robert R., 1993, Low-flow characteristics of North Carolina streams: U.S. Geological Survey Water Supply Paper 2403, 29 p.

Riggs, H.C., 1968, Some statistical tools in hydrology: U.S. Geological Survey Techniques of Water Resources Investigations, book 4, chap. A1, $39 \mathrm{p}$.

Riggs, H.C., 1968, Frequency curves: U.S. Geological Survey Techniques of Water Resources Investigations, book 4, chap. A2, $15 \mathrm{p}$.

Riggs, H.C., 1972, Low-flow investigations: U.S. Geological Survey Techniques of Water Resources Investigations, book 4, chap. B1, 8 p.

Riggs, H.C., 1973, Regional analyses of streamflow characteristics: U.S. Geological Survey Techniques of Water Resources Investigations, book 4, chap. B3, $15 \mathrm{p}$.

Riggs, H.C., and Hardison, C.H., 1973, Storage analyses for water supply: U.S. Geological Survey Techniques of Water Resources Investigations, book 4, chap. B2, $20 \mathrm{p}$.

Searcy, J.K.,1959, Flow duration Curves: U.S. Geological Survey Water-Supply Paper 1542-A, 33 p.

U.S. Geological Survey, Water resources data for Florida: U.S. Geological Survey Water-Data Report Series, published annually. 


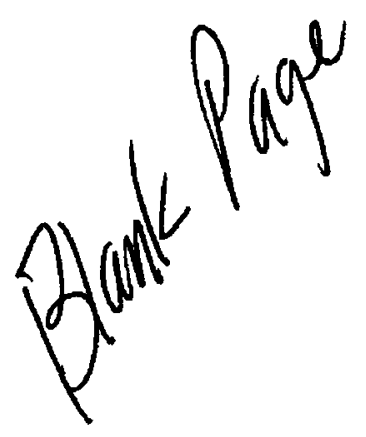

8

Statistical Summaries of Surface-Water Hydrologic Data Collected in the Suwannee River Water Management District 
STATISTICAL SUMMARIES OF HYDROLOGIC DATA FOR STREAMS 


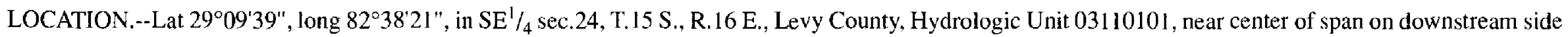
of bridge on U.S. Highways 19 and 98, just downstream from North Prong Tenmile Creek, 0.2 mi south of Lebanon Station, 9.4 mi upstream from mouth, and 13 mi northwest of Dunnellon.

DRAINAGE AREA.--26 $\mathrm{mi}^{2}$, approximately; $34 \mathrm{mi}^{2}$, approximately, includes that of Horse Hole Creek.

PERIOD OF RECORD.--October 1963 to September 1992.

GAGE.--Water-stage recorder. Datum of gage is $15.00 \mathrm{ft}$ above National Geodetic Vertical Datum of 1929. Since Feb. 26,1964, nonrecording gage at Horse Hole Creek. Datum of gage is National Geodetic Vertical Datum of 1929.

REMARKS.--Records poor. Records do not include considerable amount of water diverted naturally above station into Horse Hole Creek basin. Discharge measurements of Horse Hole Creek, drainage area $8.1 \mathrm{mi}^{2}$, approximately, are made at bridge on U.S. Highways 19 and $98,1.9$ mi south of Tenmile Creek station.

EXTREMES FOR PERIOD OF RECORD (Horse Hole Creek-02314205).--Maximum discharge measured, 1,270 $\mathrm{ft}^{3} / \mathrm{s} \mathrm{Sept.} \mathrm{11,} \mathrm{1964,} \mathrm{elevation,} \mathrm{26.56} \mathrm{ft}$ above NGVD; creek dry at times most years.

MAXIMUM, MINIMUM, AND MEAN OF MONTHLY MEAN ELEVATIONS FOR WATER YEARS 1964-1992
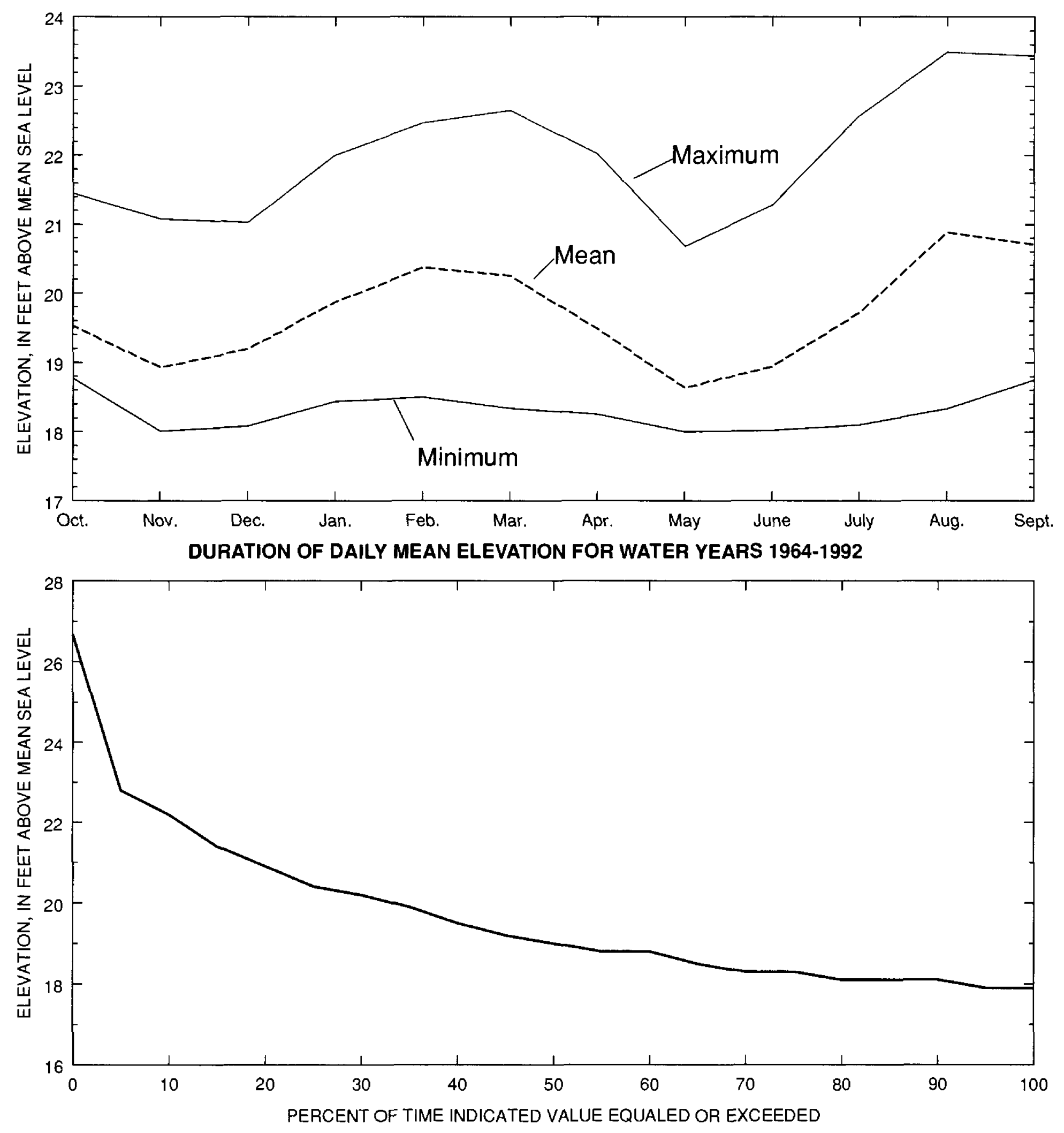
SUMMARY STATISTICS, IN CUBIC FEET PER SECOND UNLESS OTHERWISE INDICATED, FOR WATER YEARS 1964 - 1992

ANNUAL MEAN

HIGHEST ANNUAL MEAN

LOWEST ANNUAL MEAN

HIGHEST DAILY MEAN

LOWEST DAILY MEAN

ANNUAL SEVEN-DAY MINIMUM

INSTANTANEOUS PEAK FLOW

INSTANTANEOUS PEAK ELEVATION (FT)

INSTANTANEOUS LOW ELOW

ANNUAL RUNOEE (INCHES)

ANNUAL RUNOFE (CESM)

$$
\begin{array}{r}
36.8 \\
113 \\
5.89 \\
3440 \\
.00 \\
.00 \\
4290 \\
27.38 \\
.00 \\
19.21 \\
1.42
\end{array}
$$

MAXIMUM, MINIMUM, AND MEAN OF MONTHLY MEAN DISCHARGES FOR WATER YEARS 1964-1992
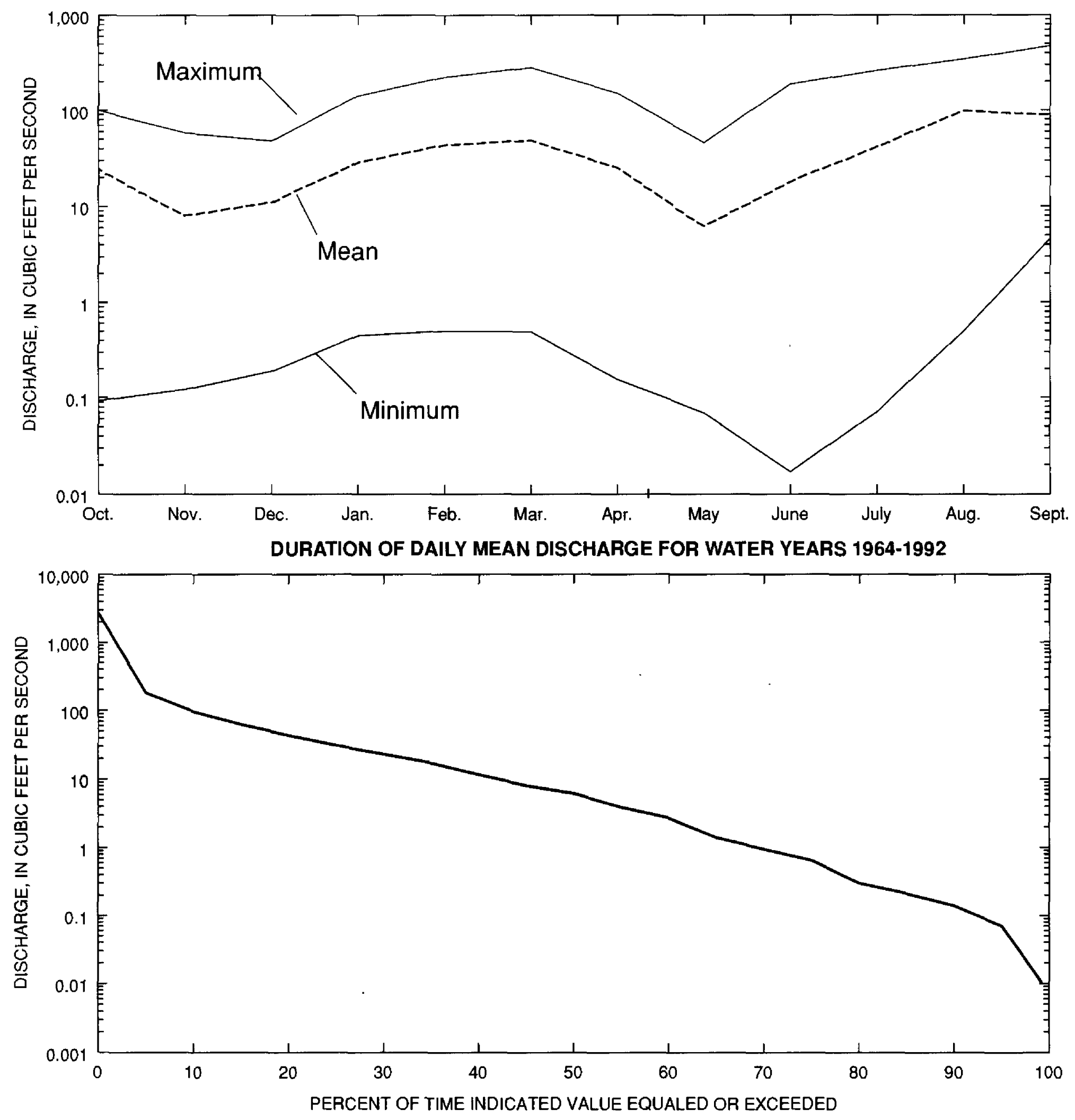
WACCASASSA RIVER BASIN

02314200 TENMILE CREEK AT LEBANON STATION, FL--Continued

SUMMARY OF MONTLY MEAN ELEVATION AND DISCHARGE STATISTICS FOR WATER YEARS 1964-1992

\begin{tabular}{lrrrrrrr} 
& \multicolumn{3}{c}{ ELEVATION } & \multicolumn{3}{c}{ DISCHARGE } \\
& \multicolumn{2}{c}{ EEET ABOVE SEA LEVEL } & \multicolumn{2}{c}{ CUBIC EEET PER SECOND } \\
\multicolumn{1}{c}{ MONTH } & MAXIMUM & MINIMUM & MEAN & MAXIMUM & MINIMUM & \multicolumn{1}{c}{ MEAN } \\
OCTOBER & 21.44 & 18.77 & 19.53 & 98.6 & .093 & 24.33 \\
NOVEMBER & 21.07 & 18.01 & 18.93 & 57.1 & .121 & 7.99 \\
DECEMBER & 21.02 & 18.08 & 19.20 & 47.0 & .188 & 11.07 \\
JANUARY & 21.99 & 18.44 & 19.87 & 139.4 & .448 & 28.71 \\
EEBRUARY & 22.46 & 18.50 & 20.37 & 219.6 & .498 & 43.30 \\
MARCH & 22.64 & 18.34 & 20.25 & 276.8 & .490 & 48.36 \\
APRIL & 22.01 & 18.25 & 19.49 & 148.1 & .155 & 25.13 \\
MAY & 20.67 & 18.00 & 18.64 & 44.9 & .069 & 6.18 \\
JUNE & 21.27 & 18.02 & 18.95 & 185.8 & .017 & 18.13 \\
JULY & 22.56 & 18.10 & 19.72 & 257.0 & .071 & 41.62 \\
AUGUST & 23.48 & 18.33 & 20.88 & 339.0 & .500 & 97.86 \\
SEPTEMBER & 23.43 & 18.75 & 20.71 & 466.8 & .462 & 88.78
\end{tabular}

\section{DURATION OF DAILY MEAN VALUES FOR WATER YEARS 1964-1992}

PERCENT

EQUAIED OR

EXCEEDED ANNUAI.

JAN

FEB

MAR

APR

MAY

JULY

AUG

SEPT

ELEVATION IN FEET ABOVE MEAN SEA LEVEI

$\begin{array}{rlll}95.0 & 17.9 & 18.0 & 18.0 \\ 90.0 & 18.1 & 18.2 & 18.1 \\ 85.0 & 18.1 & 18.2 & 18.1 \\ 80.0 & 18.1 & 18.2 & 18.1 \\ 75.0 & 18.3 & 18.3 & 18.1 \\ 70.0 & 18.3 & 18.3 & 18.3 \\ 65.0 & 18.5 & 18.5 & 18.3 \\ 50.0 & 18.8 & 18.6 & 18.3 \\ 55.0 & 18.8 & 18.8 & 18.4 \\ 50.0 & 19.0 & 19.0 & 18.6 \\ 45.0 & 19.2 & 19.3 & 18.7 \\ 40.0 & 19.5 & 19.5 & 18.8 \\ 35.0 & 19.9 & 19.8 & 19.0 \\ 30.0 & 20.2 & 20.0 & 19.1 \\ 25.0 & 20.4 & 20.3 & 19.1 \\ 20.0 & 20.9 & 20.5 & 19.3 \\ 15.0 & 21.4 & 21.0 & 19.6 \\ 10.0 & 22.2 & 21.6 & 20.0 \\ 5.0 & 22.8 & 22.5 & 20.8\end{array}$

$\begin{array}{ll}18.0 & 18.2 \\ 18.0 & 18.4 \\ 18.2 & 18.4 \\ 18.3 & 18.6 \\ 18.3 & 18.6 \\ 18.5 & 18.7 \\ 18.6 & 18.9 \\ 18.6 & 19.1 \\ 18.7 & 19.3 \\ 18.9 & 19.4 \\ 18.9 & 19.6 \\ 19.1 & 19.8 \\ 19.3 & 20.2 \\ 19.4 & 20.3 \\ 19.7 & 20.7 \\ 20.0 & 21.1 \\ 20.3 & 21.5 \\ 20.6 & 21.9 \\ 21.1 & 22.5\end{array}$

$\begin{array}{ll}18.4 & 18.2 \\ 18.5 & 18.2 \\ 18.7 & 18.4 \\ 18.9 & 18.6 \\ 19.1 & 18.8 \\ 19.3 & 19.0 \\ 19.5 & 19.2 \\ 19.7 & 19.4 \\ 19.9 & 19.8 \\ 20.1 & 20.0 \\ 20.3 & 20.2 \\ 20.5 & 20.4 \\ 20.7 & 20.6 \\ 20.9 & 20.8 \\ 21.3 & 21.3 \\ 21.5 & 21.5 \\ 22.0 & 22.2 \\ 22.4 & 22.6 \\ 22.8 & 23.1\end{array}$

$\begin{array}{ll}18.0 & 17.9 \\ 18.2 & 17.9 \\ 18.2 & 17.9 \\ 18.2 & 18.1 \\ 18.3 & 18.1 \\ 18.3 & 18.1 \\ 18.5 & 18.1 \\ 18.7 & 18.1 \\ 18.7 & 18.1 \\ 18.8 & 18.2 \\ 19.0 & 18.2 \\ 19.2 & 18.4 \\ 19.5 & 18.4 \\ 19.7 & 18.4 \\ 20.0 & 18.5 \\ 20.4 & 18.8 \\ 21.0 & 19.3 \\ 21.9 & 19.7 \\ 22.9 & 20.9\end{array}$

17.9
17.9
18.1
18.1
18.1
18.1
18.1
18.2
18.2
18.2
18.4
18.4
18.6
18.8
19.2
19.5
19.9
20.9
22.2

DISCHARGE IN CUBIC FEET PER SECOND

$\begin{array}{rrr}95.0 & 0.0 & 0.1 \\ 90.0 & 0.1 & 0.1 \\ 85.0 & 0.2 & 0.2 \\ 80.0 & 0.3 & 0.4 \\ 75.0 & 0.6 & 0.5 \\ 70.0 & 0.9 & 0.6 \\ 65.0 & 1.4 & 1.2 \\ 60.0 & 2.7 & 2.0 \\ 55.0 & 3.8 & 3.8 \\ 50.0 & 6.1 & 5.9 \\ 45.0 & 8.0 & 8.7 \\ 40.0 & 11.6 & 11.9 \\ 35.0 & 17.1 & 15.6 \\ 30.0 & 23.0 & 20.1 \\ 25.0 & 30.9 & 25.6 \\ 20.0 & 43.0 & 33.0 \\ 15.0 & 62.1 & 45.4 \\ 10.0 & 95.8 & 67.5 \\ 5.0 & 179.9 & 114.5\end{array}$

$$
\begin{array}{r}
0.1 \\
0.1 \\
0.2 \\
0.2 \\
0.2 \\
0.3 \\
0.6 \\
0.8 \\
1.4 \\
1.8 \\
2.9 \\
3.8 \\
4.7 \\
5.3 \\
6.4 \\
8.4 \\
11.1 \\
20.0 \\
38.4
\end{array}
$$

0.1
0.2
0.2
0.3
0.4
0.7
1.2
1.5
1.9
2.4
3.0
4.7
6.5
8.3
12.9
18.6
24.9
32.9
46.2

0.3
0.7
0.9
1.2
1.5
2.6
4.1
5.8
7.8
9.8
12.6
15.8
19.7
25.4
32.6
42.6
58.8
77.7
107.4

0.5
1.4
1.9
3.6
5.0
7.3
10.2
13.4
16.7
20.4
24.8
30.0
36.1
43.6
53.7
65.8
82.0
109.6
161.5

0.3
0.6
1.1
1.9
2.5
4.1
5.4
8.5
12.3
16.1
20.2
24.5
30.5
38.8
49.1
62.0
86.4
129.2
218.7

$\begin{array}{rr}0.1 & 0.0 \\ 0.1 & 0.0 \\ 0.2 & 0.0 \\ 0.3 & 0.0 \\ 0.4 & 0.0 \\ 0.5 & 0.1 \\ 0.7 & 0.1 \\ 0.9 & 0.1 \\ 1.3 & 0.2 \\ 2.4 & 0.2 \\ 3.4 & 0.2 \\ 5.2 & 0.3 \\ 8.5 & 0.3 \\ 12.8 & 0.6 \\ 17.6 & 1.3 \\ 27.0 & 2.3 \\ 40.6 & 6.7 \\ 73.6 & 13.2 \\ 145.8 & 35.0\end{array}$

0.0
0.0
0.0
0.0
0.0
0.1
0.1
0.2
0.4
0.5
0.8
1.1
2.2
4.5
6.8
10.9
18.8
33.3
90.1

0.0
0.0
0.1
0.3
0.4
0.6
0.9
1.9
3.2
5.7
9.1
13.4
18.8
26.2
38.3
55.4
80.4
128.1
186.8



FOR APR TO MAR

1 WATER YEAR RANGE 19651966

19691970 19701971

19711972 19721973 19731974 19741975 19751976

19761977 19771978 19781979 19791980 19801981

19811982

19831984

19841985

19851986

19881989 19891990 19901991

\begin{tabular}{|c|c|c|c|c|}
\hline $\begin{array}{c}1 \\
18.22 \\
18.42\end{array}$ & $\begin{array}{l}20 \\
22\end{array}$ & $\begin{array}{l}38.2 \\
18.4\end{array}$ & $\begin{array}{l}20 \\
22\end{array}$ & $\begin{array}{r}7 \\
18.2 \\
18.4\end{array}$ \\
\hline $\begin{array}{ll}18.3 & 2 \\
18.1 & 1\end{array}$ & $\begin{array}{l}21 \\
13\end{array}$ & $\begin{array}{l}18.3 \\
18.1\end{array}$ & $\begin{array}{l}21 \\
11\end{array}$ & $\begin{array}{l}18.3 \\
18.1\end{array}$ \\
\hline $\begin{array}{ll}18.1 & 1 \\
18.2 & 1 \\
18.1 & 1 \\
18.0 \\
18.0\end{array}$ & $\begin{array}{r}11 \\
18 \\
14 \\
9 \\
7\end{array}$ & $\begin{array}{l}18.1 \\
18.2 \\
18.1 \\
18.0 \\
18.0\end{array}$ & $\begin{array}{r}12 \\
19 \\
14 \\
9 \\
7\end{array}$ & $\begin{array}{l}18.1 \\
18.2 \\
18.1 \\
18.1 \\
18.0\end{array}$ \\
\hline $\begin{array}{ll}18.2 & 1 \\
17.9 & \\
18.0 & \\
18.1 & 1 \\
18.1 & 1\end{array}$ & $\begin{array}{r}17 \\
2 \\
4 \\
12 \\
15\end{array}$ & $\begin{array}{ll}18.2 & 1 \\
17.9 & \\
18.0 \\
18.1 \\
18.1 & 1\end{array}$ & $\begin{array}{r}17 \\
2 \\
4 \\
15 \\
13\end{array}$ & $\begin{array}{l}18.2 \\
17.9 \\
18.0 \\
18.2 \\
18.1\end{array}$ \\
\hline $\begin{array}{l}18.0 \\
18.0 \\
18.1 \quad 1 \\
18.0 \\
17.9\end{array}$ & $\begin{array}{r}5 \\
6 \\
16 \\
8 \\
1\end{array}$ & $\begin{array}{l}18.0 \\
18.0 \\
18.1 \\
18.0 \\
17.9\end{array}$ & $\begin{array}{r}5 \\
6 \\
16 \\
8 \\
1\end{array}$ & $\begin{array}{l}18.0 \\
18.0 \\
18.2 \\
18.0 \\
17.9\end{array}$ \\
\hline $\begin{array}{l}18.0 \\
8.21 \\
18.1\end{array}$ & $\begin{array}{r}3 \\
19 \\
10\end{array}$ & $\begin{array}{l}18.0 \\
18.2 \\
18.1\end{array}$ & $\begin{array}{r}3 \\
18 \\
10\end{array}$ & $\begin{array}{l}18.0 \\
18.2 \\
18.1\end{array}$ \\
\hline
\end{tabular}

$\begin{array}{rr} & 30 \\ 18.3 & 16 \\ 18.5 & 20 \\ 18.6 & 21 \\ 18.1 & 8 \\ 18.2 & 11 \\ 18.2 & 15 \\ 18.2 & 10 \\ 18.1 & 7 \\ 18.1 & 5 \\ 18.3 & 18 \\ 18.0 & 3 \\ 18.0 & 2 \\ 18.6 & 22 \\ 18.4 & 19 \\ 18.0 & 4 \\ 18.2 & 12 \\ 18.3 & 17 \\ 18.2 & 13 \\ 18.0 & 1 \\ 18.1 & 6 \\ 18.2 & 14 \\ 18.1 & 9\end{array}$

\begin{tabular}{rr}
\multicolumn{3}{c}{90} \\
19.1 & 19 \\
19.2 & 22 \\
19.1 & 20 \\
18.2 & 5 \\
& \\
18.2 & 6 \\
18.5 & 15 \\
18.2 & 8 \\
18.2 & 7 \\
18.2 & 3 \\
18.9 & 18 \\
18.1 & 1 \\
18.1 & 2 \\
18.9 & 17 \\
18.7 & 16 \\
& \\
18.2 & 4 \\
19.1 & 21 \\
18.5 & 12 \\
18.4 & 11 \\
18.5 & 13 \\
18.6 & 14 \\
18.3 & 10 \\
18.2 & 9
\end{tabular}

120

19.420

19.116

18.33

18.59

18.611

18.48

18.37

18.22

19.318

18.11

18.35

19.319

18.712

18.34

$19.5 \quad 21$

18.510

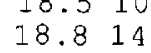

19.217

18.36

18.813
20.183 20.320

$19.5 \quad 15$ 18.88

19.112 $19.0 \quad 10$ 18.63

$\begin{array}{ll}18.6 & 4 \\ 18.8 & 7\end{array}$

19.818 18.52

19.113

$19.6 \quad 17$ 18.99

$18.6 \quad 5$ 20.422 19.616 18.76 20.019

19.514 18.51 19.111

\section{HIGHEST MEAN ELEVATION, IN FEET, AND RANKING FOR THE FOLLOWING NUMBER OF CONSECUTIVE DAYS} FOR PERIOD OCT TO SEP WATER YEAR
RANGE 19641964

19651965

$\begin{array}{ll}1966 & 1966 \\ 1969 & 1969\end{array}$

$\begin{array}{ll}1969 & 1969 \\ 1970 & 1970\end{array}$

$1971 \quad 1971$

19721972

19731973

19741974

19751975

$1976 \quad 1976$

19771977

19791979

19801980

19811981

19821982

19831983

19841984

19851985

19891989

19901990

19921992

\begin{tabular}{|c|c|c|c|c|c|}
\hline $\begin{array}{c}1 \\
27.0 \\
25.4\end{array}$ & $\begin{array}{l}1 \\
3\end{array}$ & $\begin{array}{c}3 \\
2.6 .7 \\
24.9\end{array}$ & $\frac{1}{4}$ & $\begin{array}{c}7 \\
25.4 \\
24.3\end{array}$ & $\begin{array}{l}1 \\
4\end{array}$ \\
\hline $\begin{array}{l}24.1 \\
24.2 \\
25.3\end{array}$ & $\begin{array}{r}10 \\
9 \\
4\end{array}$ & $\begin{array}{l}23.7 \\
23.9 \\
24.9\end{array}$ & $\begin{array}{r}12 \\
8 \\
3\end{array}$ & $\begin{array}{l}23.2 \\
23.3 \\
24.1\end{array}$ & $\begin{array}{r}11 \\
10 \\
6\end{array}$ \\
\hline $\begin{array}{l}24.7 \\
23.7 \\
23.3 \\
23.6 \\
24.4\end{array}$ & $\begin{array}{r}12 \\
15 \\
20 \\
16 \\
8\end{array}$ & $\begin{array}{l}23.7 \\
23.6 \\
22.9 \\
23.3 \\
23.7\end{array}$ & $\begin{array}{l}10 \\
13 \\
20 \\
16 \\
11\end{array}$ & $\begin{array}{l}23.0 \\
23.4 \\
22.8 \\
22.9 \\
22.7\end{array}$ & $\begin{array}{r}13 \\
8 \\
18 \\
15 \\
19\end{array}$ \\
\hline $\begin{array}{l}23.9 \\
23.5 \\
25.4 \\
24.8 \\
24.0\end{array}$ & $\begin{array}{r}14 \\
18 \\
2 \\
7 \\
13\end{array}$ & $\begin{array}{l}23.6 \\
23.3 \\
25.1 \\
24.7 \\
23.6\end{array}$ & $\begin{array}{r}14 \\
18 \\
2 \\
5 \\
15\end{array}$ & $\begin{array}{l}22.8 \\
22.9 \\
24.4 \\
24.4 \\
23.2\end{array}$ & $\begin{array}{r}17 \\
16 \\
2 \\
3 \\
12\end{array}$ \\
\hline $\begin{array}{l}22.9 \\
24.9 \\
24.1 \\
23.4 \\
25.0\end{array}$ & $\begin{array}{r}22 \\
6 \\
11 \\
19 \\
5\end{array}$ & $\begin{array}{l}22.3 \\
24.6 \\
23.8 \\
23.2 \\
24.6\end{array}$ & $\begin{array}{r}23 \\
7 \\
9 \\
19 \\
6\end{array}$ & $\begin{array}{l}21.3 \\
24.2 \\
23.4 \\
22.9 \\
24.0\end{array}$ & $\begin{array}{r}23 \\
5 \\
9 \\
14 \\
7\end{array}$ \\
\hline $\begin{array}{l}2.8 \\
3.5\end{array}$ & $\begin{array}{l}23 \\
17\end{array}$ & $\begin{array}{l}22.7 \\
23.3\end{array}$ & $\begin{array}{l}22 \\
17\end{array}$ & $\begin{array}{l}22.1 \\
22.5\end{array}$ & $\begin{array}{l}22 \\
20\end{array}$ \\
\hline
\end{tabular}

$\begin{array}{rr}15 & \\ 24.3 & 7 \\ 23.6 & 6 \\ 23.0 & 9 \\ 22.5 & 16 \\ 23.7 & 4 \\ 22.5 & 12 \\ 22.7 & 10 \\ 22.5 & 14 \\ 22.5 & 13 \\ 21.7 & 20 \\ 22.6 & 11 \\ 22.2 & 17 \\ 24.0 & 2 \\ 23.6 & 5 \\ 22.0 & 19 \\ 20.6 & 23 \\ 23.9 & 3 \\ 23.1 & 8 \\ 22.5 & 15 \\ 23.4 & 7 \\ 21.0 & 22 \\ 21.5 & 21 \\ 22.0 & 18\end{array}$

22.018

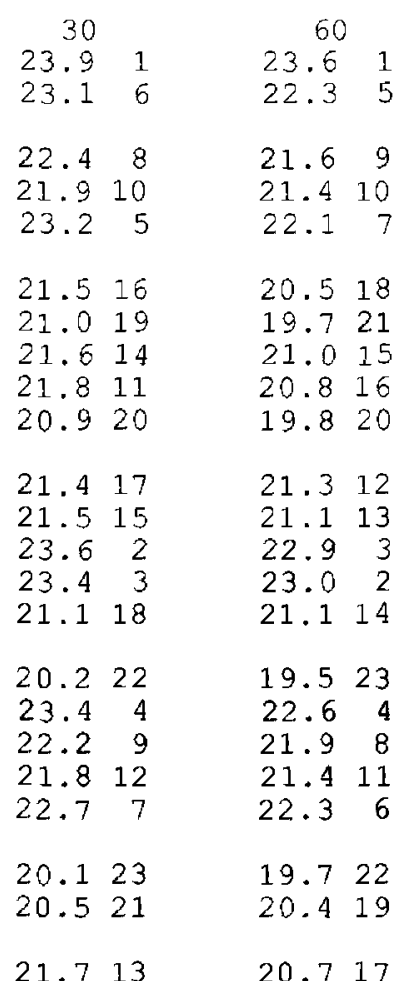

$22.5^{90}$

$\begin{array}{ll}22.5 & 2 \\ 21.7 & 6\end{array}$

21.011

$\begin{array}{rr}20.9 & 14 \\ 22.1 & 4\end{array}$

19.819

19.721

21.010

20.416

19.322

$20.9 \quad 12$

20.913

22.43

22.05

20.815

19.123

$22.7 \quad 1$

21.29

21.58

19.720

20.018

20.217
203

$\begin{array}{ll}20.9 & 4 \\ 20.5 & 8\end{array}$

20.211

$\begin{array}{ll}20.7 & 7 \\ 21.2 & 2\end{array}$

19.219

19.417

20.49

19.418
18.823

20.112

20.015

20.76

$\begin{array}{ll}20.9 & 5 \\ 20.1 & 13\end{array}$

18.922

21.21

20.410

$\begin{array}{rr}21.0 & 3 \\ 20.0 & 14\end{array}$

19.516

19.121

19.220 

CONSECUTIVE DAYS FOR PERIOD APR TO MAR

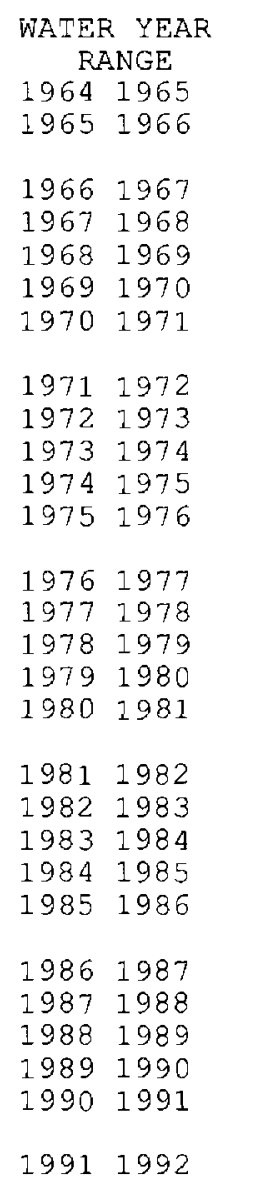

$\begin{array}{rr}3 & \\ .0000 & 1 \\ .10 & 15 \\ & \\ .10 & 16 \\ .0000 & 2 \\ .010 & 7 \\ .14 & 21 \\ .10 & 17 \\ .037 & 10 \\ .040 & 11 \\ .040 & 12 \\ .070 & 14 \\ .050 & 13 \\ & \\ .18 & 23 \\ .0000 & 3 \\ .20 & 25 \\ .31 & 27 \\ .11 & 18 \\ .0000 & 4 \\ .13 & 20 \\ .48 & 28 \\ .12 & 19 \\ .0000 & 5 \\ .027 & 9 \\ .26 & 26 \\ .16 & 22 \\ .0000 & 6 \\ .010 & 8\end{array}$

.1924

\begin{tabular}{rrrrr}
7 & & \multicolumn{2}{c}{14} & \\
.0000 & 1 & .0000 & 1 \\
.10 & 15 & .11 & 15 \\
& & & & \\
.14 & 18 & & .20 & 17 \\
.006 & 5 & .017 & 5 \\
.013 & 7 & & .016 & 4 \\
.15 & 19 & .25 & 23 \\
.12 & 16 & & .12 & 16 \\
& & & \\
.041 & 9 & .052 & 10 \\
.051 & 11 & & .054 & 11 \\
.046 & 10 & & .049 & 9 \\
.080 & 14 & & .087 & 13 \\
.059 & 12 & & .072 & 12 \\
& & & & \\
.19 & 23 & & .21 & 20 \\
.0000 & 2 & .011 & 3 \\
.21 & 25 & .22 & 21 \\
.51 & 27 & & .77 & 28 \\
.13 & 17 & & .57 & 27 \\
& & & & \\
.0000 & 3 & .0010 & 2 \\
.16 & 20 & .21 & 18 \\
.51 & 28 & & .52 & 25 \\
.16 & 21 & .30 & 24 \\
.004 & 4 & .034 & 8 \\
.073 & 13 & .094 & 14 \\
.48 & 26 & .55 & 26 \\
.19 & 22 & .25 & 22 \\
.011 & 6 & .032 & 7 \\
.016 & 8 & .024 & 6
\end{tabular}

.1924

.2119

\begin{tabular}{|c|c|c|}
\hline $\begin{array}{l}30 \\
.003 \\
.13 \quad 1\end{array}$ & $\begin{array}{r}1 \\
14\end{array}$ & $\begin{array}{r}60 \\
1.0820 \\
.52 \quad 16\end{array}$ \\
\hline $\begin{array}{l}.802 \\
.048 \\
.024 \\
1.272 \\
.171\end{array}$ & $\begin{array}{r}23 \\
6 \\
2 \\
27 \\
16\end{array}$ & $\begin{array}{rr}2.02 & 24 \\
.11 & 4 \\
.11 & 5 \\
4.18 & 28 \\
.22 & 11\end{array}$ \\
\hline $\begin{array}{l}.060 \\
.141 \\
.121 \\
.11 \\
.088\end{array}$ & $\begin{array}{r}8 \\
15 \\
13 \\
11 \\
9\end{array}$ & $\begin{array}{rr}.067 & 2 \\
.43 & 15 \\
.16 & 9 \\
.17 & 10 \\
.11 & 7\end{array}$ \\
\hline $\begin{array}{r}.362 \\
.034 \\
.221 \\
1.242 \\
1.042\end{array}$ & $\begin{array}{r}20 \\
4 \\
17 \\
26 \\
25\end{array}$ & $\begin{array}{rr}2.89 & 25 \\
.048 & 1 \\
.26 & 12 \\
1.38 & 21 \\
1.78 & 23\end{array}$ \\
\hline $\begin{array}{l}.026 \\
.672 \\
.87 \\
.53 \\
.11 \\
11\end{array}$ & $\begin{array}{l}3 \\
22 \\
24 \\
21 \\
10\end{array}$ & $\begin{array}{rr}.14 & 8 \\
3.61 & 26 \\
1.07 & 19 \\
.97 & 18 \\
1.50 & 22\end{array}$ \\
\hline $\begin{array}{r}.121 \\
1.622 \\
.321 \\
.055 \\
.035\end{array}$ & $\begin{array}{r}12 \\
28 \\
19 \\
7 \\
5\end{array}$ & $\begin{array}{rr}.28 & 13 \\
3.88 & 27 \\
.83 & 17 \\
.082 & 3 \\
.11 & 6\end{array}$ \\
\hline
\end{tabular}

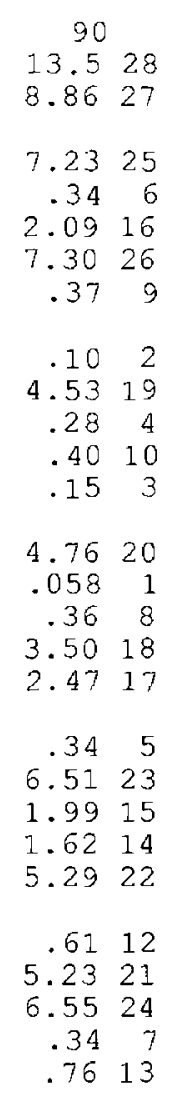

.2418

$.28 \quad 14$

.4311

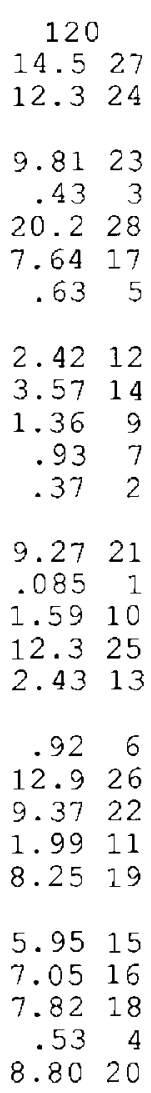

1.338
183
30.823 47.727

28.622 $\begin{array}{rr}1.02 & 1 \\ 46.2 & 26\end{array}$ 15.717 10.612

12.514 $18.3 \quad 18$ 3.024 $\begin{array}{rr}2.55 & 3 \\ 11.5 & 13\end{array}$ $22.0 \quad 20$ $3.75 \quad 5$

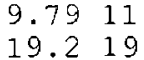
6.128 $3.81 \quad 6$ $42.8 \quad 25$ 27.921 $\begin{array}{rr}5.23 & 7 \\ 51.2 & 28\end{array}$ $38.4 \quad 24$ $6.99 \quad 10$ 14.116 $\begin{array}{rrr}2.13 & 2 \\ 13.6 & 15\end{array}$ 6.339

\section{HIGHEST MEAN DISCHARGE, IN CUBIC FEET PER SECOND, AND RANKING FOR THE FOLLOWING NUMBER OF}

\section{CONSECUTIVE DAYS FOR PERIOD OCT TO SEP}

WATER YEAR

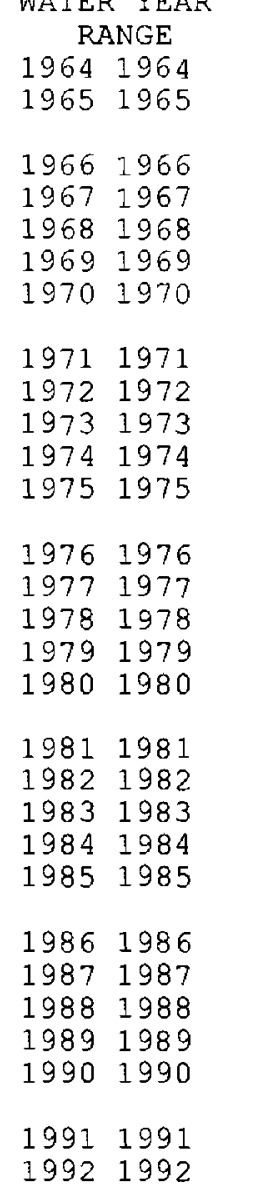

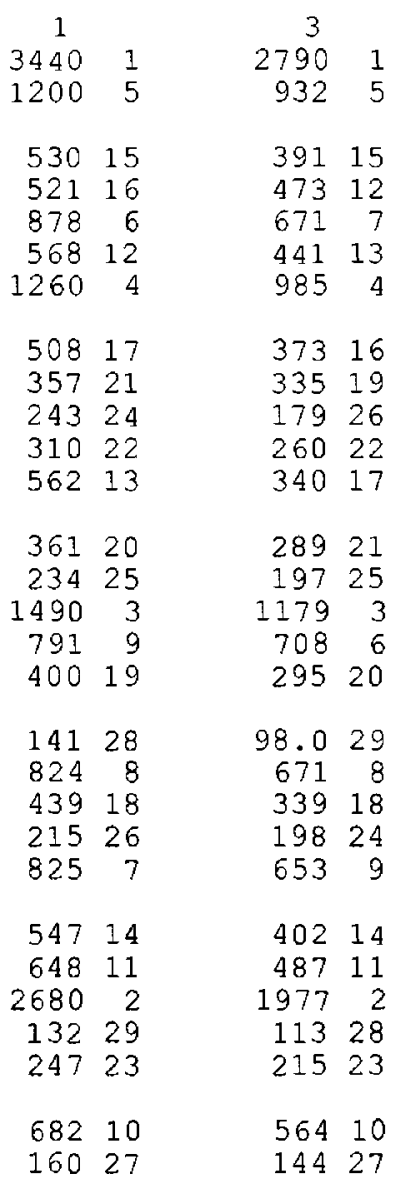

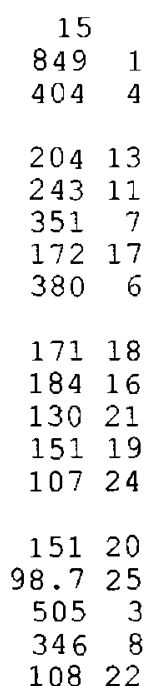

10822

60.429

5048

24018

$\begin{array}{ll}153 & 24 \\ 416 & 9\end{array}$

27514

35812

13032

86.428

13226

$399 \quad 10$

$110 \quad 27$

$\begin{array}{rr}51.5 & 28 \\ 77.8 & 27\end{array}$

2829

\begin{tabular}{|c|c|c|}
\hline $\begin{array}{c}30 \\
556 \\
286\end{array}$ & $\begin{array}{l}1 \\
4\end{array}$ & $\begin{array}{l}60 \\
517^{6} \\
204\end{array}$ \\
\hline $\begin{array}{ll}142 & 1 \\
140 & 1 \\
241 \\
123 & 1 \\
281 & \end{array}$ & $\begin{array}{r}13 \\
14 \\
8 \\
15 \\
5\end{array}$ & $\begin{array}{rr}94.9 & 13 \\
81.2 & 15 \\
133 & 10 \\
79.6 & 16 \\
157 & 8\end{array}$ \\
\hline $\begin{array}{rl}100 & 1 \\
96.4 & 1 \\
85.1 & 2 \\
103 & 1 \\
65.5 & 2\end{array}$ & $\begin{array}{l}18 \\
19 \\
21 \\
17 \\
26\end{array}$ & $\begin{array}{ll}58.1 & 21 \\
48.5 & 24 \\
54.3 & 22 \\
58.8 & 20 \\
33.9 & 27\end{array}$ \\
\hline $\begin{array}{r}86.02 \\
66.0 \\
351 \\
274 \\
70.32\end{array}$ & $\begin{array}{r}20 \\
25 \\
3 \\
7 \\
23\end{array}$ & $\begin{array}{rr}72.1 & 17 \\
50.8 & 23 \\
251 & 2 \\
212 & \\
63.2 & 19\end{array}$ \\
\hline $\begin{array}{r}25.1 \\
275 \\
120 \\
80.3 \\
195\end{array}$ & $\begin{array}{r}29 \\
6 \\
16 \\
22 \\
10\end{array}$ & $\begin{array}{rr}14.7 & 29 \\
178 & \\
93.6 & 14 \\
64.9 & 18 \\
139 & 9\end{array}$ \\
\hline $\begin{array}{r}143 \\
170 \\
390 \\
28.3 \\
44.3\end{array}$ & $\begin{array}{r}12 \\
11 \\
2 \\
28 \\
27\end{array}$ & $\begin{array}{rr}111 & 12 \\
119 & 11 \\
218 & 3 \\
16.9 & 28 \\
36.5 & 26\end{array}$ \\
\hline $\begin{array}{r}228 \\
59.9\end{array}$ & 24 & $\begin{array}{r}170 \\
43.7\end{array}$ \\
\hline
\end{tabular}

$\begin{array}{rr}120 & \\ 271 & 1 \\ 126 & 6 \\ 66.7 & 12 \\ 44.4 & 20 \\ 86.1 & 9 \\ 49.1 & 17 \\ 135 & 4 \\ 29.1 & 23 \\ 27.6 & 24 \\ 48.7 & 18 \\ 33.1 & 22 \\ 17.1 & 27\end{array}$

17.127

53.516

$\begin{array}{rr}35.1 & 21 \\ 146 & 3\end{array}$

1237
$458 \quad 19$

$\begin{array}{rr}43.2 & 22 \\ 184 & 3\end{array}$

1517

59.517

16.028
10.129

$\begin{array}{rr}186 & 2 \\ 73.7 & 14\end{array}$

$\begin{array}{ll}73.7 & 14 \\ 61.8 & 16\end{array}$

98.910

84.912

96.611

$\begin{array}{rrr}96.6 & 11 \\ 163 & 4\end{array}$

$7.58 \quad 29$

$170 \quad 2$

$\begin{array}{rr}58.0 & 15 \\ 58.2 & 14\end{array}$

$\begin{array}{ll}58.2 & 14 \\ 76.8 & 11\end{array}$

65.513

81.910

1265

$\begin{array}{ll}14.1 & 28 \\ 20.6 & 26\end{array}$

$\begin{array}{rr}132 & 8 \\ 33.3 & 25\end{array}$
$107 \quad 8$
$25.5 \quad 25$

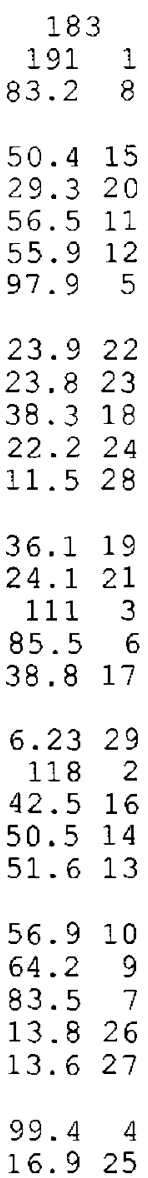


THIS PAGE INTENTIONALLY BLANK 
LOCATION.--Lat $30^{\circ} 32^{\prime} 40^{\prime \prime}$, long $82^{\circ} 44^{\prime} 02^{\prime \prime}$, in $\mathrm{SE}^{\prime} / 4$ sec. 29 , T.2 N., R. 16 E., Hamilton County, Hydrologic Unit 03110201 , at bridge on county road, 1.4 mi Upstream from mouth, 3.0 mi north of Belmont, and 12 mi east of Bakers MiII.

DRAINAGE AREA.--50 $\mathrm{mi}^{2}$, approximately.

PERIOD OF RECORD.--August 1970 to April 1976, (gage heights and discharge measurements only); May 1976 to September 1982 (discontinued). REVISED RECORDS.--WDR FL-75-1: Drainage area.

GAGE.--Water-stage recorder. Datum of gage is $86.64 \mathrm{ft}$ National Geodetic Vertical Datum of 1929. Prior to May 19, 1976, nonrecording gage at same site and datum.

REMARKS.--Records poor. Flow affected by backwater from Suwannee River at times.
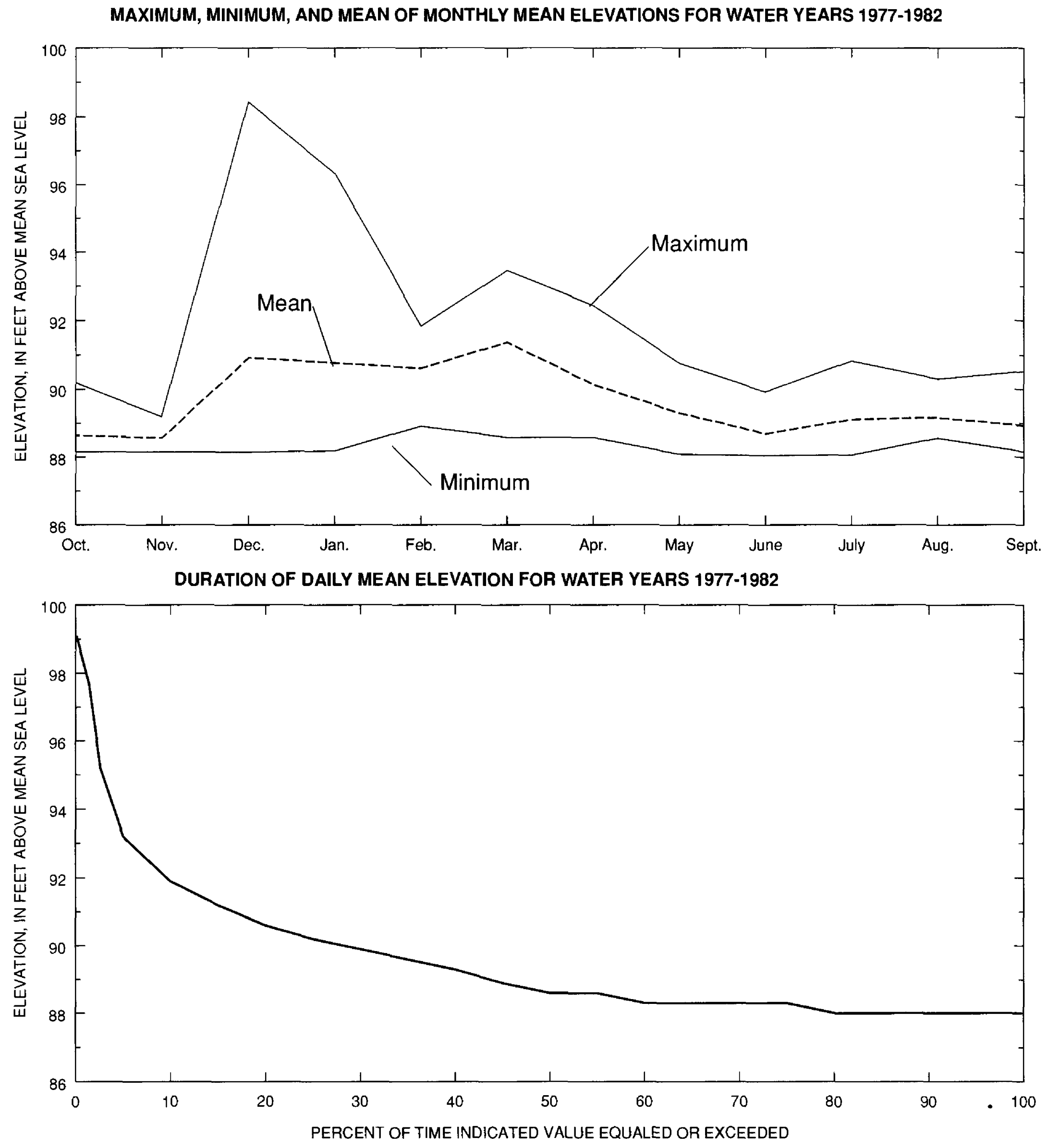
SUMMARY STATISTICS, IN CUBIC FEET PER SECOND UNLESS OTHERWISE INDICATED, FOR WATER YEARS $1977-1982$

ANNUAL MEAN

HIGHEST ANNUAL MEAN

LOWEST ANNUAL MEAN

HIGHEST DAILY MEAN

LOWEST DAILY MEAN

ANNUAL SEVEN-DAY MINIMUM

INSTANTANEOUS PEAK FLOW

INSTANTANEOUS PEAK ELEVATION (FT)

INSTANTANEOUS LOW ELOW

ANNUAL RUNOFF (INCHES)

ANNUAL RUNOFF (CFSM)
33.8

42.1

20.5

499

Mar 111980

May 71977

$500^{.00} \quad \begin{array}{llr}\text { May } & 7 & 1977 \\ & \text { Mar } 11 & 1980\end{array}$

$98.89 \quad$ Sep 111964

$.00 \quad$ May 71977

9.19

0.68

MAXIMUM, MINIMUM, AND MEAN OF MONTHLY MEAN DISCHARGES FOR WATER YEARS 1977-1982
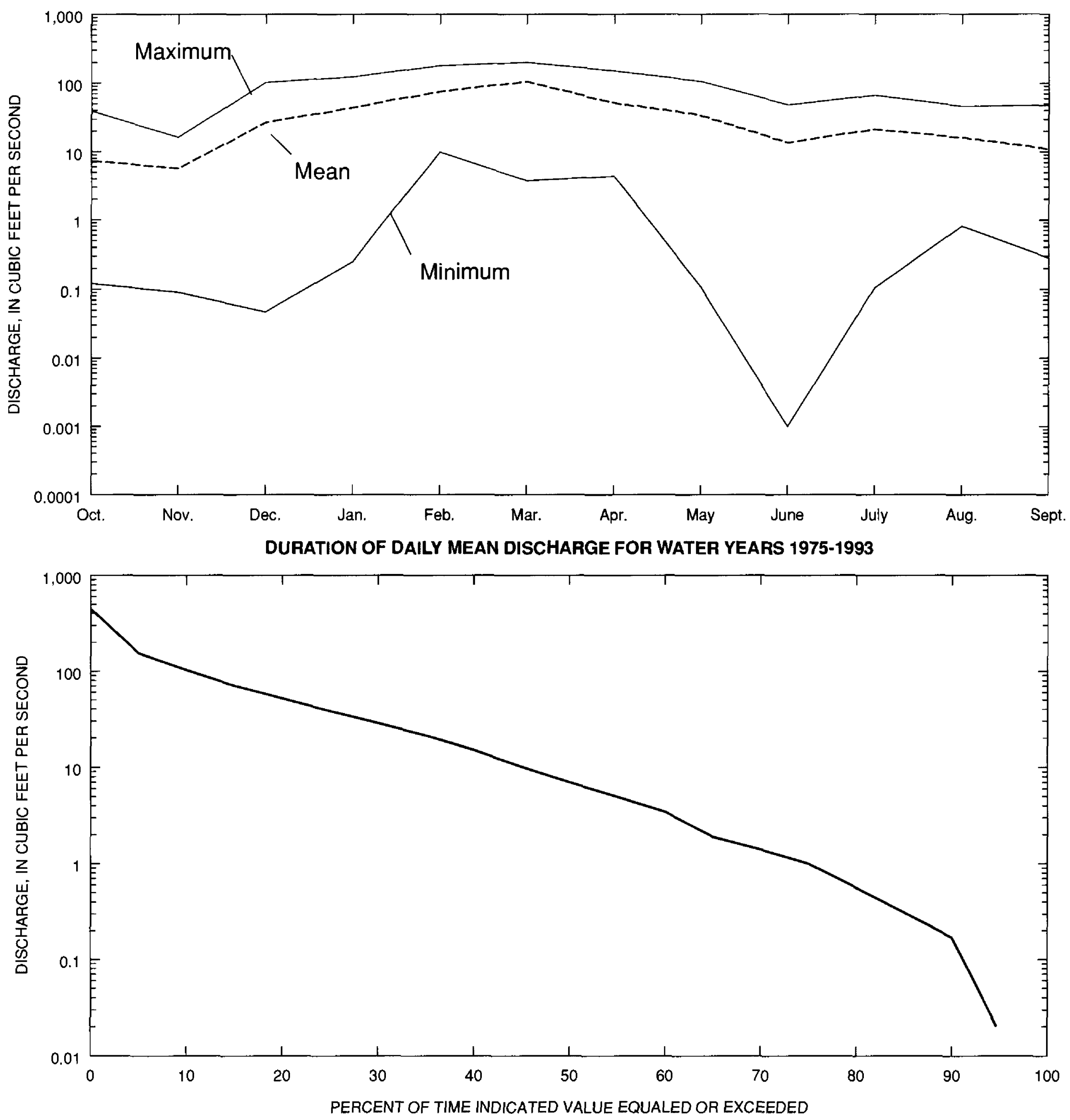
SUMMARY OF MONTHLY MEAN ELEVATION AND DISCHARGE STATISTICS FOR WATER YEARS 1977-1982

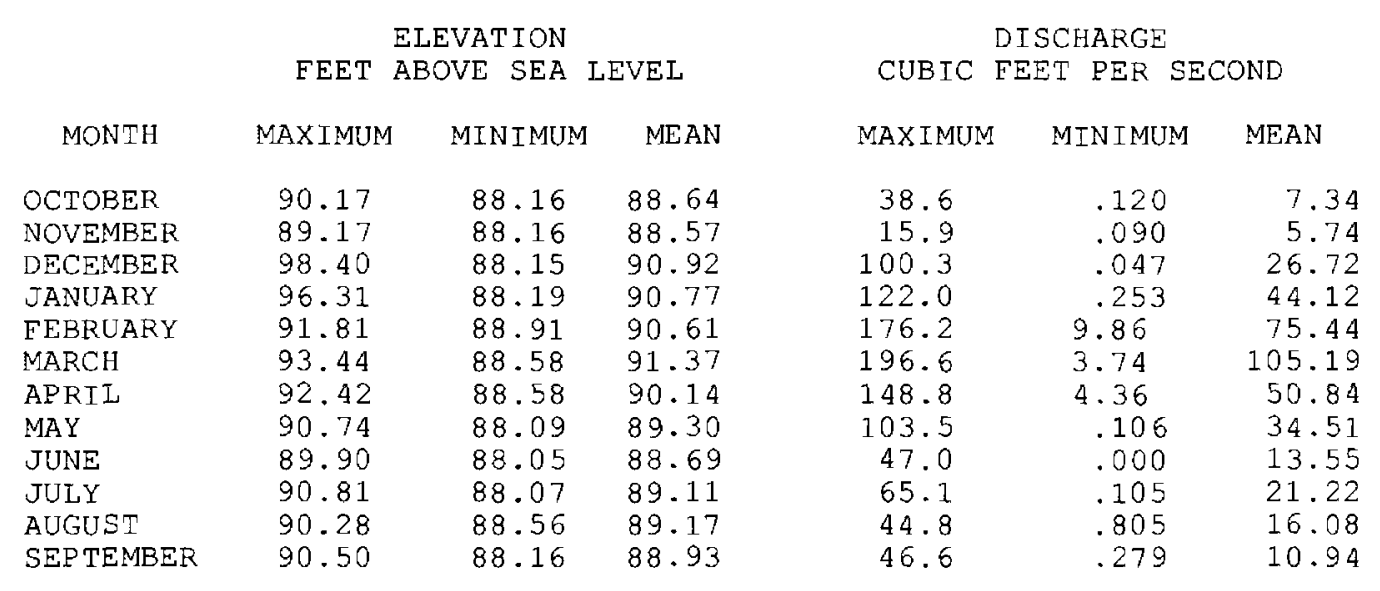

DURATION OF DAILY MEAN VALUES FOR WATER YEARS 1970-1976

PERCENT

EQUALED OR

EXCEEDED ANNUAL OCT DEC JAN FEB MAR APR MA ELEVATION IN FEET ABOVE MEAN SEA LEVEL

$\begin{array}{rll}95.0 & 88.0 & 88.1 \\ 90.0 & 88.0 & 88.1 \\ 85.0 & 88.0 & 88.1 \\ 80.0 & 88.0 & 88.1 \\ 75.0 & 88.3 & 88.1 \\ 70.0 & 88.3 & 88.1 \\ 55.0 & 88.3 & 88.1 \\ 60.0 & 88.3 & 88.1 \\ 55.0 & 88.6 & 88.3 \\ 50.0 & 88.6 & 88.3 \\ 45.0 & 88.9 & 88.3 \\ 40.0 & 89.3 & 88.3 \\ 35.0 & 89.6 & 88.4 \\ 30.0 & 89.9 & 88.4 \\ 25.0 & 90.2 & 88.5 \\ 20.0 & 90.6 & 88.7 \\ 15.0 & 91.2 & 89.1 \\ 10.0 & 91.9 & 89.6 \\ 5.0 & 93.2 & 90.6\end{array}$

$\begin{array}{lll}88.1 & 88.1 & 88.1 \\ 88.1 & 88.1 & 88.1 \\ 88.1 & 88.1 & 88.1 \\ 88.1 & 88.1 & 88.4 \\ 88.1 & 88.1 & 88.7 \\ 88.1 & 88.4 & 88.7 \\ 88.3 & 88.8 & 88.7 \\ 88.3 & 88.8 & 88.7 \\ 88.3 & 88.8 & 89.3 \\ 88.3 & 89.1 & 89.5 \\ 88.3 & 89.1 & 90.1 \\ 88.4 & 89.1 & 90.1 \\ 88.4 & 89.4 & 90.4 \\ 88.5 & 89.7 & 90.6 \\ 88.5 & 90.4 & 90.9 \\ 88.7 & 90.7 & 92.1 \\ 88.8 & 98.0 & 92.9 \\ 88.9 & 98.4 & 96.2 \\ 90.3 & 98.7 & 97.4\end{array}$

$\begin{array}{ll}88.4 & 88.2 \\ 88.5 & 88.4 \\ 88.8 & 88.9 \\ 89.6 & 89.3 \\ 89.8 & 89.6 \\ 90.0 & 90.0 \\ 90.2 & 90.3 \\ 90.2 & 90.5 \\ 90.4 & 90.9 \\ 90.6 & 91.2 \\ 90.8 & 91.4 \\ 91.3 & 91.9 \\ 91.5 & 92.1 \\ 91.5 & 92.3 \\ 91.7 & 92.6 \\ 91.9 & 93.1 \\ 92.1 & 93.8 \\ 92.5 & 94.2 \\ 92.7 & 94.7\end{array}$

$\begin{array}{ll}88.2 & 37.2 \\ 88.3 & 74.4 \\ 88.3 & 88.1 \\ 88.5 & 88.2 \\ 88.7 & 88.2 \\ 89.0 & 88.4 \\ 89.2 & 88.4 \\ 89.4 & 88.5 \\ 89.5 & 88.7 \\ 89.7 & 89.0 \\ 89.9 & 89.1 \\ 90.0 & 89.3 \\ 90.2 & 89.5 \\ 90.6 & 89.6 \\ 90.9 & 89.9 \\ 91.4 & 90.1 \\ 92.1 & 90.4 \\ 92.8 & 91.0 \\ 93.5 & 91.7\end{array}$

88.0
88.0

88.1

$88 \cdot 1$

88.1

88.2

88.3

88.3

88.4

88.5

88.7

88.9

89.1

89.3

89.7

90.1

DISCHARGE IN CUBIC FEET PER SECOND

$\begin{array}{rrrrr}95.0 & 0.0 & 0.0 & 0.0 & 0.0 \\ 90.0 & 0.1 & 0.0 & 0.0 & 0.0 \\ 85.0 & 0.3 & 0.1 & 0.0 & 0.0 \\ 80.0 & 0.5 & 0.1 & 0.2 & 1.4 \\ 75.0 & 1.0 & 0.2 & 0.4 & 1.8 \\ 70.0 & 1.4 & 0.2 & 0.5 & 2.4 \\ 65.0 & 1.9 & 0.3 & 1.2 & 7.1 \\ 60.0 & 3.4 & 0.7 & 1.2 & 8.0 \\ 55.0 & 4.9 & 0.7 & 1.6 & 9.1 \\ 50.0 & 7.0 & 0.9 & 1.6 & 10.5 \\ 45.0 & 10.1 & 1.2 & 2.0 & 12.7 \\ 40.0 & 15.1 & 1.2 & 2.0 & 14.7 \\ 35.0 & 21.1 & 2.1 & 2.6 & 17.9 \\ 30.0 & 28.5 & 2.7 & 3.4 & 27.5 \\ 25.0 & 38.0 & 2.7 & 3.4 & 41.0 \\ 20.0 & 51.4 & 5.4 & 4.8 & 51.1 \\ 15.0 & 70.5 & 12.8 & 5.7 & 62.1 \\ 10.0 & 102.6 & 20.9 & 8.6 & 78.1 \\ 5.0 & 154.6 & 47.1 & 35.0 & 132.7\end{array}$
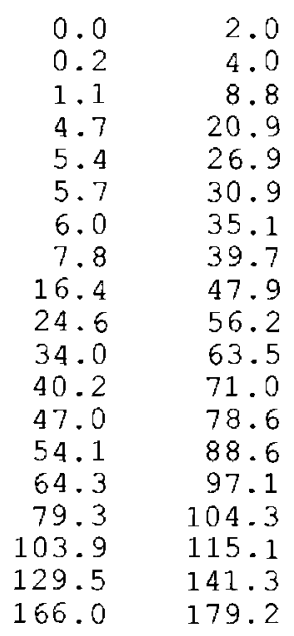

1.3
3.0
8.5
16.9
25.1
33.7
42.4
53.2
64.1
74.8
91.2
107.3
123.3
138.6
153.3
181.0
221.4
262.2
315.9

0.0
0.0
0.3
0.9
1.6
2.0
2.6
4.3
7.3
9.7
13.7
16.3
19.9
27.3
38.1
47.4
59.3
82.3
138.9

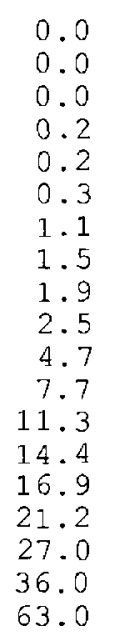

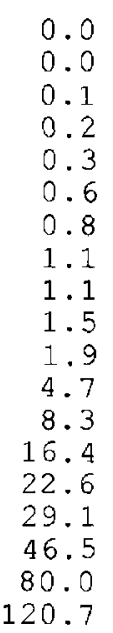

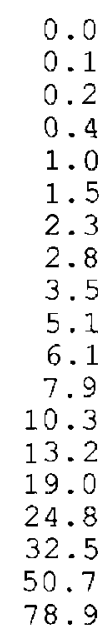

0.2
0.2
0.2
0.3
0.4
1.0
1.5
1.9
2.7
3.3
4.0
4.9
6.0
7.5
2.9
8.4
3.4
0.0
8.2 
LOWEST MEAN ELEVATION, IN FEET, AND RANKING FOR THE FOLLOWING NUMBER OF CONSECUTIVE DAYS FOR PERIOD APR TO MAR

\begin{tabular}{|c|c|c|c|c|c|c|c|c|c|c|c|c|c|c|c|c|c|}
\hline RANGE & 1 & & 3 & & 7 & & 1 & & 3 & & & & 9 & & & & \\
\hline $1977 \quad 1978$ & 88.0 & 1 & 88.0 & 1 & 88.0 & 1 & 88.0 & 1 & 88.0 & 1 & 88.1 & 1 & 88.1 & 1 & 88.1 & 1 & 88.4 \\
\hline $1978 \quad 1979$ & 88.1 & 2 & 88.1 & 2 & 88.1 & 2 & 88.1 & 2 & 88.1 & 2 & 88.1 & 2 & 88.1 & 2 & 88.1 & 2 & 88.2 \\
\hline 19791980 & 88.2 & 3 & 88.2 & 3 & 88.3 & 4 & 88.4 & 4 & 88.6 & 4 & 88.8 & 4 & 88.9 & 4 & 89.1 & 4 & 89.6 \\
\hline 9801981 & 88.2 & 4 & 88.2 & 4 & 88.2 & 3 & 88.2 & 3 & 88.3 & 3 & 88.4 & 3 & 88.4 & 3 & 88.5 & 3 & 88.6 \\
\hline
\end{tabular}

HIGHEST MEAN ELEVATION, IN FEET, AND RANKING FOR THE FOLLOWING NUMBER OF CONSECUTIVE DAYS FOR PERIOD OCT TO SEP

\begin{tabular}{|c|c|c|c|c|c|c|c|c|c|c|c|c|c|c|c|c|c|c|}
\hline & $A N G E$ & 1 & & 3 & & 7 & & 15 & & 30 & & 60 & & 9 & & 12 & & 18 \\
\hline 1977 & 1977 & 99.1 & 1 & 99.1 & 1 & 99.0 & 1 & 98.8 & 1 & 98.5 & 1 & 97.5 & 1 & 95.5 & 1 & 94.5 & 1 & 92.5 \\
\hline 1978 & 1978 & 95.0 & 3 & 95.0 & 3 & 94.8 & 3 & 94.5 & 2 & 93.5 & 2 & 92.7 & 2 & 92.3 & 2 & 91.8 & 2 & 91.0 \\
\hline 1979 & 1979 & 93.4 & 5 & 92.6 & 5 & 92.4 & 5 & 92.1 & 5 & 91.4 & 5 & 90.8 & 5 & 90.6 & 5 & 90.4 & 5 & 90.1 \\
\hline 1980 & 1980 & 96.4 & 2 & 95.9 & 2 & 95.2 & 2 & 94.1 & 3 & 93.4 & 3 & 92.6 & 3 & 92.0 & 3 & 91.6 & 3 & 90.7 \\
\hline 1981 & 1981 & 94.8 & 4 & 94.6 & 4 & 94.2 & 4 & 93.4 & 4 & 92.8 & 4 & 92.3 & 4 & 91.4 & 4 & 90.7 & 4 & 90.2 \\
\hline
\end{tabular}

LOWEST MEAN DISCHARGE, IN CUBIC FEET PER SECOND, AND RANKING FOR THE FOLLOWING NUMBER OF CONSECUTIVE DAYS FOR PERIOD APR TO MAR

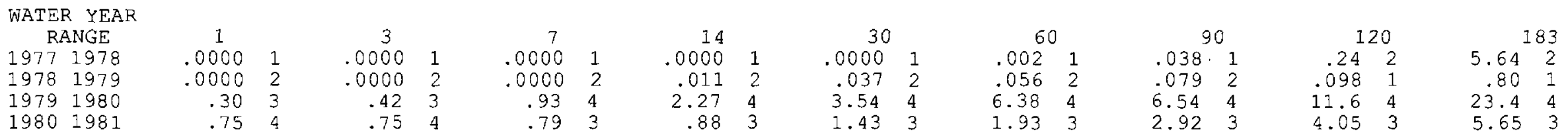

HIGHEST MEAN DISCHARGE, IN CUBIC FEET PER SECOND, AND RANKING FOR THE FOLLOWING NUMBER OF CONSECUTIVE DAYS FOR PERIOD OCT TO SEP

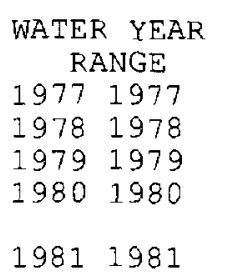

$\begin{array}{cccc}1 & & 3 & \\ 350 & 2 & 273 & 4 \\ 314 & 4 & 311 & 2 \\ 197 & 5 & 150 & 5 \\ 499 & 1 & 443 & 1 \\ 326 & 3 & 305 & 3\end{array}$

$\begin{array}{rr}7 & \\ 200 & 4 \\ 299 & 2 \\ 131 & 5 \\ 363 & 1 \\ 267 & 3\end{array}$
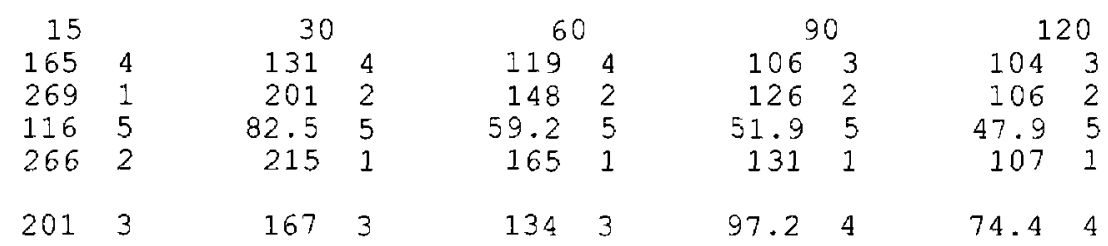

$\begin{array}{lr} & \\ 71.1 & 3 \\ 76.1 & 1 \\ 38.7 & 5 \\ 74.2 & 2 \\ 53.7 & 4\end{array}$ 
LOCATION.--Lat $30^{\circ} 30^{\prime} 26^{\prime \prime}$, long $82^{\circ} 42^{\prime} 59^{\prime \prime}$, in NE $1 / 4$ sec.9, T.I N., R.16 E., Columbia County, Hydrologic Unit 03110201 , near left bank on downstream side of bridge on State Highway 6,3.7 mi northwest of Benton, $6.4 \mathrm{mi}$ south of Florida-Georgia State Line, $13.7 \mathrm{mi}$ east of Jasper, and $196 \mathrm{mi}$, upstream from mouth.

DRAINAGE AREA.--2,090 $\mathrm{mi}^{2}$, approximately, includes part of watershed in Okefenokee Swamp which is indeterminate.

PERIOD OF RECORD.--October 1975 to 1993. Miscellaneous discharge measurements for some periods July 1934 to September 1975 . Records for December 1931 to June 1934, at site 2.0 mi upstream (at Tumer Bridge) not equivalent owing to difference in drainage areas.

GAGE.--Water-stage recorder. Datum of gage is National Geodetic Vertical Datum of 1929. Oct. 1, 1975 to Oct. 14, 1986, nonrecording gage at same site and datum. Dec. 8,1931 to June 30,1934 , nonrecording gage at site 2.0 mi upstream, datum unknown.

REMARKS.--Records good, except for estimated daily discharges, which are poor.

EXTREMES OUTSIDE PERIOD OF RECORD.--Maximum discharge measured, 27,700 ft ${ }^{3} / \mathrm{s}$ Apr. 6, 1973, gage height, $102.80 \mathrm{ft}$.
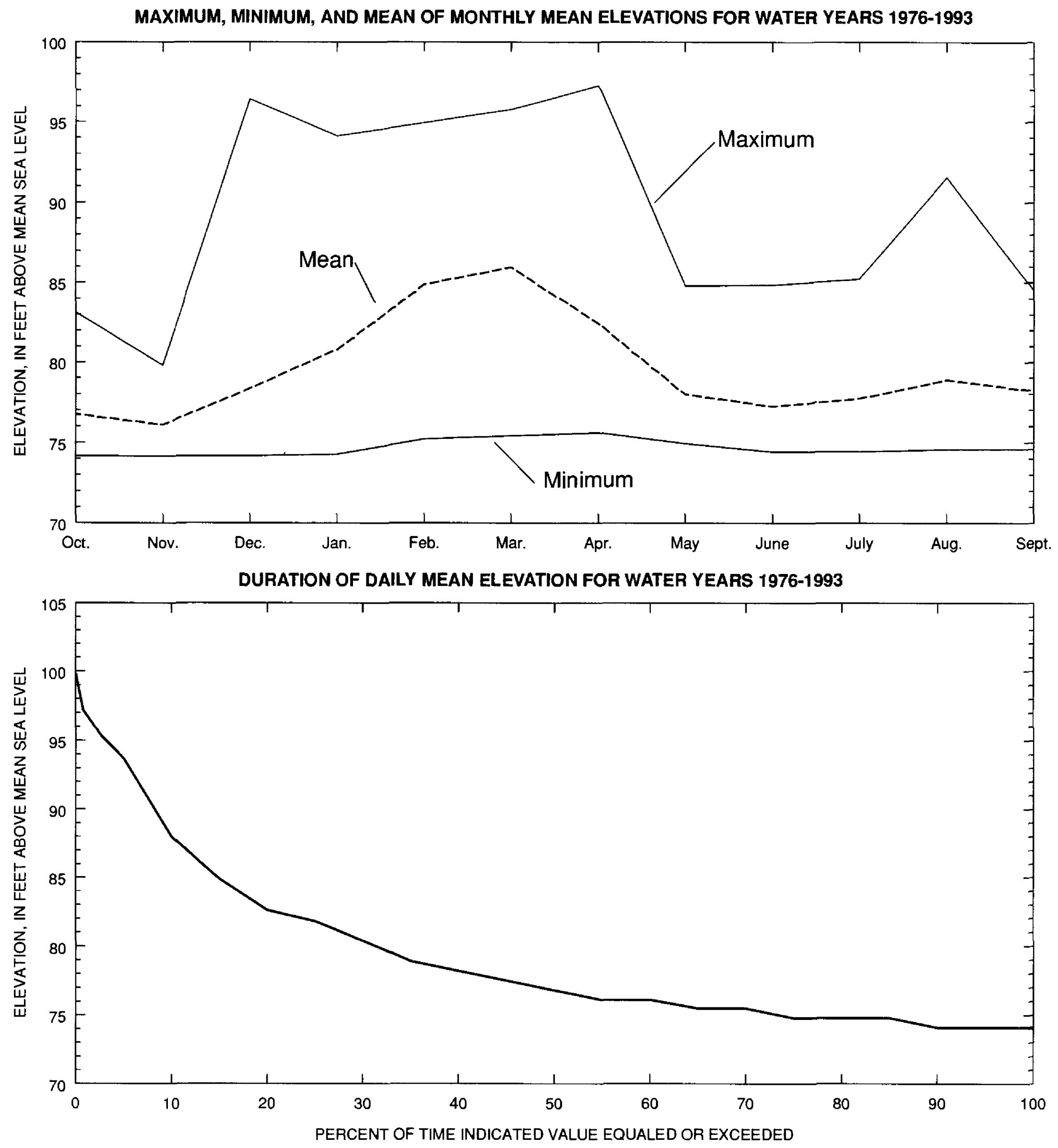
SUMMARY STATISTICS, IN CUBIC FEET PER SECOND UNLESS OTHERWISE INDICATED, FOR WATER YEARS $1976-1993$

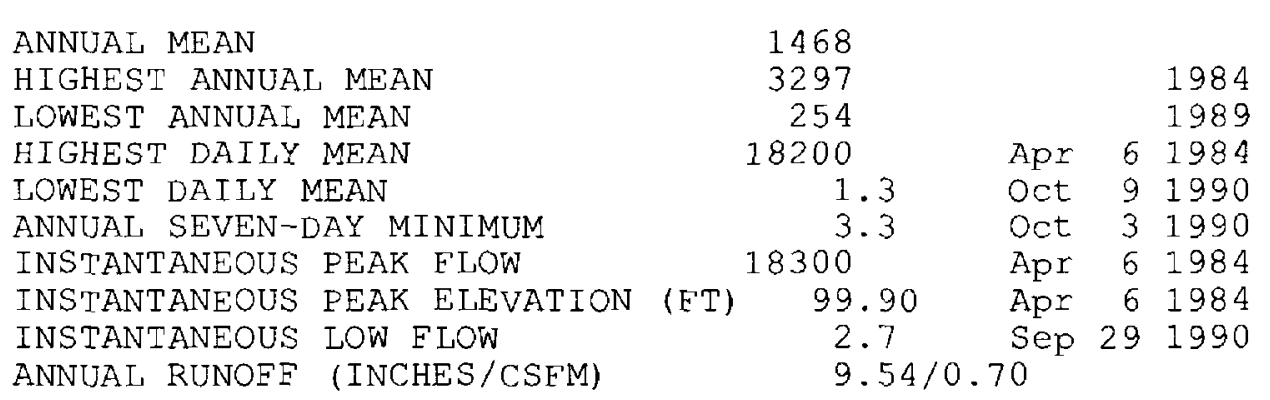

MAXIMUM, MINIMUM, AND MEAN OF MONTHLY PGPGS FOR WATER YEARS 1976-1993
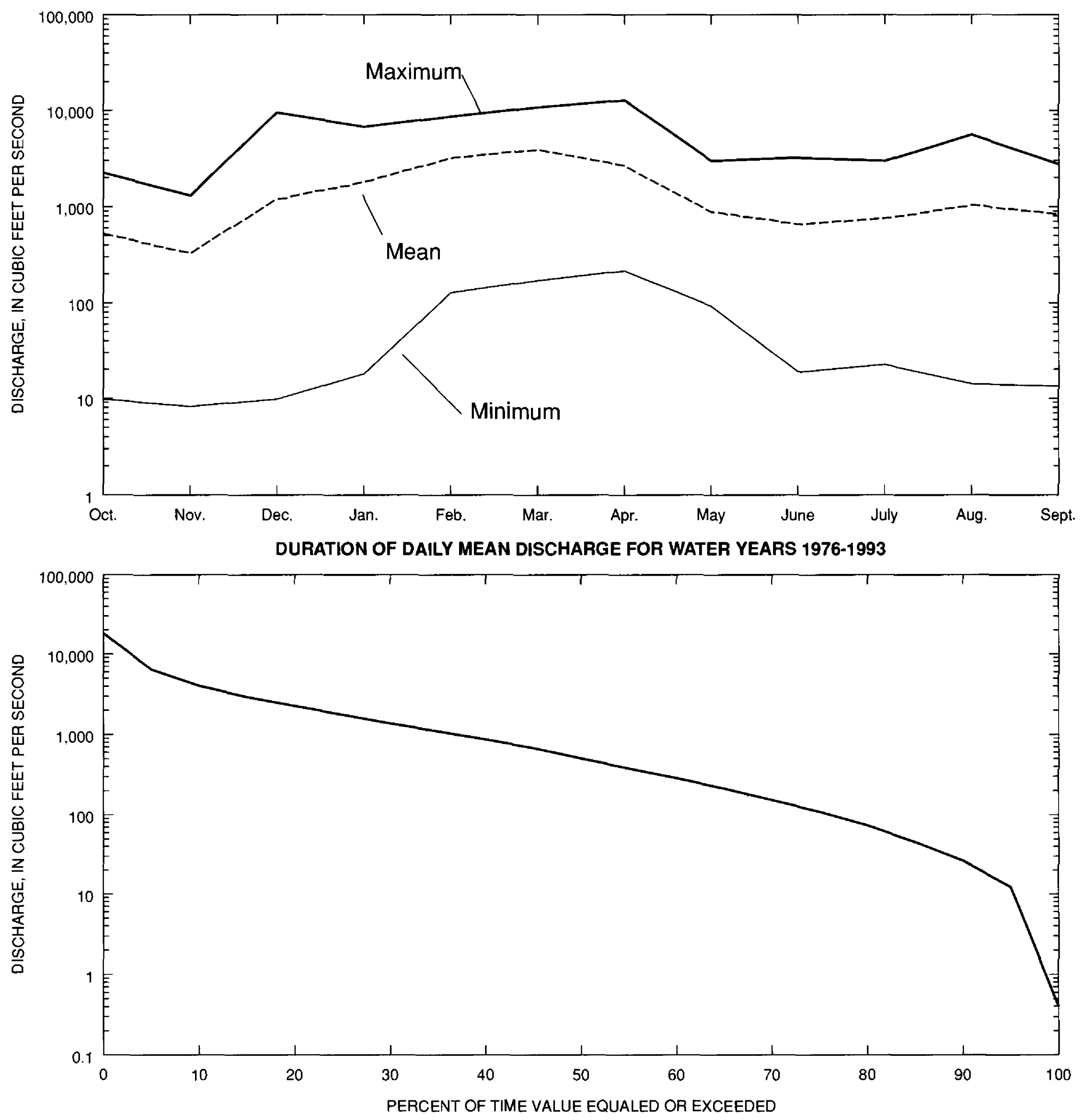
SUWANNEE RIVER BASIN

02315000 SUWANNEE RIVER NEAR BENTON, FL--Continued

SUMMARY OF MONTHLY MEAN ELEVATION AND DISCHARGE STATISTICS FOR WATER YEARS 1976-1993

PERCENT

OF TIME

EXCEEDED ANNUAL

ELEVATIONS,

FEET ABOVE SEA LEVEL
MONTH MAXIMUM MINIMUM MEAN

$\begin{array}{llll}\text { OCTOBER } & 83.07 & 74.17 & 76.73 \\ \text { NOVEMBER } & 79.75 & 74.15 & 76.08 \\ \text { DECEMBER } & 96.38 & 74.17 & 78.32 \\ \text { JANUARY } & 94.07 & 74.27 & 80.82 \\ \text { FEBRUARY } & 94.90 & 75.21 & 84.86 \\ \text { MARCH } & 95.75 & 75.41 & 85.96 \\ \text { APRIL } & 97.24 & 75.60 & 82.44 \\ \text { MAY } & 84.74 & 74.94 & 77.98 \\ \text { JUNE } & 84.80 & 74.43 & 77.23 \\ \text { JULY } & 85.18 & 74.44 & 77.71 \\ \text { AUGUST } & 91.49 & 74.58 & 78.88 \\ \text { SEPTEMBER } & 84.47 & 74.60 & 78.21\end{array}$

DISCHARGE,
CUBIC FEET PER SECOND
MAXIMUM MINIMUM MEAN

$\begin{array}{rrr}2241 & 9.77 & 524 \\ 1305 & 8.18 & 328 \\ 9472 & 9.76 & 1199 \\ 6679 & 17.9 & 1807 \\ 8574 & 128 & 3184 \\ 10750 & 171 & 3870 \\ 12760 & 215 & 2636 \\ 2979 & 92.8 & 884 \\ 3194 & 18.8 & 653 \\ 2966 & 22.5 & 756 \\ 5545 & 14.0 & 1046 \\ 2738 & 13.3 & 831\end{array}$

DURATION OF DAILY MEAN VALUES FOR WATER YEARS 1976-1993

\begin{tabular}{|c|c|c|c|c|c|c|c|c|c|c|c|c|c|}
\hline \multicolumn{14}{|c|}{ ELEVATION } \\
\hline 95.0 & 74.1 & 74.1 & 74.1 & 74.1 & 74.2 & 75.2 & 75.2 & 75.2 & 74.5 & 74.3 & 74.2 & 74.2 & 74.4 \\
\hline 90.0 & 74.1 & 74.1 & 74.1 & 74.1 & 74.7 & 75.8 & 77.0 & 75.8 & 74.5 & 74.3 & 74.2 & 74.2 & 74.4 \\
\hline 85.0 & 74.8 & 74.1 & 74.5 & 74.1 & 74.7 & 75.8 & 78.3 & 75.8 & 75.1 & 74.3 & 74.2 & 74.2 & 74.8 \\
\hline 80.0 & 74.8 & 74.4 & 74.5 & 74.1 & 75.3 & 76.4 & 79.0 & 76.5 & 75.1 & 74.8 & 74.6 & 74.7 & 74.8 \\
\hline 75.0 & 74.8 & 74.8 & 74.5 & 74.8 & 75.3 & 78.3 & 79.6 & 76.5 & 75.1 & 74.8 & 75.0 & 75.3 & 75.2 \\
\hline 70.0 & 75.5 & 74.8 & 74.5 & 74.8 & 75.3 & 79.6 & 80.3 & 77.1 & 75.1 & 74.8 & 75.0 & 75.3 & 75.7 \\
\hline 65.0 & 75.5 & 75.1 & 74.9 & 74.8 & 75.9 & 80.9 & 81.6 & 77.8 & 75.6 & 74.8 & 75.4 & 75.8 & 76.1 \\
\hline 60.0 & 76.1 & 75.1 & 74.9 & 75.4 & 77.1 & 81.6 & 82.3 & 78.5 & 75.6 & 74.8 & 75.8 & 76.4 & 76.1 \\
\hline 55.0 & 76.1 & 75.1 & 74.9 & 75.4 & 77.7 & 81.6 & 82.9 & 79.1 & 76.1 & 75.2 & 76.2 & 76.4 & 76.6 \\
\hline 50.0 & 76.8 & 75.5 & 75.2 & 76.0 & 77.7 & 82.9 & 84.3 & 79.8 & 76.1 & 75.7 & 76.2 & 77.5 & 77.0 \\
\hline 45.0 & 77.5 & 75.8 & 75.6 & 76.0 & 78.3 & 84.3 & 85.7 & 80.5 & 76.6 & 76.2 & 76.6 & 77.5 & 77.5 \\
\hline 40.0 & 78.2 & 76.2 & 75.9 & 76.6 & 79.5 & 85.7 & 86.4 & 81.2 & 77.7 & 76.7 & 77.0 & 78.0 & 77.9 \\
\hline 35.0 & 78.9 & 76.5 & 75.9 & 77.3 & 80.7 & 86.4 & 87.1 & 81.9 & 78.2 & 77.2 & 77.8 & 79.2 & 78.4 \\
\hline 30.0 & 80.4 & 76.9 & 76.3 & 77.9 & 82.6 & 87.1 & 89.3 & 83.3 & 78.2 & 77.8 & 78.6 & 79.7 & 79.3 \\
\hline 25.0 & 81.8 & 77.6 & 76.7 & 79.8 & 84.5 & 87.8 & 91.5 & 86.3 & 78.7 & 78.3 & 79.4 & 80.3 & 79.8 \\
\hline 20.0 & 82.6 & 78.7 & 77.4 & 81.8 & 87.1 & 91.5 & 93.0 & 88.5 & 79.8 & 78.8 & 80.6 & 81.5 & 80.7 \\
\hline 15.0 & 84.9 & 79.4 & 77.8 & 82.5 & 88.5 & 93.0 & 94.6 & 90.8 & 81.5 & 80.3 & 82.3 & 83.8 & 81.6 \\
\hline 10.0 & 88.0 & 80.2 & 77.8 & 83.9 & 90.5 & 94.6 & 95.3 & 94.0 & 83.2 & 81.4 & 83.6 & 85.7 & 83.1 \\
\hline 5.0 & 93.7 & 83.6 & 78.6 & 94.9 & 94.1 & 95.3 & 96.9 & 94.8 & 86.7 & 84.1 & 84.5 & 89.4 & 85.1 \\
\hline \multicolumn{14}{|c|}{ DISCHARGE IN } \\
\hline 95.0 & 19.2 & 7.6 & 8.1 & 13.9 & 27.6 & 133.4 & 205.4 & 217.7 & 49.0 & 19.3 & 16.3 & 13.7 & 20.3 \\
\hline 90.0 & 36.7 & 11.9 & 19.5 & 25.0 & 68.9 & 282.7 & 561.0 & 308.6 & 79.5 & 29.6 & 25.1 & 25.1 & 50.8 \\
\hline 85.0 & 61.0 & 17.9 & 25.9 & 35.4 & 114.0 & 397.6 & 887.2 & 388.7 & 106.7 & 46.3 & 36.1 & 34.3 & 73.8 \\
\hline 80. & 95.2 & 33.8 & 33.0 & 42.8 & 157.0 & 537.0 & 1108.1 & 445.0 & 132.8 & 61 & & 49.0 & 89.8 \\
\hline 75. & 133.3 & 54.0 & 44.0 & 53.4 & 217.4 & 1006.0 & 1283.6 & 539.0 & 156.8 & 70 & 88.0 & 76.9 & 111.7 \\
\hline 70. & 183.2 & 79.8 & 52.7 & 86.0 & 257.3 & 1327.6 & 1426.9 & 655.9 & 183.3 & 78.7 & 117.1 & 142.0 & 152.7 \\
\hline 65.0 & 249.4 & 123.2 & 66.8 & 102.3 & 340.3 & 1578.0 & 1871.0 & 807.2 & 215.8 & 90.4 & 146.9 & 191.5 & 250.9 \\
\hline 60.0 & 324.7 & 150.3 & 99.0 & 218.5 & 560.8 & 1808.5 & 2232.9 & 956.7 & 256.7 & 106.9 & 196.7 & 242.4 & 315.6 \\
\hline 55.0 & 418.1 & 178.1 & 130.6 & 259.9 & 712.7 & 1985.6 & 2432.1 & 1131.6 & 322.4 & 138.4 & 273.4 & 320.3 & 382.6 \\
\hline 50.0 & 556.9 & 213.8 & 151.1 & 303.1 & 807.5 & 2371.4 & 2737.3 & 1323.7 & 399.3 & 177.3 & 347.5 & 448.9 & 463.1 \\
\hline 45.0 & 716.1 & 270.1 & 179.9 & 380.2 & 969.9 & 2745.9 & 3216.9 & 1566.4 & 464.0 & 292.7 & 423.7 & 602.9 & 567.2 \\
\hline 40.0 & 904.9 & 341.8 & 244.8 & 494.9 & 1244.1 & 3185.8 & 3614.7 & 1814.7 & 673.8 & 408.7 & 528.1 & 768.0 & 676.6 \\
\hline 35.0 & 1131.2 & 413.6 & 313.2 & 669.9 & 1809.9 & 3514.2 & 3968.9 & 2047.8 & 811.9 & 579.2 & 660.7 & 916.7 & 775.4 \\
\hline 30.0 & 1411.9 & 549.7 & 381.0 & 904.6 & 2260.7 & 3777.3 & 4712.0 & 2388.0 & 941.2 & 705.9 & 848.7 & 1147.3 & 961.2 \\
\hline 25.0 & 1821.3 & 682.9 & 499.9 & 1324.0 & 2828.0 & 4265.0 & 5422.7 & 3308.6 & 1106.4 & 873.4 & 1088.6 & 1398.5 & 1240.0 \\
\hline 20.0 & 2285.4 & 970.6 & 622.0 & 1866.6 & 3745.8 & 5557.1 & 6180.0 & 4192.9 & 1320.9 & 1080.7 & 1403.0 & 1710.9 & 1486.9 \\
\hline 15.0 & 3005.1 & 1196.1 & 723.0 & 2158.9 & 4278.5 & 6343.7 & 7732.5 & 5222.0 & 1816.1 & 1349.6 & 1906.0 & 2357.0 & 1822.1 \\
\hline 10.0 & 4039.8 & 1475.2 & 810.1 & 2752.9 & 5113.5 & 7487.0 & 9452.2 & 7340.0 & 2312.2 & 1723.9 & 2415.4 & 3076.6 & 2243.3 \\
\hline 5.0 & 6499.6 & 2299.8 & 1029.2 & 7562.5 & 7043.8 & 9516.1 & 11266.8 & 9162.3 & 3602.1 & 2494.4 & 2899.8 & 4216.0 & 2924.2 \\
\hline
\end{tabular}

LOWEST MEAN ELEVATION, IN FEET, AND RANKING FOR THE FOLLOWING NUMBER OF CONSECUTIVE DAYS FOR PERIOD APR TO MAR

\section{WATER YEAR}

\begin{tabular}{|c|c|}
\hline & ANGE \\
\hline 1976 & 1977 \\
\hline 1977 & 1978 \\
\hline 1978 & 1979 \\
\hline 1979 & 1980 \\
\hline 1980 & 1981 \\
\hline 1981 & 1982 \\
\hline 1982 & 1983 \\
\hline 1. & 1984 \\
\hline & 1985 \\
\hline & 1986 \\
\hline 1988 & 1989 \\
\hline & 1990 \\
\hline 1990 & 1991 \\
\hline 190 & 1993 \\
\hline
\end{tabular}

$\begin{array}{rr}3 & \\ 75.4 & 13 \\ 74.2 & 2 \\ 74.1 & 1 \\ 76.4 & 14 \\ 74.4 & 6 \\ 74.2 & 3 \\ 74.5 & 7 \\ 74.6 & 9 \\ 75.0 & 11 \\ 74.7 & 10 \\ 74.3 & 5 \\ 74.6 & 8 \\ 74.2 & 4 \\ 75.3 & 12\end{array}$

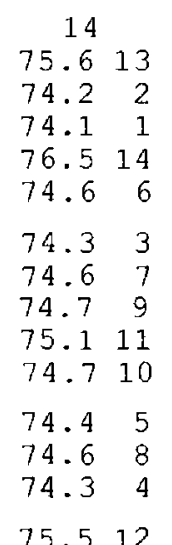

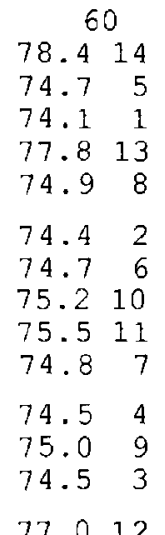

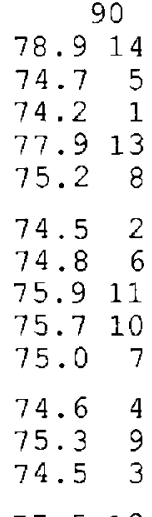

$77.5 \quad 12$

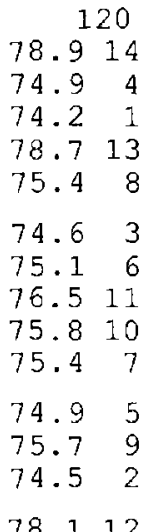

$\begin{array}{rr} & 120 \\ 78.9 & 14 \\ 74.9 & 4 \\ 74.2 & 1 \\ 78.7 & 13 \\ 75.4 & 8 \\ 74.6 & 3 \\ 75.1 & 6 \\ 76.5 & 11 \\ 75.8 & 10 \\ 75.4 & 7 \\ 74.9 & 5 \\ 75.7 & 9 \\ 74.5 & 2 \\ 78.1 & 12\end{array}$
183

80.214

74.51

$\begin{array}{rr}79.9 & 13 \\ 75.8 & 6\end{array}$

$74.6 \quad 2$

$\begin{array}{rr}76.5 & 9 \\ 77.2 & 10\end{array}$

76.08

78.311

75.75

$74.6 \quad 3$

79.412 
HIGHEST MEAN ELEVATION, IN FEET, AND RANKING FOR THE FOLLOWING NUMBER OF CONSECUTIVE DAYS FOR PERIOD OCT TO SEP

\begin{tabular}{|c|c|}
\hline WATEE & $R \quad Y E A$ \\
\hline & ANGE \\
\hline 1976 & 1976 \\
\hline 1977 & 1977 \\
\hline 1978 & 1978 \\
\hline 1979 & 1979 \\
\hline 1980 & 1980 \\
\hline 1981 & 1981 \\
\hline 1982 & 1982 \\
\hline 1983 & 1983 \\
\hline 1984 & 1984 \\
\hline 1985 & 1985 \\
\hline 1988 & 1988 \\
\hline 89 & 1989 \\
\hline 1990 & 1990 \\
\hline$y=$ & \\
\hline
\end{tabular}

92.37

$\begin{array}{ll}92.3 & 7 \\ 97.3 \quad 2\end{array}$

92.46

88.39

86.512

83.613

96.04

99.91

87.211

94.95

77.914

87.910

97.33

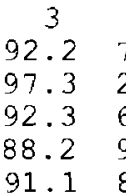

86.412

83.413

5.94

99.81

87.111

94.85

$77.8 \quad 14$

87.810

97.23

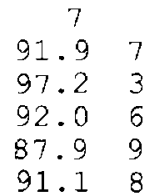

86. 312

82.813

95.84

$99.7 \quad 1$

94.75

$\begin{array}{rr}94.7 & 5 \\ 77.5 \quad 14\end{array}$

87.710

97.22

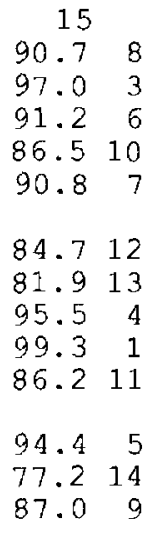

97.12

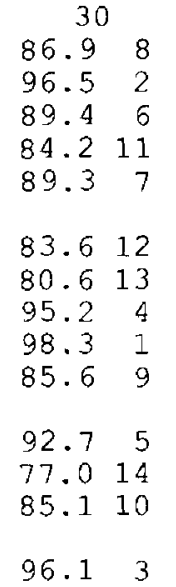

60

$\begin{array}{ll}84.8 & 8 \\ 95.4 & 2\end{array}$

88.35

82.711

88.06

81.912

79.913

94.44

$96.6 \quad 1$

87.07

$76.6 \quad 14$

82.810

95.43

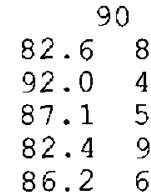

8120

90.23

85.95

82.27

$79.2 \quad 12$

80.412

$79.7 \quad 13$

93.4

94.51

79.113

91.02

$92.4 \quad 1$

83.57

81.78

76.614

81.810

76.314

80.810

92.23
183
80.7

86.64

81.27

82.86

78.312

78.611

86.83

$\begin{array}{lr}88.3 & 1 \\ 78.3 & 13\end{array}$

79.49

6.014

78.910

87.42

LOWEST MEAN DISCHARGE, IN CUBIC FEET PER SECOND, AND RANKING FOR THE FOLLOWING NUMBER OF CONSECUTIVE DAYS FOR PERIOD APR TO MAR

WATER YEAR

RANGE

$1976 \quad 1977$

19771978

19781979

19801981

19811982

19821983

19831984

19841985

$1986 \quad 1987$

19871988

19881989

19891990

19901991

19911992

19921993
1
$157 \quad 16$

5.002

7.204

35317

8.605

$\begin{array}{rr}8.60 & 5 \\ 32.0 & 10\end{array}$

38.011

96.014

58.012

$7.10 \quad 3$

14.06

$21.0 \quad 8$

14.07

88.013

15515 $\begin{array}{cc}3 \\ 160 & 16 \\ 5.97 & 2\end{array}$

$7.47 \quad 3$

37417

33.710

9.075

32.79

$\begin{array}{ll}43.0 & 11 \\ 96.7 & 14\end{array}$

64.712

7.604

14.37

22.08

1.67

88.713

15715

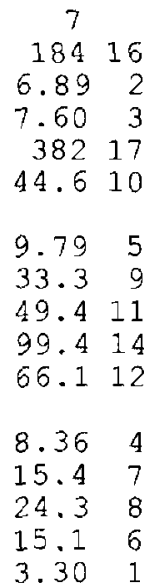

$\begin{array}{cc}14 & \\ 205 & 16\end{array}$

7.642

$408 \quad 17$

47.110

9.964

35.69

53.611

66.612

11.25

$\begin{array}{ll}17.1 & 6 \\ 27.2 & 8\end{array}$

18.17

$3.84 \quad 1$

89.913

17315

$91.8 \quad 13$

$\begin{array}{rr}196 & 15\end{array}$

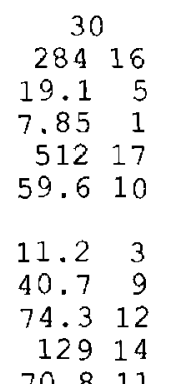

70.811

16.54

31.18

28.86
8.692

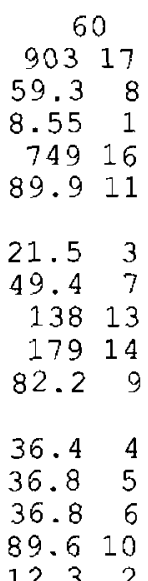

12.32

96.313

$\begin{array}{cc}960 & 15\end{array}$

$\begin{array}{ll}105 & 12 \\ 552 & 15\end{array}$
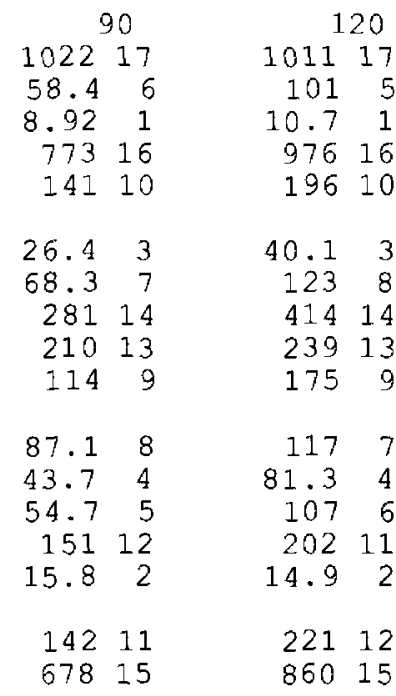

40.13

1177

81.3
107
19610

$\begin{array}{rr}123 & 8 \\ 414 & 14\end{array}$

23913

1759

20211

14.92

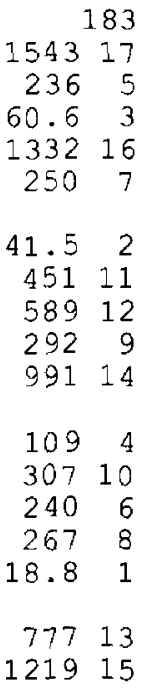

$\begin{array}{ll}221 & 12 \\ 860 & 15\end{array}$

121915

HIGHEST MEAN DISCHARGE, IN CUBIC FEET PER SECOND, AND RANKING FOR THE FOLLOWING NUMBER OF CONSECUTIVE DAYS FOR PERIOD OCT TO SEP

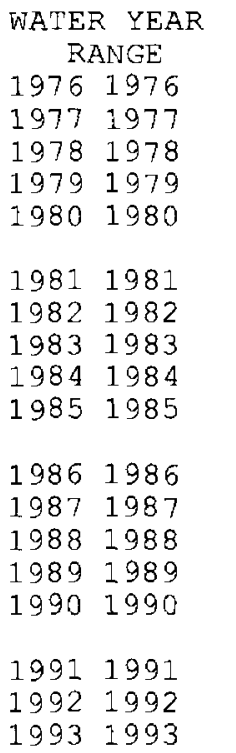

\begin{tabular}{rrrr}
1 & & \multicolumn{2}{c}{3} \\
6800 & 9 & 6757 & 9 \\
10500 & 4 & 10470 & 4 \\
6010 & 10 & 5963 & 10 \\
4190 & 12 & 4143 & 12 \\
5370 & 11 & 5317 & 11 \\
& & & \\
3510 & 15 & 3483 & 15 \\
2480 & 17 & 2387 & 17 \\
9610 & 6 & 9427 & 6 \\
18200 & 1 & 18030 & 1 \\
3690 & 14 & 3667 & 14 \\
& & & \\
12900 & 2 & 12870 & 2 \\
10300 & 5 & 10230 & 5 \\
7700 & 7 & 7657 & 7 \\
769 & 18 & 741 & 18 \\
3950 & 13 & 3940 & 13 \\
& & & \\
12000 & 3 & 11970 & 3 \\
3350 & 16 & 3323 & 16 \\
7040 & 8 & 6997 & 8
\end{tabular}
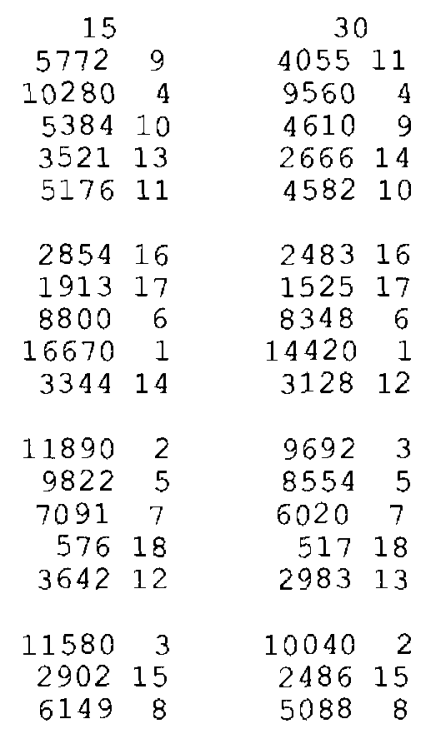

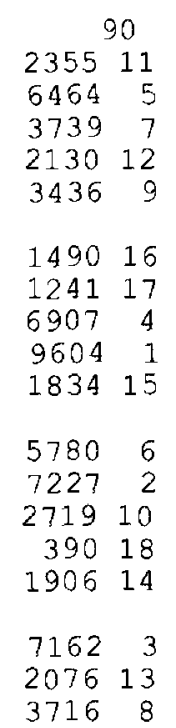

\begin{tabular}{rr}
\multicolumn{3}{c}{183} \\
1669 & 11 \\
4084 & 5 \\
2563 & 8 \\
1763 & 10 \\
2310 & 9 \\
& \\
915 & 17 \\
952 & 16 \\
4124 & 4 \\
5780 & 1 \\
991 & 15 \\
& \\
3394 & 6 \\
4323 & 3 \\
1430 & 12 \\
271 & 18 \\
1111 & 14 \\
& \\
4618 & 2 \\
1378 & 13 \\
3015 & 7
\end{tabular} 
02315200 DEEP CREEK NEAR SUWANNEE VALLEY, FL

LOCATION.--Lat $30^{\circ} 21^{\prime \prime} 55^{\prime \prime}$, long $82^{\circ} 37^{\prime} 13^{\prime \prime}$, in NW"1/4 sec.33, T.1 N., R.17 E., Columbia County, Hydrologic Unit 03110201, near left bank on upstream side of bridge on U.S. Highway 441, 4.0 mi upstream from mouth, 7.2 mi northeast of Suwannee Valley, 12.2 mi north of Lake City.

DRAINAGE AREA.--88.6 mi².

PERIOD OF RECORD.--April 1976 to September 1981, October 1990 to 1993. Miscellaneous discharge measurements for some periods prior to April 1976.

GAGE.--Water-stage recorder. Datum of gage is $82.56 \mathrm{ft}$ National Geodetic Vertical Datum of 1929 (levels by L. L. Lee and Associates).

REMARKS.--Records fair

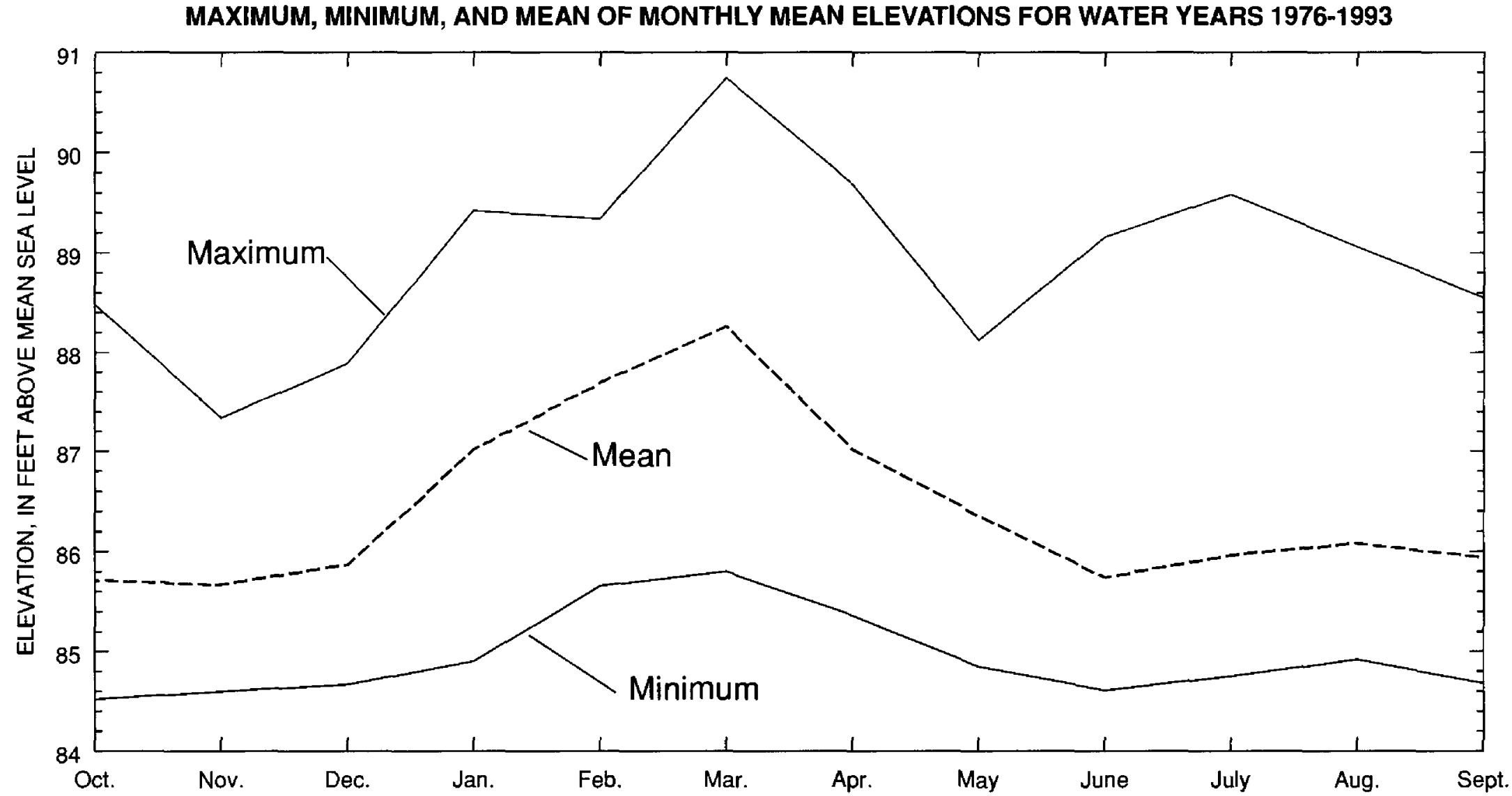

DURATION OF DAILY MEAN ELEVATION FOR WATER YEARS 1976-1993

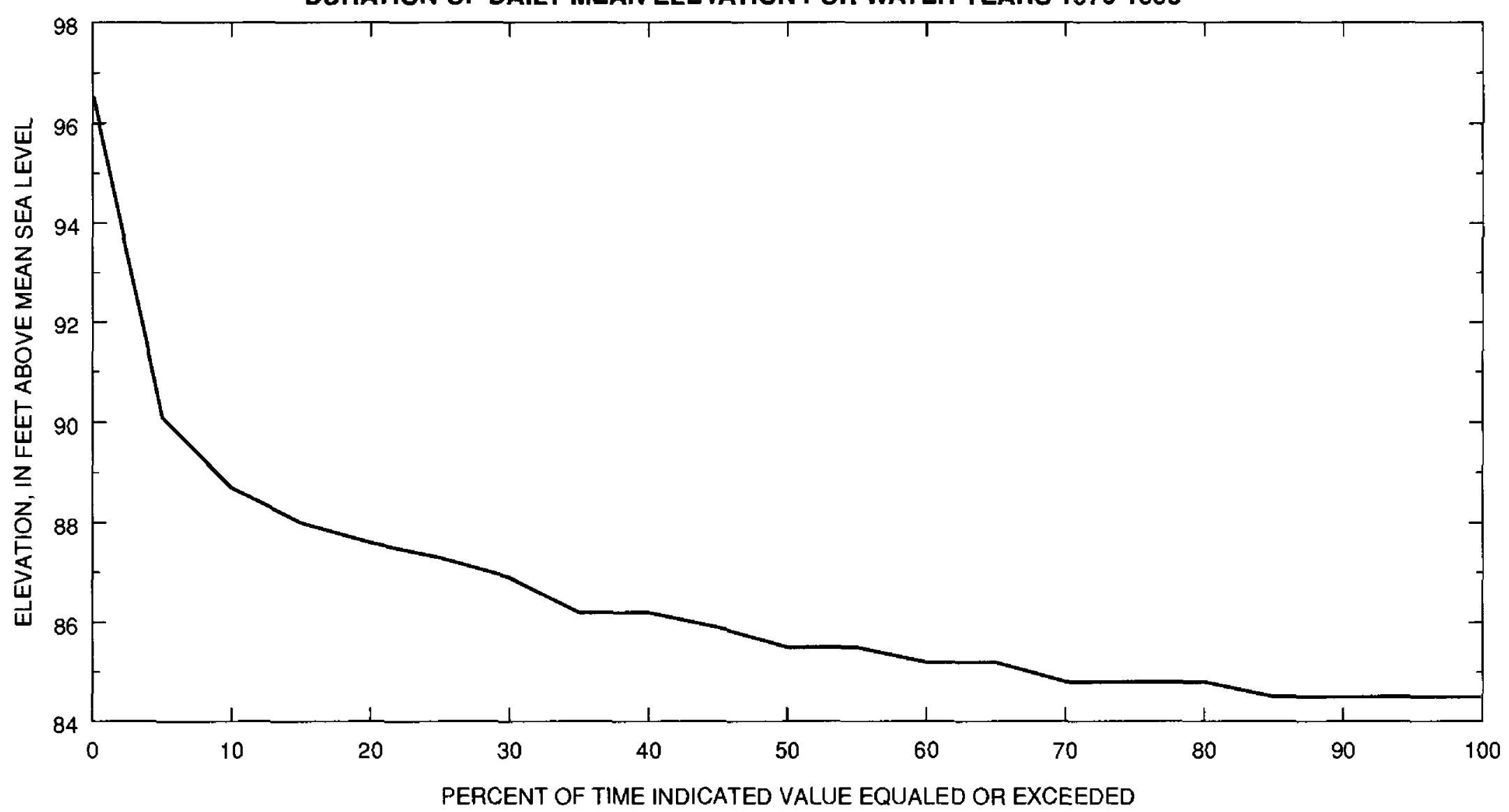


SUMMARY STATISTICS, IN CUBIC FEET PER SECOND UNLESS OTHERWISE INDICATED, FOR WATER YEARS $1976-1993$

\begin{tabular}{|c|c|c|c|}
\hline ANNUAL MEAN & 59.3 & & \\
\hline HIGHEST ANNUAL MEAN & 109 & & 1991 \\
\hline LOWEST ANNUAL MEAN & 24.1 & & 1992 \\
\hline HIGHEST DAILY MEAN & 937 & Mar 11 & 1980 \\
\hline LOWEST DAILY MEAN & .09 & Dec 22 & 1990 \\
\hline ANNUAL SEVEN-DAY MINIMUM & .09 & Dec 21 & 19 \\
\hline INSTANTANEOUS PEAK FLOW & 961 & Mar 11 & 1980 \\
\hline INSTANTANEOUS PEAK ELEVATION (FT) & 97.64 & Mar 11 & 1. \\
\hline INSTANTANEOUS LOW FLOW & .09 & Dec 22 & 1990 \\
\hline ANNUAL RUNOFF (INCHES/CSFM) & \multicolumn{3}{|c|}{$9.09 / 067$} \\
\hline
\end{tabular}

MAXIMUM, MINIMUM, AND MEAN OF MONTHLY MEAN DISCHARGES FOR WATER YEARS 1976-1993

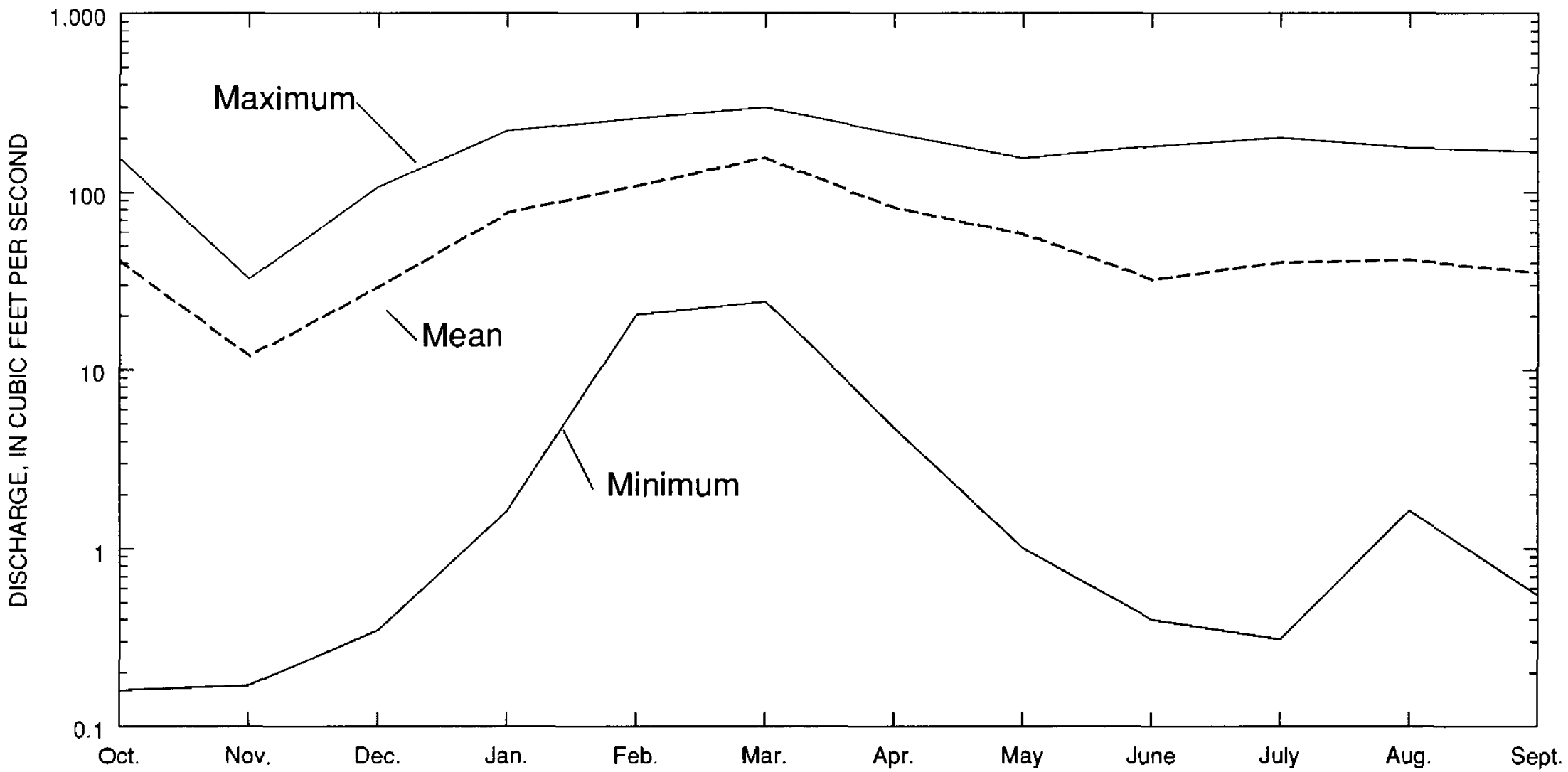

DURATION OF DAILY MEAN DISCHARGE FOR WATER YEARS 1976-1993

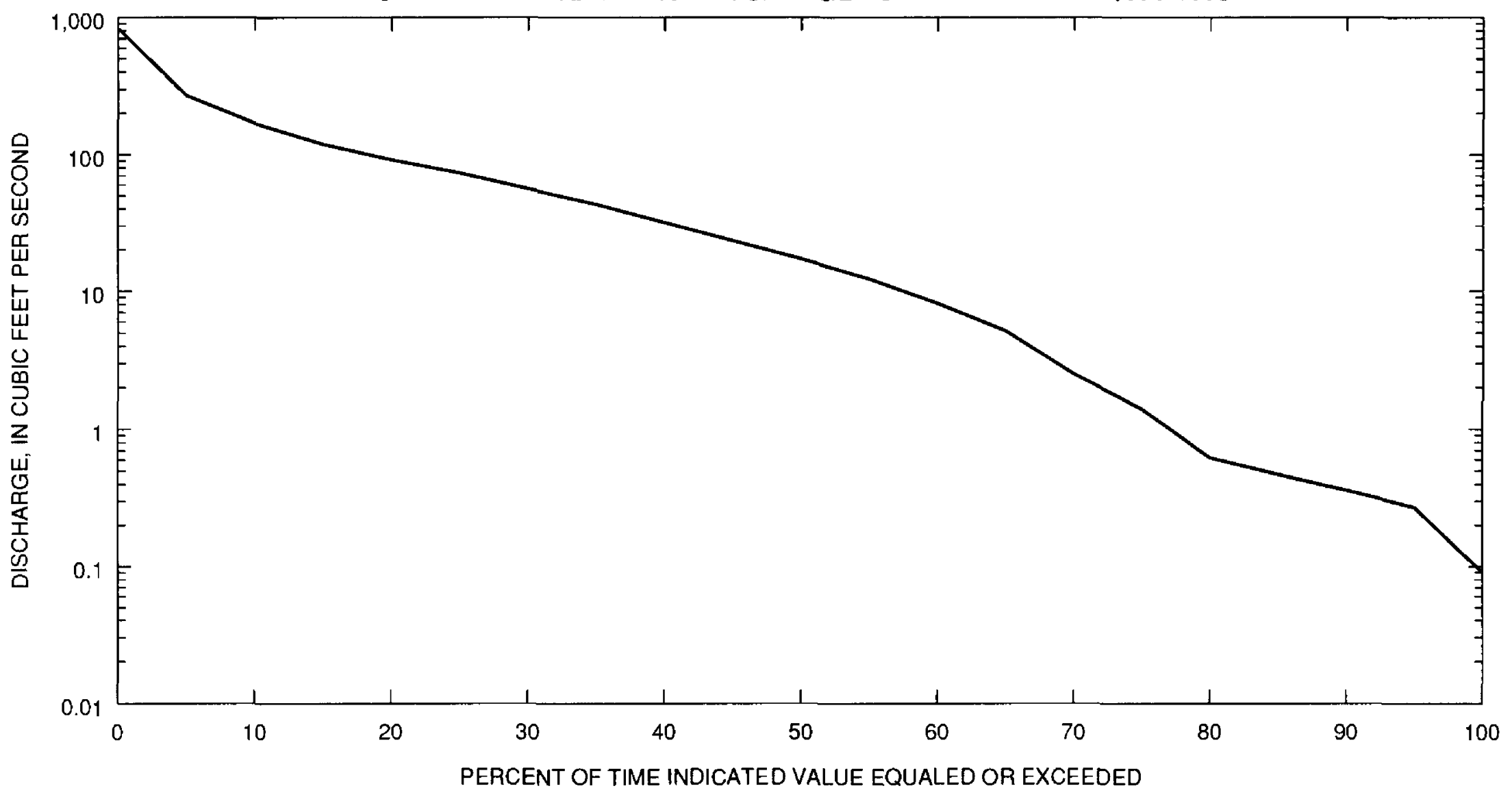


SUWANNEE RIVER BASIN

02315200 DEEP CREEK NEAR SUWANNEE VALLEY, FL--Continued

SUMMARY OF MONTHLY MEAN ELEVATION AND DISCHARGE STATISTICS FOR WATER YEARS 1976-1993

\begin{tabular}{|c|c|c|c|c|c|c|}
\hline \multirow[b]{2}{*}{ MONTH } & \multicolumn{3}{|c|}{ ELEVATIONS, } & \multicolumn{2}{|c|}{ DISCHARGE, } & $\mathrm{OND}$ \\
\hline & MAXIMUM & MINIMUM & MEAN & MAX IMUM & MINIMUM & MEAN \\
\hline OCTOBER & 88.47 & 84.52 & 85.71 & 153 & .16 & 41.2 \\
\hline NOVEMBER & 87.33 & 84.60 & 85.67 & 32.2 & .17 & 11.9 \\
\hline DECEMSER & 87.88 & 84.67 & 85.87 & 106 & .35 & 29.3 \\
\hline JANUARY & 89.41 & 84.90 & 87.02 & 218 & 1.63 & 76.7 \\
\hline FEBRUARY & 89.33 & 85.66 & 87.70 & 257 & 20.4 & 109 \\
\hline MARCH & 90.73 & 85.80 & 88.26 & 295 & 24.3 & 156 \\
\hline APRIL & 89.67 & 85.36 & 87.02 & 211 & 4.78 & 82.3 \\
\hline MAY & 88.11 & 84.85 & 86.36 & 154 & 1.01 & 58.6 \\
\hline JUNE & 89.15 & 84.61 & 85.74 & 179 & .40 & 32.4 \\
\hline JULY & 89.57 & 84.75 & 85.96 & 200 & .31 & 40.5 \\
\hline AUGUST & 89.05 & 84.92 & 86.08 & 176 & 1.64 & 41.7 \\
\hline SEPTIMBER & 88.54 & 84.68 & 85.94 & 167 & .55 & 35.3 \\
\hline
\end{tabular}

DURATION OF DAILY MEAN VALUES FOR WATER YEARS 1976-1993

PERCENT

EQUALED OR

EXCEEDED

ANNUAL OCT

NOV - DEC

ELEVATION IN EEET ABOVE MEAN SEA LEVEL

$\begin{array}{rll}95.0 & 84.5 & 84.5 \\ 90.0 & 84.5 & 84.5 \\ 85.0 & 84.5 & 84.5 \\ 80.0 & 84.8 & 84.8 \\ 75.0 & 84.8 & 84.8 \\ 70.0 & 84.8 & 84.8 \\ 65.0 & 85.2 & 84.8 \\ 60.0 & 85.2 & 84.8 \\ 55.0 & 85.5 & 85.0 \\ 50.0 & 85.5 & 85.0 \\ 45.0 & 85.9 & 85.3 \\ 40.0 & 86.2 & 85.3 \\ 35.0 & 86.2 & 85.3 \\ 30.0 & 86.9 & 85.5 \\ 25.0 & 87.3 & 85.8 \\ 20.0 & 87.6 & 86.3 \\ 15.0 & 88.0 & 87.1 \\ 10.0 & 88.7 & 88.2 \\ 5.0 & 90.7 & 90.6\end{array}$

84.6
84.6
84.8
84.8
84.8
84.9
85.0
85.2
85.2
85.3
85.3
85.4
85.6
85.8
85.9
86.0
86.2
86.3
86.5

$\begin{array}{lll}84.7 & 84.6 & 85.0 \\ 84.7 & 84.6 & 85.2 \\ 84.7 & 84.8 & 85.8 \\ 84.7 & 84.8 & 86.2 \\ 84.8 & 84.8 & 86.4 \\ 84.9 & 85.1 & 86.6 \\ 85.1 & 85.1 & 86.8 \\ 85.1 & 85.4 & 87.0 \\ 85.4 & 85.6 & 87.2 \\ 85.5 & 85.6 & 87.2 \\ 85.6 & 85.9 & 87.4 \\ 85.7 & 86.4 & 87.6 \\ 86.0 & 87.1 & 87.9 \\ 86.2 & 87.7 & 88.1 \\ 86.7 & 88.2 & 88.3 \\ 86.9 & 88.5 & 88.5 \\ 87.3 & 89.0 & 88.7 \\ 87.8 & 89.5 & 89.3 \\ 88.1 & 90.6 & 80.4\end{array}$

85.4
85.7
86.1
86.1
86.4
86.4
86.7
87.1
87.4
87.4
87.7
88.1
88.4
88.7
89.4
89.8
90.4
91.5
92.9

85.0
85.2
85.2
85.5
85.5
85.5
85.7
85.9
86.2
86.4
86.9
87.1
87.6
87.6
88.1
88.3
89.1
90.0
90.8

84.6
84.6
84.6
84.6
84.9
84.9
84.9
85.1
85.4
85.6
85.9
86.1
86.4
86.4
86.9
87.4
88.1
89.2
90.7

84.5

84.8
84.8

84.8

84.8
84.8

84.8
84.8

84.8
84.8

85.0

85.0

$85 \cdot 3$

85.3

85.6

85.6
85.8

85.8
86.9

87.5

DISCHARGE IN CUBIC FEET PER SECOND

$\begin{array}{rrr}95.0 & 0.2 & 0.1 \\ 90.0 & 0.3 & 0.1 \\ 85.0 & 0.4 & 0.2 \\ 80.0 & 0.6 & 0.3 \\ 75.0 & 1.4 & 0.4 \\ 70.0 & 2.5 & 0.6 \\ 65.0 & 5.1 & 0.6 \\ 60.0 & 8.7 & 1.8 \\ 55.0 & 12.2 & 3.0 \\ 50.0 & 17.1 & 4.3 \\ 45.0 & 23.3 & 6.7 \\ 40.0 & 31.7 & 9.6 \\ 35.0 & 43.1 & 13.5 \\ 30.0 & 56.3 & 20.5 \\ 25.0 & 73.4 & 30.0 \\ 20.0 & 91.9 & 48.5 \\ 15.0 & 119.1 & 75.0 \\ 10.0 & 169.4 & 114.0 \\ 5.0 & 268.9 & 262.6\end{array}$

$\begin{array}{rrrr}0.1 & 0.2 & 0.5 & 4.8 \\ 0.1 & 0.2 & 0.6 & 8.5 \\ 0.5 & 0.4 & 0.8 & 27.8 \\ 0.6 & 0.4 & 1.8 & 35.7 \\ 0.7 & 0.5 & 3.0 & 41.7 \\ 1.1 & 0.6 & 4.6 & 47.4 \\ 1.4 & 2.0 & 5.5 & 59.4 \\ 1.7 & 6.3 & 7.1 & 66.8 \\ 2.0 & 8.0 & 18.4 & 73.0 \\ 3.0 & 11.0 & 22.4 & 79.3 \\ 6.5 & 14.3 & 27.2 & 85.6 \\ 8.4 & 17.6 & 52.6 & 94.2 \\ 11.0 & 24.2 & 73.7 & 107.7 \\ 15.3 & 34.8 & 99.0 & 125.0 \\ 19.0 & 46.0 & 118.3 & 151.0 \\ 23.0 & 62.3 & 144.4 & 177.8 \\ 28.0 & 79.9 & 180.1 & 207.9 \\ 36.7 & 97.9 & 220.2 & 242.1 \\ 46.1 & 114.5 & 308.1 & 317.1\end{array}$

14.4
25.0
28.9
35.6
45.1
56.3
65.1
77.1
86.1
96.2
107.7
122.6
140.8
175.2
220.2
261.2
322.3
374.6
486.3

$$
\begin{array}{r}
4.5 \\
10.6 \\
14.7 \\
17.4 \\
19.9 \\
23.3 \\
29.0 \\
36.5 \\
47.9 \\
59.2 \\
73.7 \\
85.4 \\
93.6 \\
106.7 \\
128.2 \\
151.9 \\
188.7 \\
239.7
\end{array}
$$

0.2
0.3
0.3
0.4
0.5
0.8
1.7
2.6
5.4
13.0
25.6
31.4
37.6
43.8
50.0
63.3
88.3
125.4
222.4

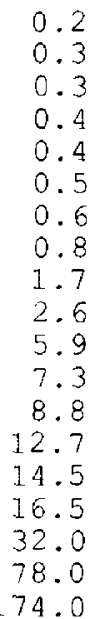

$$
\begin{array}{r}
0.2 \\
0.2 \\
0.3 \\
0.3 \\
0.3 \\
0.3 \\
0.3 \\
0.4 \\
0.5 \\
3.0 \\
5.5 \\
8.5 \\
15.1 \\
21.8 \\
33.8 \\
44.5 \\
80.0 \\
117.7
\end{array}
$$

191.7

$\begin{array}{rr}0.1 & 0.5 \\ 0.2 & 0.5 \\ 0.4 & 0.6 \\ 0.4 & 0.9 \\ 0.8 & 1.5 \\ 1.3 & 1.9 \\ 2.2 & 3.0 \\ 5.5 & 5.2 \\ 10.7 & 7.7 \\ 15.7 & 10.5 \\ 20.4 & 15.5 \\ 26.0 & 21.9 \\ 36.6 & 29.3 \\ 45.5 & 37.1 \\ 56.1 & 44.0 \\ 69.1 & 57.2 \\ 84.9 & 71.2 \\ 110.6 & 85.8 \\ 197.5 & 132.3\end{array}$


LOWEST MEAN ELEVATION, IN FEET, AND RANKING FOR THE FOLLOWING NUMBER OF CONSECUTIVE DAYS FOR PERIOD APR TO MAR

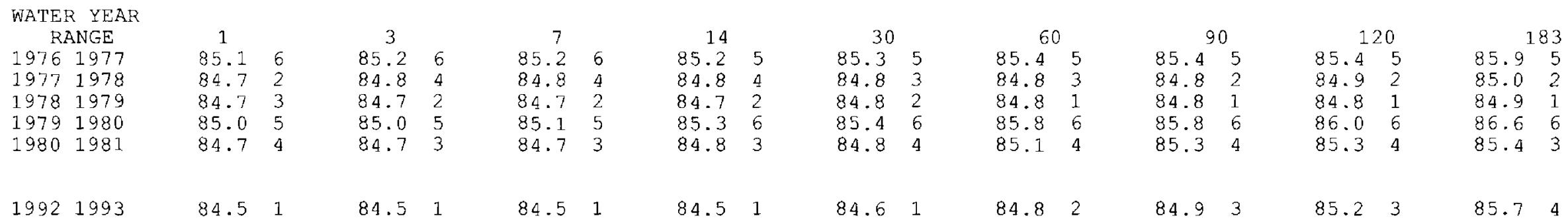

HIGHEST MEAN ELEVATION, IN FEET, AND RANKING FOR THE FOLLOWING NUMBER OF CONSECUTIVE DAYS FOR PERIOD OCT TO SEP

WATER YEAR

\begin{tabular}{|c|c|c|c|c|c|c|c|c|c|c|c|c|c|c|c|c|c|c|c|}
\hline$R$ & ANGE & 1 & & 3 & & 7 & & 15 & & 30 & & 6 & & & & & & & 183 \\
\hline 1977 & 1977 & 92.6 & 6 & 92.0 & 6 & 90.9 & 6 & 89.9 & 6 & 89.0 & 4 & 88.7 & 4 & 88.6 & 4 & 88.1 & 4 & 87.2 & 5 \\
\hline 1978 & 1978 & 93.4 & 4 & 93.0 & 4 & 91.9 & 4 & 91.1 & 3 & 90.4 & 3 & 89.9 & 3 & 89.3 & 2 & 88.8 & 2 & 88.1 & 2 \\
\hline 1979 & 1979 & 94.8 & 3 & 94.6 & 3 & 91.9 & 5 & 90.1 & 5 & 88.5 & 7 & 87.8 & 7 & 87.2 & 7 & 86.9 & 6 & 86.9 & 6 \\
\hline 1980 & 1980 & 97.5 & 1 & 96.7 & 1 & 95.6 & 1 & 93.0 & 1 & 91.7 & 1 & 90.1 & 1 & 89.1 & 3 & 88.5 & 3 & 87.7 & 3 \\
\hline 1981 & 1981 & 92.2 & 7 & 91.6 & 7 & 90.7 & 7 & 89.4 & 7 & 88.8 & 5 & 88.4 & 5 & 87.5 & 6 & 86.9 & 7 & 86.5 & 7 \\
\hline 1991 & 991 & 95.6 & 2 & 94.6 & 2 & 93.5 & 2 & 91.8 & 2 & 90.9 & 2 & 90.0 & 2 & 89.4 & 1 & 89.2 & 1 & 89.2 & \\
\hline 1993 & 1993 & 93.1 & 5 & 92.4 & 5 & 91.9 & 3 & 90.5 & 4 & 88.7 & 6 & 88.1 & 6 & 88.0 & 5 & 87.5 & 5 & 87.3 & \\
\hline
\end{tabular}

LOWEST MEAN DISCHARGE, IN CUBIC FEET PER SECOND, AND RANKING FOR THE FOLLOWING NUMBER OF CONSECUTIVE DAYS FOR PERIOD APR TO MAR

\begin{tabular}{|c|c|c|c|c|c|c|c|c|c|c|c|c|c|c|c|c|c|c|}
\hline RANGE & 1 & & 3 & & 7 & & 14 & & 30 & & 6 & & & & 1 & & & 183 \\
\hline $\begin{array}{lll}1976 & 1977\end{array}$ & .49 & 5 & .65 & 6 & 1.06 & 6 & 1.56 & 6 & 2.22 & 6 & 5.39 & 5 & 6.24 & 5 & 6.75 & 3 & 24.8 & 6 \\
\hline $1977 \quad 1978$ & .30 & 4 & .36 & 4 & .39 & 4 & .40 & 4 & .42 & 3 & .50 & 1 & .70 & 2 & .93 & 2 & 1.92 & 1 \\
\hline $1978 \quad 1979$ & .27 & 1 & .30 & 2 & .31 & 2 & .32 & 2 & .40 & 2 & .50 & 2 & .50 & 1 & .55 & 1 & 2.35 & 2 \\
\hline 19791980 & 3.10 & 7 & 3.30 & 7 & 5.20 & 7 & 9.12 & 7 & 13.2 & 7 & 24.7 & 7 & 26.2 & 7 & 32.5 & 7 & 65.1 & 7 \\
\hline 19801981 & .27 & 2 & .30 & 3 & .31 & 3 & .36 & 3 & 1.01 & 5 & 6.02 & 6 & 10.9 & 6 & 12.2 & 6 & 13.8 & 3 \\
\hline 19911992 & .52 & 6 & .52 & 5 & .52 & 5 & .53 & 5 & .95 & 4 & 1.16 & 3 & 3.03 & 4 & 9.97 & 5 & 22.5 & 4 \\
\hline 19921993 & .28 & 3 & .28 & 1 & .28 & 1 & .30 & 1 & .36 & 1 & 1.34 & 4 & 2.11 & 3 & 7.79 & 4 & 23.6 & 5 \\
\hline
\end{tabular}

HIGHEST MEAN DISCHARGE, IN CUBIC FEET PER SECOND, AND RANKING FOR THE FOLLOWING NUMBER OF CONSECUTIVE DAYS FOR PERIOD OCT TO SEP

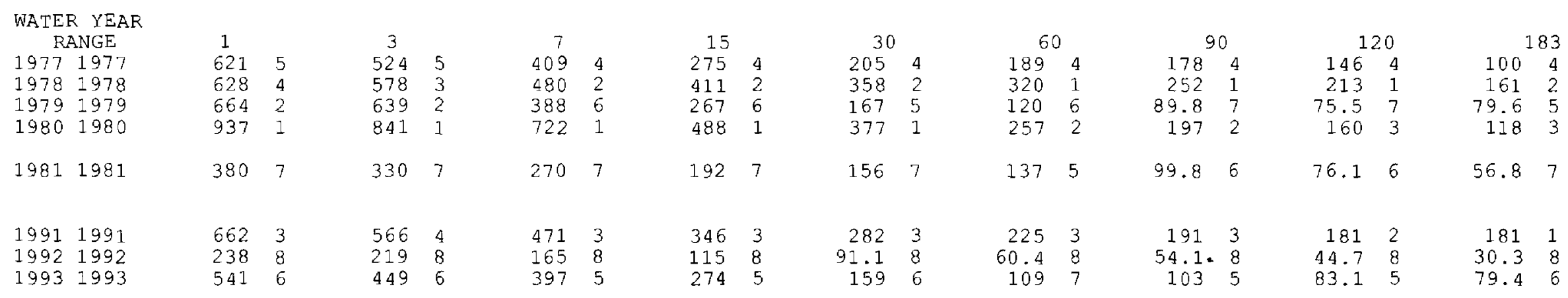


LOCATION.--30 $18^{\prime} 56^{\prime \prime}$, long $82^{\circ} 38^{\prime} 41^{\prime \prime}$, in $\mathrm{NE}^{1} / 4$ sec.18, T.2 S., R.17 E., Columbia County, Hydrologic Unit 03110201 , near center of span on downstream side of bridge on State Highway 246, $3.4 \mathrm{mi}$ upstream from mouth, 4.0 mi northeast of Suwannee Valley and 8.7 mi north of Lake City.

DRAINAGE AREA,--27.4 $\mathrm{mi}^{2}$.

PERIOD OF RECORD.--April 1976 to September 1981 (discontinued).

GAGE.--Water-stage recorder. Datum of gage is $93.54 \mathrm{ft}$ National Geodetic Vertical Datum of 1929 (levels by L. L. Lee and Associates, from Florida Department of Transportation bench mark). Prior to Apr. 8, 1976, nonrecording gage at site $0.3 \mathrm{mi}$ upstream, at datum unknown.

REMARKS.--Records poor.

MAXIMUM, MINIMUM, AND MEAN OF MONTHLY MEAN ELEVATIONS FOR WATER YEARS 1976-1981

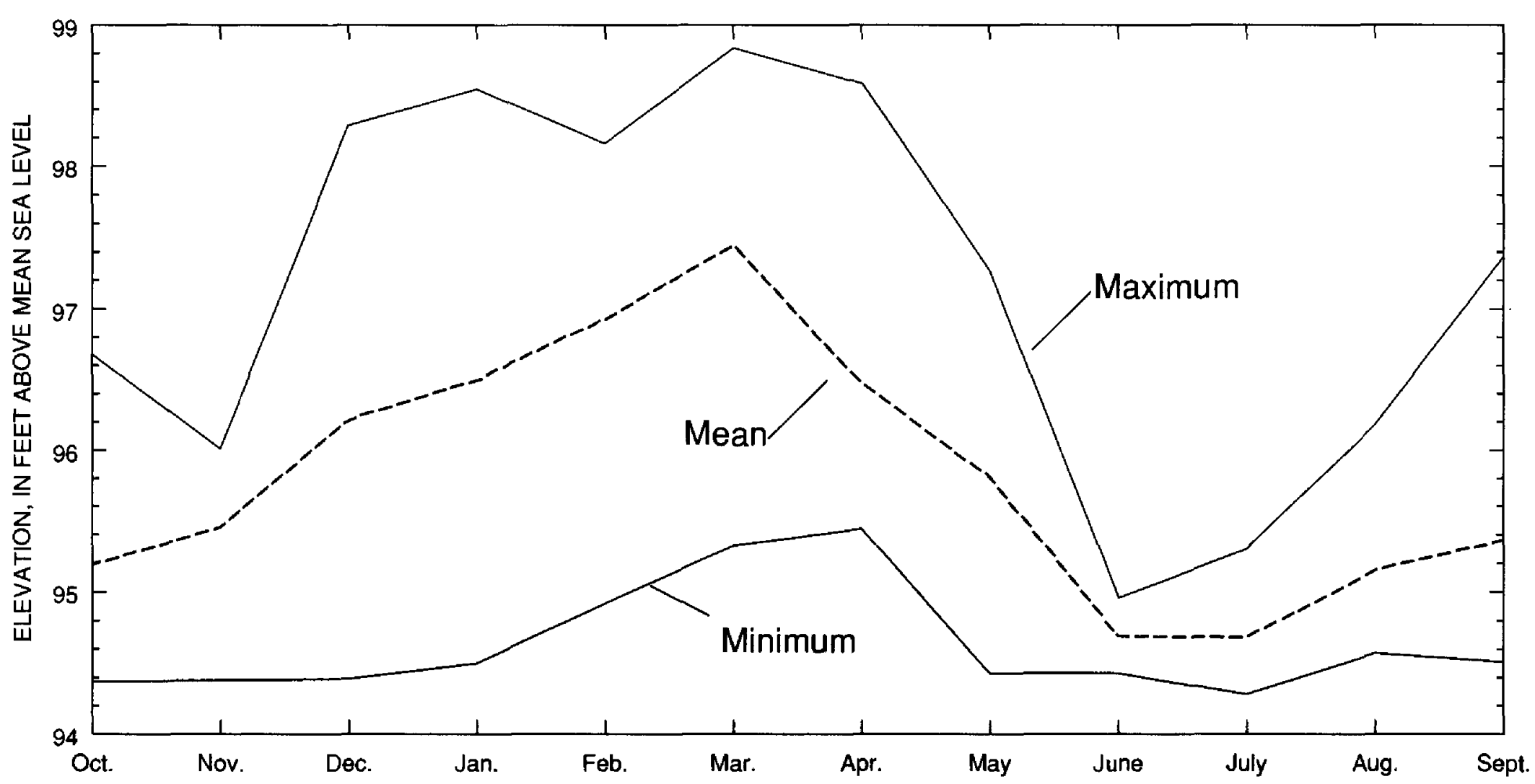

DURATION OF DAILY MEAN ELEVATION FOR WATER YEARS 1976-1981

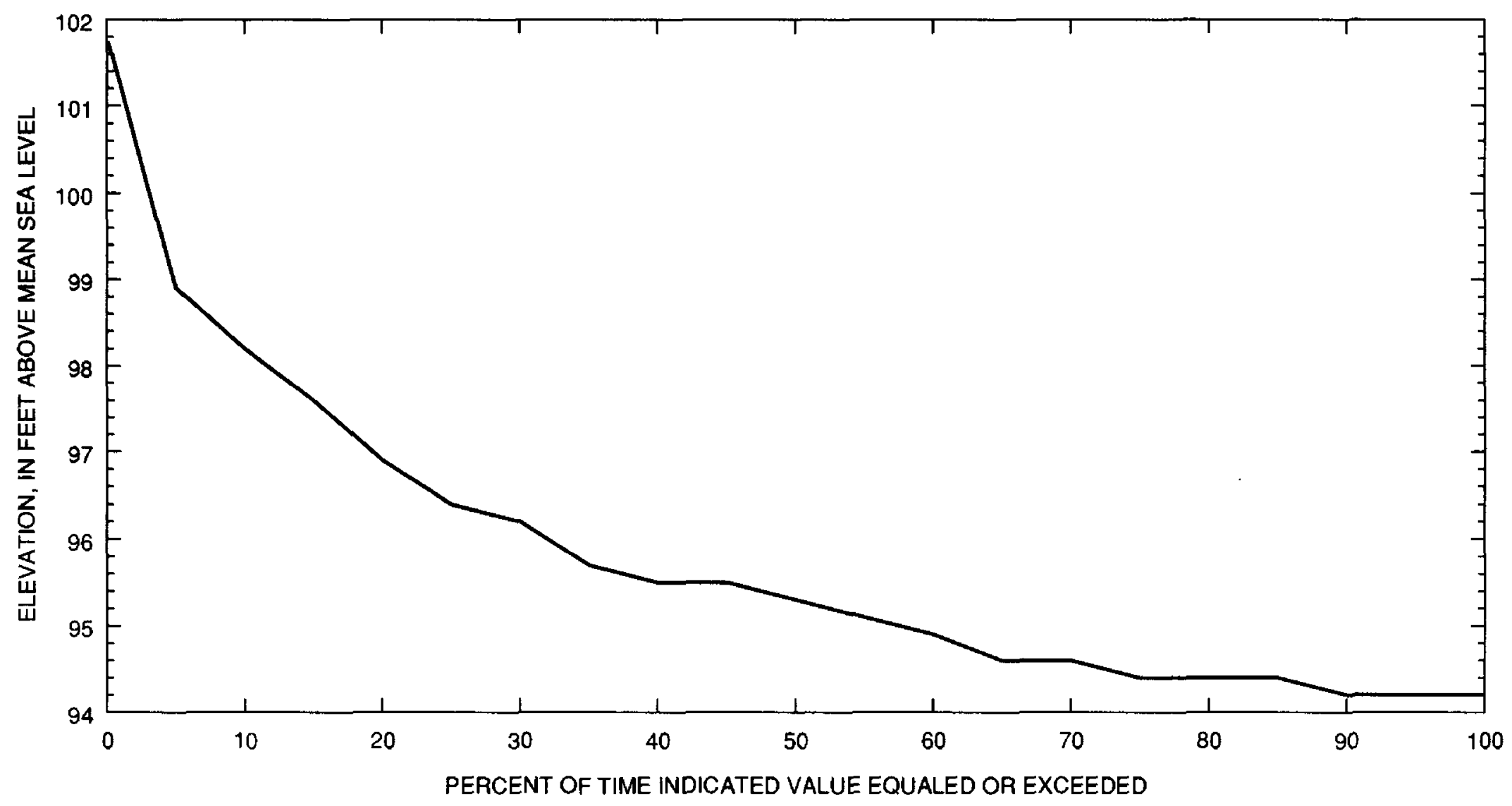


SUMMARY STATISTICS, IN CUBIC FEET PER SECOND UNLESS OTHERWISE INDICATED, FOR WATER YEARS 1976 - 1981

ANNUAL MEAN

HIGHEST ANNUAL MEAN

LOWEST ANNUAL MEAN

HIGHEST DAILY MEAN

LOWEST DAILY MEAN

ANNUAL SEVEN-DAY MINIMUM

INSTANTANEOUS PEAK FLOW

INSTANTANEOUS PEAK ELEVATION (FT)

INSTANTANEOUS LOW FLOW

ANNUAL RUNOFF (INCHES)

ANNUAL RUNOFF (CFSM)
26.6

$42.1 \quad 1978$

$13.4 \quad 1979$

$588 \quad$ Mar 111980

$.00 \quad$ May 81977

$.00 \quad$ May 81977

$762 \quad$ Mar 101980

102.78 Mar 101980

.00

May 81977

MAXIMUM, MINIMUM, AND MEAN OF MONTHLY MEAN DISCHARGES FOR WATER YEARS 1976-1981

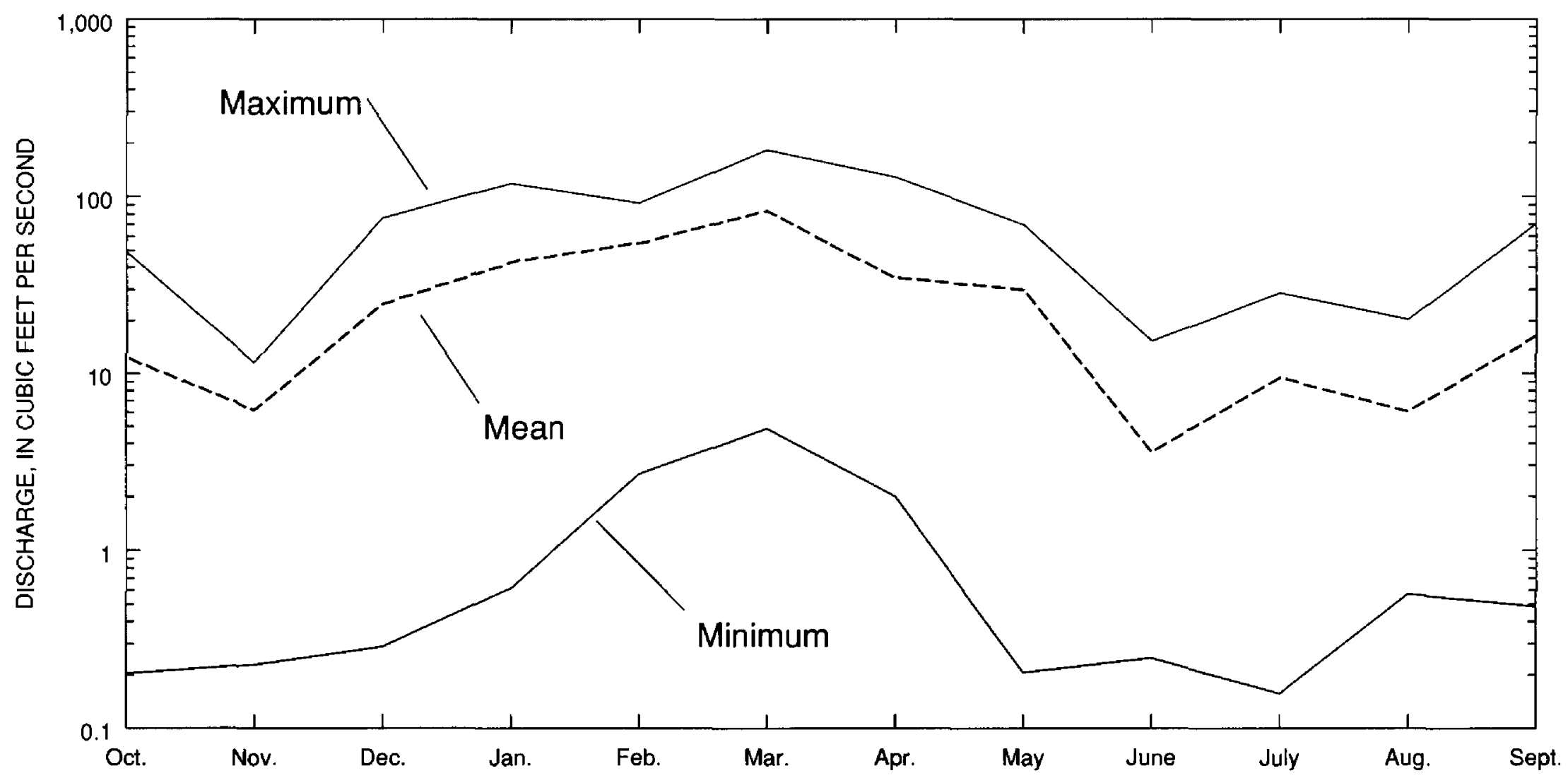

DURATION OF DAILY MEAN DISCHARGE FOR WATER YEARS 1976-1981

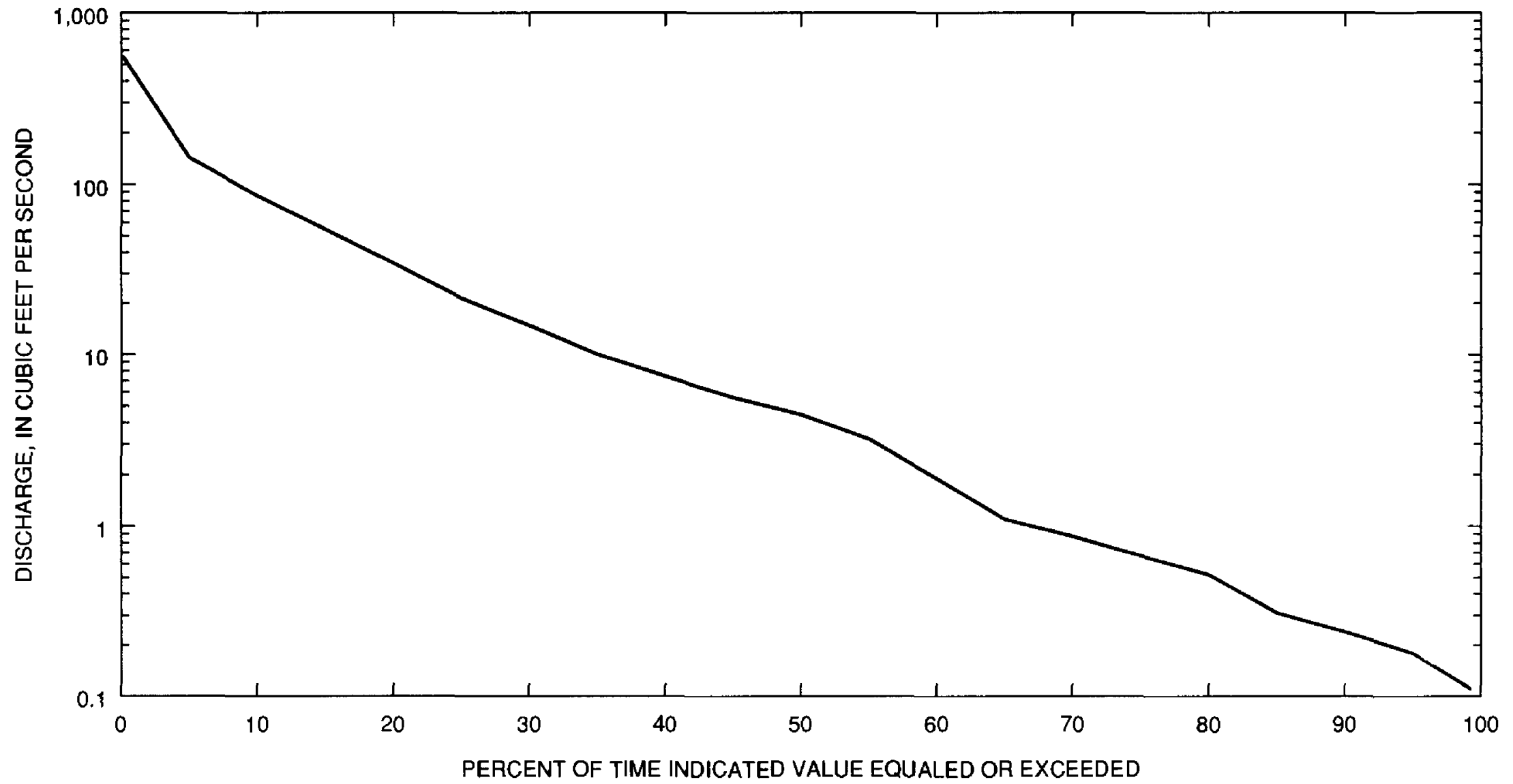


SUWANNEE RIVER BASIN

02315392 ROBINSON CREEK NEAR SUWANNEE VALLEY, FL--Continued

SUMMARY OF MONTHLY MEAN ELEVATION AND DISCHARGE STATISTICS FOR WATER YEARS 1976-1981

\begin{tabular}{lrrrrrr} 
& \multicolumn{3}{c}{ ELEVATIONS, } & \multicolumn{3}{c}{ DISCHARGE, } \\
& \multicolumn{2}{c}{ FEET ABOVE SEA LEVEL } & \multicolumn{2}{c}{ CUBIC FEET PER SECOND } \\
\multicolumn{1}{c}{ MONTH } & MAXIMUM & MINIMUM & MEAN & MAXIMUM & MINIMUM & MEAN \\
& & & & & & \\
OCTOBER & 96.67 & 94.37 & 95.19 & 48.1 & .204 & 12.37 \\
NOVEMBER & 96.00 & 94.38 & 95.45 & 11.4 & .229 & 6.18 \\
DECEMBER & 98.28 & 94.39 & 96.22 & 75.2 & .289 & 25.00 \\
JANUARY & 98.54 & 94.50 & 96.50 & 117.7 & .619 & 43.06 \\
EEBRUARY & 98.15 & 94.92 & 96.93 & 91.0 & 2.68 & 55.12 \\
MARCH & 98.83 & 95.32 & 97.45 & 181.4 & 4.86 & 83.61 \\
APRIL & 98.58 & 95.44 & 96.48 & 127.5 & 2.00 & 34.93 \\
MAY & 97.26 & 94.43 & 95.81 & 69.0 & .207 & 30.14 \\
JUNE & 94.95 & 94.43 & 94.69 & 15.1 & .249 & 3.57 \\
JULY & 95.29 & 94.28 & 94.68 & 28.4 & .157 & 9.56 \\
AUGUST & 96.17 & 94.57 & 95.15 & 20.1 & .572 & 6.06 \\
SEPTEMBER & 97.36 & 94.51 & 95.36 & 69.3 & .486 & 16.46
\end{tabular}

\section{DURATION OF DAILY MEAN VALUES FOR WATER YEARS 1976-1981}

PERCENT

OF TIME

EQUALED OR

EXCEEDED ANNUAL

NOV DEC JAN MEB MAR APR MAY JUNE

ELEVATION IN FEET ABOVE MEAN SEA LEVEI

$\begin{array}{llll}95.0 & 94.2 & 70.1 & 28.2 \\ 90.0 & 94.2 & 94.3 & 56.4 \\ 85.0 & 94.4 & 94.3 & 84.6 \\ 80.0 & 94.4 & 94.3 & 94.4 \\ 75.0 & 94.4 & 94.3 & 94.9 \\ 70.0 & 94.6 & 94.3 & 95.0 \\ 65.0 & 94.6 & 94.3 & 95.3 \\ 60.0 & 94.9 & 94.3 & 95.3 \\ 55.0 & 95.1 & 94.4 & 95.4 \\ 50.0 & 95.3 & 94.6 & 95.5 \\ 45.0 & 95.5 & 94.8 & 95.6 \\ 40.0 & 95.5 & 94.9 & 95.7 \\ 35.0 & 95.7 & 95.2 & 95.8 \\ 30.0 & 96.2 & 95.4 & 95.8 \\ 25.0 & 96.4 & 95.6 & 95.8 \\ 20.0 & 96.9 & 95.7 & 96.1 \\ 15.0 & 97.6 & 95.9 & 96.3 \\ 10.0 & 98.2 & 96.4 & 96.4 \\ 5.0 & 98.9 & 97.4 & 96.6\end{array}$

$$
\begin{aligned}
& 34.7 \\
& 69.4 \\
& 94.4 \\
& 94.4 \\
& 95.3 \\
& 95.4 \\
& 95.6 \\
& 95.6 \\
& 95.7 \\
& 95.9 \\
& 96.0 \\
& 96.3 \\
& 96.5 \\
& 96.8 \\
& 97.4 \\
& 97.8 \\
& 97.9 \\
& 98.3 \\
& 98.4
\end{aligned}
$$

94.4
94.4
94.6
94.7
95.1
95.3
95.3
95.3
95.4
95.4
96.1
97.0
97.4
97.8
97.9
98.3
98.7
98.9
99.2

$\begin{array}{lr}94.6 & 95.0 \\ 94.6 & 95.4 \\ 94.9 & 95.4 \\ 95.4 & 95.6 \\ 95.4 & 96.0 \\ 95.7 & 96.2 \\ 95.9 & 96.4 \\ 96.2 & 96.6 \\ 96.5 & 97.0 \\ 96.8 & 97.3 \\ 97.1 & 97.5 \\ 97.4 & 97.7 \\ 97.6 & 98.1 \\ 97.8 & 98.3 \\ 98.1 & 98.5 \\ 98.4 & 98.9 \\ 98.7 & 99.1 \\ 99.1 & 99.4 \\ 99.2 & 100.0\end{array}$

$\begin{array}{ll}94.8 & 94.2 \\ 95.0 & 94.2 \\ 95.2 & 94.5 \\ 95.2 & 94.5 \\ 95.3 & 94.7 \\ 95.3 & 94.8 \\ 95.5 & 95.0 \\ 95.6 & 95.1 \\ 95.6 & 95.1 \\ 95.8 & 95.5 \\ 96.0 & 95.6 \\ 96.3 & 95.8 \\ 96.6 & 95.9 \\ 96.9 & 96.1 \\ 97.3 & 96.2 \\ 97.6 & 96.5 \\ 98.4 & 97.3 \\ 99.1 & 98.3 \\ 99.4 & 98.8\end{array}$

DISCHARGE IN CUBIC FEET PER SECOND

$\begin{array}{rrr}95.0 & 0.1 & 0.1 \\ 90.0 & 0.2 & 0.1 \\ 85.0 & 0.3 & 0.1 \\ 80.0 & 0.5 & 0.2 \\ 75.0 & 0.6 & 0.2 \\ 70.0 & 0.8 & 0.3 \\ 65.0 & 1.1 & 0.4 \\ 60.0 & 1.9 & 0.5 \\ 55.0 & 3.2 & 0.5 \\ 50.0 & 4.4 & 0.8 \\ 45.0 & 5.6 & 1.0 \\ 40.0 & 7.4 & 2.0 \\ 35.0 & 10.0 & 3.0 \\ 30.0 & 14.8 & 3.7 \\ 25.0 & 21.5 & 5.6 \\ 20.0 & 34.6 & 8.8 \\ 15.0 & 54.4 & 14.4 \\ 10.0 & 85.8 & 24.6 \\ 5.0 & 143.2 & 56.8\end{array}$

0.2
0.2
0.2
0.2
0.6
1.2
2.6
3.4
4.0
4.6
4.6
4.6
5.3
7.0
7.7
9.3
12.8
17.4
22.2

0.2
0.2
0.2
0.3
6.7
7.3
7.7
8.8
10.2
11.9
13.8
16.1
19.2
23.9
43.0
52.9
62.1
75.7
93.3

$\begin{array}{rr}0.3 & 1.3 \\ 0.4 & 1.3 \\ 0.6 & 1.9 \\ 1.2 & 5.5 \\ 4.7 & 6.8 \\ 5.3 & 10.1 \\ 5.5 & 13.3 \\ 5.8 & 17.3 \\ 6.1 & 22.6 \\ 6.8 & 27.8 \\ 20.5 & 41.6 \\ 32.8 & 50.6 \\ 44.5 & 58.2 \\ 57.0 & 67.2 \\ 68.7 & 83.6 \\ 80.9 & 102.5 \\ 90.8 & 130.1 \\ 110.7 & 157.2 \\ 151.2 & 185.8\end{array}$

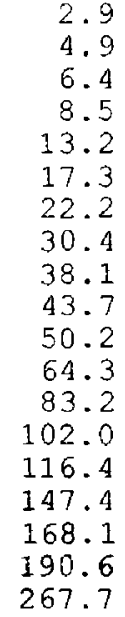

0.3
0.5
0.8
1.2
1.9
2.9
3.6
4.6
5.8
7.1
9.0
10.5
16.0
24.2
34.4
46.5
74.0
140.0
195.0

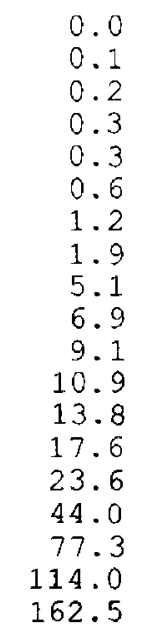
0.1
0.2
0.3
0.5
0.7
0.7
0.8
1.0
1.3
2. 7
3. 3
3. 9
3.9
15.0

0.1
0.1
0.1
0.2
0.2
0.2
0.4
0.5
0.5
0.6
0.6
0.7
0.9
1.2
1.5
3.7
21.1
33.5
50.2


LOWEST MEAN ELEVATION, IN FEET, AND RANKING FOR THE FOLLOWING NUMBER OF CONSECUTIVE DAYS FOR PERIOD APR TO MAR

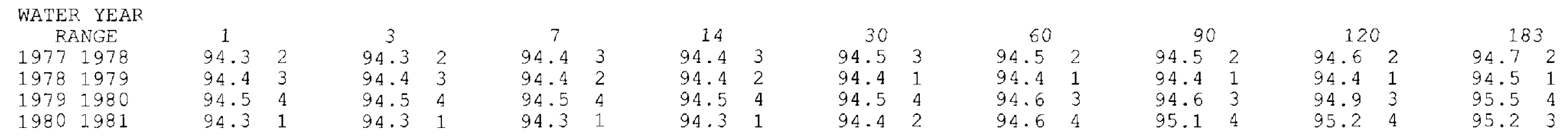

HIGHEST MEAN ELEVATION, IN FEET, AND RANKING FOR THE FOLLOWING NUMBER OF CONSECUTIVE DAYS FOR PERIOD OCT TO SEP

\begin{tabular}{|c|c|c|c|c|c|c|c|c|c|c|c|c|c|c|c|c|c|c|}
\hline $\begin{array}{l}\text { PER YEA } \\
\text { RANGE }\end{array}$ & 1 & & 3 & & 7 & & 15 & & 30 & & 60 & & 9 & & 12 & & 18 & \\
\hline $1977 \quad 1977$ & 100 & 3 & 99.8 & 4 & 99.4 & 4 & 99.0 & 3 & 98.4 & 3 & 98.2 & 3 & 98.1 & 2 & 97.7 & 2 & 97.0 & 2 \\
\hline $\begin{array}{lll}1978 & 1978\end{array}$ & 101 & 2 & 100 & 2 & 99.9 & 2 & 99.3 & 2 & 99.0 & 2 & 98.7 & 2 & 98.5 & 1 & 98.4 & 1 & 97.7 & 1 \\
\hline $1979 \quad 1979$ & 100 & 4 & 100 & 3 & 99.0 & 5 & 98.8 & 5 & 97.4 & 5 & 96.6 & 5 & 96.2 & 5 & 95.9 & 5 & 95.7 & 5 \\
\hline 19801980 & 102 & 1 & 102 & 1 & 101 & 1 & 100 & 1 & 99.6 & 1 & 98.8 & 1 & 97.9 & 3 & 97.4 & 3 & 95.8 & 3 \\
\hline $1981 \quad 1981$ & 99.9 & 5 & 99.6 & 5 & 99.5 & 3 & 98.8 & 4 & 98.3 & 4 & 97.6 & 4 & 95.9 & 4 & 96.6 & 4 & 95.3 & 4 \\
\hline
\end{tabular}

LOWEST MEAN DISCHARGE, IN CUBIC FEET PER SECOND, AND RANKING FOR THE FOLLOWING NUMBER OF CONSECUTIVE DAYS FOR PERIOD APR TO MAR

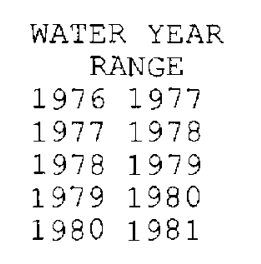

$\begin{array}{rr}1 & \\ .27 & 4 \\ .0000 & 1 \\ .19 & 2 \\ .33 & 5 \\ .25 & 3\end{array}$
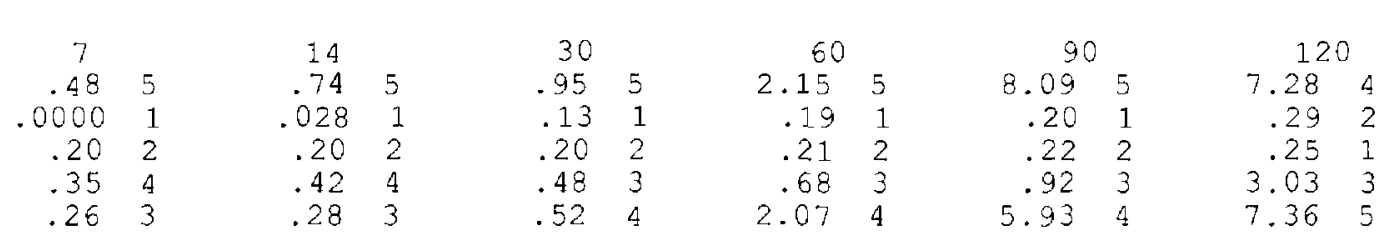

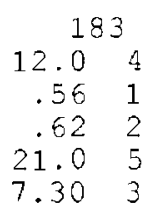

HIGHEST MEAN DISCHARGE, IN CUBIC FEET PER SECOND, AND RANKING FOR THE FOLLOWING NUMBER OF CONSECUTIVE DAYS FOR PERIOD OCT TO SEP

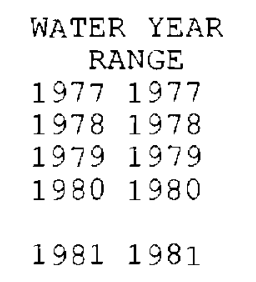

$\begin{array}{cccc}1 & & 3 & \\ 297 & 3 & 238 & 4 \\ 395 & 2 & 340 & 2 \\ 249 & 4 & 244 & 3 \\ 588 & 1 & 531 & 1 \\ 238 & 5 & 202 & 5\end{array}$

$\begin{array}{rr}15 & \\ 128 & 5 \\ 187 & 2 \\ 130 & 4 \\ 307 & 1 \\ 135 & 3\end{array}$
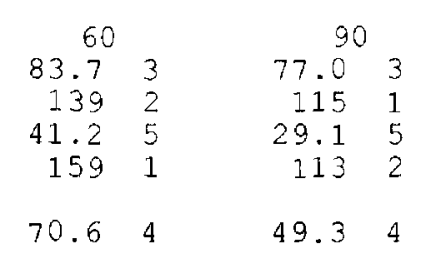

$43.5^{183} 3$ $80.3 \quad 1$

$29.6 \quad 4$ 
LOCATION.--Lat $30^{\circ} 19^{\prime} 32^{\prime \prime}$, long $82^{\circ} 44^{\prime} 18^{\prime \prime}$, in $\mathrm{SW}^{1} / 4$ sec.8, T.2 S., R.16 E., Columbia County, Hydrologic Unit 03110201 , on downstream side of bridge on

U.S. Highway 41, $1.0 \mathrm{mi}$ southeast of White Springs and $171 \mathrm{mi}$ upstream from mouth.

DRAINAGE AREA.--2,430 $\mathrm{mi}^{2}$ approximately, includes part of watershed in Okefenokee Swamp which is indeterminate.

PERIOD OF RECORD.--May 1906 to December 1908, February 1927 to 1993.

REVISED RECORDS.--WSP 1504: 1906, 1908. WSP 1905: WDR FL-75-1: Drainage area.

GAGE.--Nonrecording gage. Datum of gage is National Geodetic Vertical Datum of 1929. Prior to July 31, 1932, nonrecording gage at site 1.0 mi downstream at datum $48.54 \mathrm{ft}$. August 1, 1932 to October 10, 1979, water-stage recorder, at present site, at datum $48.54 \mathrm{ft}$. October 11, 1979 to December 1, 1983, nonrecording gage at site 2.2 miles downstream at NGVD.

REMARKS.--Records good, except for estimated daily discharges, which are fair.
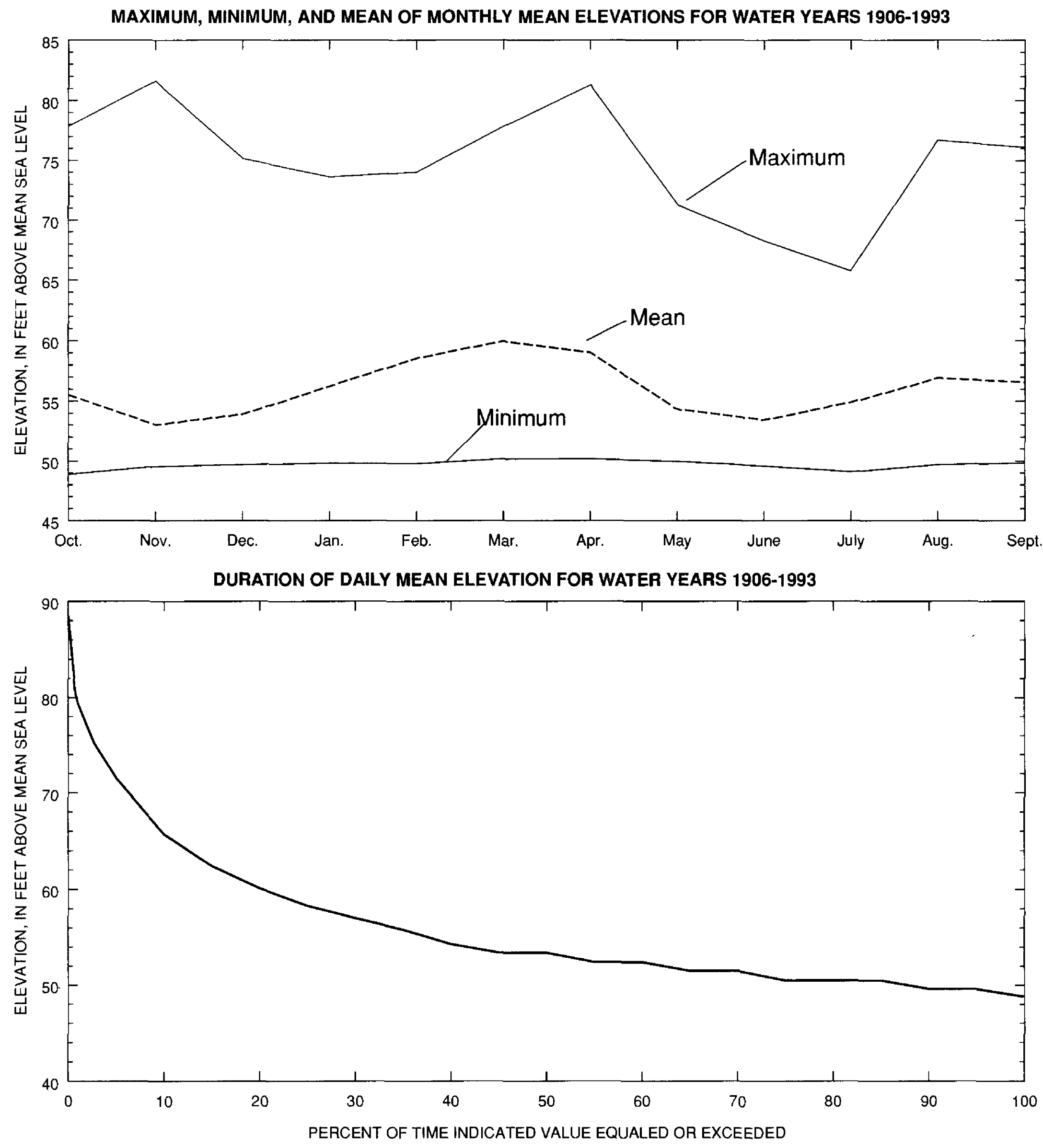
SUMMARY STATISTICS, IN CUBIC FEET PER SECOND UNLESS OTHERWISE INDICATED, FOR WATER YEARS $1906-1993$

ANNUAL MEAN

HIGHEST ANNUAL MEAN

LOWEST ANNUAL MEAN

HIGHEST DAILY MEAN

LOWEST DAILY MEAN

ANNUAL SEVEN-DAY MINIMUM

INSTANTANEOUS PEAK ELOW

INSTANTANEOUS PEAK ELEVATION

INSTANTANEOUS LOW ELOW

ANNUAL RUNOEF (INCHES/CSEM)

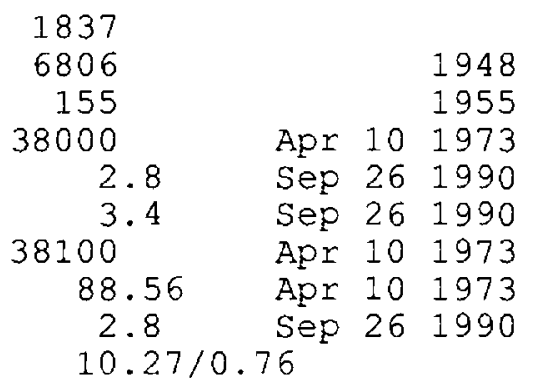

1837

15

38000

3.4

2.8

$10.27 / 0.76$

MAXIMUM, MINIMUM, AND MEAN OF MONTHLY MEAN DISCHARGES FOR WATER YEARS 1906-1993

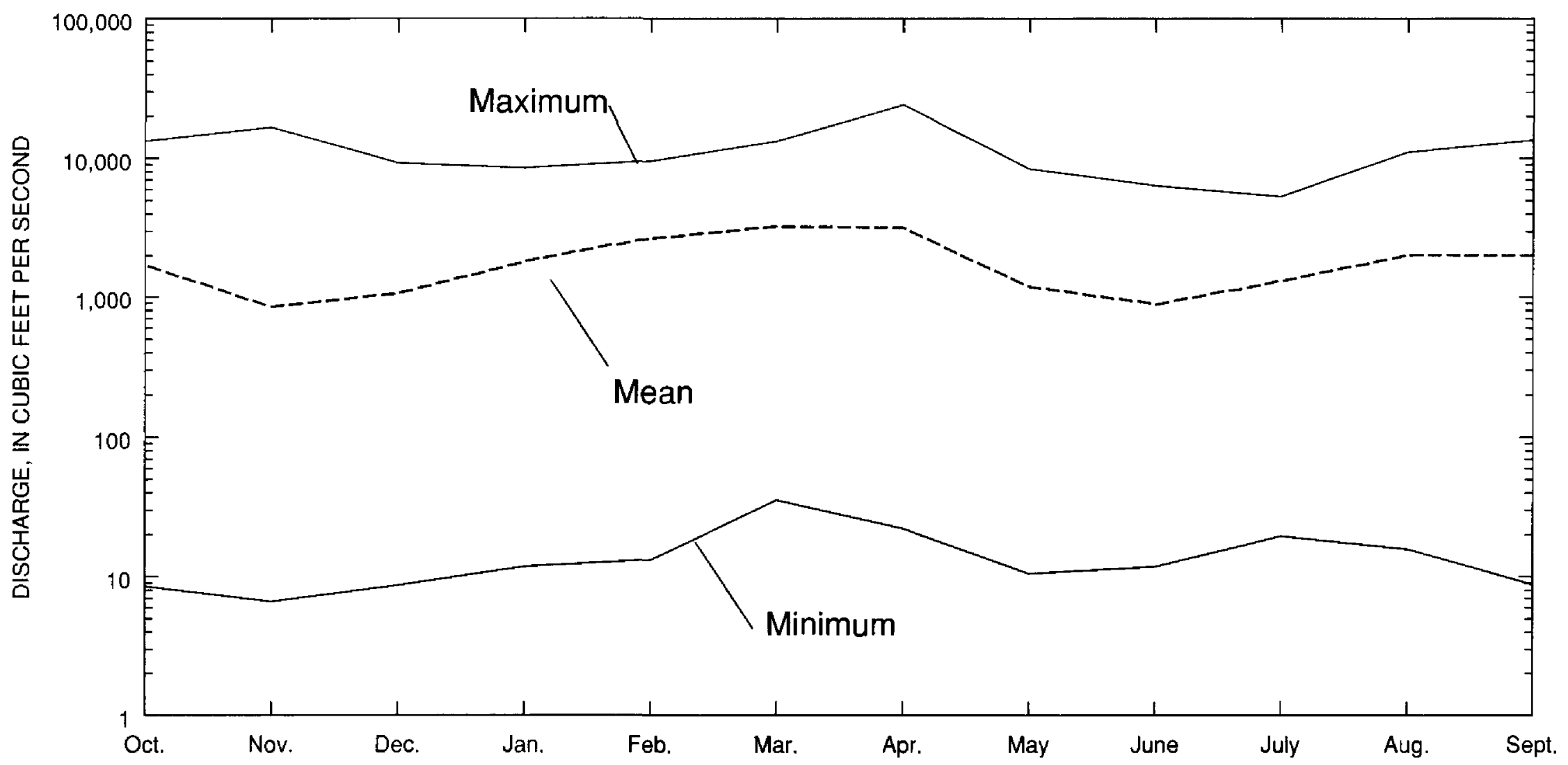

DURATION OF DAILY MEAN DISCHARGE FOR WATER YEARS 1906-1993

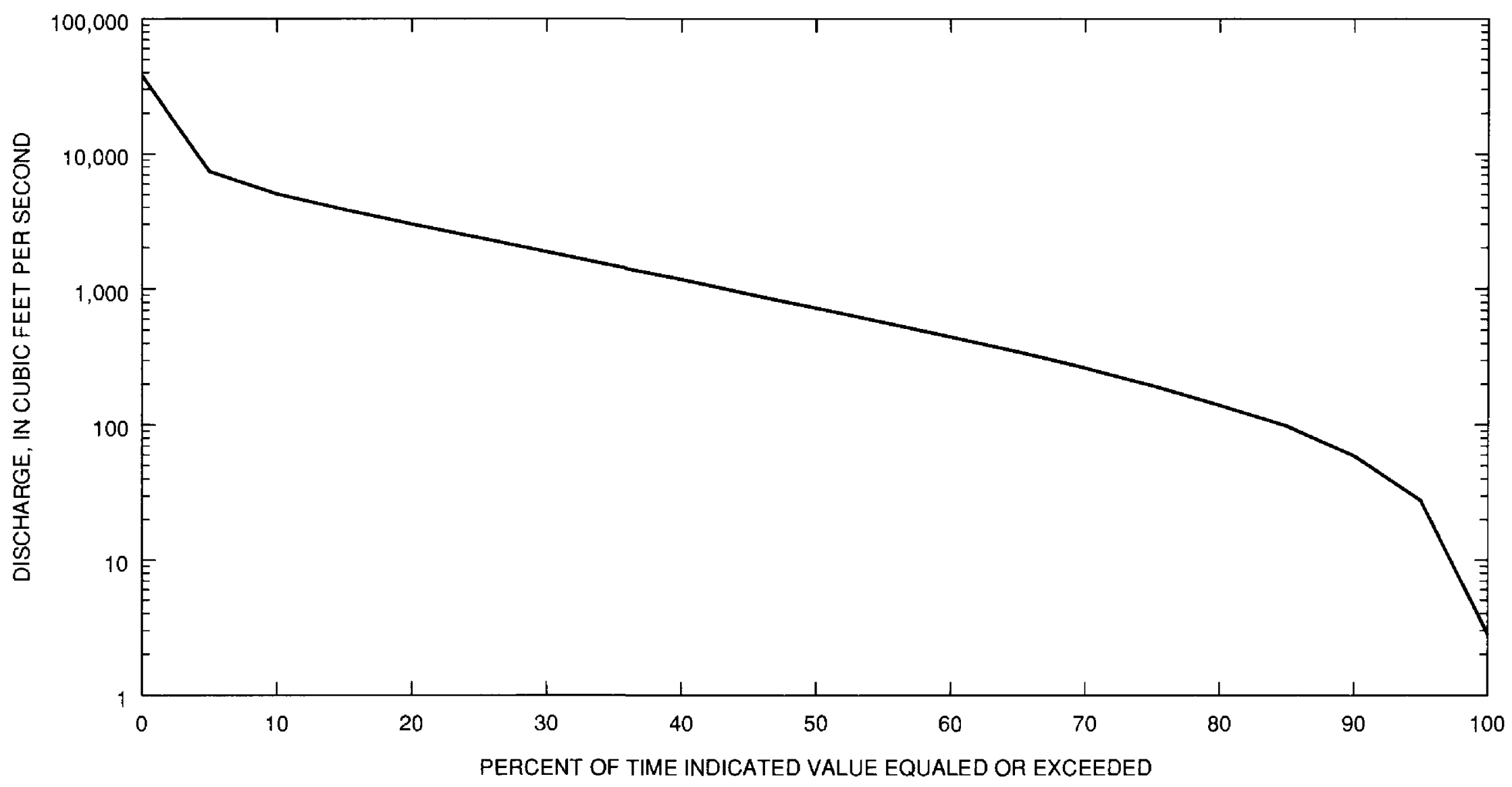




\section{SUWANNEE RIVER BASIN}

02315500 SUWANNEE RIVER AT WHITE SPRINGS, FL--Continued

SUMMARY OF MONTHLY MEAN ELEVATION AND DISCHARGE STATISTICS FOR WATER YEARS 1906-1993

\begin{tabular}{|c|c|c|c|c|c|c|}
\hline \multirow[b]{2}{*}{ MONTE } & \multicolumn{3}{|c|}{ ELEVATIONS, } & \multicolumn{3}{|c|}{ DISCHARGE, } \\
\hline & MAX IMUM & MINIMUM & MEAN & MAXIMUM & MINIMUM & MEAN \\
\hline OCTOBER & 77.79 & 48.88 & 55.52 & 13100 & 8.55 & 1720 \\
\hline NOVEMBER & 81.54 & 49.54 & 53.01 & 16450 & 6.63 & 845 \\
\hline DECEMBER & 75.11 & 49.70 & 53.91 & 9103 & 8.68 & 1060 \\
\hline JANUARY & 73.56 & 49.83 & 56.24 & 8401 & 11.8 & 1828 \\
\hline FEBRUARY & 73.97 & 49.78 & 58.56 & 9406 & 13.2 & 2664 \\
\hline MARCH & 77.78 & 50.17 & 59.96 & 13040 & 35.5 & 3254 \\
\hline APRIL & 81.23 & 50.17 & 59.03 & 23910 & 22.2 & 3200 \\
\hline MAY & 71.26 & 49.98 & 54.33 & 8288 & 10.5 & 1187 \\
\hline JUNE & 68.21 & 49.59 & 53.43 & 6317 & 11.8 & 887 \\
\hline JULY & 65.71 & 49.09 & 54.90 & 5274 & 19.6 & 1315 \\
\hline AUGUST & 76.65 & 49.69 & 56.91 & 10870 & 15.8 & 2033 \\
\hline SEPTEMBER & 76.07 & 49.84 & 56.58 & 13310 & 8.82 & 2018 \\
\hline
\end{tabular}

DURATION OF DAILY MEAN VALUES FOR WATER YEARS 1906-1908, 1927-1993

PERCENT

OE TIME

EUALED OR
EXCEEDED ANNUAL OCT
ELEVATION IN FEET ABOVE MEAN SEA LEVEL

$\begin{array}{rlll}95.0 & 49.6 & 49.6 & 49.6 \\ 90.0 & 49.6 & 49.6 & 49.6 \\ 85.0 & 50.5 & 50.4 & 49.6 \\ 80.0 & 50.5 & 50.4 & 50.4 \\ 75.0 & 50.5 & 50.4 & 50.4 \\ 70.0 & 51.5 & 51.2 & 50.4 \\ 65.0 & 51.5 & 51.2 & 50.4 \\ 60.0 & 52.4 & 51.2 & 50.4 \\ 55.0 & 52.4 & 52.1 & 51.2 \\ 50.0 & 53.4 & 52.1 & 51.2 \\ 45.0 & 53.4 & 52.9 & 51.2 \\ 40.0 & 54.3 & 53.8 & 52.1 \\ 35.0 & 55.8 & 53.8 & 52.1 \\ 30.0 & 57.0 & 54.7 & 52.1 \\ 25.0 & 58.3 & 56.5 & 52.9 \\ 20.0 & 60.1 & 58.4 & 53.8 \\ 15.0 & 62.4 & 61.6 & 54.7 \\ 10.0 & 65.7 & 64.7 & 56.5 \\ 5.0 & 71.5 & 71.9 & 60.3\end{array}$

49.3
49.9
49.9
49.9
50.6
50.6
50.6
51.3
51.3
51.3
52.1
52.1
52.8
53.5
54.3
55.8
57.3
59.0
65.0

$$
\begin{aligned}
& 49.7 \\
& 49.7 \\
& 50.4 \\
& 51.1 \\
& 51.1 \\
& 51.8 \\
& 51.8 \\
& 51.8 \\
& 52.6 \\
& 52.6 \\
& 54.1 \\
& 54.8 \\
& 56.4 \\
& 57.9 \\
& 59.6 \\
& 61.3 \\
& 63.0 \\
& 65.7 \\
& 69.4
\end{aligned}
$$

49.7
50.5
51.2
51.2
52.0
52.7
53.5
54.3
55.1
56.7
57.6
59.3
60.2
61.9
62.9
64.7
66.6
69.3
73.2

50.6
50.6
51.4
52.2
53.0
53.9
54.7
54.7
55.6
56.4
58.2
59.1
61.0
61.9
63.9
66.8
69.9
74.3
77.5

49.9
50.8
50.8
51.7
51.7
52.6
53.5
53.5
54.4
55.4
56.3
57.9
59.1
60.7
63.0
65.9
70.0
73.5
77.6

49.5
50.2
50.2
50.2
50.9
50.9
50.9
51.7
51.7
52.4
52.4
53.2
54.0
54.8
54.8
56.4
57.2
58.9
64.3

DISCHARGE IN CUBIC FEET PER SECOND

$\begin{array}{rrr}95.0 & 27.7 & 21.4 \\ 90.0 & 59.2 & 42.4 \\ 85.0 & 97.8 & 74.5 \\ 80.0 & 139.8 & 106.8 \\ 75.0 & 194.6 & 147.1 \\ 70.0 & 263.6 & 201.7 \\ 65.0 & 346.0 & 260.3 \\ 60.0 & 444.7 & 339.8 \\ 55.0 & 569.5 & 426.0 \\ 50.0 & 727.4 & 527.1 \\ 45.0 & 915.7 & 661.3 \\ 40.0 & 1168.3 & 836.4 \\ 35.0 & 1467.2 & 1082.0 \\ 30.0 & 1857.1 & 1427.9 \\ 25.0 & 2359.2 & 1906.4 \\ 20.0 & 2982.8 & 2578.0 \\ 15.0 & 3825.9 & 3437.1 \\ 10.0 & 5060.1 & 4573.0 \\ 5.0 & 7399.6 & 7393.6\end{array}$

17.0
32.2
52.7
75.3
100.4
121.1
141.6
162.1
192.5
238.5
314.9
401.1
502.0
637.8
800.8
974.9
1272.5
1703.4
3152.0

21.3
31.6
56.9
74.4
97.4
122.9
153.9
204.5
272.6
329.1
401.9
507.0
644.4
818.4
1080.9
1537.8
2186.1
2824.4
4855.8

28.9
54.3
116.9
161.3
224.8
306.3
373.2
478.0
563.0
725.1
912.2
1253.5
1649.8
2250.0
2906.3
3624.9
4374.2
5345.4
6988.7

$\begin{array}{rr}45.5 & 77.2 \\ 116.2 & 189.4 \\ 190.9 & 302.6 \\ 292.1 & 430.7 \\ 404.2 & 693.0 \\ 558.8 & 946.6 \\ 798.4 & 1182.3 \\ 1074.6 & 1408.4 \\ 1444.6 & 1640.0 \\ 1866.9 & 2037.4 \\ 2277.6 & 2521.5 \\ 2709.3 & 2958.7 \\ 3248.8 & 3390.3 \\ 3714.6 & 3865.0 \\ 4230.5 & 4532.8 \\ 4785.9 & 5494.5 \\ 5735.2 & 6658.0 \\ 6750.4 & 8569.4 \\ 8164.1 & 10541.3\end{array}$

49.8
134.8
214.1
303.9
427.6
589.5
770.7
979.8
1208.0
1456.1
1806.5
2245.7
2707.4
3268.3
4044.5
5192.6
6693.5
8365.3
10802.8

22.0
52.8
87.2
120.5
161.4
206.0
255.8
322.9
427.2
531.0
648.3
794.7
967.8
1188.1
1459.4
1779.0
2203.3
2912.0
4735.8

20.2
41.0
62.9
87.3
107.2
130.3
162.8
198.1
237.6
291.5
371.9
446.2
585.4
739.8
958.0
1239.8
1672.1
2579.3
4195.5
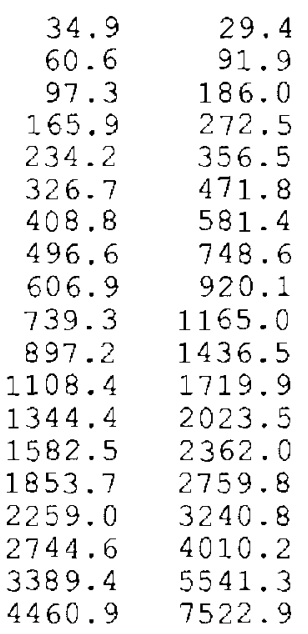

58.7
97.0
148.0
205.9
286.6
412.8
544.5
682.3
816.4
1002.5
1219.2
1437.9
1745.8
2111.0
2528.3
3296.5
4131.2
5189.8
7332.9 
THIS PAGE INTENTIONALLY BLANK 
HIGHEST MEAN ELEVATION, IN FEET, AND RANKING FOR THE FOLLOWING NUMBER OF CONSECUTIVE DAYS FOR PERIOD OCT TO SEP

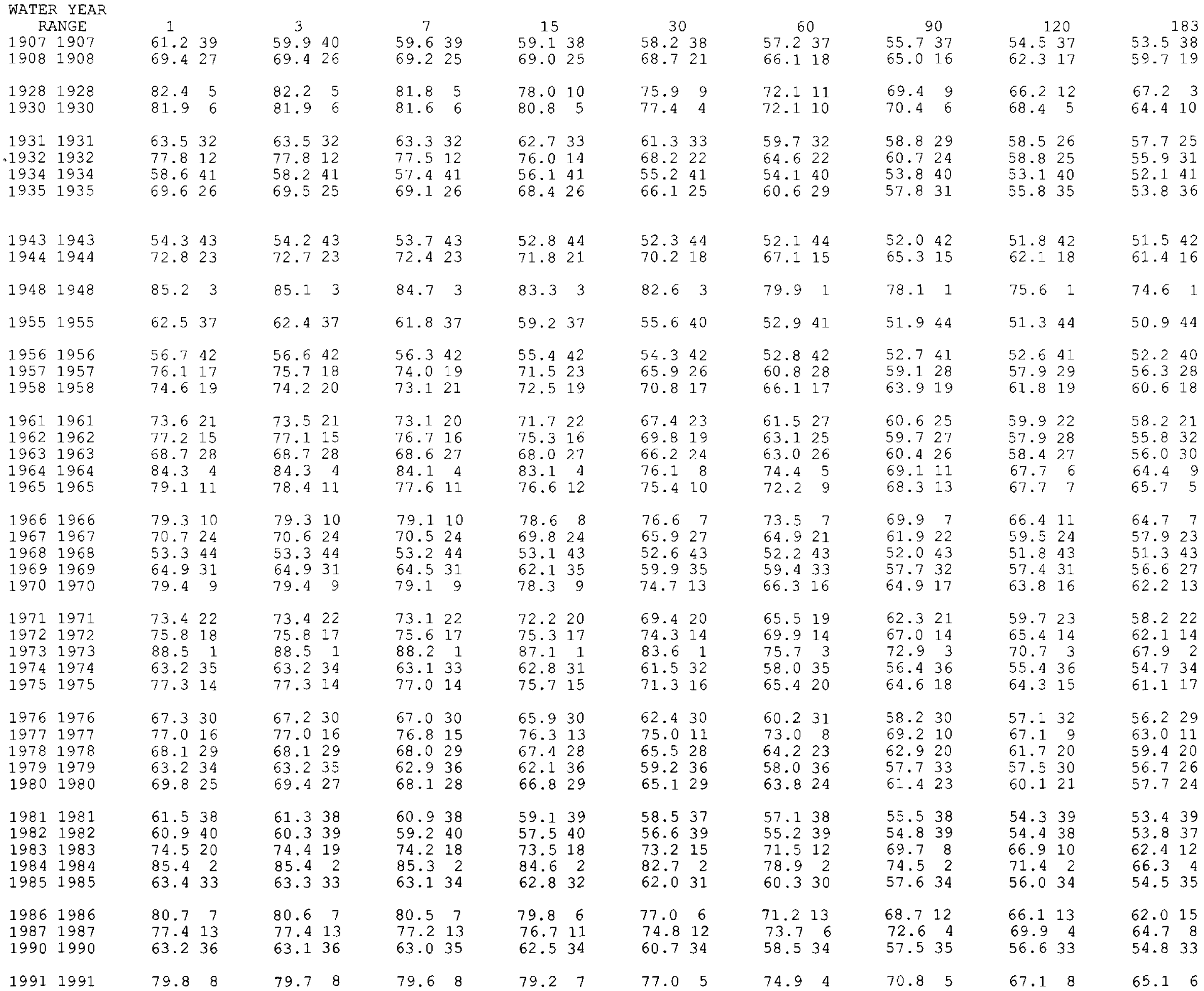



CONSECUTIVE DAYS FOR PERIOD APR TO MAR

WATER YEAR

RANGE
19071908

19271928

19281929

19291930

19301931

19311932

19321933

19331934

19341935
19351936

$1936 \quad 1937$

19371938

19381939

19391940

19401941

19411942

19421943

19431944

19441945

19451946

$\begin{array}{lll}1946 & 1947\end{array}$

19471948

19481949

19491950

19501951

195: 1952

19521953

19531954

19541955

19551956

19561957

19571958

19581959

19591960

19601961

$\begin{array}{lll}1961 & 1962\end{array}$

19621963

19631964

19641965

19651966

19661967

19671968

19681969

19691970

19701971

$1971 \quad 1972$

19721973

19731974

$1974 \quad 1975$

19751976

$1976 \quad 1977$

19771978

19781979

19791980

19801981

$1981 \quad 1982$

19821983

19831984

19841985

19851986

19861987

19871988

19881989

19891990

19901991

19911992

19921993 $\begin{array}{rr}1 & \\ 18.0 & 16 \\ 12.0 & 10 \\ 520 & 65 \\ 520 & 66 \\ 300 & 61 \\ & \\ 4.80 & 2 \\ 6.60 & 3 \\ 35.0 & 22 \\ 8.50 & 6 \\ 7.60 & 5\end{array}$

$32.0 \quad 20$

22356
16.014

10842

$17.0 \quad 15$

11.09

44.027

$\begin{array}{rr}9.50 & 8 \\ 135 & 46\end{array}$

54.031

$\begin{array}{lll}328 & 63\end{array}$

51155
102

$\begin{array}{ll}211 & 55 \\ 102 & 41\end{array}$

90.036

34.021

38.023

47.028

8.70
$7.50 \quad 4$

14.012

24957

$66.0 \quad 33$

310
258
259

$94.0 \quad 38$

94.039

38.024

17250

18151

$250 \quad 58$

41.025

31.019

13547
14248

$\begin{array}{rr}168 & 49\end{array}$

$90.0 \quad 37$

$120 \quad 43$

$130 \quad 45$

26860

18853

19.017

13.011

26.018

15.013

73.0
1214

$198 \quad 54$

79.035

52.030

47.029

55.032

41.026

$94.0 \quad 40$

18552
18.015

12.010

$540 \quad 65$

540
300

5.072

$\begin{array}{rr}6.60 & 3 \\ 36.3 & 22\end{array}$

8.676

7.604

33.020

22656
16.713

10942

17.014

11.39

$\begin{array}{rr}44.7 & 27 \\ 9.80 & 8\end{array}$

$\begin{array}{rr}9.80 & 8 \\ 142 & 46\end{array}$

57.731

$328 \quad 63$

59567

22255

105
100

34.721

38.724

49.029

$\begin{array}{ll}9.10 & 7 \\ 7.90 & 5\end{array}$

$14.0 \quad 12$

26658

66.033

$314 \quad 62$

26859

96.038

96.739

38.323

19051

$255 \quad 57$

41.725

$32.7 \quad 19$

$146 \quad 48$

18050

91.736

$\begin{array}{lll}125 & 44\end{array}$

$130 \quad 45$

$270 \quad 60$

19453

19.317

46664

28.018

18.316

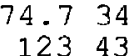

20054

$82.7 \quad 35$

53.330

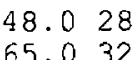

42.326

2.931

$94.7 \quad 37$

19452

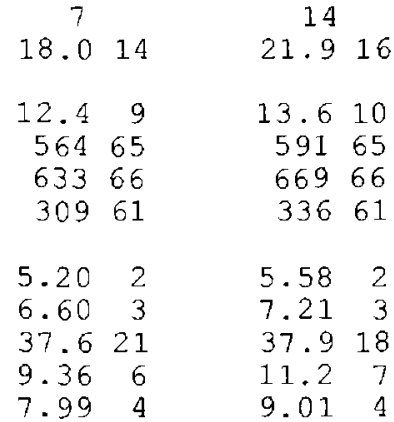

34.018

24155

18.115

11241

12.410

$\begin{array}{ll}12.4 & 10 \\ 46.6 & 27\end{array}$

10.37

15948

65.731

33163

$658 \quad 67$

24156

11542
11240

37.320

42.725

57.330

$\begin{array}{ll}10.3 & 8 \\ 8.70 & 5\end{array}$

14.312

29760

$68.6 \quad 32$

$329 \quad 62$

28559

10138

10439

40.323

$\begin{array}{ll}191 & 49 \\ 200 & 50\end{array}$

25957

42.424
37.622

$\begin{array}{rr}37.6 & 22 \\ 149 & 46\end{array}$

15047

20952

$97.4 \quad 37$

$\begin{array}{ll}131 & 44 \\ 136 & 45\end{array}$

$\begin{array}{ll}136 & 45 \\ 279 & 58\end{array}$

$220 \quad 54$

20.116

13.111

$\begin{array}{ll}521 & 64\end{array}$

21.917

77.734

20551

88.035

54.129

51.428

$71.0 \quad 33$

45.926

3.371

$96.6 \quad 36$

21253

41.121

27956

21.615

12540
17.513

13.29

47.926

10.76

$300 \quad 58$

77.133

33762

80067

$278 \quad 55$

127
146

40.019

42.923

68.130

$\begin{array}{ll}11.4 & 8 \\ 9.64 & 5\end{array}$

14.612

$326 \quad 60$

$73.0 \quad 31$

$\begin{array}{ll}371 & 63 \\ 307 & 59\end{array}$

$108 \quad 39$

10537

42.122

$\begin{array}{ll}221 & 49 \\ 234 & 51\end{array}$

26854

43.425

4.3 24

15346

23952

$105 \quad 38$

13944

13943

28557

24653

21.414

13.911

40.020

23.417

84.034
13742

21648

89.435

59.929

59.628

73.032

3.861

98.436

23350
30
70.426

$\begin{array}{rl}60 \\ 139 & 34 \\ 49.1 & 16 \\ 947 & 62 \\ 1240 & 66 \\ 842 & 60\end{array}$

6.502

9.933

38.417

11.74

66.025

33254

30.416

$\begin{array}{rl}145 \quad 40 \\ 18.2 & 12\end{array}$

$15.8 \quad 10$

52.023

12.35 

CONSECUTIVE DAYS FOR PERIOD OCT TO SEP

WATER YEAR RANGE 19071907 19081908
385059 665041
3
$3190 \quad 63$

664040 $\begin{array}{rr}7 & \\ 3070 & 62\end{array}$

657338
15
$2851 \quad 60$

$6474 \quad 36$
30
245359
636531

636531
60
204557

541323
$1544 \quad 58$

501220
$1183^{120} 60$

400024
84860

306028

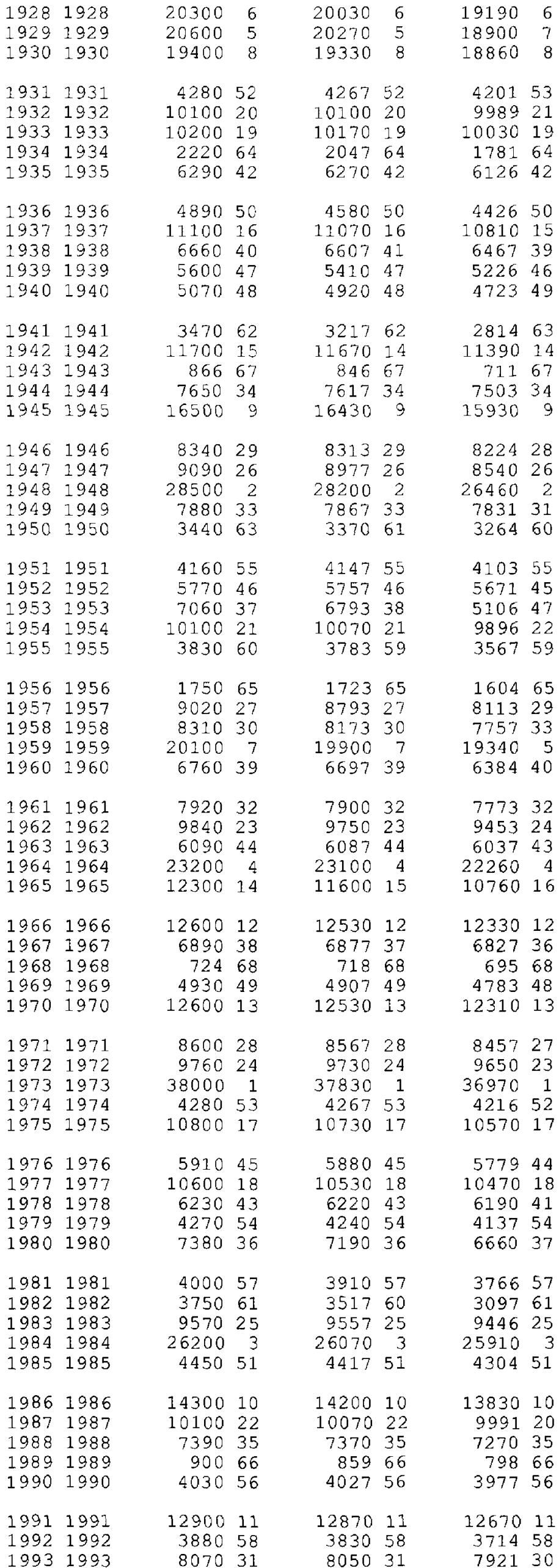

\begin{tabular}{|c|c|c|c|}
\hline 4160 & 9 & 10180 & \\
\hline 17160 & 6 & 13330 & \\
\hline 16910 & 7 & 12880 & \\
\hline 3969 & 52 & 3457 & \\
\hline 9315 & 22 & 6142 & \\
\hline 9585 & 20 & 8492 & \\
\hline 1347 & 64 & 1089 & \\
\hline 5845 & 40 & 5007 & \\
\hline 4161 & 49 & 3799 & \\
\hline 9683 & 19 & 7540 & \\
\hline 6127 & 37 & 4737 & \\
\hline 4464 & 47 & 4147 & \\
\hline 4072 & 51 & 2931 & \\
\hline 2124 & 63 & 1389 & \\
\hline 10410 & 14 & 8677 & \\
\hline 491 & 68 & 360 & \\
\hline 7267 & 31 & 6669 & \\
\hline 14530 & 8 & 11680 & \\
\hline 7876 & 27 & 7105 & \\
\hline 5295 & 45 & 5005 & \\
\hline 21550 & 3 & 18880 & \\
\hline 7656 & 28 & 6998 & \\
\hline 3016 & 59 & 2245 & \\
\hline 3905 & 53 & 2927 & \\
\hline 5535 & 43 & 4901 & \\
\hline 4712 & 46 & 4284 & \\
\hline 9309 & 23 & 7074 & \\
\hline 2669 & 61 & 1491 & \\
\hline 1314 & 65 & 967 & \\
\hline 7149 & 33 & 5100 & \\
\hline 7510 & 29 & 6871 & \\
\hline 17840 & 5 & 14920 & \\
\hline 5564 & 42 & 5376 & \\
\hline 7232 & 32 & 5647 & \\
\hline 8757 & 25 & 6629 & \\
\hline 5827 & 41 & 5176 & \\
\hline 19620 & 4 & 13310 & \\
\hline 9991 & 16 & 9173 & \\
\hline 11810 & 12 & 10260 & \\
\hline 6559 & 35 & 5108 & \\
\hline 648 & 67 & 528 & \\
\hline 3693 & 56 & 3013 & \\
\hline 11500 & 13 & 9402 & \\
\hline 8047 & 26 & 6840 & \\
\hline 9475 & 21 & 9009 & \\
\hline 33410 & 1 & 24060 & \\
\hline 4116 & 50 & 3582 & \\
\hline 9867 & 17 & 7817 & \\
\hline 5358 & 44 & 3997 & \\
\hline 10170 & 15 & 9502 & \\
\hline 5911 & 39 & 5180 & \\
\hline 3809 & 54 & 2771 & \\
\hline 6079 & 38 & 5418 & \\
\hline 3093 & 58 & 2872 & \\
\hline 2495 & 62 & 2177 & \\
\hline 9094 & 24 & 8951 & \\
\hline 23720 & 2 & 19380 & \\
\hline 4213 & 48 & 3872 & \\
\hline 12660 & 10 & 10780 & \\
\hline 9771 & 18 & 8951 & \\
\hline 7129 & 34 & 6519 & \\
\hline 734 & 66 & 663 & \\
\hline 3796 & 55 & 3149 & \\
\hline 12390 & 11 & 1100 & \\
\hline 3336 & 57 & 2831 & \\
\hline 7417 & 30 & 6246 & \\
\hline
\end{tabular}

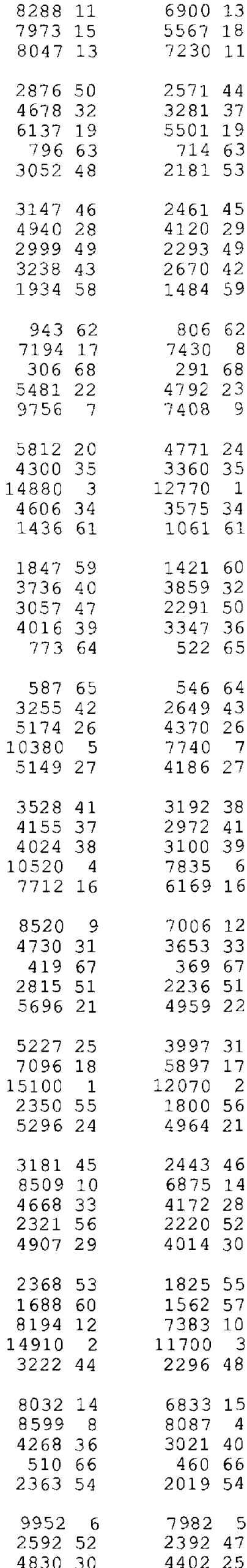

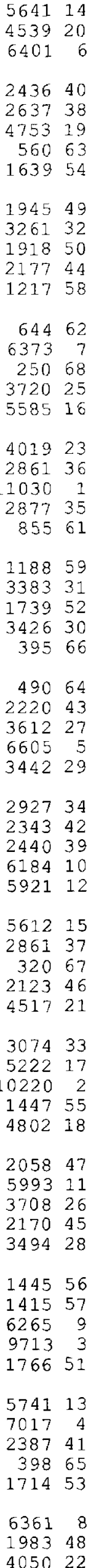

60534

481212

$2168 \quad 38$

173542

361220

37664

137851

267430

143850

92059

$467 \quad 62$

$4527 \quad 13$

21068

$3436 \quad 23$

383919

337524

238635

109801

$2447 \quad 34$

115956

331225

151149

2491
273

$406 \quad 63$

$1686 \quad 43$

318727

$\begin{array}{lr}5300 & 7 \\ 3286 & 26\end{array}$

235936

164946

$1670 \quad 45$

$\begin{array}{ll}5615 & 5 \\ 5169 & 9\end{array}$

489811

$2284 \quad 37$

24267

83940

388418

248333 $4029 \quad 17$

81142

121354

358021

175441

$4406 \quad 15$

288129

$1008 \quad 39$

264931

113357

$1268 \quad 52$

$4521 \quad 14$

70213
125753

408116

501610

$1628 \quad 47$

32565
119655

$5360 \quad 6$

156648

344922 
THIS PAGE INTENTIONALLY BLANK 
LOCATION.--Lat $30^{\circ} 23^{\prime} 34^{\prime \prime}$, long $82^{\circ} 56^{\prime} 00^{\prime \prime}$, in NE $1 / 4 \mathrm{sec} .20$. T.1 S., R. 14 E.. Suwannee County, Hydrologic Unit 03110201 , on left bank $0.2 \mathrm{mi}$ upstream from springs at town of Suwannee Springs, $7.5 \mathrm{mi}$ north of Live Oak, and $150 \mathrm{mi}$ upstream from mouth.

DRAINAGE AREA.--2,630 $\mathrm{mi}^{2}$, approximately, includes part of watershed in Okefenokee Swamp, which is indeterminate.

PERIOD OF RECORD.--October 1974 to 1993. Prior to November 1960, six miscellaneous discharge measurements made in 1906.1951 and 1956. November 1960 to September 1974 (gage heights and discharge measurements only).

REVISED RECORDS.--WSP 2105: WDR FL-75-1: Drainage area.

GAGE.--Water-stage recorder. Datum of gage is National Geodetic Vertical Datum of 1929 (Florida Department of Transportation bench mark). Dec. 13 , 1960 to Apr. 26, 1972 and Aug. 21, 1974 to May 8, 1980, auxiliary nonrecording gage 0.3 mi downstream from base gage at same datum. Since Oct. 1, 1982 water stage recorder at Suwannee River at Ellaville (02319500), about $23 \mathrm{mi}$. downstream from base gage, used to determine discharge during backwater conditions.

REMARKS.--Records good, except for estimated daily discharges, which are fair. Flow affected by backwater from Withlacoochee and Alapaha Rivers at times,

MAXIMUM, MINIMUM, AND MEAN OF MONTHLY MEAN ELEVATIONS FOR WATER YEARS 1970-1993

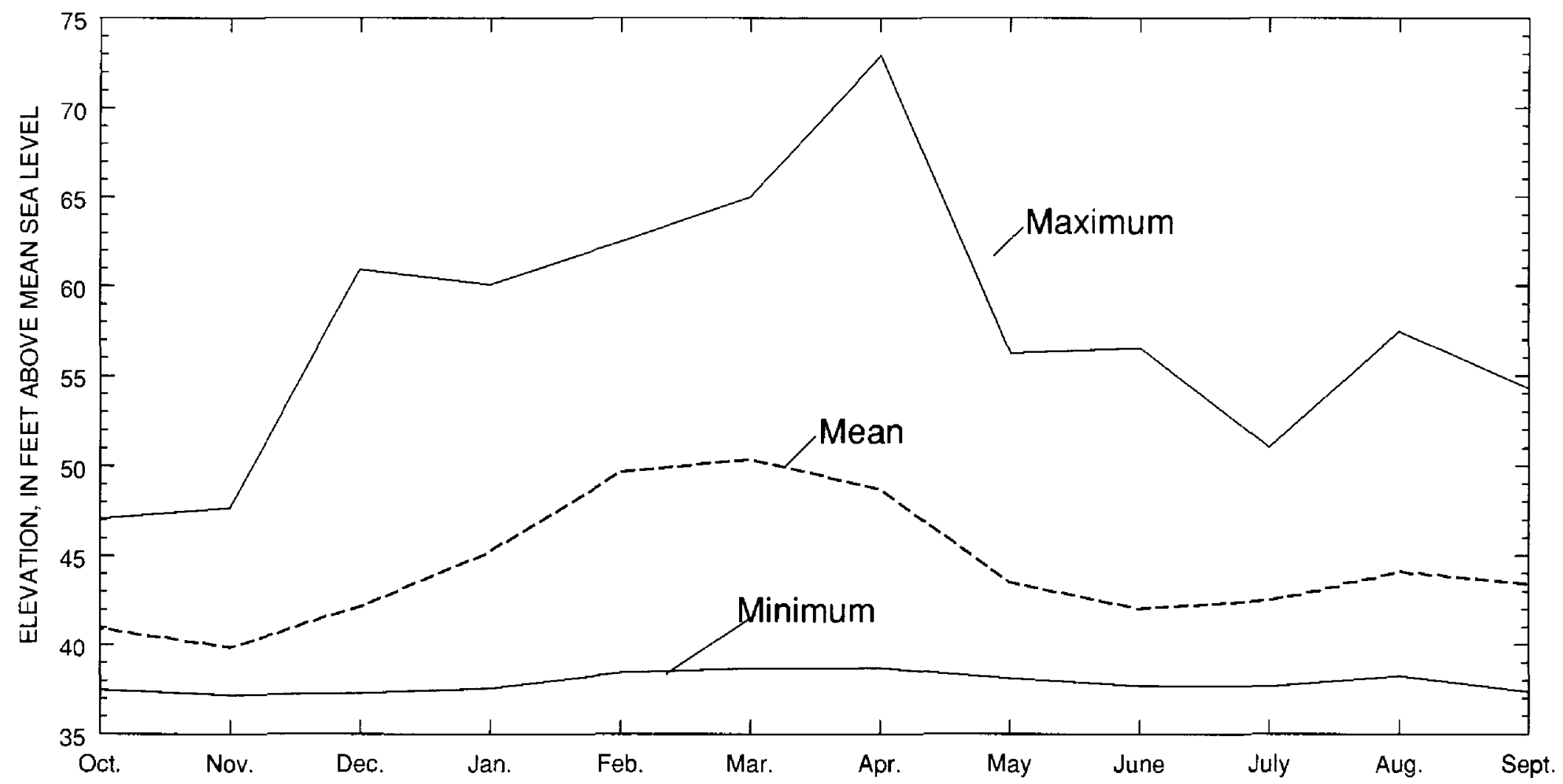

DURATION OF DAILY MEAN ELEVATION FOR WATER YEARS 1970-1993

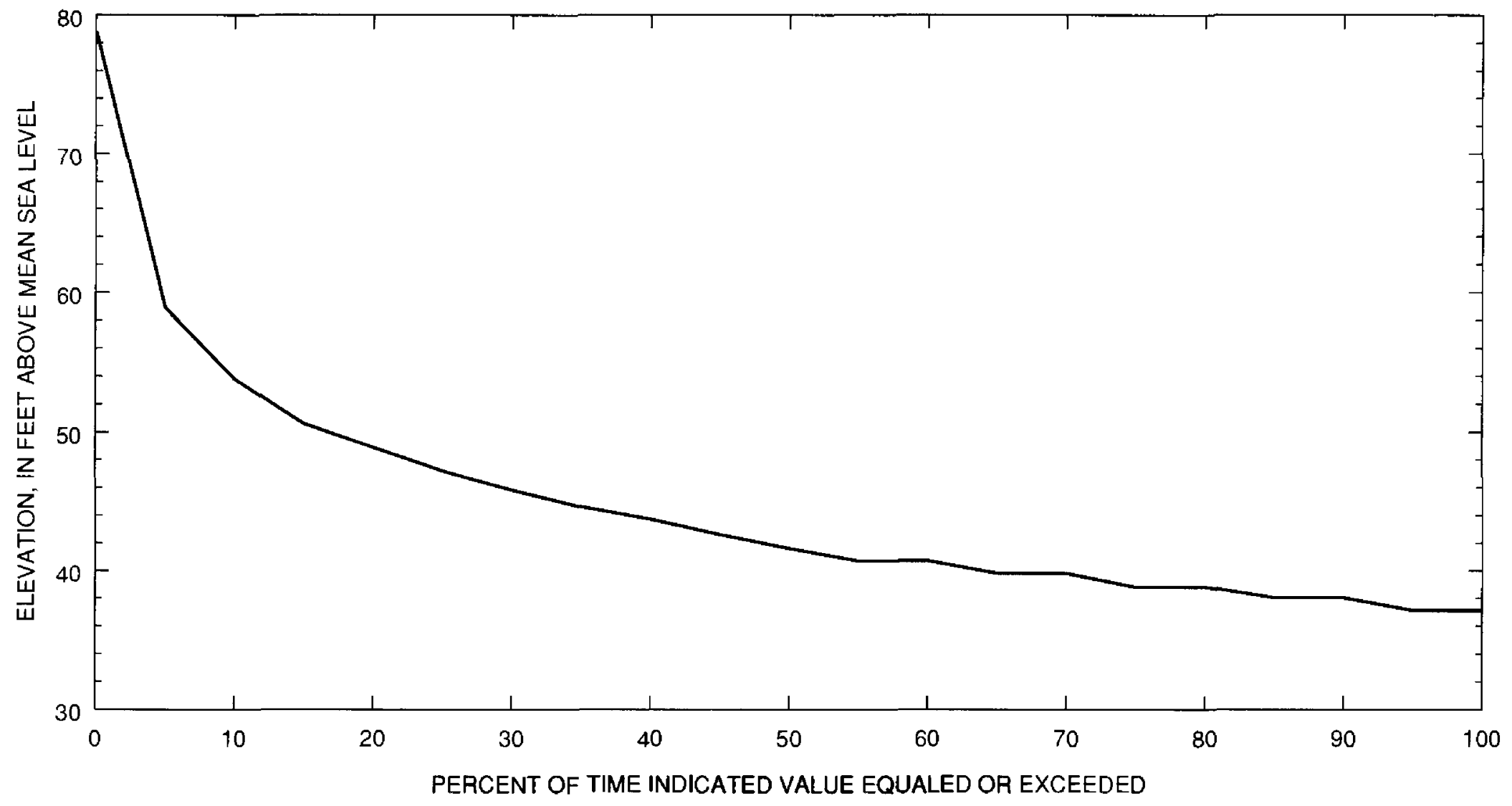


SUMMARY STATISTICS, IN CUBIC FEET PER SECOND UNLESS OTHERWISE INDICATED, FOR WATER YEARS 1975 - 1993

\begin{tabular}{|c|c|c|c|}
\hline NNUAL MEAN & 1852 & & \\
\hline HIGHEST ANNUAL MEAN & 3761 & & 1991 \\
\hline LOWEST ANNUAL MEAN & 340 & & 9 \\
\hline HIGHEST DAILY MEAN & 17800 & Apr 10 & \\
\hline LOWEST DAILY MEAN & 49 & Oct 5 & \\
\hline AL SEVEN-DF & 5 & Sep 29 & \\
\hline NTANEOUS PEAK ELOW & 30100 & Apr 12 & 1 \\
\hline INSTANTANEOUS PEAK ELEVATION & 78.91 & Apr 12 & \\
\hline TANTANEOUS LOW FLOW & 46 & oct & 0 \\
\hline NNUAL RUNOFE (INCHES/CSFM) & (. & & \\
\hline
\end{tabular}

MAXIMUM, MINIMUM, AND MEAN OF MONTHLY MEAN DISCHARGES FOR WATER YEARS 1975-1993

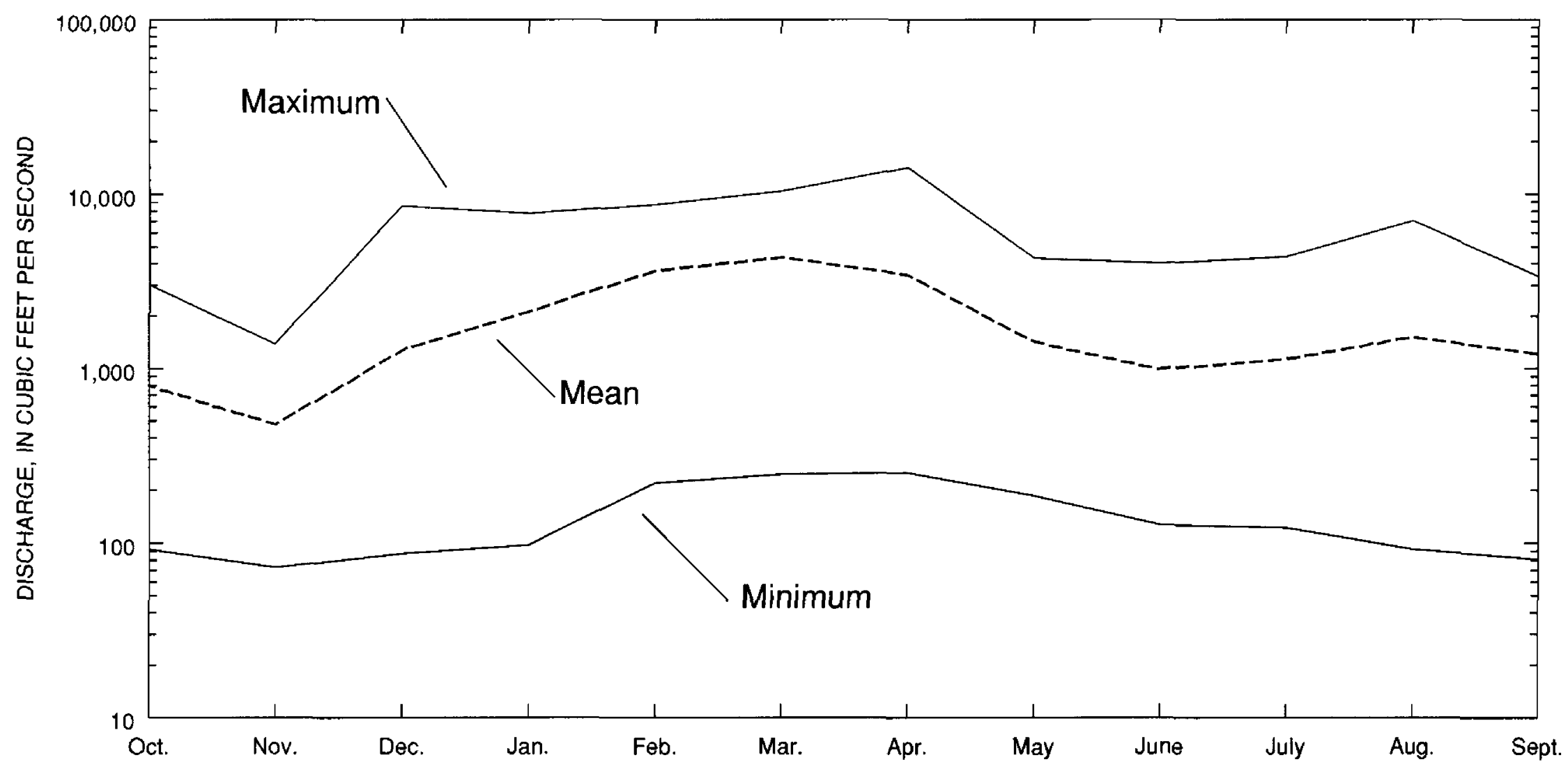

DURATION OF DAILY MEAN DISCHARGE FOR WATER YEARS 1975-1993

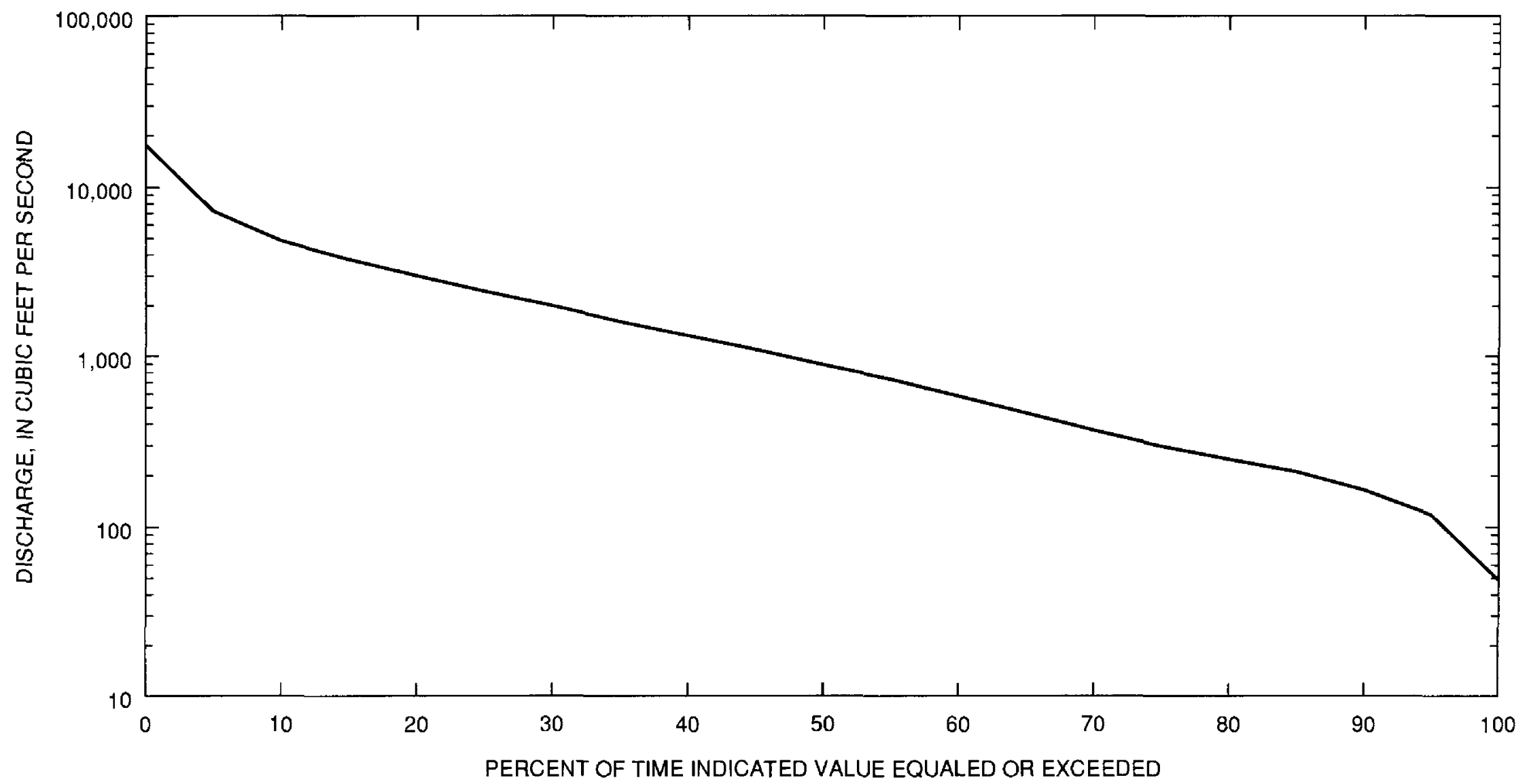



FOR WATER YEARS 1975-1993

PERCENT

OF TIME

EQUALED OR

EXCEEDED ANNUAL
ELEVATIONS,

FEET ABOVE SEA LEVEL

\begin{tabular}{lccc}
\multicolumn{1}{c}{ MONTH } & MAXIMUM & MINIMUM & MEAN \\
OCTOBER & 47.03 & 37.46 & 40.92 \\
NOVEMBER & 47.61 & 37.16 & 39.85 \\
DECEMBER & 60.86 & 37.28 & 42.15 \\
JANUARY & 60.00 & 37.54 & 45.25 \\
FEBRUARY & 62.42 & 38.44 & 49.67 \\
MARCH & 64.94 & 38.65 & 50.35 \\
APRIL & 72.81 & 38.69 & 48.72 \\
MAY & 56.18 & 38.11 & 43.49 \\
JUNE & 56.47 & 37.66 & 42.03 \\
JULY & 50.98 & 37.64 & 42.50 \\
AUGUST & 57.39 & 38.23 & 44.07 \\
SEPTEMBER & 54.24 & 37.35 & 43.41
\end{tabular}

DISCHARGE,

CUBIC FEET PER SECOND

$\begin{array}{rrr}\text { MAXIMUM } & \text { MTNIMUM } & \text { MEAN } \\ 2983 & 92.0 & 798 \\ 1369 & 72.7 & 475 \\ 8474 & 87.1 & 1278 \\ 7684 & 97.3 & 2124 \\ 8629 & 220 & 3631 \\ 10260 & 246 & 4315 \\ 14030 & 251 & 3439 \\ 4229 & 186 & 1433 \\ 3960 & 127 & 987 \\ 4331 & 122 & 1130 \\ 6963 & 92.4 & 1517 \\ 3331 & 80.9 & 1216\end{array}$

DURATION OF DAILY MEAN VALUES FOR WATER YEARS 1975-1993

NOV DEC JAN FEB MAR APR MAY JUNE JULY AUG SEPT

ELEVATION IN FEET ABOVE MEAN SEA LEVEL

$\begin{array}{rlll}95.0 & 37.1 & 37.2 & 37.1 \\ 90.0 & 38.0 & 37.5 & 37.5 \\ 85.0 & 38.0 & 37.9 & 37.8 \\ 80.0 & 38.8 & 38.3 & 37.8 \\ 75.0 & 38.8 & 38.3 & 38.2 \\ 70.0 & 39.8 & 38.7 & 38.5 \\ 65.0 & 39.8 & 39.0 & 38.5 \\ 60.0 & 40.7 & 39.0 & 38.5 \\ 55.0 & 40.7 & 39.8 & 38.9 \\ 50.0 & 41.6 & 39.8 & 38.9 \\ 45.0 & 42.6 & 40.2 & 39.2 \\ 40.0 & 43.7 & 40.6 & 39.6 \\ 35.0 & 44.6 & 41.0 & 39.9 \\ 30.0 & 45.8 & 41.8 & 40.3 \\ 25.0 & 47.2 & 42.2 & 40.7 \\ 20.0 & 48.9 & 42.6 & 41.1 \\ 15.0 & 50.6 & 43.5 & 41.4 \\ 10.0 & 53.8 & 44.8 & 42.2 \\ 5.0 & 58.9 & 47.9 & 44.2\end{array}$

$\begin{array}{ll}37.1 & 37.2 \\ 37.1 & 38.4 \\ 37.7 & 39.0 \\ 37.7 & 39.0 \\ 38.4 & 40.2 \\ 38.4 & 40.2 \\ 39.0 & 40.8 \\ 39.0 & 41.4 \\ 39.6 & 42.1 \\ 39.6 & 42.7 \\ 40.3 & 43.4 \\ 41.0 & 44.7 \\ 42.3 & 46.1 \\ 42.3 & 48.3 \\ 44.4 & 49.8 \\ 45.9 & 51.3 \\ 45.7 & 52.9 \\ 47.5 & 54.5 \\ 50.7 & 59.8\end{array}$

$$
\begin{aligned}
& 37.9 \\
& 40.1 \\
& 40.8 \\
& 42.3 \\
& 43.1 \\
& 44.8 \\
& 45.6 \\
& 45.6 \\
& 47.3 \\
& 48.2 \\
& 50.0 \\
& 50.9 \\
& 50.9 \\
& 51.9 \\
& 54.3 \\
& 56.5 \\
& 58.9 \\
& 51.3 \\
& 63.4
\end{aligned}
$$

39.0
41.1
42.6
43.3
44.1
44.9
45.7
47.3
48.1
49.0
49.9
50.7
51.6
53.5
54.4
55.4
57.7
60.6
64.1

38.4
40.1
40.1
41.0
41.9
41.9
42.8
43.7
43.7
44.7
46.8
47.9
49.1
50.6
53.1
56.1
59.9
62.8
65.3

37.7
38.3
38.3
39.0
39.0
39.7
40.4
41.1
41.1
41.8
42.5
43.3
44.0
44.0
45.6
46.3
47.2
48.8
54.1

DISCHARGE IN CUBIC EEET PER SECOND

$\begin{array}{rrr}95.0 & 118.5 & 88.1 \\ 90.0 & 165.0 & 108.5 \\ 85.0 & 211.7 & 138.0 \\ 80.0 & 249.3 & 172.4 \\ 75.0 & 297.1 & 206.5 \\ 70.0 & 367.2 & 252.5 \\ 65.0 & 464.8 & 288.5 \\ 60.0 & 585.2 & 330.1 \\ 55.0 & 732.0 & 398.1 \\ 50.0 & 888.6 & 463.1 \\ 45.0 & 1094.5 & 529.4 \\ 40.0 & 1323.4 & 620.3 \\ 35.0 & 1602.6 & 758.5 \\ 30.0 & 1994.4 & 926.1 \\ 25.0 & 2438.9 & 1088.7 \\ 20.0 & 3007.3 & 1223.5 \\ 15.0 & 3764.1 & 1427.4 \\ 10.0 & 4841.3 & 1860.4 \\ 5.0 & 7230.1 & 3120.9\end{array}$

$\begin{array}{rrr}72.8 & 90.6 & 118.8 \\ 83.1 & 109.3 & 217.2 \\ 124.8 & 135.2 & 255.3 \\ 169.7 & 174.0 & 284.0 \\ 200.5 & 218.1 & 330.9 \\ 220.1 & 243.8 & 403.5 \\ 239.7 & 267.0 & 635.1 \\ 253.4 & 308.8 & 761.5 \\ 268.1 & 373.6 & 884.9 \\ 291.6 & 472.3 & 1047.5 \\ 339.1 & 553.6 & 1201.2 \\ 404.7 & 659.8 & 1489.5 \\ 469.4 & 834.6 & 2044.1 \\ 560.5 & 1103.0 & 2449.1 \\ 673.9 & 1454.7 & 3446.2 \\ 779.2 & 2082.7 & 4341.9 \\ 902.2 & 2420.6 & 4917.3 \\ 1060.0 & 2940.1 & 5841.4 \\ 1336.7 & 5381.6 & 7579.4\end{array}$

$\begin{array}{rrr}239.9 & 296.2 & 273.2 \\ 536.2 & 725.5 & 515.1 \\ 639.9 & 1268.0 & 622.5 \\ 853.1 & 1542.6 & 740.6 \\ 1420.2 & 1772.5 & 902.7 \\ 1759.3 & 2080.0 & 1056.4 \\ 2021.2 & 2483.8 & 1240.6 \\ 2229.9 & 2809.7 & 1480.0 \\ 2474.9 & 3073.7 & 1753.5 \\ 3048.7 & 3319.2 & 2130.6 \\ 3547.8 & 3676.9 & 2536.4 \\ 3962.9 & 4096.0 & 2867.3 \\ 4346.4 & 4689.6 & 3220.0 \\ 4702.1 & 5625.0 & 3711.2 \\ 5056.7 & 6473.0 & 4459.2 \\ 5867.9 & 7055.1 & 5395.0 \\ 6619.0 & 8241.5 & 7597.8 \\ 8119.5 & 9554.5 & 8958.1 \\ 9130.4 & 11205.4 & 10191.7\end{array}$

173.3
193.6
218.1
269.6
354.8
410.9
461.5
553.6
713.1
844.9
1076.8
1331.8
1516.3
1701.2
2005.2
2334.7
2781.2
3367.7
4514.0

134.8
148.6
164.4
187.7
224.1
290.7
329.4
353.2
426.9
497.1
650.7
921.0
1020.7
1146.8
1273.7
1445.2
1725.0
2075.0
3835.6

$\begin{array}{rr}129.3 & 103.0 \\ 140.3 & 151.8 \\ 159.0 & 170.5 \\ 216.5 & 198.5 \\ 268.1 & 246.4 \\ 299.6 & 331.5 \\ 328.9 & 441.7 \\ 365.6 & 559.9 \\ 426.4 & 689.5 \\ 551.5 & 836.4 \\ 800.0 & 952.1 \\ 942.0 & 1226.7 \\ 1116.9 & 1427.4 \\ 1393.4 & 1836.9 \\ 1707.6 & 2282.7 \\ 2065.3 & 2702.0 \\ 2564.1 & 3205.4 \\ 2952.0 & 3716.6 \\ 3608.7 & 5909.9\end{array}$

5909.9

\section{LOWEST MEAN ELEVATION, IN FEET, AND RANKING FOR THE FOLLOW
FOR PERIOD APR TO MAR}

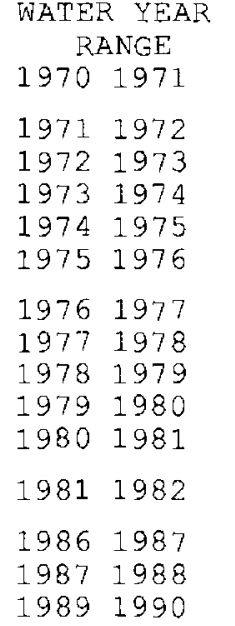

\begin{tabular}{rrrrr}
1 & & \multicolumn{2}{c}{3} \\
39.2 & 14 & & 39.3 & 14 \\
38.9 & 12 & & 39.0 & 12 \\
38.4 & 9 & & 38.4 & 9 \\
38.6 & 10 & & 38.7 & 10 \\
38.2 & 7 & & 38.2 & 7 \\
39.2 & 13 & & 39.2 & 13 \\
38.7 & 11 & & 38.7 & 11 \\
38.0 & 5 & & 38.0 & 5 \\
37.1 & 1 & & 37.1 & 1 \\
39.9 & 15 & & 40.0 & 15 \\
37.9 & 4 & & 37.9 & 4 \\
37.3 & 2 & & 37.3 & 2 \\
38.0 & 6 & & 38.0 & 6 \\
38.3 & 8 & 38.3 & 8 \\
37.6 & 3 & 37.6 & 3
\end{tabular}

$\begin{array}{rr}7 & \\ 39.3 & 14 \\ 39.1 & 12 \\ 38.4 & 9 \\ 38.7 & 10 \\ 38.2 & 7 \\ 39.3 & 13 \\ 38.8 & 11 \\ 38.0 & 5 \\ 37.1 & 1 \\ 40.2 & 15 \\ 37.9 & 4 \\ 37.4 & 2 \\ 38.0 & 6 \\ 38.3 & 8 \\ 37.6 & 3\end{array}$

$\begin{array}{rr}14 & \\ 39.3 & 13 \\ 39.3 & 14 \\ 38.5 & 9 \\ 38.7 & 10 \\ 38.2 & 7 \\ 39.3 & 12 \\ 39.0 & 11 \\ 38.1 & 5 \\ 37.1 & 1 \\ 40.5 & 15 \\ 38.0 & 4 \\ 37.4 & 2 \\ 38.1 & 6 \\ 38.4 & 8 \\ 37.6 & 3\end{array}$

\begin{tabular}{rr}
\multicolumn{3}{c}{30} \\
39.4 & 12 \\
39.6 & 14 \\
38.6 & 9 \\
38.8 & 10 \\
38.3 & 7 \\
39.3 & 11 \\
39.5 & 13 \\
38.2 & 5 \\
37.2 & 1 \\
40.8 & 15 \\
38.1 & 4 \\
37.5 & 2 \\
38.2 & 6 \\
38.5 & 8 \\
37.7 & 3
\end{tabular}

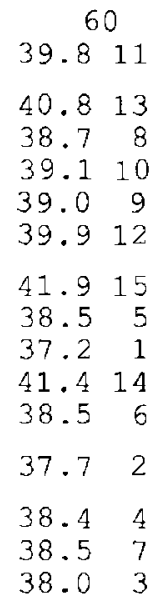
$40.2^{90} 11$
41.914
39.18
39.49
$\begin{array}{ll}39.8 & 10 \\ 40.5 & 12\end{array}$
42.315
$\begin{array}{lll}38.5 & 4 \\ 37.3 & 1\end{array}$
41.513
39.07
37.82
$\begin{array}{ll}39.0 & 6 \\ 38.6 & 5\end{array}$

120

$\begin{array}{ll}39.6 & 8 \\ 39.8 & 9\end{array}$

40.811

41.312

$\begin{array}{rr}42.3 & 13 \\ 38.9 & 5\end{array}$

37.31

42.314

38.12

39.06

$\begin{array}{ll}38.6 & 4\end{array}$ 
HIGHEST MEAN ELEVATION, IN FEET, AND RANKING FOR THE FOLLOWING NUMBER OF CONSECUTIVE DAYS FOR PERIOD OCT TO SEP

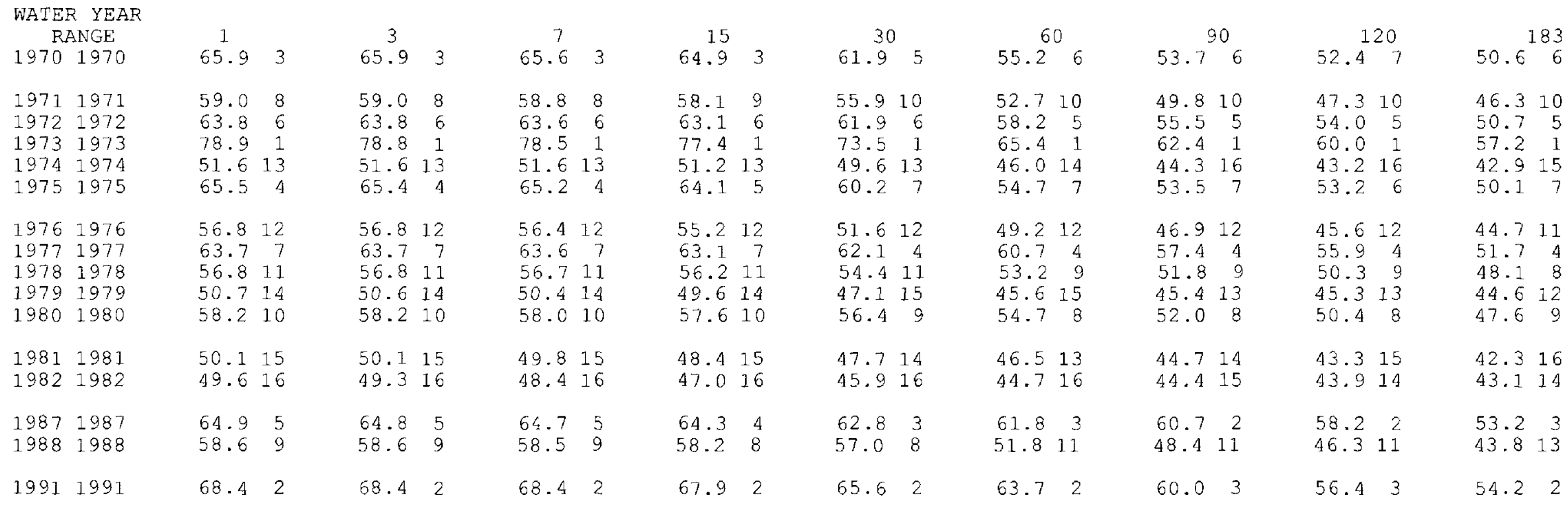

LOWEST MEAN DISCHARGE, IN CUBIC FEET PER SECOND, AND RANKING FOR THE FOLLOWING NUMBER OF CONSECUTIVE DAYS FOR PERIOD APR TO MAR

WATER YEAR

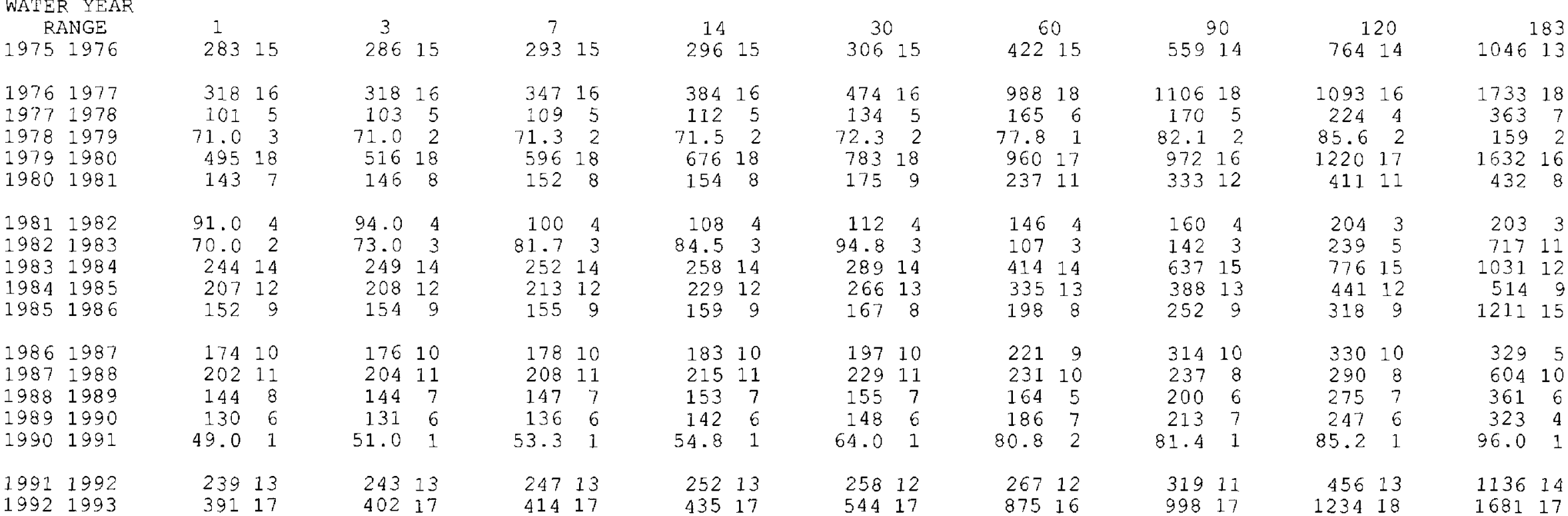

HIGHEST MEAN DISCHARGE, IN CUBIC FEET PER SECOND, AND RANKING FOR THE FOLLOWING NUMBER OF CONSECUTIVE DAYS FOR PERIOD OCT TO SEP

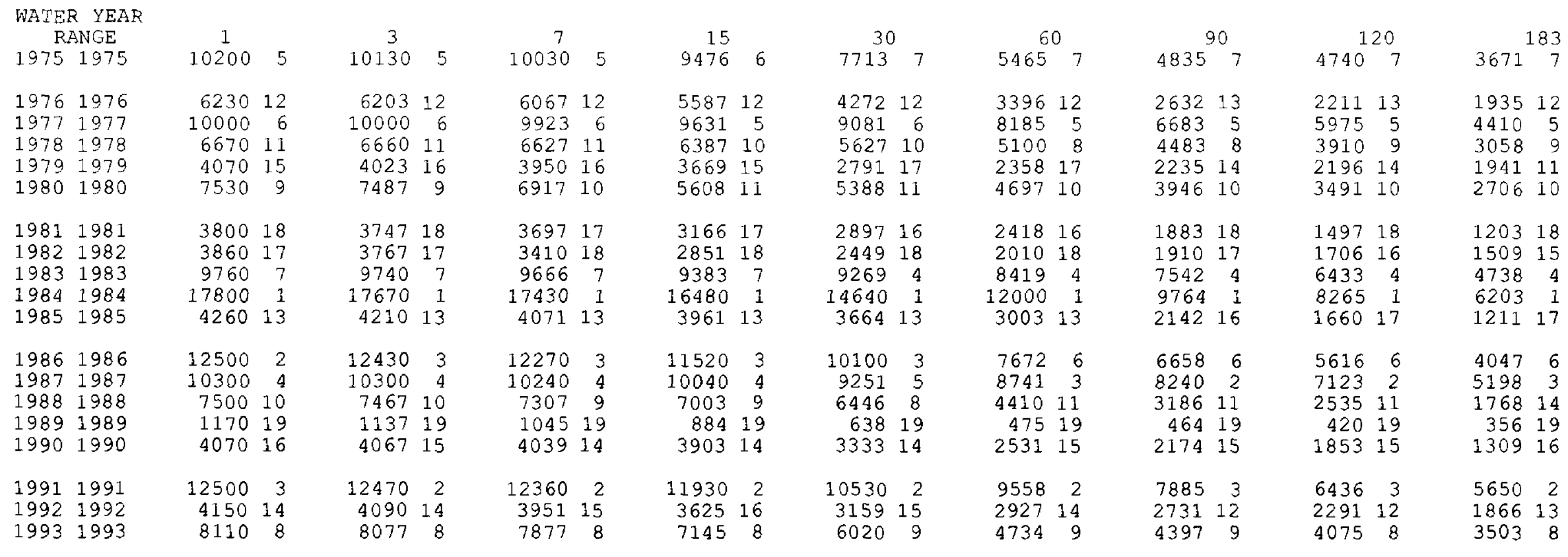


LOCATION.--Lat $30^{\circ} 35^{\prime} 53^{\prime \prime}$, long $83^{\circ} 04^{\prime} 24^{\prime \prime}$, in SW${ }^{1} / 4$ sec.1, T.2 N., R.12 E., Hamilton County, Hydrologic Unit 03110202, near left bank on downstream side of bridge on State Highway I50, $150 \mathrm{ft}$ upstream from Southern Railroad bridge, 1,400 ft downstream from Apalahoochee River, 1.5 mi south of FloridaGeorgia State line, and $1.6 \mathrm{mi}$ southeast of Jennings, and 20.1 mi upstream from mouth.

DRAINAGE AREA.--1,680 $\mathrm{mi}^{2}$, approximately.

PERIOD OF RECORD.--July 1976 to September 1984; October 1984 to September 1985 (gage height and peak discharge only); October 1985 to September 1987 (discontinued). Prior to July 28, 1976 (one miscellaneous discharge measurement in 1923, three in 1928 and six made by Suwannee River Water Management District in 1976).

GAGE.--Water-stage recorder. Datum of gage is $58.22 \mathrm{ft}$ above National Geodetic Vertical Datum of 1929 (Florida Department of Transportation bench mark). Prior to August 18, 1928, nonrecording gage at site $150 \mathrm{ft}$ downstream at datum unknown.

REMARKS.--Records fair.

EXTREMES OUTSIDE PERIOD OF RECORD.--Maximum measured discharge, 17,900 $\mathrm{ft}^{3} / \mathrm{s}$ May 2, 1928, gage height not determined.

MAXIMUM, MINIMUM, AND MEAN OF MONTHEY MEAN ELEVATIONS FOR WATER YEARS 1976-1987
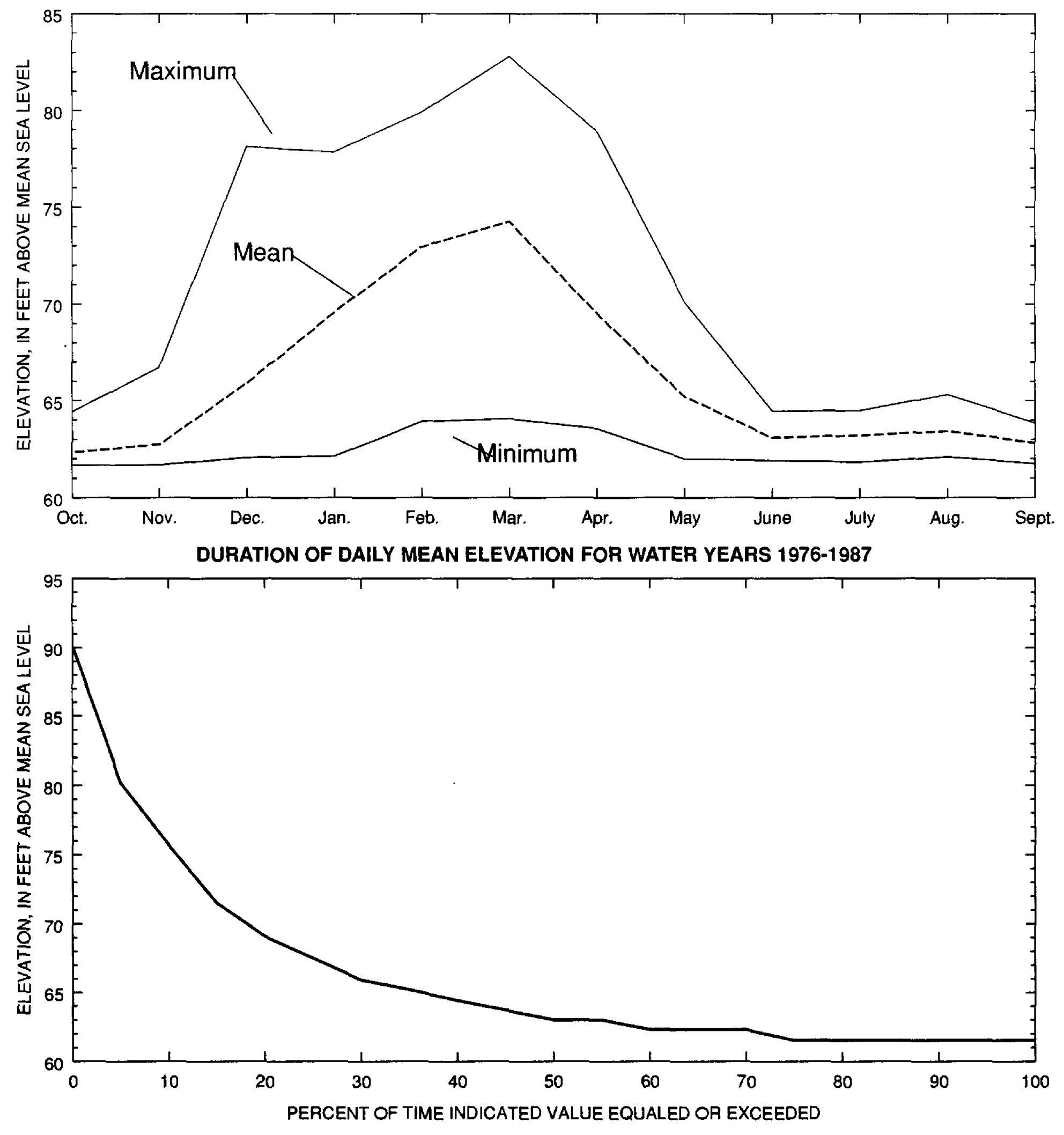
SUMMARY STATISTICS, IN CUBIC FEET PER SECOND UNLESS OTHERWISE INDICATED, FOR WATER YEARS 1976 - 1987

ANNUAL MEAN

HIGHEST ANNUAL MEAN

LOWEST ANNUAL MEAN

HIGHEST DAILY MEAN

LOWEST DAILY MEAN

ANNUAL SEVEN-DAY MINIMUM

INSTANTANEOUS PEAK ELOW

INSTANTANEOUS PEAK ELEVATION (ET)

INSTANTANEOUS LOW FLOW

ANNUAL RUNOFE (INCHES)

ANNUAL RUNOFE (CESM)

1674
2766
273
16100
34
40
18800
90.32
31
13.53
1.00

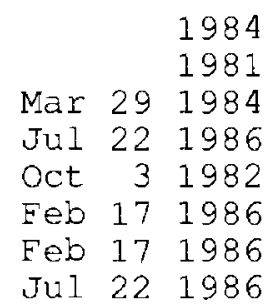

Jul $22 \quad 1986$

MAXIMUM, MINIMUM, AND MEAN OF MONTHLY MEAN DISCHARGES FOR WATER YEARS 1976-1987
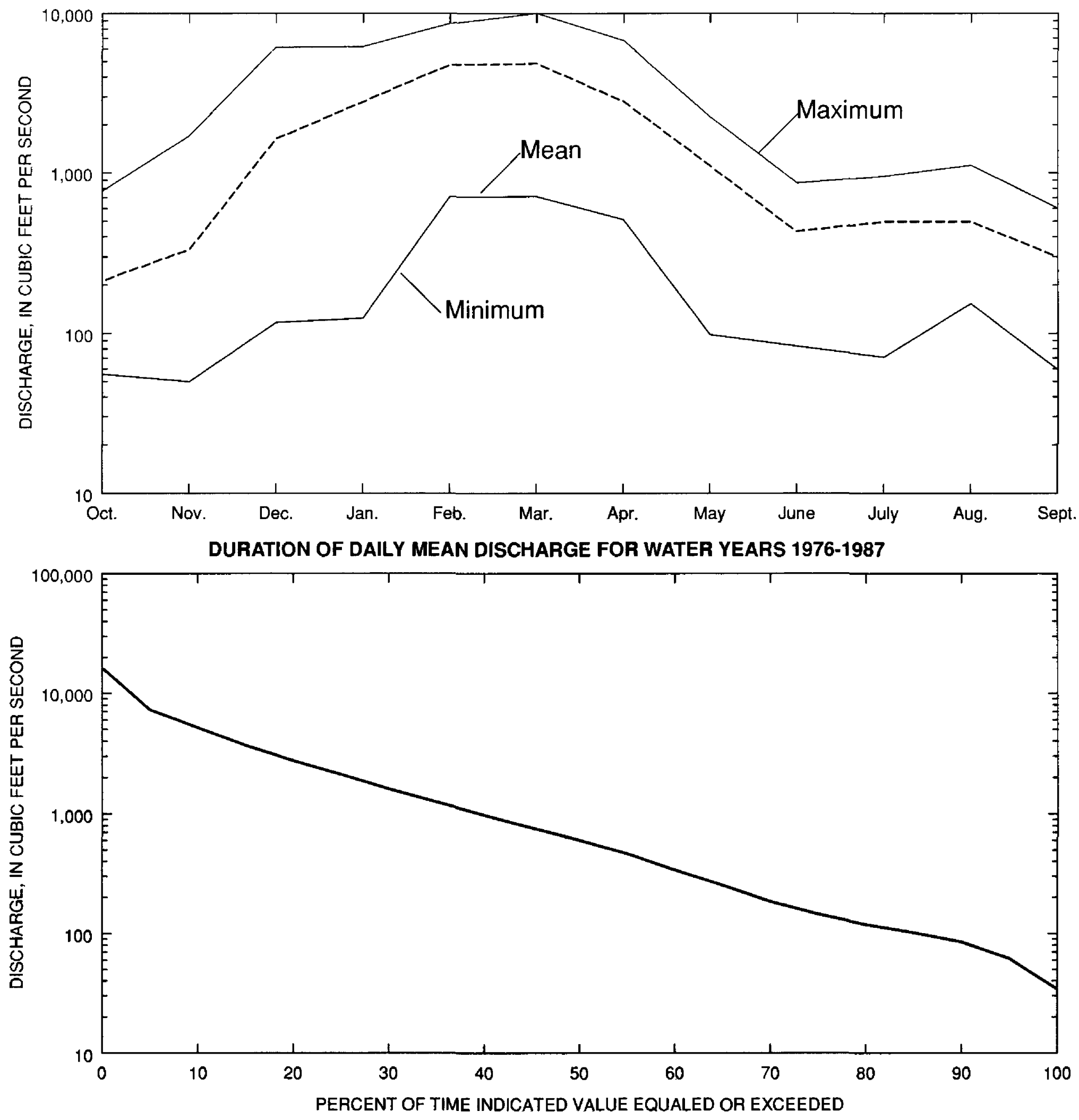
SUWANNEE RIVER BASIN

02317620 ALAPAHA RIVER NEAR JENNINGS, FL--Continued

SUMMARY OF MONTHLY MEAN ELEVATION AND DISCHARGE STATISTICS FOR WATER YEARS 1976-1987

EJ.EVATIONS,

FEET ABOVE SEA LEVEL

\begin{tabular}{lccr}
\multicolumn{1}{c}{ MONTH } & MAXIMUM & MINIMUM & MEAN \\
& & & \\
OCTOBER & 64.38 & 61.67 & 62.33 \\
NOVEMBER & 66.69 & 61.68 & 62.74 \\
DECEMBER & 78.07 & 62.06 & 65.93 \\
JANUARY & 77.79 & 62.12 & 69.59 \\
FEBRUARY & 79.88 & 63.92 & 72.98 \\
MARCH & 82.75 & 64.06 & 74.25 \\
APRIL & 78.86 & 63.54 & 69.50 \\
MAY & 70.06 & 61.98 & 65.22 \\
JUNE & 64.41 & 61.89 & 63.08 \\
JULY & 64.44 & 61.81 & 63.19 \\
AUGUST & 65.28 & 62.08 & 63.42 \\
SEPTEMBER & 63.80 & 61.74 & 62.81
\end{tabular}

DISCHARGE,

CUBIC FEET PER SECOND

\begin{tabular}{rrr} 
MAXIMUM & MINIMUM & \multicolumn{1}{c}{ MEAN } \\
759.5 & 55.6 & 213.15 \\
1683 & 49.9 & 332.68 \\
6081 & 116.6 & 1629.72 \\
6147 & 124.0 & 2798.30 \\
8541 & 707.6 & 4776.76 \\
9935 & 712.1 & 4870.51 \\
6693 & 512.9 & 2826.82 \\
2222 & 98.0 & 1107.02 \\
852.5 & 82.9 & 431.32 \\
942.4 & 71.0 & 496.22 \\
1105 & 152.6 & 497.15 \\
594.5 & 59.1 & 299.15
\end{tabular}

\section{DURATION OF DAILY MEAN VALUES FOR WATER YEARS 1976-1987}

PERCEN'T
OF TIME

EQUALED OR

$\begin{array}{ccc}\text { EQUALED OR } & & \\ \text { EXCEEDED } & \text { ANNUAL } & \text { OCT } \\ & & \\ & & \\ & & \\ 95.0 & 61.5 & 61.5 \\ 90.0 & 61.5 & 61.5 \\ 85.0 & 61.5 & 61.7 \\ 80.0 & 61.5 & 61.7 \\ 75.0 & 61.5 & 61.7 \\ 70.0 & 62.3 & 61.7 \\ 65.0 & 62.3 & 61.7 \\ 60.0 & 62.3 & 61.8 \\ 55.0 & 63.0 & 61.8 \\ 50.0 & 63.0 & 62.0 \\ 45.0 & 63.7 & 62.0 \\ 40.0 & 64.4 & 62.0 \\ 35.0 & 65.2 & 62.0 \\ 30.0 & 65.9 & 62.1 \\ 25.0 & 67.5 & 62.4 \\ 20.0 & 69.1 & 62.6 \\ 15.0 & 71.5 & 62.8 \\ 10.0 & 75.7 & 63.7 \\ 5.0 & 80.2 & 65.1\end{array}$

NOV

DEC

JAN

FEB

MAR

ELEVATION IN FEET ABOVE MEAN SEA LEVEE

$\begin{array}{lllll}61.6 & 61.9 & 62.0 & 63.0 & 63.5 \\ 61.6 & 61.9 & 62.0 & 64.4 & 65.6 \\ 61.6 & 61.9 & 64.1 & 65.9 & 66.3 \\ 61.6 & 61.9 & 64.1 & 66.6 & 67.0 \\ 61.6 & 61.9 & 64.7 & 68.1 & 67.7 \\ 61.6 & 61.9 & 65.8 & 68.9 & 69.1 \\ 61.6 & 62.4 & 66.4 & 69.6 & 69.8 \\ 61.6 & 63.0 & 66.9 & 69.6 & 70.6 \\ 61.6 & 63.0 & 68.1 & 70.4 & 71.3 \\ 62.2 & 64.0 & 68.7 & 71.2 & 73.6 \\ 62.2 & 64.6 & 69.3 & 72.8 & 75.2 \\ 62.2 & 65.1 & 69.9 & 73.6 & 76.0 \\ 62.2 & 66.2 & 70.4 & 74.5 & 77.6 \\ 62.2 & 66.2 & 71.1 & 76.1 & 79.2 \\ 62.8 & 67.9 & 72.3 & 77.9 & 80.1 \\ 62.8 & 68.5 & 74.2 & 79.6 & 80.9 \\ 63.3 & 72.0 & 75.4 & 81.4 & 81.8 \\ 63.9 & 75.1 & 77.4 & 84.2 & 81.8 \\ 64.5 & 77.7 & 79.4 & 86.1 & 84.4\end{array}$

DISCHARGE IN CUBIC FEET PER SECOND

$\begin{array}{rrr}95.0 & 61.7 & 42.7 \\ 90.0 & 85.1 & 46.4 \\ 85.0 & 101.9 & 50.2 \\ 80.0 & 119.1 & 54.7 \\ 75.0 & 145.1 & 59.8 \\ 70.0 & 185.0 & 65.8 \\ 65.0 & 251.8 & 74.1 \\ 60.0 & 338.9 & 84.8 \\ 55.0 & 463.5 & 91.0 \\ 50.0 & 602.4 & 97.5 \\ 45.0 & 763.7 & 104.3 \\ 40.0 & 975.5 & 112.6 \\ 35.0 & 1249.7 & 124.5 \\ 30.0 & 1606.2 & 153.5 \\ 25.0 & 2114.4 & 196.7 \\ 20.0 & 2754.5 & 239.4 \\ 15.0 & 3677.3 & 317.5 \\ 10.0 & 5157.7 & 604.0 \\ 5.0 & 7258.3 & 1062.5\end{array}$

$\begin{array}{rr}50.8 & 100.6 \\ 55.4 & 108.5 \\ 63.2 & 118.3 \\ 73.0 & 138.3 \\ 83.7 & 163.1 \\ 94.5 & 272.7 \\ 102.0 & 343.0 \\ 109.8 & 428.0 \\ 122.2 & 611.5 \\ 135.4 & 928.1 \\ 149.2 & 1185.4 \\ 170.5 & 1434.0 \\ 203.8 & 1611.2 \\ 239.1 & 1792.5 \\ 281.2 & 2209.6 \\ 391.8 & 2908.3 \\ 506.6 & 3960.0 \\ 645.2 & 4703.7 \\ 989.0 & 5816.4\end{array}$

121.2
168.0
759.2
875.0
1091.4
1470.0
1702.5
1937.6
2195.8
2521.9
2722.8
3002.2
3327.3
3601.5
4017.4
4508.9
5150.8
5904.2
6945.6

$\begin{array}{rrr}723.4 & 587.5 & 266.0 \\ 1320.8 & 1240.0 & 421.0 \\ 1650.7 & 1678.3 & 602.0 \\ 2177.8 & 1923.3 & 758.9 \\ 2432.5 & 2182.0 & 935.5 \\ 2649.6 & 2595.0 & 1090.7 \\ 2840.9 & 2827.5 & 1247.8 \\ 3073.0 & 3176.0 & 1381.1 \\ 3348.9 & 3551.5 & 1493.7 \\ 3827.1 & 4289.0 & 1645.0 \\ 4296.6 & 4943.3 & 1970.7 \\ 4689.1 & 5454.3 & 2176.7 \\ 5031.6 & 5969.2 & 2381.2 \\ 5644.0 & 6786.0 & 3197.5 \\ 6882.6 & 7230.3 & 4156.0 \\ 7520.2 & 7667.8 & 5708.6 \\ 8388.6 & 8085.4 & 6761.7 \\ 9838.2 & 8503.1 & 7216.7 \\ 11794.5 & 10442.9 & 8270.0\end{array}$

90.8
104.3
117.9
140.2
172.1
248.0
310.5
403.7
633.7
798.0
930.3
1085.7
1186.7
1331.7
1701.2
2074.4
2353.4
2622.6
3286.4

73.4
80.7
91.3
104.2
127.2
156.3
194.3
247.7
296.9
342.8
389.0
452.0
492.8
572.1
615.0
681.0
778.3
900.5
1102.5

56.3
67.0
82.1
100.0
119.6
132.9
158.7
204.0
280.0
368.4
485.3
537.7
582.9
651.0
728.5
847.6
1006.7
1211.3
1400.0

89.8
102.7
116.6
128.8
147.2
175.8
207.6
234.6
264.8
297.3
360.4
418.5
494.0
598.1
716.7
840.8
990.2
212.8
597.1
APR

SEPT

$\begin{array}{lll}62.3 & 61.8 & 61.7 \\ 62.9 & 61.8 & 61.7 \\ 63.5 & 61.8 & 61.8 \\ 64.1 & 62.1 & 61.8 \\ 64.8 & 62.1 & 61.8 \\ 64.8 & 62.5 & 62.0 \\ 65.4 & 62.9 & 62.2 \\ 65.4 & 62.9 & 62.4 \\ 66.0 & 63.3 & 62.5 \\ 66.7 & 64.1 & 62.9 \\ 67.3 & 64.4 & 62.9 \\ 68.0 & 64.8 & 63.2 \\ 68.6 & 65.2 & 63.2 \\ 70.7 & 65.6 & 63.4 \\ 72.8 & 66.4 & 63.8 \\ 76.4 & 67.6 & 63.9 \\ 78.6 & 68.5 & 64.1 \\ 79.4 & 69.3 & 64.5 \\ 81.8 & 70.6 & 64.8\end{array}$

$61 \cdot 9$
61.9
61.9
62.1
62.1
62.4
62.4
62.6
62.6
62.8
63.0
63.2
63.4
63.9
64.1
64.3
64.7
65.2
66.5
$61 \cdot 6$
$61 \cdot 7$
$61 \cdot 7$
$61 \cdot 9$
62.0
$62 \cdot 0$
62.2
62.3
62.3
62.4
62.4
62.7
62.9
63.2
63.3
63.6
63.7
64.0
64.7

52.9

65.0

79.6
97.0

114.4

128.2

148.7

165.1

180.4

202.1

235.6

285.6

323.5

436.7

436.7
515.0

571.1

647.4
775.9 
SUWANNEE RIVER BASIN

02317620 ALAPAHA RIVER NEAR JENNINGS, FL-Continued

LOWEST MEAN ELEVATION, IN FEET, AND RANKING FOR THE FOLLOWING NUMBER OF CONSECUTIVE DAYS FOR PERIOD APR TO MAR

WATER YEAR

RANGE

$\begin{array}{ll}1977 & 1978 \\ 1978 & 1979\end{array}$

1978
1979
1980

19801981

19811982

1
61.75

61.64

62.16

61.63

$$
\begin{array}{cc}
3 & \\
61.8 & 5 \\
61.6 & 4 \\
62.1 & 6 \\
61.6 & 2
\end{array}
$$

7
$61.8 \quad 5$

14
61.85

61.74

62.36

$\begin{array}{ll} & 30 \\ 61.9 & 5 \\ 61.7 & 2\end{array}$

$\begin{array}{ll}61.7 & 4 \\ 62.2 & 6\end{array}$

61.51

61.61

61.61

$61.6 \quad 1$

$62.6 \quad 6$

61.73

$62.0^{60} 5$

$61.6 \quad 1$

$\begin{array}{ll}63.2 & 6 \\ 61.7 & 2\end{array}$

\begin{tabular}{ll}
\multicolumn{2}{c}{90} \\
62.1 & 5 \\
61.8 & 1 \\
63.5 & 6 \\
61.9 & 3
\end{tabular}

$62.3^{120} 5$

61.91

19861987

61.63

61.63

61.63

61.74

61.73

61.92

62.03

61.92

61.94

62.04

$62.6^{183}$

62.54

$\begin{array}{ll}63.7 & 6 \\ 62.0 & 3\end{array}$

62.01

62.02

HIGHEST MEAN ELEVATION, IN FEET, AND RANKING FOR THE FOLLOWING NUMBER OF CONSECUTIVE DAYS FOR PERIOD OCT TO SEP

WATER YEAR

\begin{tabular}{|c|c|}
\hline $\begin{array}{l}\mathrm{E} \\
\mathrm{R}\end{array}$ & INGE \\
\hline 1977 & 1977 \\
\hline 1978 & 1978 \\
\hline 1979 & 1979 \\
\hline 1980 & 1980 \\
\hline 1981 & 1981 \\
\hline 1982 & 1982 \\
\hline 36 & 86 \\
\hline 1987 & \\
\hline
\end{tabular}

$\begin{array}{cc}1 & \\ 83.9 & 5 \\ 87.7 & 2 \\ 82.8 & 6 \\ 84.1 & 4 \\ 67.5 & 8 \\ 75.7 & 7 \\ 90.1 & 1 \\ 85.4 & 3\end{array}$

$\begin{array}{cccc}3 & & 7 & \\ 83.3 & 5 & 81.4 & 6 \\ 87.6 & 2 & 87.1 & 2 \\ 82.6 & 6 & 81.8 & 5 \\ 83.7 & 4 & 83.0 & 4 \\ 67.2 & 8 & 65.4 & 8 \\ 75.6 & 7 & 75.3 & 7 \\ 89.8 & 1 & 88.8 & 1 \\ 85.2 & 3 & 84.4 & 3\end{array}$

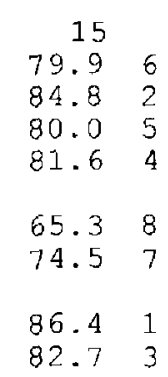

\begin{tabular}{cccc}
30 & \multicolumn{2}{c}{60} \\
78.9 & 5 & 77.7 & 2 \\
79.5 & 4 & 76.6 & 4 \\
77.5 & 6 & 73.3 & 6 \\
81.4 & 1 & 76.7 & 3 \\
64.9 & 8 & 64.6 & 8 \\
72.5 & 7 & 71.4 & 7 \\
80.4 & 3 & 74.9 & 5 \\
80.8 & 2 & 79.9 & 1
\end{tabular}
$74.9^{90}$
$\begin{array}{ll}74.1 & 3 \\ 71.0 & 6\end{array}$
$71.0 \quad 6$
64.18
$73.8 \quad 5$

75.120

72.25

70.16

63.78

$\begin{array}{lll}69.87 & 69.37\end{array}$

$72.5 \quad 3$

$72.0 \quad \begin{array}{r}183 \\ 7\end{array}$

70.23

$\begin{array}{ll}67.9 & 6 \\ 69.8 & 4\end{array}$

63.18

67.57

69.35

LOWEST MEAN DISCHARGE, IN CUBIC FEET PER SECOND, AND RANKING FOR THE FOLLOWING NUMBER OF CONSECUTIVE DAYS FOR PERIOD APR TO MAR

WATER YEAR

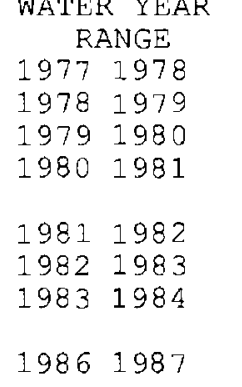

$\begin{array}{rr}1 & \\ 65.0 & 5 \\ 41.0 & 4 \\ 123 & 8 \\ 40.0 & 3 \\ 39.0 & 2 \\ 84.0 & 7 \\ 65.0 & 6 \\ 34.0 & 1\end{array}$

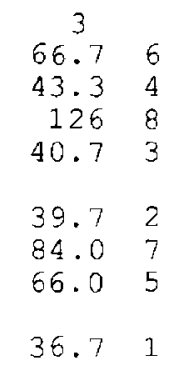

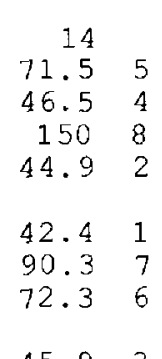

45.93

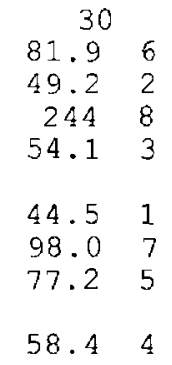

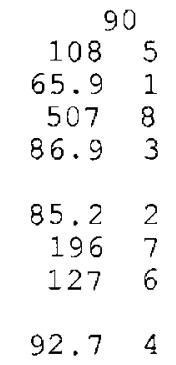

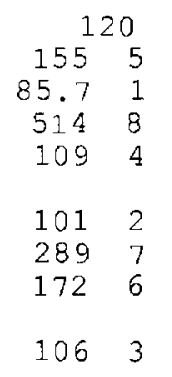

$\begin{array}{lr}258 & 183 \\ 25 & 5 \\ 240 & 4 \\ 575 & 8 \\ 114 & 3 \\ 100 & 1 \\ 505 & 7 \\ 330 & 6 \\ 106 & 2\end{array}$

HIGHEST MEAN DISCHARGE, IN CUBIC FEET PER SECOND, AND RANKING FOR THE FOLLOWING NUMBER OF CONSECUTIVE DAYS FOR PERIOD OCT TO SEP

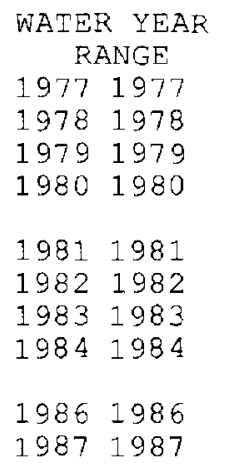

WATER YEAR
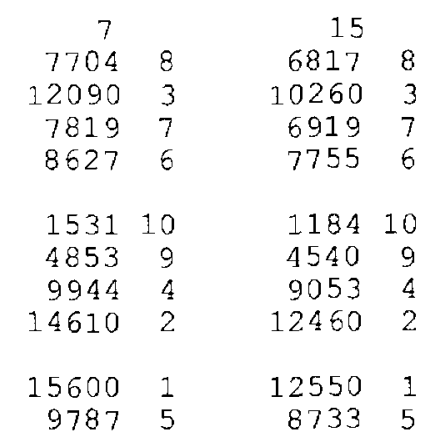
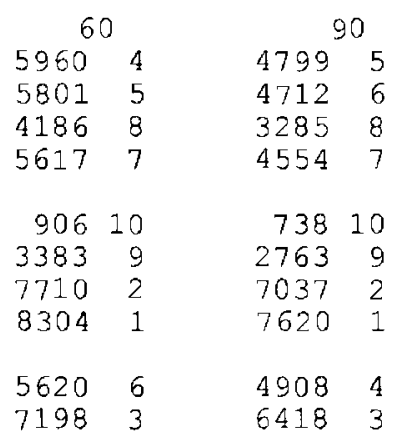

$3660^{183} 4$

30995

$\begin{array}{ll}2111 & 8 \\ 2883 & 7\end{array}$

$436 \quad 10$

$\begin{array}{rr}436 & 10 \\ 19519\end{array}$

$4257 \quad 2$
5031

29236 


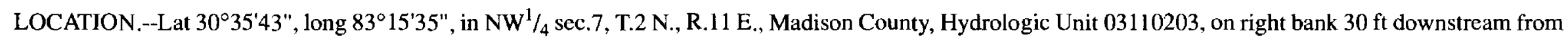
bridge, $0.1 \mathrm{mi}$ downstream from small tributary, $0.3 \mathrm{mi}$ west of Bellville, $5.6 \mathrm{mi}$ east of Pinetta, and $22 \mathrm{mi}$ upstream from mouth.

DRAINAGE AREA.--2,120 $\mathrm{mi}^{2}$, approximately.

PERIOD OF RECORD.--October 1931 to 1993. Monthly discharge only for October and November 1931, published in WSP 1304.

REVISED RECORDS.--WSP 972: 1941-42. WSP 1905: Drainage area.

GAGE.- Water-stage recorder. Datum of gage is $47.21 \mathrm{ft}$ above National Geodetic Vertical Datum of 1929 (levels by Corps of Engineers). Oct. 11, 1931 to Dec. 3, 1941, nonrecording gage at same site and datum. Dec. 3, 1941 to Aug. 2, 1972, water-stage recorder at same site and datum. Aug. 2, 1972 to Apr. 22,1986, nonrecording gage at same site and datum.

REMARKS.--Records good.

EXTREMES OUTSIDE PERIOD OF RECORD.--Flood in August 1928 reached a stage of $36.75 \mathrm{ft}$ from floodmarks, discharge, $53,600 \mathrm{ft}^{3} / \mathrm{s}$.
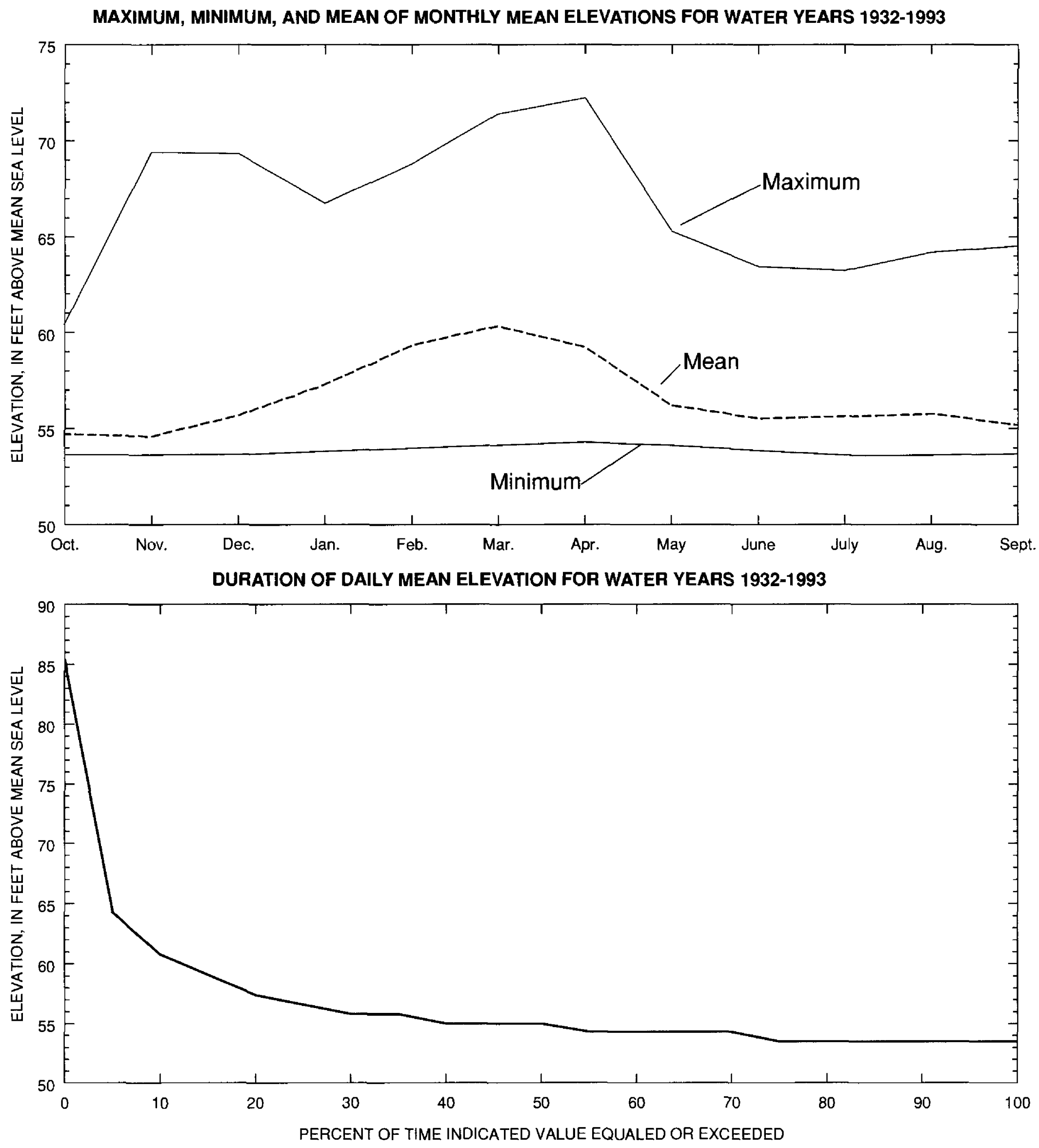
SUMMARY STATISTICS, IN CUBIC FEET PER SECOND UNLESS OTHERWISE INDICATED, FOR WATER YEARS $1932 \cdot 1993$

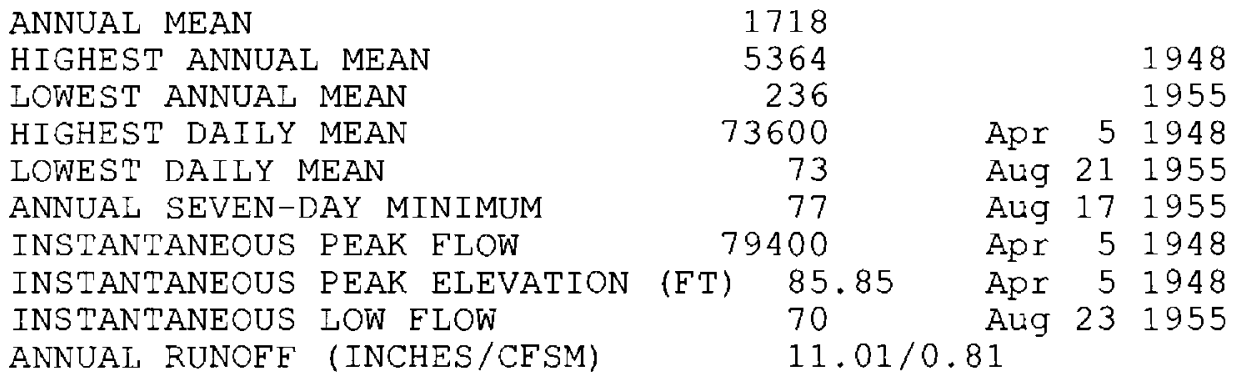

MAXIMUM, MINIMUM, AND MEAN OF MONTHLY MEAN DISCHARGES FOR WATER YEARS 1932-1993

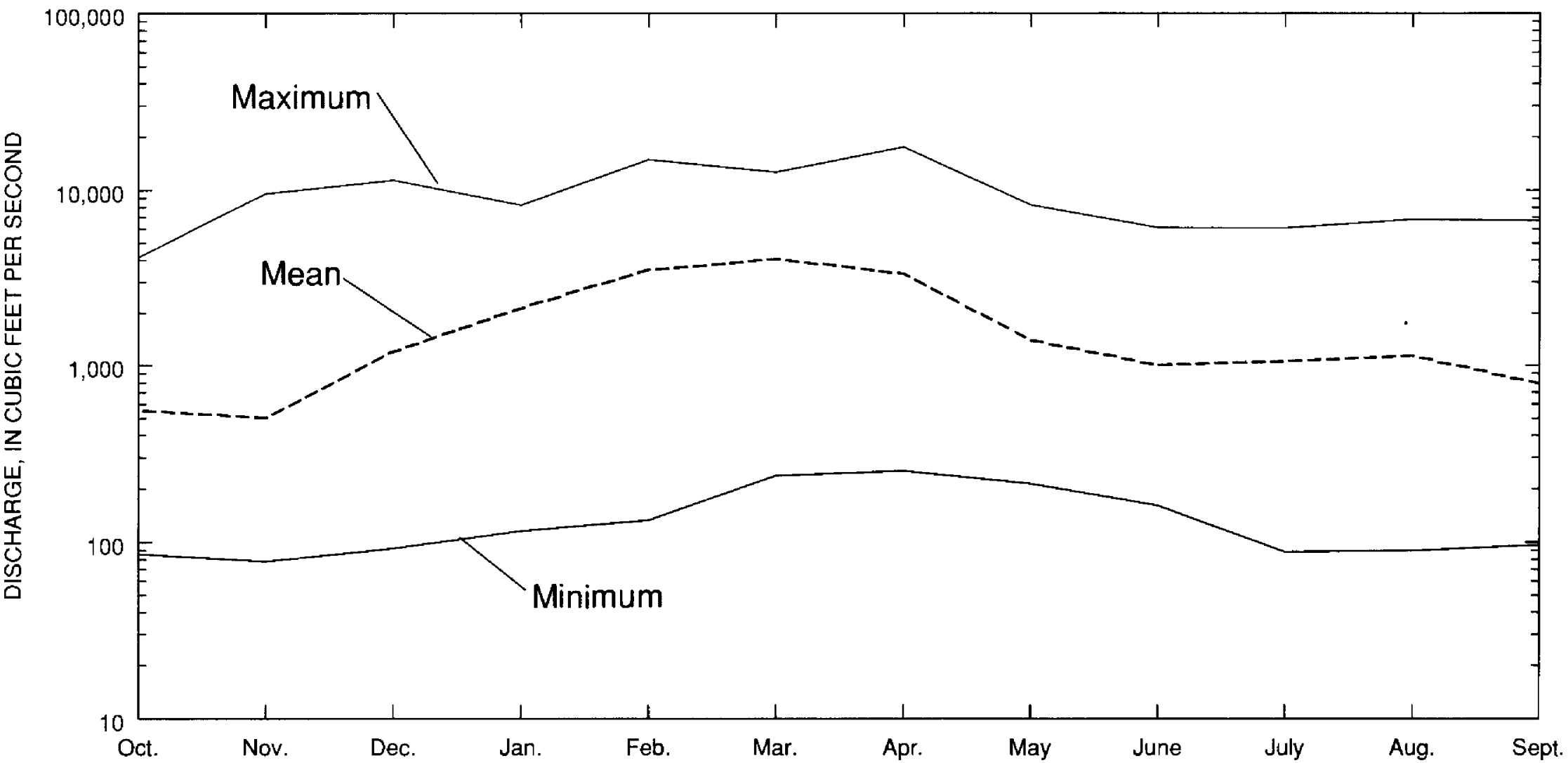

DURATION OF DAILY MEAN DISCHARGE FOR WATER YEARS 1932-1993

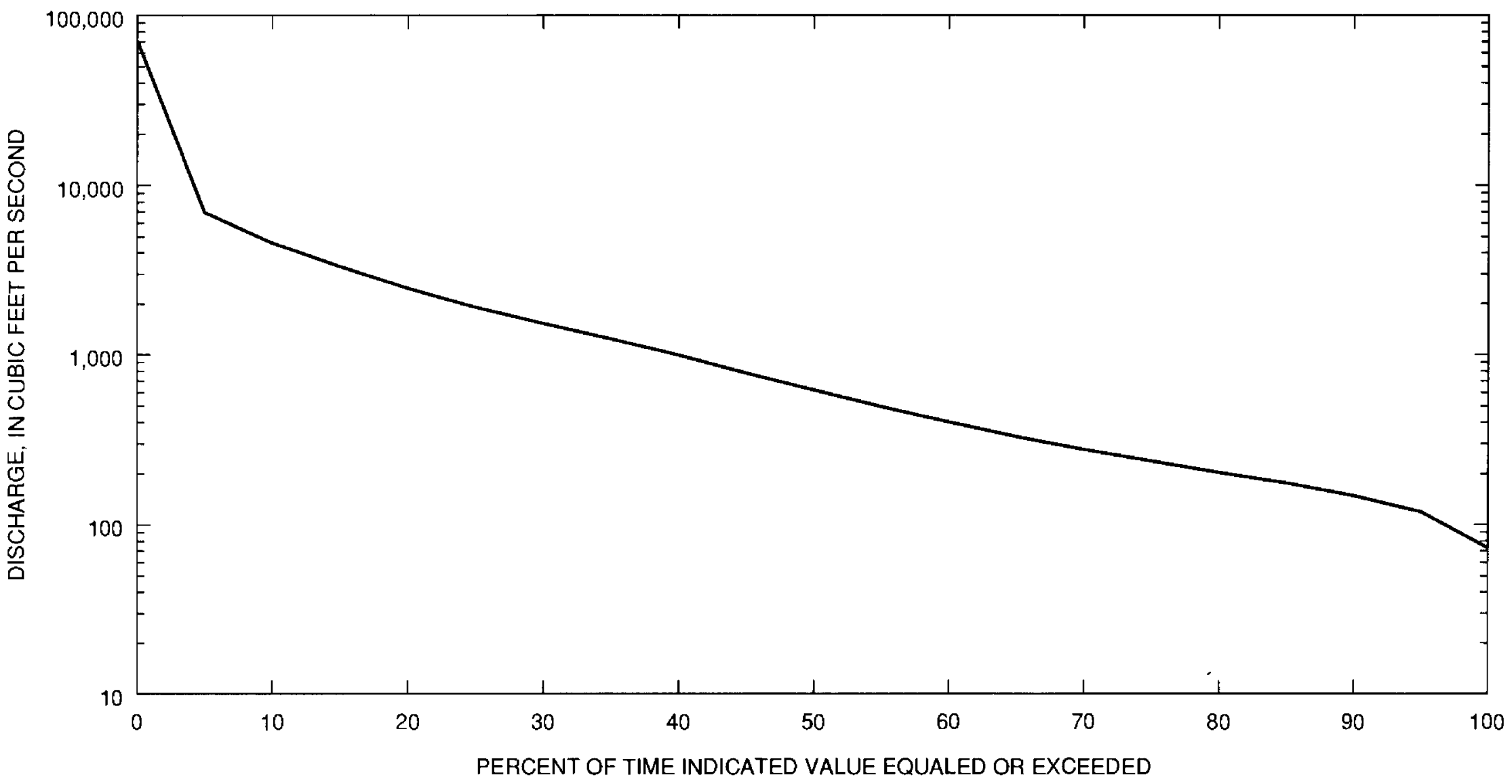



FOR WATER YEARS 1932-1993

\begin{tabular}{lccccrr} 
& \multicolumn{3}{c}{ ELEVATIONS, } & \multicolumn{3}{c}{ DISCHARGE, } \\
& \multicolumn{2}{c}{ FEET ABOVE SEA LEVEL } & \multicolumn{2}{c}{ CUBIC FEET PER SECOND } \\
\multicolumn{1}{c}{ MONTH } & MAXIMUM & MINIMUM & MEAN & MAXIMUM & MINIMUM & MEAN \\
& & & & & & \\
OCTOBER & 60.39 & 53.63 & 54.71 & 4096 & 85.7 & 555 \\
NOVEMBER & 69.39 & 53.63 & 54.59 & 9450 & 78.1 & 503 \\
DECEMBER & 69.31 & 53.66 & 55.70 & 11280 & 92.4 & 1205 \\
JANUARY & 66.72 & 53.82 & 57.29 & 8134 & 116 & 2140 \\
EEBRUARY & 68.78 & 53.98 & 59.34 & 14720 & 133 & 3516 \\
MARCH & 71.38 & 54.14 & 60.31 & 12500 & 238 & 4067 \\
ARRII & 72.19 & 54.29 & 59.23 & 17320 & 253 & 3349 \\
MAY & 65.27 & 54.13 & 56.20 & 8154 & 214 & 1407 \\
JUNE & 63.41 & 53.87 & 55.53 & 6043 & 161 & 1005 \\
JULY & 63.20 & 53.61 & 55.63 & 6003 & 88.3 & 1055 \\
AUGUST & 64.16 & 53.62 & 55.76 & 6759 & 89.7 & 1137 \\
SEPTEMBER & 64.48 & 53.69 & 55.19 & 6625 & 96.5 & 792
\end{tabular}

\section{DURATION OF DAILY MEAN VALUES FOR WATER YEARS 1932-1993}

PERCENT OF TIME

EQJALED OR

EXCFEDED ANNUAL OCT

$\begin{array}{rlll}95.0 & 53.5 & 53.6 & 53.6 \\ 90.0 & 53.5 & 53.6 & 53.6 \\ 85.0 & 53.5 & 53.6 & 53.6 \\ 80.0 & 53.5 & 53.6 & 53.6 \\ 75.0 & 53.5 & 53.6 & 53.6 \\ 70.0 & 54.3 & 53.9 & 53.6 \\ 55.0 & 54.3 & 53.9 & 53.6 \\ 60.0 & 54.3 & 53.9 & 53.6 \\ 55.0 & 54.3 & 53.9 & 53.6 \\ 50.0 & 55.0 & 53.9 & 53.6 \\ 45.0 & 55.0 & 53.9 & 53.6 \\ 40.0 & 55.0 & 54.3 & 54.1 \\ 35.0 & 55.8 & 54.3 & 54.1 \\ 30.0 & 55.8 & 54.3 & 54.1 \\ 25.0 & 56.6 & 54.7 & 54.1 \\ 20.0 & 57.4 & 55.0 & 54.1 \\ 15.0 & 59.1 & 55.4 & 54.6 \\ 10.0 & 60.8 & 55.8 & 55.2 \\ 5.0 & 64.3 & 55.9 & 56.8\end{array}$

DEC JAN

ELEVATION IN FEET ABOVE MEAN SEA LEVEL

$\begin{array}{llllll}53.6 & 53.5 & 53.8 & 54.6 & 53.9 & 53.9 \\ 53.6 & 53.5 & 53.8 & 55.3 & 54.6 & 53.9 \\ 53.6 & 53.5 & 54.6 & 55.3 & 54.6 & 53.9 \\ 53.6 & 54.2 & 54.6 & 55.3 & 54.6 & 53.9 \\ 53.6 & 54.2 & 55.4 & 56.1 & 54.6 & 53.9 \\ 53.6 & 54.2 & 55.4 & 56.1 & 55.4 & 53.9 \\ 53.6 & 54.9 & 56.1 & 56.8 & 55.4 & 54.5 \\ 53.6 & 54.9 & 56.9 & 56.8 & 56.2 & 54.5 \\ 53.6 & 55.6 & 56.9 & 57.6 & 56.2 & 54.5 \\ 53.6 & 55.6 & 57.7 & 57.6 & 56.9 & 54.5 \\ 54.3 & 56.3 & 58.5 & 58.4 & 56.9 & 55.2 \\ 54.3 & 56.3 & 58.5 & 59.2 & 57.7 & 55.2 \\ 54.3 & 57.1 & 59.3 & 60.0 & 58.5 & 55.8 \\ 55.0 & 57.8 & 60.2 & 60.8 & 58.5 & 55.8 \\ 55.7 & 57.8 & 61.0 & 61.6 & 60.2 & 56.5 \\ 56.5 & 58.6 & 61.9 & 63.3 & 61.9 & 56.5 \\ 57.2 & 60.1 & 62.7 & 65.9 & 64.5 & 57.2 \\ 59.5 & 62.4 & 65.4 & 68.6 & 67.3 & 58.6 \\ 61.9 & 64.9 & 69.1 & 73.3 & 72.8 & 52.2\end{array}$

53.
53.
54.
54.
54.
54.
54.
54.
54.
54.
54.
54.
55.
5.2
55.
56.
57.
50.5

DISCHARGE IN CUBIC EEET PER SECOND

$\begin{array}{rrr}95.0 & 119.3 & 101.4 \\ 90.0 & 148.0 & 115.2 \\ 85.0 & 175.7 & 126.8 \\ 80.0 & 203.2 & 137.9 \\ 75.0 & 236.6 & 152.2 \\ 70.0 & 277.2 & 164.9 \\ 55.0 & 330.0 & 176.7 \\ 60.0 & 401.5 & 192.8 \\ 55.0 & 494.6 & 212.8 \\ 50.0 & 620.2 & 235.0 \\ 45.0 & 780.2 & 262.3 \\ 40.0 & 992.1 & 296.2 \\ 35.0 & 1236.2 & 341.8 \\ 30.0 & 1523.4 & 406.6 \\ 25.0 & 1906.8 & 494.9 \\ 20.0 & 2463.6 & 622.9 \\ 15.0 & 3336.1 & 801.3 \\ 10.0 & 4583.0 & 1126.4 \\ 5.0 & 6941.5 & 1993.5\end{array}$

96.3
108.7
118.9
128.9
139.0
148.0
156.4
164.8
175.8
187.8
203.8
225.9
248.9
275.7
315.9
389.7
515.2
893.7
1793.0

$\begin{array}{rr}102.9 & 141.6 \\ 117.8 & 193.8 \\ 130.7 & 231.9 \\ 145.9 & 272.1 \\ 161.6 & 335.6 \\ 177.5 & 425.3 \\ 194.0 & 612.6 \\ 213.9 & 807.7 \\ 235.5 & 1003.7 \\ 262.9 & 1205.5 \\ 307.8 & 1456.1 \\ 390.1 & 1748.4 \\ 519.5 & 2053.8 \\ 729.2 & 2408.3 \\ 1101.6 & 2835.4 \\ 1503.7 & 3393.6 \\ 2044.5 & 4267.0 \\ 3514.5 & 5566.0 \\ 5392.1 & 7253.5\end{array}$

$\begin{array}{rrr}212.5 & 438.5 & 299.5 \\ 307.2 & 718.2 & 413.2 \\ 528.8 & 948.6 & 515.7 \\ 694.3 & 1152.7 & 628.8 \\ 898.3 & 1335.6 & 763.0 \\ 1189.5 & 1516.5 & 937.5 \\ 1508.0 & 1747.9 & 1120.9 \\ 1844.3 & 2030.1 & 1310.9 \\ 2199.3 & 2282.1 & 1505.6 \\ 2579.2 & 2560.1 & 1753.9 \\ 3024.4 & 2890.0 & 2044.6 \\ 3383.3 & 3302.6 & 2384.1 \\ 3771.7 & 3761.5 & 2820.6 \\ 4180.5 & 4312.1 & 3248.7 \\ 4745.4 & 5036.6 & 4137.5 \\ 5383.8 & 6020.0 & 5345.4 \\ 6093.0 & 7422.8 & 6555.0 \\ 7496.3 & 9371.6 & 8020.0 \\ 9882.0 & 13040.6 & 10923.1\end{array}$

192.7
222.2
248.3
276.4
310.5
351.8
399.9
455.3
526.6
632.9
765.3
925.9
1132.0
1326.6
1519.7
1775.3
2367.9
3310.9
5402.3

146.6
173.2
195.5
218.2
242.5
272.1
307.5
345.9
383.4
432.1
497.3
579.9
684.5
824.0
1018.5
1263.2
1580.5
2333.9
4284.2

136.1

159.5

177.2

201.2

296.9

358.0

358.0
415.5

493.1

573.1

681.9

797.0

948.7

1102.1

1271.8

1481.8

1812.9

3691.7

$\begin{array}{rr}116.3 & 107.6 \\ 162.7 & 128.9 \\ 202.9 & 153.5 \\ 239.5 & 175.1 \\ 274.6 & 195.5 \\ 318.6 & 219.0 \\ 373.8 & 247.2 \\ 447.8 & 275.7 \\ 533.9 & 308.0 \\ 628.4 & 346.8 \\ 731.3 & 395.9 \\ 844.1 & 461.4 \\ 996.0 & 534.8 \\ 1175.0 & 622.5 \\ 1362.3 & 725.7 \\ 1595.8 & 944.1 \\ 1914.3 & 1372.9 \\ 2537.2 & 1895.9 \\ 4168.0 & 3250.0\end{array}$




\begin{tabular}{|c|c|c|c|c|c|c|c|c|c|c|}
\hline RA & ANGE & 1 & 3 & 7 & 14 & 30 & 60 & 90 & 120 & 183 \\
\hline $\begin{array}{l}1932 \\
1933 \\
1934 \\
1935\end{array}$ & $\begin{array}{l}1933 \\
1934 \\
1935 \\
1936\end{array}$ & $\begin{array}{ll}54.0 & 39 \\
53.8 & 21 \\
53.7 & 11 \\
53.7 & 13\end{array}$ & $\begin{array}{ll}54.0 & 40 \\
53.8 & 23 \\
53.7 & 11 \\
53.8 & 13\end{array}$ & $\begin{array}{ll}54.1 & 41 \\
53.9 & 21 \\
53.7 & 11 \\
53.8 & 14\end{array}$ & $\begin{array}{rr}54.2 & 41 \\
53.9 & 21 \\
53.7 & 8 \\
53.8 & 14\end{array}$ & $\begin{array}{rr}54.3 & 44 \\
53.9 & 21 \\
53.7 & 5 \\
53.9 & 17\end{array}$ & $\begin{array}{rr}54.5 & 42 \\
53.9 & 15 \\
53.7 & 4 \\
54.1 & 34\end{array}$ & $\begin{array}{rr}55.4 & 50 \\
53.9 & 10 \\
53.8 & 6 \\
54.3 & 35\end{array}$ & $\begin{array}{rr}55.4 & 50 \\
53.9 & 8 \\
53.9 & 6 \\
54.6 & 35\end{array}$ & $\begin{array}{ll}56.4 & 48 \\
54.2 & 13 \\
54.1 & 11 \\
56.5 & 49\end{array}$ \\
\hline $\begin{array}{l}1936 \\
1937\end{array}$ & $\begin{array}{l}1937 \\
1938\end{array}$ & $\begin{array}{ll}54.0 & 40 \\
54.2 & 50\end{array}$ & 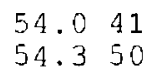 & $\begin{array}{ll}54.1 & 42 \\
54.5 & 51\end{array}$ & $\begin{array}{ll}54.1 & 40 \\
54.6 & 51\end{array}$ & $\begin{array}{ll}54.2 & 38 \\
54.7 & 50\end{array}$ & $\begin{array}{l}54.644 \\
54.849\end{array}$ & $\begin{array}{ll}54.6 & 41 \\
55.2 & 49\end{array}$ & $\begin{array}{ll}54.8 & 43 \\
55.4 & 49\end{array}$ & $\begin{array}{ll}55.3 & 39 \\
55.7 & 44\end{array}$ \\
\hline $\begin{array}{l}1942 \\
1943 \\
1944\end{array}$ & $\begin{array}{l}1943 \\
1944 \\
1945\end{array}$ & $\begin{array}{rr}53.8 & 15 \\
53.6 & 3 \\
54.1 & 43\end{array}$ & $\begin{array}{rrr}53.8 & 15 \\
53.6 & 3 \\
54.1 & 43\end{array}$ & $\begin{array}{rr}53.8 & 15 \\
53.6 & 3 \\
54.2 & 43\end{array}$ & $\begin{array}{rr}53.8 & 15 \\
53.7 & 3 \\
54.2 & 47\end{array}$ & $\begin{array}{rr}53.8 & 14 \\
53.7 & 3 \\
54.4 & 47\end{array}$ & $\begin{array}{rr}53.9 & 16 \\
53.7 & 3 \\
54.8 & 48\end{array}$ & $\begin{array}{rr}54.1 & 24 \\
53.7 & 3 \\
55.0 & 45\end{array}$ & $\begin{array}{lr}54.1 & 20 \\
53.8 & 4 \\
55.3 & 46\end{array}$ & $\begin{array}{rr}54.3 & 18 \\
54.0 & 6 \\
55.3 & 37\end{array}$ \\
\hline $\begin{array}{l}1947 \\
1948 \\
1949\end{array}$ & $\begin{array}{l}1948 \\
1949 \\
1950\end{array}$ & $\begin{array}{ll}54.0 & 42 \\
54.4 & 51 \\
54.2 & 47\end{array}$ & $\begin{array}{ll}54.0 & 42 \\
54.4 & 51 \\
54.2 & 47\end{array}$ & $\begin{array}{ll}54.1 & 40 \\
54.5 & 50 \\
54.2 & 45\end{array}$ & $\begin{array}{ll}54.1 & 39 \\
54.5 & 50 \\
54.2 & 45\end{array}$ & 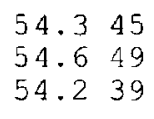 & 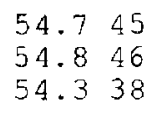 & $\begin{array}{ll}54.7 & 43 \\
55.1 & 46 \\
54.3 & 34\end{array}$ & $\begin{array}{ll}54.8 & 41 \\
55.3 & 48 \\
54.3 & 29\end{array}$ & 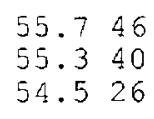 \\
\hline $\begin{array}{l}1951 \\
1952 \\
1954 \\
1955\end{array}$ & $\begin{array}{l}1952 \\
1953 \\
1955 \\
1956\end{array}$ & $\begin{array}{rr}53.7 & 10 \\
53.8 & 24 \\
53.6 & 2 \\
53.5 & 1\end{array}$ & $\begin{array}{rr}53.7 & 9 \\
53.8 & 24 \\
53.6 & 2 \\
53.5 & 1\end{array}$ & $\begin{array}{rr}53.7 & 9 \\
53.9 & 25 \\
53.6 & 2 \\
53.5 & 1\end{array}$ & $\begin{array}{rr}53.7 & 9 \\
53.9 & 24 \\
53.6 & 2 \\
53.5 & 1\end{array}$ & $\begin{array}{rr}53.8 & 13 \\
53.9 & 25 \\
53.6 & 2 \\
53.6 & 1\end{array}$ & $\begin{array}{rr}54.0 & 21 \\
53.9 & 17 \\
53.7 & 2 \\
53.6 & 1\end{array}$ & $\begin{array}{rr}54.0 & 17 \\
54.0 & 15 \\
53.7 & 2 \\
53.6 & 1\end{array}$ & $\begin{array}{rr}54.0 & 14 \\
54.0 & 16 \\
53.7 & 2 \\
53.7 & 1\end{array}$ & $\begin{array}{rr}54.1 & 12 \\
54.1 & 8 \\
53.7 & 2 \\
53.7 & 1\end{array}$ \\
\hline $\begin{array}{l}1956 \\
1957 \\
1958 \\
1959 \\
1960\end{array}$ & $\begin{array}{l}1957 \\
1958 \\
1959 \\
1960 \\
1961\end{array}$ & $\begin{array}{rr}53.6 & 4 \\
54.0 & 41 \\
53.8 & 26 \\
54.2 & 48 \\
53.9 & 32\end{array}$ & $\begin{array}{rr}53.6 & 4 \\
54.0 & 39 \\
53.9 & 26 \\
54.2 & 48 \\
53.9 & 31\end{array}$ & $\begin{array}{rr}53.7 & 4 \\
54.0 & 39 \\
53.9 & 26 \\
54.2 & 48 \\
53.9 & 31\end{array}$ & $\begin{array}{rr}53.7 & 6 \\
54.2 & 42 \\
53.9 & 25 \\
54.3 & 48 \\
53.9 & 31\end{array}$ & $\begin{array}{ll}53.8 & 10 \\
54.4 & 46 \\
53.9 & 23 \\
54.4 & 48 \\
54.0 & 28\end{array}$ & $\begin{array}{ll}54.0 & 26 \\
54.9 & 50 \\
53.9 & 19 \\
54.8 & 47 \\
54.0 & 31\end{array}$ & $\begin{array}{ll}54.0 & 21 \\
55.6 & 51 \\
53.9 & 13 \\
55.2 & 48 \\
54.2 & 32\end{array}$ & $\begin{array}{ll}54.1 & 19 \\
56.1 & 51 \\
54.0 & 15 \\
55.2 & 45 \\
54.3 & 30\end{array}$ & $\begin{array}{ll}54.2 & 15 \\
56.6 & 50 \\
54.4 & 21 \\
55.7 & 45 \\
54.6 & 27\end{array}$ \\
\hline $\begin{array}{l}1961 \\
1962 \\
1963 \\
1964 \\
1965\end{array}$ & $\begin{array}{l}1962 \\
1963 \\
1964 \\
1965 \\
1966\end{array}$ & $\begin{array}{rr}53.9 & 28 \\
53.8 & 17 \\
53.7 & 6 \\
54.6 & 52 \\
54.2 & 46\end{array}$ & $\begin{array}{rr}53.9 & 28 \\
53.8 & 18 \\
53.7 & 5 \\
54.7 & 52 \\
54.2 & 45\end{array}$ & $\begin{array}{rr}53.9 & 27 \\
53.8 & 19 \\
53.7 & 5 \\
54.7 & 52 \\
54.2 & 46\end{array}$ & $\begin{array}{rr}53.9 & 22 \\
53.8 & 18 \\
53.7 & 4 \\
55.0 & 52 \\
54.2 & 44\end{array}$ & $\begin{array}{rr}53.9 & 24 \\
53.9 & 18 \\
53.7 & 4 \\
55.3 & 52 \\
54.2 & 40\end{array}$ & $\begin{array}{rr}53.9 & 18 \\
54.0 & 24 \\
53.8 & 7 \\
55.9 & 52 \\
54.3 & 39\end{array}$ & $\begin{array}{rr}54.0 & 16 \\
54.1 & 27 \\
53.9 & 9 \\
57.3 & 52 \\
54.6 & 40\end{array}$ & $\begin{array}{rr}54.1 & 21 \\
54.2 & 24 \\
54.0 & 9 \\
57.7 & 52 \\
54.6 & 37\end{array}$ & $\begin{array}{ll}54.3 & 16 \\
54.2 & 14 \\
54.4 & 22 \\
58.0 & 52 \\
55.3 & 38\end{array}$ \\
\hline $\begin{array}{l}1966 \\
1967 \\
1968 \\
1969 \\
1970\end{array}$ & $\begin{array}{l}1967 \\
1968 \\
1969 \\
1970 \\
1971\end{array}$ & $\begin{array}{rr}54.2 & 45 \\
53.8 & 22 \\
53.7 & 5 \\
53.8 & 23 \\
54.0 & 38\end{array}$ & $\begin{array}{ll}54.2 & 46 \\
53.8 & 21 \\
53.7 & 10 \\
53.8 & 22 \\
54.0 & 38\end{array}$ & $\begin{array}{ll}54.2 & 47 \\
53.9 & 22 \\
53.7 & 10 \\
53.8 & 20 \\
54.0 & 37\end{array}$ & $\begin{array}{ll}54.2 & 46 \\
53.9 & 23 \\
53.7 & 10 \\
53.8 & 19 \\
54.0 & 37\end{array}$ & $\begin{array}{rr}54.3 & 42 \\
53.9 & 22 \\
53.7 & 6 \\
53.9 & 19 \\
54.1 & 37\end{array}$ & $\begin{array}{rr}54.3 & 40 \\
53.9 & 13 \\
53.8 & 6 \\
54.0 & 29 \\
54.2 & 35\end{array}$ & $\begin{array}{rr}54.4 & 39 \\
53.9 & 11 \\
53.8 & 5 \\
54.4 & 38 \\
54.2 & 29\end{array}$ & $\begin{array}{rr}54.4 & 34 \\
54.0 & 12 \\
53.8 & 5 \\
54.7 & 38 \\
54.4 & 32\end{array}$ & $\begin{array}{rr}55.1 & 34 \\
54.1 & 10 \\
53.9 & 4 \\
54.7 & 28 \\
55.4 & 42\end{array}$ \\
\hline $\begin{array}{l}1971 \\
1972 \\
1973 \\
1974 \\
1975\end{array}$ & $\begin{array}{l}1972 \\
1973 \\
1974 \\
1975 \\
1976\end{array}$ & $\begin{array}{rr}53.8 & 18 \\
53.7 & 7 \\
54.0 & 37 \\
53.9 & 34 \\
54.2 & 44\end{array}$ & $\begin{array}{rr}53.8 & 17 \\
53.7 & 6 \\
54.0 & 37 \\
53.9 & 34 \\
54.2 & 44\end{array}$ & $\begin{array}{rr}53.8 & 16 \\
53.7 & 6 \\
54.0 & 36 \\
54.0 & 34 \\
54.2 & 44\end{array}$ & $\begin{array}{rr}53.8 & 17 \\
53.7 & 5 \\
54.0 & 35 \\
54.0 & 36 \\
54.2 & 43\end{array}$ & $\begin{array}{rr}53.9 & 16 \\
53.8 & 9 \\
54.0 & 34 \\
54.0 & 35 \\
54.3 & 41\end{array}$ & $\begin{array}{rr}53.9 & 14 \\
53.8 & 8 \\
54.0 & 32 \\
54.1 & 33 \\
54.3 & 41\end{array}$ & $\begin{array}{rr}54.1 & 28 \\
53.9 & 8 \\
54.2 & 30 \\
54.3 & 36 \\
54.6 & 42\end{array}$ & $\begin{array}{ll}54.8 & 42 \\
54.0 & 10 \\
54.3 & 28 \\
55.0 & 44 \\
54.7 & 40\end{array}$ & $\begin{array}{ll}55.6 & 43 \\
54.5 & 25 \\
54.9 & 32 \\
55.3 & 41 \\
55.2 & 35\end{array}$ \\
\hline $\begin{array}{l}1976 \\
1977 \\
1978 \\
1979 \\
1980\end{array}$ & $\begin{array}{l}1977 \\
1978 \\
1979 \\
1980 \\
1981\end{array}$ & 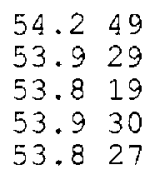 & $\begin{array}{ll}54.2 & 49 \\
53.9 & 29 \\
53.8 & 19 \\
53.9 & 30 \\
53.9 & 27\end{array}$ & $\begin{array}{ll}54.3 & 49 \\
53.9 & 29 \\
53.8 & 17 \\
53.9 & 30 \\
53.9 & 28\end{array}$ & $\begin{array}{ll}54.5 & 49 \\
53.9 & 28 \\
53.8 & 16 \\
53.9 & 30 \\
53.9 & 26\end{array}$ & $\begin{array}{ll}54.7 & 51 \\
53.9 & 27 \\
53.8 & 15 \\
54.0 & 36 \\
54.0 & 29\end{array}$ & $\begin{array}{ll}55.0 & 51 \\
54.0 & 25 \\
53.9 & 11 \\
54.2 & 37 \\
54.0 & 22\end{array}$ & $\begin{array}{ll}55.1 & 47 \\
54.1 & 25 \\
53.9 & 12 \\
54.4 & 37 \\
54.0 & 18\end{array}$ & $\begin{array}{ll}55.3 & 47 \\
54.2 & 25 \\
54.0 & 13 \\
54.6 & 36 \\
54.0 & 17\end{array}$ & $\begin{array}{rr}56.6 & 51 \\
54.3 & 19 \\
54.3 & 20 \\
54.9 & 31 \\
54.1 & 7\end{array}$ \\
\hline $\begin{array}{l}1981 \\
1982 \\
1983 \\
1984\end{array}$ & $\begin{array}{l}1982 \\
1983 \\
1984 \\
1985\end{array}$ & $\begin{array}{rr}53.7 & 8 \\
53.8 & 16 \\
53.9 & 31 \\
54.0 & 36\end{array}$ & $\begin{array}{rr}53.7 & 8 \\
53.8 & 16 \\
53.9 & 32 \\
54.0 & 35\end{array}$ & $\begin{array}{rr}53.7 & 7 \\
53.8 & 18 \\
53.9 & 32 \\
54.0 & 35\end{array}$ & $\begin{array}{ll}53.7 & 11 \\
53.9 & 20 \\
53.9 & 32 \\
54.0 & 34\end{array}$ & $\begin{array}{rr}53.7 & 8 \\
53.9 & 20 \\
54.0 & 31 \\
54.0 & 33\end{array}$ & $\begin{array}{ll}53.9 & 12 \\
53.9 & 20 \\
54.2 & 36 \\
54.0 & 30\end{array}$ & $\begin{array}{ll}54.0 & 14 \\
54.2 & 31 \\
54.2 & 33 \\
54.0 & 19\end{array}$ & $\begin{array}{ll}54.0 & 11 \\
54.4 & 33 \\
54.4 & 31 \\
54.1 & 22\end{array}$ & $\begin{array}{lr}54.0 & 5 \\
54.9 & 33 \\
54.9 & 30 \\
54.4 & 24\end{array}$ \\
\hline $\begin{array}{l}1986 \\
1987 \\
1988 \\
1989 \\
1990\end{array}$ & $\begin{array}{l}1987 \\
1988 \\
1989 \\
1990 \\
1991\end{array}$ & $\begin{array}{rr}53.7 & 12 \\
53.8 & 14 \\
53.8 & 20 \\
53.8 & 25 \\
53.7 & 9\end{array}$ & $\begin{array}{rr}53.7 & 12 \\
53.8 & 14 \\
53.8 & 20 \\
53.8 & 25 \\
53.7 & 7\end{array}$ & $\begin{array}{rr}53.7 & 12 \\
53.8 & 13 \\
53.9 & 23 \\
53.9 & 24 \\
53.7 & 8\end{array}$ & $\begin{array}{rr}53.8 & 12 \\
53.8 & 13 \\
53.9 & 27 \\
53.9 & 29 \\
53.7 & 7\end{array}$ & $\begin{array}{rr}53.8 & 11 \\
53.8 & 12 \\
53.9 & 26 \\
54.0 & 30 \\
53.7 & 7\end{array}$ & $\begin{array}{rr}53.8 & 10 \\
53.8 & 9 \\
54.0 & 23 \\
54.0 & 27 \\
53.7 & 5\end{array}$ & $\begin{array}{rr}54.1 & 26 \\
53.8 & 7 \\
54.0 & 20 \\
54.0 & 22 \\
53.8 & 4\end{array}$ & $\begin{array}{rr}54.2 & 26 \\
53.9 & 7 \\
54.1 & 18 \\
54.2 & 27 \\
53.8 & 3\end{array}$ & $\begin{array}{rr}54.3 & 17 \\
54.1 & 9 \\
54.4 & 23 \\
55.2 & 36 \\
53.8 & 3\end{array}$ \\
\hline $\begin{array}{l}1991 \\
1992\end{array}$ & $\begin{array}{l}1992 \\
1993\end{array}$ & $\begin{array}{ll}53.9 & 33 \\
53.9 & 35\end{array}$ & $\begin{array}{ll}53.9 & 33 \\
54.0 & 36\end{array}$ & $\begin{array}{ll}53.9 & 33 \\
54.0 & 38\end{array}$ & $\begin{array}{ll}54.0 & 33 \\
54.1 & 38\end{array}$ & $\begin{array}{lll}54.0 & 32 \\
54.3 & 43\end{array}$ & $\begin{array}{ll}54.0 & 28 \\
54.5 & 43\end{array}$ & $\begin{array}{ll}54.1 & 23 \\
54.7 & 44\end{array}$ & $\begin{array}{ll}54.2 & 23 \\
54.7 & 39\end{array}$ & $\begin{array}{ll}55.7 & 47 \\
54.8 & 29\end{array}$ \\
\hline
\end{tabular}



FOR PERIOD OCT TO SEP

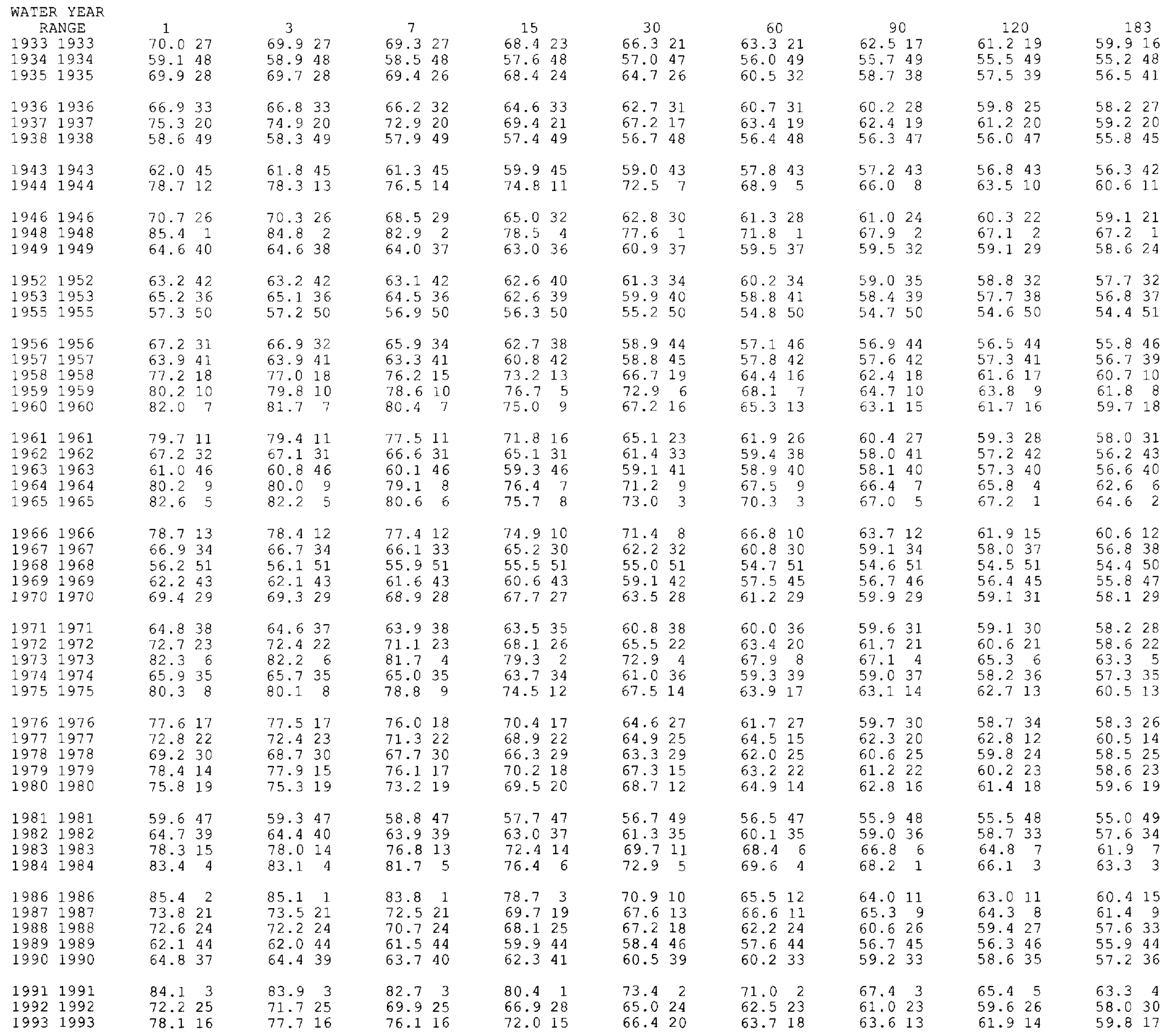



CONSECUTIVE DAYS FOR PERIOD APR TO MAR

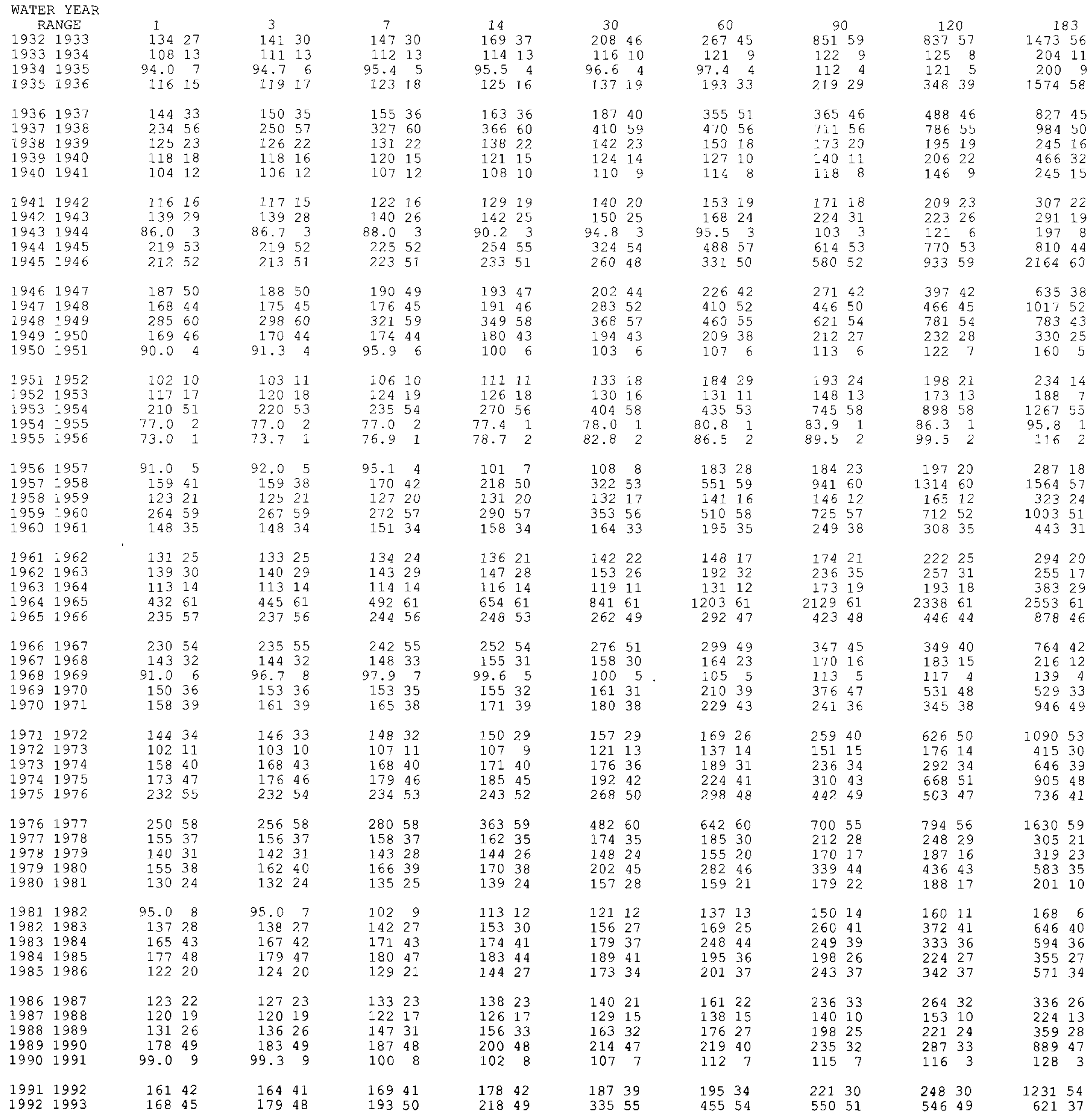


HIGHEST MEAN DISCHARGE, IN CUBIC FEET PER SECOND, AND RANKING FOR THE FOLLOWING NUMBER OF CONSECUTIVE DAYS FOR PERIOD OCT TO SEP

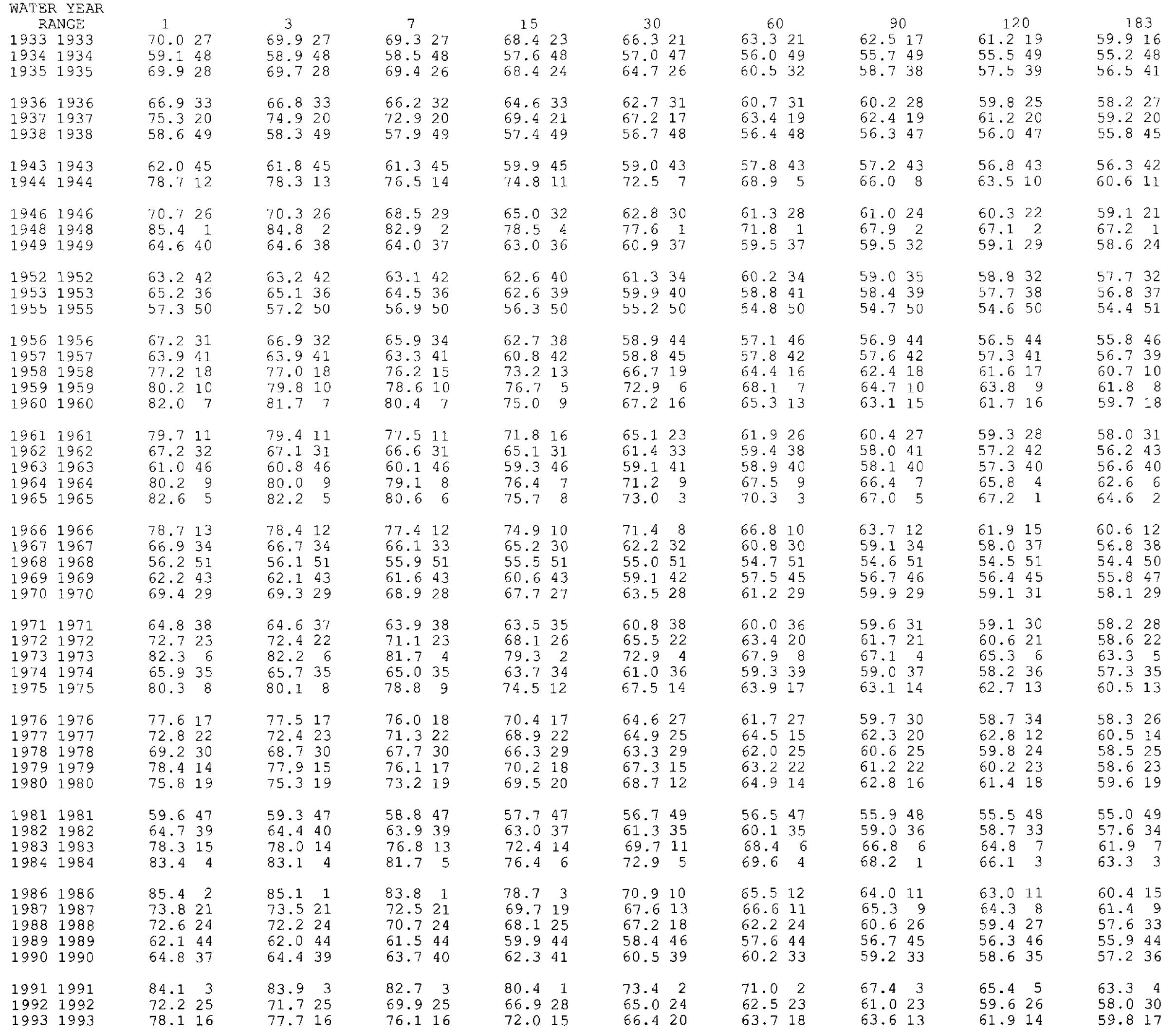


THIS PAGE INTENTIONALLY BLANK 
LOCATION.--Lat $30^{\circ} 23^{\prime} 04^{\prime \prime}$, long $83^{\circ} 10^{\prime} 19^{\prime \prime}$, in NE $1 / 4$ sec.24, T.1 S., R.11 E., Suwannee County, Hydrologic Unit 03110205 , on left bank at Ellaville, 100 ft upstream from Seaboard Air Line Railroad bridge, $200 \mathrm{ft}$ downstream from Withlacoochee River, $900 \mathrm{ft}$ upstream from bridge on U.S. Highway 90 , and 127 mi upstream from mouth.

DRAINAGE AREA.--6,970 $\mathrm{mi}^{2}$, approximately, includes part of watershed in Okefenokee Swamp which is indeterminate.

PERIOD OF RECORD.--January 1927 to 1993.

REVISED RECORDS.--WSP 1905: WDR FL-75-1: Drainage area.

GAGE.--Water-stage recorder. Datum of gage is $27.22 \mathrm{ft}$ above National Geodetic Vertical Datum of 1929 . Prior to June 20, 1932 , nonrecording gage at same site and datum. Nov. 8,1955 to Sept. 30,1970 , nonrecording gage 1.1 mi downstream from base gage at datum $2.67 \mathrm{ft}$ lower, used as supplementary gage when flow was less than $4,800 \mathrm{ft}^{3} / \mathrm{s}$.

REMARKS.--Records good above $5.000 \mathrm{cfs}$, and fair below. Since Nov. 7, 1953, slight regulation at low water caused by diversions above control $0.7 \mathrm{mi}$ down stream from gage by a steam-electric powerplant for cooling of condensers. Total diverted flow is returned to river below control and is included in station record. Records include flow of large spring on left bank about $200 \mathrm{ft}$ downstream; spring flow may reverse during high stages.
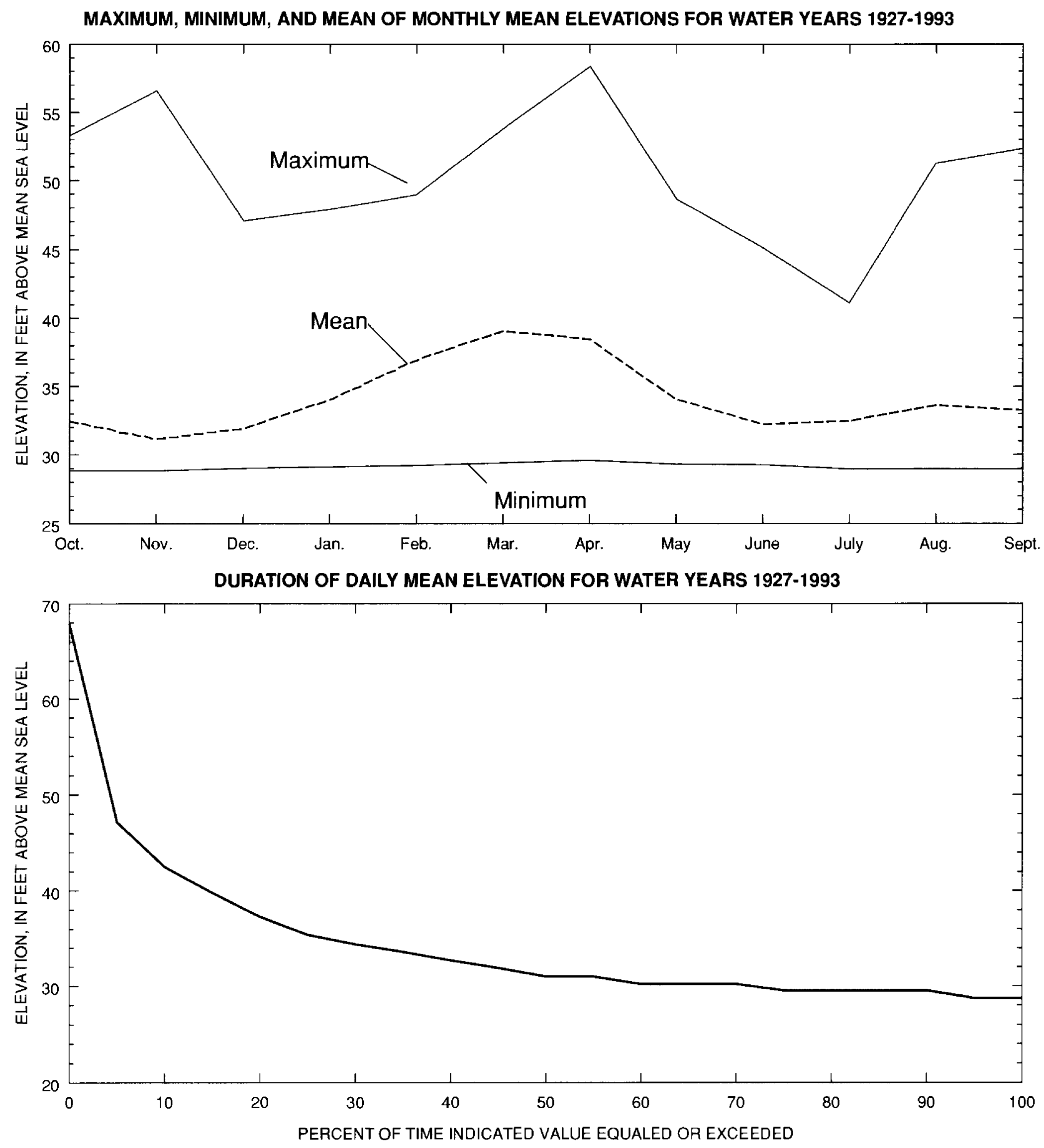
SUMMARY STATISTICS, IN CUBIC FEET PER SECOND UNLESS OTHERWISE INDICATED, FOR WATER YEARS $1927-1993$

\begin{tabular}{|c|c|c|c|c|}
\hline ANNUAL MEAN & 6528 & & & \\
\hline HIGHEST ANNUAL MEAN & 19710 & & & 1948 \\
\hline LOWEST ANNUAL MEAN & 1296 & & & \\
\hline HIGHEST DAILY MEAN & 94 & Apr & 8 & \\
\hline LOWEST DAILY MEAN & & Nov & 8 & \\
\hline I SEVEN-DAY MINIMUM & & Nov & 3 & \\
\hline INSTANTANEOUS PEAK FLOW & 95300 & Apr & 7 & O \\
\hline INSTANTANEOUS PEAK ELEVATION (FT) & 68.10 & Apr & 7 & \\
\hline INSTANTANEOUS LOW FLOW & & Nov & 8 & 99 \\
\hline ANNUAI RUNOFF (INCHES/CFSM) & \multicolumn{4}{|c|}{$12.73 / 0.94$} \\
\hline
\end{tabular}
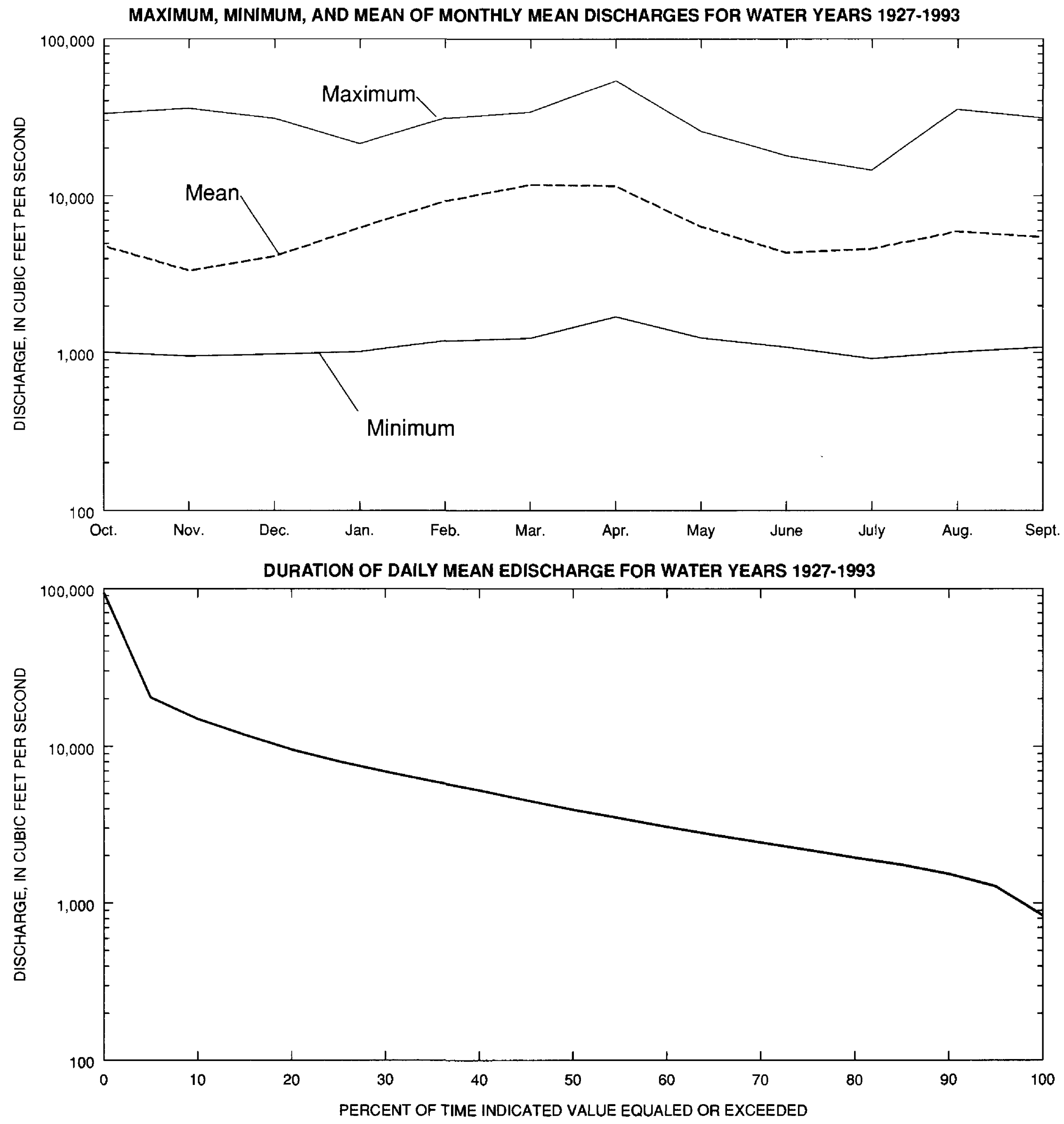

FOR WATER YEARS 1927-1993

ELEVATIONS,
FEET ABOVE SEA LEVEL

\begin{tabular}{lccc}
\multicolumn{1}{c}{ MONTH } & MAXIMUM & MINIMUM & MEAN \\
OCTOBER & 53.24 & 28.83 & 32.44 \\
NOVEMBER & 56.51 & 28.81 & 31.13 \\
DECEMBER & 46.99 & 29.00 & 31.90 \\
JANUARY & 47.83 & 29.11 & 34.03 \\
FEBRUARY & 48.90 & 29.21 & 36.95 \\
MARCH & 53.75 & 29.40 & 39.05 \\
APRII & 58.30 & 29.58 & 38.47 \\
MAY & 48.59 & 29.30 & 34.08 \\
JUNE & 45.06 & 29.26 & 32.22 \\
JULY & 41.06 & 28.95 & 32.47 \\
AUGUST & 51.20 & 28.97 & 33.62 \\
SEPTEMBER & 52.31 & 28.94 & 33.25
\end{tabular}

DISCHARGE,
CUBIC FEE'T PER SECOND

\begin{tabular}{crr} 
MAXIMUM & MINIMUM & \multicolumn{1}{c}{ MEAN } \\
32940 & 1006 & 4826 \\
35590 & 951 & 3367 \\
30600 & 978 & 4148 \\
21150 & 1014 & 6280 \\
30720 & 1189 & 9281 \\
33680 & 1240 & 11760 \\
53180 & 1702 & 11510 \\
25380 & 1245 & 6375 \\
17800 & 1084 & 4366 \\
14380 & 917 & 4619 \\
34990 & 1010 & 5944 \\
30760 & 1082 & 5468
\end{tabular}

PERCENT

EQUALED OR

QQUALED OR

DURATION OF DAILY MEAN VALUES FOR WATER YEARS 1927-1993

NOV DEC JAN FEB MAR APR MAY JUNE JULY AUG SEPT

ELEVATION IN FEET ABOVE MEAN SEA LEVEL

$\begin{array}{rlll}95.0 & 28.7 & 28.7 & 28.7 \\ 90.0 & 29.5 & 29.3 & 28.7 \\ 85.0 & 29.5 & 29.3 & 29.3 \\ 80.0 & 29.5 & 29.3 & 29.3 \\ 75.0 & 29.5 & 29.3 & 29.3 \\ 70.0 & 30.2 & 29.3 & 29.3 \\ 65.0 & 30.2 & 30.0 & 29.3 \\ 60.0 & 30.2 & 30.0 & 29.3 \\ 55.0 & 31.0 & 30.0 & 29.3 \\ 50.0 & 31.0 & 30.0 & 29.9 \\ 45.0 & 31.9 & 30.6 & 29.9 \\ 40.0 & 32.7 & 30.6 & 29.9 \\ 35.0 & 33.6 & 31.3 & 30.6 \\ 30.0 & 34.4 & 32.0 & 30.6 \\ 25.0 & 35.4 & 32.0 & 30.6 \\ 20.0 & 37.3 & 32.7 & 31.2 \\ 15.0 & 39.8 & 34.9 & 31.9 \\ 10.0 & 42.5 & 37.2 & 32.6 \\ 5.0 & 47.2 & 44.2 & 34.7\end{array}$

$\begin{array}{lll}28.8 & 29.0 & 29.1 \\ 28.8 & 29.5 & 29.8 \\ 28.8 & 29.5 & 29.8 \\ 29.4 & 29.5 & 30.5 \\ 29.4 & 30.1 & 30.5 \\ 29.4 & 30.1 & 31.2 \\ 29.4 & 30.1 & 32.6 \\ 29.4 & 30.7 & 33.4 \\ 29.4 & 30.7 & 34.9 \\ 30.0 & 31.3 & 35.7 \\ 30.0 & 32.5 & 36.6 \\ 30.0 & 33.1 & 37.4 \\ 30.7 & 33.8 & 38.3 \\ 31.3 & 35.1 & 40.0 \\ 32.0 & 36.4 & 40.9 \\ 32.7 & 37.8 & 41.9 \\ 34.1 & 40.1 & 44.0 \\ 35.5 & 42.4 & 45.7 \\ 40.3 & 44.9 & 48.8\end{array}$

$$
\begin{aligned}
& 29.9 \\
& 30.5 \\
& 31.2 \\
& 31.2 \\
& 31.9 \\
& 33.4 \\
& 34.1 \\
& 34.9 \\
& 35.7 \\
& 37.3 \\
& 38.1 \\
& 39.8 \\
& 40.7 \\
& 41.7 \\
& 42.6 \\
& 44.5 \\
& 47.2 \\
& 51.0 \\
& 53.8
\end{aligned}
$$

29.9
29.9
30.7
30.7
31.5
32.3
33.1
34.0
34.9
35.8
36.7
37.7
39.7
41.1
43.1
45.2
48.0
50.3
53.0

29.1
29.7
29.7
29.7
30.3
30.3
31.0
31.0
31.6
32.3
32.3
33.0
33.7
35.1
35.9
36.7
38.2
39.9

29.4
29.4
29.4
29.8
29.8
29.8
30.3
30.3
30.8
30.8
31.3
31.3
31.8
32.3
32.8
33.3
34.4
36.6
40.2

28.9
29.4
29.4
29.9
29.9
30.4
30.4
30.4
30.9
30.9
31.4
31.9
32.4
33.0
33.5
34.1
35.2
37.0
39.5

28.9
28.9
29.6
29.6
30.4
30.4
30.4
31.1
31.1
31.8
31.8
32.6
33.4
33.4
34.2
35.9
36.8
39.5
45.2

DISCHARGE IN CUBIC FEET PER SECOND

$\begin{array}{rrr}95.0 & 1273.2 & 1170.2 \\ 90.0 & 1528.7 & 1374.7 \\ 85.0 & 1746.5 & 1561.7 \\ 80.0 & 1938.4 & 1687.2 \\ 75.0 & 2171.2 & 1798.4 \\ 70.0 & 2427.1 & 1959.5 \\ 65.0 & 2713.6 & 2124.8 \\ 60.0 & 3063.3 & 2294.9 \\ 55.0 & 3482.5 & 2501.0 \\ 50.0 & 3946.4 & 2763.1 \\ 45.0 & 4548.8 & 3099.3 \\ 40.0 & 5264.3 & 3554.1 \\ 35.0 & 6007.2 & 4008.4 \\ 30.0 & 6924.0 & 4518.3 \\ 25.0 & 8028.0 & 5137.0 \\ 20.0 & 9539.5 & 6105.2 \\ 15.0 & 11854.4 & 7644.7 \\ 10.0 & 14919.4 & 9759.7 \\ 5.0 & 20359.1 & 17341.2\end{array}$

1058.0
1190.0
1296.6
1443.3
1561.6
1655.4
1766.1
1882.9
2008.2
2141.4
2333.7
2563.5
2843.4
3173.4
3571.4
4117.1
4861.8
6005.6
7670.0

1053.1
1188.3
1333.8
1453.2
1593.4
1708.1
1813.6
1968.7
2111.7
2233.9
2382.2
2552.7
3262.2
3871.8
4681.8
5554.9
6579.9
8466.5
13130.0

$\begin{array}{rrr}1254.6 & 1591.7 & 2061.8 \\ 1490.0 & 1829.6 & 2859.5 \\ 1729.2 & 2074.3 & 3389.1 \\ 1923.8 & 2466.2 & 3950.2 \\ 2084.1 & 3166.4 & 4751.5 \\ 2270.9 & 3793.2 & 5694.9 \\ 2545.3 & 4753.1 & 6478.6 \\ 2997.7 & 6174.5 & 7389.2 \\ 3526.0 & 7099.8 & 8401.8 \\ 3996.8 & 7860.9 & 9509.3 \\ 5082.8 & 8671.8 & 10723.0 \\ 5836.4 & 9632.7 & 12030.2 \\ 6673.9 & 10949.1 & 13227.3 \\ 7665.4 & 12347.1 & 14397.5 \\ 8563.2 & 13555.3 & 15875.4 \\ 10272.8 & 14744.5 & 17656.4 \\ 12520.9 & 16501.1 & 20243.6 \\ 15018.8 & 18523.0 & 24919.9 \\ 18150.0 & 22232.8 & 29924.3\end{array}$

$\begin{array}{rr}2015.2 & 1446.7 \\ 2456.8 & 1714.2 \\ 2838.9 & 1982.5 \\ 3329.5 & 2268.7 \\ 4164.1 & 2567.3 \\ 5014.2 & 2860.6 \\ 5766.6 & 3170.7 \\ 6395.2 & 3579.8 \\ 7104.1 & 4097.0 \\ 7957.9 & 4681.8 \\ 9207.0 & 5256.8 \\ 10590.0 & 5816.2 \\ 11812.8 & 6464.1 \\ 13318.9 & 7315.9 \\ 15429.3 & 8282.7 \\ 18004.3 & 9233.1 \\ 21549.5 & 10679.8 \\ 24668.4 & 12480.5\end{array}$

1294.6

1517.0

1757.1

2158.9

2356.1

2735.0

2984.6
3267.6

3571.7

3903.4

4222.1
4654.9

4654.9
5151.9

5786.0

8684.0

29065.3

12480.5
17680.6

12557.1

1405.6
1605.5
1806.7
1964.5
2153.9
2433.1
2695.7
2960.3
3226.5
3591.3
3937.3
4310.5
4765.6
5377.2
6040.0
6699.1
7699.3
9219.2
12047.2

1355.0

1672.4
1964.6

1964.6
2393.5

2595.1

2806.3

3024.7

3379.5
3810.1

3810.1
4252.5

4730.4

5258.7

5789.2

6350.5
6992.4

6992.4
7819.2

9106.4

$17595.7 \quad 11755.0$

\section{LOWEST MEAN ELEVATION AND RANKING FOR THE FOLLOWING NUMBER OF CONSECUTIVE DAYS
FOR PERIOD APR TO MAR}

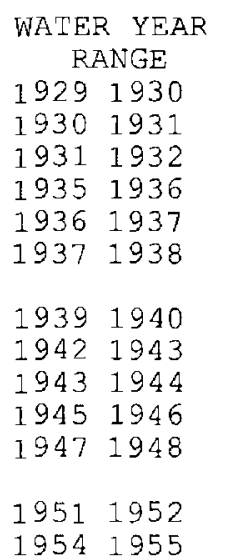

$\begin{array}{cc}3 & \\ 32.2 & 44 \\ 30.2 & 36 \\ 29.3 & 13 \\ 29.3 & 10 \\ 29.8 & 28 \\ 30.5 & 40 \\ & \\ 29.7 & 23 \\ 29.8 & 27 \\ 29.3 & 11 \\ 30.2 & 37 \\ 30.9 & 42 \\ 29.6 & 19 \\ 29.0 & 3\end{array}$

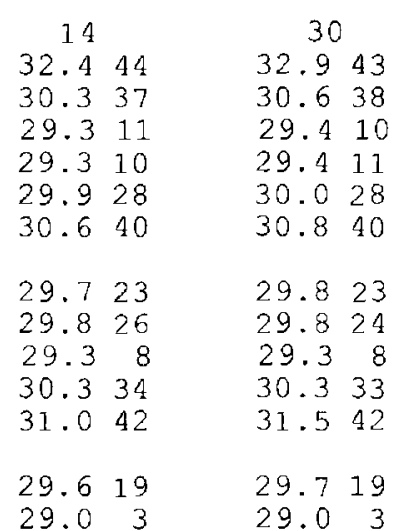

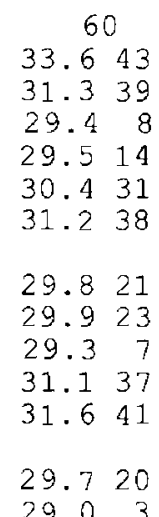

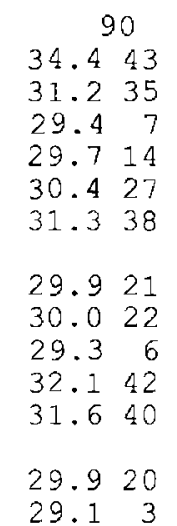

$\begin{array}{rr} & 120 \\ 35.1 & 43 \\ 31.5 & 36 \\ 29.5 & 7 \\ 29.9 & 16 \\ 30.6 & 25 \\ 31.5 & 35 \\ 30.1 & 21 \\ 30.2 & 22 \\ 29.4 & 6 \\ 33.2 & 42 \\ 31.9 & 39 \\ 30.1 & 20 \\ 29 & 3\end{array}$
28.8 29.4 30.0 30.0 30.0 30.6 30.6 31.2 31.2 31.9 32.5 33.2 35.3 36.8 39.1 42.4 


\section{LOWEST MEAN ELEVATION AND RANKING FOR THE FOLLOWING NUMBER OF CONSECUTIVE DAYS}

\begin{tabular}{|c|c|}
\hline $\begin{array}{r}\text { WATE } \\
R\end{array}$ & $\begin{array}{l}\text { R YEA } \\
\text { ANGE }\end{array}$ \\
\hline 1959 & 1960 \\
\hline 1960 & 1961 \\
\hline 1961 & 1962 \\
\hline 1962 & 1963 \\
\hline 1963 & 1964 \\
\hline 1964 & 1965 \\
\hline 1965 & 1966 \\
\hline 1966 & 1967 \\
\hline 1967 & 1968 \\
\hline 1968 & 1969 \\
\hline 1969 & 1970 \\
\hline 1970 & 1971 \\
\hline 1971 & 1972 \\
\hline 1972 & 1973 \\
\hline 1973 & 1974 \\
\hline 1974 & 1975 \\
\hline 1975 & 1976 \\
\hline 1976 & 1977 \\
\hline 1977 & 1978 \\
\hline 1978 & 1979 \\
\hline 1979 & 1980 \\
\hline 1980 & 1981 \\
\hline 1981 & 1982 \\
\hline 1983 & 1984 \\
\hline 1985 & 1986 \\
\hline 1986 & 1987 \\
\hline 1987 & 1988 \\
\hline 1988 & 1989 \\
\hline 1990 & 1991 \\
\hline 1991 & 1992 \\
\hline & \\
\hline
\end{tabular}
FOR PERIOD APR TO MAR--Continued

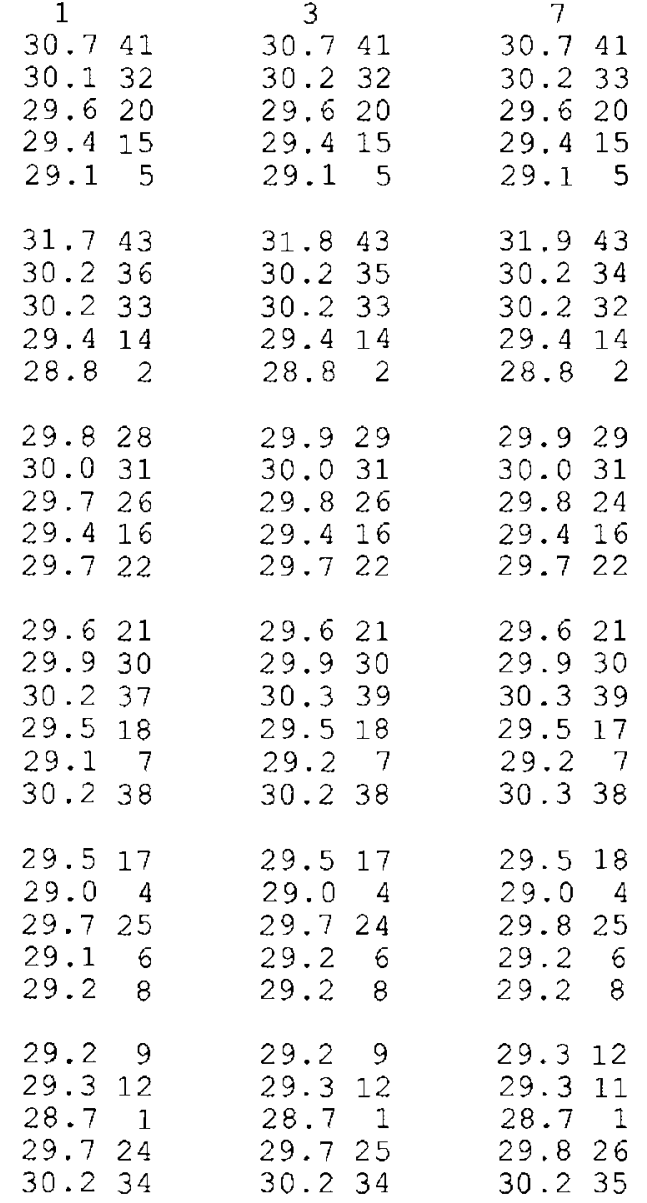

$\begin{array}{rr}14 & \\ 30.8 & 41 \\ 30.2 & 32 \\ 29.7 & 20 \\ 29.4 & 15 \\ 29.1 & 5 \\ 32.1 & 43 \\ 30.3 & 35 \\ 30.2 & 33 \\ 29.4 & 14 \\ 28.8 & 2 \\ 30.0 & 31 \\ 30.0 & 30 \\ 29.8 & 25 \\ 29.4 & 16 \\ 29.7 & 22 \\ 29.7 & 21 \\ 29.9 & 29 \\ 30.4 & 38 \\ 29.5 & 18 \\ 29.2 & 7 \\ 30.5 & 39 \\ & \\ 29.5 & 17 \\ 29.1 & 4 \\ 29.8 & 24 \\ 29.2 & 6 \\ 29.3 & 9 \\ 29.3 & 13 \\ 29.3 & 12 \\ 28.7 & 1 \\ 29.8 & 27 \\ 30.3 & 36 \\ & \end{array}$

\begin{tabular}{lr}
\multicolumn{3}{c}{30} \\
31.3 & 41 \\
30.2 & 31 \\
29.7 & 20 \\
29.5 & 16 \\
29.2 & 5 \\
& \\
33.0 & 44 \\
30.4 & 34 \\
30.3 & 32 \\
29.4 & 14 \\
28.9 & 2 \\
& \\
30.4 & 35 \\
30.0 & 30 \\
30.0 & 27 \\
29.5 & 15 \\
29.7 & 22 \\
& \\
29.7 & 21 \\
30.0 & 29 \\
30.6 & 39 \\
29.6 & 18 \\
29.2 & 6 \\
30.6 & 37 \\
29.6 & 17 \\
29.1 & 4 \\
29.9 & 25 \\
29.3 & 7 \\
29.4 & 12 \\
& \\
29.4 & 13 \\
29.3 & 9 \\
28.8 & 1 \\
29.9 & 26 \\
30.5 & 36
\end{tabular}

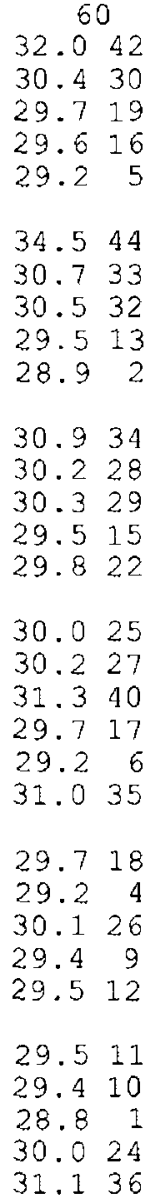

\begin{tabular}{rr}
\multicolumn{2}{r}{90} \\
31.9 & 41 \\
30.8 & 30 \\
29.9 & 19 \\
29.7 & 15 \\
29.4 & 8 \\
& \\
37.5 & 44 \\
31.3 & 37 \\
31.0 & 33 \\
29.6 & 12 \\
29.0 & 2 \\
& \\
31.2 & 36 \\
30.5 & 28 \\
30.9 & 31 \\
29.6 & 13 \\
30.1 & 23 \\
30.2 & 25 \\
30.7 & 29 \\
31.4 & 39 \\
29.8 & 17 \\
29.3 & 5 \\
31.2 & 34 \\
29.8 & 18 \\
29.2 & 4 \\
30.3 & 26 \\
29.6 & 10 \\
29.7 & 16 \\
29.5 & 9 \\
29.6 & 11 \\
28.8 & 1 \\
30.1 & 24 \\
31.0 & 32
\end{tabular}

\begin{tabular}{lrrr}
\multicolumn{1}{r}{120} & \multicolumn{2}{r}{183} \\
32.1 & 40 & 32.7 & 34 \\
31.5 & 34 & 32.4 & 31 \\
30.0 & 18 & 30.7 & 19 \\
29.9 & 17 & 30.0 & 13 \\
29.5 & 8 & 30.1 & 15 \\
39.2 & 44 & 40.8 & 44 \\
31.5 & 37 & 32.7 & 36 \\
31.2 & 30 & 33.2 & 38 \\
29.7 & 11 & 29.8 & 7 \\
29.1 & 2 & 29.2 & 2 \\
& & & \\
31.4 & 32 & 32.5 & 32 \\
30.9 & 27 & 32.5 & 33 \\
32.3 & 41 & 33.3 & 39 \\
29.8 & 13 & 30.5 & 17 \\
30.4 & 23 & 31.2 & 23 \\
& & & \\
31.0 & 28 & 31.9 & 27 \\
31.2 & 29 & 32.2 & 29 \\
31.5 & 38 & 33.7 & 40 \\
30.0 & 19 & 29.9 & 11 \\
29.4 & 5 & 29.8 & 8 \\
31.4 & 33 & 31.7 & 26 \\
& & & \\
29.8 & 14 & 29.9 & 9 \\
29.3 & 4 & 29.3 & 4 \\
30.6 & 26 & 31.4 & 24 \\
29.8 & 12 & 31.1 & 21 \\
29.8 & 15 & 29.9 & 10 \\
29.6 & 9 & 30.1 & 14 \\
29.7 & 10 & 30.0 & 12 \\
28.9 & 1 & 28.9 & 1 \\
30.5 & 24 & 33.2 & 37 \\
31.3 & 31 & 31.6 & 25 \\
& & &
\end{tabular}

HIGHEST MEAN ELEVATION, IN FEET, AND RANKING FOR THE FOLLOWING NUMBER OF CONSECUTIVE DAYS

WATER YEAR

19301930

19311931

19321932
19351935

19361936 19371937

19381938

19421942

19431943

19441944

19461946

19481948

19511951

19531953

19571957

19591959

19601960

19611961

19621962

19641964

19651965

19661966

19671967

19691969

19701970

19711971

19721972

19731973

19741974

19751975

19761976 19771977

19781978

19791979

19801980

19811981

$\begin{array}{lll}1982 & 1982\end{array}$

19841984
64.23

$\begin{array}{lll}54.1 & 12\end{array}$

37.945

42.933

45.629

$42.4 \quad 34$

50.919

40.341

2.115

$34.6 \quad 47$

50.721

44.931

68.01

38.244

$41.2 \quad 39$

$\begin{array}{lll}42.0 & 35 \\ 59 & 0 & 7\end{array}$

55.010

50.820

47.626

40.540

$56.8 \quad 8$

$55.8 \quad 9$

$44.2 \quad 32$

30.948

$38.6 \quad 43$

1.416

41.737

50.918

65.02

$41.7 \quad 36$

54.013

48.624

49.623

46.227

45.728

50.022

36.146

39.642

60.76

FOR PERIOD OCT TO SEP

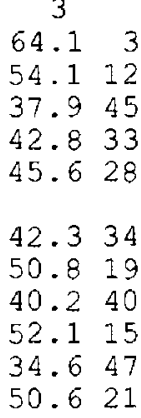

$44.8 \quad 31$

38.144

$\begin{array}{ll}39.9 & 41 \\ 42.0 & 35\end{array}$

59.07

54.911

50.720

47.626

40.539

56.78

55.89

$44.2 \quad 32$

$\begin{array}{ll}30.9 & 48 \\ 38.6 & 43\end{array}$

51.416

41.736

50.918

64.92

41.637

48.524

49.623

46.227

45.529

50.022

36.146

$\begin{array}{rr}39.5 & 42 \\ 60.6 & 6\end{array}$
15
$61.3 \quad 3$

$63.5 \quad 3$

$53.7 \quad 13$

$37.8 \quad 45$

42.733
45.528

42.234

50.419

$40.0 \quad 40$

51.815

$\begin{array}{ll}34.5 & 47 \\ 50.4 & 20\end{array}$

44.431

$66.6 \quad 1$

37.944

39.441

$41.8 \quad 35$

$\begin{array}{lll}58.8 & 7\end{array}$

50.221

47.326

40.439

$54.8 \quad 10$

55.59

44.132

30.748

$38.4 \quad 43$

51.216

$41.5 \quad 36$

$50.7 \quad 17$

64.52

$41.3 \quad 37$

48.024

49.523

$\begin{array}{lll}46.1 & 27\end{array}$

45.129

49.822

35.946

39.342 $\begin{array}{lll}52.2 & 13 \\ 37.4 & 44\end{array}$

42.133

45.028

$41.9 \quad 34$

49.022

$39.4 \quad 40$

51.115

33.947
49.7

$43.2 \quad 32$

$\begin{array}{lll}43.2 & 32 \\ 37.0 & 1\end{array}$

37.145

38.642

40.536

58.07

52.412

48.423

46.325

40.239

54.98

.010

54.89

43.531
$30.5 \quad 48$

37.743

50.516

$40.8 \quad 35$

50.017

63.22

40.437
52.811

46.226

$\begin{array}{ll}46.2 & 26 \\ 49.1 & 21\end{array}$

45.527

43.730
49.119

34.946

$\begin{array}{ll}34.9 & 46 \\ 38.7 & 41\end{array}$

38.7
59.8

\begin{abstract}
30
56.84
$\begin{array}{ll}50.0 & 12 \\ 36.4 & 43\end{array}$

$38.9 \quad 36$

$42.2 \quad 29$

40.933

46.621

$37.4 \quad 41$

49.513

$\begin{array}{lll}33.7 & 47\end{array}$
\end{abstract}

$\begin{array}{rr}41.4 & 31 \\ 61.2 & 1\end{array}$

$35.6 \quad 45$

36.742

38.138

56.15

48.017

44.824

43.326

39.834

52.010

53.18

$52.6 \quad 9$

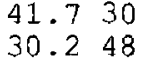

36.144

47.619

39.235

48.216

$\begin{array}{ll}49.62 & 2\end{array}$

$\begin{array}{ll}37.5 & 40 \\ 49.1 & 14\end{array}$

42.328

$48.5 \quad 15$

48.515
43.525

41.332

47.918

33.946

37.539
51 60

$51.9 \quad 5$

$\begin{array}{rr}47.2 & 13 \\ 35.6 & 39 \\ 37.1 & 36\end{array}$

37.136

36.937

39.130

43.021

35.043

46.115

$32.6 \quad 47$
46.2

40.028

$\begin{array}{ll}40.0 & 28 \\ 36.8 & 1\end{array}$

33.646

35.042

35.241

50.77

$45.8 \quad 17$

40.327

38.931

38.233

48.210

51.7

48.89

$41.6 \quad 24$

30.048

34.044

37.734

45.816

52.74

35.3
43.90

39.729

39.72

47.6112
42.323

38.332

45.218

33.845

36.438

$\begin{array}{rr}36.4 & 38 \\ 55.1 & 2\end{array}$

479

$46.0 \quad 10$

$35.2 \quad 37$

$\begin{array}{ll}35.1 & 38 \\ 34.7 & 40\end{array}$

38.528

41.421

$\begin{array}{ll}44.0 & 43 \\ 44.6 & 14 \\ 32.6 & 47\end{array}$

$32.2 \quad 47$

43.018

$39.6 \quad 24$

$53.6 \quad 1$

$32.4 \quad 46$

$\begin{array}{ll}34.6 & 41 \\ 34.2 & 42\end{array}$

46.79

43.616

38.529 
HIGHEST MEAN ELEVATION, IN FEET, AND RANKING FOR THE FOLLOWING NUMBER OF CONSECUTIVE DAYS FOR PERIOD OCT TO SEP

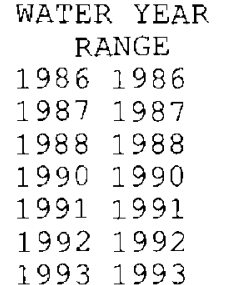

$\begin{array}{cr}1 & \\ 61.8 & 4 \\ 52.3 & 14 \\ 48.3 & 25 \\ 41.3 & 38 \\ 60.8 & 5 \\ 45.1 & 30 \\ 51.3 & 17\end{array}$
$61^{3} .64$

$52.3 \quad 14$

48.225

41.238

60.75

45.030

51.117

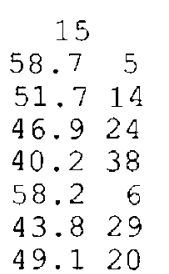

49.120

$\begin{array}{cr}30 \\ 54.0 & 7 \\ 50.3 & 11 \\ 45.7 & 23 \\ 38.6 & 37 \\ 54.5 & 6 \\ 42.4 & 27 \\ 46.1 & 22\end{array}$

50.618

$\begin{array}{rr}7 & \\ 60.9 & 4 \\ 52.1 & 14 \\ 47.9 & 25 \\ 41.0 & 38 \\ 60.1 & 6 \\ 44.7 & 30 \\ 50.6 & 18\end{array}$

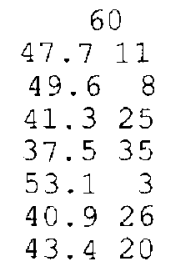

\begin{tabular}{rr} 
& \multicolumn{2}{c}{90} \\
45.3 & 12 \\
48.6 & 6 \\
38.9 & 27 \\
36.5 & 34 \\
49.8 & 4 \\
39.5 & 25 \\
43.2 & 17
\end{tabular}

$\begin{array}{rr} & 120 \\ 43.5 & 12 \\ 46.6 & 7 \\ 37.0 & 29 \\ 35.4 & 34 \\ 46.9 & 5 \\ 37.7 & 25\end{array}$

41.617
183

39.613

42.19

$34.6 \quad 32$

33.536

$\begin{array}{rr}44.2 & 6 \\ 35.4 & 27\end{array}$

38.817

LOWEST MEAN DISCHARGE, IN CUBIC FEET PER SECOND, AND RANKING FOR THE FOLLOWING NUMBER OF CONSECUTIVE DAYS FOR PERIOD APR TO MAR

WATER YEAR RANGE

19281929

19291930

19311932

19331934

19341935

19351936

19361937

19371938

19381939

19391940

19401941

19411942

19421943

19441945

19451946

19461947

19471948

19481949

19491950

19501951

19511952

19521953

19531954

1955

19561957

19571958

19581959

9591960

9601961

19611962

9621963

9631964

9641965

9651966

19661967

19671968

9681969

9691970

19701971

19711972

$1972 \quad 1973$

19731974

$1974 \quad 1975$

19751976

$1976 \quad 1977$

$1977 \quad 1978$

19781979

19791980

$1980 \quad 1981$

19811982

19821983

19831984

9841985

19851986

19861987

19871988

19881989

19891990

19901991

$1991 \quad 1992$

19921993

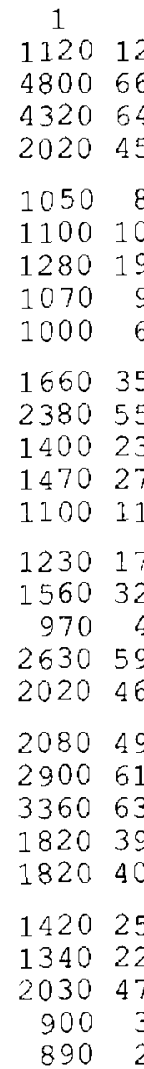

$1160 \quad 13$

269060

$1580 \quad 33$

299062

600

1690
15002
1190

19014

433065

$2340 \quad 54$

45026

984

206048

$1930 \quad 44$

153029

$1760 \quad 38$

166036

210050

252058

$1620 \quad 34$

120016

$\begin{array}{ll}2510 & 57 \\ 1550 & 31\end{array}$

$1040 \quad 7$

$1540 \quad 30$

9204

1890
1190
15

$1320 \quad 21$

125018

14024

8351

$1870 \quad 41$

248056
3

484066

440065
$2020 \quad 45$

10508

$1100 \quad 10$

131320

10076

166035

$2400 \quad 55$

140723

148027

110311

$1237 \quad 17$

$1560 \quad 31$

265359

$2060 \quad 47$

$2080 \quad 49$

290061

$3360 \quad 63$

$1820 \quad 39$

182040

$1420 \quad 25$

134022

$2050 \quad 46$

8902

116013

273060

$1640 \quad 34$

302362

169357

151328

119714

436764

5

234354
145726

9845

$2077 \quad 48$
218351

$1950 \quad 44$

154029

176738

166736

210350

255758

162733

123016

254057

156032

10477

$1553 \quad 30$

193043

$\begin{array}{ll}1897 & 42 \\ 1210 & 15\end{array}$

132721

125318

1417
1290
89

8461

187741

250056

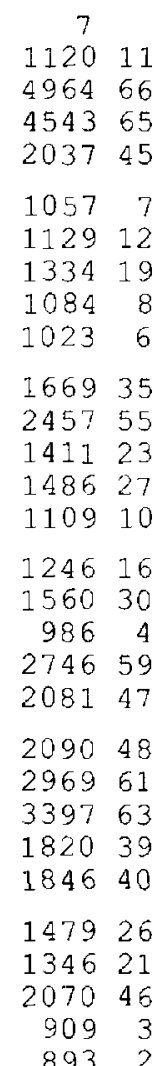

14

$5146 \quad 66$

467165

212345

10577

116112

133818

10496

169435

259455

143623

$1502 \quad 27$

$1262 \quad 17$

156029

9884

210145

$2130 \quad 47$

$3058 \quad 60$

$3516 \quad 63$

$1833 \quad 39$

185840

149626

$1358 \quad 19$

213248

9052

116013

284460

166334

303362

$1703 \quad 37$

153128

$1206 \quad 14$

443064

54

236354
145925
996

9965

211749

218951

$1954 \quad 44$

155029

38

166936
212750

$2650 \quad 58$

$1643 \quad 33$

125617

260757

159432

10999

$1574 \quad 31$

195142

195143

$12141 \mathrm{~b}$

$1370 \quad 22$

133920

$1439 \quad 24$

132118

188341

256156

117113

314861

$1671 \quad 33$

227452

$1721 \quad 37$

153928

122614

$4603 \quad 64$

240754

$2405 \quad 53$

$1468 \quad 24$

10095

226551

200143

156730

179638

170436

214549

$2675 \quad 56$

168334

125716

278758

159831

$11: 3 \quad 9$

198142

202944

125015

$\begin{array}{lll}1418 & 21\end{array}$

137920

$1484 \quad 25$

$\begin{array}{rr}1424 & 22 \\ 885 & 1\end{array}$

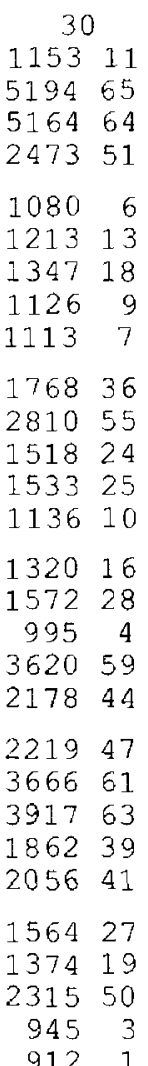

9121 
HIGHEST MEAN DISCHARGE, IN CUBIC FEET PER SECOND, AND RANKING FOR THE FOLLOWING NUMBER OF CONSECUTIVE DAYS FOR PERIOD OCT TO SEP

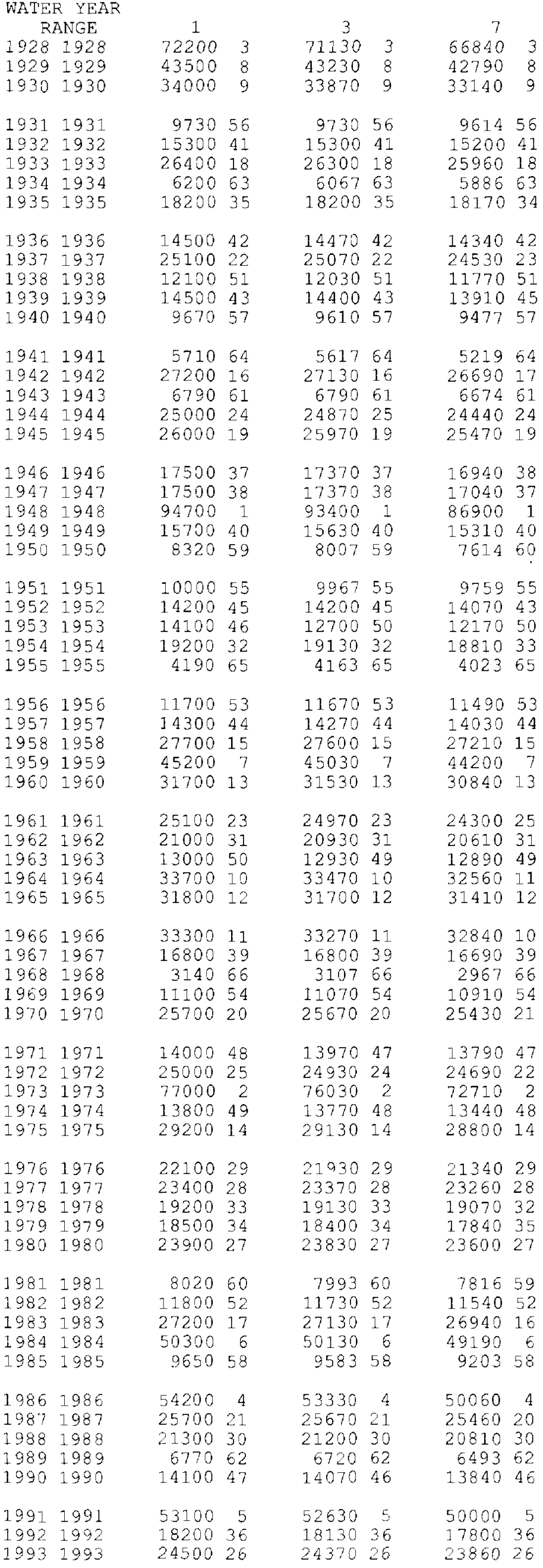

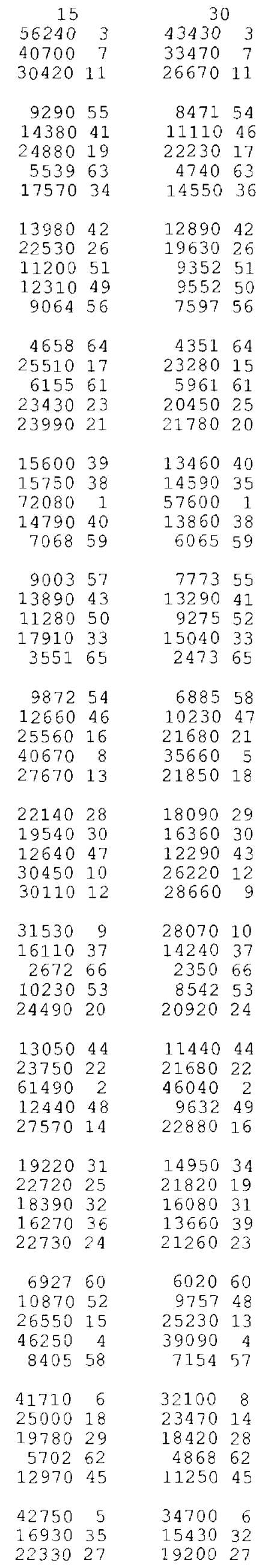

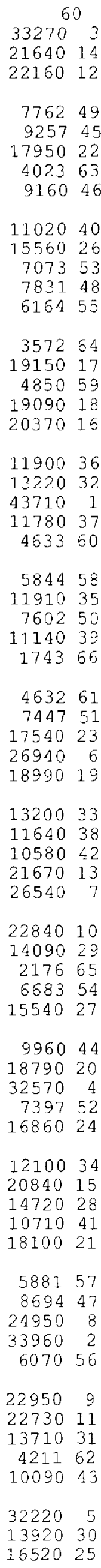

$25880^{90}$

25880

2031011

737146

723347

1670017

$\begin{array}{ll}3817 & 62 \\ 6792 & 50\end{array}$

1046036

1375027

611753

6695
5595
55

309364

1736015

434259

1565022

1617021

1168031

1097035

$\begin{array}{lll}10970 & 35 \\ 11920 & 30\end{array}$

383461

458658

$10290 \quad 37$

$7198 \quad 48$

917140

157666

434060

642652

1555023

215609

1644018

1132033

925439

887844

2038010

225806

1931013

115803

221065

566454

1381026

904643

1636019

$27670 \quad 3$

1485024

969138

1798014

$13050 \quad 28$
$9126 \quad 41$

1478025

502356

800345

224707

285502

481157

$19520 \quad 12$

216508

1127034

366563

908242

$27100 \quad 4$

1219029
1626020
120
$21140 \quad 6$

1466018

1825011

710646

627349

$14690 \quad 17$

345562
538253

927438

556752

612750

485155

282464

1555015

409257

$13050 \quad 24$

$12700 \quad 25$

1102029

935436

$31030 \quad 1$

1086030
348261

399559

1018033

632347

1022032

151366

403458

596251

1399021

$19700 \quad 7$

1399022

$10070 \quad 34$

760344

750145

196208

$21400 \quad 5$

$16010 \quad 14$

$9533 \quad 35$

212465

1954

1228026

851140

1456020

$24470 \quad 3$

1486016

820341

$17480 \quad 12$

1163028
$8726 \quad 39$

1310023

423956

$7762 \quad 43$

1942010

$24680 \quad 2$

394960

1715013

194309

932137

326063

$7837 \quad 42$

$23080 \quad 4$

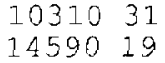

$21490^{183} 2$

$15250 \quad 9$

$14390 \quad 12$

$6335 \quad 42$

$4677 \quad 53$

1185018

297253
417355

707140

476052

476052
598545

$4388 \quad 54$

$2700 \quad 64$

$500 \quad 21$
$3655 \quad 56$

365556

173019

1010026

968827

$\begin{array}{lll}7517 \quad 37 \\ 31500 & 19\end{array}$

$31500 \quad 1$

63629

352357

347659

888331

566648

$418 \quad 32$

145066

348758

547249

1257016

1129023

814833

$5808 \quad 47$

599244

$15630 \quad 8$

$14130 \quad 13$

743738

196065

516451

1094024

$7849 \quad 35$

1135022

$20340 \quad 3$

521450

1197017

756536

1363014

964328

729939

1019025

347460

627343

1497010

193704

333861

$12790 \quad 15$

1471011

688441

306262

591346

$19220 \quad 5$

800134
1169020 
LOCATION.--Lat $30^{\circ} 05^{\prime} 59^{\prime \prime}$, long $83^{\circ} 10^{\prime} 18^{\prime \prime}$, in NE $\frac{1}{4}$ sec.36, T.4 S., R.11 E., Suwannee County, Hydrologic Unit 03110205, near right bank on upstream side of bridge on State Highway 51, 1.6 mi south of Luraville, $10.8 \mathrm{mi}$ south of Dowling Park and $97 \mathrm{mi}$ upstream from mouth.

DRAINAGE AREA.--7,330 $\mathrm{mi}^{2}$ approximately, includes part of watershed in Okefenokee Swamp which is indeterminate.

PERIOD OF RECORD.--February 1927 to December 1937. April 1956, January and March 1976 (one to two discharge measurements made each year); May to August 1977, January 1978 to 1983 (monthly gage heights and discharge measurements only).

REVISED RECORDS.--WDR FL-78-4: Drainage area.

GAGE.--Nonrecording gage. Datum of gage is National Geodetic Vertical Datum of 1929. February 1927 to December 1937, nonrecording gage at site 0.8 mi downstream at datum of $16.49 \mathrm{ft}$ lower.

REMARKS.--Records fair.

MAXIMUM, MINIMUM, AND MEAN OF MONTHLY MEAN ELEVATIONS FOR WATER YEARS 1927-1937
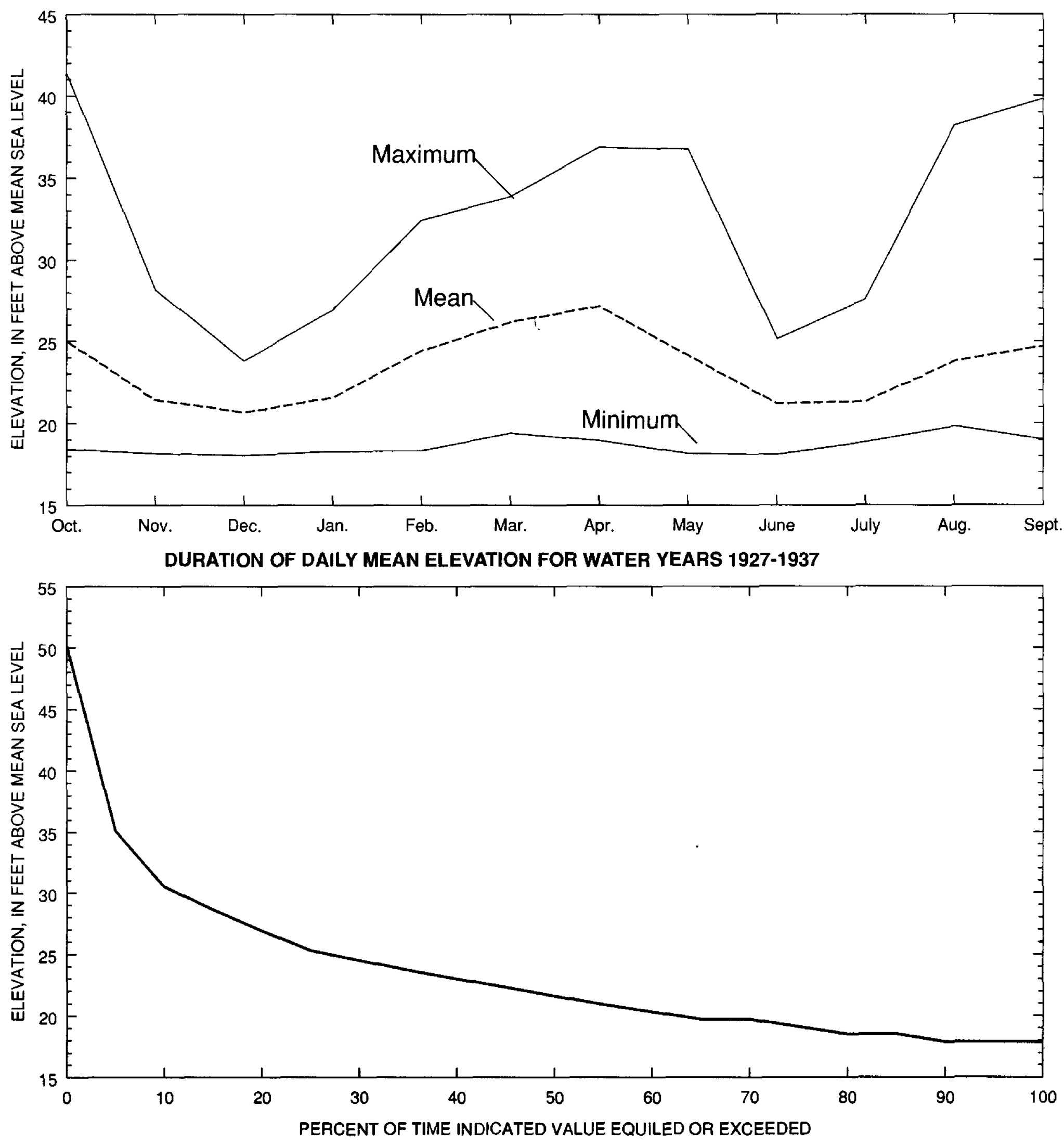
SUMMARY STATISTICS

(CUBIC FEET PER SECOND)

WATER YEARS 1927 - 1937

WATER YEARS 1937 - 1973

ANNUAL MEAN

HIGHEST ANNUAL MEAN

LOWEST ANNUAL MEAN

HIGHEST DAILY MEAN

TONEST DAIIY MEAN

ANNUAL SEVEN-DAY MINIMUM

INSTANTANEOUS PEAK FLOW

INSTANTANEOUS PEAK ELEVATION (FT)

ANNUAL RUNOFF (INCHES)

7162

12570

2709

66000

1290

1300

66000

50.20

13.37

$\begin{array}{lll} & & 1929 \\ & & 1934 \\ \text { Aug } 24 & 1928 \\ \text { Jun 25 } & 1935 \\ \text { Jun 25 } & 1935 \\ \text { Aug 24 } & 1928 \\ \text { Aug 24 } & 1928\end{array}$

90000

Apr 51948

MAXIMUM, MINIMUM, AND MEAN OF MONTHLY MEAN DISCHARGES FOR WATER YEARS 1927-1937

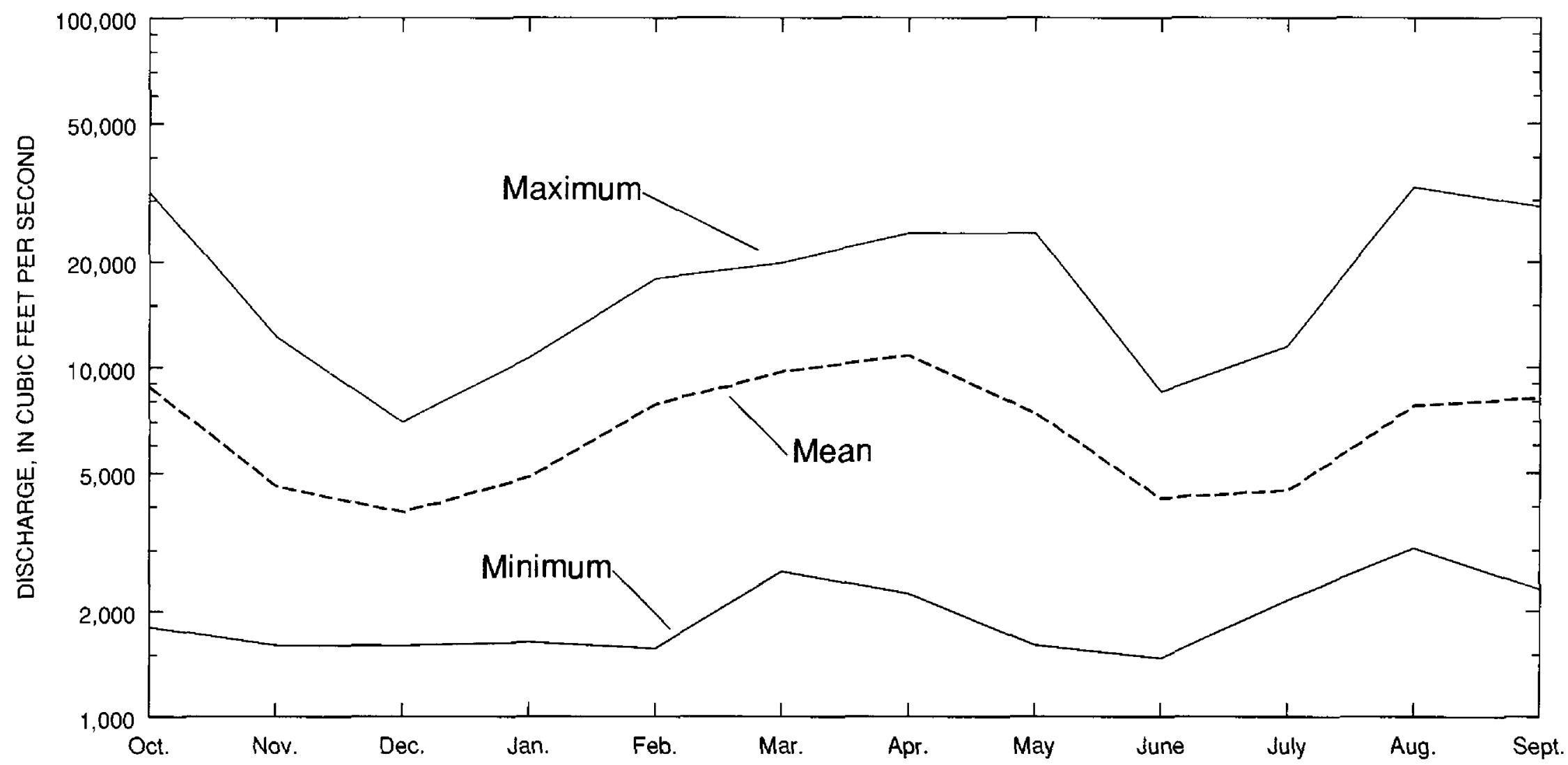

DURATION OF DAILY MEAN DISCHARGE FOR WATER YEARS 1927-1937

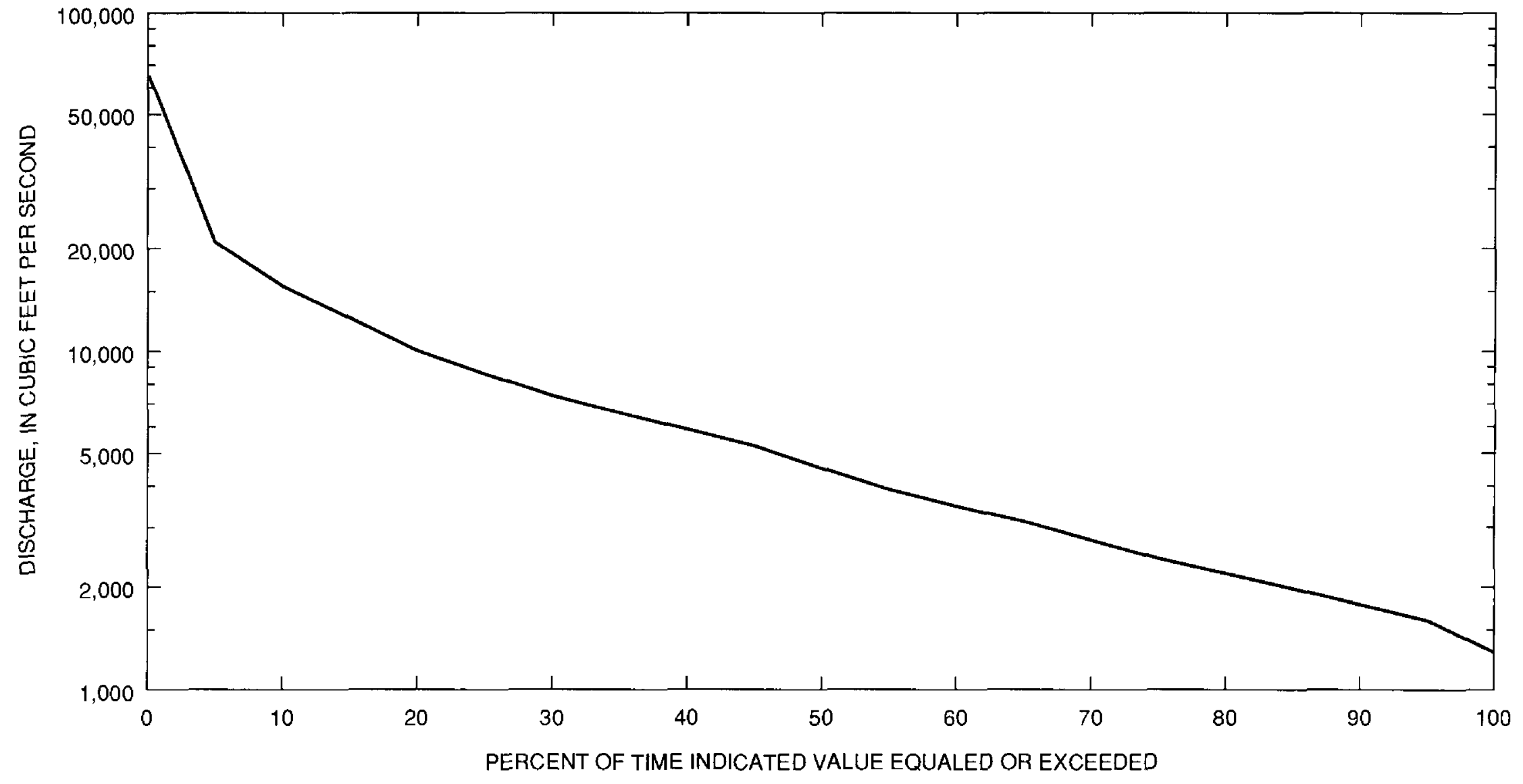


SUWANNEE RIVER BASIN

02320000 SUWANNEE RIVER AT LURAVILLE, FL--Continued

SUMMARY OF MONTHLY MEAN ELEVATION AND DISCHARGE STATISTICS FOR WATER YEARS 1927-1937

\begin{tabular}{|c|c|c|c|c|c|c|}
\hline \multirow[b]{2}{*}{ MONTH } & \multicolumn{3}{|c|}{$\begin{array}{ll} & \text { ELEVAT IONS, } \\
\text { FEET ABOVE SEA LEVEL }\end{array}$} & \multicolumn{3}{|c|}{ DISCHARGE, } \\
\hline & MAXIMUM & MTNTMUM & MEAN & MAXIMUM & MINIMUM & MEAN \\
\hline OCTOBER & 41.32 & 18.40 & 25.01 & 31460 & 1800 & 8809.73 \\
\hline NOVEMBER & 28.14 & 18.15 & 21.43 & 12180 & 1600 & 4590.73 \\
\hline DECEMBER & 23.73 & 18.04 & 20.66 & 6936 & 1595 & 3854.55 \\
\hline JANUARY & 26.87 & 18.27 & 21.56 & 10580 & 1630 & 4879.20 \\
\hline FEBRUARY & 32.38 & 18.32 & 24.41 & 17810 & 1565 & 7850.91 \\
\hline MARCH & 33.82 & 19.38 & 26.18 & 19760 & 2612 & 9701.73 \\
\hline APRIL & 36.84 & 18.94 & 27.14 & 24050 & 2248 & 10811.36 \\
\hline MAY & 36.71 & 18.15 & 24.11 & 24060 & 1600 & 7429.18 \\
\hline JUNE & 25.08 & 18.09 & 21.21 & 8453 & 1467 & 4231.54 \\
\hline JUZY & 27.54 & 18.85 & 21.33 & 11430 & 2155 & 4454.54 \\
\hline AUGUST & 38.16 & 19.81 & 23.76 & 32590 & 3045 & 7781.36 \\
\hline SEPTEMBER & 39.78 & 19.01 & 24.69 & 28650 & 2316 & 8192.73 \\
\hline
\end{tabular}

\section{DURATION OF DAILY MEAN VALUES FOR WATER YEARS 1927-1937}

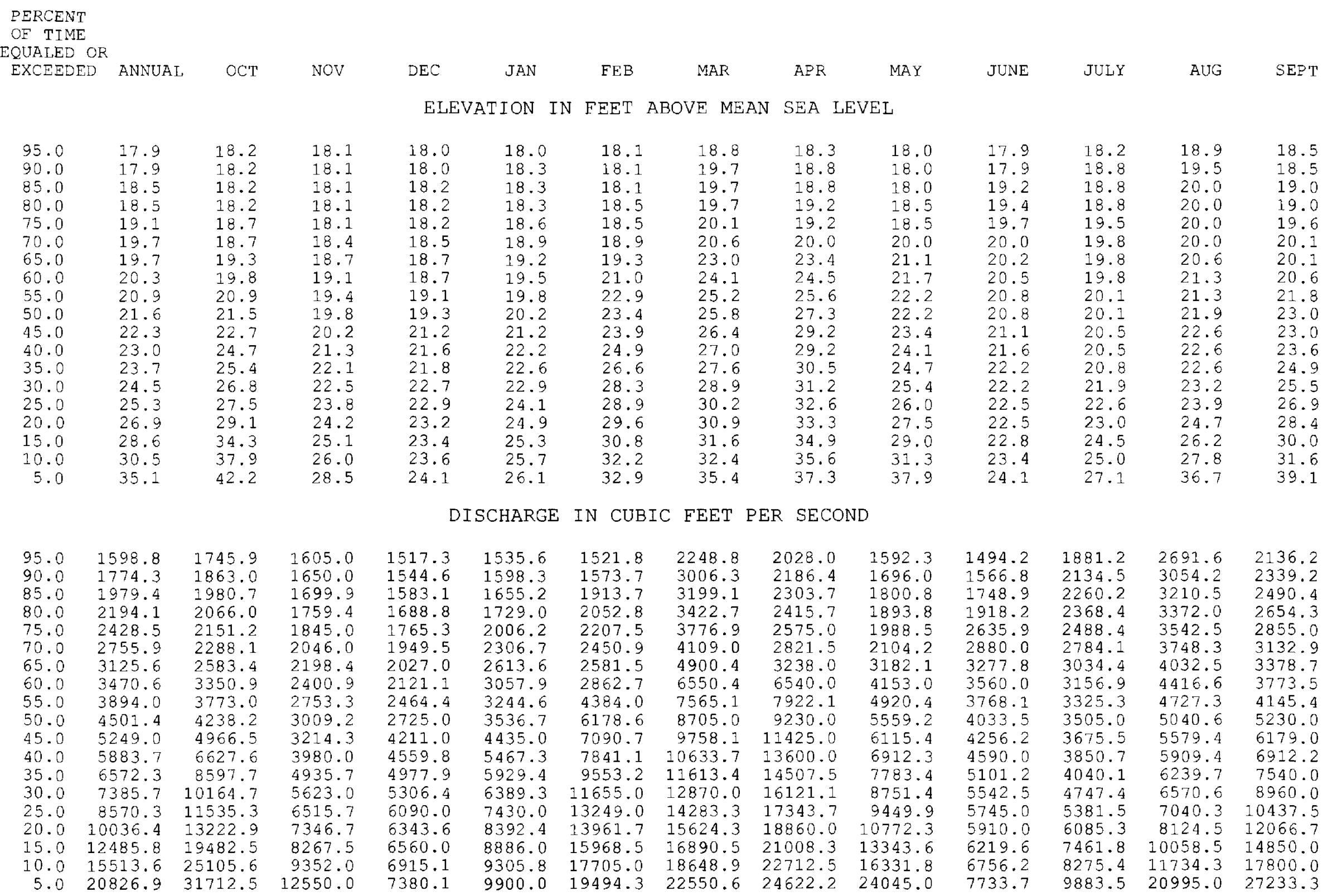



FOR PERIOD APR TO MAR

WATER YEAR

RANGE

19281929

19301931

19311932

19321933

19331934

19351936

$1936 \quad 1937$

$\begin{array}{cccccc}1 & & 3 & & 7 & \\ 22.8 & 8 & 22.8 & 8 & 22.9 & 8 \\ 21.2 & 7 & 22.5 & 7 & 22.5 & 7 \\ 19.7 & 6 & 19.7 & 6 & 19.8 & 6 \\ 18.0 & 2 & 18.0 & 2 & 18.0 & 2 \\ 18.0 & 3 & 18.0 & 3 & 18.0 & 3 \\ 18.3 & 4 & 18.3 & 4 & 18.3 & 4 \\ 17.9 & 1 & 17.9 & 1 & 17.9 & 1\end{array}$

18.85

18.85

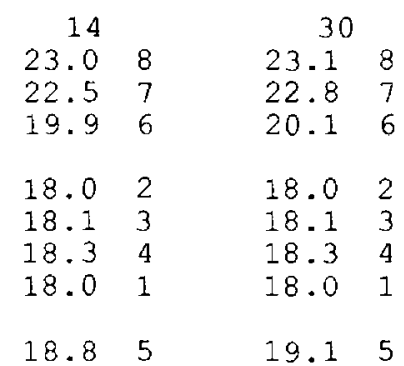

$18.8 \quad 5$

18.85

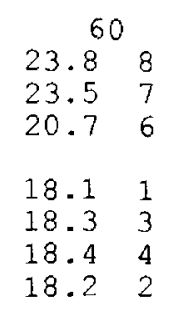

19.55

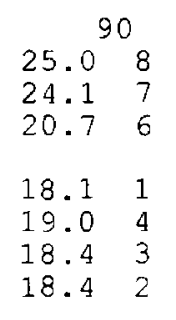

20.15

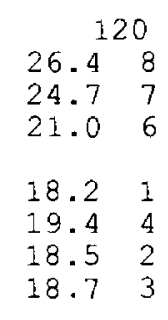

$20.4 \quad 5$

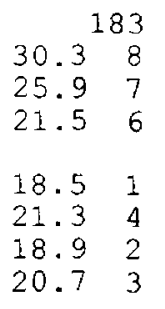

21.45

HIGHEST MEAN ELEVATION, IN FEET, AND RANKING FOR THE FOLLOWING NUMBER OF CONSECUTIVE DAYS FOR PERIOD OCT TO SEP

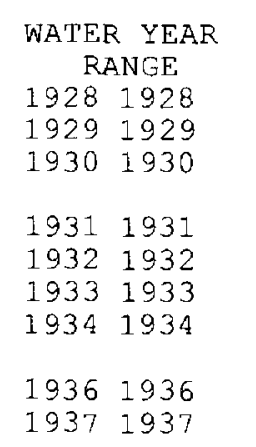

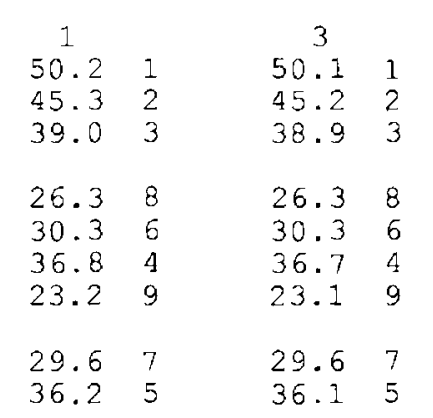

$\begin{array}{rr}15 & \\ 48.0 & 1 \\ 44.2 & 2 \\ 37.9 & 3 \\ 26.0 & 8 \\ 29.1 & 7 \\ 36.0 & 4 \\ 22.5 & 9 \\ 29.2 & 6 \\ 35.1 & 5\end{array}$

$\begin{array}{ll}30 & \\ 43.8 & 1 \\ 41.6 & 2 \\ 37.1 & 3 \\ 25.2 & 8 \\ 27.0 & 7 \\ 34.7 & 4 \\ 21.8 & 9 \\ 28.5 & 6 \\ 33.5 & 5\end{array}$

\begin{tabular}{llll}
\multicolumn{2}{c}{60} & \multicolumn{2}{c}{90} \\
39.1 & 1 & 35.3 & 1 \\
35.0 & 2 & 31.7 & 4 \\
34.8 & 3 & 33.4 & 2 \\
& & & \\
24.6 & 8 & 24.2 & 7 \\
25.3 & 7 & 23.6 & 8 \\
32.4 & 4 & 31.8 & 3 \\
21.1 & 9 & 20.9 & 9 \\
& & & \\
27.2 & 6 & 26.8 & 6 \\
30.8 & 5 & 29.3 & 5
\end{tabular}

\begin{tabular}{llll}
\multicolumn{2}{c}{120} & \multicolumn{3}{c}{183} \\
32.7 & 1 & 33.3 & 1 \\
30.2 & 3 & 30.3 & 2 \\
32.3 & 2 & 29.5 & 3 \\
& & & \\
24.0 & 7 & 23.3 & 7 \\
22.7 & 8 & 21.3 & 8 \\
30.2 & 4 & 27.8 & 4 \\
20.6 & 9 & 20.0 & 9 \\
& & & \\
25.7 & 6 & 23.8 & 6 \\
27.9 & 5 & 25.7 & 5
\end{tabular}

LOWEST MEAN DISCHARGE, IN CUBIC FEET PER SECOND, AND RANKING FOR THE FOLLOWING NUMBER OF CONSECUTIVE DAYS FOR PERIOD APR TO MAR

\begin{tabular}{|c|c|c|c|c|c|c|c|c|c|c|c|c|c|c|c|c|c|c|}
\hline RANGE & 1 & & 3 & & 7 & & 14 & & 30 & & 60 & & 9 & 90 & & 20 & & 183 \\
\hline 19271928 & 1630 & 6 & 1630 & 6 & 1630 & 6 & 1664 & 6 & 1689 & 6 & 1717 & 6 & 1805 & 5 & 1956 & 4 & 2356 & 4 \\
\hline 19281929 & 5950 & 10 & 5983 & 10 & 60211 & 10 & 6171 & 10 & 62951 & 10 & 7056 & 10 & 8418 & 10 & 10090 & 10 & 15490 & 10 \\
\hline 19291930 & 5650 & 9 & 5650 & 9 & 5650 & 9 & 5679 & 9 & 6021 & 9 & 6679 & 9 & 7311 & 9 & 8077 & 9 & 9476 & 9 \\
\hline 19301931 & 2910 & 8 & 2910 & 8 & 2974 & 8 & 3058 & 8 & 3282 & 8 & 3883 & 8 & 3873 & 8 & 4149 & 8 & 4623 & 7 \\
\hline 19311932 & 1490 & 3 & 1490 & 3 & 1490 & 3 & 1490 & 2 & 1509 & 2 & 1539 & 1 & 1589 & 1 & 1676 & 2 & 1848 & 1 \\
\hline 19321933 & 1490 & 4 & 1513 & 4 & 1540 & 4 & 1550 & 4 & 1574 & 5 & 1702 & 5 & 2395 & 6 & 2673 & 6 & 4703 & 8 \\
\hline 19331934 & 1490 & 5 & 1537 & 5 & 1550 & 5 & 1555 & 5 & 1558 & 3 & 1611 & 4 & 1705 & 3 & 1794 & 3 & 2121 & 3 \\
\hline $1934 \quad 1935$ & 1470 & 2 & 1470 & 2 & 1470 & 2 & 1502 & 3 & 1563 & 4 & 1582 & 3 & 1601 & 2 & 1674 & 1 & 1945 & 2 \\
\hline 19351936 & 1290 & 1 & 1290 & 1 & 1.303 & 1 & 1335 & 1 & 1392 & 1 & 1558 & 2 & 1730 & 4 & 1972 & 5 & 4223 & 6 \\
\hline $1936 \quad 1937$ & 2010 & 7 & 2010 & 7 & 2010 & 7 & 2016 & 7 & 2100 & 7 & 2601 & 7 & 2626 & 7 & 2866 & 7 & 3218 & 5 \\
\hline
\end{tabular}

HIGHEST MEAN DISCHARGE AND RANKING FOR THE FOLLOWING NUMBER OF CONSECUTIVE DAYS FOR PERIOD OCT TO SEP

\footnotetext{
WATER YEAR

RANGE
19281928

19281928

19301930

$1931 \quad 1931$

19321932

19331933

19341934

19351935

19361936
}

$\begin{array}{rrrr}1 & & 3 & \\ 66000 & 1 & 65600 & 1 \\ 39800 & 2 & 39400 & 2 \\ 27200 & 3 & 27130 & 3 \\ & & & \\ 9810 & 9 & 9810 & 9 \\ 14800 & 7 & 14800 & 7 \\ 24000 & 4 & 23870 & 4 \\ 6560 & 10 & 6487 & 10 \\ 17500 & 6 & 17500 & 6 \\ 13900 & 8 & 13900 & 8 \\ 23300 & 5 & 23170 & 5\end{array}$
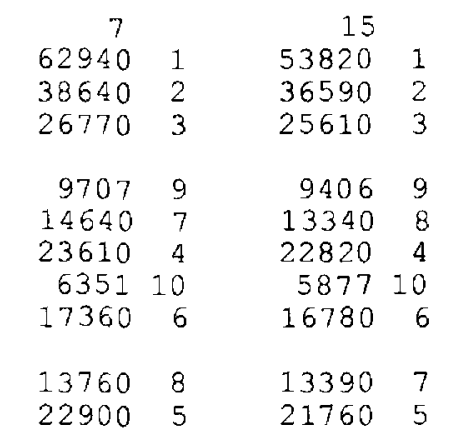

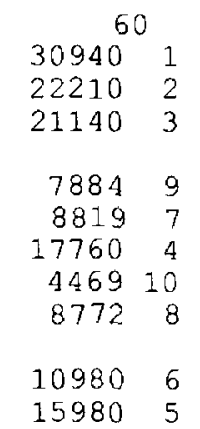

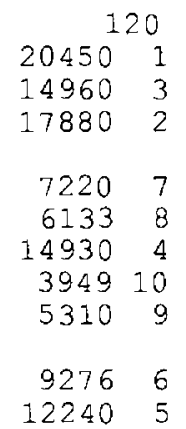

\begin{tabular}{rr}
\multicolumn{3}{r}{183} \\
20570 & 1 \\
15460 & 2 \\
14300 & 3 \\
& \\
6519 & 7 \\
4703 & 8 \\
12030 & 4 \\
3385 & 10 \\
4223 & 9 \\
7228 & 6 \\
9650 & 5
\end{tabular} 
LOCATION.--Lat $29^{\circ} 57^{\prime} 20^{\prime \prime}$, long $82^{\circ} 55^{\prime} 40^{\prime \prime}$, in NE ${ }^{1} / 4$ sec.20, T.6 S., R. 14 E., Suwannee County, Hydrologic Unit 03110205, near left bank on upstream side of bridge on U.S. Highway 27 at Branford, 10.2 mi upstream from Santa Fe River and 75 mi upstream from mouth.

DRAINAGE AREA.--7,880 $\mathrm{mi}^{2}$, includes part of watershed in Okefenokee Swamp which is indeterminate.

PERIOD OF RECORD.--July 193 I to 1993.

REVISED RECORDS.--WSP 1905: WDR FL-75-1: Drainage area.

GAGE.--Nonrecording gage. Datum of gage is $4.81 \mathrm{ft}$ above National Geodetic Vertical Datum of 1929.

REMARKS,--Records are good.

EXTREMES OUTSIDE PERIOD OF RECORD.--Flood of August 1928 reached a stage of $32.0 \mathrm{ft}$ from floodmark; discharge, $65,000 \mathrm{ft}^{3} / \mathrm{s}$ computed on basis of measured crest flow at Ellaville (station 02319500).
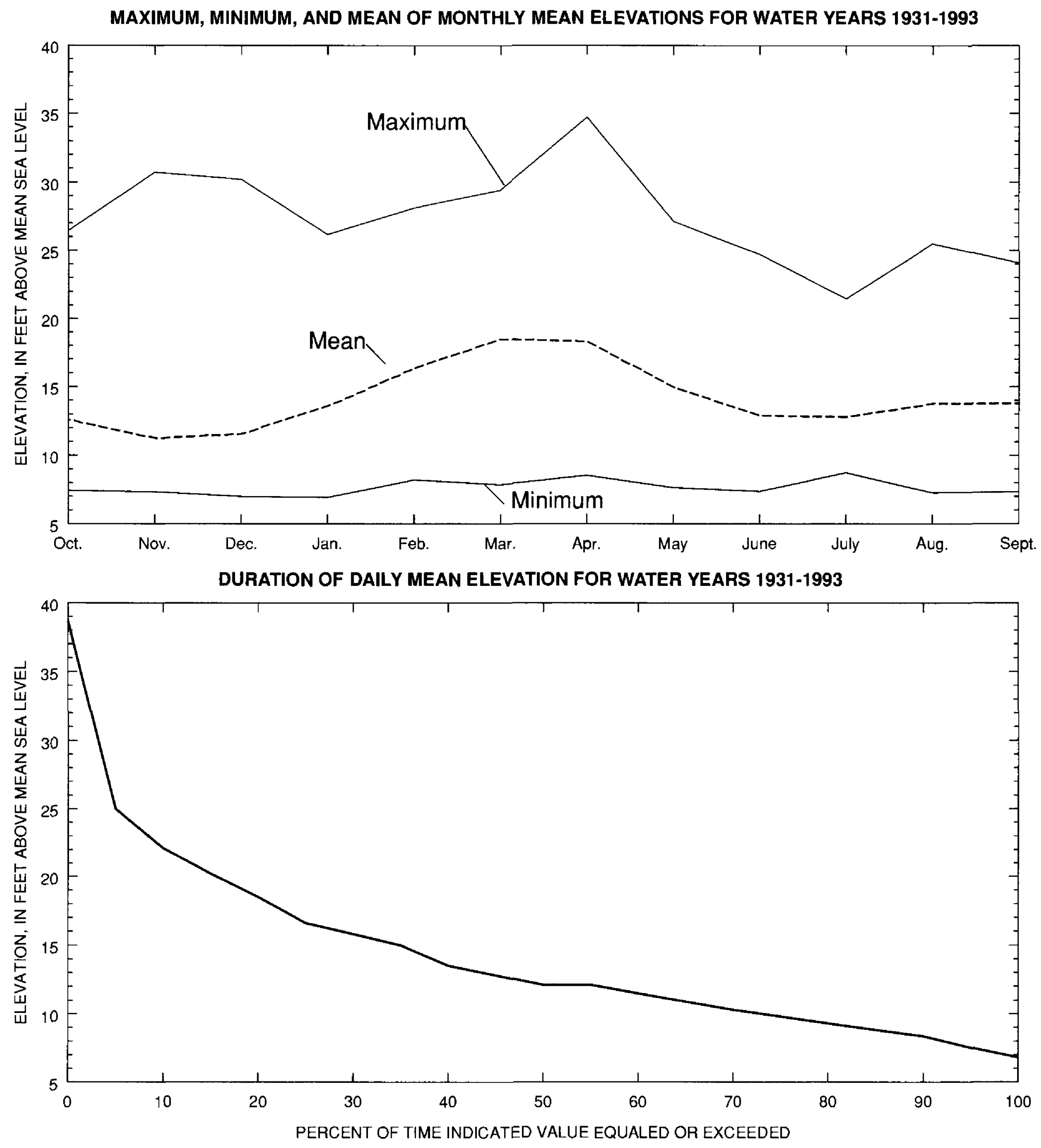
SUMMARY STATISTICS, IN CUBIC FEET PER SECOND UNLESS OTHERWISE INDICATED, FOR WATER YEARS 1931 - 1993

ANNUAL MEAN

LOWEST ANNUAL MEAN

HIGHEST DAILY MFAN

LOWEST DAILY MEAN

ANNUAL SEVEN-DAY MINIMUM

INSTANTANEOUS PEAK FLOW

INSTANTANEOUS PEAK ELEVATION

INSTANTANEOUS LOW FLOW

ANNUAL RUNOFE (INCHES/CFSM)
HIGHEST ANNUAL MEAN

$\begin{array}{rrrr}7046 & & & \\ 19260 & & & 1948 \\ 1950 & & & 1955 \\ 82800 & \text { Apr } 11 & 1948 \\ 1530 & \text { JuI } & 1 & 1955 \\ 1550 & \text { Jan } 8 & 1956 \\ 83900 & \text { Apr } 11 & 1948 \\ \text { (FT) } 38.88 & \text { Apr } 11 & 1948 \\ 1530 & \text { JuI } 1 & 1955 \\ 12.15 / 0.89 & & \end{array}$

MAXIMUM, MINIMUM, AND MEAN OF MONTHLY MEAN DISCHARGES FOR WATER YEARS $1931-1993$

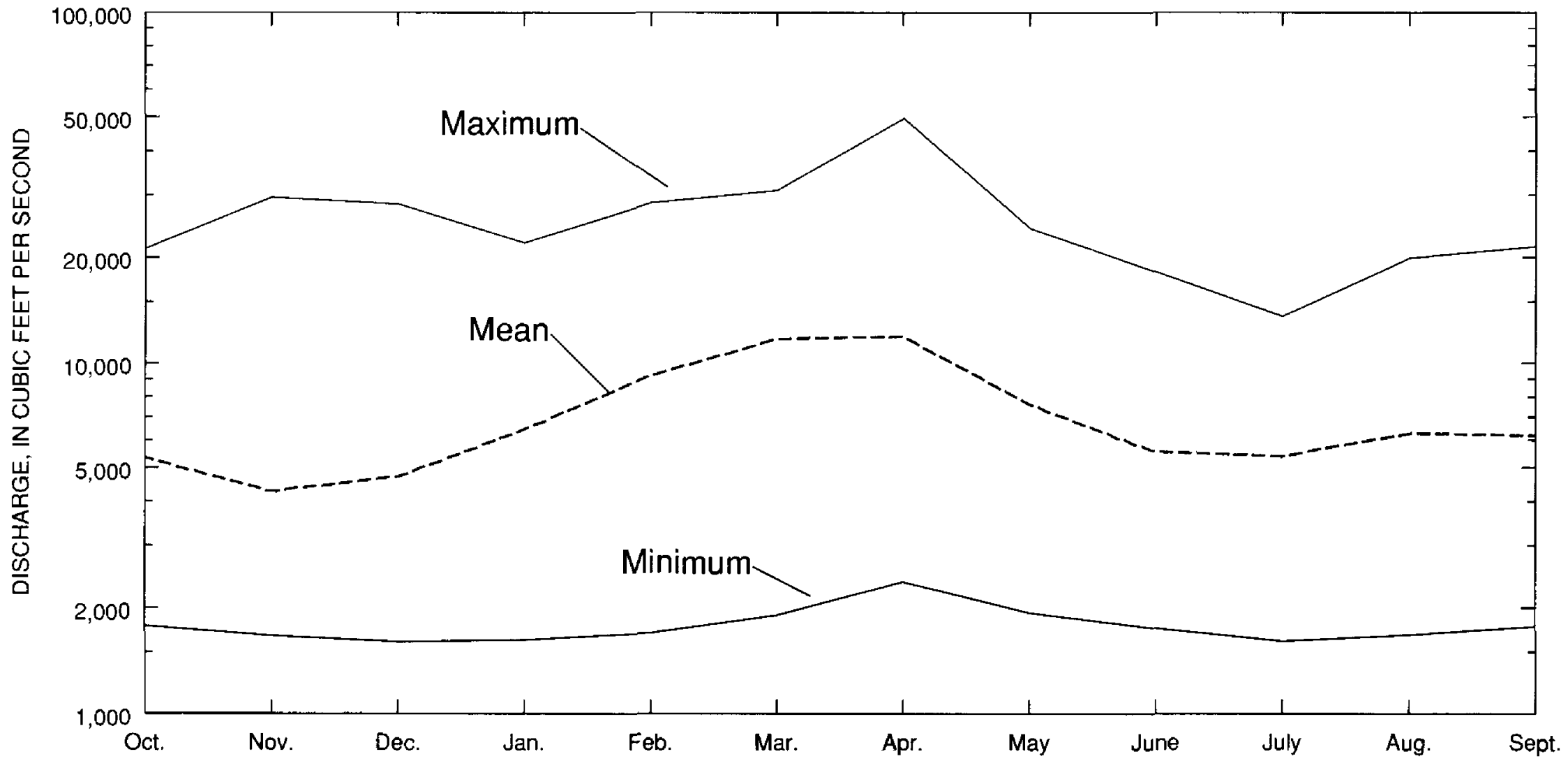

DURATION OF DAILY MEAN DISCHARGE FOR WATER YEARS 1931-1993

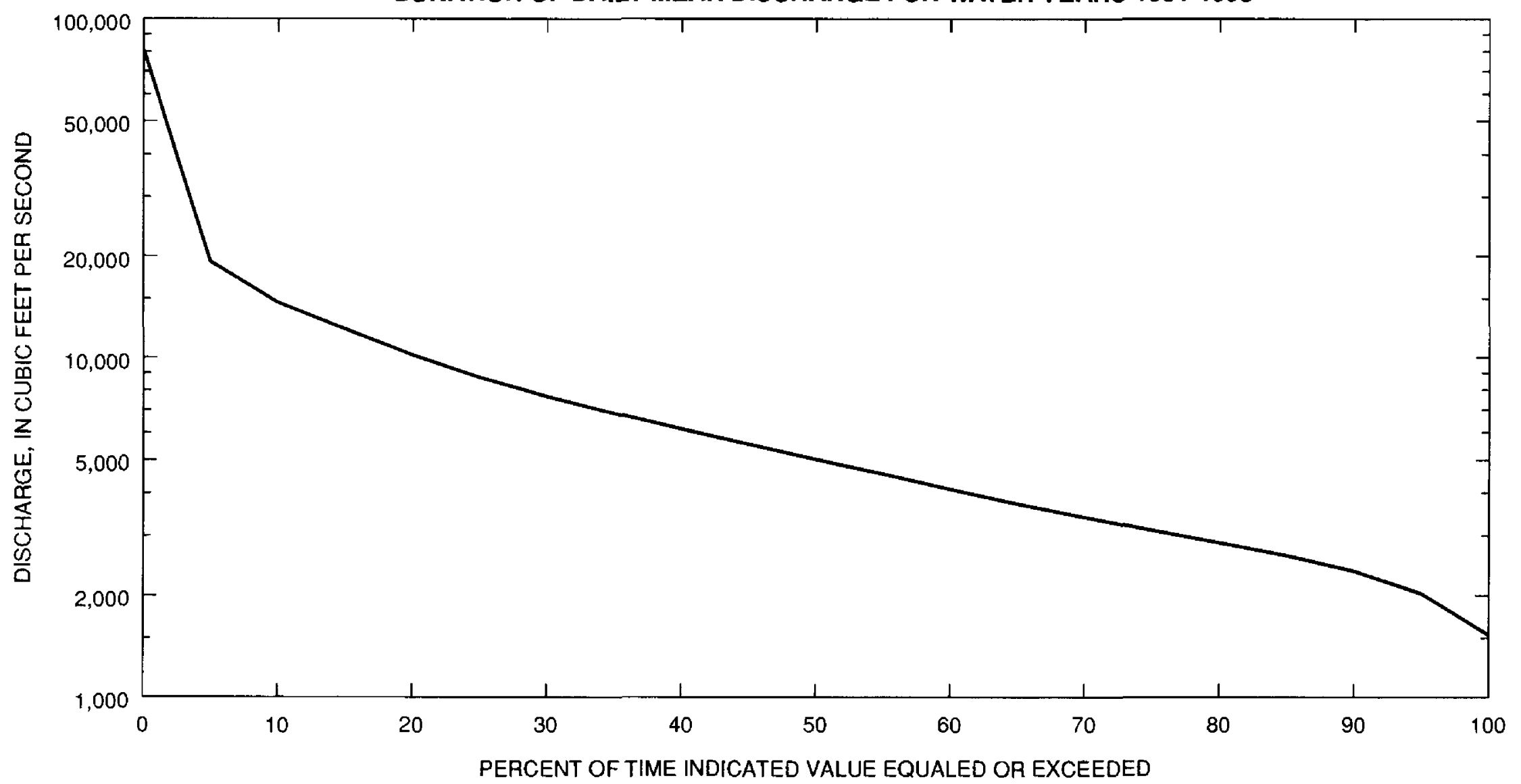


SUWANNEE RIVER BASIN

02320500 SUWANNEE RIVER AT BRANFORD, FL--Continued

\section{SUMMARY OF MONTHEY MEAN ELEVATION AND DISCHARGE STATISTICS} FOR WATER YEARS 1931-1993

\begin{tabular}{|c|c|c|c|c|c|c|}
\hline \multirow[b]{2}{*}{ MONTH } & \multicolumn{3}{|c|}{$\begin{array}{l}\text { ELEVATIONS, } \\
\text { FEET ABOVE SEA LEVE,L }\end{array}$} & \multicolumn{3}{|c|}{$\begin{array}{ll} & \text { DISCHARGE, } \\
\text { CUBIC FEET PER SÉCOND }\end{array}$} \\
\hline & MAXIMUM & MINIMUM & MEAN & MAXIMUM & MINIMUM & MEAN \\
\hline OCTOBER & 26.37 & 7.44 & 12.59 & 21020 & 1778 & 5331 \\
\hline NOVEMBER & 30.65 & 7.34 & 11.25 & 29380 & 1666 & 4269 \\
\hline DECEMBER & 30.15 & 7.00 & 11.58 & 28130 & 1602 & 4703 \\
\hline JANUARY & 26.12 & 6.93 & 13.60 & 21830 & 1623 & 6463 \\
\hline FEBRUARY & 28.07 & 8.22 & 16.36 & 28370 & 1699 & 9200 \\
\hline MARCH & 29.38 & 7.87 & 18.47 & 30710 & 1905 & 11720 \\
\hline APRII & 34.64 & 8.53 & 18.30 & 49040 & 2366 & 11920 \\
\hline MAY & 27.09 & 7.65 & 14.96 & 24020 & 1937 & 7609 \\
\hline JUNE & 24.66 & 7.38 & 12.90 & 18120 & 1752 & 5544 \\
\hline JULY & 21.41 & 8.73 & 12.80 & 13510 & 1610 & 5372 \\
\hline AUGUST & 25.44 & 7.28 & 13.77 & 19810 & 1678 & 6275 \\
\hline SEPTEMBER & 24.04 & 7.33 & 13.76 & 21340 & 1769 & 6190 \\
\hline
\end{tabular}

\section{DURATION OF DAILY MEAN VALUES FOR WATER YEARS 1931-1993}

\begin{tabular}{|c|c|c|}
\hline PERCENT & & \\
\hline OF TIME & & \\
\hline EQUALED & & \\
\hline $\begin{array}{c}\text { OR } \\
\text { EXCEEDED }\end{array}$ & ANNUAL & OCT \\
\hline 95.0 & 7.5 & 7.8 \\
\hline 90.0 & 8.3 & 8.1 \\
\hline 85.0 & 8.8 & 8.8 \\
\hline 80.0 & 9.3 & 8.8 \\
\hline 75.0 & 9.8 & 9.2 \\
\hline 70.0 & 10.3 & 9.6 \\
\hline 65.0 & 10.9 & 10.1 \\
\hline 60.0 & 11.5 & 20.1 \\
\hline 55.0 & 12.1 & 20.9 \\
\hline 50.0 & 12.1 & 11.4 \\
\hline 45.0 & 12.8 & 11.9 \\
\hline 40.0 & 13.5 & 12.4 \\
\hline 35.0 & 15.0 & 13.5 \\
\hline 30.0 & 15.8 & 24.1 \\
\hline 25.0 & 16.6 & 24.7 \\
\hline 20.0 & 18.5 & 16.0 \\
\hline 15.0 & 20.2 & 16.6 \\
\hline 10.0 & 22.1 & 18.1 \\
\hline 5.0 & 25.0 & 19.7 \\
\hline
\end{tabular}

$\begin{array}{rrr}95.0 & 2029.0 & 1942.5 \\ 90.0 & 2358.5 & 2265.6 \\ 85.0 & 2617.8 & 2597.1 \\ 80.0 & 2858.8 & 2768.0 \\ 75.0 & 3108.4 & 2896.5 \\ 70.0 & 3393.1 & 3043.9 \\ 65.0 & 3714.8 & 3204.7 \\ 60.0 & 4091.9 & 3414.9 \\ 55.0 & 4549.9 & 3709.1 \\ 50.0 & 5007.5 & 4048.0 \\ 45.0 & 5538.0 & 4472.2 \\ 40.0 & 6134.0 & 4920.6 \\ 35.0 & 6819.9 & 5381.0 \\ 30.0 & 7658.4 & 5858.1 \\ 25.0 & 8715.6 & 6447.7 \\ 20.0 & 10151.9 & 7246.9 \\ 15.0 & 12175.6 & 8584.4 \\ 10.0 & 14585.8 & 9930.4 \\ 5.0 & 19298.5 & 12464.1\end{array}$

\begin{tabular}{|c|c|c|}
\hline \multirow[t]{2}{*}{ NOV } & DEC & JAN \\
\hline & \multicolumn{2}{|c|}{ ELEVATION } \\
\hline 7.3 & 7.1 & 7.4 \\
\hline 7.7 & 7.5 & 8.1 \\
\hline 8.0 & 7.8 & 8.5 \\
\hline 8.4 & 8.2 & 8.9 \\
\hline 8.8 & 8.6 & 9.3 \\
\hline 8.8 & 9.0 & 9.8 \\
\hline 9.2 & 9.0 & 9.8 \\
\hline 9.2 & 9.4 & 10.8 \\
\hline 9.7 & 9.9 & 11.3 \\
\hline 10.2 & 10.4 & 11.8 \\
\hline 10.6 & 10.4 & 12.4 \\
\hline 11.1 & 10.9 & 13.6 \\
\hline 11.6 & 11.4 & 14.2 \\
\hline 12.2 & 11.9 & 14.9 \\
\hline 12.8 & 12.5 & 16.3 \\
\hline 12.8 & 13.1 & 17.9 \\
\hline 13.4 & 14.3 & 19. \\
\hline 14.6 & 15.7 & 21. \\
\hline 16.0 & 19.8 & \\
\hline
\end{tabular}

$\begin{array}{rr}\text { FEB } & \text { MAR } \\ \text { FEET } & \text { ABOVE MEAN } \\ & \\ 8.0 & 8.8 \\ 8.8 & 9.7 \\ 9.2 & 10.7 \\ 9.7 & 11.8 \\ 10.7 & 13.0 \\ 11.7 & 13.6 \\ 12.3 & 14.9 \\ 14.1 & 15.7 \\ 14.8 & 16.4 \\ 15.5 & 18.0 \\ 16.3 & 18.9 \\ 17.1 & 19.8 \\ 17.9 & 21.0 \\ 19.7 & 22.0 \\ 21.0 & 22.7 \\ 22.1 & 24.1 \\ 23.1 & 25.5 \\ 24.3 & 27.9 \\ 26.2 & 29.5\end{array}$

$\begin{array}{rr}\text { APR } & \text { MAY } \\ \text { SEA LEVEL } \\ 8.8 & 8.0 \\ 9.7 & 8.7 \\ 10.8 & 9.1 \\ 11.9 & 10.0 \\ 12.5 & 10.5 \\ 13.8 & 10.9 \\ 14.5 & 11.4 \\ 15.2 & 12.5 \\ 16.0 & 13.0 \\ 16.8 & 13.6 \\ 17.6 & 14.8 \\ 19.4 & 15.5 \\ 20.8 & 16.2 \\ 21.7 & 16.9 \\ 22.9 & 17.7 \\ 24.2 & 19.3 \\ 25.8 & 20.2 \\ 27.7 & 22.0 \\ 29.7 & 25.0\end{array}$

DISCHARGE IN CUBIC FEET PER SECOND

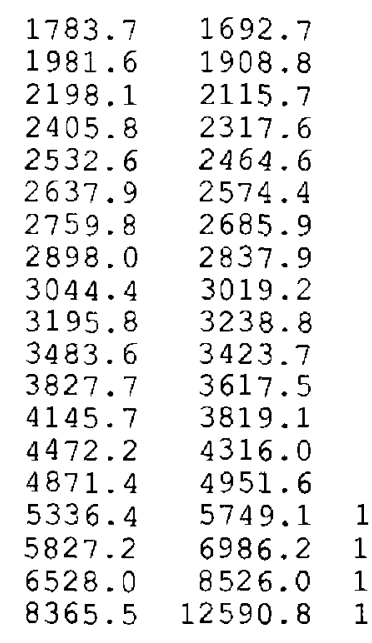

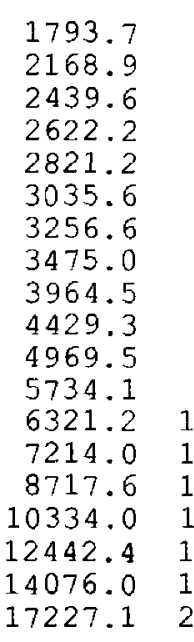

$\begin{array}{rrrr}2126.5 & 2722.5 & 2676.9 & 2223.2 \\ 2521.0 & 3334.6 & 3224.5 & 2537.5 \\ 2831.4 & 3802.3 & 3798.0 & 2929.2 \\ 3112.3 & 4537.2 & 4539.0 & 3288.5 \\ 3801.2 & 5506.4 & 5282.7 & 3654.2 \\ 4419.0 & 6195.3 & 6066.6 & 4117.9 \\ 5081.9 & 6969.8 & 6793.5 & 4588.7 \\ 6306.2 & 7879.8 & 7508.1 & 5068.3 \\ 7306.0 & 8829.5 & 8316.6 & 5536.3 \\ 8003.9 & 10015.5 & 9244.1 & 6207.1 \\ 8572.0 & 11390.6 & 10282.4 & 6923.7 \\ 9189.5 & 12466.4 & 11604.8 & 7556.6 \\ 10521.3 & 13442.7 & 12959.2 & 8138.0 \\ 11920.2 & 14396.3 & 13941.5 & 8807.8 \\ 13272.0 & 15601.4 & 15678.6 & 9620.4 \\ 14548.9 & 17579.7 & 17904.8 & 10857.7 \\ 15845.5 & 19679.1 & 20472.7 & 12265.7 \\ 17771.9 & 24320.3 & 23604.3 & 14487.3 \\ 21712.3 & 27879.1 & 27838.3 & 18745.2\end{array}$

2122.4

2429.3
2629.4 2911.9 3188.3 3448.8 3734.1 3973.8 4254.5 4591.3 4942.4 $5231 \cdot 7$ 5608.2 6124.0 6613.7 7264.6 8419.2 $\begin{array}{llllllll}8365.5 & 12590.8 & 17227.1 & 21712.3 & 27879.1 & 27838.3 & 18745.2 & 12961.5\end{array}$

$\begin{array}{rrr}2233.5 & 2152.6 & 2168.8 \\ 2450.1 & 2495.1 & 2379.1 \\ 2747.5 & 2799.8 & 2805.1 \\ 2946.5 & 3197.5 & 3108.3 \\ 3124.9 & 3581.7 & 3353.7 \\ 3323.4 & 3804.7 & 3590.0 \\ 3579.4 & 4131.0 & 3863.8 \\ 3852.6 & 4531.0 & 4220.5 \\ 4282.2 & 4902.8 & 4608.6 \\ 4611.5 & 5303.7 & 4934.5 \\ 4904.5 & 5612.8 & 5377.5 \\ 5217.8 & 5991.0 & 5873.6 \\ 5584.6 & 6411.2 & 6375.5 \\ 5964.8 & 6866.7 & 6883.8 \\ 6626.4 & 7477.1 & 7445.8 \\ 7404.4 & 8611.9 & 8318.7 \\ 8386.2 & 9480.5 & 10025.5 \\ 9905.5 & 10709.1 & 11884.6 \\ 12008.7 & 15606.9 & 14893.2\end{array}$


LOWEST MEAN ELEVATION, IN FEET, AND RANKING FOR THE FOLLOWING NUMBER OF CONSECUTIVE DAYS FOR PERIOD APR TO MAR

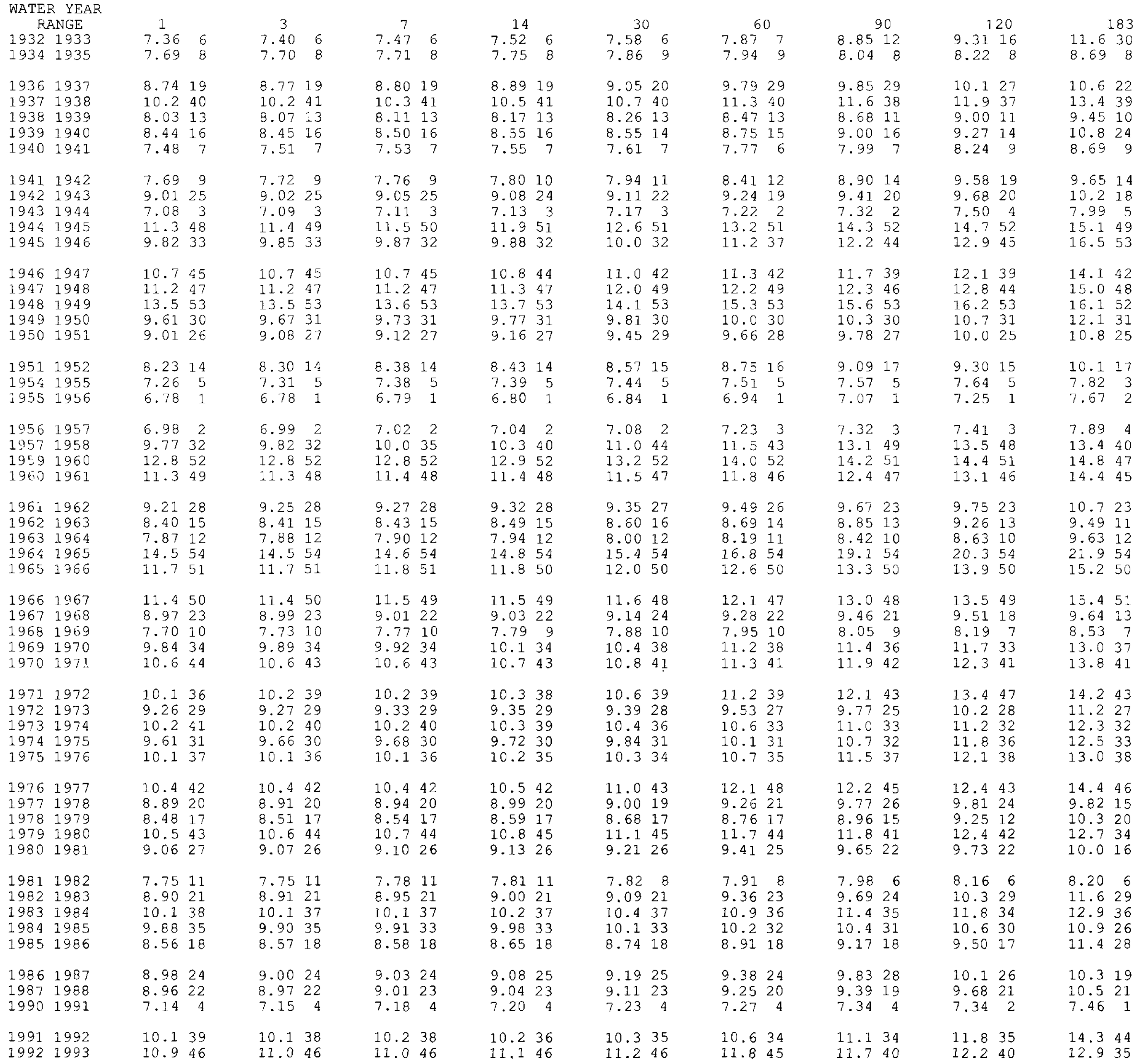


HIGHEST MEAN ELEVATION, IN FEET, AND RANKING FOR THE FOLLOWING NUMBER OF CONSECUTIVE DAYS FOR PERIOD OCT TO SEP

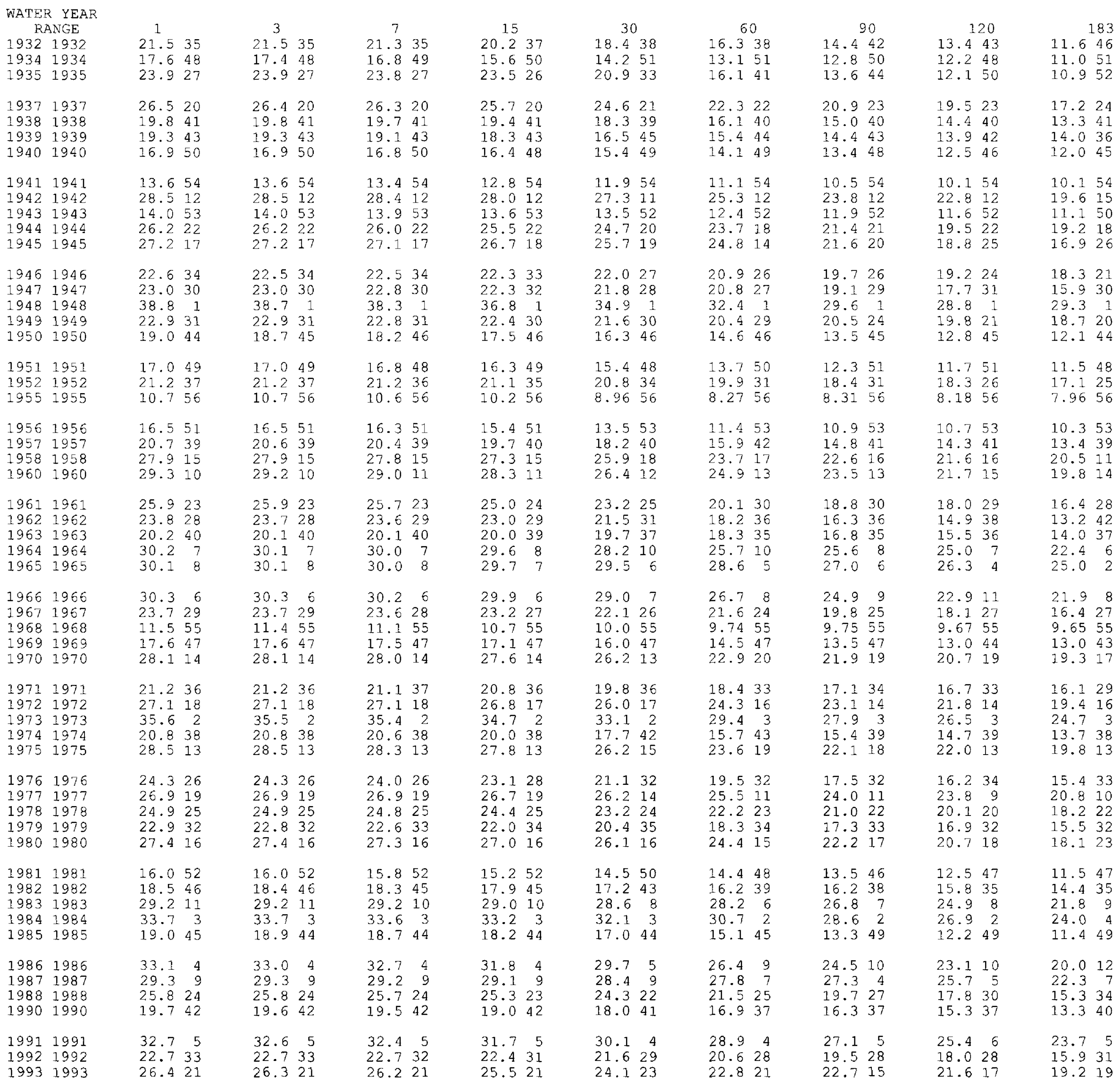


LOWEST MEAN DISCHARGE, IN CUBIC FEET PER SECOND, AND RANKING FOR THE FOLLOWING NUMBER OF CONSECUTIVE DAYS FOR PERIOD APR TO MAR

\begin{tabular}{|c|c|c|c|c|c|c|c|c|c|c|c|c|c|c|c|c|c|c|c|}
\hline $\mathrm{RA}$ & ANGE & 1 & & 3 & & 7 & & 14 & & 30 & & 6 & & 90 & & 12 & & & 83 \\
\hline 1932 & 1933 & 1790 & 7 & 1790 & 7 & 1824 & 7 & 1854 & 7 & 1890 & 7 & 2077 & 11 & 2783 & 26 & 3080 & 30 & 5034 & 41 \\
\hline 1933 & 1934 & 2060 & 15 & 2060 & 15 & 2060 & 14 & 2069 & 14 & 2080 & 13 & 2151 & 13 & 2237 & 12 & 2348 & 11 & 2719 & 11 \\
\hline 1934 & 1935 & 1950 & 11 & 1950 & 11 & 1950 & 9 & 1975 & 11 & 2027 & 12 & 2065 & 9 & 2116 & 8 & 2213 & 8 & 2471 & 8 \\
\hline 1935 & 1935 & 1820 & 8 & 1833 & 8 & 1860 & 8 & 1884 & 8 & 1913 & 8 & 2033 & 8 & 2159 & 9 & 2372 & 12 & 4410 & 35 \\
\hline 1936 & 1937 & 2470 & 29 & 2503 & 30 & 2521 & 29 & 2568 & 31 & 2668 & 32 & 3142 & 37 & 3199 & 36 & 3403 & 36 & 3761 & 29 \\
\hline 1937 & 1938 & 3400 & 48 & 3400 & 48 & 3454 & 48 & 3562 & 49 & 3743 & 51 & 4170 & 49 & 4424 & 50 & 4633 & 45 & 5887 & 45 \\
\hline 1938 & 1939 & 2100 & 16 & 2133 & 16 & 2157 & 16 & 2182 & 16 & 2227 & 17 & 2344 & 16 & 2458 & 14 & 2655 & 14 & 2942 & 13 \\
\hline 1939 & 1940 & 2300 & 22 & 2320 & 22 & 2343 & 22 & 2377 & 22 & 2388 & 21 & 2489 & 21 & 2642 & 21 & 2815 & 21 & 3988 & 32 \\
\hline 1940 & 1941 & 1940 & 9 & 1940 & 9 & 1954 & 11 & 1965 & 9 & 1990 & 10 & 2069 & 10 & 2184 & 10 & 2327 & 9 & 2607 & 10 \\
\hline 1941 & 1942 & 1990 & 13 & 1990 & 13 & 2019 & 13 & 2040 & 13 & 2118 & 14 & 2382 & 17 & 2601 & 19 & 3022 & 28 & 3061 & 15 \\
\hline 1942 & 1943 & 2570 & 33 & 2570 & 33 & 2596 & 33 & 2613 & 33 & 2630 & 30 & 2715 & 28 & 2830 & 28 & 3020 & 27 & 3308 & 21 \\
\hline 1943 & 1944 & 1610 & 4 & 1610 & 4 & 1610 & 3 & 1616 & 3 & 1630 & 3 & 1647 & 3 & 1685 & 3 & 1787 & 4 & 2104 & 5 \\
\hline 1944 & 1945 & 4100 & 57 & 4127 & 57 & 4230 & 57 & 4509 & 58 & 5012 & 59 & 5467 & 58 & 6286 & 59 & 6621 & 59 & 6925 & 58 \\
\hline 1945 & 1946 & 3070 & 42 & 3097 & 42 & 3127 & 42 & 3133 & 42 & 3241 & 42 & 4204 & 51 & 5165 & 56 & 5880 & 57 & 8553 & 60 \\
\hline 1946 & 1947 & 3580 & 53 & 3580 & 52 & 3580 & 52 & 3603 & 50 & 3687 & 49 & 3955 & 47 & 4244 & 44 & 4520 & 43 & 6163 & 49 \\
\hline 1947 & 1948 & 4300 & 58 & 4300 & 58 & 4334 & 58 & 4409 & 57 & 4736 & 57 & 4926 & 57 & 4946 & 55 & $528 I$ & 54 & 7499 & 59 \\
\hline 1948 & 1949 & 5050 & 60 & 5083 & 60 & 5129 & 60 & 5241 & 60 & 5541 & 60 & 6460 & 60 & 6595 & 60 & 7006 & 60 & 6901 & 56 \\
\hline 1949 & 1950 & 2740 & 36 & 2767 & 36 & 2786 & 36 & 2800 & 36 & 2821 & 36 & 2922 & 35 & 3067 & 35 & 3300 & 34 & 4476 & 37 \\
\hline 1950 & 1951 & 2460 & 28 & 2500 & 29 & 2537 & 31 & 2554 & 30 & 2754 & 35 & 2848 & 33 & 2870 & 29 & 2966 & 26 & 3521 & 25 \\
\hline 1951 & 1952 & 2200 & 18 & 2253 & 18 & 2301 & 21 & 2325 & 21 & 2402 & 22 & 2501 & 22 & 2739 & 25 & 2895 & 24 & 3536 & 26 \\
\hline 1952 & 1953 & 2150 & 17 & 2170 & 17 & 2184 & 17 & 2191 & 17 & 2215 & 16 & 2281 & 14 & 2358 & 13 & 2493 & 13 & 2583 & 9 \\
\hline 1953 & 1954 & 3140 & 43 & 3160 & 44 & 3176 & 44 & 3249 & 45 & 3430 & 46 & 3889 & 46 & 4503 & 51 & 5180 & 53 & 6521 & 52 \\
\hline 1954 & 1955 & 1580 & 3 & 1603 & 3 & 1629 & 4 & 1634 & 4 & 1650 & 4 & 1684 & 4 & 1709 & 4 & 1743 & 2 & 1831 & 2 \\
\hline 1955 & 1956 & 1530 & 1 & 1537 & 1 & 1550 & 1 & 1554 & 1 & 1575 & 1 & 1619 & 2 & 1667 & 1 & 1750 & 3 & 2049 & 4 \\
\hline 1956 & 1957 & 1640 & 6 & 1647 & 6 & 1664 & 6 & 1675 & 5 & 1695 & 5 & 1778 & 6 & 1826 & 6 & 1874 & 5 & 2177 & 6 \\
\hline 1957 & 1958 & 3540 & 52 & 3583 & 53 & 3746 & 55 & 3976 & 56 & 4514 & 56 & 4913 & 56 & 5952 & 58 & 6558 & 58 & 6234 & 51 \\
\hline 1958 & 1959 & 2660 & 35 & 2673 & 35 & 2681 & 35 & 2704 & 35 & 2735 & 34 & 2855 & 34 & 2998 & 32 & 3106 & 31 & 3906 & 31 \\
\hline 1959 & 1950 & 4440 & 59 & 4460 & 59 & 4467 & 59 & 4513 & 59 & 4800 & 58 & 5570 & 59 & 5482 & 57 & 5692 & 56 & 6083 & 48 \\
\hline 1960 & 1961 & 3630 & 55 & 3640 & 55 & 3670 & 54 & 3698 & 54 & 3726 & 50 & 3957 & 48 & 4413 & 49 & 4992 & 50 & 6066 & 47 \\
\hline 1961 & 1962 & 2610 & 34 & 2633 & 34 & 2647 & 34 & 2676 & 34 & 2694 & 33 & 2779 & 32 & 2885 & 30 & 2936 & 25 & 3672 & 27 \\
\hline 1962 & 1963 & 2250 & 19 & 2260 & 19 & 2271 & 18 & 2310 & 19 & 2375 & 20 & 2429 & 19 & 2533 & 17 & 2810 & 18 & 2965 & 14 \\
\hline 1963 & 1954 & 1980 & 12 & 1987 & 12 & 1999 & 12 & 2011 & 12 & 2014 & 11 & 2085 & 12 & 2219 & 11 & 2341 & 10 & 3067 & 16 \\
\hline 1964 & 1965 & 6370 & 61 & 6403 & 61 & 6471 & 61 & 6665 & 61 & 7130 & 61 & 8378 & 61 & 11030 & 61 & 12930 & 61 & 15010 & 61 \\
\hline 1965 & 1966 & 3600 & 54 & 3620 & 54 & 3626 & 53 & 3661 & 53 & 3797 & 52 & 4172 & 50 & 4672 & 53 & 5131 & 52 & 6233 & 50 \\
\hline 1966 & 1967 & 3440 & 49 & 3447 & 49 & 3471 & 49 & 3495 & 48 & 3552 & 47 & 3843 & 45 & 4530 & 52 & 4898 & 49 & 6748 & 54 \\
\hline 1967 & 1968 & 2400 & 25 & 2.410 & 25 & 2423 & 25 & 2435 & 25 & 2494 & 25 & 2579 & 25 & 2686 & 22 & 2775 & 17 & 2867 & 12 \\
\hline 1968 & 1969 & 1630 & 5 & 1637 & 5 & 1659 & 5 & 1679 & 6 & 1705 & 6 & 1727 & 5 & 1782 & 5 & 1889 & 6 & 2019 & 3 \\
\hline 1969 & 1970 & 3270 & 47 & 3303 & 47 & 3320 & 47 & 3459 & 47 & 3676 & 48 & 4239 & 52 & 4399 & 48 & 4629 & 44 & 5608 & 44 \\
\hline 1970 & 1971 & 3240 & 46 & 3243 & 46 & 3267 & 46 & 3299 & 46 & 3392 & 45 & 3725 & 44 & 4145 & 42 & 4485 & 42 & 5890 & 46 \\
\hline 1971 & 1972 & 2860 & 38 & 2913 & 40 & 2927 & 40 & 2979 & 40 & 3193 & 41 & 3683 & 43 & 4318 & 46 & 5452 & 55 & 6610 & 53 \\
\hline 1972 & 1973 & 2450 & 27 & 2460 & 27 & 2489 & 27 & 2499 & 27 & 2523 & 27 & 2596 & 26 & 2724 & 24 & 3050 & 29 & 3786 & 30 \\
\hline 1973 & 1974 & 2780 & 37 & 2787 & 37 & 2797 & 37 & 2814 & 37 & 2862 & 37 & 3011 & 36 & 3313 & 37 & 3560 & 38 & 4449 & 36 \\
\hline 1974 & 1975 & 2870 & 39 & 2897 & 39 & 2907 & 38 & 2929 & 38 & 2990 & 38 & 3163 & 38 & 3547 & 39 & 4232 & 40 & 5003 & 40 \\
\hline 1975 & 1976 & 3140 & 44 & 3153 & 43 & 3174 & 43 & 3198 & 43 & 3290 & 44 & 3617 & 42 & 4185 & 43 & 4669 & 46 & 5431 & 43 \\
\hline 1976 & 1977 & 3510 & 51 & 3517 & 50 & 3563 & 51 & 3615 & 51 & 3961 & 55 & 4872 & 55 & 4920 & $5 A$ & 5082 & 51 & 6921 & 57 \\
\hline 1977 & 1978 & 2490 & 30 & 2507 & 31 & 2521 & 30 & 2553 & 29 & 2561 & 28 & 2741 & 29 & 3025 & 34 & 3127 & 32 & 3088 & 17 \\
\hline 1978 & 1979 & 2260 & 20 & 2277 & 21 & 2294 & 20 & 2321 & 20 & 2367 & 19 & 2416 & 18 & 2525 & 16 & 2714 & 15 & 3484 & 24 \\
\hline 1979 & 1980 & 3470 & 50 & 3523 & 51 & 3556 & 50 & 3655 & 52 & 3822 & 53 & 4282 & 53 & 4333 & 47 & 4779 & 48 & 5046 & 42 \\
\hline 1980 & 1981 & 2410 & 26 & 2417 & 26 & 2433 & 26 & 2451 & 26 & 2496 & 26 & 2611 & 27 & 2805 & 27 & 2887 & 23 & 3112 & 18 \\
\hline 1981 & 1982 & 1940 & 10 & 1940 & 10 & 1953 & 10 & 1965 & 10 & 1972 & 9 & 2017 & 7 & 2054 & 7 & 2148 & 7 & 2179 & 7 \\
\hline 1982 & 1983 & 2490 & 31 & 2497 & 28 & 2520 & 28 & 2549 & 28 & 2599 & 29 & 2753 & 30 & 2960 & 31 & 3380 & 35 & 4289 & 34 \\
\hline 1983 & 1984 & 2870 & 40 & 2883 & 38 & 2916 & 39 & 2958 & 39 & 3043 & 39 & 3333 & 40 & 3655 & 40 & 3950 & 39 & 4861 & 38 \\
\hline 1984 & 1985 & 3060 & 41 & 3073 & 41 & 3086 & 41 & 3125 & 41 & 3187 & 40 & 3265 & 39 & 3381 & 38 & 3520 & 37 & 3756 & 28 \\
\hline 1985 & 1986 & 2260 & 21 & 2267 & 20 & 2274 & 19 & 2306 & 18 & 2360 & 18 & 2459 & 20 & 2618 & 20 & 2825 & 22 & 4249 & 33 \\
\hline 1986 & 1387 & 2540 & 32 & 2550 & 32 & 2566 & 32 & 2592 & 32 & 2654 & 31 & 2767 & 31 & 3023 & 33 & 3155 & 33 & 3279 & 20 \\
\hline 1987 & 1988 & 2340 & 23 & 2350 & 23 & 2366 & 23 & 2383 & 23 & 2424 & 23 & 2503 & 23 & 2600 & 18 & 2813 & 20 & 3436 & 22 \\
\hline 1988 & 1989 & 2360 & 24 & 2367 & 24 & 2383 & 24 & 2407 & 24 & 2447 & 24 & 2521 & 24 & 2708 & 23 & 2810 & 19 & 3219 & 19 \\
\hline 1989 & 1990 & 1990 & 14 & 2037 & 14 & 2071 & 15 & 2114 & 15 & 2165 & 15 & 2303 & 15 & 2502 & 15 & 2730 & 16 & 3447 & 23 \\
\hline 1990 & 1991 & 1550 & 2 & 1553 & 2 & 1567 & 2 & 1576 & 2 & 1592 & 2 & 1618 & 1 & 1677 & 2 & 1689 & 1 & 1780 & 1 \\
\hline 1991 & 1992 & 3180 & 45 & 3190 & 45 & 3214 & 45 & 3235 & 44 & 3282 & 43 & 3498 & 41 & 3828 & 41 & 4371 & 41 & 6884 & 55 \\
\hline 92 & 1993 & 3660 & 56 & 3723 & 56 & 3763 & 56 & 3839 & 55 & 3889 & 54 & 4347 & 54 & 4270 & 45 & 4711 & 47 & 5001 & 39 \\
\hline
\end{tabular}



CONSECUTIVE DAYS FOR PERIOD OCT TO SEP

\begin{tabular}{|c|c|c|c|c|c|c|c|c|c|}
\hline \\
\hline \multicolumn{2}{|c|}{ RANGE } & \multicolumn{2}{|l|}{1} & \multicolumn{2}{|l|}{3} & \multicolumn{2}{|l|}{7} & \multirow{2}{*}{\multicolumn{2}{|c|}{$\begin{array}{r}15 \\
3050\end{array}$}} \\
\hline 1932 & & 14800 & 37 & 14730 & 37 & 14490 & 37 & & \\
\hline 1933 & 1933 & 24100 & 16 & 23900 & 16 & 23440 & 16 & 22610 & 1 \\
\hline 1934 & 1934 & 8350 & 56 & 7870 & 57 & 7436 & 57 & 6667 & 5 \\
\hline 1935 & 1935 & 16200 & 34 & 16130 & 34 & 15990 & 34 & 15530 & 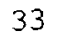 \\
\hline 1936 & 1936 & 14200 & 39 & 14130 & 39 & 14000 & 39 & 13640 & \\
\hline 1937 & 1937 & 22700 & 19 & 22700 & 19 & 22410 & 19 & 21410 & \\
\hline 1938 & 1938 & 12100 & 47 & 12100 & 47 & 11970 & 47 & 11590 & \\
\hline 1939 & 1939 & 13300 & 42 & 13230 & 42 & 12970 & 42 & 12030 & \\
\hline 1940 & 1940 & 8900 & 55 & 8867 & 55 & 8824 & 55 & 8578 & \\
\hline 1941 & 1941 & 5790 & 60 & 5763 & 60 & 5671 & 60 & 5521 & \\
\hline 1942 & 1942 & 24900 & 13 & 24830 & 13 & 24610 & 13 & 23980 & \\
\hline 1943 & 1943 & 6730 & 59 & 6730 & 59 & 6614 & 59 & 6347 & \\
\hline 1944 & 1944 & 21700 & 24 & 21630 & 24 & 21290 & 24 & 20510 & \\
\hline 1945 & 1945 & 22500 & 20 & 22430 & 20 & 22170 & 21 & 21450 & \\
\hline 1946 & 1946 & 14700 & 38 & 14570 & 38 & 14490 & 38 & 14270 & \\
\hline 1947 & 1947 & 16500 & 33 & 16430 & 33 & 16210 & 33 & 15470 & \\
\hline 1948 & 1948 & 82800 & $I$ & 80970 & 1 & 76830 & 1 & 63150 & \\
\hline 1949 & 1949 & 15600 & 35 & 15570 & 35 & 15410 & 35 & 14960 & \\
\hline 1950 & 1950 & 10800 & 49 & 10400 & 50 & 9849 & 51 & 9061 & \\
\hline 1951 & 1951 & 9480 & 53 & 9447 & 53 & 9255 & 53 & 8774 & \\
\hline 1952 & 1952 & 13700 & 40 & 13700 & 40 & 13660 & 40 & 13630 & \\
\hline 1953 & 1953 & 11600 & 48 & 11530 & 48 & 11370 & 48 & 10960 & \\
\hline 1954 & 1954 & 16700 & 32 & 16700 & 31 & 16540 & 31 & 16080 & \\
\hline 1955 & 1955 & 4320 & 61 & 4313 & 61 & 4213 & 61 & 3877 & \\
\hline 1956 & 1956 & 9170 & 54 & 9133 & 54 & 8973 & 54 & 8190 & \\
\hline 1957 & 1957 & 13200 & 43 & 13130 & 43 & 12930 & 43 & 12190 & \\
\hline 1958 & 1958 & 23400 & 17 & 23370 & 17 & 23140 & 17 & 22220 & 1 \\
\hline 1959 & 1959 & 34100 & 6 & 34030 & 6 & 33630 & 6 & 32300 & \\
\hline 1960 & 1960 & 25400 & 12 & 25270 & 12 & 24860 & 12 & 23420 & \\
\hline 1961 & 1961 & 19800 & 26 & 19800 & 26 & 19510 & 26 & 18530 & 2 \\
\hline 1962 & 1962 & 16800 & 31 & 16700 & 32 & 16470 & 32 & 15820 & \\
\hline 1963 & 1963 & 12600 & 45 & 12600 & 45 & 12560 & 45 & 12470 & \\
\hline 1964 & 1964 & 28700 & 8 & 28630 & 8 & 28340 & 8 & 27350 & \\
\hline 1965 & 1965 & 28100 & 9 & 28030 & 9 & 27870 & 9 & 27300 & \\
\hline 1966 & 1966 & 29100 & 7 & 29000 & 7 & 28810 & 7 & 28170 & \\
\hline 1967 & 1967 & 17000 & 30 & 17000 & 30 & 16860 & 30 & 16300 & \\
\hline 1968 & 1968 & 3560 & 62 & 3517 & 62 & 3444 & 62 & 3265 & \\
\hline 1969 & 1969 & 9520 & 52 & 9500 & 52 & 9416 & 52 & 8993 & \\
\hline 1970 & 1970 & 24300 & 15 & 24300 & 15 & 24110 & 15 & 23430 & \\
\hline 1971 & 1971 & 13700 & 41 & 13670 & 41 & 13530 & 41 & 13060 & \\
\hline 1972 & 1972 & 22500 & 21 & 22430 & 21 & 22300 & 20 & 21790 & \\
\hline 1973 & 1973 & 54700 & 2 & 54470 & 2 & 53290 & 2 & 48750 & \\
\hline 1974 & 1974 & 12500 & 46 & 12470 & 46 & 12310 & 46 & 11730 & 4 \\
\hline 1975 & 1975 & 24900 & 14 & 24830 & 14 & 24560 & 14 & 23560 & \\
\hline 1976 & 1976 & 17800 & 28 & 17730 & 28 & 17310 & 29 & 16070 & 3 \\
\hline 1977 & 1977 & 21800 & 23 & 21730 & 23 & 21660 & 22 & 21340 & 2 \\
\hline 1978 & 1978 & 18600 & 27 & 18600 & 27 & 18460 & 27 & 17920 & 2 \\
\hline 1979 & 1979 & 15500 & 36 & 15470 & 36 & 15230 & 36 & 14370 & 3 \\
\hline 1980 & 1980 & 23300 & 18 & 23270 & 18 & 23110 & 18 & 22420 & \\
\hline 1981 & 1981 & 8170 & 57 & 8147 & 56 & 8003 & 56 & 7459 & 5 \\
\hline 1982 & 1982 & 10200 & 51 & 10170 & 51 & 10040 & 50 & 9611 & \\
\hline 1983 & 1983 & 26900 & 11 & 26900 & 11 & 26840 & 11 & 26550 & \\
\hline 1984 & 1984 & 41400 & 3 & 41370 & 3 & 41000 & 3 & 39390 & \\
\hline 1985 & 1985 & 10800 & 50 & 10670 & 49 & 10490 & 49 & 9923 & 4 \\
\hline 1986 & 1986 & 38500 & 4 & 38230 & 4 & 37230 & 4 & 34230 & \\
\hline 1987 & 1987 & 27100 & 10 & 27100 & 10 & 26960 & 10 & 26590 & 1 \\
\hline 1988 & 1988 & 20400 & 25 & 20370 & 25 & 20140 & 25 & 19410 & 2 \\
\hline 1989 & 1989 & 6830 & 58 & 6780 & 58 & 6651 & 58 & 6193 & \\
\hline 1990 & 1990 & 12900 & 44 & 12870 & 44 & 12690 & 44 & 12170 & \\
\hline 1991 & 1991 & 37700 & 5 & 37530 & 5 & 37110 & 5 & 35470 & \\
\hline 1992 & 1992 & 17700 & 29 & 17700 & 29 & 17610 & 28 & 17110 & 2 \\
\hline 1993 & 1993 & 22000 & 22 & 21930 & 22 & 21630 & 23 & 20510 & \\
\hline
\end{tabular}

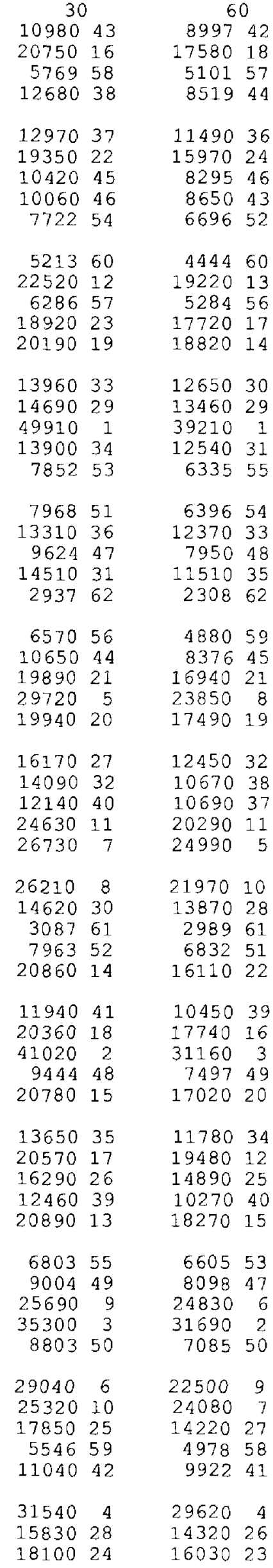

\begin{tabular}{rr}
\multicolumn{2}{c}{90} \\
7300 & 47 \\
16920 & 14 \\
4933 & 56 \\
6596 & 49 \\
& \\
10920 & 34 \\
14020 & 24 \\
7307 & 46 \\
7632 & 43 \\
6098 & 50
\end{tabular}

394660

1708013

489757

1481022

1520020

$11540 \quad 31$

1160030

315301

1256027

542754

$5255 \quad 55$

1095033

751744

976536

216262

451558

735245

1534019

$\begin{array}{ll}15570 & 17\end{array}$

1097032

886741

923239

$20260 \quad 8$

219007

$18970 \quad 11$

1177029

301161

593651

1456023

927238

1603016

26870

$7208 \quad 48$

1505021

$9863 \quad 35$

1742012

1348025

918940

1535018

$5872 \quad 52$

812142

22280

$27100 \quad 2$

572353

1931010

$23100 \quad 5$

1209028

450659

$9297 \quad 37$

$25540 \quad 4$

12830
16160
120

644348

1502014

455757

542750

983235

1236024

671846

$7096 \quad 43$

538551

$3656 \quad 60$

1571013

468655

$12720 \quad 22$

1227025

1104028

$10130 \quad 32$

$28810 \quad 1$

177026

485954

467656

1079029

686345

1054030

211762

$4350 \quad 58$

692544

$13940 \quad 18$

$\begin{array}{rr}13610 & 19\end{array}$

1002033

758942

803840

191108

209605

1622012

997934

293561

560649

1309021

880836

$14370 \quad 17$

$24060 \quad 2$
6629

1478016

863738

1714010

1251023

877737

1350020

$5137 \quad 52$

777041

194507

$23950 \quad 3$
$4883 \quad 53$

1709011

206406

1022031

410659
828039

$22260 \quad 4$

$11080 \quad 27$

1479015
183

$5034 \quad 49$

1236014

$\begin{array}{ll}3881 & 58 \\ 4410 & 52\end{array}$

$7883 \quad 36$

996926

$5845 \quad 47$

$6883 \quad 39$

502150

350560

$12240 \quad 16$

428254

$11760 \quad 19$

1017025

996727

838433

287701

1063023

445251

$4190 \quad 56$

966628

$6521 \quad 42$

918929

207062

395757 623444

$12740 \quad 13$

158207

1141021

842932 607845 665940

$15740 \quad 8$

$19280 \quad 3$

1458010

812434

286761

562548

1194018

847331

1175020

$\begin{array}{rr}20430 & 2 \\ 5856 & 46\end{array}$

1231015

$7884 \quad 35$

1377011

1057024

$7555 \quad 38$

1072022

432853

659041

153409

$19260 \quad 4$
424955

1333012 160906

781837

382159

651843

188005

878930

1202017 
THIS PAGE INTENTIONALLY BLANK 
LOCATION.--Lat $29^{\circ} 50^{\prime} 46^{\prime \prime}$, long $82^{\circ} 13^{\prime} 11^{\prime \prime}$, in NE $1 / 4 \mathrm{sec} .32$, T.7 S., R.21 E., Alachua County, Hydrologic Unit 03110206, near left bank on upstream side of bridge on State Highway 225, $1.0 \mathrm{mi}$ south of Graham, $1.5 \mathrm{mi}$ upstream from Sampson River, and 71 mi upstream from mouth.

DRAINAGE AREA.--94.9 $\mathrm{mi}^{2}$.

PERIOD OF RECORD.--August 1957 to 1993.

REVISED RECORDS.--WSP 2105: Drainage area,

GAGE.--Water-stage recorder. Datum of gage is $103.55 \mathrm{ft}$ above National Geodetic Vertical Datum of 1929.

REMARKS.--Records good except for those below $2 \mathrm{ft}^{3} / \mathrm{s}$, which are poor. Records do not include natural diversions during periods of high stages from Santa Fe Lake through Lochloosa Creek in St. Johns River basin.
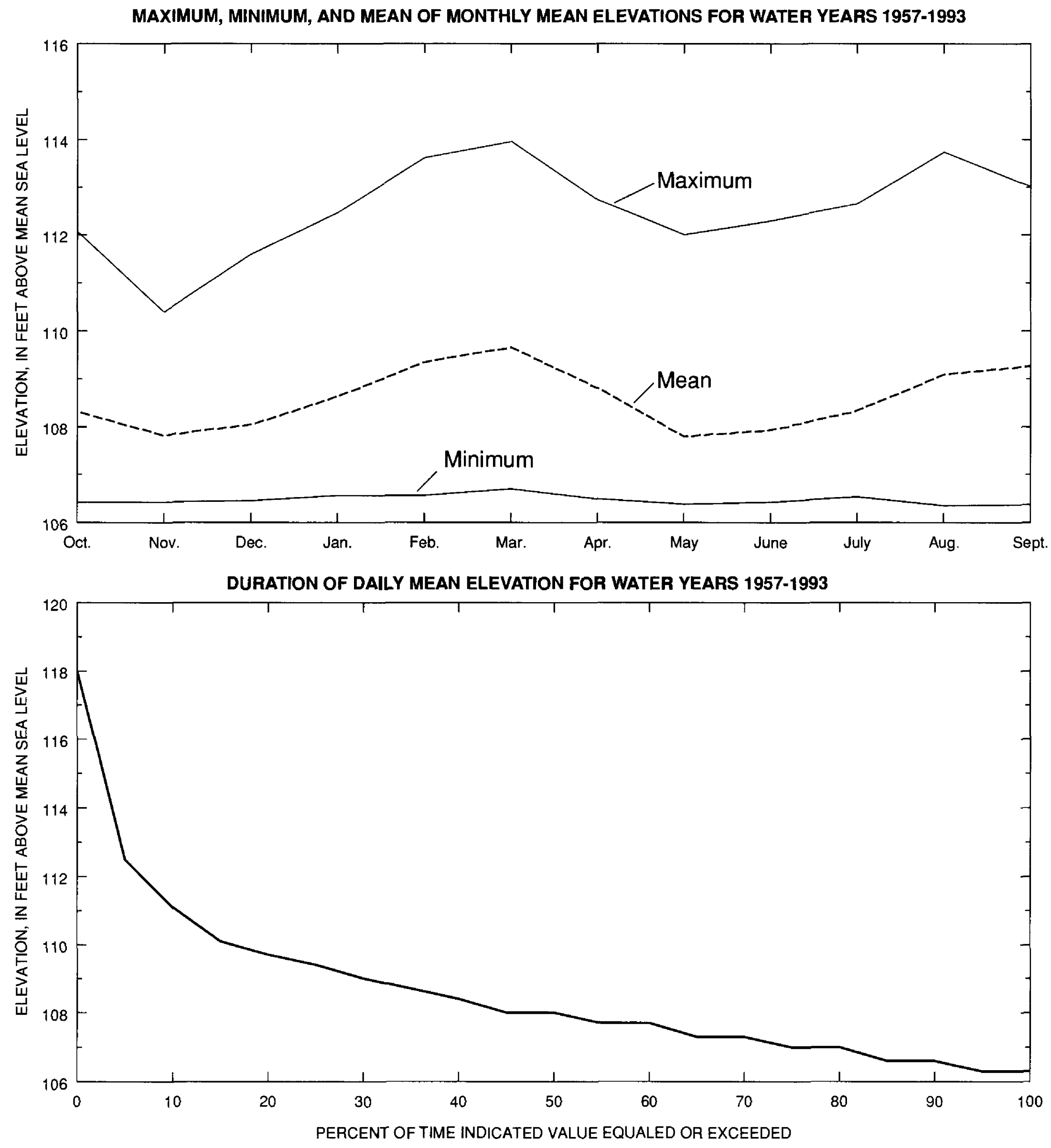
SUMMARY STATISTICS, IN CUBIC FEET PER SECOND UNLESS OTHERWISE INDICATED, FOR WATER YEARS 1957 - 1993

ANNUAL MEAN

HIGHEST ANNUAL MEAN

LOWEST ANNUAL MEAN

HIGHEST DAILY MEAN

LOWEST DAILY MEAN

ANNUAL SEVEN-DAY MINTMUM

INSTANTANEOUS PEAK FLOW

INSTANTANEOUS PEAK ELEVAT

INSTANTANEOUS LOW FLOW

ANNUAL RUNOFF (INCHES/CFSM)

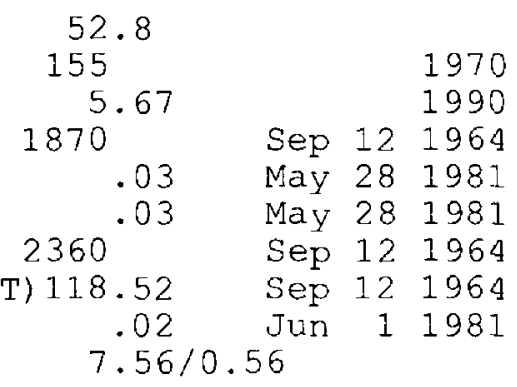

52.8

90

May 281981

May 281981

Sep 121964

Jun 11981

$7.56 / 0.56$

MAXIMUM, MINIMUM, AND MEAN OF MONTHLY MEAN ELEVATIONS FOR WATER YEARS 1957-1993

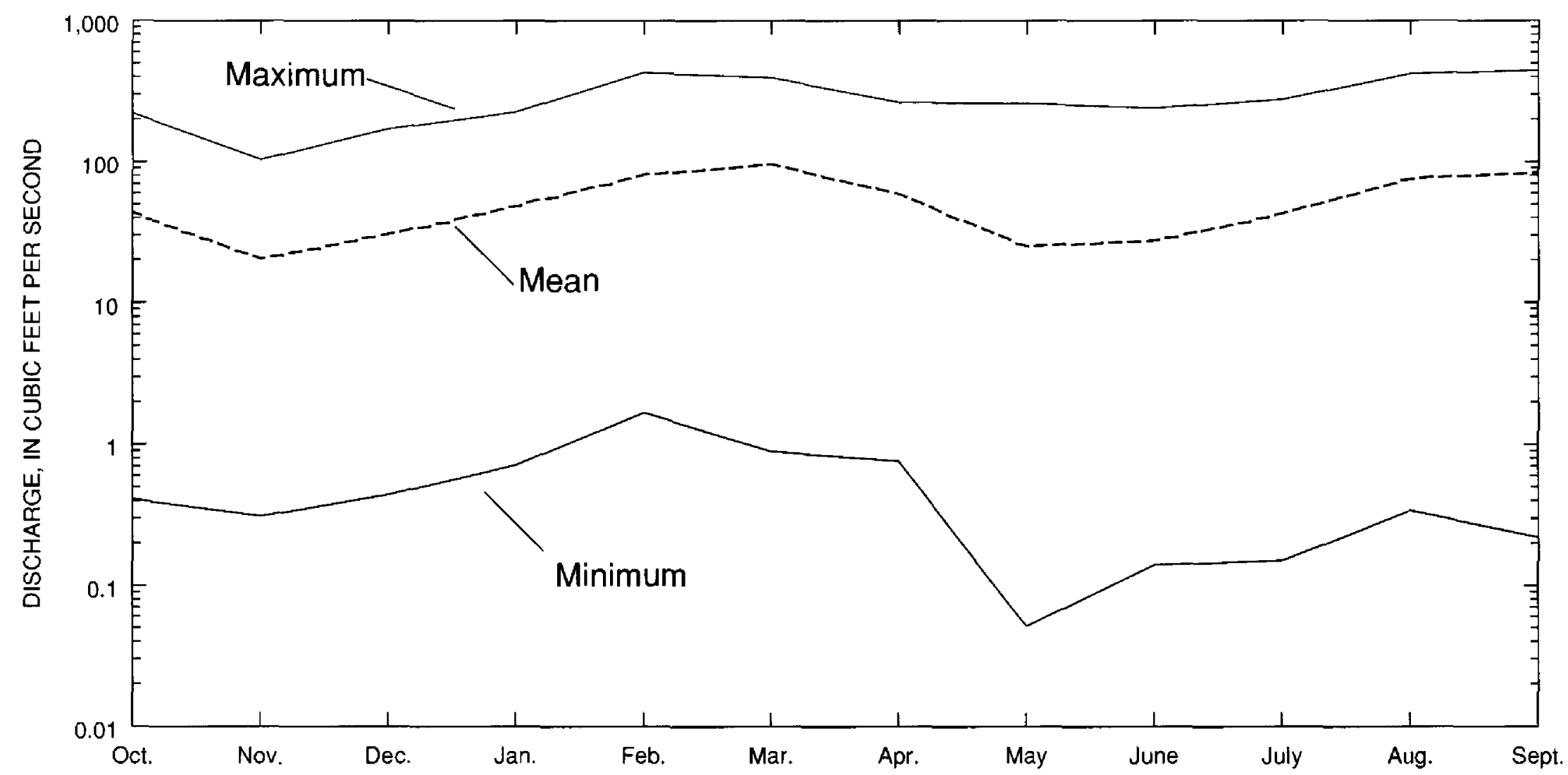

DURATION OF DAILY MEAN ELEVATION FOR WATER YEARS 1957-1993

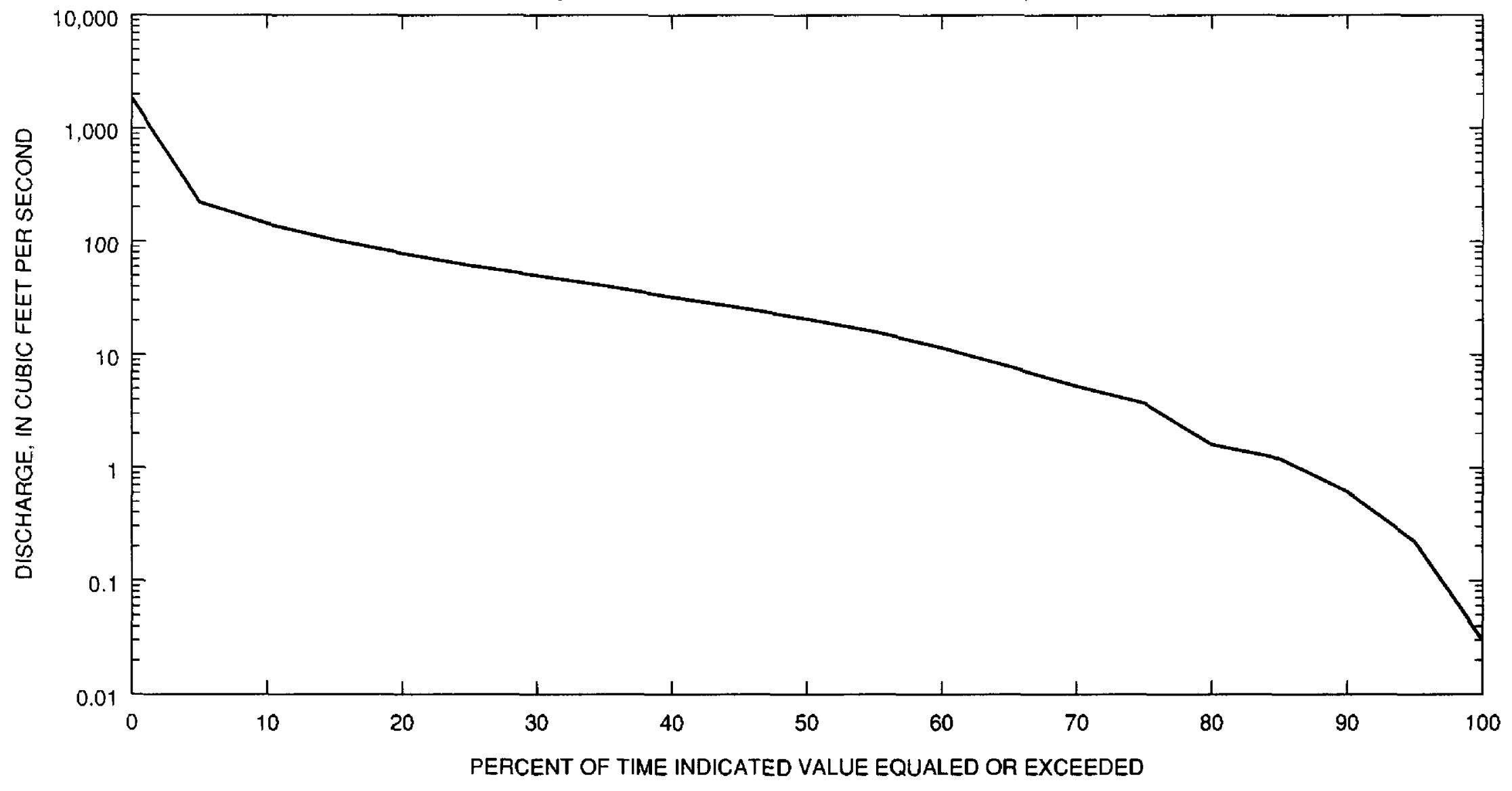


SUWANNEE RIVER BASIN

02320700 SANTA FE RIVER NEAR GRAHAM, FL--Continued

SUMMARY OF MONTHLY MEAN ELEVATION AND DISCHARGE STATISTICS FOR WATER YEARS 1957-1993

\begin{tabular}{|c|c|c|c|c|c|c|}
\hline \multirow[b]{2}{*}{ MONTH } & \multicolumn{3}{|c|}{$\begin{array}{l}\text { ELEVATIONS, } \\
\text { EET ABOVE SEA LEVEL }\end{array}$} & \multicolumn{3}{|c|}{$\begin{array}{ll} & \text { DISCHARGE, } \\
\text { CUBIC FEET PER SE }\end{array}$} \\
\hline & MAXIMUM & MIN IMUM & MEAN & MAXIMUM & MINIMUM & MEAN \\
\hline OCTOBER & 112.05 & 106.42 & 108.32 & 218 & .41 & 43.9 \\
\hline NOVEMBER & 110.38 & 106.42 & 107.82 & 102 & .31 & 20.4 \\
\hline DECEMBER & 111.58 & 106.45 & 108.04 & 168 & .44 & 30.6 \\
\hline JANUARY & 112.45 & 106.56 & 108.64 & 221 & .71 & 48.7 \\
\hline FEBRUARY & 113.61 & 106.56 & 109.35 & 420 & 1.67 & 80.6 \\
\hline MARCH & 113.95 & 106.70 & 109.66 & 387 & .89 & 95.9 \\
\hline APRII & 112.72 & 106.49 & 108.82 & 259 & .76 & 59.0 \\
\hline MAY & 111.99 & 106.38 & 107.80 & 254 & .051 & 25.1 \\
\hline JUNE & 112.28 & 106.42 & 107.93 & 236 & .14 & 27.3 \\
\hline JULY & 112.64 & 106.53 & 108.34 & 271 & .25 & 42.7 \\
\hline AUGUST & 113.73 & 106.35 & 109,10 & 414 & .34 & 76.6 \\
\hline SEPTEMBER & 113.00 & 106.37 & 109.27 & 436 & .22 & 83.4 \\
\hline
\end{tabular}

DURATION OF DAILY MEAN VALUES FOR WATER YEARS 1957-1993

PERCENT

OF TIME

ANNUAL OCF NOV

DEC

JAN

FEB MAR

APR MAY

JUNE

JULY

AUG

SEPT

$\begin{array}{rlllll}95.0 & 106.3 & 106.4 & 106.4 & 106.4 & 106.4 \\ 90.0 & 106.6 & 106.6 & 106.6 & 106.6 & 106.5 \\ 85.0 & 106.6 & 106.6 & 106.6 & 106.6 & 106.9 \\ 80.0 & 107.0 & 106.6 & 106.8 & 106.9 & 107.2 \\ 75.0 & 107.0 & 106.9 & 107.0 & 107.2 & 107.4 \\ 70.0 & 107.3 & 107.2 & 107.2 & 107.2 & 107.4 \\ 65.0 & 107.3 & 107.5 & 107.2 & 107.2 & 107.4 \\ 60.0 & 107.7 & 107.8 & 107.4 & 107.2 & 107.7 \\ 55.0 & 107.7 & 107.8 & 107.4 & 107.4 & 107.9 \\ 50.0 & 108.0 & 108.1 & 107.4 & 107.4 & 108.2 \\ 45.0 & 108.0 & 108.1 & 107.6 & 107.7 & 108.2 \\ 40.0 & 108.4 & 108.1 & 107.8 & 107.7 & 108.4 \\ 35.0 & 108.7 & 108.3 & 107.8 & 108.0 & 108.7 \\ 30.0 & 109.0 & 108.3 & 108.0 & 108.0 & 109.0 \\ 25.0 & 109.4 & 108.6 & 108.2 & 108.5 & 109.2 \\ 20.0 & 109.7 & 108.9 & 108.4 & 108.8 & 109.5 \\ 15.0 & 110.1 & 109.5 & 109.0 & 109.1 & 110.0 \\ 10.0 & 111.1 & 110.7 & 109.4 & 109.6 & 111.1 \\ 5.0 & 112.5 & 111.9 & 110.0 & 110.7 & 112.1\end{array}$

106.5
106.8
107.1
107.4
107.7
108.1
108.1
108.4
108.4
108.7
108.7
109.0
109.4
109.7
110.3
110.7
111.3
112.0
113.3

106.5
107.1
107.4
107.8
107.8
108.1
108.4
108.4
108.7
109.0
109.4
109.7
110.0
110.3
111.0
111.6
112.0
113.0
114.0

$\begin{array}{ll}106.4 & 190.6 \\ 106.6 & 106.4 \\ 106.9 & 106.6 \\ 107.2 & 106.6 \\ 107.5 & 106.9 \\ 107.5 & 106.9 \\ 107.7 & 106.9 \\ 108.0 & 107.2 \\ 108.0 & 107.2 \\ 108.3 & 107.2 \\ 108.6 & 107.5 \\ 108.8 & 107.5 \\ 108.8 & 107.8 \\ 109.1 & 108.1 \\ 109.7 & 108.4 \\ 110.0 & 108.7 \\ 110.5 & 108.7 \\ 111.1 & 109.3 \\ 113.1 & 109.9\end{array}$

106.3
106.3
106.6
106.6
106.6
106.9
106.9
107.1
107.1
107.4
107.4
107.6
107.9
107.9
108.2
108.7
109.3
109.8
111.1

106.4
106.4
106.7
106.7
106.7
106.9
106.9
107.5
107.5
107.8
108.1
108.4
108.7
109.0
109.0
109.3
109.6
110.5
111.6

106.3 $\begin{array}{ll}106.6 & 106.3\end{array}$ $106.6 \quad 107.0$ $106.6 \quad 107.3$ $\begin{array}{ll}107.2 & 107.7 \\ 107.8 & 108.0\end{array}$ $\begin{array}{ll}107.8 & 108.0 \\ 108.1 & 108.0\end{array}$ $108.4 \quad 108.4$ $108.7 \quad 108.7$ $108.7 \quad 108.7$ $108.9 \quad 109.0$ $109.3 \quad 109.0$ $109.5 \quad 109.4$ $109.8 \quad 109.7$ $110.1 \quad 110.1$ $110.4 \quad 110.4$ $111.0 \quad 110.8$ DISCHARGE IN CUBIC FEET PER SECOND

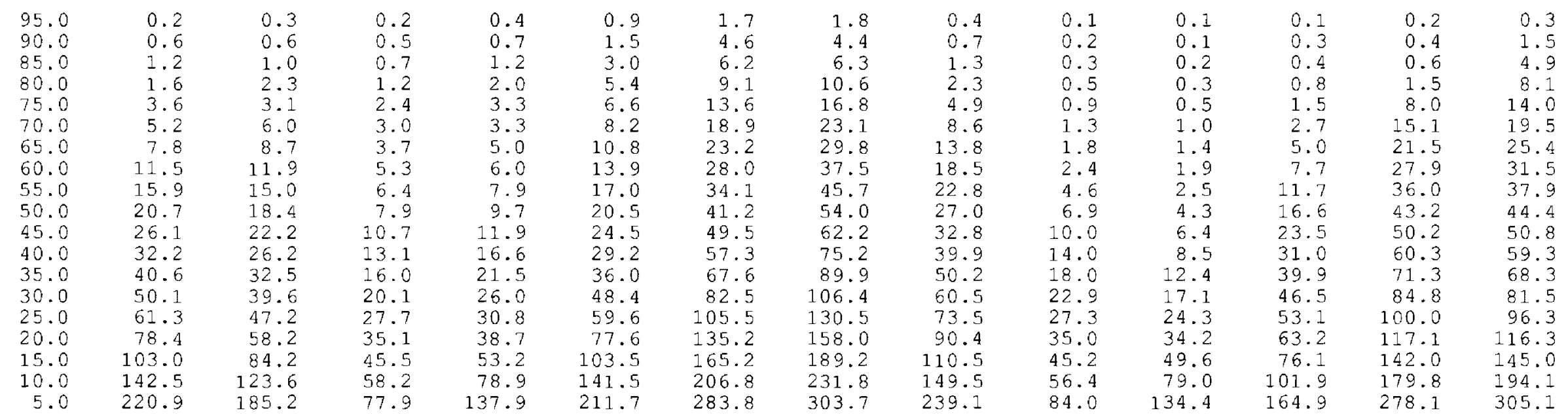



FOR PERIOD APR TO MAR

\begin{tabular}{|c|c|c|c|c|c|c|c|c|c|c|c|c|c|c|c|c|c|}
\hline $\begin{array}{r}\text { WATE } \\
R \\
1959 \\
1960\end{array}$ & $\begin{array}{l}R \text { YEAR } \\
\text { ANGE } \\
1960 \\
1961\end{array}$ & $\begin{array}{c}1 \\
108 \\
108\end{array}$ & $\begin{array}{l}26 \\
25\end{array}$ & $\begin{array}{c}3 \\
108 \\
108\end{array}$ & $\begin{array}{l}26 \\
25\end{array}$ & $\begin{array}{r}7 \\
108 \\
108\end{array}$ & $\begin{array}{l}26 \\
25\end{array}$ & $\begin{array}{r}14 \\
108 \\
108\end{array}$ & & $\begin{array}{c}30 \\
1092 \\
108\end{array}$ & & $\begin{array}{r}60 \\
1092 \\
1092\end{array}$ & $\begin{array}{l}109^{90} \\
109\end{array}$ & $\begin{array}{l}90 \\
25 \\
26\end{array}$ & $\begin{array}{r}12 \\
1092 \\
1102\end{array}$ & & $\begin{array}{r}18 \\
1102 \\
1102\end{array}$ \\
\hline $\begin{array}{l}1961 \\
1962 \\
1963 \\
1964 \\
1965\end{array}$ & $\begin{array}{l}1962 \\
1963 \\
1964 \\
1965 \\
1965\end{array}$ & $\begin{array}{l}108 \\
107 \\
107 \\
107 \\
107\end{array}$ & $\begin{array}{l}23 \\
20 \\
18 \\
21 \\
22\end{array}$ & $\begin{array}{l}108 \\
107 \\
107 \\
107 \\
108\end{array}$ & $\begin{array}{l}23 \\
20 \\
18 \\
21 \\
22\end{array}$ & $\begin{array}{l}108 \\
107 \\
107 \\
107 \\
108\end{array}$ & $\begin{array}{l}23 \\
20 \\
18 \\
21 \\
22\end{array}$ & $\begin{array}{l}108 \\
107 \\
107 \\
107 \\
108\end{array}$ & $\begin{array}{l}23 \\
20 \\
18 \\
21 \\
22\end{array}$ & $\begin{array}{l}108 \\
107 \\
107 \\
108 \\
108\end{array}$ & $\begin{array}{l}23 \\
19 \\
17 \\
21 \\
22\end{array}$ & $\begin{array}{l}108 \\
107 \\
107 \\
108 \\
108\end{array}$ & $\begin{array}{l}108 \\
107 \\
107 \\
108 \\
108\end{array}$ & $\begin{array}{l}22 \\
16 \\
13 \\
21 \\
23\end{array}$ & $\begin{array}{ll}108 & 2 \\
108 & 1 \\
108 & 1 \\
108 & 2 \\
1092\end{array}$ & $\begin{array}{l}21 \\
13 \\
19 \\
22 \\
23\end{array}$ & $\begin{array}{ll}108 & 20 \\
108 & 13 \\
108 & 19 \\
109 & 23 \\
109 & 22\end{array}$ \\
\hline $\begin{array}{l}1969 \\
1970\end{array}$ & $\begin{array}{l}1970 \\
1971\end{array}$ & $\begin{array}{l}107 \\
107\end{array}$ & $\begin{array}{l}17 \\
19\end{array}$ & $\begin{array}{l}107 \\
107\end{array}$ & $\begin{array}{l}17 \\
19\end{array}$ & $\begin{array}{l}107 \\
107\end{array}$ & $\begin{array}{l}15 \\
19\end{array}$ & $\begin{array}{l}107 \\
107\end{array}$ & $\begin{array}{l}14 \\
19\end{array}$ & $\begin{array}{l}107 \\
107\end{array}$ & $\begin{array}{l}14 \\
18\end{array}$ & $\begin{array}{ll}107 & 1 \\
107 & 1\end{array}$ & $\begin{array}{l}107 \\
107\end{array}$ & $\begin{array}{l}14 \\
15\end{array}$ & $\begin{array}{l}1081 \\
108\end{array}$ & $\begin{array}{l}16 \\
15\end{array}$ & $\begin{array}{ll}108 & 17 \\
108 & 11\end{array}$ \\
\hline $\begin{array}{l}1971 \\
1972 \\
1973 \\
1974 \\
1975\end{array}$ & $\begin{array}{l}1972 \\
1973 \\
1974 \\
1975 \\
1976\end{array}$ & $\begin{array}{l}107 \\
108 \\
107 \\
107 \\
107\end{array}$ & $\begin{array}{l}15 \\
24 \\
13 \\
16 \\
12\end{array}$ & $\begin{array}{l}107 \\
108 \\
107 \\
107 \\
107\end{array}$ & $\begin{array}{l}14 \\
24 \\
13 \\
16 \\
12\end{array}$ & $\begin{array}{l}107 \\
108 \\
107 \\
107 \\
107\end{array}$ & $\begin{array}{l}13 \\
24 \\
14 \\
16 \\
11\end{array}$ & $\begin{array}{l}107 \\
108 \\
107 \\
107 \\
107\end{array}$ & $\begin{array}{l}12 \\
24 \\
13 \\
15 \\
11\end{array}$ & $\begin{array}{l}107 \\
108 \\
107 \\
107 \\
107\end{array}$ & $\begin{array}{l}11 \\
24 \\
13 \\
16 \\
12\end{array}$ & $\begin{array}{ll}107 & 1 \\
108 & 2 \\
107 & 1 \\
107 & 1 \\
107 & 1\end{array}$ & $\begin{array}{l}107 \\
109 \\
108 \\
107 \\
107\end{array}$ & $\begin{array}{r}9 \\
24 \\
19 \\
17 \\
11\end{array}$ & $\begin{array}{ll}107 & 1 \\
109 & 2 \\
108 & 1 \\
108 & 1 \\
107 & 1\end{array}$ & $\begin{array}{l}10 \\
25 \\
14 \\
18 \\
12\end{array}$ & $\begin{array}{ll}108 & 1 \\
110 & 24 \\
108 & 10 \\
108 & 16 \\
108 & 12\end{array}$ \\
\hline $\begin{array}{l}1976 \\
1977 \\
1978\end{array}$ & $\begin{array}{l}1977 \\
1978 \\
1979\end{array}$ & $\begin{array}{l}107 \\
107 \\
107\end{array}$ & $\begin{array}{r}10 \\
9 \\
11\end{array}$ & $\begin{array}{l}107 \\
107 \\
107\end{array}$ & $\begin{array}{r}10 \\
9 \\
11\end{array}$ & $\begin{array}{l}207 \\
107 \\
107\end{array}$ & $\begin{array}{r}10 \\
9 \\
12\end{array}$ & $\begin{array}{l}107 \\
107 \\
107\end{array}$ & $\begin{array}{r}9 \\
8 \\
16\end{array}$ & $\begin{array}{l}107 \\
107 \\
1072\end{array}$ & $\begin{array}{r}10 \\
7 \\
20\end{array}$ & $\begin{array}{l}107 \\
107 \\
1082\end{array}$ & $\begin{array}{l}107 \\
107 \\
108\end{array}$ & $\begin{array}{r}7 \\
6 \\
20\end{array}$ & $\begin{array}{l}107 \\
107 \\
108\end{array}$ & $\begin{array}{r}7 \\
5 \\
20\end{array}$ & $\begin{array}{lr}107 & 5 \\
107 & 2 \\
109 & 21\end{array}$ \\
\hline $\begin{array}{l}1981 \\
1982 \\
1984\end{array}$ & $\begin{array}{l}1982 \\
1983 \\
1985\end{array}$ & $\begin{array}{l}106 \\
107 \\
107\end{array}$ & $\begin{array}{r}3 \\
14 \\
7\end{array}$ & $\begin{array}{l}106 \\
107 \\
107\end{array}$ & $\begin{array}{r}3 \\
15 \\
7\end{array}$ & $\begin{array}{l}106 \\
107 \\
107\end{array}$ & $\begin{array}{r}3 \\
17 \\
7\end{array}$ & $\begin{array}{l}106 \\
107 \\
107\end{array}$ & $\begin{array}{r}3 \\
17 \\
10\end{array}$ & $\begin{array}{l}106 \\
107 \quad 1 \\
107\end{array}$ & $\begin{array}{r}3 \\
15 \\
9\end{array}$ & $\begin{array}{l}106 \\
107 \quad 1 \\
107\end{array}$ & $\begin{array}{l}106 \\
107 \\
107\end{array}$ & $\begin{array}{r}2 \\
18 \\
8\end{array}$ & $\begin{array}{l}107 \\
108 \\
107\end{array}$ & $\begin{array}{r}2 \\
17 \\
8\end{array}$ & $\begin{array}{l}107 \\
108 \\
107\end{array}$ \\
\hline $\begin{array}{l}1987 \\
1988 \\
1989 \\
1990\end{array}$ & $\begin{array}{l}1988 \\
1989 \\
1990 \\
1991\end{array}$ & $\begin{array}{l}107 \\
106 \\
106 \\
106\end{array}$ & $\begin{array}{l}8 \\
6 \\
1 \\
2\end{array}$ & $\begin{array}{l}107 \\
106 \\
106 \\
106\end{array}$ & $\begin{array}{l}8 \\
6 \\
1 \\
2\end{array}$ & $\begin{array}{l}107 \\
106 \\
106 \\
106\end{array}$ & $\begin{array}{l}8 \\
6 \\
2 \\
1\end{array}$ & $\begin{array}{l}107 \\
106 \\
106 \\
106\end{array}$ & $\begin{array}{l}7 \\
6 \\
2 \\
1\end{array}$ & $\begin{array}{l}107 \\
107 \\
106 \\
106\end{array}$ & $\begin{array}{l}8 \\
5 \\
2 \\
1\end{array}$ & $\begin{array}{l}107 \\
107 \\
106 \\
106\end{array}$ & $\begin{array}{l}107 \\
107 \\
106 \\
106\end{array}$ & $\begin{array}{r}10 \\
5 \\
3 \\
1\end{array}$ & $\begin{array}{l}107 \\
107 \\
107 \\
106\end{array}$ & $\begin{array}{l}9 \\
6 \\
3 \\
1\end{array}$ & $\begin{array}{l}107 \\
107 \\
107 \\
106\end{array}$ \\
\hline $\begin{array}{l}1991 \\
1992\end{array}$ & $\begin{array}{l}1992 \\
1993\end{array}$ & $\begin{array}{l}106 \\
106\end{array}$ & $\begin{array}{l}4 \\
5\end{array}$ & $\begin{array}{l}106 \\
106\end{array}$ & $\begin{array}{l}4 \\
5\end{array}$ & $\begin{array}{l}106 \\
106\end{array}$ & $\begin{array}{l}4 \\
5\end{array}$ & $\begin{array}{l}106 \\
106\end{array}$ & $\begin{array}{l}4 \\
5\end{array}$ & $\begin{array}{l}106 \\
107\end{array}$ & $\begin{array}{l}4 \\
6\end{array}$ & $\begin{array}{l}106 \\
107 \quad 1\end{array}$ & $\begin{array}{l}106 \\
107\end{array}$ & $\begin{array}{r}4 \\
12\end{array}$ & $\begin{array}{l}107 \\
107 \quad 1\end{array}$ & $\begin{array}{r}4 \\
11\end{array}$ & $\begin{array}{ll}107 & 3 \\
108 & 15\end{array}$ \\
\hline
\end{tabular}

HIGHEST MEAN ELEVATION, IN FEET, AND RANKING FOR THE FOLLOWING NUMBER OF CONSECUTIVE DAYS FOR PERIOD OCT TO SEP

WATER YEAR

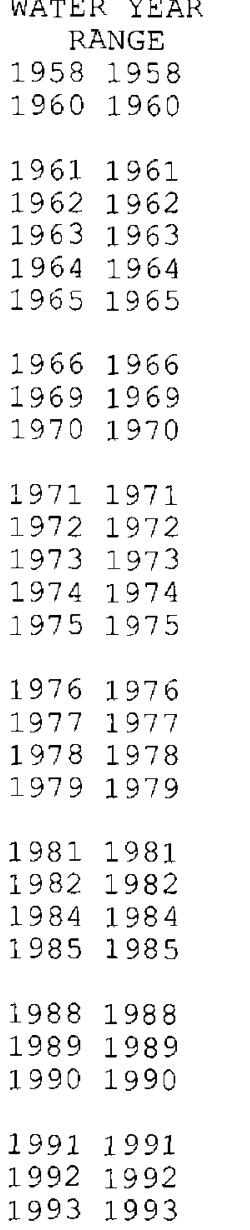

$\begin{array}{rrrrrr}1 & & 3 & & 7 & \\ 114 & 16 & 114 & 16 & 113 & 16 \\ 117 & 3 & 116 & 3 & 115 & 4 \\ 116 & 6 & 116 & 6 & 115 & 8 \\ 112 & 23 & 111 & 23 & 111 & 23 \\ 111 & 25 & 111 & 25 & 110 & 25 \\ 118 & 1 & 118 & 1 & 117 & 1 \\ 115 & 14 & 115 & 14 & 114 & 15 \\ 116 & 9 & 116 & 9 & 115 & 7 \\ 113 & 21 & 113 & 20 & 112 & 20 \\ 118 & 2 & 117 & 2 & 116 & 2 \\ 113 & 19 & 112 & 21 & 112 & 21 \\ 116 & 7 & 116 & 7 & 115 & 5 \\ 116 & 10 & 116 & 8 & 115 & 6 \\ 115 & 15 & 114 & 15 & 114 & 14 \\ 114 & 18 & 113 & 18 & 112 & 17 \\ 110 & 26 & 110 & 26 & 110 & 26 \\ 110 & 27 & 109 & 27 & 109 & 27 \\ 116 & 4 & 116 & 4 & 115 & 3 \\ 113 & 20 & 113 & 19 & 112 & 18 \\ 111 & 24 & 111 & 24 & 111 & 24 \\ 115 & 13 & 115 & 13 & 114 & 13 \\ 115 & 11 & 115 & 11 & 115 & 11 \\ 115 & 12 & 115 & 12 & 114 & 12 \\ 116 & 8 & 115 & 10 & 115 & 10 \\ 109 & 28 & 109 & 28 & 109 & 28 \\ 109 & 29 & 109 & 29 & 108 & 29 \\ 113 & 22 & 112 & 22 & 112 & 22 \\ 114 & 17 & 114 & 17 & 112 & 19 \\ 116 & 5 & 116 & 5 & 115 & 9\end{array}$

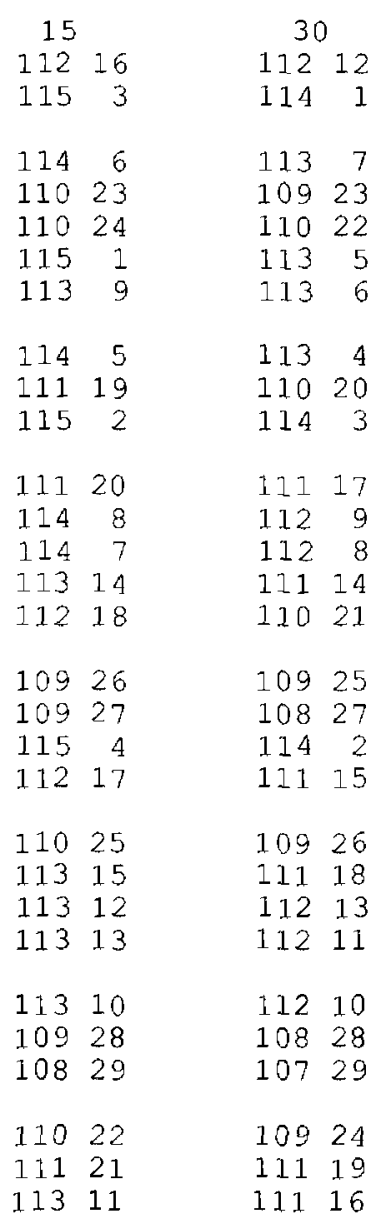

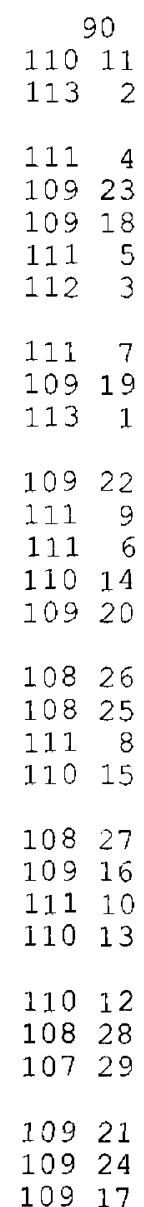

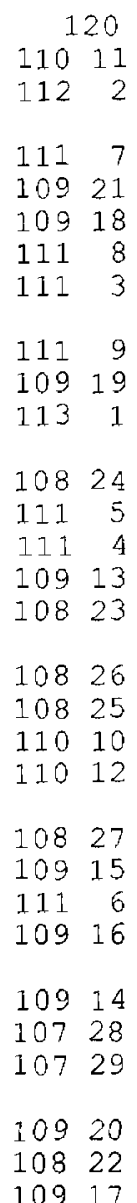

183

$\begin{array}{rr}110 & -0 \\ 111 & 2\end{array}$

1108

10819

10821

$\begin{array}{ll}110 & 9 \\ 111 & 3\end{array}$

1114

1121

10823

1105

10916

10822

10825

10826

$\begin{array}{ll}110 & 11 \\ 109 & 12\end{array}$

10727

10913

$\begin{array}{rr}110 & 7 \\ 108 & 20\end{array}$

10915

10728

10729

10917

10824 
LOWEST MEAN DISCHARGE, IN CUBIC FEET PER SECOND, AND RANKING FOR THE FOLLOWING NUMBER OF

WATER YEAR

RANGE

19581959

19591960

19601961

$1961 \quad 1962$

19631964

19641965

19651966

19661967

19671968

19681969

19691970

$1970 \quad 1971$

19711972

19721973

19731974

19741975

19751976

19761977

19771978

19781979

19791980

19801981

19811982

19821983

19831984

19841985

19861987

19871988

19881989

19891990

19901991

19911992

19921993 CONSECUTIVE DAYS FOR PERIOD APR TO MAR

\begin{tabular}{rrrr}
1 & & \multicolumn{3}{r}{3} \\
1.90 & 27 & 2.07 & 27 \\
13.0 & 35 & 13.3 & 35 \\
4.00 & 32 & 4.27 & 32 \\
.50 & 18 & .57 & 19 \\
.30 & 15 & .40 & 17 \\
.10 & 7 & .10 & 7 \\
2.90 & 30 & 3.33 & 30 \\
1.10 & 24 & 1.20 & 24 \\
6.50 & 33 & 6.70 & 33 \\
.58 & 22 & .58 & 20 \\
.060 & 2 & .060 & 2 \\
.090 & 6 & .097 & 6 \\
1.50 & 25 & 1.63 & 25 \\
& & & \\
.22 & 13 & .25 & 13 \\
11.0 & 34 & 11.7 & 34 \\
1.70 & 26 & 1.80 & 26 \\
.31 & 16 & .33 & 15 \\
.55 & 20 & .58 & 21 \\
.16 & 11 & .17 & 11 \\
.070 & 4 & .080 & 5 \\
2.50 & 29 & 2.70 & 29 \\
.060 & 3 & .060 & 3 \\
.18 & 12 & .23 & 12 \\
.030 & 1 & .030 & 1 \\
2.20 & 28 & 2.37 & 28 \\
3.40 & 31 & 3.77 & 31 \\
.56 & 21 & .59 & 22 \\
.11 & 9 & .11 & 9 \\
.52 & 19 & .53 & 18 \\
.37 & 17 & .38 & 16 \\
.25 & 14 & .26 & 14 \\
.070 & 5 & .070 & 4 \\
.10 & 8 & .11 & 8 \\
.12 & 10 & .12 & 10 \\
.59 & 23 & .71 & 23
\end{tabular}

14
$2.71 \quad 27$

2.7127
15.935

15.9
7.69
33

1.1822

.6318

.113
.11

4.1028

$1.78 \quad 24$

7.6132

$.74 \quad 19$

.168

$\begin{array}{rr}.13 & 5 \\ 1.82 & 25\end{array}$

.3211

14.234

2.3526

.5216

1.1521

.2210

$\begin{array}{rr}.10 & 2 \\ 5.80 & 30\end{array}$

.3613

$.38 \quad 14$

.0391

4.2929

7.2131

.8220

$.57 \quad 17$

.5717
$.41 \quad 15$

.3412

.157

13

.199
1.6023
6. 30

6.1028

19.934

$2.72 \quad 23$

.8716

.347

4.9127

3.88 26

8.0530

1.0520

.3611

2.0622

.3610

21.135

.9318

1.3721

.348

$\begin{array}{rr}.11 & 2 \\ .93 & 31\end{array}$

.9331

.5413

$.047 \quad 1$

$7.38 \quad 29$

$11.4 \quad 33$

.8817

.6815

.6815
.5514
.35

.359

.18
.15

$\begin{array}{rr}.26 & 6 \\ 2.98 & 24\end{array}$
12.60

12.6
$22.2 \quad 32$

$\begin{array}{ll}22.2 & 32 \\ 28.5 & 34\end{array}$

8.5224

I. 2815

.417

10.2
12.126

12.329

2.5521

.5711
2.2519

3.6822

.439

29.035

6.2423

.9413
7.78

.162

13.331

1. 4216

.093

9.2525

23.233

1.7417

.213

1.1314

2. 3420

.396

.35
.224

.4510
$1 i .927$
90

20.929

$24.7 \quad 32$

40.034

10.624

1. $47 \quad 12$

1.7914

22.430

22.931

14.226

4.7821

1.0910

4.0219

4.4820

$.66 \quad 6$

$40.3 \quad 35$

9.0823

3.4416

3.9818

.655

$\begin{array}{rr}.19 & 2 \\ 17 & 27\end{array}$

3.7517

089

$\begin{array}{rr}0899 & 1 \\ 13.6 & 25\end{array}$

27.533

$1.73 \quad 13$

1.1111

1.8115

5.7922

.544

.949
.323

$\begin{array}{rr}.84 & 8 \\ 18.3 \quad 28\end{array}$
25.120

35.93

35.9
554

10.723

2.448

12.424

27.030

$38.3 \quad 33$

25.627

6.0913

8.0618

$7.80 \quad 17$

6.3014

2.247

$56.3 \quad 35$

8.5020

8.4719

.834

.191

9.6221

.813

2.056

18.825

2.52

6.6716

$4.80 \quad 12$

6.4215

1.595

2.8010

$\begin{array}{ll}3.05 & 11 \\ 18.8 & 26\end{array}$
183

31.224

85.033

85.934

14.815 15.116

10435

56.430

50.128 6.546 25.321

$23.4 \quad 19$

11.010

$26.5 \quad 23$ $78.4 \quad 32$

11.611

22.318

3.374

.652

26.122

14.514

35.326

35.125

4.795

12.912

10.99

$12.9 \quad 13$

9.398

$7.75 \quad 7$

41.427 
HIGHEST MEAN DISCHARGE, IN CUBIC FEET PER SECOND, AND RANKING FOR THE FOLLOWING NUMBER OF

WATER YEAR$$
\text { RANGE }
$$

19581958

19591959

19601960

$196] 1961$

19621962

19631963

19641964

19651965

19661966

19671967

19681968

19691969

$1970 \quad 1970$

$1971 \quad 1971$

19721972

19731973

$1974 \quad 1974$

19751975

19761976

19771977

$1978 \quad 1978$

19791979

19801980

19811981

19821982

19831983

19841984

19851985

19861986

19871987

19881988

19891989

19901990

19911991

19921992

19931993 CONSECUTIVE DAYS FOR PERIOD OCT TO SEP

$\begin{array}{rrrrrrrr}3 & & 3 & & 7 & 15 \\ 343 & 24 & 299 & 23 & 228 & 23 & 197 & 24 \\ 984 & 6 & 911 & 5 & 813 & 3 & 555 & 3 \\ 979 & 7 & 852 & 7 & 626 & 7 & 441 & 6 \\ & & & & & & & \\ 811 & 8 & 682 & 8 & 501 & 11 & 384 & 10 \\ 206 & 30 & 157 & 30 & 143 & 30 & 101 & 30 \\ 132 & 32 & 119 & 32 & 90.6 & 32 & 73.3 & 32 \\ 1870 & 1 & 1710 & 1 & 1238 & 1 & 756 & 1 \\ 393 & 19 & 358 & 19 & 300 & 22 & 266 & 16 \\ & & & & & & & \\ 630 & 12 & 565 & 12 & 494 & 12 & 383 & 11 \\ 383 & 20 & 352 & 21 & 303 & 21 & 207 & 22 \\ 1090 & 3 & 999 & 3 & 787 & 4 & 483 & 5 \\ 234 & 29 & 217 & 28 & 186 & 28 & 156 & 26 \\ 1790 & 2 & 1520 & 2 & 1020 & 2 & 623 & 2 \\ 242 & 27 & 206 & 29 & 169 & 29 & 142 & 27 \\ 689 & 10 & 622 & 10 & 543 & 9 & 389 & 9 \\ 618 & 13 & 596 & 11 & 515 & 10 & 390 & 8 \\ 381 & 21 & 356 & 20 & 316 & 19 & 230 & 20 \\ 300 & 25 & 246 & 25 & 214 & 25 & 172 & 25 \\ & & & & & & & \\ 91.0 & 33 & 85.3 & 33 & 69.7 & 33 & 61.8 & 33 \\ 85.0 & 34 & 74.3 & 34 & 62.9 & 34 & 46.5 & 35 \\ 1060 & 4 & 945 & 4 & 757 & 5 & 532 & 4 \\ 258 & 26 & 244 & 26 & 227 & 24 & 197 & 23 \\ 431 & 17 & 400 & 17 & 329 & 17 & 226 & 21 \\ 145 & 31 & 141 & 31 & 113 & 31 & 76.5 & 31 \\ 443 & 16 & 406 & 16 & 343 & 16 & 237 & 18 \\ 353 & 22 & 325 & 22 & 309 & 20 & 256 & 17 \\ 545 & 14 & 517 & 14 & 419 & 14 & 279 & 14 \\ 506 & 15 & 488 & 15 & 397 & 15 & 273 & 15 \\ 400 & 18 & 376 & 18 & 324 & 18 & 236 & 19 \\ 650 & 11 & 559 & 13 & 458 & 13 & 350 & 12 \\ 751 & 9 & 667 & 9 & 562 & 8 & 348 & 13 \\ 79.0 & 35 & 72.7 & 35 & 62.1 & 35 & 48.7 & 34 \\ 51.0 & 36 & 48.7 & 36 & 41.0 & 36 & 27.7 & 36 \\ & & & & & & & \\ 240 & 28 & 217 & 27 & 186 & 27 & 126 & 29 \\ 347 & 23 & 292 & 24 & 212 & 26 & 142 & 28 \\ 1000 & 5 & 900 & 6 & 647 & 6 & 393 & 7\end{array}$

\begin{tabular}{|c|c|c|c|}
\hline 30 & & 6 & \\
\hline 171 & 18 & 123 & 20 \\
\hline 394 & 4 & 273 & 4 \\
\hline 380 & 5 & 283 & 2 \\
\hline 262 & 9 & 240 & 7 \\
\hline 66.1 & 31 & 43.7 & 31 \\
\hline 70.3 & 30 & 52.3 & 30 \\
\hline 436 & 1 & 259 & 6 \\
\hline 236 & 12 & 195 & 0 \\
\hline 286 & 8 & 193 & \\
\hline 151 & 22 & 105 & 23 \\
\hline 289 & 7 & 169 & 12 \\
\hline 102 & 27 & 77.5 & 26 \\
\hline 405 & 3 & 369 & 1 \\
\hline 122 & 26 & 73.7 & 27 \\
\hline 258 & 10 & 187 & 10 \\
\hline 253 & 11 & 370 & 11 \\
\hline 147 & 23 & 118 & 21 \\
\hline 101 & 28 & 63.0 & 29 \\
\hline 45.4 & 33 & 27.2 & 34 \\
\hline 39.8 & 35 & 36.2 & 33 \\
\hline 423 & 2 & 274 & \\
\hline 156 & 20 & 116 & 22 \\
\hline 155 & 21 & 143 & 17 \\
\hline 50.3 & 32 & 42.6 & 32 \\
\hline 144 & 24 & 92.2 & 24 \\
\hline 188 & 17 & 146 & 15 \\
\hline 201 & 16 & 165 & 14 \\
\hline 213 & 15 & 166 & 13 \\
\hline 161 & 19 & 143 & 18 \\
\hline 312 & 6 & 265 & \\
\hline 216 & 14 & 145 & 16 \\
\hline 41.4 & 34 & 25.0 & 35 \\
\hline 16.8 & 36 & 11.5 & 36 \\
\hline 76.6 & 29 & 69.1 & 28 \\
\hline 138 & 25 & 85.8 & 2 \\
\hline 224 & 13 & 128 & 1 \\
\hline
\end{tabular}

\begin{tabular}{rr}
\multicolumn{3}{c}{90} \\
94.1 & 20 \\
268 & 2 \\
249 & 3
\end{tabular}

1806

47.530

$\begin{array}{rr}478 & 7\end{array}$

16110

$\begin{array}{rr}149 & 11\end{array}$

$121 \quad 15$

55.227

3281

49.329

1649

7.728

49.928

19.235

$30.9 \quad 32$

2015

93.621

12016

28.933

$77.0 \quad 23$

12314

14112

11917

13193

$\begin{array}{rr}117 & 4\end{array}$

11418

19.834

10.836

58.126

63.52

94.919 $\begin{array}{rr} & 120 \\ 75.4 & 21 \\ 245 & 2 \\ 195 & 3\end{array}$

13810

26.131

37.928

$\begin{array}{rr}135 & 11 \\ 150 & 7\end{array}$

12312

59.924

91.216

42.627

$37.0 \quad 30$

1625

1498

68.423

37.929

18.834

24.632

1546

$90.3 \quad 1.7$

11613

$21.7 \quad 33$

72.922

$100 \quad 15$

1479

89.618

$107 \quad 14$

$182 \quad 4$

87.919

17.135

10.436

57.825

56.026

77.820
183

$69.6 \quad 19$

$\begin{array}{ll}190 & 2 \\ 161 & 3\end{array}$

98.012

19.131

25.730

$10 \leq 11$

11310

49.324

60.121

34.427

2391

28.828

$136 \quad 4$

1167

45.325

27.329

15.933

$16.3 \quad 32$

1139

69.818

91.814

15.734

70.117

$79.8 \quad 16$

1158

59.522

$96.8 \quad 13$

1276

61.320

12.735

54.623

41.426

80.915 
SUWANNEE RIVER BASIN

02321000 NEW RIVER NEAR LAKE BUTLER, FL

LOCATION.-Lat $29^{\circ} 59^{\prime} 53^{\prime \prime}$, long $82^{\circ} 16^{\prime} 27^{\prime \prime}$, in SW $1 / 4$ sec. 2, T.6. S., R.20 E., Union County, Hydrologic unit 03110206, near right bank on downstream side of bridge on State Highway 100, 4.4 miles southeast of Lake Butler.

DRAINAGE AREA.--191 $\mathrm{mi}^{2}$.

PERIOD OF RECORD.--January 1950 to September 1971, June 1973 to May 1977, periodic discharge measurements. October 1990 to Septernber 1991 , October 1992 to 1993.

REVISED RECORDS.--WRD FLA. 1968 Drainage area.

GAGE.--Water-stage recorder. Datum of gage is $83.8 \mathrm{ft}$ above National Geodetic Vertical Datum of 1929.

REMARKS.--Records good.
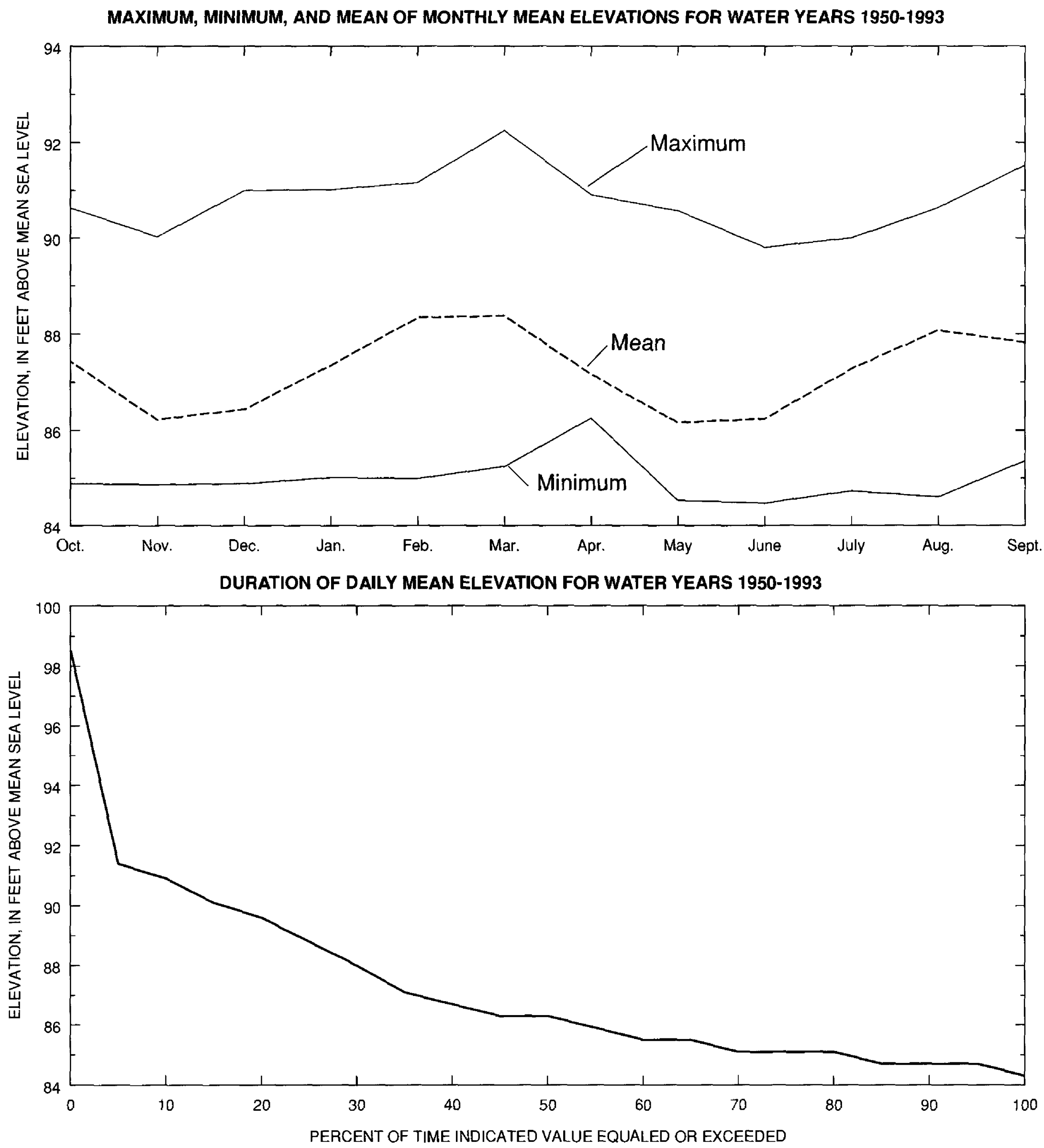
SUMMARY STATISTICS, IN CUBIC FEET PER SECOND UNLESS OTHERWISE INDICATED, FOR WATER YEARS $1950-1993$

ANNUAL MEAN

HIGHEST ANNUAL MEAN

LOWEST ANNUAL MEAN

HIGHEST DAILY MEAN

LOWEST DAILY MEAN

ANNUAL SEVEN-DAY MINIMUM

INSTANTANEOUS PEAK FLOW

INSTANTANEOUS PEAK ELEVA

INSTANTANEOUS LOW FLOW

ANNUAL RUNOFF (INCHES/CFSM)

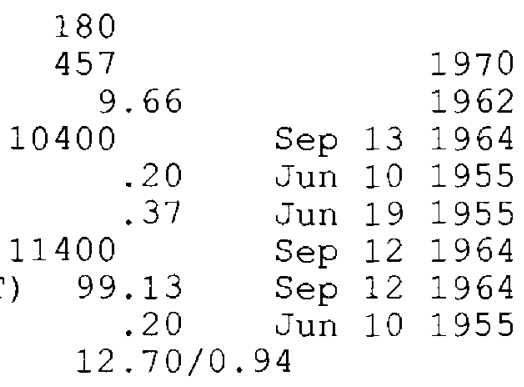

MAXIMUM, MINIMUM, AND MEAN OF MONTHLY MEAN DISCHARGES FOR WATER YEARS 1950-1993

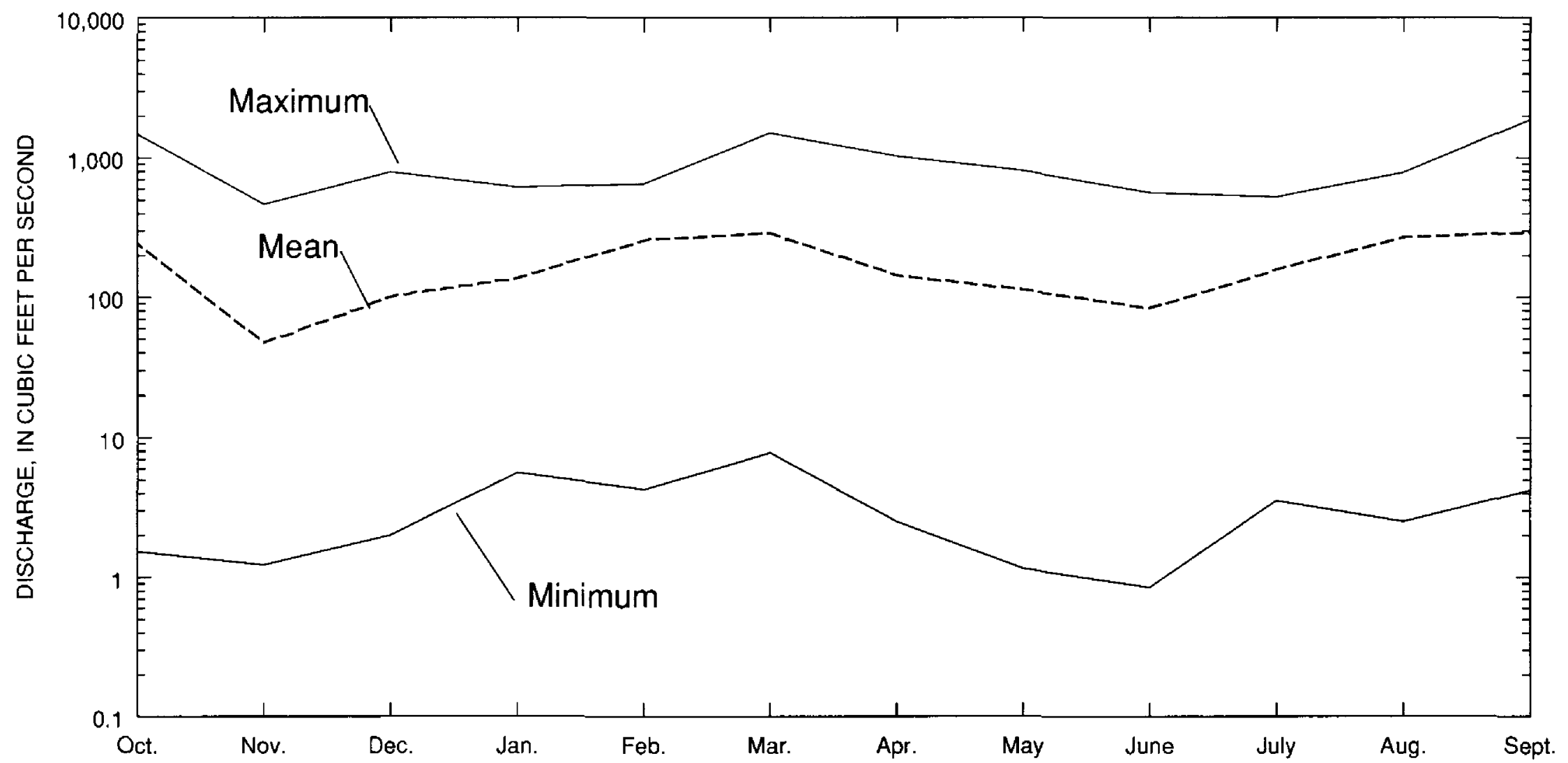

DURATION OF DAILY MEAN DISCHARGE FOR WATER YEARS 1950-1993

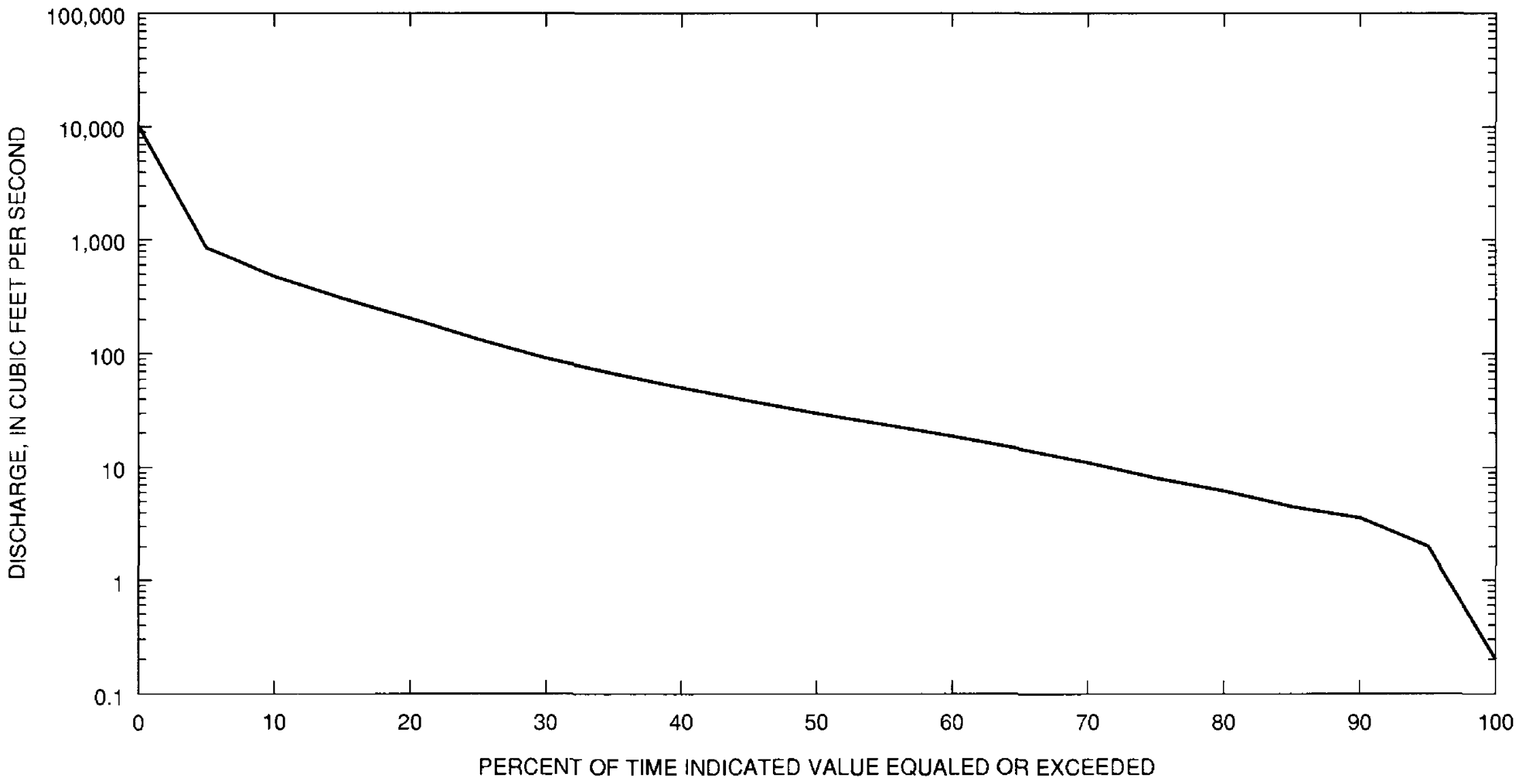


ELEVATIONS,

FEET ABOVE SEA LEVEI

\begin{tabular}{lccr}
\multicolumn{1}{c}{ MONTH } & MAXIMUM & MINIMUM & MEAN \\
OCTOBER & 90.62 & 84.88 & 87.43 \\
NOVEMBER & 90.01 & 84.86 & 86.22 \\
DECEMBER & 90.98 & 84.88 & 86.44 \\
JANUARY & 91.00 & 85.01 & 87.35 \\
FEBRUARY & 91.15 & 84.99 & 88.34 \\
MARCH & 92.23 & 85.24 & 88.37 \\
APRII & 90.89 & 86.25 & 87.16 \\
MAY & 90.56 & 84.53 & 86.16 \\
JUNE & 89.79 & 84.47 & 86.24 \\
JULY & 89.99 & 84.73 & 87.28 \\
AUGUST & 90.62 & 84.60 & 88.07 \\
SEPTEMBER & 91.51 & 85.36 & 87.82
\end{tabular}

DISCHARGE, CUBIC FEET PER SECOND

MAXIMUM MLNIMUM MEAN

$\begin{array}{rrr}1461 & 1.53 & 243.44 \\ 459.1 & 1.23 & 47.12 \\ 780.8 & 2.01 & 101.38 \\ 606.9 & 5.64 & 137.43 \\ 642.8 & 4.28 & 259.02 \\ 1491 & 7.82 & 289.42 \\ 1014 & 2.52 & 144.45 \\ 800.7 & 1.16 & 113.29 \\ 556.3 & .847 & 83.35 \\ 518.7 & 3.55 & 158.87 \\ 772.1 & 2.51 & 269.79 \\ 1845 & 4.20 & 291.43\end{array}$

DURATION OF DAILY MEAN VALUES FOR WATER YEARS 1950-1993

OF TIME

ANNUAL OCT NOV

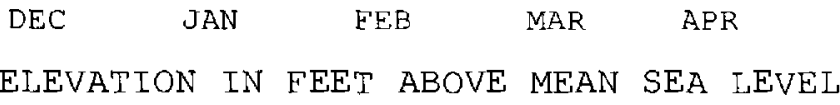

$\begin{array}{rlll}95.0 & 84.7 & 84.6 & 84 . \\ 90.0 & 84.7 & 84.6 & 84 . \\ 85.0 & 84.7 & 85.0 & 84 . \\ 80.0 & 85.1 & 85.0 & 85 . \\ 75.0 & 85.1 & 85.4 & 85 . \\ 70.0 & 85.1 & 85.4 & 85 . \\ 65.0 & 85.5 & 85.7 & 85 . \\ 60.0 & 85.5 & 86.1 & 85 . \\ 55.0 & 85.9 & 86.5 & 85 . \\ 50.0 & 86.3 & 86.5 & 85 . \\ 45.0 & 86.3 & 86.9 & 85 . \\ 40.0 & 86.7 & 87.2 & 86 . \\ 35.0 & 87.1 & 87.6 & 86 . \\ 30.0 & 88.0 & 88.0 & 86 . \\ 25.0 & 88.8 & 88.7 & 86 . \\ 20.0 & 89.6 & 89.9 & 86 . \\ 15.0 & 90.1 & 90.7 & 86 . \\ 10.0 & 90.9 & 91.4 & 87 . \\ 5.0 & 91.4 & 91.8 & 89 .\end{array}$

84.8
84.8
84.8
85.0
85.0
85.0
85.3
85.3
85.5
85.7
85.7
86.0
86.0
86.2
86.4
86.7
86.9
87.4
89.1

$\begin{array}{ll}84.8 & 84.8 \\ 84.8 & 84.8 \\ 84.8 & 85.0 \\ 85.1 & 85.3 \\ 85.1 & 85.5 \\ 85.1 & 85.8 \\ 85.1 & 85.8 \\ 85.3 & 86.0 \\ 85.6 & 86.2 \\ 85.6 & 86.5 \\ 85.6 & 86.7 \\ 85.8 & 86.9 \\ 85.8 & 87.2 \\ 86.1 & 87.4 \\ 86.4 & 88.2 \\ 86.9 & 89.1 \\ 88.7 & 89.9 \\ 90.3 & 90.4 \\ 91.4 & 91.4\end{array}$

$\begin{array}{ll}85.1 & 84.8 \\ 85.4 & 85.1 \\ 85.7 & 85.4 \\ 86.0 & 85.4 \\ 86.3 & 85.7 \\ 86.3 & 86.0 \\ 86.9 & 86.6 \\ 86.9 & 86.9 \\ 87.3 & 87.5 \\ 87.6 & 88.2 \\ 88.2 & 88.8 \\ 88.8 & 89.1 \\ 89.1 & 89.7 \\ 89.8 & 90.0 \\ 90.1 & 90.4 \\ 90.4 & 90.7 \\ 90.8 & 91.0 \\ 91.1 & 91.6 \\ 91.7 & 92.3\end{array}$
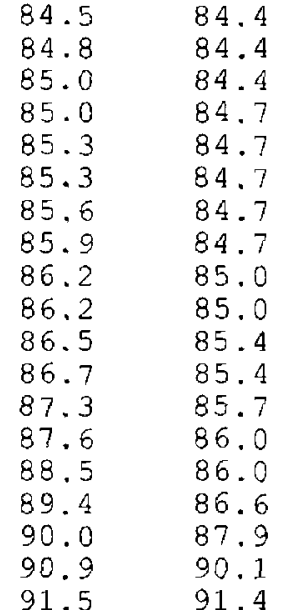

DISCHARGE IN CUBIC FEET PER SECOND

95.0
90.0
85.0
80.0
75.0
70.0
65.0
60.0
55.0
50.0
45.0
40.0
35.0
30.0
25.0
20.0
15.0
10.0
5.0

$$
\begin{array}{r}
2.0 \\
3.5 \\
4.4 \\
6.1 \\
8.0 \\
11.0 \\
14.5 \\
18.7 \\
23.8 \\
30.0 \\
38.7 \\
50.6 \\
66.9 \\
91.2 \\
133.7 \\
203.8 \\
304.5 \\
475.4 \\
850.8
\end{array}
$$

$$
\begin{array}{r}
2.4 \\
3.5 \\
4.4 \\
5.8 \\
10.1 \\
14.5 \\
21.1 \\
28.9 \\
37.1 \\
44.9 \\
52.3 \\
62.4 \\
74.8 \\
99.0 \\
150.1 \\
251.0 \\
414.5 \\
731.7 \\
183.4
\end{array}
$$

\section{0}

3.8

5.6
6.5

6.5
7.7

8.5
10.8

13.2

15.4

18.3

21.2
24.1

28.1
32.7

38.5

47.9

70.0
154.3

$\begin{array}{rr}3.2 & 4.8 \\ 4.5 & 5.8 \\ 4.9 & 7.9 \\ 5.5 & 11.5 \\ 6.6 & 14.3 \\ 7.2 & 16.5 \\ 8.5 & 19.5 \\ 10.5 & 23.2 \\ 13.1 & 28.8 \\ 14.6 & 35.2 \\ 16.0 & 43.5 \\ 18.6 & 51.4 \\ 21.4 & 60.4 \\ 24.1 & 73.1 \\ 28.3 & 92.7 \\ 40.1 & 141.6 \\ 103.5 & 214.4 \\ 268.3 & 330.4 \\ 632.7 & 624.6\end{array}$

4.8
7.3
15.6
21.2
25.9
31.9
39.6
47.6
58.0
71.0
88.4
126.3
173.5
223.8
279.6
372.4
473.6
628.6
1005.6

6.2
7.7
10.5
14.1
17.6
21.8
28.0
40.5
54.9
74.9
103.3
135.2
188.5
233.5
292.5
357.8
506.0
764.6

2.1
2.7
3.4
5.3
7.4
9.2
12.1
14.6
19.0
25.3
32.4
41.2
52.5
66.9
92.2
147.3
239.4
421.5

1.4
1.8
1.8
2.3
2.3
3.1
3.1
4.3
4.8
5.9
7.8
10.1
15.3
21.1
27.8
40.2
64.8
236.1
655.6

0.6
1.1
2.0
2.7
2.7
3.8
4.3
5.3
7.2
9.5
12.1
16.0
21.5
33.1
53.7
80.7
119.0
189.9
373.6

$$
\begin{array}{r}
1.7 \\
2.8 \\
5.3 \\
8.5 \\
11.2 \\
15.9 \\
23.8 \\
31.0 \\
39.8 \\
50.9 \\
63.3 \\
78.5 \\
98.7 \\
126.8 \\
187.0 \\
262.6 \\
356.5 \\
468.3 \\
705.9
\end{array}
$$

$\begin{array}{rr}3.2 & 3.9 \\ 4.8 & 5.3 \\ 6.4 & 9.8 \\ 11.6 & 16.2 \\ 18.8 & 22.1 \\ 27.4 & 28.0 \\ 40.5 & 34.3 \\ 56.1 & 41.1 \\ 75.8 & 49.1 \\ 103.2 & 59.5 \\ 127.9 & 77.6 \\ 160.3 & 96.0 \\ 199.7 & 127.1 \\ 240.1 & 176.0 \\ 309.9 & 238.5 \\ 396.0 & 321.3 \\ 519.3 & 430.4 \\ 718.0 & 617.5 \\ 184.4 & 1174.0\end{array}$

LOWEST MEAN ELEVATION, IN FEET, AND RANKING FOR THE FOLLOWING NUMBER OF CONSECUTIVE DAYS FOR PERIOD APR TO MAR

WATER YEAR

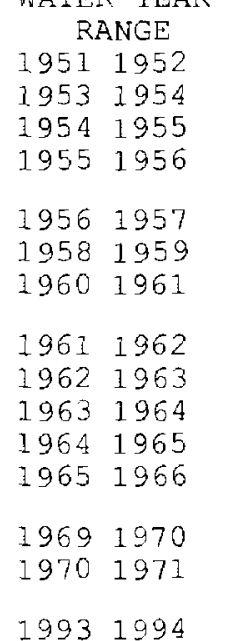

$\begin{array}{lr}3 & \\ 84.5 & 4 \\ 84.6 & 6 \\ 84.3 & 1 \\ 84.3 & 2 \\ 84.5 & 3 \\ 84.6 & 8 \\ 84.8 & 11 \\ 84.6 & 9 \\ 84.6 & 5 \\ 84.6 & 7 \\ 84.8 & 12 \\ 84.7 & 10 \\ 84.9 & 13 \\ 85.0 & 14 \\ 85.3 & 15\end{array}$

$\begin{array}{rr}14 & \\ 84.6 & 3 \\ 84.6 & 7 \\ 84.4 & 1 \\ 84.4 & 2 \\ 84.6 & 4 \\ 84.7 & 9 \\ 84.9 & 12 \\ 84.7 & 10 \\ 84.6 & 6 \\ 84.6 & 5 \\ 84.9 & 11 \\ 84.7 & 8 \\ 85.1 & 13 \\ 85.1 & 14 \\ 85.3 & 15\end{array}$

\begin{tabular}{lr}
\multicolumn{3}{c}{30} \\
84.6 & 3 \\
84.9 & 9 \\
84.5 & 1 \\
84.5 & 2 \\
84.7 & 5 \\
84.8 & 8 \\
85.0 & 11 \\
84.9 & 10 \\
84.6 & 4 \\
84.7 & 6 \\
85.0 & 12 \\
84.8 & 7 \\
85.2 & 14 \\
85.2 & 13 \\
85.3 & 15
\end{tabular}

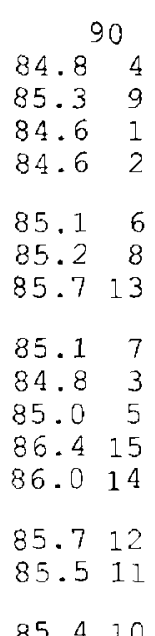

$\begin{array}{lr}84.8 & 90 \\ 85.3 & 9 \\ 84.6 & 1 \\ 84.6 & 2 \\ 85.1 & 6 \\ 85.2 & 8 \\ 85.7 & 13 \\ 85.1 & 7 \\ 84.8 & 3 \\ 85.0 & 5 \\ 86.4 & 15 \\ 86.0 & 14 \\ 85.7 & 12 \\ 85.5 & 11 \\ 85.4 & 10\end{array}$
84.6
85.0
85.0
85.4
85.8
85.8
86.2
86.6
86.6
87.0
87.4
87.8
88.2
89.0
89.4
90.3
90.3
91.1
91.5 

FOR PERIOD OCT TO SEP

\begin{tabular}{|c|c|}
\hline WATE & $\begin{array}{l}R \text { YEA } \\
\text { ANGE }\end{array}$ \\
\hline 1951 & 1951 \\
\hline 1952 & 1952 \\
\hline 1954 & 1954 \\
\hline 1955 & 1955 \\
\hline 1956 & 1956 \\
\hline 1957 & 1957 \\
\hline 1960 & 1960 \\
\hline 1961 & 1961 \\
\hline 1962 & 1962 \\
\hline 1963 & 1963 \\
\hline 1964 & 1964 \\
\hline 1965 & 1965 \\
\hline 1966 & 1966 \\
\hline 1969 & 1969 \\
\hline 1970 & 1970 \\
\hline 1971 & 1971 \\
\hline 1991 & 1991 \\
\hline 1993 & 1993 \\
\hline
\end{tabular}

$\begin{array}{lr}1 & \\ 95.2 & 4 \\ 91.6 & 15 \\ 93.6 & 10 \\ 90.5 & 17 \\ 91.4 & 16 \\ 94.2 & 7 \\ 94.8 & 6 \\ 92.9 & 12 \\ 88.6 & 18 \\ 92.3 & 14 \\ 98.6 & 1 \\ 93.0 & 11 \\ 94.1 & 8 \\ 92.6 & 13 \\ 95.7 & 3 \\ 93.8 & 9 \\ 94.8 & 5 \\ 98.0 & 2\end{array}$

$\begin{array}{rrrrr}3 & & 7 & \\ 94.8 & 4 & & 94.0 & 3 \\ 91.5 & 15 & & 91.1 & 15 \\ 93.2 & 10 & & 92.7 & 9 \\ 90.1 & 17 & & 89.8 & 17 \\ & & & & \\ 91.1 & 16 & & 91.1 & 16 \\ 94.0 & 7 & 92.9 & 8 \\ 94.5 & 5 & 93.4 & 6 \\ & & & & \\ 92.6 & 12 & & 92.4 & 11 \\ 88.0 & 18 & & 87.0 & 18 \\ 92.0 & 14 & 91.7 & 14 \\ 98.0 & 1 & 96.1 & 1 \\ 92.9 & 11 & 92.2 & 12 \\ & & & & \\ 93.8 & 8 & 93.1 & 7 \\ 92.4 & 13 & 92.1 & 13 \\ 95.3 & 3 & 93.9 & 4 \\ & & & \\ 93.5 & 9 & 92.6 & 10 \\ 94.3 & 6 & 93.8 & 5 \\ 97.2 & 2 & 94.9 & 2\end{array}$

\begin{tabular}{rrrrr} 
& & \multicolumn{2}{c}{30} \\
92.4 & 6 & & 90.3 & 13 \\
90.9 & 15 & & 89.5 & 14 \\
91.9 & 10 & & 91.5 & 5 \\
88.7 & 17 & 87.6 & 17 \\
& & & & \\
90.5 & 16 & & 88.5 & 16 \\
91.5 & 13 & 89.5 & 15 \\
91.8 & 11 & 91.3 & 6 \\
& & & & \\
92.3 & 7 & 90.5 & 12 \\
86.0 & 18 & 85.7 & 18 \\
91.2 & 14 & 90.9 & 9 \\
93.5 & 1 & 91.7 & 3 \\
91.9 & 9 & 91.2 & 7 \\
& & & & \\
92.5 & 5 & 91.6 & 4 \\
91.7 & 12 & 90.6 & 11 \\
92.6 & 4 & 91.8 & 2 \\
92.1 & 8 & 91.0 & 8 \\
92.9 & 3 & 91.8 & 1 \\
92.9 & 2 & 90.7 & 10
\end{tabular}

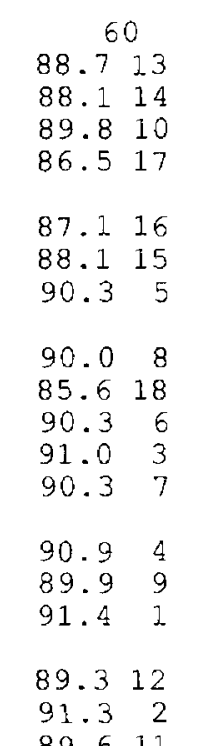

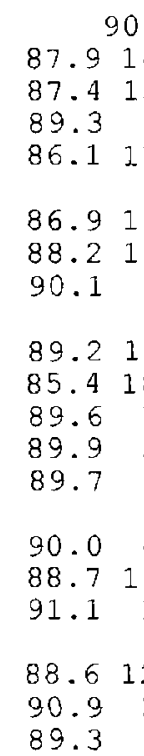

\begin{tabular}{lr}
\multicolumn{3}{r}{120} \\
87.5 & 14 \\
87.3 & 15 \\
89.2 & 6 \\
85.8 & 17 \\
86.5 & 16 \\
87.9 & 12 \\
89.2 & 7 \\
& \\
88.4 & 10 \\
85.4 & 18 \\
88.6 & 9 \\
89.4 & 5 \\
89.8 & 4 \\
89.9 & 3 \\
88.1 & 11 \\
90.9 & 1 \\
87.8 & 13 \\
90.6 & 2 \\
88.6 & 8
\end{tabular}
CONSECUTIVE DAYS FOR PERIOD APR TO MAR

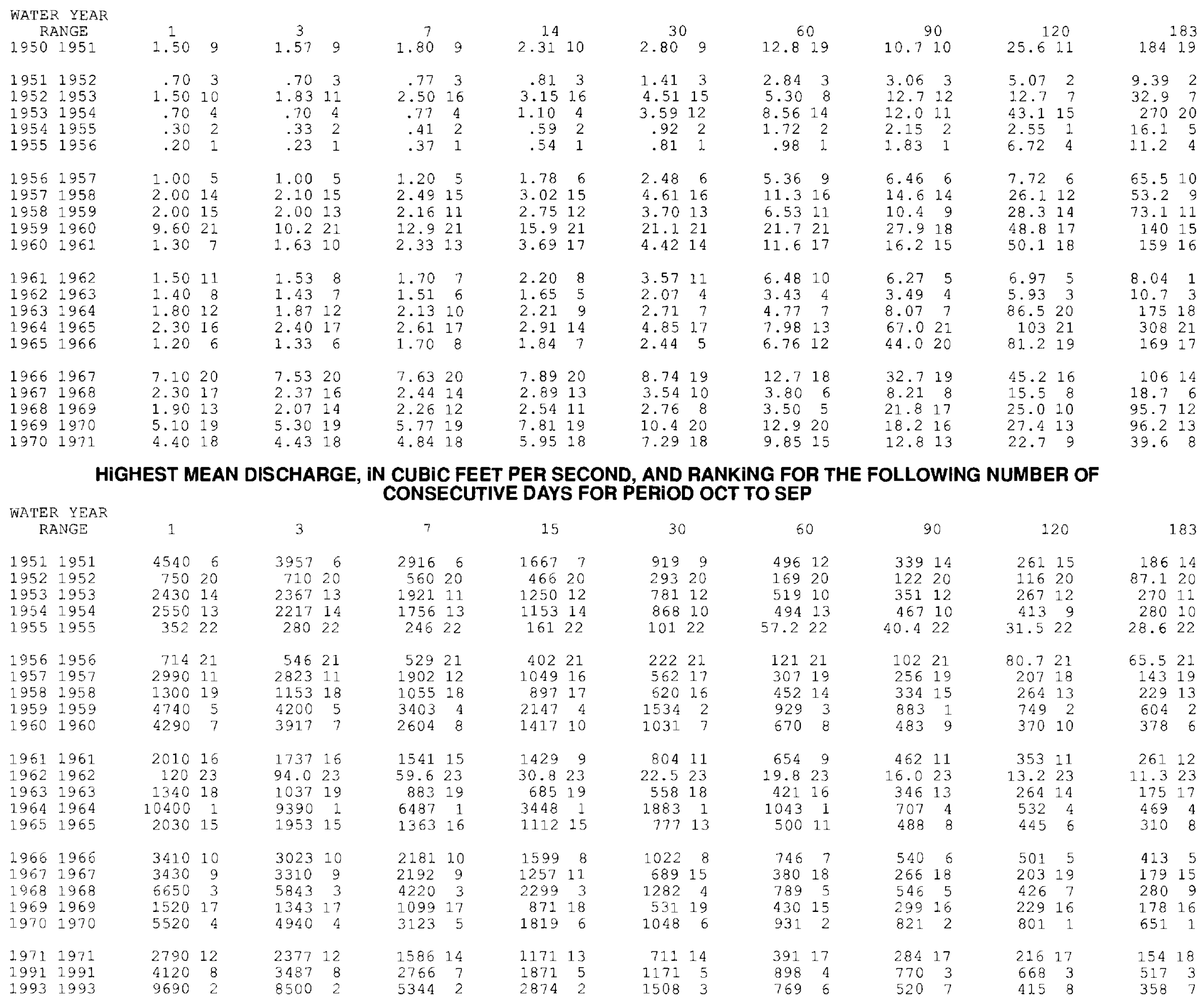


LOCATION.--Lat 2955'18", long 82²5'35", in SE $1 / 4$ sec.32, T.6 S., R.19 E., Alachua County, Hydrologic Unit 03110206, near center of span on downstream side of bridge on State Highway 121, $0.5 \mathrm{mi}$ south of Worthington Springs, $0.8 \mathrm{mi}$ downstream from New River, and $5 \mathrm{I}$ mi upstream from mouth.

DRAINAGE AREA.--575 $\mathrm{mi}^{2}$.

PERIOD OF RECORD.--October 1931 to 1993 . Published as "near Worthington" prior to October 1965. Monthly discharge only for October 1931, published in WSP 1304.

REVISED RECORDS.--WSP 2105: WDR FL-76-4: Drainage area.

GAGE.--Water-stage recorder. Datum of gage is $42.74 \mathrm{ft}$ above National Geodetic Vertical Datum of 1929 (levels by Corps of Engineers). Prior to Jan. 16, 1939 , nonrecording gage at site 0.2 mi downstream at present datum; Jan. 16, 1939 to July 23, I953, nonrecording gage at present site and datum.

REMARKS.--Records good, except estimated periods which are fair. Records do not include diversions,during periods of high stages, from Santa Fe Lake to Lochloosa Creek in St. Johns River Basin.
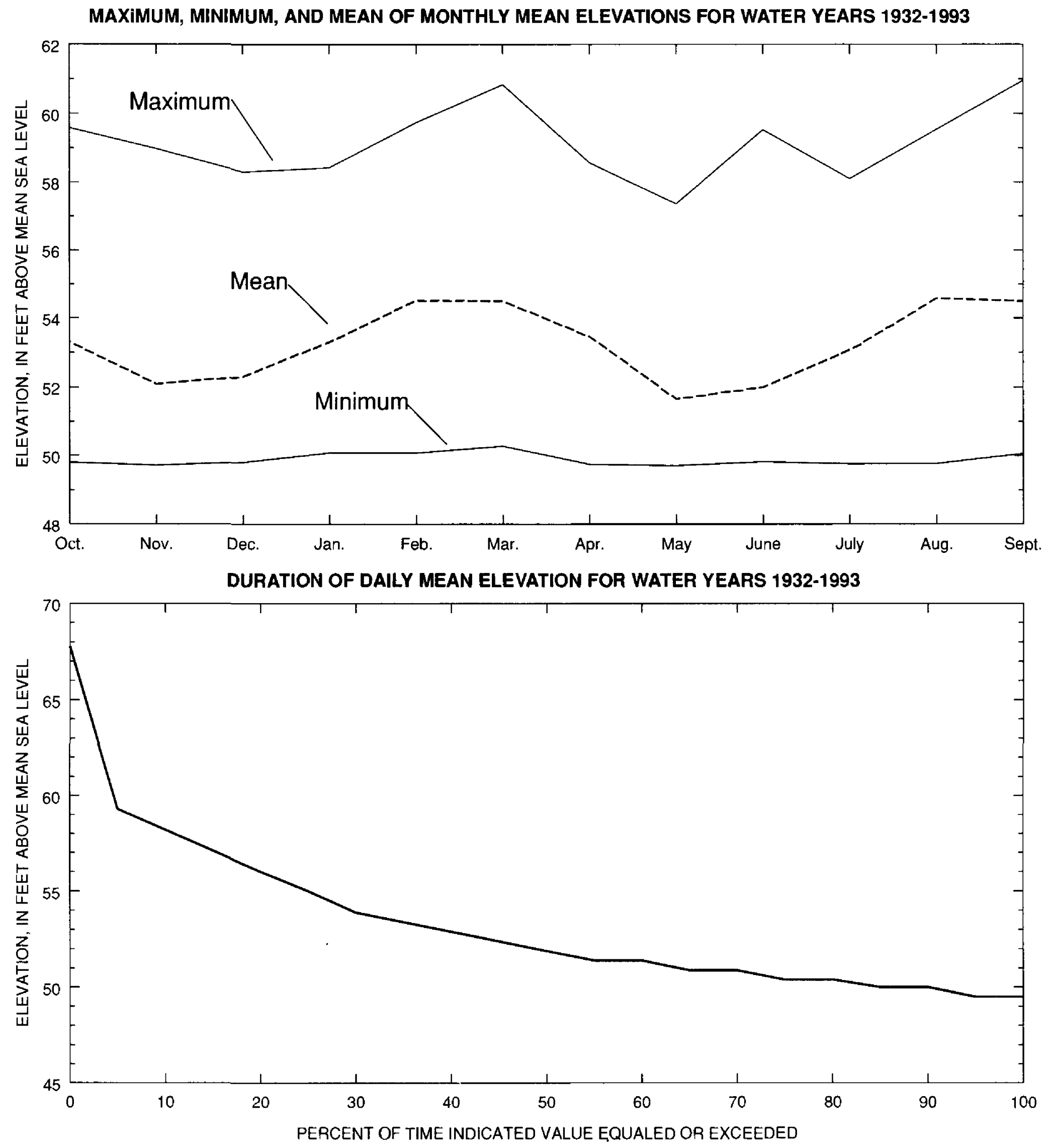
SUMMARY STATISTICS, IN CUBIC FEET PER SECOND UNLESS OTHERWISE INDICATED, FOR WATER YEARS 1932 - 1993

$\begin{array}{lcccc}\text { ANNUAL MEAN } & 437 & & \\ \text { HIGHEST ANNUAL MEAN } & 1163 & & 1948 \\ \text { LOWEST ANNUAL MEAN } & 54.8 & & 1956 \\ \text { HIGHEST DAILY MEAN } & 19000 & \text { Sep } 13 & 1964 \\ \text { LOWEST DAILY MEAN } & .60 & \text { Jun } 24 & 1955 \\ \text { ANNUAL SEVEN-DAY MINIMUM } & 1.3 & \text { Jun } 20 & 1955 \\ \text { INSTANTANEOUS PEAK ELOW } & 20000 & \text { Sep } 13 & 1964 \\ \text { INSTANTANEOUS PEAK ELEVATION } & \text { (FT) } & 71.14 & \text { Sep } 13 & 1964 \\ \text { INSTANTANEOUS LOW ELOW } & & .50 & \text { Jun } 24 & 1955 \\ \text { ANNUAL RUNOEE (INCHES/CFSM) } & 10.33 / 0.76 & \end{array}$

MAXIMUM, MINIMUM, AND MEAN OF MONTHLY MEAN DISCHARGES FOR WATER YEARS 1932-1993
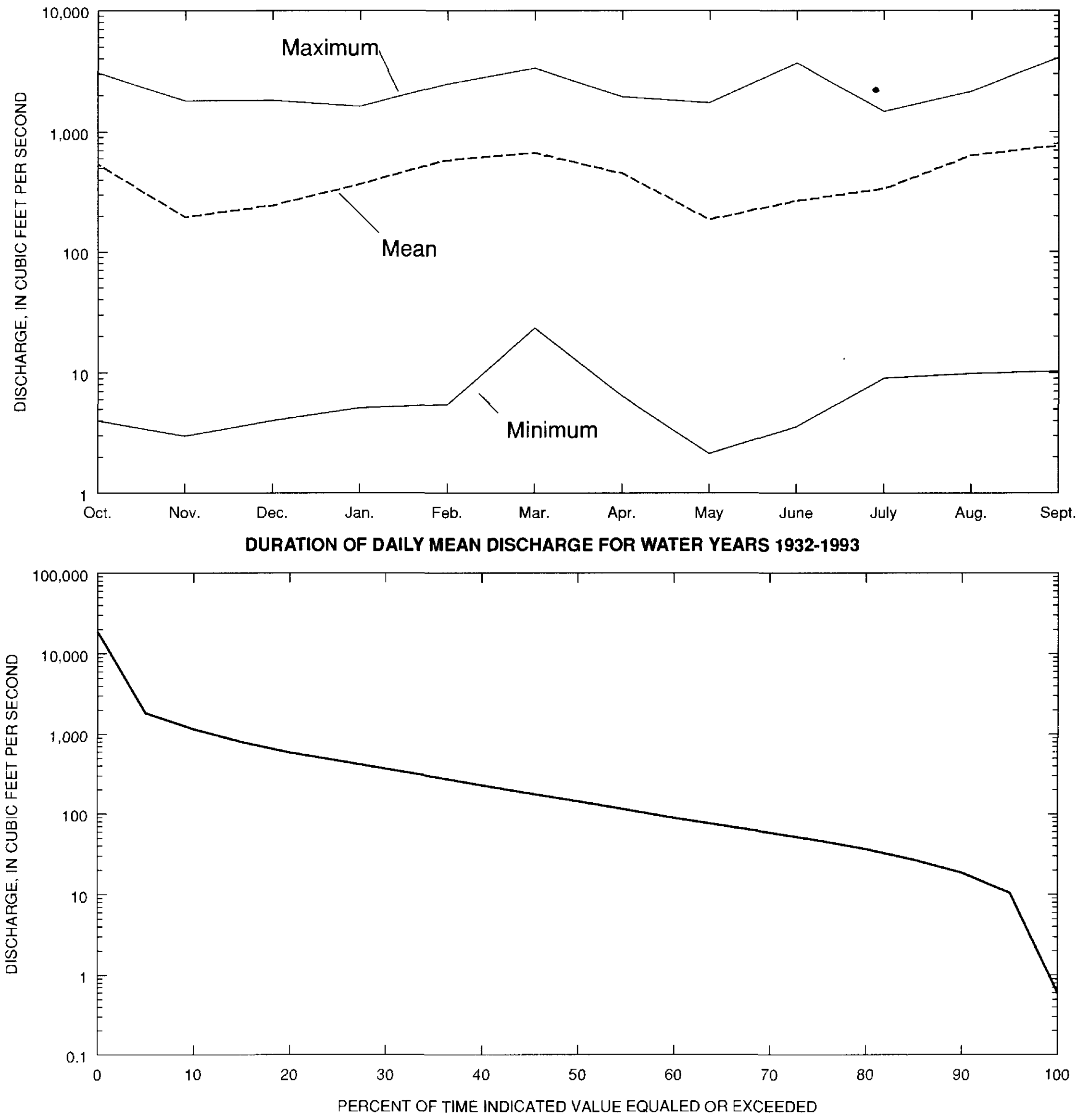
SUMMARY OF MONTHLY MEAN ELEVATION AND DISCHARGE STATISTICS FOR WATER YEARS 1932-1993

\begin{tabular}{lcccccc} 
& \multicolumn{3}{c}{ ELEVATIONS, } & \multicolumn{3}{c}{ DISCHARGE, } \\
& \multicolumn{2}{c}{ FEET ABOVE SEA LEVEL } & \multicolumn{2}{c}{ CUBIC FEET PER SECOND } \\
\multicolumn{1}{c}{ MONTH } & MAXIMUM & MINIMUM & MEAN & MAXIMUM & MINIMUM & MEAN \\
& & & & & & \\
OCTOBER & 59.57 & 49.80 & 53.31 & 3043 & 4.00 & 539 \\
NOVEMBER & 58.96 & 49.72 & 52.09 & 1788 & 2.98 & 196 \\
DECEMBER & 58.28 & 49.80 & 52.30 & 1801 & 4.00 & 246 \\
JANUARY & 58.40 & 50.08 & 53.29 & 1607 & 5.12 & 368 \\
FEBRUARY & 59.72 & 50.08 & 54.51 & 2461 & 5.44 & 586 \\
MARCH & 60.81 & 50.28 & 54.49 & 3303 & 23.4 & 668 \\
APRIL & 58.54 & 49.74 & 53.45 & 1927 & 6.41 & 454 \\
MAY & 57.34 & 49.71 & 51.66 & 1716 & 2.13 & 187 \\
JUNE & 59.51 & 49.82 & 52.00 & 3646 & 3.58 & 267 \\
JULY & 58.08 & 49.76 & 53.06 & 1459 & 9.05 & 339 \\
AUGUST & 59.52 & 49.77 & 54.58 & 2137 & 9.86 & 539 \\
SEPTEMBER & 60.93 & 50.06 & 54.49 & 4033 & 10.3 & 767
\end{tabular}

\section{- DURATION OF DAILY MEAN VALUES FOR WATER YEARS 1932-1993}

PERCENT

OF TIME

EQUAIED OR

DEC JAN FEB MAR APR MAY

ELEVATION IN FEET ABOVE MEAN SEA LEVEL

$\begin{array}{rllll}95.0 & 49.5 & 49.6 & 49.7 & 49.7 \\ 90.0 & 50.0 & 50.1 & 50.0 & 50.1 \\ 85.0 & 50.0 & 50.1 & 50.0 & 50.1 \\ 80.0 & 50.4 & 50.6 & 50.3 & 50.4 \\ 75.0 & 50.4 & 50.6 & 50.3 & 50.4 \\ 70.0 & 50.9 & 51.1 & 50.6 & 50.4 \\ 65.0 & 50.9 & 51.1 & 50.6 & 50.8 \\ 60.0 & 51.4 & 51.1 & 50.9 & 50.8 \\ 55.0 & 51.4 & 51.5 & 50.9 & 51.1 \\ 50.0 & 51.9 & 52.0 & 51.3 & 51.5 \\ 45.0 & 52.4 & 52.5 & 51.6 & 51.5 \\ 40.0 & 52.9 & 53.0 & 51.6 & 51.8 \\ 35.0 & 53.4 & 53.5 & 51.9 & 51.8 \\ 30.0 & 53.9 & 54.0 & 52.3 & 52.2 \\ 25.0 & 55.0 & 55.1 & 52.6 & 52.9 \\ 20.0 & 56.0 & 55.6 & 53.0 & 53.6 \\ 15.0 & 57.1 & 56.6 & 53.7 & 54.8 \\ 10.0 & 58.2 & 58.3 & 54.7 & 56.3 \\ 5.0 & 59.3 & 59.9 & 57.2 & 57.8\end{array}$

$\begin{array}{lll}50.1 & 49.9 & 50.3 \\ 50.5 & 50.3 & 50.3 \\ 50.5 & 50.8 & 50.7 \\ 50.5 & 51.2 & 50.7 \\ 50.8 & 51.6 & 51.2 \\ 50.8 & 52.0 & 51.6 \\ 51.2 & 52.4 & 51.6 \\ 51.5 & 52.9 & 52.1 \\ 51.5 & 53.3 & 53.0 \\ 51.9 & 53.8 & 53.5 \\ 52.6 & 54.2 & 54.4 \\ 52.9 & 54.7 & 54.9 \\ 53.7 & 55.1 & 55.9 \\ 54.0 & 56.0 & 56.4 \\ 54.8 & 56.9 & 57.4 \\ 55.5 & 57.4 & 57.9 \\ 56.7 & 57.9 & 58.4 \\ 57.8 & 58.4 & 59.4 \\ 58.6 & 59.3 & 59.9\end{array}$

$\begin{array}{ll}49.9 & 49.5 \\ 49.9 & 49.9 \\ 49.9 & 49.9 \\ 50.3 & 49.9 \\ 50.7 & 49.9 \\ 50.7 & 50.3 \\ 51.1 & 50.3 \\ 51.5 & 50.3 \\ 51.9 & 50.7 \\ 52.4 & 50.7 \\ 52.8 & 51.1 \\ 53.6 & 51.1 \\ 54.0 & 51.5 \\ 54.5 & 51.9 \\ 55.3 & 52.3 \\ 56.2 & 52.7 \\ 57.1 & 53.1 \\ 58.0 & 53.9 \\ 59.4 & 56.5\end{array}$

DISCHARGE IN CUBIC FEET PER SECOND

$\begin{array}{rrr}95.0 & 10.5 & 11.1 \\ 90.0 & 18.7 & 22.9 \\ 85.0 & 27.0 & 35.0 \\ 80.0 & 36.6 & 44.3 \\ 75.0 & 47.1 & 54.4 \\ 70.0 & 58.6 & 65.1 \\ 65.0 & 72.2 & 76.9 \\ 60.0 & 89.2 & 93.6 \\ 55.0 & 113.5 & 115.4 \\ 50.0 & 143.3 & 144.5 \\ 45.0 & 179.4 & 183.6 \\ 40.0 & 227.5 & 232.5 \\ 35.0 & 289.6 & 286.5 \\ 30.0 & 367.9 & 358.8 \\ 25.0 & 466.7 & 452.9 \\ 20.0 & 592.7 & 559.7 \\ 15.0 & 803.3 & 806.5 \\ 10.0 & 1155.8 & 1291.6 \\ 5.0 & 1840.0 & 2328.3\end{array}$

$\begin{array}{rr}11.7 & 11.5 \\ 18.8 & 17.9 \\ 23.1 & 24.2 \\ 27.6 & 30.7 \\ 33.6 & 37.6 \\ 39.1 & 45.0 \\ 46.2 & 52.9 \\ 54.2 & 61.8 \\ 63.8 & 71.3 \\ 75.8 & 82.1 \\ 90.9 & 96.8 \\ 109.6 & 114.6 \\ 130.1 & 133.7 \\ 158.2 & 158.9 \\ 193.5 & 201.3 \\ 235.9 & 283.7 \\ 291.7 & 438.7 \\ 406.4 & 621.0 \\ 840.3 & 1118.6\end{array}$

$$
\begin{array}{r}
16.5 \\
26.9 \\
43.3 \\
53.3 \\
61.2 \\
71.0 \\
83.4 \\
99.2 \\
122.6 \\
150.7 \\
187.4 \\
232.0 \\
274.9 \\
342.9 \\
417.7 \\
538.4 \\
712.6 \\
008.5 \\
519.8
\end{array}
$$

24.4
39.3
56.8
79.0
113.6
146.1
177.3
213.1
253.5
302.0
357.3
420.8
505.2
648.2
791.1
956.7
1168.5
1472.7
2006.3

27.9
38.8
51.3
63.7
80.6
99.2
122.3
156.3
200.7
264.9
343.2
426.2
520.7
634.9
782.2
991.5
1318.2
1823.2
2477.5

10.0
17.5
23.8
32.8
43.3
58.4
74.5
92.1
115.1
148.0
192.4
245.4
310.9
396.1
495.0
648.0
879.0
1236.3
1937.4

5.0
8.2
11.5
15.1
18.7
22.8
27.9
34.4
42.4
51.6
61.6
74.1
89.7
110.9
140.5
184.4
234.2
343.6
741.5

4.3
7.4
10.8
14.8
18.8
23.7
29.1
34.8
41.3
48.2
55.7
64.8
82.1
112.9
167.8
256.1
424.0
655.8
1156.4

9.2
16.9
26.1
35.1
47.1
56.7
69.7
88.7
131.7
174.7
213.7
265.4
319.8
381.6
461.4
549.2
681.4
866.5
1281.3

$\begin{array}{rr}22.9 & 26.5 \\ 44.2 & 49.1 \\ 65.9 & 75.7 \\ 97.1 & 103.3 \\ 126.5 & 136.8 \\ 155.6 & 172.3 \\ 189.0 & 211.3 \\ 232.9 & 256.1 \\ 286.0 & 300.8 \\ 348.9 & 345.9 \\ 422.4 & 403.8 \\ 502.6 & 462.9 \\ 591.0 & 539.3 \\ 741.4 & 620.2 \\ 881.8 & 756.4 \\ 1091.4 & 949.8 \\ 1312.5 & 1274.2 \\ 1637.7 & 1806.3 \\ 2166.6 & 2724.4\end{array}$



FOR PERIOD APR TO MAR

WATER YEAR

WATER YEAR RANGE

19481949

19491950

$\begin{array}{ll}1958 & 1959 \\ 1960 & 1961\end{array}$

$1961 \quad 1962$

19651966

19691970

19701971

19711972

19721973

19741975

$1977 \quad 1978$

19781979

19791980

19801981

19811982

19831984

19851986

19881989

19891990

19911992
50.316

50.519

50.110

50.0
50.318

49.84

50.111

50.315

$50.7 \quad 22$

$\begin{array}{ll}50.0 & 9 \\ 50.6 & 21\end{array}$

50.317

50.214

$49.6 \quad 2$

50.213

50.212

49.51

50.823

49.63

$49.9 \quad 7$
49.9

50.520
$5^{3} \cdot 5 \quad 18$

$\begin{array}{ll}50.5 & 18 \\ 50.5 & 19\end{array}$

50.110

$\begin{array}{cc}7 \\ 50.6 & 20 \\ 50.6 & 19\end{array}$

$\begin{array}{ll}50.6 & 19 \\ 50.2 & 10\end{array}$

\subsection{8}

49.84

50.111

$50.3 \quad 15$

50.19

$50.6 \quad 21$

50.214

$49.8 \quad 3$

50.213

50.212

49.95

49.51

$50.8 \quad 23$

49.72

$\begin{array}{ll}49.97 & 7\end{array}$

50.520

49.94

50.211

50.315

50.721

$\begin{array}{rr}50.1 & 9 \\ 50.7 & 22\end{array}$

50.316

50.314

$49.8 \quad 3$

50.213

50.212

49.95

$49.6 \quad 1$

50.923

49.72

50.07

50.518

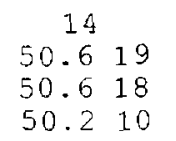

$\begin{array}{llll}50.0 & 8 & 50.1 & 8\end{array}$

$\begin{array}{ll}50.0 & 4 \\ 50.2 & 9\end{array}$

$50.4 \quad 15$

50.721

$\begin{array}{ll}50.3 & 14 \\ 50.7 & 22\end{array}$

50.313

50.416

49.93

$50.2 \quad 11$

50.312

50.05

$49.6 \quad 1$

51.123

49.82

50.07

50.617

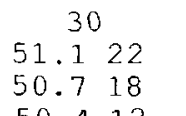

60
51.621
51.0
50.618

$51.5^{90} 18$

$\begin{array}{rr}51.6 & 19 \\ 50.8 & 8\end{array}$

50.412

$\begin{array}{rr}50.3 & 8 \\ 50.8 & 20\end{array}$

$\begin{array}{rr}50.6 & 9 \\ 51.8 & 22\end{array}$

50.911

52.220

$\begin{array}{ll}50.2 & 7 \\ 50.3 & 9\end{array}$

50.6111

$\begin{array}{lr}50.6 & 6 \\ 52.3 & 22\end{array}$
50.616

50.819

$\begin{array}{ll}50.5 & 14 \\ 50.9 & 21\end{array}$

50.413

50.515

50.02

50.310

50.411

$49.6 \quad 1$

$51.8 \quad 23$

50.03

$\begin{array}{ll}50.1 & 6 \\ 50.1 & 4\end{array}$

50.617
$50.816 \quad 51.014$

50.613

51.420

50.610

50.714

50.02

50.57

50.58

$49.7 \quad 1$

53.123

50.26

50.25

50.12

50.715

\subsection{6}

$\begin{array}{ll}50.8 & 10 \\ 52.3 & 21\end{array}$

51.115

51.013

50.12

50.77

$\begin{array}{rr}51.3 & 17 \\ 50.1 & 3\end{array}$

$49.8 \quad 1$

53.523

50.89

$\begin{array}{ll}50.3 & 5 \\ 50.3 & 4\end{array}$

50.912
51.8120

183
2.8

$53.021 \quad 54.222$

51.1992 .19

$51.514 \quad 52.511$

$52.919 \quad 54.121$

$\begin{array}{lrrr}50.7 & 4 & 50.9 & 3 \\ 53.1 & 22 & 54.4 & 23\end{array}$

$51.413 \quad 52.715$

51.412

51.18

53.020

51.716

50.12

51.07

52.018

50.1 .1

50.13

53.623

51.311

$\begin{array}{ll}50.7 & 6 \\ 50.7 & 5\end{array}$

51.615

$\begin{array}{rr}52.7 & 15 \\ 51.9 & 8\end{array}$

$52.6 \quad 13$

53.820

51.34

51.45

52.512

$53.2 \quad 17$

$50.6 \quad 1$

53.819

$53.7 \quad 18$

$\begin{array}{ll}51.7 & 7 \\ 51.5 & 6\end{array}$

52.410

HIGHEST MEAN ELEVATION, IN FEET, AND RANKING FOR THE FOLLOWING NUMBER OF CONSECUTIVE DAYS FOR PERIOD OCT TO SEP

WATER YEAR RANGE
+9341934

$\lcm{9381938}$

19481948

19491949

19501950

19561956

19581958

19591959

19611961

19621962

19651965

19661965

19691969

19701970

19711971

$1972 \quad 1972$

19731973

19741974

19751975

19771977

19781978

19791979

19801980

19811981

19831983

19851985

19861986

19891989

19911991

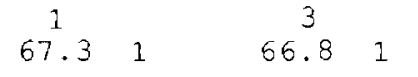

63.410

63.19

$\begin{array}{rr}7 & \\ 65.1 & 1\end{array}$

62.010

67.22

$\begin{array}{ll}63.8 & 8 \\ 66.7 & 3\end{array}$

66.62

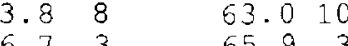

55.529

64.825

62.015

$\begin{array}{ll}56.5 & 27 \\ 61.6 & 17\end{array}$

63.69

60.323

66.44

60.821

$62.4 \quad 14$

63.819

61.020

$59.8 \quad 24$

63.113

60.622

53.311

59.126

61.618

54.16

61.616

55.328

63.112

55.329

59.724

61.515

55.828

61.418

53.28

$60.2 \quad 23$

65.94

60.621

$62.1 \quad 14$

63.57

60.620

59.625

62.813

60.522

63.011

58.726

51.416

63.76

61.417

56.327

62.812

64.62

62.19

55.228

$\begin{array}{ll}5.5 & 24\end{array}$ 

CONSECUTIVE DAYS FOR PERIOD APR TO MAR

\begin{tabular}{|c|c|c|c|c|c|}
\hline \multicolumn{6}{|c|}{ WATER YEAR } \\
\hline & ANGE & 1 & & 3 & \\
\hline 1932 & 1933 & 1.30 & 4 & 1.37 & 3 \\
\hline 1933 & 1934 & 9.00 & 26 & 10.3 & 28 \\
\hline 1934 & 1935 & 5.50 & 17 & 6.90 & 21 \\
\hline 1935 & 1936 & 1.90 & 6 & 2.00 & 6 \\
\hline 1936 & 1937 & 5.10 & 15 & 5.63 & 15 \\
\hline 1937 & 1938 & 10.0 & 28 & 10.3 & 29 \\
\hline 1938 & 1939 & 3.30 & 11 & 4.33 & 13 \\
\hline 1939 & 1940 & 10.0 & 29 & 10.3 & 30 \\
\hline 1940 & 1941 & 8.10 & 23 & 9.23 & 24 \\
\hline 1941 & 1942 & 1.90 & 7 & 2.13 & 7 \\
\hline 1942 & 1943 & 18.0 & 40 & 19.0 & 42 \\
\hline 1943 & 1944 & 5.60 & 18 & 6.10 & 18 \\
\hline 1944 & 1945 & 18.0 & 41 & 19.3 & 44 \\
\hline 1945 & 1946 & 2.80 & 9 & 3.73 & 10 \\
\hline 1946 & 1947 & 34.0 & 57 & 37.0 & 56 \\
\hline 1947 & 1948 & 27.0 & 51 & 39.3 & 58 \\
\hline 1948 & 1949 & 17.0 & 38 & 18.0 & 39 \\
\hline 1949 & 1950 & 13.0 & 32 & 14.3 & 33 \\
\hline 1950 & 1951 & 2.60 & 8 & 2.83 & 8 \\
\hline 1951 & 1952 & 3.10 & 10 & 3.27 & 9 \\
\hline 1952 & 1953 & 7.30 & 22 & 7.30 & 22 \\
\hline 1953 & 1954 & 21.0 & 49 & 21.7 & 48 \\
\hline 1954 & 1955 & 5.70 & 19 & 5.77 & 17 \\
\hline 1955 & 1956 & .60 & 1 & .80 & 1 \\
\hline 1956 & 1957 & 1.00 & 2 & 1.00 & 2 \\
\hline 1957 & 1958 & 16.0 & 36 & 16.3 & 36 \\
\hline 1958 & 1959 & 16.0 & 37 & 16.7 & 37 \\
\hline 1959 & 1960 & 81.0 & 61 & 81.7 & 61 \\
\hline 1960 & 1961 & 30.0 & 53 & 32.7 & 53 \\
\hline 1961 & 1962 & 13.0 & 33 & 13.7 & 32 \\
\hline 1962 & 1963 & 3.90 & 12 & 4.10 & 11 \\
\hline 1963 & 1964 & 8.80 & 25 & 10.0 & 25 \\
\hline 1964 & 1965 & 18.0 & 42 & 18.3 & 41 \\
\hline 1965 & 1966 & 11.0 & 31 & 11.3 & 31 \\
\hline 1966 & 1967 & 50.0 & 59 & 50.7 & 59 \\
\hline 1967 & 1968 & 9.70 & 27 & 10.2 & 27 \\
\hline 1968 & 1969 & 6.20 & 21 & 6.33 & 19 \\
\hline 1969 & 1970 & 19.0 & 44 & 19.7 & 45 \\
\hline 1970 & 1971 & 31.0 & 54 & 31.0 & 52 \\
\hline 1971 & 1972 & 8.30 & 24 & 8.87 & 23 \\
\hline 1972 & 1973 & 33.0 & 56 & 34.0 & 55 \\
\hline 1973 & 1974 & 19.0 & 45 & 19.7 & 46 \\
\hline 1974 & 1975 & 17.0 & 39 & 17.3 & 38 \\
\hline 1975 & 1976 & 27.0 & 52 & 27.7 & 51 \\
\hline 1976 & 1977 & 15.0 & 34 & 15.3 & 35 \\
\hline 1977 & 1978 & 5.30 & 16 & 5.63 & 16 \\
\hline 1978 & 1979 & 19.0 & 46 & 19.0 & 43 \\
\hline 1979 & 1980 & 19.0 & 47 & 19.7 & 47 \\
\hline 1980 & 1981 & 10.0 & 30 & 10.0 & 26 \\
\hline 1981 & 1982 & 4.10 & 13 & 4.17 & 12 \\
\hline 1982 & 1983 & 35.0 & 58 & 37.3 & 57 \\
\hline 1983 & 1984 & 51.0 & 60 & 54.0 & 60 \\
\hline 1984 & 1985 & 20.0 & 48 & 22.7 & 50 \\
\hline 1985 & 1986 & 5.90 & 20 & 6.37 & 20 \\
\hline 1986 & 1987 & 15.0 & 35 & 15.0 & 34 \\
\hline 1987 & 1988 & 18.0 & 43 & 18.0 & 40 \\
\hline 1988 & 1989 & 5.00 & 14 & 5.13 & 14 \\
\hline 1989 & 1990 & 1.20 & 3 & 1.40 & 4 \\
\hline 1990 & 1991 & 1.50 & 5 & 1.57 & 5 \\
\hline 1991 & 199. & 22.0 & 50 & 22.3 & 49 \\
\hline 1992 & 1993 & 32.0 & 55 & 33.3 & 54 \\
\hline
\end{tabular}

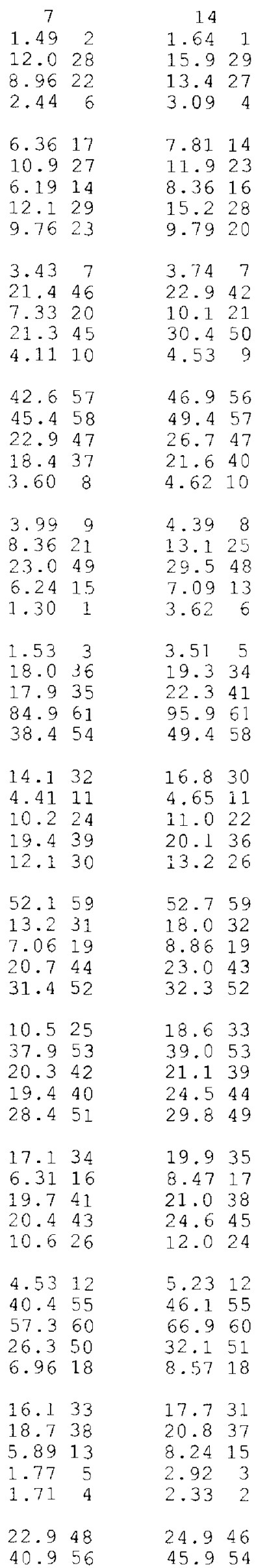

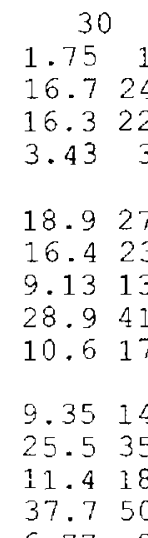

6.779

51.555

92.059

37.549

35.848

7.7511

6.748

18.826

48.954

4.695

7.1110

24.934

30.643

12861

60.456

$24.8 \quad 33$

6.557

16.825

$23.6 \quad 31$

21.329

$61.6 \quad 57$

20.028

10.216

31.745

33.947

$22.6 \quad 30$

48.653

$23.6 \quad 32$

27.939

33.446

29.142

11.619

26.036

$28.7 \quad 40$

14.620

5.936

48.352

$\begin{array}{ll}44.2 & 51 \\ 16.2 & 21\end{array}$

26.938

31.044

8.93
4.48

$\begin{array}{ll}4.98 & 2\end{array}$

$26.2 \quad 37$

26.2
$73.3 \quad 58$

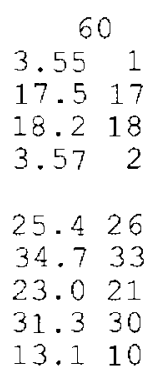

13.110

24.925

$33.1 \quad 32$

14.012

56.848
-5.415

58.051

13458

55.947

$\begin{array}{ll}49.1 & 42 \\ 20.2 & 20\end{array}$

12. 48

23.222

10656
11.37

$11.3 \quad$
6.53

24.723

$51.4 \quad 44$

49.343

13859
12157

44.141

13.211

19.119

41.338

58.550

80.854

28.427

$-4.413$

43.740
$41.6 \quad 39$

29.328

75.653

32.931

37.236

57.049

91.355

12.79

$35.8 \quad 34$

$36.2 \quad 35$

8.286

55.146

$\begin{array}{ll}71.9 & 52 \\ 24.7 & 24\end{array}$

38.937

53.245

15.616

4.513

30.829

17060

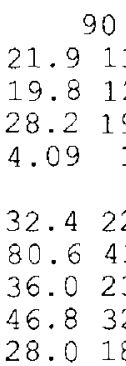

28.018

$114 \quad 48$

37.024

19.011

15455
8.530

13051

12950

10947

58.936

22.514

18.39

26.216

12249

$\begin{array}{ll}11.7 & 4 \\ 11.8 & 5\end{array}$

30.020

81.644

78.842

$178 \quad 58$

14854

46.231

27.117

31.821

17357

19059

$138 \quad 53$

42.529

38.325

58.234

52.433

39.726

16056

68.339

58.635

83.545

13152

16.68

46.130

95.346

9.003

73.341

33261

69.240

$63.5 \quad 37$

42.128

63.938

25.315

14.56

41.527
24260
120

26.27

$31.0 \quad 12$

26.38

56.421

12841

56.522

64.524

$296 \quad 58$

36.215

29.811

28656
81.930

22351

$\begin{array}{ll}223 & 5 \\ 170 & 47\end{array}$

33060

85.832
15845

33.314

27.810

4654

$\begin{array}{ll}13.4 & 3 \\ 12.2 & 2\end{array}$

38,16

$113 \quad 39$

$129 \quad 42$

22050

23352

$47.9 \quad 19$

47.418

16946

29659

28055

18849

91.436

$74.2 \quad 27$

88.934

72.526

56.823

24353

75.128

$110 \quad 38$

13943

17948

17.65

66.925

15344

$16.7 \quad 4$

26.99

11440

3466

77.4
$103 \quad 37$

49.420

85.331

42.417

52.313

$88.8 \quad 33$

88.8
29357
183
13225

41.03

42.24

64.512

46549

12523

14927
18729

29139

64.311

59.19

57956
$358 \quad 44$

$540 \quad 53$

$568 \quad 55$

58157

19531
54254

65.513

56.38

73260

25.22

80.314

$182 \quad 28$

$266 \quad 37$

$\begin{array}{lll}90 & 52\end{array}$

46950

$\begin{array}{lll}3.0 & 10 \\ 110 & 20\end{array}$

33043

$889 \quad 61$

$476 \quad 51$

39247

5.3
329
22

$224 \quad 34$

11221

$290 \quad 38$

$448 \quad 48$

35.115

23936

$206 \quad 33$

$127 \quad 24$

$228 \quad 35$

48.85

$50.4 \quad 6$

31540

$104 \quad 19$

$\begin{array}{ll}104 & 19 \\ 644 & 59\end{array}$

13626

11422

96.217

98.418

14.91

19932

61158 
SUWANNEE RIVER BASIN

HIGHEST MEAN DISCHARGE, IN CUBIC FEET PER SECOND, AND RANKING FOR THE FOLLOWING NUMBER OF CONSECUTIVE DAYS FOR PERIOD OCT TO SEP

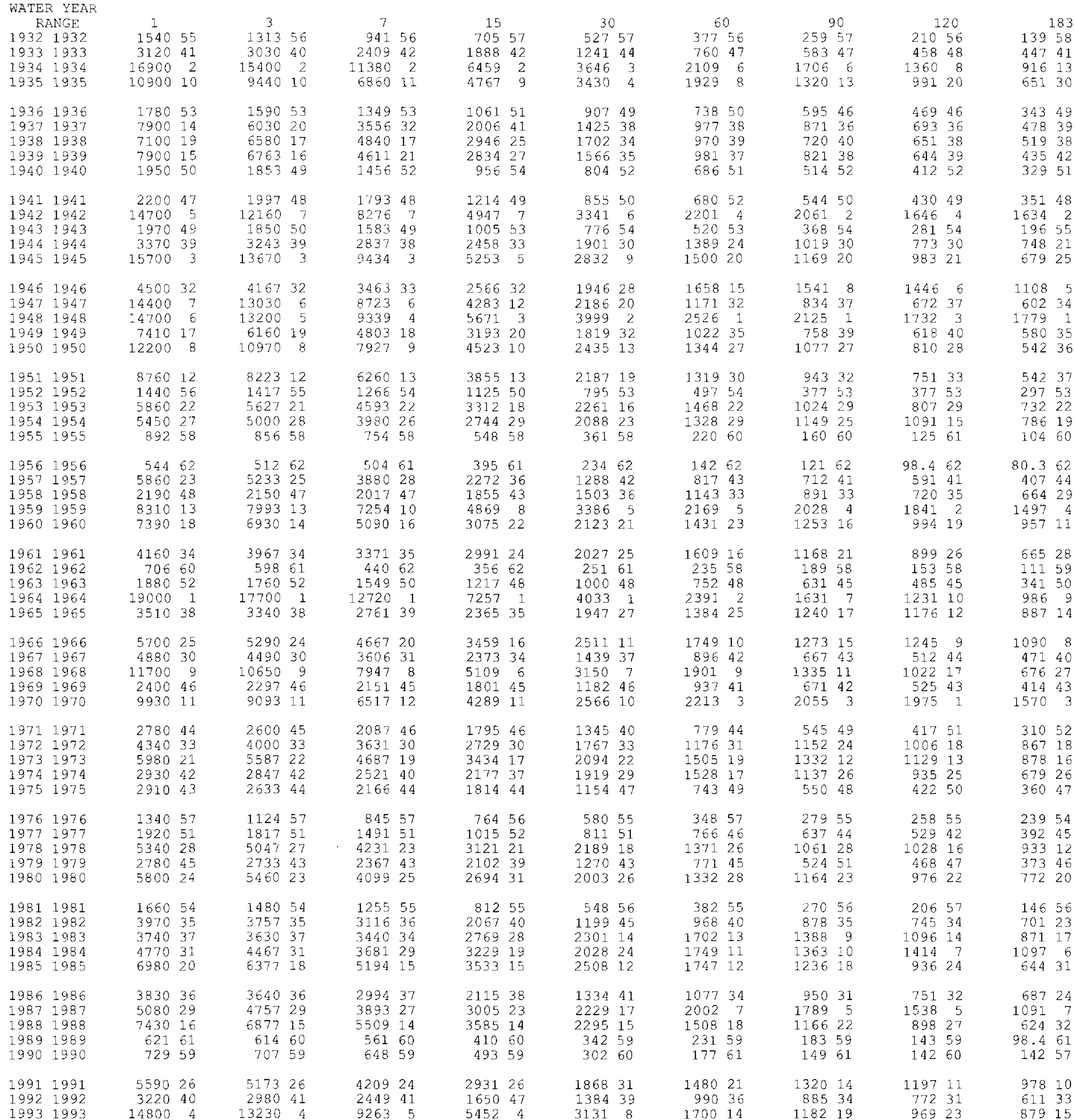


LOCATION.--Lat $30^{\circ} 03^{\prime}$, long $82^{\circ} 25^{\prime}$, in sec.16, T.5 S., R.19 E., near center of span on downstream side of bridge on State Highway 100 at Guilford, 5 miles northwest of town of Lake Butler, Union County.

DRAINAGE AREA.--27 $\mathrm{mi}^{2}$. approximately.

PERIOD OF RECORD.--August 1957 to September 1959.

GAGE.--Water-stage recorder. Datum of gage is $109.56 \mathrm{ft}$ above National Geodetic Vertical Datum of I929 (Florida Geodetic Survey bench mark). REMARKS.--Records fair.

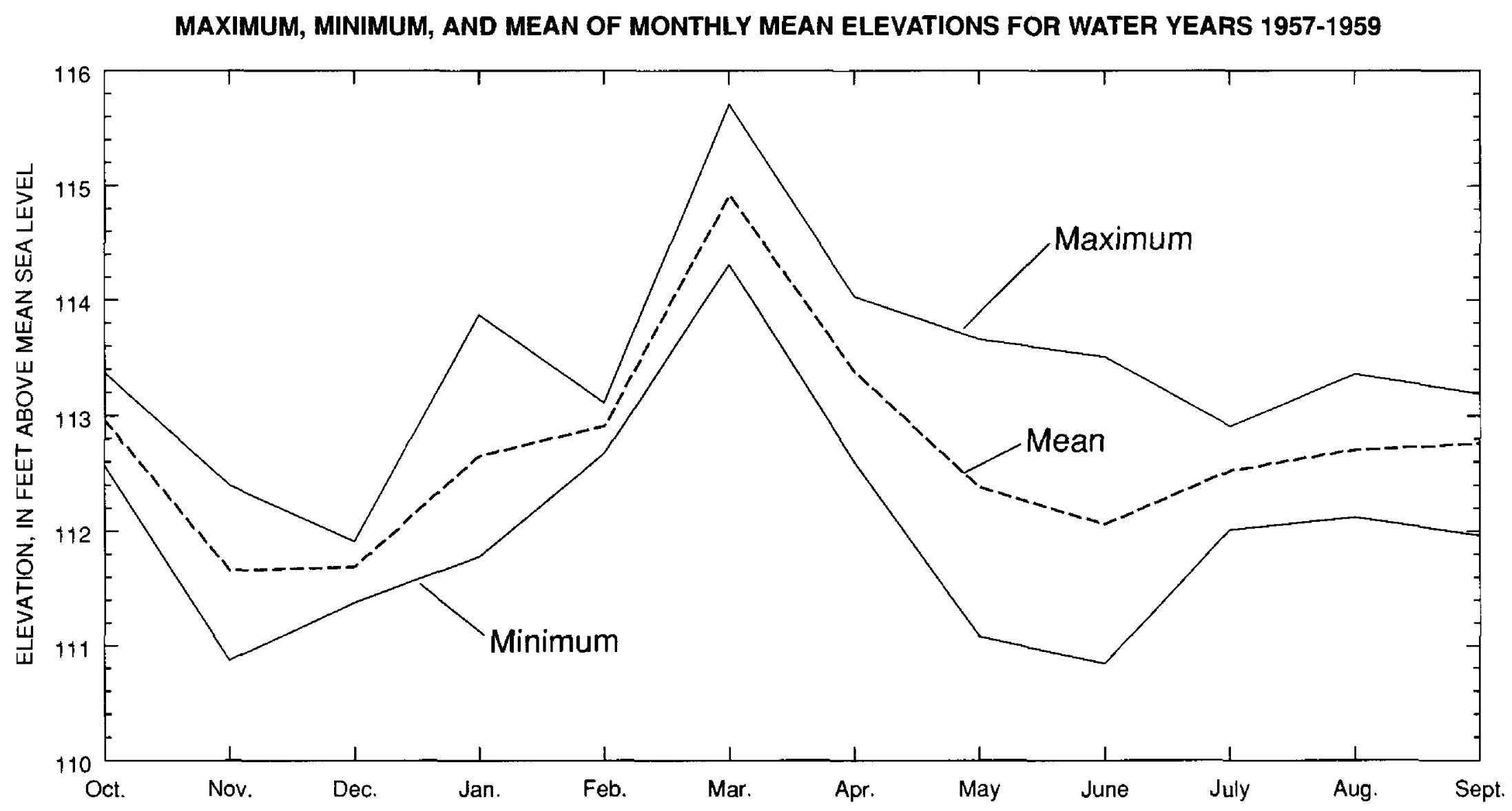

DURATION OF DAILY MEAN ELEVATION FOR WATER YEARS 1957-1959

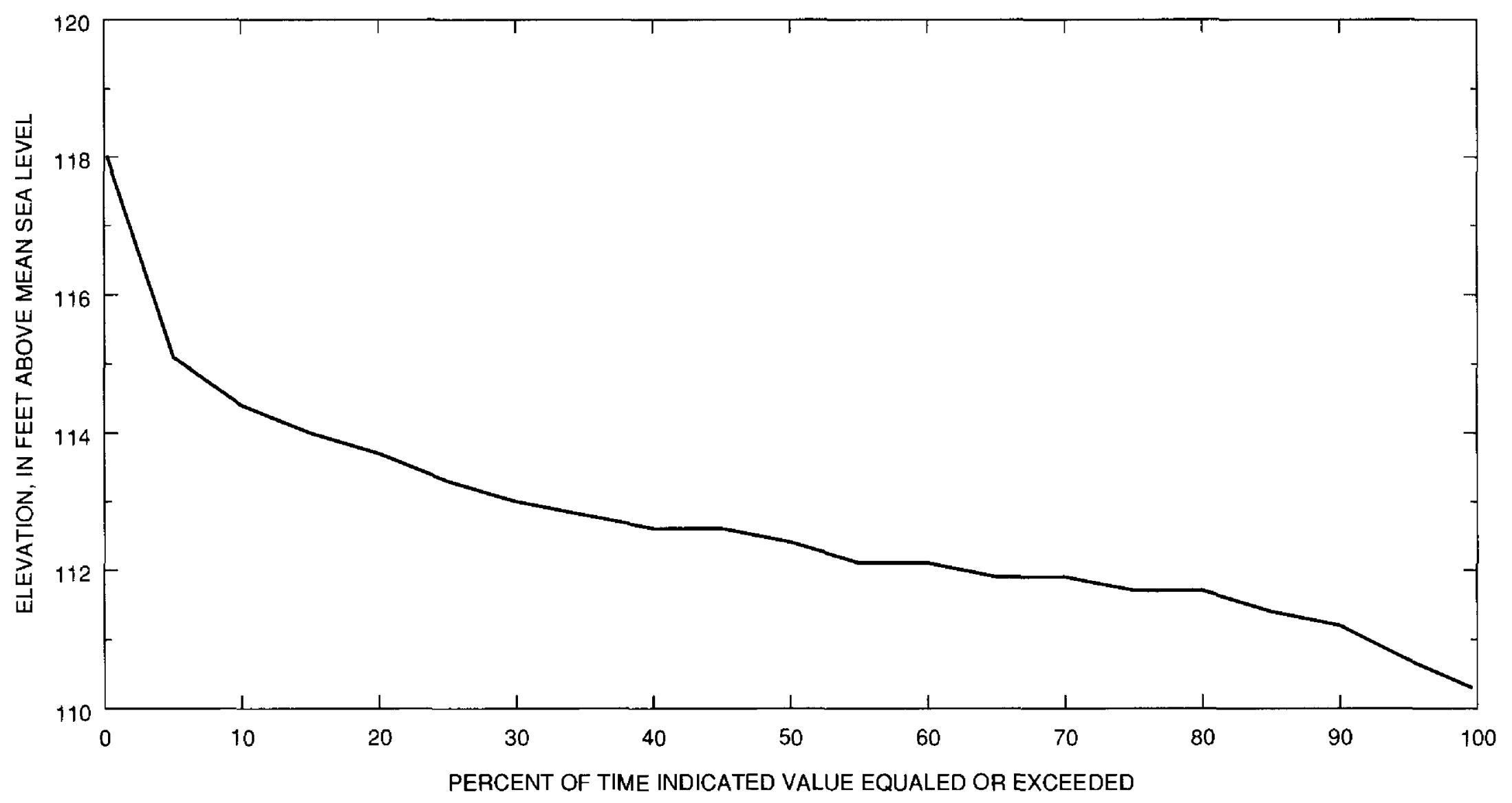


SUMMARY STATISTICS (CUBIC FEET PER SECOND)

ANNUAL MEAN

HIGHEST ANNUAL MEAN

LOWEST ANNUAL MEAN

HIGHEST DAILY MEAN

LOWEST DAILY MEAN

ANNUAL SEVEN-DAY. MINIMUM

INSTANTANEOUS PEAK FLOW

INSTANTANEOUS PEAK ELEVATION (ET)

INSTANTANEOUS LOW FLOW

ANNUAL RUNOFF (INCHES)

ANNUAL RUNOFE (CF SM)
WATER YEARS $1957-1960$

WATER YEARS $1961-1982$

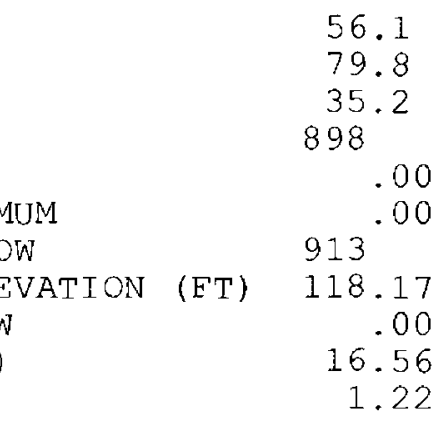

$\begin{array}{lll} & & 1959 \\ & 1958 \\ \text { Mar } 18 & 1960 \\ \text { Sep } & 30 & 1958 \\ \text { Sep } & 30 & 1958 \\ \text { Mar } 18 & 1960 \\ \text { Mar } 18 & 1960 \\ \text { Sep } 30 & 1958\end{array}$

1880

120.18

Sep 131964

Sep $13 \quad 1964$

MAXIMUM, MINIMUM, AND MEAN OF MONTHLY MEAN DISCHARGES FOR WATER YEARS 1957-1959

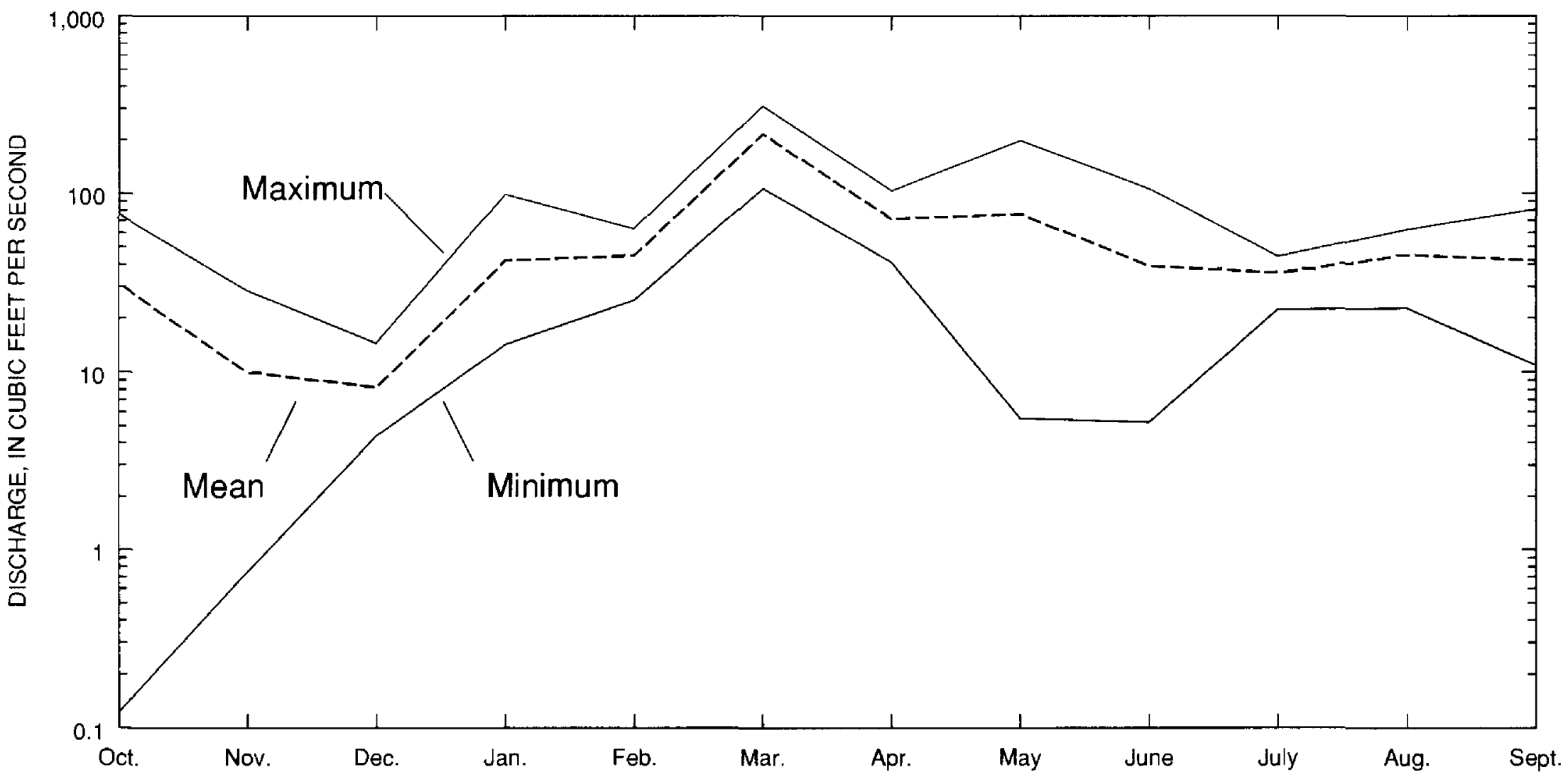

DURATION OF DAILY MEAN DISCHARGE FOR WATER YEARS 1957-1959

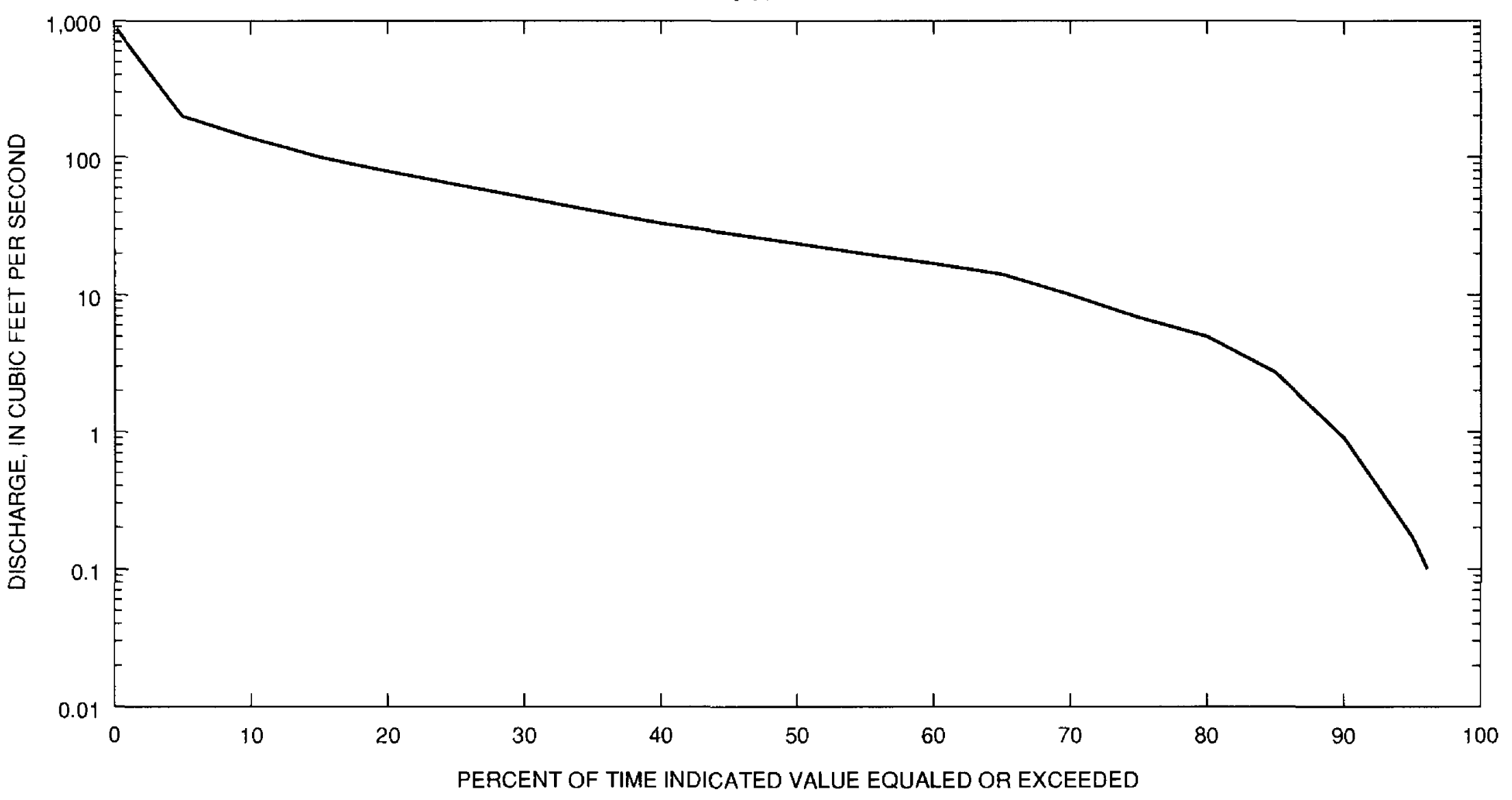


SUWANNEE RIVER BASIN

02321700 SWIFT CREEK NEAR LAKE BUTLER, FL--Continued

\section{SUMMARY OF MONTHLY MEAN ELEVATION AND DISCHARGE STATISTICS} FOR WATER YEARS 1957-1960

\begin{tabular}{|c|c|c|c|c|c|c|}
\hline \multirow[b]{2}{*}{ MONTH } & \multicolumn{3}{|c|}{$\begin{array}{ll} & \text { ELEVATIONS, } \\
\text { EEET ABOVE SEA LEVE, }\end{array}$} & \multicolumn{3}{|c|}{$\begin{array}{l}\text { DISCHARGE, } \\
\text { FEET PER SECOND }\end{array}$} \\
\hline & MAXIMUM & MIN IMUM & MEAN & MAXIMUM & MINIMUM & MEAN \\
\hline OC'T'OBER & 113.36 & 112.57 & 112.96 & 75.3 & .123 & 31.47 \\
\hline NOVEMBER & 112.39 & 110.87 & 111.66 & 28.1 & .747 & 9.98 \\
\hline DECEMBER & 111.90 & 111.38 & 111.69 & 14.3 & 4.38 & 8.19 \\
\hline JANUARY & 113.86 & 111.78 & 112.65 & 97.1 & 14.2 & 41.93 \\
\hline EEBRUARY & 113.10 & 112.67 & 112.91 & 62.1 & 25.1 & 44.60 \\
\hline MARCH & 115.69 & 114.31 & 114.92 & 305.5 & 105.9 & 215.87 \\
\hline APRIL & 114.02 & 112.60 & 113.38 & 101.6 & 41.2 & 71.50 \\
\hline MAY & 113.65 & 111.08 & 112.38 & 195.5 & 5.49 & 76.03 \\
\hline JUNE & 113.50 & 110.84 & 112.06 & 103.8 & 5.21 & 39.19 \\
\hline JULY & 112.90 & 112.01 & 112.52 & 43.7 & 22.3 & 35.70 \\
\hline AUGUST & 113.35 & 112.12 & 112.70 & 61.5 & 22.7 & 44.97 \\
\hline SEPTEMBER & 113.18 & 111.96 & 112.76 & 80.1 & 10.9 & 42.07 \\
\hline
\end{tabular}

DURATION OF DAILY MEAN VALUES FOR WATER YEARS 1937-1960

PERCENT

EOUATED OR

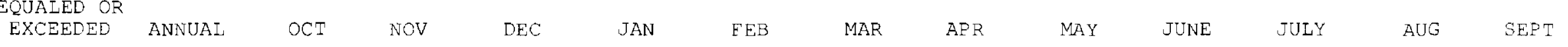

ELEVATION IN FEET ABOVE MEAN SEA LEVEL

$\begin{array}{rlll}95.0 & 110.7 & 111.9 & 110.7 \\ 90.0 & 111.2 & 112.0 & 110.8 \\ 85.0 & 111.4 & 112.0 & 110.8 \\ 80.0 & 111.7 & 112.1 & 110.8 \\ 75.0 & 111.7 & 112.2 & 110.9 \\ 70.0 & 111.9 & 112.3 & 111.3 \\ 65.0 & 111.9 & 112.4 & 111.6 \\ 60.0 & 112.1 & 112.4 & 111.6 \\ 55.0 & 112.1 & 112.6 & 111.7 \\ 50.0 & 112.4 & 112.7 & 111.7 \\ 45.0 & 112.6 & 112.9 & 111.8 \\ 40.0 & 112.6 & 113.0 & 111.8 \\ 35.0 & 112.8 & 113.1 & 111.8 \\ 30.0 & 113.0 & 113.3 & 111.9 \\ 25.0 & 113.3 & 113.5 & 112.1 \\ 20.0 & 113.7 & 113.6 & 112.2 \\ 15.0 & 114.0 & 113.9 & 112.4 \\ 10.0 & 114.4 & 114.4 & 112.7 \\ 5.0 & 115.1 & 114.9 & 112.8\end{array}$

110.9
111.0
111.5
111.6
111.6
111.6
111.6
111.7
111.7
111.8
111.8
111.8
111.8
111.9
111.9
111.9
111.9
111.9
112.0

111.4
111.7
111.7
111.8
111.8
111.9
111.9
111.9
112.0
112.4
112.4
112.6
112.7
112.8
113.1
113.4
113.8
114.2
115.1

112.1
112.2
112.3
112.3
112.4
112.5
112.5
112.6
112.6
112.7
112.7
112.8
112.9
112.9
113.2
113.3
113.6
113.7
114.4
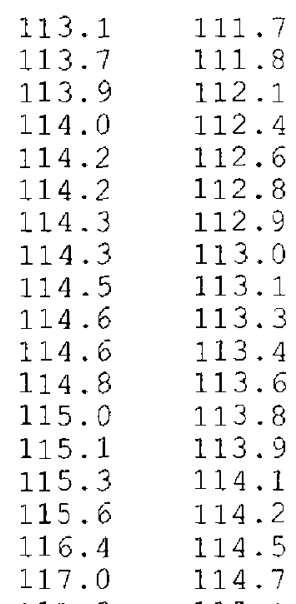

110.4
110.6
110.8
111.3
111.3
111.5
111.5
111.7
111.7
111.9
111.9
111.9
112.1
112.4
112.6
112.8
113.5
115.3
116.8

07.2
110.3
110.3
110.3
110.6
111.3
111.3
111.5
111.5
111.7
111.8
112.0
112.2
112.4
112.5
112.9
113.9
114.8
115.2

111.1
111.2
111.3
111.4
112.0
112.0
112.1
112.4
112.5
112.6
112.7
112.8
113.0
113.1
113.2
113.3
113.3
113.5

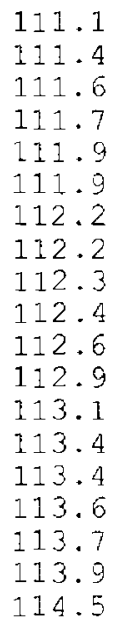

110.9

DISCHARGE IN CUBIC FEET PER SECOND

$\begin{array}{rrrrrrr}95.0 & 0.1 & 0.0 & 0.1 & 0.7 & 3.1 & 15.2 \\ 90.0 & 0.9 & 0.0 & 0.1 & 1.5 & 6.8 & 17.4 \\ 85.0 & 2.7 & 0.0 & 0.2 & 2.3 & 7.9 & 18.7 \\ 80.0 & 4.9 & 0.0 & 0.2 & 3.4 & 9.8 & 20.2 \\ 75.0 & 6.8 & 0.0 & 0.3 & 3.9 & 13.1 & 21.4 \\ 70.0 & 10.0 & 0.1 & 0.4 & 4.4 & 15.2 & 22.8 \\ 65.0 & 14.2 & 2.5 & 0.5 & 5.1 & 16.2 & 24.2 \\ 60.0 & 16.9 & 5.9 & 0.7 & 5.1 & 17.2 & 25.6 \\ 55.0 & 19.9 & 7.2 & 0.7 & 5.8 & 18.1 & 26.8 \\ 50.0 & 23.7 & 12.5 & 1.1 & 7.6 & 18.9 & 30.5 \\ 45.0 & 28.1 & 18.9 & 1.4 & 8.4 & 21.1 & 33.6 \\ 40.0 & 33.1 & 22.7 & 1.6 & 9.1 & 24.2 & 38.7 \\ 35.0 & 41.0 & 27.5 & 4.2 & 10.0 & 27.6 & 43.5 \\ 30.0 & 51.0 & 37.6 & 17.5 & 11.3 & 32.2 & 51.0 \\ 25.0 & 63.2 & 44.2 & 19.9 & 12.4 & 48.6 & 56.7 \\ 20.0 & 78.9 & 49.3 & 22.4 & 13.8 & 67.3 & 66.5 \\ 15.0 & 100.0 & 60.5 & 27.6 & 15.3 & 84.1 & 71.9 \\ 10.0 & 137.9 & 96.8 & 33.0 & 16.9 & 104.9 & 79.0 \\ 5.0 & 199.0 & 151.1 & 43.0 & 0.0 & 173.1 & 129.7\end{array}$

$\begin{array}{rr}57.9 & 12.8 \\ 69.9 & 15.0 \\ 84.1 & 20.5 \\ 90.9 & 25.0 \\ 98.1 & 30.7 \\ 105.8 & 33.0 \\ 112.3 & 38.5 \\ 119.2 & 47.0 \\ 127.7 & 52.5 \\ 136.4 & 57.0 \\ 145.3 & 68.7 \\ 154.6 & 75.0 \\ 175.9 & 86.0 \\ 193.2 & 94.5 \\ 216.2 & 100.7 \\ 262.1 & 105.7 \\ 425.0 & 127.0 \\ 612.4 & 156.0 \\ 681.2 & 181.0\end{array}$

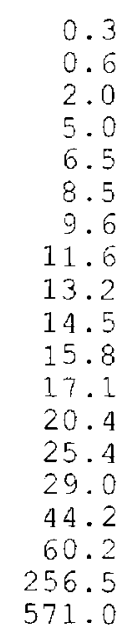
0.0
0.0
0.1
0.1
1.2
1.9
1.9
2.5
5.6
7.1
9.
9.1
12.5
16.4
20.1
24.0
28.5
38.0
144.3
198.4


LOWEST MEAN ELEVATION, IN FEET, AND RANKING FOR THE FOLLOWING NUMBER OF CONSECUTIVE DAYS FOR PERIOD APR TO MAR

WATER YEAR RANGE $\begin{array}{ccc}1 & & 3 \\ 111 & 1 & 111\end{array}$ $\begin{array}{rr}7 & \\ 11 & 1\end{array}$ $\begin{array}{rr}14 \\ 111 & 1\end{array}$ $112^{30} 1$ $112^{60} 1$ $112^{90} 1$ $112^{120} 1$ $112^{183} 1$

HIGHEST MEAN ELEVATION, IN FEET, AND RANKING FOR THE FOLLOWING NUMBER OF CONSECUTIVE DAYS FOR PERIOD OCT TO SEP

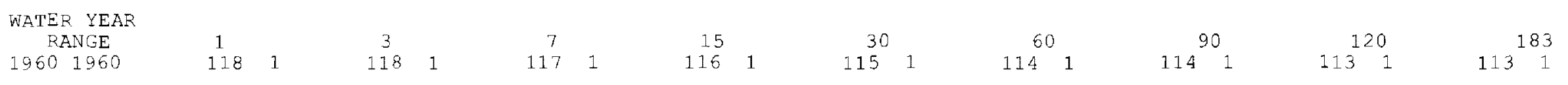

LOWEST MEAN DISCHARGE, IN CUBIC FEET PER SECOND, AND RANKING FOR THE FOLLOWING NUMBER OF CONSECUTIVE DAYS FOR PERIOD APR TO MAR

\begin{tabular}{|c|c|c|c|c|c|c|c|c|c|c|c|c|c|}
\hline RANGE & 1 & & 3 & & 7 & & 14 & & 30 & 60 & 90 & 120 & 183 \\
\hline $1958 \quad 1959$ & .0000 & 1 & .0000 & 1 & .0000 & 1 & .0000 & 1 & .12 & .43 & 1.05 & 3.94 & 20.2 \\
\hline 19591960 & 2.00 & 2 & 2.23 & 2 & 2.79 & 2 & 4.29 & 2 & 13.7 & 14.1 & 17.9 & 24.3 & 38.3 \\
\hline
\end{tabular}

HIGHEST MEAN DISCHARGE, IN CUBIC FEET PER SECOND, AND RANKING FOR THE FOLLOWING NUMBER OF CONSECUTIVE DAYS FOR PERIOD OCT TO SEP 
LOCATION.--Lat $30^{\circ} 00^{\prime}$, long $82^{\circ} 34^{\prime}$, in sec.36, T.5 S., R. 17 E., on upstream side of bridge on State Highway $238,1.5$ miles west of Providence, Union County, 6.1 miles upstream from mouth, and 13.8 miles west of Lake Butler

DRAINAGE AREA.-- $88 \mathrm{mi}^{2}$, approximately.

PERIOD OF RECORD.--October 1957 to September 1959.

GAGE.--Water-stage recorder. Datum of gage is $53.35 \mathrm{ft}$ above National Geodetic Vertical Datum of 1929 (State Road Department bench mark).

REMARKS.--Records fair.
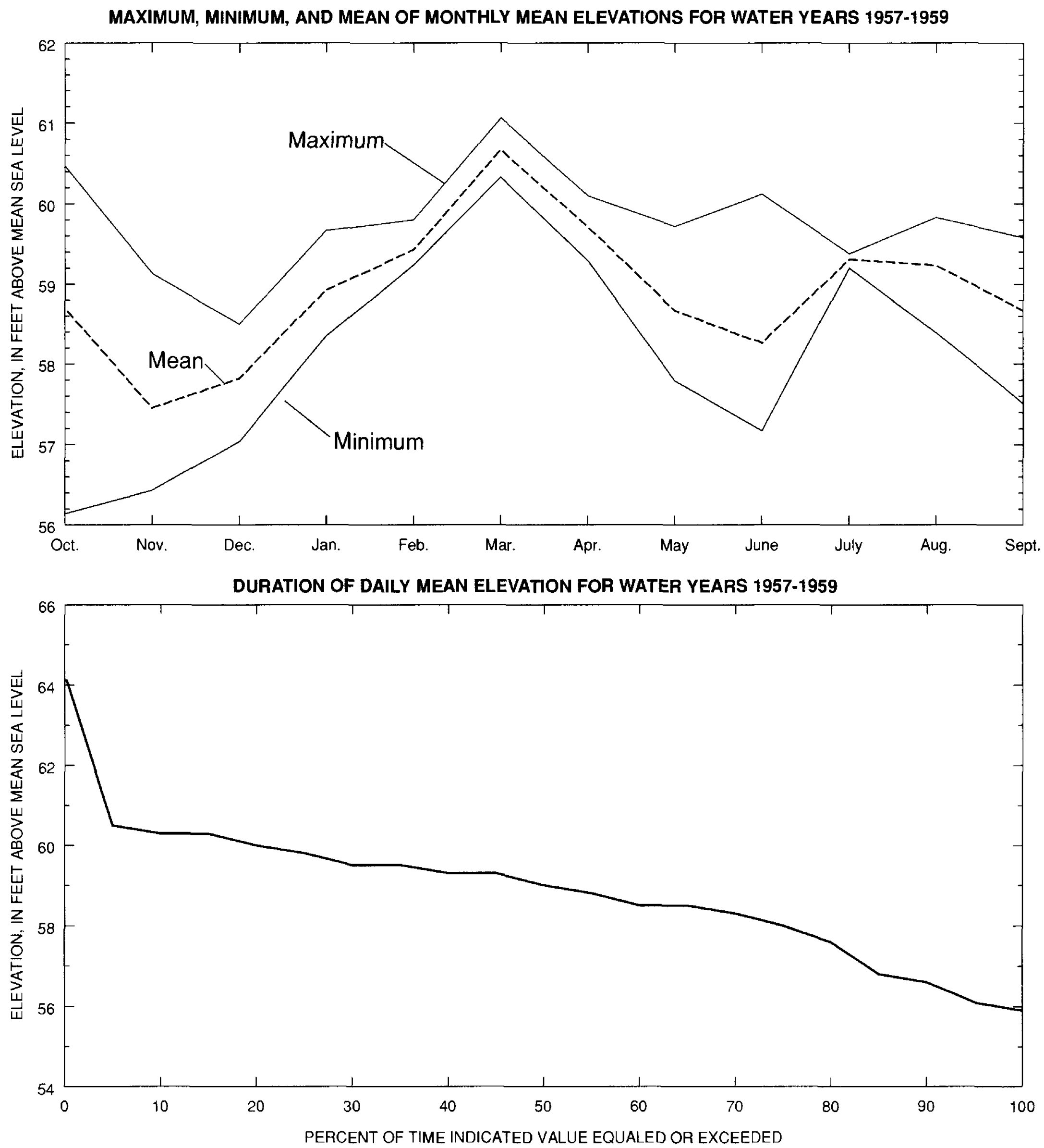
SUWANNEE RIVER BASIN

(2321800 OLUSTEE CREEK NEAR PROVIDENCE, FL--Continued

SUMMARY STATISTICS (CUBIC FEET PER SECOND)

ANNUAL MEAN

HIGHEST ANNUAL MEAN

LOWEST ANNUAL MEAN

HIGHEST DAILY MFAN

LOWEST DAILY MEAN

ANNUAL SEVEN-DAY MINIMUM

INSTANTANEOUS PEAK FLOW

-NSTANTANEOUS PEAK ETFVATTON (ET)

INSTANTANEOUS LOW FLOW

ANNUAL RUNOFF (INCHES)

ANNUAL RUNOFE (CFSM)

175
233
115
5410
.20
.31
5690
65.04
.20
14.55
1.07

WATER YEARS 1961 - 1972

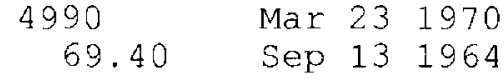

MAXIMUM, MiNIMUM, AND MEAN OF MONTHLY MEAN DISCHARGES FOR WATER YEARS 1957-1959

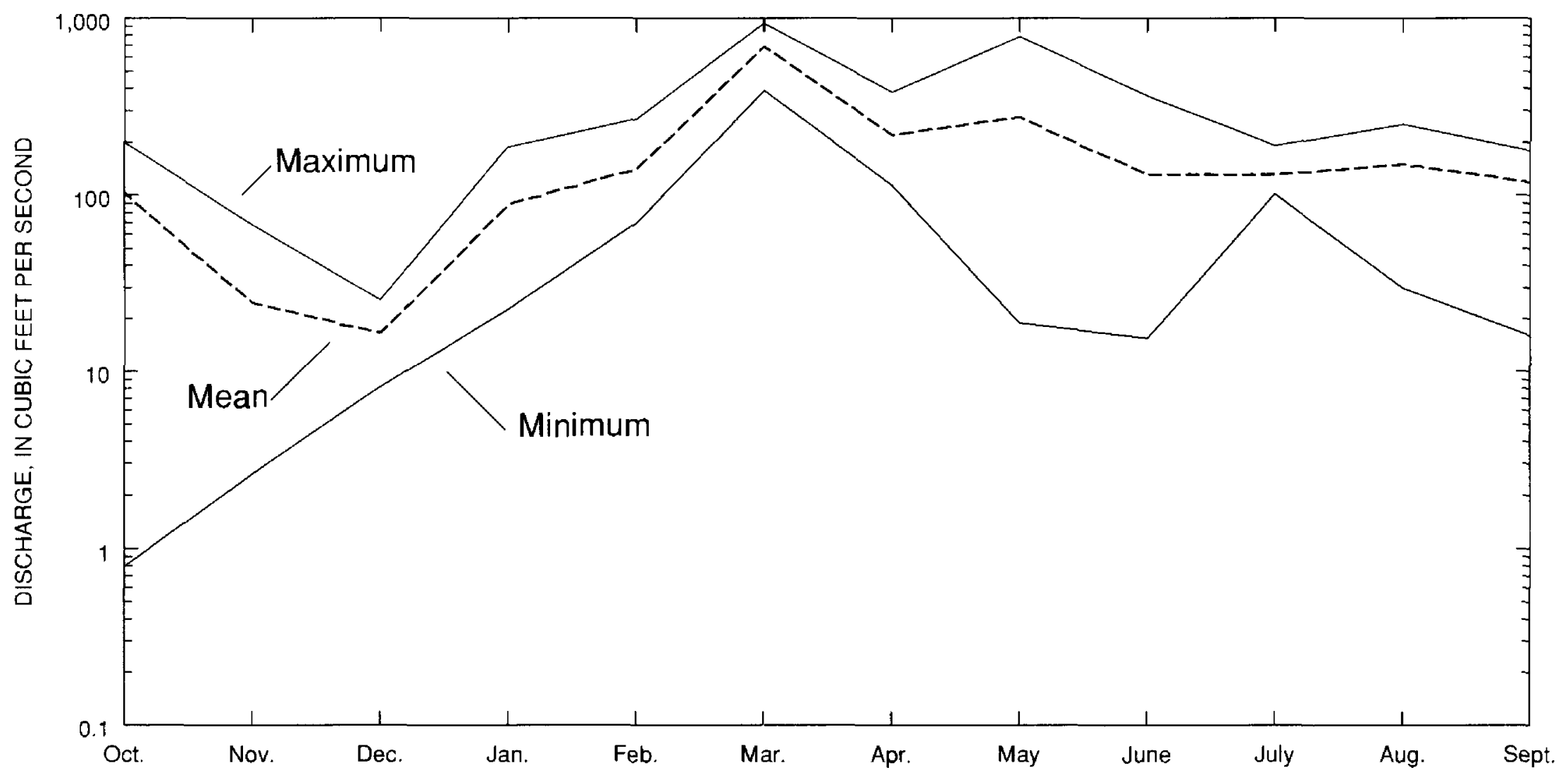

DURATION OF DAILY MEAN DISCHARGE FOR WATER YEARS 1957-1959

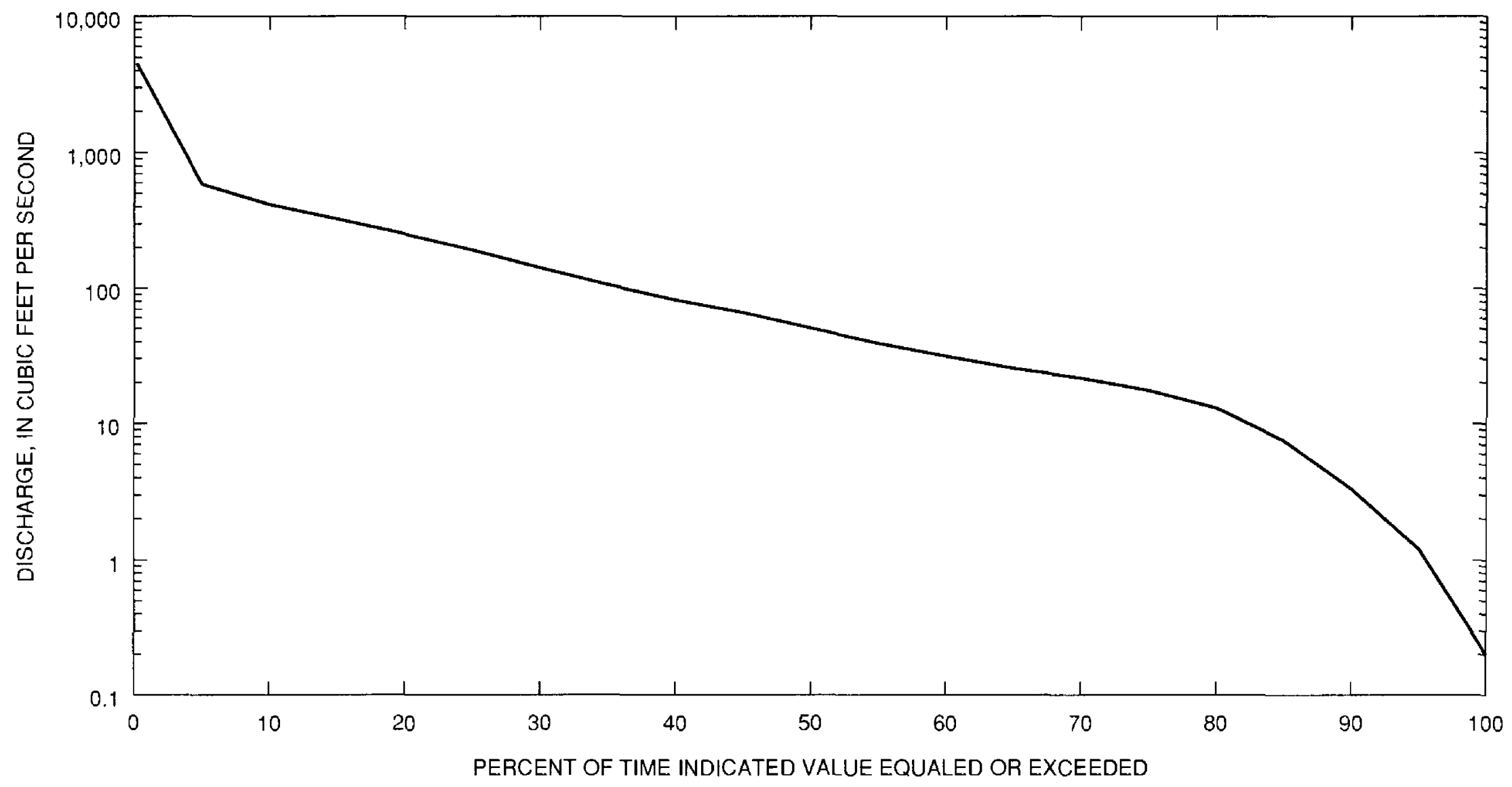


SUWANNEE RIVER BASIN

02321800 OLUSTEE CREEK NEAR PROVIDENCE, FL--Continued

SUMMARY OF MONTHLY MEAN ELEVATION AND DISCHARGE STATISTICS FOR WATER YEARS 1975-1960

\begin{tabular}{|c|c|c|c|c|c|c|}
\hline MONTH & MAXIMUM & MIN I MUM & MEAN & MAXIMJM & MIN IMUM & MEAN \\
\hline OCTOBER & 50.46 & 56.14 & 58.70 & 196.5 & .794 & 102.70 \\
\hline NOVEMBER & 59.12 & 56.43 & 57.45 & 67.3 & 2.62 & 24.61 \\
\hline DECEMBER & 58.49 & 57.04 & 57.82 & 25.1 & 8.14 & 16.48 \\
\hline JANUARY & 59.66 & 58.35 & 58.93 & 185.2 & 22.6 & 88.90 \\
\hline FEBRUARY & 59.79 & 59.24 & 59.43 & 265.6 & 69.4 & 140.47 \\
\hline MARCH & 61.05 & 60.33 & 60.67 & 919.3 & 388.4 & 687.73 \\
\hline APRIL & 60.09 & 59.29 & 59.71 & 375.6 & 114.6 & 219.40 \\
\hline MAY & 59.71 & 57.79 & 58.67 & 772.4 & 18.8 & 274.53 \\
\hline JUNE & 50.11 & 57.17 & 58.26 & 357.0 & 15.4 & 131.13 \\
\hline JULY & 59.37 & 59.20 & 59.31 & 188.6 & 101.4 & 130.47 \\
\hline AUGUST & 59.82 & 58.39 & 59.23 & 248.0 & 29.8 & 149.03 \\
\hline SEPTEMBER & 59.57 & 57.51 & 58.67 & 176.4 & 15.9 & 118.10 \\
\hline
\end{tabular}

\section{DURATION OF DAILY MEAN VALUES FOR WATER YEARS 1931-1960}

PERCENT

OF TIME
EQUALED OR

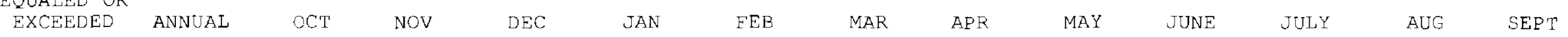

ELEVATION IN FEET ABOVE MEAN SEA LEVEL

$\begin{array}{rl}95.0 & 56.1 \\ 90.0 & 56.6 \\ 85.0 & 56.8 \\ 80.0 & 57.6 \\ 75.0 & 58.0 \\ 70.0 & 58.3 \\ 65.0 & 58.5 \\ 60.0 & 58.5 \\ 55.0 & 58.8 \\ 50.0 & 59.0 \\ 45.0 & 59.3 \\ 40.0 & 59.3 \\ 35.0 & 59.5 \\ 30.0 & 59.5 \\ 25.0 & 59.8 \\ 20.0 & 50.0 \\ 15.0 & 60.3 \\ 10.0 & 60.3 \\ 5.0 & 60.5\end{array}$

$\begin{array}{ll}55.9 & 56.1 \\ 56.1 & 56.1 \\ 56.1 & 56.3 \\ 56.1 & 56.4 \\ 56.2 & 56.5 \\ 56.2 & 56.6 \\ 58.3 & 56.7 \\ 58.6 & 56.7 \\ 59.0 & 56.7 \\ 59.5 & 56.7 \\ 59.8 & 56.8 \\ 60.3 & 56.9 \\ 60.4 & 57.0 \\ 60.4 & 58.5 \\ 60.4 & 58.8 \\ 60.4 & 58.9 \\ 60.4 & 59.0 \\ 60.4 & 59.1 \\ 60.4 & 59.5\end{array}$

$\begin{array}{lll}56.3 & 57.5 & 58.7 \\ 56.4 & 57.8 & 58.8 \\ 57.3 & 58.0 & 58.8 \\ 57.3 & 58.2 & 58.9 \\ 57.4 & 58.3 & 59.0 \\ 57.5 & 58.4 & 59.1 \\ 57.6 & 58.6 & 59.2 \\ 57.7 & 58.6 & 59.2 \\ 57.8 & 58.7 & 59.3 \\ 57.8 & 58.9 & 59.3 \\ 57.9 & 59.1 & 59.4 \\ 58.2 & 59.2 & 59.4 \\ 58.2 & 59.3 & 59.5 \\ 58.2 & 59.4 & 59.5 \\ 58.3 & 59.5 & 59.7 \\ 58.4 & 59.5 & 59.7 \\ 58.6 & 59.7 & 59.8 \\ 58.6 & 59.9 & 60.2 \\ 58.7 & 60.2 & 60.5\end{array}$

$\begin{array}{ll}59.7 & 58.4 \\ 60.0 & 58.7 \\ 60.0 & 58.9 \\ 60.1 & 59.2 \\ 60.1 & 59.3 \\ 60.3 & 59.4 \\ 60.3 & 59.4 \\ 60.4 & 59.5 \\ 60.4 & 59.6 \\ 60.4 & 59.7 \\ 60.4 & 59.8 \\ 60.4 & 59.8 \\ 60.5 & 59.9 \\ 60.6 & 60.0 \\ 60.6 & 60.0 \\ 60.9 & 60.1 \\ 61.3 & 60.2 \\ 62.0 & 60.5 \\ 62.6 & 60.8\end{array}$

56.5
56.9
57.4
57.6
57.8
57.8
58.1
58.3
58.3
58.3
58.5
58.5
58.7
58.7
58.9
59.2
59.2
50.6
62.2

56.2
56.4
56.5
56.5
56.5
56.7
56.8
56.9
57.4
57.8
59.0
59.1
59.3
59.4
59.7
59.9
60.0
60.5
60.8

58.0
58.3
58.6
58.8
58.9
59.0
59.1
59.1
59.2
59.3
59.5
59.5
59.6
59.7
59.8
59.9
59.9
60.1
60.2

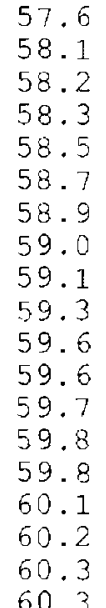

DISCHARGE IN CUBIC FEET PER SECOND

$\begin{array}{rrr}95.0 & 1.2 & 0.3 \\ 90.0 & 3.3 & 0.4 \\ 85.0 & 7.5 & 0.5 \\ 80.0 & 13.0 & 0.6 \\ 75.0 & 17.5 & 0.8 \\ 70.0 & 21.6 & 1.1 \\ 65.0 & 25.5 & 7.2 \\ 60.0 & 31.3 & 12.6 \\ 55.0 & 39.0 & 19.1 \\ 50.0 & 50.4 & 24.7 \\ 45.0 & 66.0 & 28.3 \\ 40.0 & 82.2 & 36.6 \\ 35.0 & 106.5 & 53.3 \\ 30.0 & 141.7 & 77.5 \\ 25.0 & 191.7 & 106.0 \\ 20.0 & 252.9 & 169.0 \\ 15.0 & 324.4 & 248.3 \\ 10.0 & 416.6 & 375.9 \\ 5.0 & 588.3 & 542.8\end{array}$

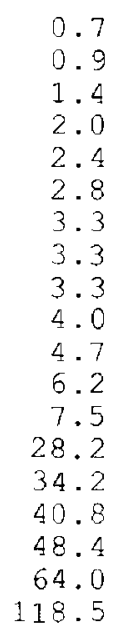

$\begin{array}{rr}1.4 & 11.8 \\ 2.0 & 15.8 \\ 10.5 & 18.0 \\ 11.4 & 20.3 \\ 12.1 & 21.9 \\ 12.6 & 23.4 \\ 13.1 & 25.8 \\ 13.9 & 28.8 \\ 14.8 & 34.1 \\ 15.6 & 45.0 \\ 17.1 & 62.0 \\ 18.7 & 76.5 \\ 19.9 & 84.4 \\ 21.0 & 95.5 \\ 22.4 & 114.9 \\ 24.0 & 131.5 \\ 26.0 & 166.2 \\ 27.8 & 218.2 \\ 39.8 & 337.3\end{array}$

$\begin{array}{rr}32.7 & 144.4 \\ 35.1 & 238.7 \\ 40.5 & 265.7 \\ 43.6 & 300.7 \\ 47.2 & 321.6 \\ 53.0 & 344.9 \\ 60.1 & 371.4 \\ 66.8 & 388.5 \\ 74.5 & 404.6 \\ 80.5 & 421.3 \\ 84.7 & 442.9 \\ 93.7 & 464.4 \\ 108.3 & 491.8 \\ 114.0 & 521.3 \\ 119.7 & 608.0 \\ 138.0 & 726.0 \\ 171.7 & 1108.2 \\ 304.5 & 1790.0 \\ 491.5 & 2539.3\end{array}$

$\begin{array}{rr}26.0 & 2.4 \\ 33.7 & 5.3 \\ 43.5 & 11.4 \\ 66.0 & 14.4 \\ 77.0 & 16.3 \\ 86.0 & 18.3 \\ 95.0 & 20.4 \\ 108.5 & 22.4 \\ 128.5 & 23.8 \\ 142.0 & 25.3 \\ 162.5 & 26.8 \\ 176.5 & 28.8 \\ 192.3 & 33.4 \\ 222.6 & 41.2 \\ 241.9 & 51.6 \\ 278.0 & 63.4 \\ 328.0 & 78.2 \\ 469.0 & 606.3 \\ 671.7 & 2285.0\end{array}$

1.2
1.5
1.8
2.3
2.3
3.4
4.2
6.9
10.5
18.0
51.7
60.0
72.6
91.0
160.2
206.0
282.0
521.5
684.2

17.3
24.3
29.9
35.1
40.2
46.9
52.4
64.1
80.4
90.8
111.4
126.1
146.4
178.6
216.2
236.5
263.6
297.7
345.1

$\begin{array}{rr}14.3 & 1.9 \\ 20.2 & 4.1 \\ 22.0 & 7.5 \\ 24.4 & 10.0 \\ 27.2 & 12.7 \\ 32.3 & 16.0 \\ 38.4 & 20.5 \\ 53.4 & 25.7 \\ 77.3 & 34.2 \\ 96.2 & 48.0 \\ 131.4 & 68.5 \\ 146.1 & 89.5 \\ 171.5 & 118.7 \\ 195.0 & 157.7 \\ 222.4 & 209.5 \\ 277.3 & 249.7 \\ 326.2 & 287.9 \\ 364.7 & 336.7 \\ 434.7 & 417.4\end{array}$


LOWEST MEAN ELEVATION, IN FEET, AND RANKING FOR THE FOLLOWING NUMBER OF CONSECUTIVE DAYS FOR PERIOD APR TO MAR

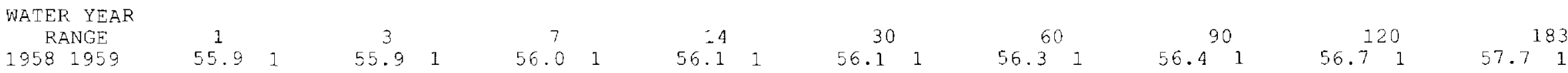

HIGHEST MEAN ELEVATION, IN FEET, AND RANKING FOR THE FOLLOWING NUMBER OF CONSECUTIVE DAYS FOR PERIOD OCT TO SEP

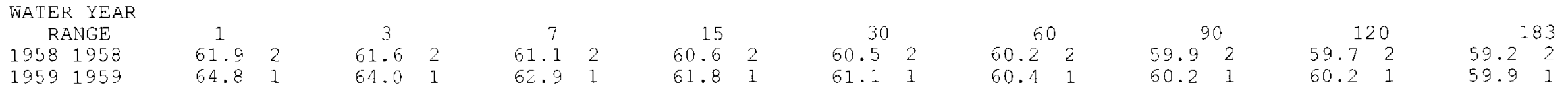

LOWEST MEAN DISCHARGE, IN CUBIC FEET PER SECOND, AND RANKING FOR THE FOLLOWING NUMBER OF CONSECUTIVE DAYS FOR PERIOD APR TO MAR

WATER YEAR

RANGE

19581959

$\begin{array}{cccc}1 & & 3 \\ .20 & 1 & .20 & 1 \\ 7.50 & 2 & 7.70 & 2\end{array}$

$\begin{array}{rr}7 & \\ .31 & 1 \\ 9.34 & 2\end{array}$

$\begin{array}{rr}14 & \\ .56 & 1 \\ 11.2 & 2\end{array}$

\begin{tabular}{cccc}
30 & \multicolumn{2}{c}{60} \\
.79 & 1 & 1.70 & 1 \\
21.3 & 2 & 23.6 & 2
\end{tabular}

$2.52^{90} 1$

$5.81^{120} 1$

$\begin{array}{rl}183 \\ 47.5 & 1\end{array}$

19591960

HIGHEST MEAN DISCHARGE, IN CUBIC FEET PER SECOND, AND RANKING FOR THE FOLLOWING NUMBER OF CONSECUTIVE DAYS FOR PERIOD OCT TO SEP

WATER YEAR

RANGE
19581958

19581958

19591959

$\begin{array}{cccc}1 & & 3 & \\ 1720 & 3 & 1349 & 3 \\ 5410 & 1 & 4383 & 1 \\ 3670 & 2 & 3403 & 2\end{array}$

$\begin{array}{rr}7 & \\ 974 & 3 \\ 2957 & 1 \\ 2230 & 2\end{array}$

$\begin{array}{rr}15 & \\ 622 & 3 \\ 1747 & 1 \\ 1257 & 2\end{array}$

$\begin{array}{rr}30 & \\ 429 & 3 \\ 1087 & 1 \\ 861 & 2\end{array}$

$\begin{array}{cc}60 \\ 391 & 3 \\ 604 & 1 \\ 539 & 2\end{array}$

$\begin{array}{ll}287^{90} & 3 \\ 643 & 1 \\ 385 & 2\end{array}$

$\begin{array}{ll}120 \\ 233 & 3 \\ 568 & 1 \\ 294 & 2\end{array}$

$\begin{array}{lr} & 183 \\ 182 & 3 \\ 415 & 1 \\ 237 & 2\end{array}$ 
LOCATION.--Lat $29^{\circ} 51^{\prime}$, long $82^{\circ} 38^{\prime}$, in sec. 29 . T.7 S., R. 17 E., near right bank at upstream side of bridge on U.S. Highway $27,150 \mathrm{ft}$ upstream from Atlantic

Coast Line Railroad bridge and 2 miles northwest of High Springs, Alachua County.

DRAINAGE AREA.--950 $\mathrm{mi}^{2}$, approximately.

PERIOD OF RECORD.--January 1931 to September 1971.

GAGE.--Water-stage recorder. Datum of gage is $26.36 \mathrm{ft}$ above National Geodetic Vertical Datum of 1929 (levels by Florida State Road Depattment). Prior to Jan. 9, 1933, staff gage at same site and datum.

AVERAGE DISCHARGE.--28 years, $763 \mathrm{ft}^{3} / \mathrm{s}$

REMARKS.--Records good.
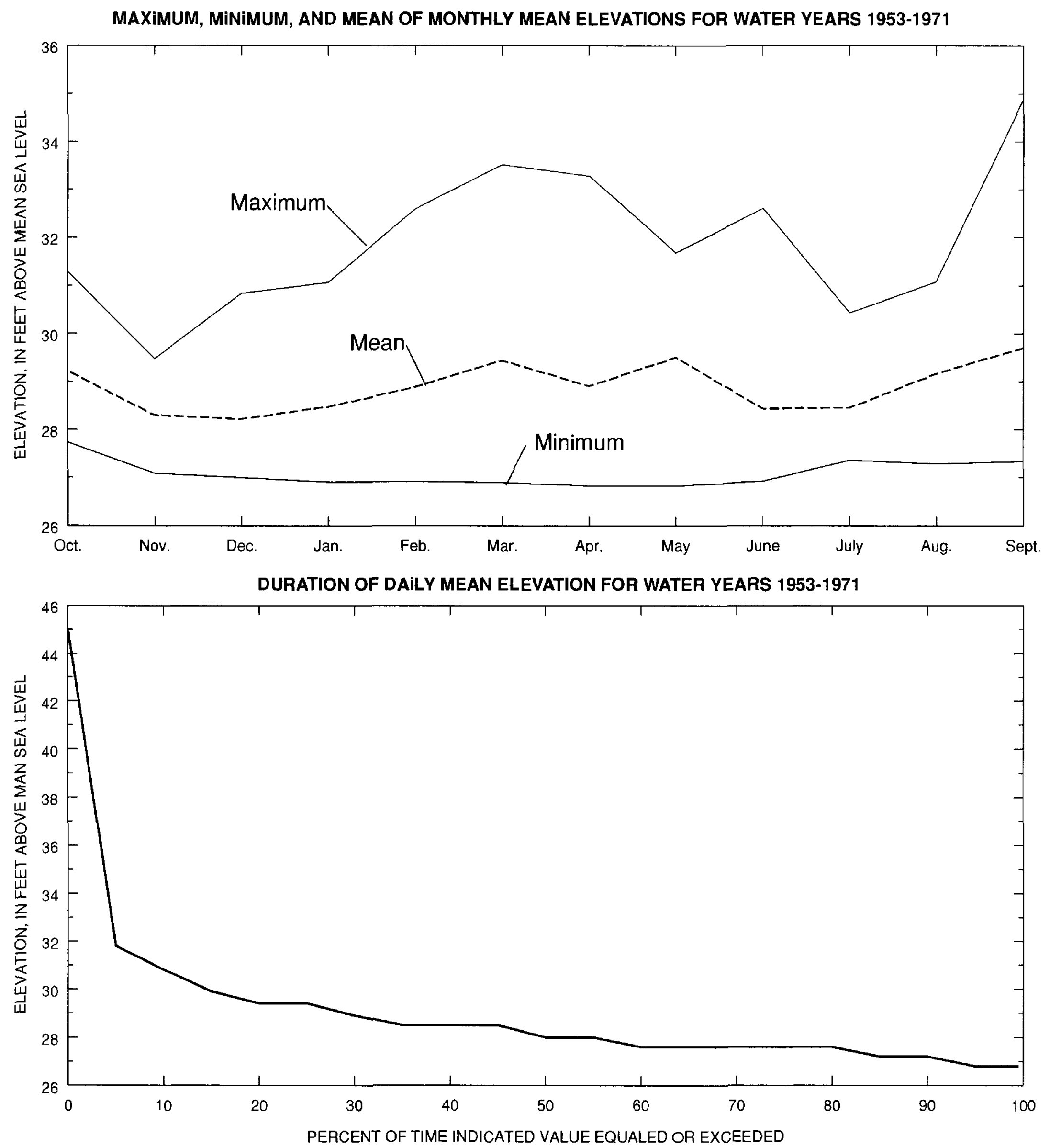
SUMMARY STATISTICS, IN CUBIC FEET PER SECOND UNLESS OTHERWISE INDICATED, FOR WATER YEARS $1931-1971$

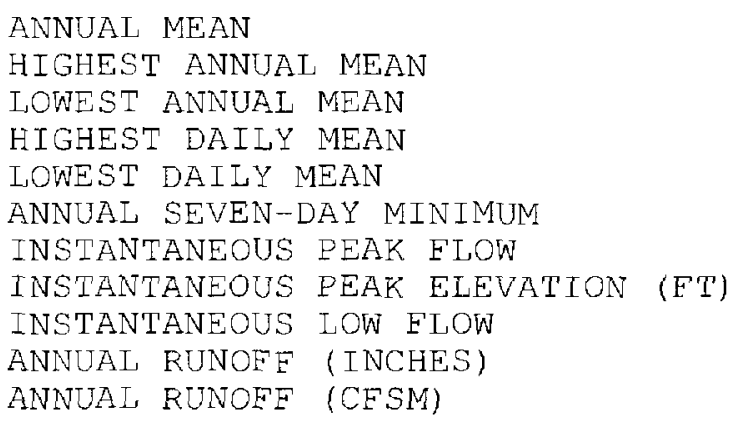

$\begin{array}{rlrl}846 & & & \\ 2143 & & & 1948 \\ 81.7 & & & 1956 \\ 19600 & \text { Sep } 15 & 1964 \\ 31 & \text { Apr } 29 & 1956 \\ 31 & \text { Apr } 28 & 1956 \\ 20000 & \text { Sep } 15 & 1964 \\ 45.32 & \text { Sep } 16 & 1964 \\ 31 & \text { Apr } 28 & 1956 \\ 12.10 & & & \\ .89 & & & \end{array}$

MAXIMUM, MINIMUM, AND MEAN OF MONTHLY MEAN DISCHARGES FOR WATER YEARS 1931-1971

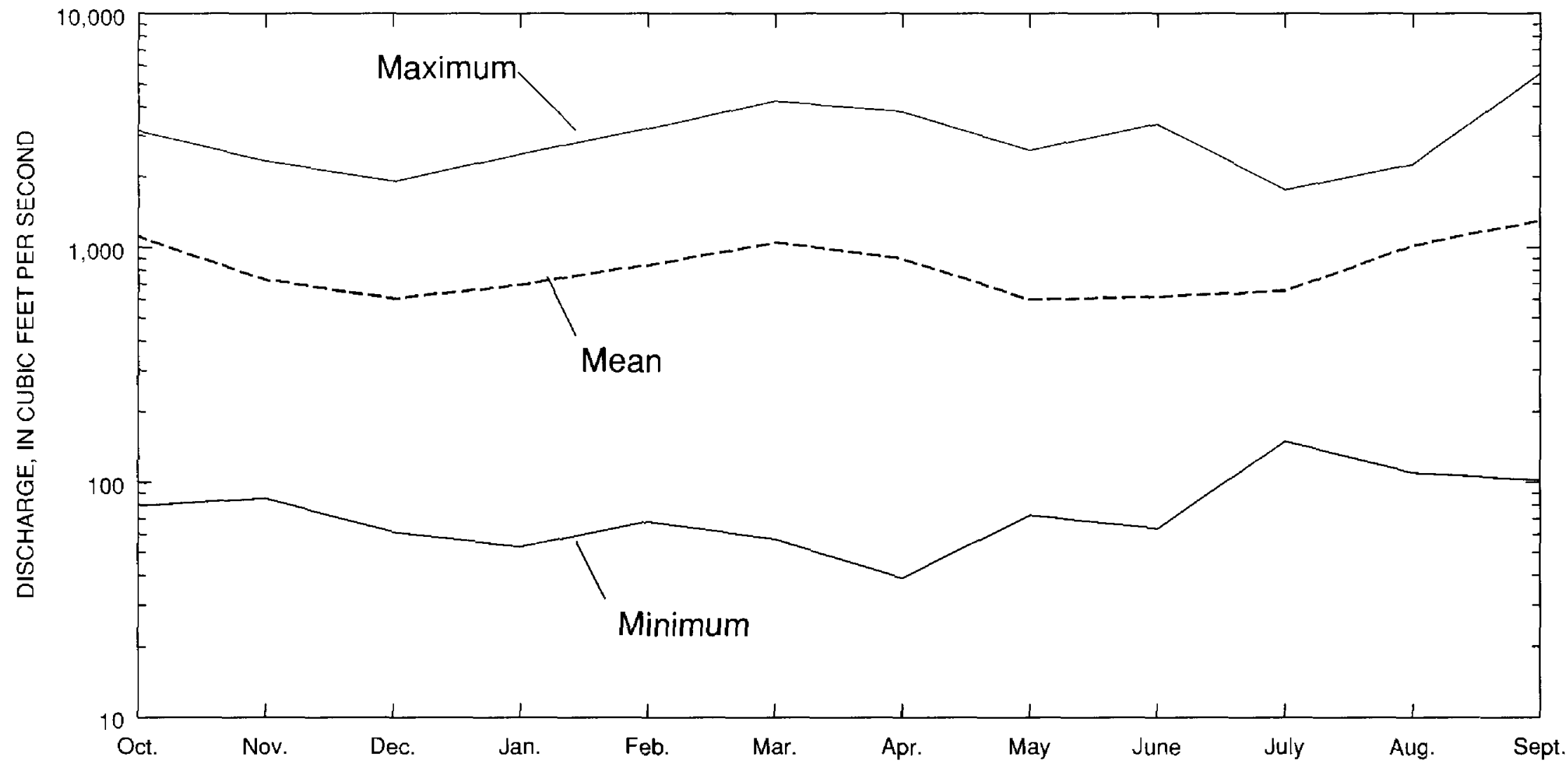

DURATION OF DAILY MEAN DISCHARGE FOR WATER YEARS 1931-1971

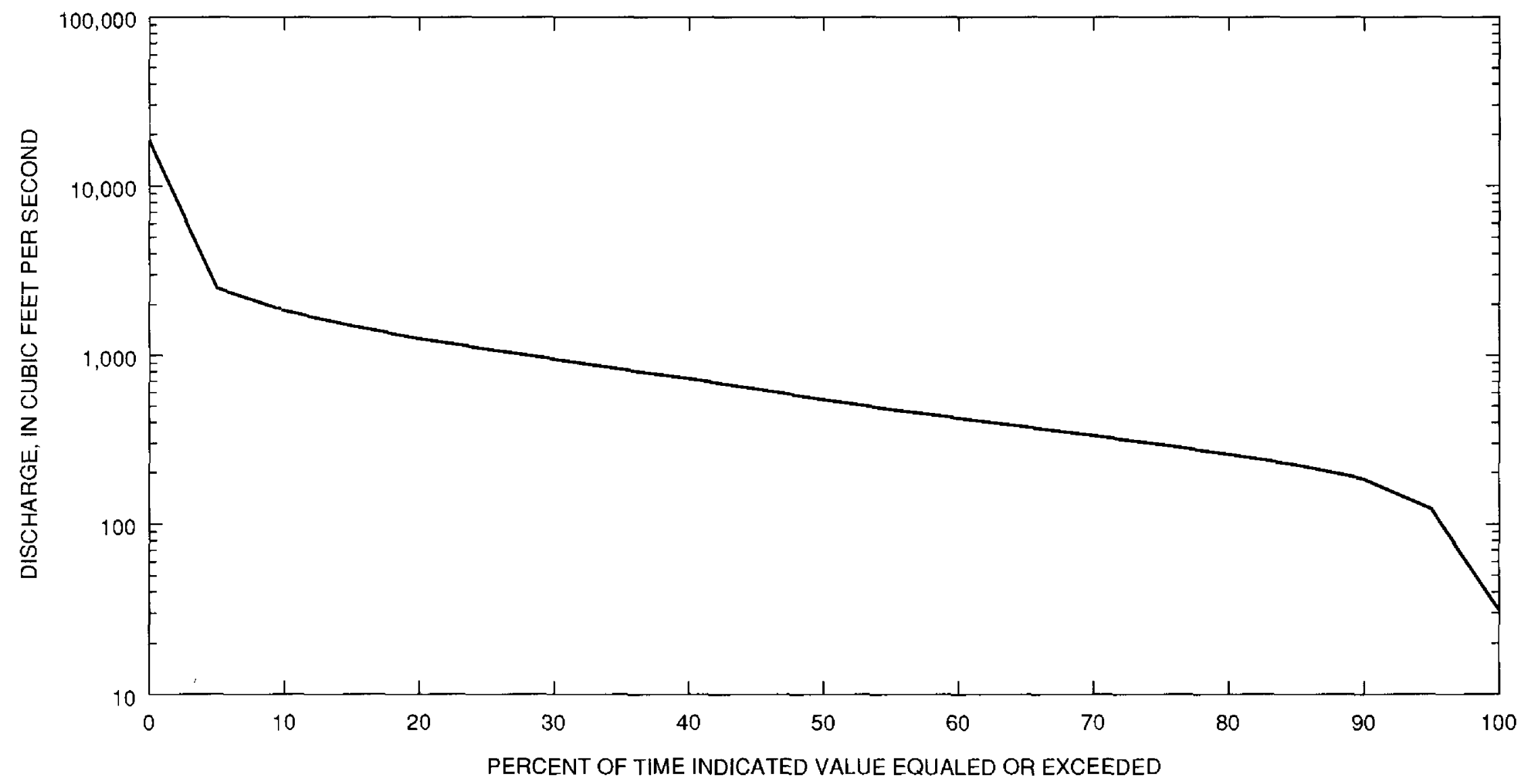


SUWANNEE RIVER BASIN

02322000 SANTA FE RIVER NEAR HIGH SPRINGS. FL--Continued

\section{SUMMARY OF MONTHLY MEAN ELEVATION AND DISCHARGE STATISTICS} FOR WATER YEARS 1975-1993

\begin{tabular}{lrrrrrrr} 
& \multicolumn{3}{c}{ ELEVATIONS, } & \multicolumn{3}{c}{ DISCHARGE, } \\
& \multicolumn{2}{c}{ FEET ABOVE SEA LEVEL } & \multicolumn{3}{c}{ CUBIC EEET FER SECOND } \\
\multicolumn{1}{c}{ MONTH } & MAXIMUM & MINIMUM & MEAN & MAXIMUM & MINIMUM & \multicolumn{1}{c}{ MEAN } \\
& & & & & & \\
OCTOBER & 31.27 & 27.73 & 29.22 & 3125 & 79.4 & 1114.60 \\
NOVEMBER & 29.46 & 27.08 & 28.30 & 2318 & 85.4 & 729.27 \\
DECEMBER & 30.83 & 26.99 & 28.22 & 1886 & 61.2 & 602.89 \\
JANUARY & 31.06 & 26.90 & 28.48 & 2480 & 52.8 & 692.96 \\
FEBRUARY & 32.58 & 26.91 & 28.89 & 3197 & 68.1 & 845.63 \\
MARCH & 33.51 & 26.89 & 29.44 & 4188 & 57.1 & 1050.94 \\
APRIL & 33.27 & 26.82 & 28.91 & 3776 & 38.9 & 898.67 \\
MAY & 31.67 & 26.82 & 29.51 & 2578 & 72.5 & 599.33 \\
JUNE & 32.59 & 26.92 & 28.44 & 3344 & 63.6 & 616.07 \\
JULY & 30.42 & 27.35 & 28.46 & 1743 & 150.0 & 653.55 \\
AUGUST & 31.07 & 27.28 & 29.17 & 2243 & 110.2 & 1021.53 \\
SEPTEMBER & 34.85 & 27.33 & 29.70 & 5481 & 102.0 & 1300.58
\end{tabular}

DURATION OF DAILY MEAN VALUES FOR WATER YEARS 1931-1993

PERCENT

OF TIME

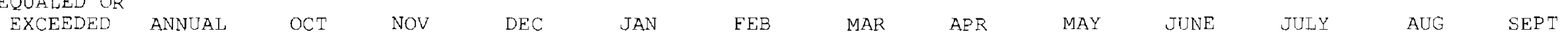
ELEVATION IN FEET ABOVE MEAN SEA LEVEL

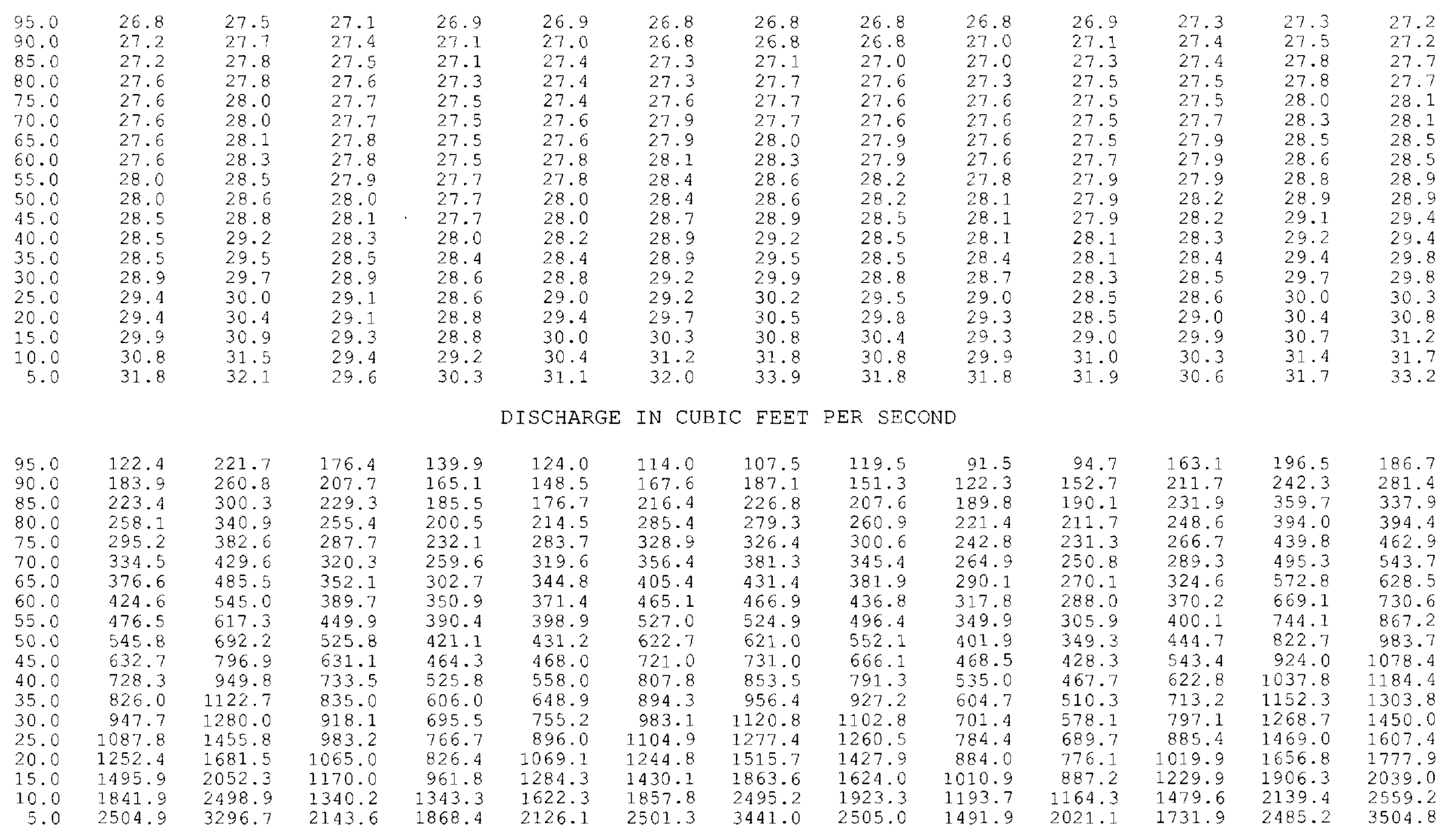

\section{LOWEST MEAN ELEVATION, IN FEET, AND RANKING FOR THE FOLLOWING NUMBER OF CONSECUTIVE DAYS FOR PERIOD APR TO MAR}

WATER YEAR
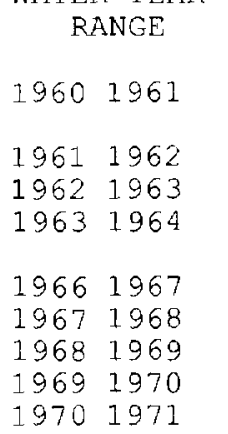

$\begin{array}{cc}1 & \\ 28.3 & 8 \\ 27.2 & 3 \\ 27.1 & 1 \\ 27.2 & 2 \\ 28.6 & 9 \\ 27.7 & 7 \\ 27.4 & 4 \\ 27.6 & 6 \\ 27.5 & 5\end{array}$

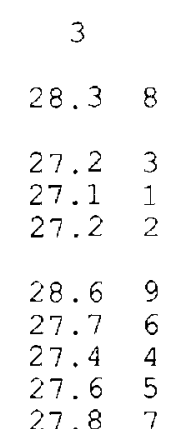

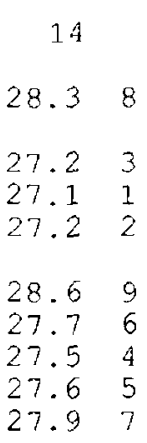

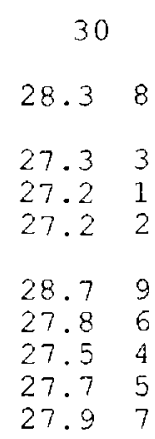

60

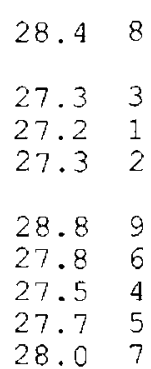

\begin{tabular}{cc}
\multicolumn{3}{c}{90} \\
28.6 & 8 \\
27.4 & 2 \\
27.2 & 1 \\
27.4 & 3 \\
28.8 & 9 \\
27.8 & 6 \\
27.6 & 4 \\
27.8 & 5 \\
28.0 & 7
\end{tabular}

120

$28.7 \quad 8$

27.52

$\begin{array}{ll}27.2 & 1 \\ 27.7 & 4\end{array}$

$29.1 \quad 9$

$\begin{array}{ll}27.8 & 5 \\ 27.6 & 3\end{array}$

27.96

27.96 

FOR PERIOD OCT TO SEP

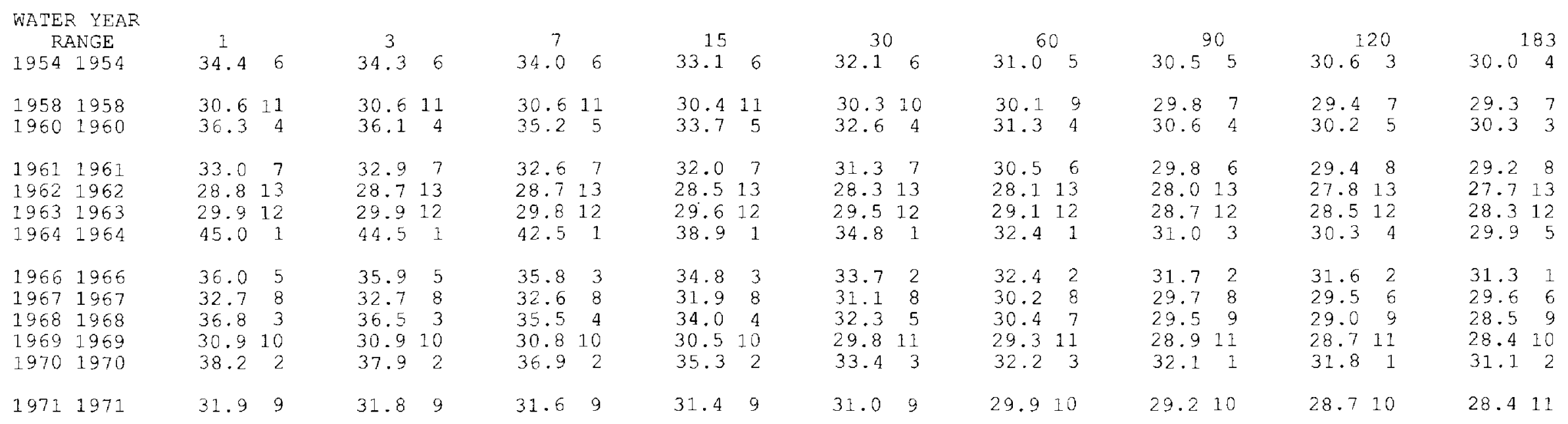

LOWEST MEAN DISCHARGE, IN CUBIC FEET PER SECOND, AND RANKING FOR THE FOLLOWING NUMBER OF CONSECUTIVE DAYS FOR PERIOD APR TO MAR

\begin{tabular}{|c|c|c|c|c|c|c|c|c|c|c|c|c|c|c|c|c|c|c|c|}
\hline & ANGE & 1 & & 3 & & 7 & & 14 & & 30 & & 60 & 0 & & 90 & & 20 & & 183 \\
\hline 1931 & 1932 & 105 & 6 & 110 & 6 & 112 & 6 & 113 & 6 & 117 & 6 & 125 & 6 & 135 & 5 & 154 & 6 & 188 & 4 \\
\hline 1932 & 1933 & 78.0 & 4 & 78.7 & 4 & 82.3 & 5 & 82.3 & 5 & 86.2 & 5 & 102 & 5 & 120 & 4 & 136 & 4 & 257 & 7 \\
\hline 1933 & 1934 & 172 & 14 & 174 & 14 & 177 & 13 & 179 & 13 & 183 & 13 & 189 & 11 & 197 & 9 & 208 & 9 & 283 & 9 \\
\hline 1934 & 1935 & 153 & 11 & 155 & 10 & 158 & 10 & 162 & 11 & 171 & 10 & 196 & 13 & 217 & 10 & 221 & 10 & 294 & 10 \\
\hline 1935 & 1936 & 79.0 & 5 & 79.0 & 5 & 79.9 & 4 & 80.6 & 4 & 83.6 & 4 & 90.1 & 3 & 102 & 3 & 126 & 3 & 821 & 30 \\
\hline 1936 & 1937 & 253 & 25 & 254 & 25 & 256 & 25 & 258 & 24 & 275 & 24 & 322 & 26 & 360 & 26 & 389 & 24 & 407 & 15 \\
\hline 1937 & 1938 & 332 & 31 & 335 & 31 & 338 & 31 & 344 & 31 & 370 & 32 & 429 & 32 & 510 & 33 & 564 & 30 & 926 & 33 \\
\hline 1938 & 1939 & 209 & 18 & 212 & 19 & 213 & 19 & 218 & 19 & 237 & 20 & 255 & 18 & 278 & 16 & 300 & 15 & 448 & 17 \\
\hline 1939 & 1940 & 211 & 19 & 211 & 18 & 212 & 18 & 214 & 18 & 217 & 16 & 228 & 17 & 297 & 19 & 319 & 18 & 422 & 16 \\
\hline 1940 & 1941 & 175 & 15 & 177 & 15 & 181 & 15 & 183 & 14 & 189 & 15 & 204 & 15 & 237 & 15 & 281 & 14 & 386 & 12 \\
\hline 1941 & 1942 & 191 & 17 & 194 & 17 & 196 & 17 & 202 & 17 & 223 & 18 & 261 & 19 & 335 & 22 & 571 & 31 & 697 & 26 \\
\hline 1942 & 1943 & 292 & 28 & 295 & 28 & 298 & 28 & 305 & 28 & 317 & 27 & 323 & 27 & 342 & 24 & 362 & 20 & 456 & 18 \\
\hline 1943 & 1944 & 125 & 7 & 128 & 7 & 129 & 7 & 130 & 7 & 132 & 7 & 137 & 7 & 140 & 6 & 143 & 5 & 183 & 3 \\
\hline 1944 & 1945 & 212 & 20 & 213 & 20 & 215 & 20 & 220 & 20 & 230 & 19 & 266 & 21 & 370 & 28 & 612 & 33 & 922 & 32 \\
\hline 1945 & 1946 & 161 & 12 & 164 & 12 & 167 & 12 & 170 & 12 & 182 & 12 & 194 & 12 & 221 & 11 & 270 & 13 & 618 & 24 \\
\hline 1946 & 1947 & 336 & 32 & 338 & 32 & 341 & 32 & 355 & 32 & 363 & 31 & 478 & 35 & 549 & 34 & 624 & 34 & 935 & 34 \\
\hline 1947 & 1948 & 321 & 30 & 323 & 30 & 325 & 30 & 332 & 30 & 355 & 30 & 403 & 30 & 412 & 30 & 461 & 28 & 785 & 28 \\
\hline 1948 & 1949 & 767 & 40 & 767 & 40 & 775 & 40 & 786 & 40 & 810 & 40 & 893 & 39 & 1026 & 40 & 1185 & 40 & 1393 & 39 \\
\hline 1949 & 1950 & 397 & 35 & 403 & 35 & 415 & 35 & 426 & 35 & 430 & 35 & 453 & 33 & 503 & 32 & 585 & 32 & 765 & 27 \\
\hline 1950 & 1951 & 272 & 27 & 273 & 27 & 274 & 27 & 275 & 25 & 281 & 25 & 298 & 24 & 313 & 21 & 408 & 26 & 803 & 29 \\
\hline 1951 & 1952 & 235 & 24 & 235 & 24 & 239 & 23 & 240 & 23 & 255 & 22 & 265 & 20 & 289 & 17 & 303 & 16 & 347 & 11 \\
\hline 1952 & 1953 & 180 & 16 & 181 & 16 & 183 & 16 & 184 & 15 & 188 & 14 & 198 & 14 & 233 & 14 & 247 & 11 & 252 & 6 \\
\hline 1953 & 1954 & 228 & 23 & 233 & 23 & 254 & 24 & 297 & 27 & 327 & 28 & 344 & 29 & 378 & 29 & 486 & 29 & 895 & 31 \\
\hline 1954 & 1955 & 127 & 8 & 128 & 8 & 129 & 8 & 132 & 8 & 136 & 8 & 146 & 8 & 162 & 7 & 186 & 7 & 215 & 5 \\
\hline 1955 & 1956 & 46.0 & 2 & 47.3 & 2 & 47.9 & 2 & 49.6 & 2 & 52.7 & 2 & 55.1 & 1 & 59.2 & 2 & 59.5 & 1 & 67.2 & 1 \\
\hline 1956 & 1957 & 31.0 & 1 & 31.0 & 1 & 31.3 & 1 & 33.9 & 1 & 36.6 & 1 & 55.5 & 2 & 58.3 & 1 & 90.0 & 2 & 96.2 & 2 \\
\hline 1957 & 1958 & 72.0 & 3 & 73.3 & 3 & 73.7 & 3 & 74.3 & 3 & 78.8 & 3 & 90.7 & 4 & 349 & 25 & 370 & 22 & 478 & 20 \\
\hline 1958 & 1959 & 259 & 26 & 261 & 26 & 270 & 26 & 281 & 26 & 309 & 26 & 319 & 25 & 339 & 23 & 376 & 23 & 574 & 22 \\
\hline 1959 & 1950 & 627 & 37 & 629 & 36 & 636 & 36 & 647 & 36 & 679 & 37 & 727 & 37 & 770 & 36 & 871 & 37 & 1115 & 35 \\
\hline 1960 & 1961 & 626 & 36 & 636 & 37 & 637 & 37 & 647 & 37 & 665 & 36 & 709 & 36 & 783 & 37 & 839 & 36 & 1115 & 36 \\
\hline 1961 & 1962 & 220 & 21 & 224 & 22 & 226 & 21 & 235 & 22 & 269 & 23 & 272 & 23 & 291 & 18 & 316 & 17 & 406 & 14 \\
\hline 1962 & 1963 & 152 & 10 & 157 & 11 & 159 & 11 & 162 & 10 & 164 & 9 & 175 & 9 & 187 & 8 & 208 & 8 & 278 & 8 \\
\hline 1963 & 1964 & 145 & 9 & 148 & 9 & 151 & 9 & 158 & 9 & 172 & 11 & 185 & 10 & 226 & 12 & 327 & 19 & 473 & 19 \\
\hline 1964 & 1965 & 381 & 34 & 383 & 34 & 385 & 34 & 389 & 34 & 403 & 34 & 468 & 34 & 662 & 35 & 746 & 35 & 1635 & 40 \\
\hline 1965 & 1966 & 731 & 39 & 733 & 39 & 738 & 39 & 750 & 39 & 794 & 39 & 924 & 40 & 1010 & 39 & 1128 & 39 & 1365 & 38 \\
\hline 1966 & 1967 & 664 & 38 & 674 & 38 & 680 & 38 & 689 & 38 & 717 & 38 & 760 & 38 & 792 & 38 & 923 & 38 & 1217 & 37 \\
\hline 1967 & 1968 & 305 & 29 & 308 & 29 & 310 & 29 & 316 & 29 & 330 & 29 & 340 & 28 & 364 & 27 & 363 & 21 & 394 & 13 \\
\hline 1968 & 1969 & 168 & 13 & 171 & 13 & 180 & 14 & 192 & 16 & 217 & 17 & 219 & 16 & 231 & 13 & 251 & 12 & 682 & 25 \\
\hline 1969 & 1970 & 221 & 22 & 222 & 21 & 228 & 22 & 235 & 21 & 241 & 21 & 266 & 22 & 311 & 20 & 389 & 25 & 577 & 23 \\
\hline 1970 & 1971 & 350 & 33 & 355 & 33 & 361 & 33 & 372 & 33 & 399 & 33 & 415 & 31 & 439 & 31 & 436 & 27 & 525 & 21 \\
\hline
\end{tabular}


SUWANNEE RIVER BASIN

02322000 SANTA FE RIVER NEAR HIGH SPRINGS. FL--Continued

HIGHEST MEAN DISCHARGE, IN CUBIC FEET PER SECOND, AND RANKING FOR THE FOLLOWING NUMBER OF CONSECUTIVE DAYS FOR PERIOD OCT TO SEP

WATER YEAR

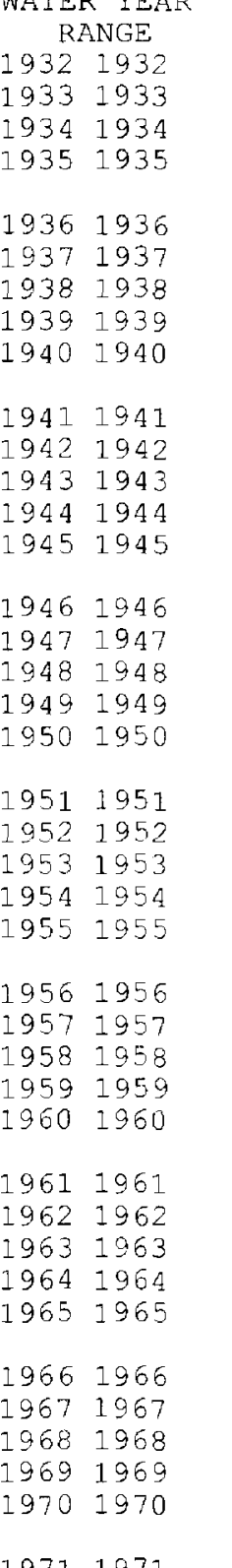

\begin{tabular}{rrrrrr}
1 & \multicolumn{3}{c}{3} & \multicolumn{3}{r}{} \\
1230 & 35 & 1170 & 36 & 1065 & 37 \\
2760 & 28 & 2720 & 28 & 2520 & 27 \\
11500 & 3 & 11030 & 3 & 8783 & 3 \\
6720 & 12 & 6400 & 12 & 5521 & 11 \\
& & & & & \\
2940 & 26 & 2760 & 26 & 2426 & 28 \\
3330 & 22 & 3177 & 22 & 2827 & 25 \\
4610 & 16 & 4470 & 16 & 4031 & 16 \\
4280 & 17 & 4047 & 17 & 3510 & 20 \\
1470 & 33 & 1420 & 33 & 1323 & 34 \\
& & & & & \\
2400 & 30 & 2380 & 30 & 2211 & 30 \\
7840 & 7 & 7360 & 8 & 5903 & 7 \\
1150 & 37 & 1140 & 37 & 1095 & 36 \\
2820 & 27 & 2760 & 27 & 2683 & 26 \\
9170 & 4 & 8807 & 4 & 7053 & 4 \\
3140 & 23 & 3107 & 23 & 2969 & 21 \\
8680 & 5 & 7720 & 6 & 5246 & 12 \\
12700 & 2 & 12200 & 2 & 9736 & 2 \\
5080 & 15 & 4863 & 15 & 4347 & 15 \\
7600 & 9 & 7200 & 9 & 5857 & 8 \\
7170 & 10 & 6777 & 10 & 5713 & 9 \\
1220 & 36 & 1213 & 35 & 1194 & 35 \\
3790 & 20 & 3733 & 20 & 3541 & 19 \\
4030 & 18 & 3943 & 18 & 3701 & 17 \\
608 & 39 & 600 & 39 & 573 & 39 \\
294 & 40 & 292 & 40 & 278 & 40 \\
3490 & 21 & 3377 & 21 & 2951 & 22 \\
1750 & 32 & 1740 & 32 & 1714 & 32 \\
7700 & 8 & 7423 & 7 & 6754 & 6 \\
5680 & 13 & 5490 & 14 & 4757 & 14 \\
3140 & 24 & 3103 & 24 & 2924 & 23 \\
890 & 38 & 873 & 38 & 828 & 38 \\
1400 & 34 & 1390 & 34 & 1340 & 33 \\
19600 & 1 & 17770 & 1 & 13960 & 1 \\
3960 & 19 & 3907 & 19 & 3651 & 18 \\
5640 & 14 & 5567 & 13 & 5234 & 13 \\
3000 & 25 & 2983 & 25 & 2899 & 24 \\
6930 & 11 & 6560 & 11 & 5570 & 10 \\
2050 & 31 & 2013 & 31 & 1944 & 31 \\
8570 & 6 & 8083 & 5 & 6926 & 5 \\
2560 & 29 & 2513 & 29 & 2376 & 29
\end{tabular}

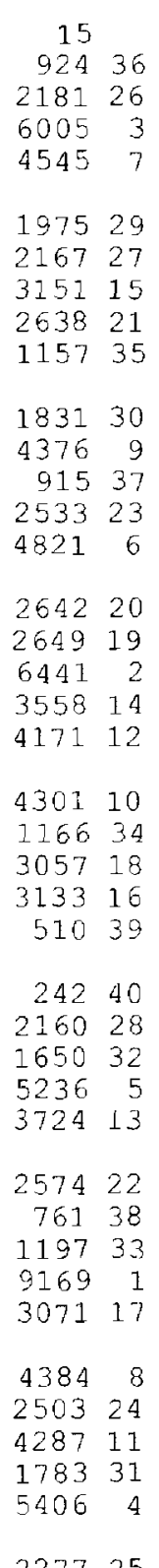

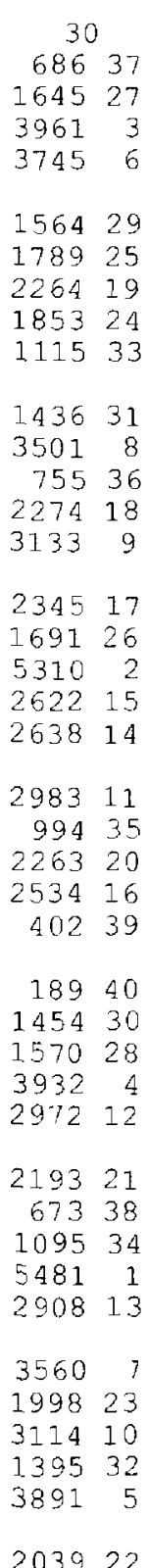

203922

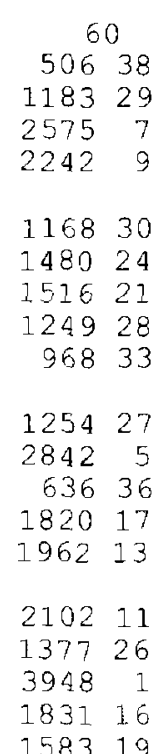

158319

$2101 \quad 12$

78035

152920

191315

30239

14940

106732

150023

$2964 \quad 3$

221510

$1763 \quad 18$

$578 \quad 37$

90534

34512

24738

$2778 \quad 6$

150722

191714

1126
2952

144225

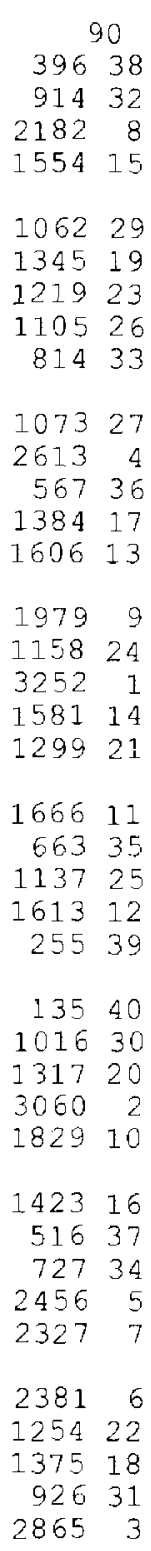

$2865 \quad 3$

106828

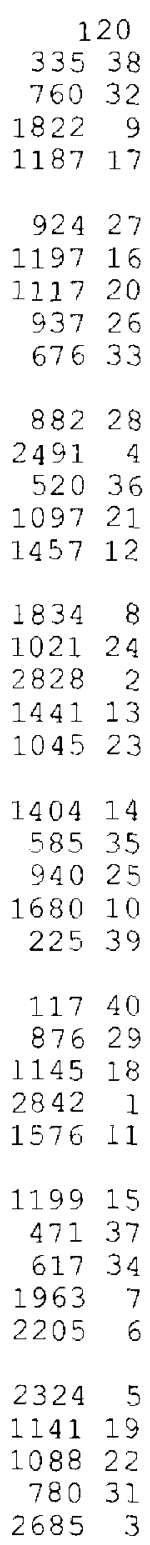

86130 $\begin{array}{rr} & 183 \\ 257 & 38 \\ 788 & 27 \\ 1267 & 12 \\ 821 & 24 \\ & \\ 905 & 22 \\ 935 & 21 \\ 967 & 19 \\ 696 & 30 \\ 585 & 33 \\ & \\ 702 & 28 \\ 2469 & 2 \\ 453 & 36 \\ 1006 & 18 \\ 1157 & 14 \\ & \\ 1457 & 9 \\ 965 & 20 \\ 2741 & 1 \\ 1392 & 10 \\ 803 & 25 \\ & \\ 1098 & 16 \\ 556 & 34 \\ 895 & 23 \\ 1361 & 11 \\ 234 & 39 \\ 96.2 & 40 \\ 606 & 32 \\ 1079 & 17 \\ 2345 & 3 \\ 1632 & 8 \\ 1211 & 15 \\ 403 & 37 \\ 524 & 35 \\ 1635 & 7 \\ 1946 & 6 \\ & \\ 2145 & 5 \\ 1221 & 13 \\ 800 & 26 \\ 702 & 29 \\ 2211 & 4 \\ & \end{array}$

68831 
THIS PAGE INTENTIONALLY BLANK 


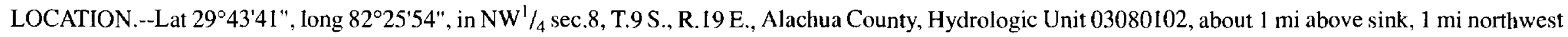
of University of Florida Agricultural Experiment Station, $6.0 \mathrm{mi}$ southeast of Alachua and $7.6 \mathrm{mi}$ northwest of Gainesville.

DRAINAGE AREA.--5.12 $\mathrm{mi}^{2}$, of which $2.5 \mathrm{mi}^{2}$ are noncontributing at low and medium discharge.

PERIOD OF RECORD.--June 1984 to 1993.

REVISED RECORDS.--WDR FL-88-4: 1985, 1987(M), WDR FL-92-4: Drainage Area.

GAGE.--Water-stage recorder. Elevation of the gage is $105 \mathrm{ft}$ above National Geodetic Vertical Datum of 1929, from topographic map.

REMARKS.--Records fair except for estimated daily discharges which are poor.
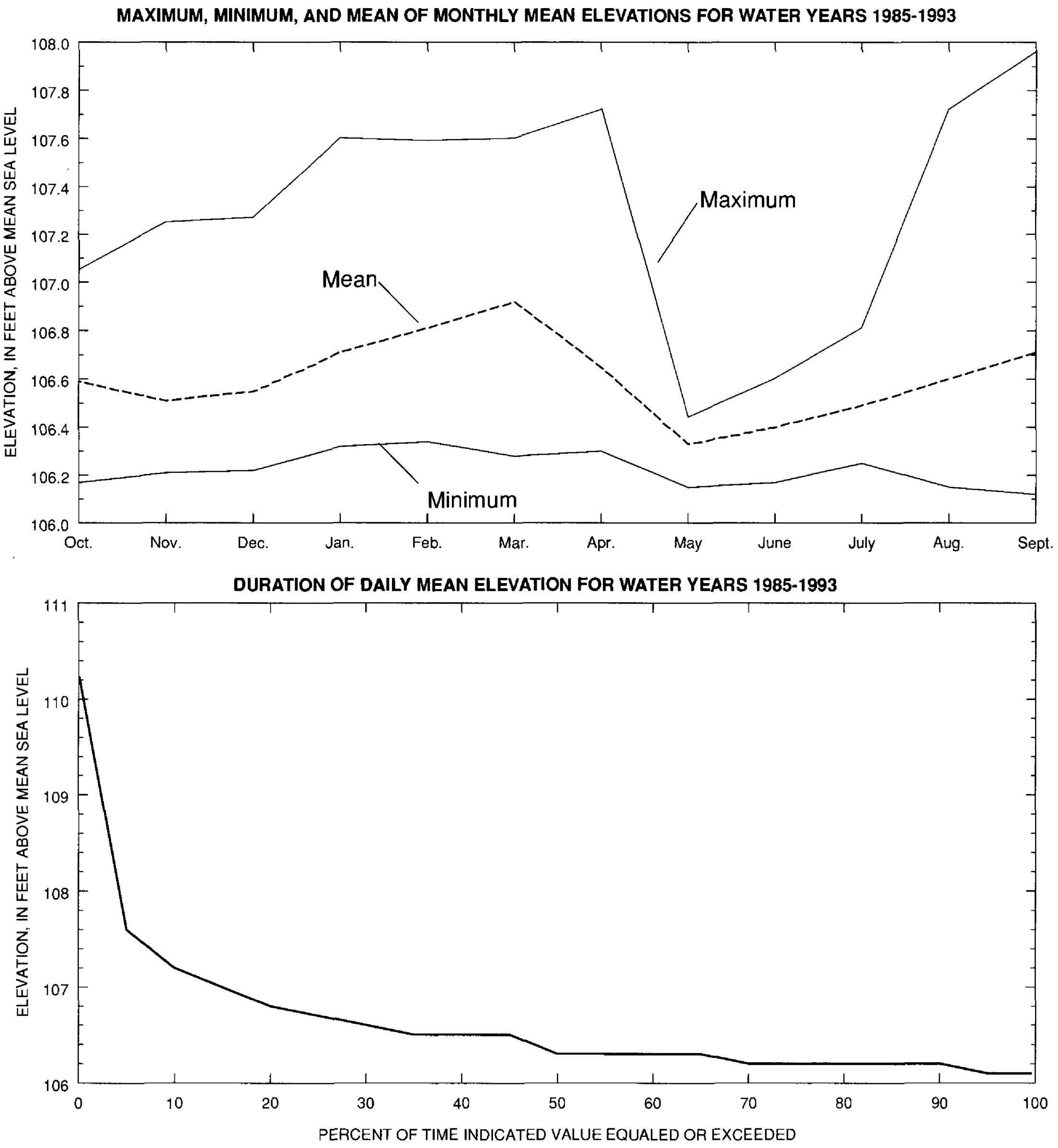
SUMMARY STATISTICS, IN CUBIC FEET PER SECOND UNLESS OTHERWISE INDICATED, FOR WATER YEARS $1985-1993$

ANNUAL MEAN

HIGHEST ANNUAL MEAN

LOWEST ANNUAL MEAN

HIGHEST DAILY MEAN

LOWEST DAILY MEAN

ANNUAL SEVEN $\rightarrow$ DAY MINIMUM

INSTANTANEOUS PEAK FLOW

INSTANTANEOUS PEAK FTEVATTON (FT)

INSTANTANEOUS LOW FLOW

ANNUAL RUNOFE (INCHES/CFSM)

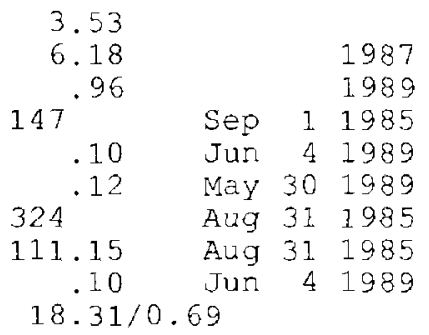

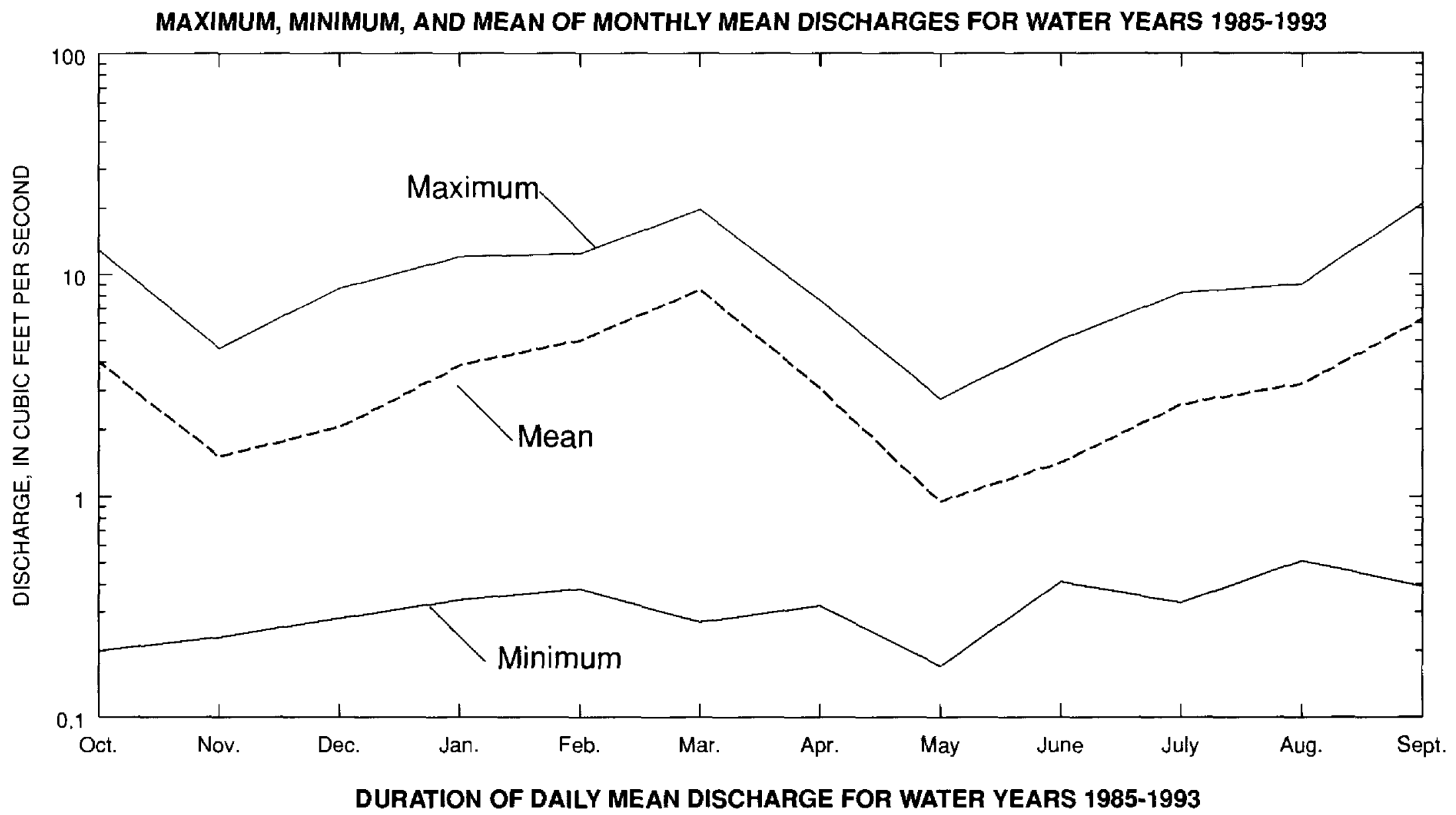

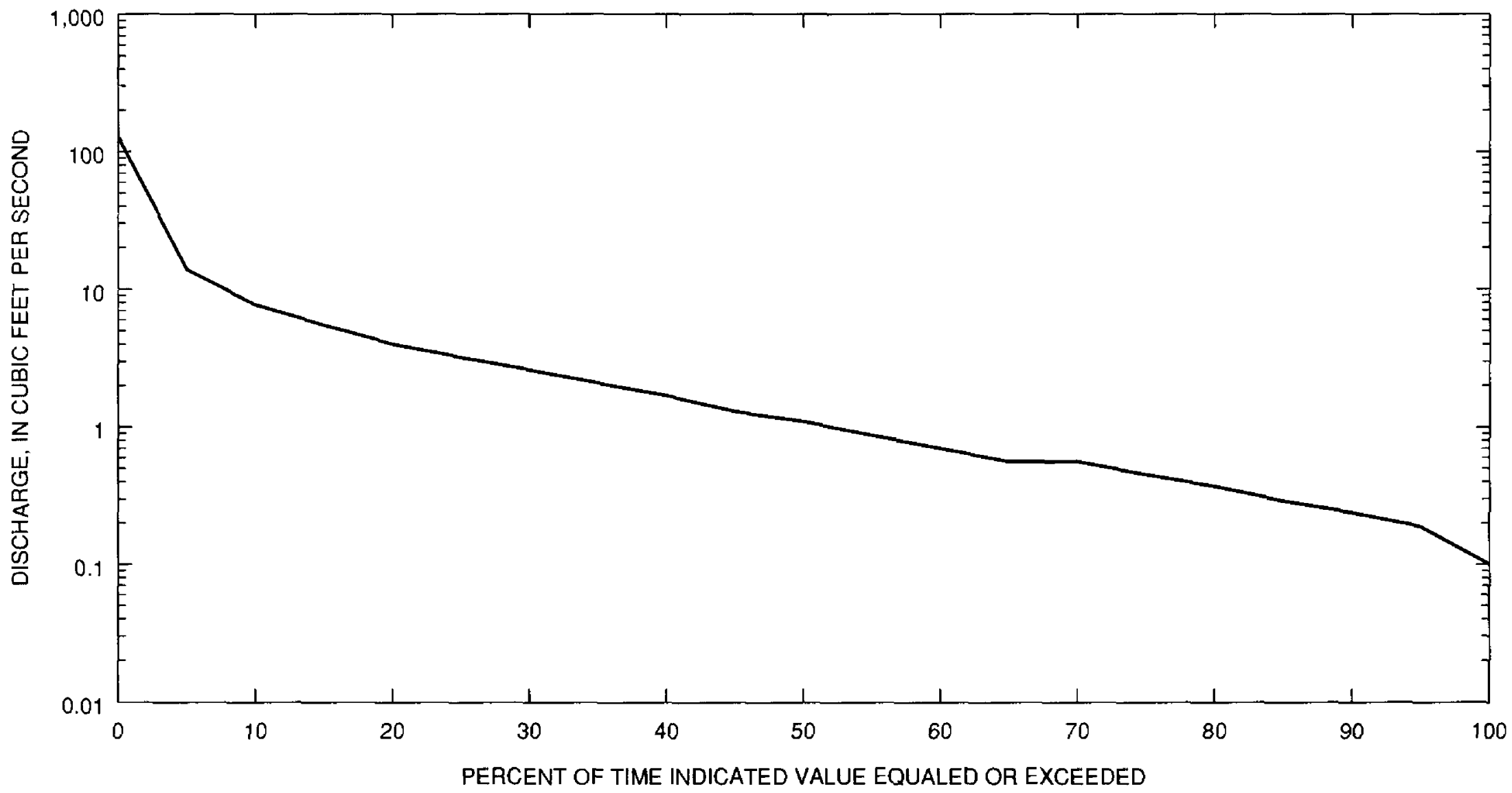


SUWANNEE RIVER BASIN

02322016 BLUES CREEK NEAR GAINESVILLE, FL--Continued

SUMMARY OF MONTHLY MEAN ELEVATION AND DISCHARGE STATISTICS FOR WATER YEARS 1985-1993

\begin{tabular}{lcccccc} 
& \multicolumn{3}{c}{ ELEVATIONS, } & \multicolumn{3}{c}{ DISCHARGE, } \\
& \multicolumn{3}{c}{ FEET ABOVE SEA LEVEL } & CUBIC FEET FER SECOND \\
MONTH & MAXIMUM & MINIMUM & MEAN & MAXIMUM & MINIMUM & MEAN \\
OCTOBER & 107.05 & 106.17 & 106.59 & 12.8 & .20 & 4.01 \\
NOVEMBER & 107.25 & 106.21 & 106.51 & 4.57 & .23 & 1.51 \\
DECEMBER & 107.27 & 106.22 & 106.55 & 8.56 & .28 & 2.05 \\
JANUARY & 107.60 & 106.32 & 106.71 & 11.9 & .34 & 3.88 \\
EEBRUARY & 107.59 & 106.34 & 106.81 & 12.3 & .38 & 5.00 \\
MARCH & 107.60 & 106.28 & 106.92 & 19.5 & .27 & 8.52 \\
APRIL & 107.72 & 106.30 & 106.65 & 7.52 & .32 & 3.06 \\
MAY & 106.44 & 106.15 & 106.33 & 2.71 & .17 & .95 \\
JUNE & 106.60 & 106.17 & 106.40 & 5.00 & .41 & 1.42 \\
JULY & 106.81 & 106.25 & 106.49 & 8.17 & .33 & 2.59 \\
AUGUST & 107.72 & 106.15 & 106.60 & 8.97 & .51 & 3.20 \\
SEFTEMBER & 107.96 & 106.12 & 106.71 & 20.7 & .39 & 6.25
\end{tabular}

DURATION OF DAILY MEAN VALUES FOR WATER YEARS 1985-1993

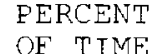

ELEVATION IN FEET ABOVE MEAN SEA LEVEL

$\begin{array}{lllll}95.0 & 106.1 & 106.2 & 106.2 & 106.2 \\ 90.0 & 106.2 & 106.2 & 106.2 & 106.2 \\ 85.0 & 106.2 & 106.2 & 106.2 & 106.2 \\ 80.0 & 106.2 & 106.2 & 106.2 & 106.2 \\ 75.0 & 106.2 & 106.3 & 106.3 & 106.3 \\ 70.0 & 106.2 & 106.4 & 106.3 & 106.3 \\ 65.0 & 106.3 & 106.4 & 106.3 & 106.3 \\ 60.0 & 106.3 & 106.4 & 106.3 & 106.4 \\ 55.0 & 106.3 & 106.4 & 106.3 & 106.5 \\ 50.0 & 106.3 & 106.4 & 106.3 & 106.5 \\ 45.0 & 106.5 & 106.5 & 106.4 & 106.5 \\ 40.0 & 106.5 & 106.5 & 106.4 & 106.6 \\ 35.0 & 106.5 & 106.6 & 106.5 & 106.6 \\ 30.0 & 106.6 & 106.7 & 106.6 & 106.6 \\ 25.0 & 106.7 & 106.9 & 106.6 & 106.7 \\ 20.0 & 106.8 & 106.9 & 106.7 & 106.8 \\ 15.0 & 107.0 & 107.0 & 106.7 & 106.8 \\ 10.0 & 107.2 & 107.1 & 107.0 & 107.1 \\ 5.0 & 107.6 & 107.6 & 107.1 & 107.2\end{array}$

$\begin{array}{lll}106.2 & 106.3 & 106.2 \\ 106.3 & 106.3 & 106.2 \\ 106.3 & 106.3 & 106.2 \\ 106.3 & 106.3 & 106.4 \\ 106.3 & 106.3 & 106.4 \\ 106.3 & 106.4 & 106.4 \\ 106.4 & 106.4 & 106.5 \\ 106.4 & 106.5 & 106.6 \\ 106.5 & 106.5 & 106.7 \\ 106.5 & 106.6 & 106.8 \\ 106.5 & 106.6 & 106.9 \\ 106.5 & 106.7 & 106.9 \\ 106.7 & 106.8 & 107.0 \\ 106.7 & 106.9 & 107.1 \\ 106.9 & 107.1 & 107.3 \\ 107.2 & 107.2 & 107.4 \\ 107.3 & 107.4 & 107.6 \\ 107.5 & 107.6 & 107.8 \\ 107.9 & 107.9 & 108.3\end{array}$

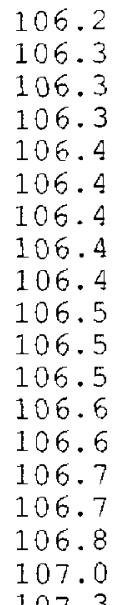

106.2
106.2
106.2
106.3
106.3
106.3
106.3
106.3
106.3
106.3
106.3
106.4
106.4
106.4
106.4
106.4
106.4
106.5
106.5

106.1
106.2
106.2
106.2
106.3
106.3
106.3
106.3
106.3
106.3
106.3
106.4
106.4
106.5
106.5
106.5
106.6
106.7
106.9
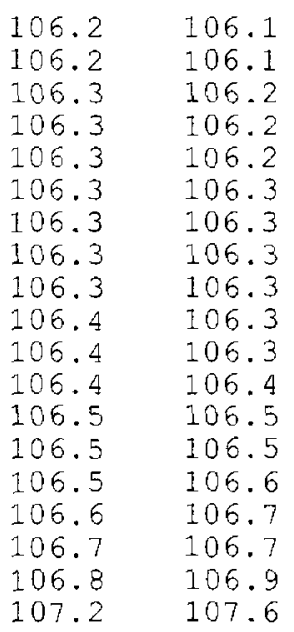

106.1

DISCHARGE IN CUBIC FEET PER SECOND

$\begin{array}{rrrrr}95.0 & 0.1 & 0.1 & 0.2 & 0.2 \\ 90.0 & 0.2 & 0.1 & 0.2 & 0.3 \\ 85.0 & 0.2 & 0.1 & 0.3 & 0.4 \\ 80.0 & 0.3 & 0.2 & 0.4 & 0.5 \\ 75.0 & 0.4 & 0.3 & 0.4 & 0.5 \\ 70.0 & 0.5 & 0.5 & 0.5 & 0.5 \\ 65.0 & 0.5 & 0.6 & 0.5 & 0.9 \\ 60.0 & 0.7 & 0.9 & 0.5 & 1.1 \\ 55.0 & 0.8 & 1.1 & 0.6 & 1.2 \\ 50.0 & 1.1 & 1.4 & 0.9 & 1.2 \\ 45.0 & 1.3 & 1.7 & 1.0 & 1.4 \\ 40.0 & 1.7 & 2.0 & 1.2 & 1.6 \\ 35.0 & 2.1 & 2.4 & 1.4 & 1.6 \\ 30.0 & 2.6 & 2.4 & 1.6 & 1.6 \\ 25.0 & 3.2 & 2.9 & 1.9 & 1.8 \\ 20.0 & 4.0 & 3.6 & 2.1 & 2.0 \\ 15.0 & 5.5 & 4.3 & 2.1 & 2.9 \\ 10.0 & 7.7 & 7.0 & 2.5 & 5.5 \\ 5.0 & 13.8 & 20.4 & 3.8 & 7.1\end{array}$

$\begin{array}{rr}0.3 & 0.3 \\ 0.3 & 0.4 \\ 0.4 & 0.5 \\ 0.5 & 0.5 \\ 0.7 & 0.7 \\ 0.8 & 1.2 \\ 1.1 & 1.8 \\ 1.3 & 2.5 \\ 1.5 & 2.8 \\ 1.9 & 2.8 \\ 2.2 & 3.3 \\ 2.6 & 3.8 \\ 2.9 & 4.3 \\ 3.9 & 5.0 \\ 4.5 & 5.8 \\ 5.9 & 7.0 \\ 7.5 & 8.6 \\ 9.7 & 12.9 \\ 14.5 & 19.3\end{array}$

$\begin{array}{rr}0.3 & 0.2 \\ 0.4 & 0.2 \\ 0.5 & 0.4 \\ 0.5 & 0.6 \\ 0.7 & 0.8 \\ 1.2 & 1.4 \\ 1.8 & 2.5 \\ 2.5 & 3.0 \\ 2.8 & 3.6 \\ 2.8 & 4.3 \\ 3.3 & 4.3 \\ 3.8 & 5.9 \\ 4.3 & 6.8 \\ 5.0 & 8.1 \\ 5.8 & 9.9 \\ 7.0 & 12.6 \\ 8.6 & 16.8 \\ 12.9 & 21.5 \\ 19.3 & 31.1\end{array}$

$\begin{array}{ll}0.2 & 0.2 \\ 0.2 & 0.2 \\ 0.4 & 0.4 \\ 0.6 & 0.5 \\ 0.8 & 0.7 \\ 1.4 & 0.8 \\ 2.5 & 1.1 \\ 3.0 & 1.4 \\ 3.6 & 1.6 \\ 4.3 & 1.6 \\ 4.3 & 1.9 \\ 5.9 & 2.2 \\ 6.8 & 2.2 \\ 8.1 & 2.7 \\ 9.9 & 3.1 \\ 2.6 & 3.7 \\ 16.8 & 4.4 \\ 21.5 & 6.2 \\ 31.1 & 10.3\end{array}$

$$
\begin{aligned}
& 0.1 \\
& 0.1 \\
& 0.2 \\
& 0.2 \\
& 0.3 \\
& 0.3 \\
& 0.3 \\
& 0.3 \\
& 0.4 \\
& 0.4 \\
& 0.5 \\
& 0.6 \\
& 0.7 \\
& 1.1 \\
& 1.2 \\
& 1.4 \\
& 1.7 \\
& 2.4 \\
& 3.1
\end{aligned}
$$

$\begin{array}{lr}0.1 & 0.2 \\ 0.1 & 0.2 \\ 0.2 & 0.3 \\ 0.2 & 0.3 \\ 0.3 & 0.3 \\ 0.3 & 0.4 \\ 0.3 & 0.4 \\ 0.4 & 0.5 \\ 0.4 & 0.6 \\ 0.5 & 0.7 \\ 0.5 & 0.8 \\ 0.6 & 1.1 \\ 0.7 & 1.1 \\ 0.8 & 1.5 \\ 1.2 & 1.7 \\ 1.6 & 2.3 \\ 1.9 & 3.6 \\ 2.9 & 5.4 \\ 4.7 & 12.2\end{array}$


LOWEST MEAN ELEVATION, IN FEET, AND RANKING FOR THE FOLLOWING NUMBER OF CONSECUTIVE DAYS FOR PERIOD APR TO MAR

WATER YEAR

RANGE

19861987

19881989

19901991

19911992

$\begin{array}{cccc}1 & & 3 & \\ 106 & 4 & 106 & 4 \\ 106 & 3 & 106 & 3 \\ 106 & 2 & 106 & 1 \\ 106 & 1 & 106 & 2\end{array}$

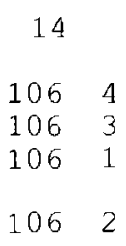

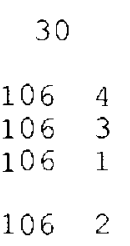

60

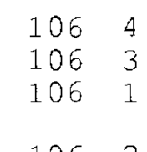

90

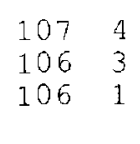

1062

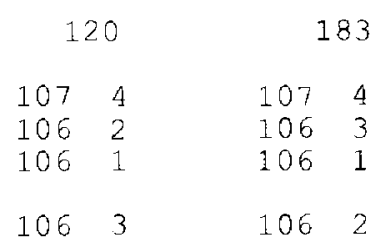

HIGHEST MEAN ELEVATION, IN FEET, AND RANKING FOR THE FOLLOWING NUMBER OF CONSECUTIVE DAYS FOR PERIOD OCT TO SEP

WATER YEAR

$$
\text { RANGE }
$$

19861986

19881988

19901990

$1991 \quad 1991$

$\begin{array}{cccc}1 & & 3 & \\ 109 & 3 & 109 & 2 \\ 110 & 1 & 110 & 1 \\ 108 & 4 & 108 & 4 \\ 109 & 2 & 108 & 3\end{array}$

$\begin{array}{cccc}7 & & 15 & \\ 108 & 2 & 108 & 2 \\ 109 & 1 & 108 & 1 \\ 107 & 4 & 107 & 4 \\ 108 & 3 & 107 & 3\end{array}$

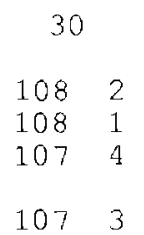

1073

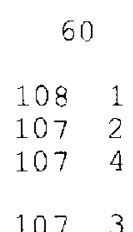

1073

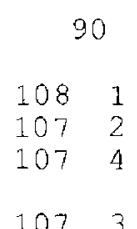

1073

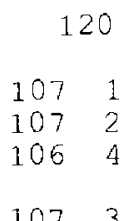

1073 $\begin{array}{rr}183 \\ 07 & 1 \\ 07 & 2 \\ 06 & 4 \\ & \\ 07 & 3\end{array}$

LOWEST MEAN DISCHARGE, IN CUBIC FEET PER SECOND, AND RANKING FOR THE FOLLOWING NUMBER OF CONSECUTIVE DAYS FOR PERIOD APR TO MAR

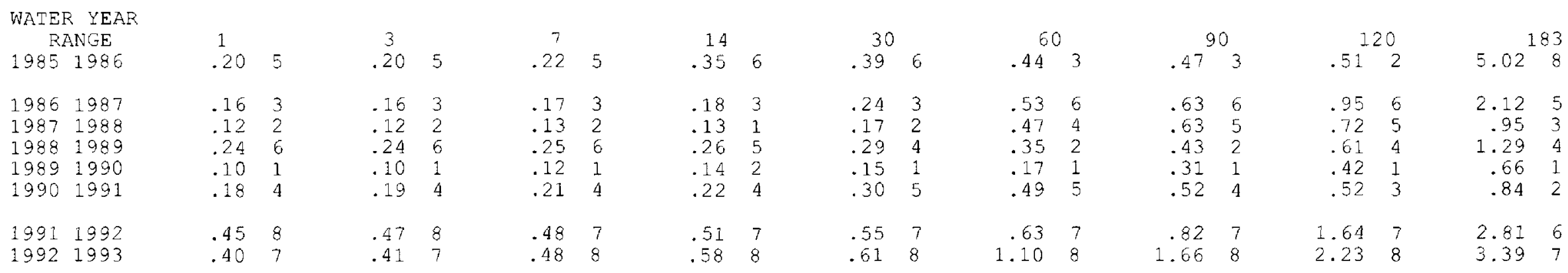

HIGHEST MEAN DISCHARGE, IN CUBIC FEET PER SECOND, AND RANKING FOR THE FOLLOWING NUMBER OF CONSECUTIVE DAYS FOR PERIOD OCT TO SEP
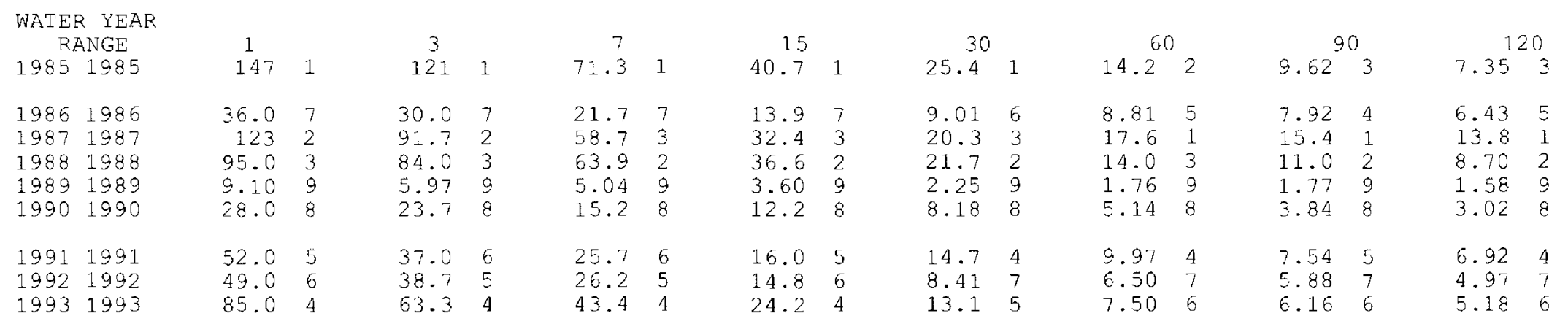

$\begin{array}{lr}5.02 & 183 \\ 5.46 & 5 \\ 10.9 & 1 \\ 6.13 & 3 \\ 1.26 & 9 \\ 2.55 & 8 \\ 6.87 & 2 \\ 3.92 & 7 \\ 5.60 & 4\end{array}$ 


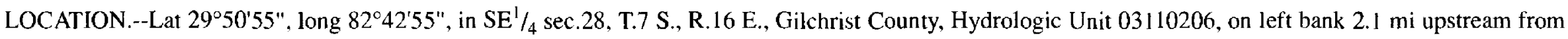
bridge on State Highway 47, $5.1 \mathrm{mi}$ south of Fort White, and $18 \mathrm{mi}$ upstream from mouth.

DRAINAGE AREA.--1,017 $\mathrm{mi}^{2}$.

PERIOD OF RECORD.--October 1927 to January 1930, June 1932 to September 1993.

REVISED RECORDS.--WDR FL-75-1: Drainage area.

GAGE.--Water-stage recorder. Datum of gage is $20.86 \mathrm{ft}$ above National Geodetic Vertical Datum of 1929 (levels by Corps of Engineers). Prior to June 3, 1932 , nonrecording gage at several sites within $200 \mathrm{ft}$ of present site at various datums. Oct. 1, 1947 to Feb. 10, 1949, auxiliary nonrecording gage and since Feb. 11, 1949, auxiliary water-stage recorder at bridge on U.S. Highway 129, 16 mi downstream from base gage at datum $3.5 \mathrm{ft}$ above National Geodetic Vertical Datum of 1929.

REMARKS.--Records fair, except for estimated daily discharges, which are poor.

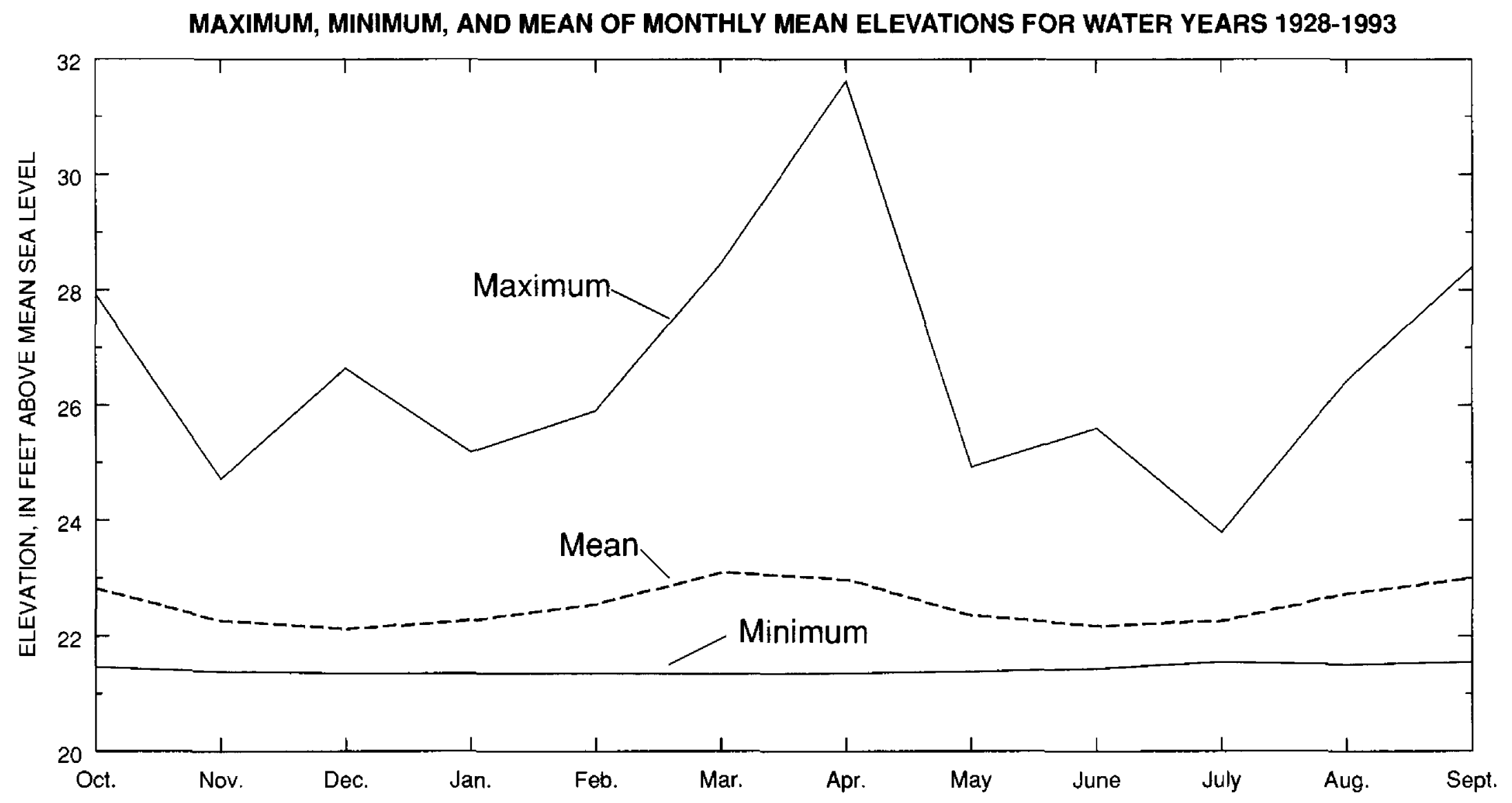

DURATION OF DAILY MEAN ELEVATION FOR WATER YEARS 1928-1993

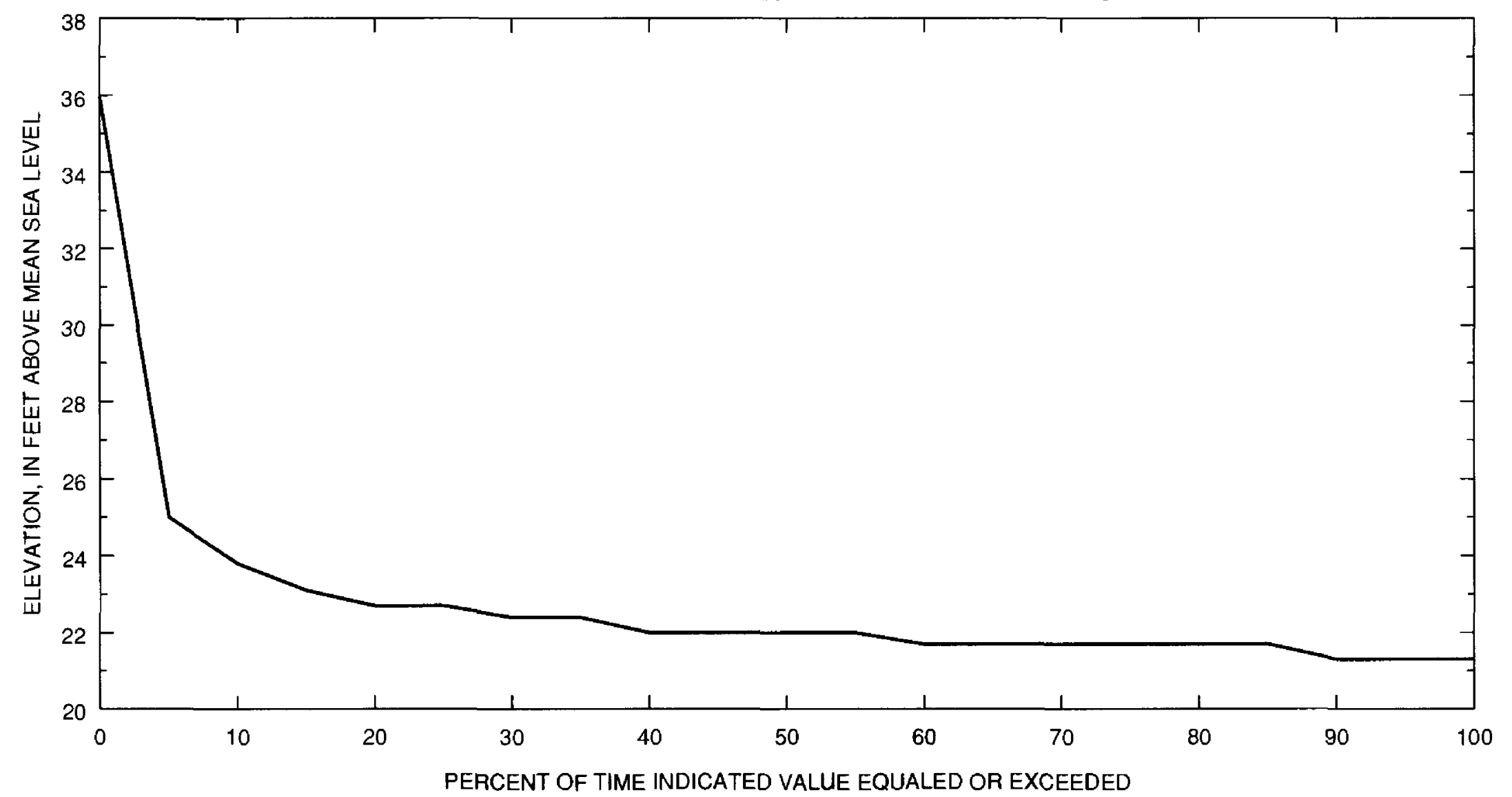


SUMMARY STATISTICS, IN CUBIC FEET PER SECOND UNLESS OTHERWISE INDICATED, FOR WATER YEARS $1928-1993$

\begin{tabular}{|c|c|c|c|}
\hline ANNUAL MEAN & 1602 & & \\
\hline HIGHEST ANNUAL MEAN & 3112 & & 19 \\
\hline LOWEST ANNUAL MEAN & 724 & & \\
\hline HIGHEST DAILY MEAN & 6900 & Sep 16 & \\
\hline T DAILY MEAN & 6 & $\operatorname{Jan} 7$ & \\
\hline$-D A$ & & Jan & \\
\hline ANTANEOUS PEAK FLOW & 17000 & Sep 16 & \\
\hline JTANEOUS PEAK ELEVATIOI & $(\mathrm{FT})$ & Sep 16 & \\
\hline NSTANTANEOUS LOW FLOW & 508 & $\operatorname{Jan}$ & 1 \\
\hline NNUAL RUNOFF (INCHES/CFSM) & 21. & & \\
\hline
\end{tabular}

MAXIMUM, MINIMUM, AND MEAN OF MONTHLY MEAN DISCHARGES FOR WATER YEARS 1928-1993

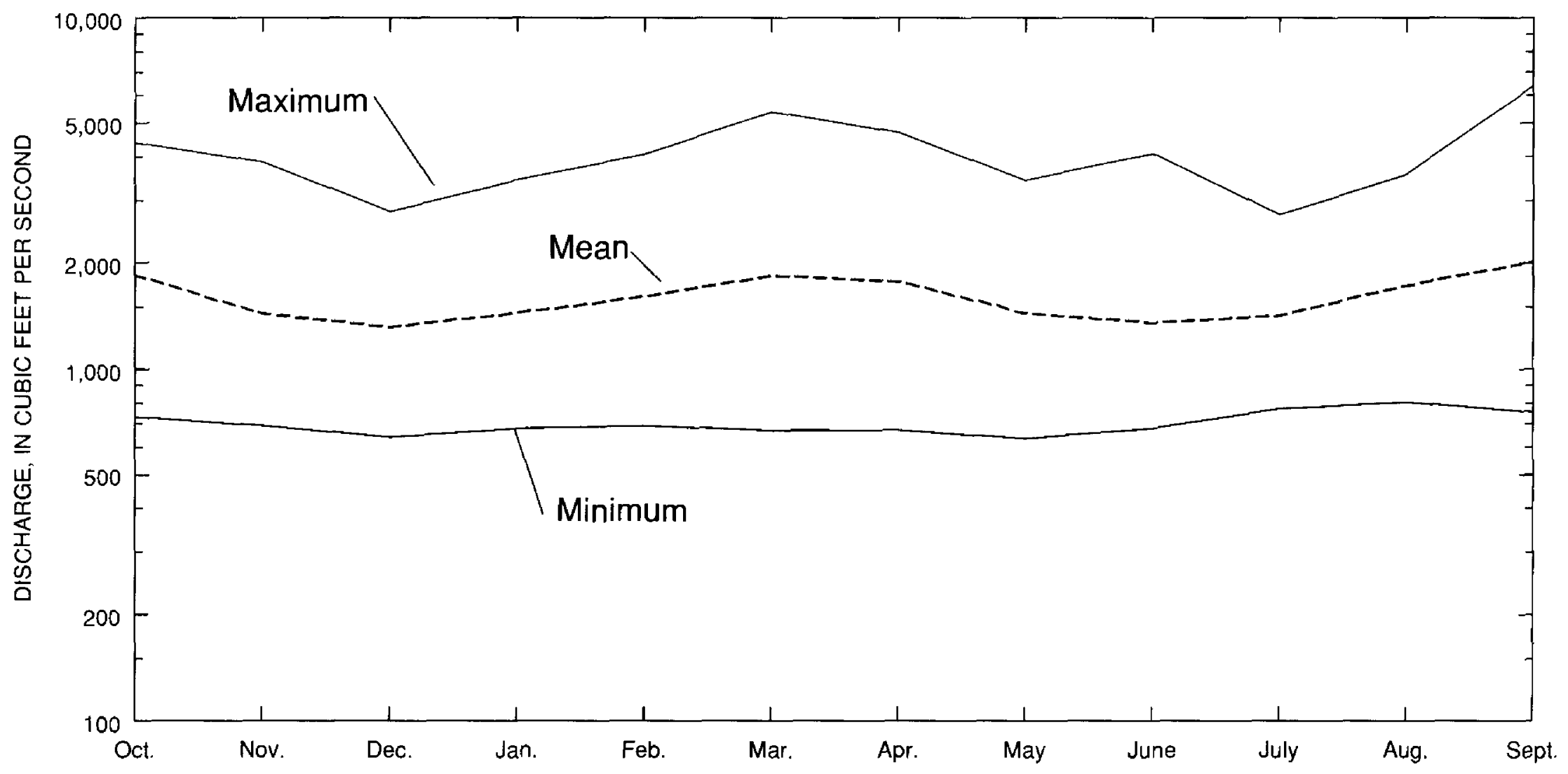

DURATION OF DAILY MEAN DISCHARGE FOR WATER YEARS 1928-1993

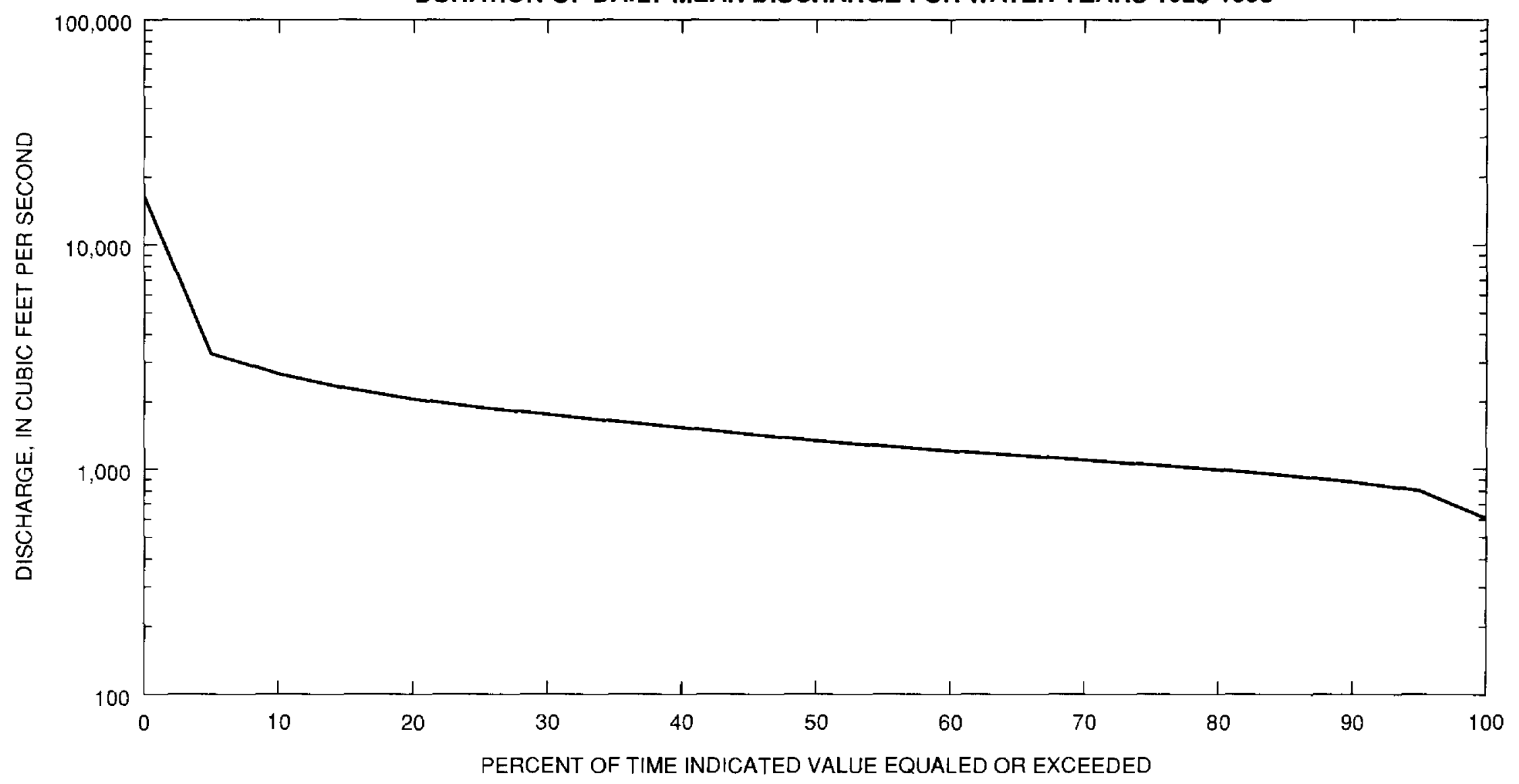


SUWANNEE RIVER BASIN

02322500 SANTA FE RIVER NEAR FORT WHITE, FL--Continued

SUMMARY OF MONTHLY MEAN ELEVATION AND DISCHARGE STATISTICS FOR WATER YEARS 1928-1993

\begin{tabular}{lcccccc}
\multicolumn{3}{c}{ ELEVATION, } & \multicolumn{3}{c}{ DISCHARGE, } \\
& EEET ABOVE SEA LEVEL & & CUBIC FEET PER SECOND \\
\multicolumn{1}{c}{ MONTH } & MAXIMUM & MINIMUM & MEAN & MAXIMUM & MINIMUM & MEAN \\
OCTOBER & 27.89 & 21.46 & 22.82 & 4357 & 730 & 1846 \\
NOVEMBER & 24.69 & 21.38 & 22.27 & 3840 & 691 & 1436 \\
DECEMBER & 26.62 & 21.35 & 22.12 & 2778 & 641 & 1314 \\
JANUARY & 25.17 & 21.35 & 22.27 & 3415 & 678 & 1441 \\
FEBRUARY & 25.89 & 21.35 & 22.55 & 4044 & 691 & 1608 \\
MARCH & 28.44 & 21.34 & 23.10 & 5345 & 670 & 1843 \\
APRIL & 31.58 & 21.35 & 22.98 & 4668 & 671 & 1775 \\
MAY & 24.90 & 21.38 & 22.36 & 3409 & 536 & 1442 \\
JUNE & 25.58 & 21.43 & 22.17 & 4063 & 679 & 1352 \\
JULY & 23.76 & 21.55 & 22.26 & 2728 & 773 & 1420 \\
AUGUST & 26.40 & 21.50 & 22.73 & 3545 & 808 & 1734 \\
SEPTEMBER & 28.38 & 21.55 & 23.00 & 6344 & 756 & 2020
\end{tabular}

\section{DURATION OF DAILY MEAN VALUES FOR WATER YEARS 1928-1993}

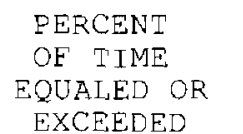

EQUALED OR
EXCEEDED

ANNUAL

OCT

NOV

DEC

JAN

$F \Xi B$

MAR

APR

JUNE

JULY

AUG

SEPT ELEVATION IN FEET ABOVE MEAN SEA LEVEL

$\begin{array}{rl}95.0 & 21.3 \\ 90.0 & 21.3 \\ 85.0 & 21.7 \\ 80.0 & 21.7 \\ 75.0 & 21.7 \\ 70.0 & 21.7 \\ 65.0 & 21.7 \\ 60.0 & 21.7 \\ 55.0 & 22.0 \\ 50.0 & 22.0 \\ 45.0 & 22.0 \\ 40.0 & 22.0 \\ 35.0 & 22.4 \\ 30.0 & 22.4 \\ 25.0 & 22.7 \\ 20.0 & 22.7 \\ 15.0 & 23.1 \\ 10.0 & 23.8 \\ 5.0 & 25.0\end{array}$

$\begin{array}{ll}21.6 & 21.4 \\ 21.6 & 21.5 \\ 21.8 & 21.5 \\ 21.8 & 21.5 \\ 21.8 & 21.7 \\ 21.8 & 21.7 \\ 21.8 & 21.7 \\ 22.0 & 21.7 \\ 22.0 & 21.7 \\ 22.0 & 21.9 \\ 22.3 & 21.9 \\ 22.3 & 21.9 \\ 22.5 & 22.1 \\ 22.7 & 22.3 \\ 23.0 & 22.5 \\ 23.5 & 22.7 \\ 24.2 & 22.7 \\ 25.0 & 22.9 \\ 26.3 & 23.8\end{array}$

$\begin{array}{lll}21.3 & 21.3 & 21.5 \\ 21.5 & 21.5 & 21.5 \\ 21.5 & 21.5 & 21.5 \\ 21.5 & 21.5 & 21.7 \\ 21.7 & 21.7 & 21.7 \\ 21.7 & 21.7 & 21.7 \\ 21.7 & 21.7 & 21.9 \\ 21.7 & 21.8 & 21.9 \\ 21.8 & 21.8 & 22.0 \\ 21.8 & 21.8 & 22.0 \\ 21.8 & 21.8 & 22.2 \\ 22.0 & 22.0 & 22.4 \\ 22.0 & 22.0 & 22.4 \\ 22.0 & 22.3 & 22.6 \\ 22.2 & 22.5 & 22.8 \\ 22.3 & 22.7 & 23.0 \\ 22.5 & 23.0 & 23.3 \\ 22.7 & 23.4 & 23.9 \\ 23.4 & 24.3 & 25.1\end{array}$

21.3
21.6
21.6
21.6
21.6
21.6
21.9
21.9
21.9
22.2
22.2
22.5
22.8
23.1
23.4
24.0
25.3
26.3
27.0

$21 \cdot 3$
$21 \cdot 3$
$21 \cdot 6$
$21 \cdot 6$
$21 \cdot 6$
$21 \cdot 6$
$21 \cdot 6$
22.0
$22 \cdot 0$
22.0
$22 \cdot 3$
22.3
22.6
22.9
$23 \cdot 3$
23.6
$24 \cdot 3$
25.4
26.5

21.3
21.5
21.5
21.5
21.5
21.7
21.7
21.7
21.9
21.9
21.9
22.2
22.2
22.4
22.6
22.8
23.0
23.2
24.8

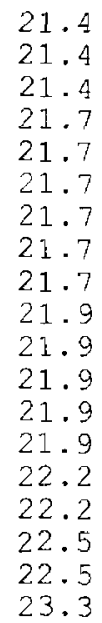

DISCHARGE IN CUBIC EEET PER SECOND

$\begin{array}{rr}95.0 & 800.8 \\ 90.0 & 877.3 \\ 85.0 & 937.7 \\ 80.0 & 993.7 \\ 75.0 & 1045.9 \\ 70.0 & 1097.7 \\ 65.0 & 1150.4 \\ 60.0 & 1203.2 \\ 55.0 & 1256.1 \\ 50.0 & 1333.5 \\ 45.0 & 1425.4 \\ 40.0 & 1526.1 \\ 35.0 & 1628.5 \\ 30.0 & 1744.6 \\ 25.0 & 1879.8 \\ 20.0 & 2052.9 \\ 15.0 & 2294.6 \\ 10.0 & 2658.2 \\ 5.0 & 3283.3\end{array}$

$\begin{array}{rrr}800.9 & 767.9 & 761.9 \\ 973.4 & 893.4 & 858.0 \\ 1044.8 & 927.2 & 898.4 \\ 1103.2 & 966.7 & 928.0 \\ 1158.3 & 1016.8 & 956.8 \\ 1222.1 & 1084.0 & 1004.1 \\ 1284.5 & 1124.9 & 1050.6 \\ 1342.0 & 1158.5 & 1096.7 \\ 1403.1 & 1191.2 & 1149.7 \\ 1467.8 & 1246.7 & 1202.1 \\ 1545.1 & 1315.6 & 1251.7 \\ 1634.3 & 1387.4 & 1294.4 \\ 1742.0 & 1469.4 & 1345.3 \\ 1926.6 & 1582.7 & 1408.6 \\ 2157.6 & 1718.2 & 1525.3 \\ 2399.7 & 1866.4 & 1634.7 \\ 2799.3 & 1985.7 & 1725.2 \\ 3330.3 & 2131.4 & 1858.5 \\ 9081.9 & 2502.2 & 2465.6\end{array}$

$\begin{array}{rr}751.7 & 832.5 \\ 860.4 & 873.8 \\ 898.2 & 952.8 \\ 955.9 & 1021.3 \\ 1010.9 & 1082.7 \\ 1056.6 & 1122.0 \\ 1097.2 & 1162.9 \\ 1132.4 & 1246.7 \\ 1172.5 & 1320.8 \\ 1217.3 & 1403.9 \\ 1267.7 & 1505.2 \\ 1338.2 & 1589.1 \\ 1457.7 & 1672.4 \\ 1551.9 & 1770.3 \\ 1670.1 & 1916.3 \\ 1826.2 & 2072.3 \\ 2095.9 & 2323.3 \\ 2361.8 & 2680.2 \\ 2808.5 & 3186.7\end{array}$

$\begin{array}{rr}809.5 & 820.2 \\ 908.4 & 905.9 \\ 972.5 & 963.4 \\ 1033.3 & 1016.1 \\ 1097.1 & 1069.0 \\ 1166.4 & 1120.2 \\ 1210.2 & 1169.1 \\ 1252.7 & 1236.3 \\ 1333.6 & 1327.3 \\ 1463.9 & 1426.0 \\ 1591.6 & 1540.8 \\ 1738.5 & 1675.9 \\ 1888.2 & 1828.9 \\ 2046.5 & 2012.3 \\ 2223.0 & 2227.8 \\ 2461.2 & 2455.7 \\ 2802.3 & 2786.9 \\ 3305.4 & 3088.6 \\ 4108.0 & 3784.8\end{array}$

781.1
856.5
897.5
945.9
988.4
1026.9
1075.1
1128.1
1180.1
1241.1
1314.4
1387.8
1485.3
1598.0
1743.2
1872.2
2012.6
2195.3
2662.8

752.7
834.7
879.5
919.1
958.3
993.1
1028.0
1063.9
1100.4
1140.2
1194.5
1254.6
1319.8
1432.4
1539.1
1635.9
1741.7
1912.2
2608.9
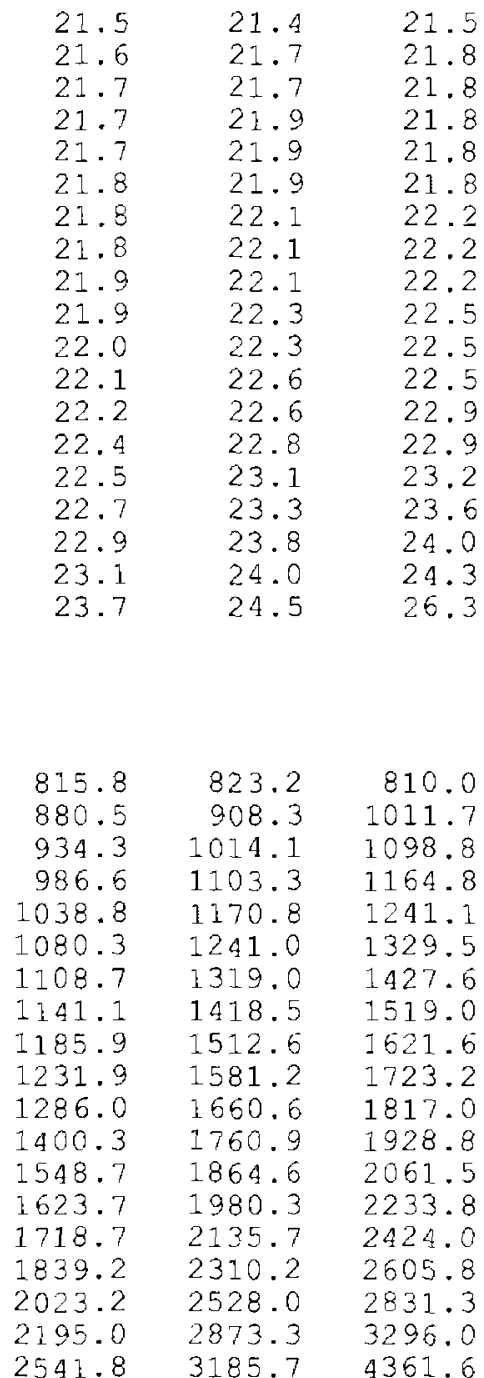

$24.5 \quad 26.3$ 
LOWEST MEAN ELEVATION, IN FEET, AND RANKING FOR THE FOLLOWING NUMBER OF CONSECUTIVE DAYS FOR PERIOD APR TO MAR

WATER YEAR 19281929 19341935 19351936

19361937

19371938

19381939

19391940

$1941 \quad 1942$

19421943

19431944

19451946

19561957

19581959

19611962

19621963

19631964

19641965

19651966

19661967

19671968

$1974 \quad 1975$

19751976

19761977

19771978

19781979

19801981

$1981 \quad 1982$

19831984

19841985

$1986 \quad 1987$

19871988

19881989

19891990

19911992

\begin{tabular}{|c|c|c|c|c|c|}
\hline $\begin{array}{c}1 \\
21.7\end{array}$ & 20 & $\begin{array}{c}3 \\
21.7\end{array}$ & 19 & $\begin{array}{r}7 \\
21.8\end{array}$ & 21 \\
\hline 21.61 & 12 & 21.6 & 12 & 21.6 & 12 \\
\hline 21.4 & 4 & 21.4 & 4 & 21.5 & 4 \\
\hline 21.82 & 27 & 21.8 & 28 & 21.8 & 28 \\
\hline $22.0=$ & 32 & 22.0 & 32 & 22.0 & 32 \\
\hline 21.71 & 18 & 21.7 & 18 & 21.7 & 18 \\
\hline 21.71 & 16 & 21.7 & 16 & 21.7 & 16 \\
\hline 21.72 & 21 & 21.7 & 21 & 21.7 & 20 \\
\hline 21.82 & 25 & 21.8 & 25 & 21.8 & 25 \\
\hline 21.51 & 10 & 21.5 & 10 & 21.5 & 10 \\
\hline 21.71 & 17 & 21.7 & 17 & 21.7 & 17 \\
\hline 21.3 & 1 & 21.3 & I & 21.3 & 2 \\
\hline 21.82 & 28 & 21.8 & 27 & 21.8 & 27 \\
\hline 21.61 & 15 & 21.6 & 15 & 21.6 & 15 \\
\hline 21.51 & 11 & 21.5 & 11 & 21.6 & 11 \\
\hline 21.5 & 9 & 21.5 & 9 & 21.5 & 9 \\
\hline 21.93 & 30 & 21.9 & 30 & 21.9 & 30 \\
\hline 22.33 & 35 & 22.3 & 35 & 22.3 & 35 \\
\hline 22.33 & 34 & 22.3 & 34 & 22.3 & 34 \\
\hline 21.82 & 26 & 21.8 & 26 & 21.8 & 26 \\
\hline 21.5 & 7 & 21.5 & 7 & 21.5 & 7 \\
\hline 21.61 & 14 & 21.6 & 14 & 21.6 & 14 \\
\hline 21.5 & 6 & 21.5 & 6 & 21.5 & 6 \\
\hline 21.4 & 3 & 21.4 & 3 & 21.4 & 3 \\
\hline 21.71 & 19 & 21.7 & 20 & 21.7 & 19 \\
\hline 21.82 & 24 & 21.8 & 24 & 21.8 & 24 \\
\hline 21.61 & 13 & 21.6 & 13 & 21.6 & 13 \\
\hline 21.3 & 2 & 21.3 & 2 & 21.3 & 1 \\
\hline 22.1 & 33 & 22.1 & 33 & 22.1 & 33 \\
\hline 21.93 & 31 & 21.9 & 31 & 21.9 & 31 \\
\hline 21.82 & 23 & 21.8 & 23 & 21.8 & 22 \\
\hline 21.92 & 29 & 21.9 & 29 & 21.9 & 29 \\
\hline 21.82 & 22 & 21.8 & 22 & 21.8 & 23 \\
\hline 21.5 & 5 & 21.5 & 5 & 21.5 & 5 \\
\hline 21.5 & 8 & 21.5 & 8 & 21.5 & 8 \\
\hline
\end{tabular}

\begin{tabular}{rr}
\multicolumn{3}{c}{30} \\
22.5 & 35 \\
21.6 & 12 \\
21.5 & 4 \\
& \\
21.9 & 27 \\
22.0 & 31 \\
21.7 & 19 \\
21.7 & 16 \\
& \\
21.8 & 20 \\
21.8 & 24 \\
21.5 & 10 \\
21.7 & 17 \\
21.3 & 1 \\
21.9 & 26 \\
& \\
21.7 & 15 \\
21.6 & 11 \\
21.5 & 8 \\
21.9 & 28 \\
22.4 & 34 \\
22.4 & 33 \\
21.8 & 25 \\
& \\
21.5 & 9 \\
21.6 & 14 \\
21.5 & 6 \\
21.4 & 3 \\
21.7 & 18 \\
21.8 & 23 \\
21.6 & 13 \\
21.3 & 2 \\
22.2 & 32 \\
21.9 & 30 \\
21.8 & 21 \\
21.9 & 29 \\
21.8 & 22 \\
21.5 & 5 \\
21.5 & 7 \\
&
\end{tabular}

60

22.734

$\begin{array}{rrr}21.6 & 12\end{array}$

21.927

22.131

$21.8 \quad 19$

21.716

21.820

$21.8 \quad 24$

21.69

$21.7 \quad 17$

21.31

21.926

$21.7 \quad 14$

21.611

21.58

$22.0 \quad 30$

22.735

$22.4 \quad 33$

21.825

$\begin{array}{rr}21.5 & 6 \\ 21.7 & 15\end{array}$

21.610

21.53

$\begin{array}{rr}21.5 & 3 \\ 21.7 & 18\end{array}$

21.823

21.613

21.42

$22.3 \quad 32$

22.028

21.822

22.029

$21.8 \quad 21$

21.55

21.57
90

22.835

$21.6 \quad 12$

22.027

22.230

21.819

21.818

21.923

$21.8 \quad 22$

$21.6 \quad 6$

21.816

$21.4 \quad 1$

21.926

$21.7 \quad 14$

21.68

21.610

22.432

22.834

$22.5 \quad 33$

21.924

21.69

$21.7 \quad 15$

$21.6 \quad 11$

21.54

21.817

21.925

21.613

$21.4 \quad 2$

22.431

22.029

$21.8 \quad 20$

22.028

$21.8 \quad 21$

21.67
120

22.934

$\begin{array}{rr}21.7 & 11 \\ 21.5 & 4\end{array}$

22.026

$22.3 \quad 30$

$21.8 \quad 17$

21.923

$22.2 \quad 29$

21.921

$21.6 \quad 6$

21.816

$\begin{array}{rr}21.4 & 1 \\ 22.0 & 25\end{array}$

$21.7 \quad 14$

21.67

21.815

22.732

23.035

22.733

21.922

$21.7 \quad 9$

21.713

$21.7 \quad 10$

21.53

21.818

21.924

21.712

$21.4 \quad 2$

22.431

22.027

21.919

22.028

21.920

21.55

21.68
183
23.35

21.89

$22.5 \quad 29$

22.021

22.731

22.019

22.020

22.328

22.018

$21.6 \quad 4$

$22.2 \quad 27$

$\begin{array}{rr}21.5 & 2 \\ 22.2 & 25\end{array}$

21.913

21.77

23.834
$23.4 \quad 33$

23.132

21.914

21.811

21.812

$21.7 \quad 6$

$21.5 \quad 3$

22.017

$\begin{array}{ll}22.1 & 22 \\ 21.8 & 10\end{array}$

$21.5 \quad 1$

22.124

22.016

$22.2 \quad 26$

22.123

21.65

$21.8 \quad 8$ 
HIGHEST MEAN ELEVATION, IN FEET, AND RANKING FOR THE FOLLOWING NUMBER OF CONSECUTIVE DAYS FOR PERIOD OCT TO SEP

\begin{tabular}{|c|c|c|c|c|c|c|c|c|c|c|c|c|c|c|c|c|c|c|}
\hline & ANGE & 1 & & 3 & & & & 15 & & & & 6 & & & 30 & & 20 & 183 \\
\hline 1929 & 1929 & 29.3 & 4 & 29.3 & 4 & 29.2 & 4 & 29.0 & 3 & 28.0 & 3 & 25.7 & 8 & 24.7 & 8 & 24.3 & 7 & 23.96 \\
\hline $\begin{array}{l}1934 \\
1935\end{array}$ & $\begin{array}{l}1934 \\
1935\end{array}$ & $\begin{array}{l}31.7 \\
29.0\end{array}$ & $\begin{array}{l}2 \\
5\end{array}$ & $\begin{array}{l}31.3 \\
28.8\end{array}$ & $\begin{array}{l}2 \\
5\end{array}$ & $\begin{array}{l}30.0 \\
28.4\end{array}$ & $\begin{array}{l}3 \\
6\end{array}$ & $\begin{array}{l}28.0 \\
27.8\end{array}$ & $\begin{array}{l}5 \\
6\end{array}$ & $\begin{array}{l}25.9 \\
26.8\end{array}$ & $\begin{array}{r}11 \\
8\end{array}$ & $\begin{array}{l}24.4 \\
24.6\end{array}$ & $\begin{array}{l}12 \\
10\end{array}$ & $\begin{array}{l}24.0 \\
23.6\end{array}$ & $\begin{array}{l}10 \\
13\end{array}$ & $\begin{array}{l}23.6 \\
23.1\end{array}$ & $\begin{array}{l}10 \\
15\end{array}$ & $\begin{array}{ll}22.9 & 13 \\
22.5 & 20\end{array}$ \\
\hline $\begin{array}{l}1936 \\
1937 \\
1938 \\
1939 \\
1940\end{array}$ & $\begin{array}{l}1936 \\
1937 \\
1938 \\
1939 \\
1940\end{array}$ & $\begin{array}{l}26.4 \\
25.3 \\
28.6 \\
26.3 \\
23.0\end{array}$ & $\begin{array}{r}14 \\
20 \\
8 \\
16 \\
32\end{array}$ & $\begin{array}{l}26.1 \\
25.2 \\
26.7 \\
26.2 \\
23.0\end{array}$ & $\begin{array}{l}16 \\
20 \\
13 \\
15 \\
32\end{array}$ & $\begin{array}{l}25.6 \\
24.8 \\
26.4 \\
25.6 \\
22.9\end{array}$ & $\begin{array}{l}16 \\
21 \\
13 \\
15 \\
33\end{array}$ & $\begin{array}{l}24.8 \\
24.5 \\
25.5 \\
24.7 \\
22.7\end{array}$ & $\begin{array}{l}17 \\
21 \\
13 \\
19 \\
32\end{array}$ & $\begin{array}{l}24.0 \\
24.0 \\
24.4 \\
23.7 \\
22.7\end{array}$ & $\begin{array}{l}20 \\
21 \\
15 \\
22 \\
31\end{array}$ & $\begin{array}{l}23.3 \\
23.5 \\
23.5 \\
23.0 \\
22.5\end{array}$ & $\begin{array}{l}21 \\
17 \\
16 \\
23 \\
29\end{array}$ & $\begin{array}{l}22.9 \\
23.4 \\
23.1 \\
22.8 \\
22.3\end{array}$ & $\begin{array}{l}20 \\
15 \\
19 \\
22 \\
29\end{array}$ & $\begin{array}{l}22.7 \\
23.1 \\
22.9 \\
22.6 \\
22.2\end{array}$ & $\begin{array}{l}20 \\
14 \\
18 \\
21 \\
29\end{array}$ & $\begin{array}{ll}22.7 & 18 \\
22.8 & 17 \\
22.7 & 19 \\
22.3 & 21 \\
22.1 & 27\end{array}$ \\
\hline $\begin{array}{l}1942 \\
1943 \\
1944\end{array}$ & $\begin{array}{l}1942 \\
1943 \\
1944\end{array}$ & $\begin{array}{l}28.9 \\
22.8 \\
25.0\end{array}$ & $\begin{array}{r}6 \\
34 \\
21\end{array}$ & $\begin{array}{l}28.7 \\
22.8 \\
25.0\end{array}$ & $\begin{array}{l}7 \\
34 \\
21\end{array}$ & $\begin{array}{l}27.8 \\
22.7 \\
24.9\end{array}$ & $\begin{array}{l}7 \\
34 \\
20\end{array}$ & $\begin{array}{l}27.1 \\
22.5 \\
24.7\end{array}$ & $\begin{array}{r}8 \\
34 \\
18\end{array}$ & $\begin{array}{l}26.9 \\
22.3 \\
24.4\end{array}$ & $\begin{array}{r}6 \\
34 \\
16\end{array}$ & $\begin{array}{l}26.1 \\
22.2 \\
23.7\end{array}$ & $\begin{array}{r}4 \\
33 \\
14\end{array}$ & $\begin{array}{l}25.4 \\
22.1 \\
23.2\end{array}$ & $\begin{array}{r}4 \\
33 \\
17\end{array}$ & $\begin{array}{l}25.2 \\
22.1 \\
22.8\end{array}$ & $\begin{array}{r}4 \\
33 \\
19\end{array}$ & $\begin{array}{rr}25.0 & 1 \\
22.0 & 33 \\
22.9 & 16\end{array}$ \\
\hline 1952 & 1952 & 23.1 & 31 & 23.1 & 30 & 23.1 & 30 & 23.0 & 30 & 22.8 & 29 & 22.5 & 28 & 22.4 & 27 & 22.3 & 26 & 22.226 \\
\hline 1956 & 1956 & 21.9 & 37 & 21.9 & 37 & 21.9 & 37 & 21.8 & 37 & 21.8 & 37 & 21.7 & 37 & 21.7 & 37 & 21.6 & 37 & $21.5 \quad 37$ \\
\hline $\begin{array}{l}1961 \\
1962 \\
1963 \\
1964 \\
1965\end{array}$ & $\begin{array}{l}1961 \\
1962 \\
1963 \\
1964 \\
1965\end{array}$ & $\begin{array}{l}25.3 \\
22.5 \\
22.9 \\
36.1 \\
27.8\end{array}$ & $\begin{array}{r}19 \\
35 \\
33 \\
1 \\
12\end{array}$ & $\begin{array}{l}25.3 \\
22.5 \\
22.9 \\
35.8 \\
27.6\end{array}$ & $\begin{array}{r}19 \\
36 \\
33 \\
1 \\
11\end{array}$ & $\begin{array}{l}25.2 \\
22.5 \\
22.9 \\
34.6 \\
27.3\end{array}$ & $\begin{array}{r}19 \\
36 \\
32 \\
1 \\
11\end{array}$ & $\begin{array}{l}25.0 \\
22.4 \\
22.8 \\
32.1 \\
27.0\end{array}$ & $\begin{array}{r}16 \\
35 \\
31 \\
1 \\
10\end{array}$ & $\begin{array}{l}24.5 \\
22.3 \\
22.7 \\
28.4 \\
26.8\end{array}$ & $\begin{array}{r}14 \\
35 \\
30 \\
2 \\
7\end{array}$ & $\begin{array}{l}23.7 \\
22.1 \\
22.5 \\
26.0 \\
26.3\end{array}$ & $\begin{array}{r}15 \\
35 \\
30 \\
6 \\
3\end{array}$ & $\begin{array}{l}23.3 \\
22.0 \\
22.2 \\
24.7 \\
25.5\end{array}$ & $\begin{array}{r}16 \\
34 \\
31 \\
7 \\
3\end{array}$ & $\begin{array}{l}23.1 \\
22.0 \\
22.1 \\
24.0 \\
25.5\end{array}$ & $\begin{array}{r}16 \\
34 \\
32 \\
8 \\
3\end{array}$ & $\begin{array}{rr}22.9 & 15 \\
21.9 & 34 \\
22.0 & 32 \\
23.8 & 8 \\
25.0 & 2\end{array}$ \\
\hline $\begin{array}{l}1966 \\
1967 \\
1969\end{array}$ & $\begin{array}{l}1966 \\
1967 \\
1969\end{array}$ & $\begin{array}{l}28.7 \\
25.5 \\
23.7\end{array}$ & $\begin{array}{r}7 \\
18 \\
25\end{array}$ & $\begin{array}{l}28.7 \\
25.6 \\
23.7\end{array}$ & $\begin{array}{r}6 \\
18 \\
24\end{array}$ & $\begin{array}{l}28.6 \\
25.5 \\
23.6\end{array}$ & $\begin{array}{r}5 \\
17 \\
24\end{array}$ & $\begin{array}{l}28.1 \\
25.1 \\
23.4\end{array}$ & $\begin{array}{r}4 \\
15 \\
25\end{array}$ & $\begin{array}{l}27.5 \\
24.2 \\
23.0\end{array}$ & $\begin{array}{r}4 \\
17 \\
25\end{array}$ & $\begin{array}{l}26.0 \\
23.5 \\
22.7\end{array}$ & $\begin{array}{r}5 \\
18 \\
25\end{array}$ & $\begin{array}{l}25.3 \\
23.1 \\
22.5\end{array}$ & $\begin{array}{r}5 \\
18 \\
25\end{array}$ & $\begin{array}{l}25.1 \\
23.0 \\
22.3\end{array}$ & $\begin{array}{r}5 \\
17 \\
25\end{array}$ & $\begin{array}{rr}24.7 & 4 \\
23.1 & 10 \\
22.2 & 25\end{array}$ \\
\hline $\begin{array}{l}1971 \\
1974 \\
1975\end{array}$ & $\begin{array}{l}1971 \\
1974 \\
1975\end{array}$ & $\begin{array}{l}24.6 \\
24.2 \\
23.7\end{array}$ & $\begin{array}{l}22 \\
23 \\
27\end{array}$ & $\begin{array}{l}24.6 \\
24.2 \\
23.6\end{array}$ & $\begin{array}{l}22 \\
23 \\
26\end{array}$ & $\begin{array}{l}24.5 \\
24.0 \\
23.6\end{array}$ & $\begin{array}{l}22 \\
23 \\
25\end{array}$ & $\begin{array}{l}24.3 \\
23.7 \\
23.3\end{array}$ & $\begin{array}{l}22 \\
23 \\
26\end{array}$ & $\begin{array}{l}24.1 \\
23.5 \\
22.9\end{array}$ & $\begin{array}{l}18 \\
23 \\
28\end{array}$ & $\begin{array}{l}23.3 \\
23.2 \\
22.4\end{array}$ & $\begin{array}{l}20 \\
22 \\
31\end{array}$ & $\begin{array}{l}22.8 \\
22.8 \\
22.3\end{array}$ & $\begin{array}{l}21 \\
23 \\
30\end{array}$ & $\begin{array}{l}22.5 \\
22.5 \\
22.2\end{array}$ & $\begin{array}{l}23 \\
24 \\
30\end{array}$ & $\begin{array}{ll}22.3 & 22 \\
22.2 & 23 \\
22.1 & 28\end{array}$ \\
\hline $\begin{array}{l}1976 \\
1977 \\
1978 \\
1979 \\
1980\end{array}$ & $\begin{array}{l}1976 \\
1977 \\
1978 \\
1979 \\
1980\end{array}$ & $\begin{array}{l}23.2 \\
23.6 \\
25.7 \\
23.5 \\
26.4\end{array}$ & $\begin{array}{l}30 \\
28 \\
17 \\
29 \\
15\end{array}$ & $\begin{array}{l}23.1 \\
23.5 \\
25.7 \\
23.3 \\
26.2\end{array}$ & $\begin{array}{l}31 \\
28 \\
17 \\
29 \\
14\end{array}$ & $\begin{array}{l}22.9 \\
23.5 \\
25.4 \\
23.2 \\
25.9\end{array}$ & $\begin{array}{l}31 \\
26 \\
18 \\
29 \\
14\end{array}$ & $\begin{array}{l}22.7 \\
23.5 \\
24.6 \\
23.1 \\
25.2\end{array}$ & $\begin{array}{l}33 \\
24 \\
20 \\
29 \\
14\end{array}$ & $\begin{array}{l}22.5 \\
23.2 \\
24.1 \\
22.6 \\
24.9\end{array}$ & $\begin{array}{l}33 \\
24 \\
19 \\
32 \\
13\end{array}$ & $\begin{array}{l}22.2 \\
23.0 \\
23.4 \\
22.3 \\
24.3\end{array}$ & $\begin{array}{l}34 \\
24 \\
19 \\
32 \\
13\end{array}$ & $\begin{array}{l}22.0 \\
22.8 \\
23.4 \\
22.2 \\
23.7\end{array}$ & $\begin{array}{l}35 \\
24 \\
14 \\
32 \\
12\end{array}$ & $\begin{array}{l}21.9 \\
22.6 \\
23.2 \\
22.2 \\
23.3\end{array}$ & $\begin{array}{l}35 \\
22 \\
13 \\
31 \\
12\end{array}$ & $\begin{array}{ll}21.8 & 35 \\
22.2 & 24 \\
23.1 & 11 \\
22.1 & 31 \\
22.9 & 14\end{array}$ \\
\hline $\begin{array}{l}1981 \\
1983 \\
1984 \\
1986 \\
1987 \\
1988 \\
1989\end{array}$ & $\begin{array}{l}1981 \\
1983 \\
1984 \\
1986 \\
1987 \\
1988 \\
1989\end{array}$ & $\begin{array}{l}22.5 \\
25.9 \\
30.3 \\
28.0 \\
27.9 \\
28.5 \\
23.7\end{array}$ & $\begin{array}{r}36 \\
13 \\
3 \\
10 \\
11 \\
9 \\
26\end{array}$ & $\begin{array}{l}22.5 \\
26.9 \\
30.3 \\
27.9 \\
27.9 \\
28.4 \\
23.6\end{array}$ & $\begin{array}{r}35 \\
12 \\
3 \\
9 \\
10 \\
8 \\
27\end{array}$ & $\begin{array}{l}22.5 \\
26.8 \\
30.2 \\
27.7 \\
27.7 \\
27.8 \\
23.5\end{array}$ & $\begin{array}{r}35 \\
12 \\
2 \\
10 \\
9 \\
8 \\
28\end{array}$ & $\begin{array}{l}22.3 \\
26.6 \\
29.8 \\
27.1 \\
27.6 \\
26.7 \\
23.3\end{array}$ & $\begin{array}{r}36 \\
12 \\
2 \\
9 \\
7 \\
11 \\
27\end{array}$ & $\begin{array}{l}22.2 \\
26.3 \\
28.8 \\
26.1 \\
27.1 \\
25.5 \\
22.9\end{array}$ & $\begin{array}{r}36 \\
9 \\
1 \\
10 \\
5 \\
12 \\
26\end{array}$ & $\begin{array}{l}22.0 \\
25.9 \\
27.5 \\
24.8 \\
26.7 \\
24.5 \\
22.6\end{array}$ & $\begin{array}{r}36 \\
7 \\
1 \\
9 \\
2 \\
11 \\
27\end{array}$ & $\begin{array}{l}21.9 \\
25.0 \\
26.3 \\
24.2 \\
26.3 \\
23.9 \\
22.4\end{array}$ & $\begin{array}{r}36 \\
6 \\
1 \\
9 \\
2 \\
11 \\
28\end{array}$ & $\begin{array}{l}21.8 \\
24.4 \\
25.7 \\
23.7 \\
25.5 \\
23.5 \\
22.2\end{array}$ & $\begin{array}{r}36 \\
6 \\
1 \\
9 \\
2 \\
11 \\
28\end{array}$ & $\begin{array}{rr}21.8 & 36 \\
23.8 & 7 \\
24.8 & 3 \\
23.3 & 9 \\
24.5 & 5 \\
23.0 & 12 \\
22.1 & 30\end{array}$ \\
\hline 992 & 1992 & 23.7 & 24 & 23.7 & 25 & 23.5 & 27 & 23.2 & 28 & 22.9 & 27 & 22.6 & 26 & 22.4 & 26 & 22.3 & 27 & 22.129 \\
\hline
\end{tabular}



CONSECUTIVE DAYS FOR PERIOD APR TO MAR

\begin{tabular}{|c|c|c|c|c|c|c|c|c|c|c|c|c|c|c|c|c|c|c|c|}
\hline $\begin{array}{r}\mathrm{R} \\
1928\end{array}$ & $\begin{array}{l}\text { ANGE } \\
1929\end{array}$ & 1000 & 34 & $\begin{array}{c}3 \\
1000\end{array}$ & 34 & $\begin{array}{r}7 \\
1016\end{array}$ & 34 & $\begin{array}{c}14 \\
1058\end{array}$ & 40 & $\begin{array}{r}30 \\
1543^{30}\end{array}$ & 58 & $1704^{66}$ & $0_{59}$ & $1826^{9}$ & $\begin{array}{l}90 \\
59\end{array}$ & $1880^{1}$ & $\begin{array}{l}20 \\
58\end{array}$ & 2395 & $\begin{array}{r}183 \\
60\end{array}$ \\
\hline $\begin{array}{l}1933 \\
1934 \\
1935\end{array}$ & $\begin{array}{l}1934 \\
1935 \\
1936\end{array}$ & $\begin{array}{l}838 \\
764 \\
590\end{array}$ & $\begin{array}{r}15 \\
7 \\
6\end{array}$ & $\begin{array}{l}845 \\
764 \\
692\end{array}$ & $\begin{array}{r}15 \\
7 \\
5\end{array}$ & $\begin{array}{l}845 \\
766 \\
695\end{array}$ & $\begin{array}{r}15 \\
7 \\
6\end{array}$ & $\begin{array}{l}852 \\
767 \\
699\end{array}$ & $\begin{array}{r}15 \\
7 \\
6\end{array}$ & $\begin{array}{l}858 \\
784 \\
712\end{array}$ & $\begin{array}{c}13 \\
8 \\
6\end{array}$ & $\begin{array}{l}858 \\
828 \\
731\end{array}$ & $\begin{array}{c}12 \\
8 \\
6\end{array}$ & $\begin{array}{l}875 \\
866 \\
745\end{array}$ & $\begin{array}{r}10 \\
9 \\
5\end{array}$ & $\begin{array}{l}891 \\
899 \\
767\end{array}$ & $\begin{array}{r}9 \\
10 \\
5\end{array}$ & $\begin{array}{r}970 \\
1012 \\
1464\end{array}$ & $\begin{array}{r}8 \\
13 \\
43\end{array}$ \\
\hline $\begin{array}{l}1936 \\
1937 \\
1938 \\
1939 \\
1940\end{array}$ & $\begin{array}{l}1937 \\
1938 \\
1939 \\
1940 \\
1941\end{array}$ & $\begin{array}{r}1020 \\
1140 \\
954 \\
862 \\
864\end{array}$ & $\begin{array}{l}35 \\
50 \\
30 \\
18 \\
19\end{array}$ & $\begin{array}{r}1020 \\
1167 \\
954 \\
866 \\
864\end{array}$ & $\begin{array}{l}35 \\
52 \\
29 \\
19 \\
18\end{array}$ & $\begin{array}{r}1026 \\
1174 \\
957 \\
872 \\
868\end{array}$ & $\begin{array}{l}37 \\
52 \\
29 \\
20 \\
18\end{array}$ & $\begin{array}{r}1029 \\
1177 \\
962 \\
881 \\
875\end{array}$ & $\begin{array}{l}34 \\
52 \\
29 \\
20 \\
19\end{array}$ & $\begin{array}{c}1050 \\
1194 \\
975 \\
900 \\
886\end{array}$ & $\begin{array}{l}35 \\
51 \\
29 \\
19 \\
18\end{array}$ & $\begin{array}{r}1063 \\
1244 \\
994 \\
920 \\
909\end{array}$ & $\begin{array}{l}33 \\
51 \\
26 \\
18 \\
17\end{array}$ & $\begin{array}{r}1105 \\
1317 \\
1032 \\
1004 \\
949\end{array}$ & $\begin{array}{l}35 \\
52 \\
25 \\
23 \\
16\end{array}$ & $\begin{array}{r}1123 \\
1388 \\
1064 \\
1118 \\
987\end{array}$ & $\begin{array}{l}30 \\
50 \\
25 \\
29 \\
16\end{array}$ & $\begin{array}{l}1156 \\
1737 \\
1204 \\
1218 \\
1078\end{array}$ & $\begin{array}{l}21 \\
52 \\
24 \\
27 \\
15\end{array}$ \\
\hline $\begin{array}{l}1941 \\
1942 \\
1943 \\
1944 \\
1945\end{array}$ & $\begin{array}{l}1942 \\
1943 \\
1944 \\
1945 \\
1946\end{array}$ & $\begin{array}{r}912 \\
1030 \\
810 \\
903 \\
930\end{array}$ & $\begin{array}{l}25 \\
39 \\
10 \\
23 \\
27\end{array}$ & $\begin{array}{r}917 \\
1037 \\
810 \\
903 \\
933\end{array}$ & $\begin{array}{l}25 \\
40 \\
10 \\
23 \\
27\end{array}$ & $\begin{array}{r}923 \\
1050 \\
813 \\
903 \\
934\end{array}$ & $\begin{array}{l}25 \\
40 \\
10 \\
23 \\
27\end{array}$ & $\begin{array}{r}929 \\
1056 \\
817 \\
908 \\
937\end{array}$ & $\begin{array}{l}25 \\
39 \\
10 \\
23 \\
27\end{array}$ & $\begin{array}{r}943 \\
1066 \\
820 \\
925 \\
943\end{array}$ & $\begin{array}{l}24 \\
37 \\
10 \\
22 \\
25\end{array}$ & $\begin{array}{r}998 \\
1075 \\
830 \\
967 \\
957\end{array}$ & $\begin{array}{r}27 \\
36 \\
9 \\
23 \\
22\end{array}$ & $\begin{array}{r}1070 \\
1100 \\
828 \\
1136 \\
987\end{array}$ & $\begin{array}{r}28 \\
33 \\
6 \\
40 \\
19\end{array}$ & $\begin{array}{r}1293 \\
1134 \\
833 \\
1409 \\
1032\end{array}$ & $\begin{array}{r}48 \\
31 \\
6 \\
52 \\
21\end{array}$ & $\begin{array}{r}1437 \\
1242 \\
874 \\
1645 \\
1376\end{array}$ & $\begin{array}{r}41 \\
29 \\
5 \\
49 \\
37\end{array}$ \\
\hline $\begin{array}{l}1946 \\
1947 \\
1948 \\
1949 \\
1950\end{array}$ & $\begin{array}{l}1947 \\
1948 \\
1949 \\
1950 \\
1951\end{array}$ & $\begin{array}{l}1060 \\
1050 \\
1540 \\
1180 \\
1020\end{array}$ & $\begin{array}{l}43 \\
41 \\
61 \\
54 \\
36\end{array}$ & $\begin{array}{l}1073 \\
1050 \\
1640 \\
1187 \\
1020\end{array}$ & $\begin{array}{l}44 \\
41 \\
61 \\
55 \\
36\end{array}$ & $\begin{array}{l}1077 \\
1053 \\
1641 \\
1200 \\
1020\end{array}$ & $\begin{array}{l}44 \\
41 \\
51 \\
55 \\
35\end{array}$ & $\begin{array}{l}1087 \\
1059 \\
1656 \\
1216 \\
1023\end{array}$ & $\begin{array}{l}44 \\
41 \\
61 \\
55 \\
32\end{array}$ & $\begin{array}{l}1110 \\
1086 \\
1688 \\
1225 \\
1038\end{array}$ & $\begin{array}{l}45 \\
41 \\
60 \\
54 \\
32\end{array}$ & $\begin{array}{l}1270 \\
1145 \\
1793 \\
1255 \\
1068\end{array}$ & $\begin{array}{l}54 \\
46 \\
60 \\
52 \\
34\end{array}$ & $\begin{array}{l}1345 \\
1162 \\
1863 \\
1315 \\
1087\end{array}$ & $\begin{array}{l}53 \\
42 \\
60 \\
51 \\
30\end{array}$ & $\begin{array}{l}1426 \\
1219 \\
1979 \\
1402 \\
1176\end{array}$ & $\begin{array}{l}53 \\
42 \\
60 \\
51 \\
38\end{array}$ & $\begin{array}{l}1745 \\
1506 \\
2209 \\
1606 \\
1564\end{array}$ & $\begin{array}{l}53 \\
44 \\
58 \\
47 \\
46\end{array}$ \\
\hline $\begin{array}{l}1951 \\
1952 \\
1953 \\
1954 \\
1955\end{array}$ & $\begin{array}{l}1952 \\
1953 \\
1954 \\
1955 \\
1956\end{array}$ & $\begin{array}{r}1020 \\
896 \\
930 \\
851 \\
658\end{array}$ & $\begin{array}{r}37 \\
22 \\
28 \\
16 \\
4\end{array}$ & $\begin{array}{r}1020 \\
896 \\
933 \\
857 \\
658\end{array}$ & $\begin{array}{r}37 \\
22 \\
28 \\
16 \\
4\end{array}$ & $\begin{array}{r}1021 \\
898 \\
956 \\
863 \\
661\end{array}$ & $\begin{array}{r}36 \\
22 \\
28 \\
16 \\
4\end{array}$ & $\begin{array}{r}1028 \\
902 \\
1030 \\
866 \\
662\end{array}$ & $\begin{array}{r}33 \\
22 \\
35 \\
16 \\
4\end{array}$ & $\begin{array}{r}1047 \\
909 \\
1080 \\
874 \\
671\end{array}$ & $\begin{array}{r}34 \\
21 \\
39 \\
16 \\
4\end{array}$ & $\begin{array}{r}1074 \\
922 \\
1094 \\
885 \\
677\end{array}$ & $\begin{array}{r}35 \\
19 \\
38 \\
14 \\
3\end{array}$ & $\begin{array}{r}1091 \\
941 \\
1125 \\
898 \\
680\end{array}$ & $\begin{array}{c}31 \\
15 \\
39 \\
13 \\
2\end{array}$ & $\begin{array}{r}1097 \\
959 \\
1235 \\
923 \\
580\end{array}$ & $\begin{array}{c}28 \\
14 \\
43 \\
12 \\
1\end{array}$ & $\begin{array}{r}1108 \\
976 \\
1679 \\
974 \\
694\end{array}$ & $\begin{array}{r}17 \\
10 \\
50 \\
9 \\
1\end{array}$ \\
\hline $\begin{array}{l}1956 \\
1957 \\
1958 \\
1959 \\
1960\end{array}$ & $\begin{array}{l}1957 \\
1958 \\
1959 \\
1960 \\
1961\end{array}$ & $\begin{array}{r}633 \\
617 \\
1050 \\
1430 \\
1480\end{array}$ & $\begin{array}{r}3 \\
2 \\
42 \\
57 \\
58\end{array}$ & $\begin{array}{r}636 \\
617 \\
1050 \\
1437 \\
1487\end{array}$ & $\begin{array}{r}3 \\
2 \\
42 \\
57 \\
58\end{array}$ & $\begin{array}{r}639 \\
623 \\
1054 \\
1443 \\
1489\end{array}$ & $\begin{array}{r}3 \\
2 \\
42 \\
57 \\
58\end{array}$ & $\begin{array}{r}648 \\
626 \\
1066 \\
1456 \\
1499\end{array}$ & $\begin{array}{r}3 \\
2 \\
42 \\
57 \\
58\end{array}$ & $\begin{array}{r}669 \\
635 \\
1086 \\
1495 \\
1518\end{array}$ & $\begin{array}{r}3 \\
2 \\
42 \\
56 \\
57\end{array}$ & $\begin{array}{r}686 \\
659 \\
1096 \\
1550 \\
1570\end{array}$ & $\begin{array}{c}4 \\
2 \\
39 \\
56 \\
57\end{array}$ & $\begin{array}{r}697 \\
1070 \\
1118 \\
1595 \\
1645\end{array}$ & $\begin{array}{r}3 \\
29 \\
37 \\
55 \\
57\end{array}$ & $\begin{array}{r}728 \\
1093 \\
1155 \\
1706 \\
1694\end{array}$ & $\begin{array}{r}3 \\
27 \\
35 \\
57 \\
56\end{array}$ & $\begin{array}{r}754 \\
1214 \\
1314 \\
1938 \\
1993\end{array}$ & $\begin{array}{r}3 \\
26 \\
32 \\
55 \\
56\end{array}$ \\
\hline $\begin{array}{l}1961 \\
1962 \\
1963 \\
1964 \\
1965\end{array}$ & $\begin{array}{l}1962 \\
1963 \\
1964 \\
1965 \\
1955\end{array}$ & $\begin{array}{r}984 \\
872 \\
768 \\
1170 \\
1520\end{array}$ & $\begin{array}{r}33 \\
20 \\
8 \\
53 \\
60\end{array}$ & $\begin{array}{r}984 \\
872 \\
773 \\
1170 \\
1520\end{array}$ & $\begin{array}{r}32 \\
20 \\
9 \\
53 \\
59\end{array}$ & $\begin{array}{r}987 \\
872 \\
775 \\
1176 \\
1529\end{array}$ & $\begin{array}{r}31 \\
19 \\
9 \\
53 \\
59\end{array}$ & $\begin{array}{r}996 \\
874 \\
777 \\
1179 \\
1546\end{array}$ & $\begin{array}{r}30 \\
18 \\
9 \\
53 \\
59\end{array}$ & $\begin{array}{r}1013 \\
880 \\
794 \\
1192 \\
1609\end{array}$ & $\begin{array}{r}30 \\
17 \\
9 \\
50 \\
59\end{array}$ & $\begin{array}{r}1024 \\
888 \\
850 \\
1263 \\
1805\end{array}$ & $\begin{array}{l}30 \\
16 \\
10 \\
53 \\
61\end{array}$ & $\begin{array}{r}1047 \\
898 \\
920 \\
1465 \\
1915\end{array}$ & $\begin{array}{l}27 \\
11 \\
14 \\
54 \\
61\end{array}$ & $\begin{array}{r}1081 \\
920 \\
1005 \\
1538 \\
2056\end{array}$ & $\begin{array}{l}26 \\
11 \\
17 \\
54 \\
61\end{array}$ & $\begin{array}{r}1194 \\
976 \\
1154 \\
2438 \\
2314\end{array}$ & $\begin{array}{l}23 \\
11 \\
20 \\
61 \\
59\end{array}$ \\
\hline $\begin{array}{l}1966 \\
1967 \\
1968 \\
1969 \\
1970\end{array}$ & $\begin{array}{l}1967 \\
1968 \\
1969 \\
1970 \\
1971\end{array}$ & $\begin{array}{r}1510 \\
1090 \\
980 \\
1090 \\
1140\end{array}$ & $\begin{array}{l}59 \\
46 \\
32 \\
47 \\
51\end{array}$ & $\begin{array}{r}1523 \\
1090 \\
983 \\
1090 \\
1147\end{array}$ & $\begin{array}{l}60 \\
46 \\
31 \\
47 \\
50\end{array}$ & $\begin{array}{r}1547 \\
1099 \\
993 \\
1093 \\
1149\end{array}$ & $\begin{array}{l}60 \\
47 \\
33 \\
46 \\
50\end{array}$ & $\begin{array}{l}1596 \\
1111 \\
1003 \\
1099 \\
1154\end{array}$ & $\begin{array}{l}60 \\
47 \\
31 \\
46 \\
50\end{array}$ & $\begin{array}{l}1688 \\
1141 \\
1013 \\
1105 \\
1180\end{array}$ & $\begin{array}{l}61 \\
47 \\
31 \\
44 \\
49\end{array}$ & $\begin{array}{l}1696 \\
1142 \\
1017 \\
1131 \\
1223\end{array}$ & $\begin{array}{l}58 \\
45 \\
29 \\
43 \\
48\end{array}$ & $\begin{array}{l}1755 \\
1165 \\
1029 \\
1183 \\
1238\end{array}$ & $\begin{array}{l}58 \\
44 \\
24 \\
46 \\
49\end{array}$ & $\begin{array}{l}1905 \\
1169 \\
1049 \\
1251 \\
1247\end{array}$ & $\begin{array}{l}59 \\
37 \\
24 \\
45 \\
44\end{array}$ & $\begin{array}{l}2202 \\
1208 \\
1539 \\
1407 \\
1358\end{array}$ & $\begin{array}{l}57 \\
25 \\
45 \\
38 \\
35\end{array}$ \\
\hline $\begin{array}{l}1971 \\
1972 \\
1973 \\
1974 \\
1975\end{array}$ & $\begin{array}{l}1972 \\
1973 \\
1974 \\
1975 \\
1975\end{array}$ & $\begin{array}{r}950 \\
1150 \\
854 \\
819 \\
911\end{array}$ & $\begin{array}{l}29 \\
52 \\
17 \\
11 \\
24\end{array}$ & $\begin{array}{r}955 \\
1150 \\
862 \\
823 \\
914\end{array}$ & $\begin{array}{l}30 \\
51 \\
17 \\
11 \\
24\end{array}$ & $\begin{array}{r}959 \\
1157 \\
863 \\
826 \\
920\end{array}$ & $\begin{array}{l}30 \\
51 \\
17 \\
11 \\
24\end{array}$ & $\begin{array}{r}961 \\
1169 \\
873 \\
830 \\
925\end{array}$ & $\begin{array}{l}28 \\
51 \\
17 \\
11 \\
24\end{array}$ & $\begin{array}{r}968 \\
1195 \\
903 \\
861 \\
947\end{array}$ & $\begin{array}{l}28 \\
52 \\
20 \\
14 \\
27\end{array}$ & $\begin{array}{r}978 \\
1234 \\
939 \\
935 \\
1005\end{array}$ & $\begin{array}{l}25 \\
49 \\
21 \\
20 \\
28\end{array}$ & $\begin{array}{r}998 \\
1282 \\
987 \\
977 \\
1035\end{array}$ & $\begin{array}{l}21 \\
50 \\
20 \\
18 \\
26\end{array}$ & $\begin{array}{l}1042 \\
1322 \\
1012 \\
1010 \\
1019\end{array}$ & $\begin{array}{l}22 \\
49 \\
19 \\
18 \\
20\end{array}$ & $\begin{array}{l}1442 \\
1616 \\
1087 \\
1132 \\
1144\end{array}$ & $\begin{array}{l}42 \\
48 \\
16 \\
18 \\
19\end{array}$ \\
\hline $\begin{array}{l}1976 \\
1977 \\
1978 \\
1979 \\
1980\end{array}$ & $\begin{array}{l}1977 \\
1978 \\
1979 \\
1980 \\
1981\end{array}$ & $\begin{array}{r}835 \\
768 \\
1020 \\
1080 \\
835\end{array}$ & $\begin{array}{r}13 \\
9 \\
38 \\
45 \\
14\end{array}$ & $\begin{array}{r}841 \\
768 \\
1030 \\
1083 \\
835\end{array}$ & $\begin{array}{r}14 \\
8 \\
38 \\
45 \\
13\end{array}$ & $\begin{array}{r}842 \\
768 \\
1039 \\
1087 \\
839\end{array}$ & $\begin{array}{r}14 \\
8 \\
39 \\
45 \\
13\end{array}$ & $\begin{array}{r}846 \\
769 \\
1039 \\
1088 \\
842\end{array}$ & $\begin{array}{r}14 \\
8 \\
37 \\
45 \\
13\end{array}$ & $\begin{array}{r}865 \\
775 \\
1042 \\
1096 \\
850\end{array}$ & $\begin{array}{r}15 \\
7 \\
33 \\
43 \\
12\end{array}$ & $\begin{array}{r}887 \\
807 \\
1060 \\
1114 \\
874\end{array}$ & $\begin{array}{r}15 \\
7 \\
32 \\
42 \\
13\end{array}$ & $\begin{array}{r}952 \\
836 \\
1091 \\
1163 \\
898\end{array}$ & $\begin{array}{r}17 \\
7 \\
32 \\
43 \\
12\end{array}$ & $\begin{array}{r}979 \\
858 \\
1143 \\
1212 \\
924\end{array}$ & $\begin{array}{r}15 \\
7 \\
32 \\
41 \\
13\end{array}$ & $\begin{array}{r}988 \\
886 \\
1250 \\
1326 \\
1029\end{array}$ & $\begin{array}{r}12 \\
6 \\
30 \\
33 \\
14\end{array}$ \\
\hline $\begin{array}{l}1981 \\
1982 \\
1983 \\
1984 \\
1985\end{array}$ & $\begin{array}{l}1982 \\
1983 \\
1984 \\
1985 \\
1986\end{array}$ & $\begin{array}{r}674 \\
971 \\
1350 \\
1180 \\
1100\end{array}$ & $\begin{array}{l}5 \\
31 \\
56 \\
55 \\
48\end{array}$ & $\begin{array}{r}674 \\
985 \\
1350 \\
1183 \\
1103\end{array}$ & $\begin{array}{l}5 \\
33 \\
56 \\
54 \\
48\end{array}$ & $\begin{array}{r}677 \\
989 \\
1366 \\
1186 \\
1107\end{array}$ & $\begin{array}{l}5 \\
32 \\
56 \\
54 \\
48\end{array}$ & $\begin{array}{r}684 \\
1041 \\
1384 \\
1193 \\
1111\end{array}$ & $\begin{array}{r}5 \\
38 \\
56 \\
54 \\
48\end{array}$ & $\begin{array}{r}689 \\
1067 \\
1433 \\
1208 \\
1121\end{array}$ & $\begin{array}{r}5 \\
38 \\
55 \\
53 \\
46\end{array}$ & $\begin{array}{r}704 \\
1099 \\
1547 \\
1240 \\
1139\end{array}$ & $\begin{array}{r}5 \\
40 \\
55 \\
50 \\
44\end{array}$ & $\begin{array}{r}718 \\
1137 \\
1603 \\
1237 \\
1175\end{array}$ & $\begin{array}{r}4 \\
41 \\
56 \\
48 \\
45\end{array}$ & $\begin{array}{r}744 \\
1196 \\
1624 \\
1251 \\
1204\end{array}$ & $\begin{array}{r}4 \\
39 \\
55 \\
46 \\
40\end{array}$ & $\begin{array}{r}791 \\
1414 \\
1708 \\
1312 \\
1760\end{array}$ & $\begin{array}{r}4 \\
39 \\
51 \\
31 \\
54\end{array}$ \\
\hline $\begin{array}{l}1986 \\
1987 \\
1988 \\
1989 \\
1990\end{array}$ & $\begin{array}{l}1987 \\
1988 \\
1989 \\
1990 \\
1991\end{array}$ & $\begin{array}{r}1070 \\
1110 \\
1030 \\
821 \\
608\end{array}$ & $\begin{array}{r}44 \\
49 \\
40 \\
12 \\
1\end{array}$ & $\begin{array}{r}1070 \\
1117 \\
1030 \\
823 \\
608\end{array}$ & $\begin{array}{r}43 \\
49 \\
39 \\
12 \\
1\end{array}$ & $\begin{array}{r}1074 \\
1123 \\
1030 \\
827 \\
611\end{array}$ & $\begin{array}{r}43 \\
49 \\
38 \\
12 \\
1\end{array}$ & $\begin{array}{r}1079 \\
1131 \\
1036 \\
837 \\
615\end{array}$ & $\begin{array}{r}43 \\
49 \\
36 \\
12 \\
1\end{array}$ & $\begin{array}{r}1085 \\
1153 \\
1055 \\
840 \\
626\end{array}$ & $\begin{array}{r}40 \\
48 \\
36 \\
11 \\
1\end{array}$ & $\begin{array}{r}1105 \\
1197 \\
1080 \\
857 \\
644\end{array}$ & $\begin{array}{r}41 \\
47 \\
37 \\
11 \\
1\end{array}$ & $\begin{array}{r}1121 \\
1212 \\
1106 \\
866 \\
663\end{array}$ & $\begin{array}{r}38 \\
47 \\
36 \\
8 \\
1\end{array}$ & $\begin{array}{r}1151 \\
1272 \\
1146 \\
878 \\
684\end{array}$ & $\begin{array}{r}34 \\
47 \\
33 \\
8 \\
2\end{array}$ & $\begin{array}{r}1240 \\
1426 \\
1346 \\
949 \\
720\end{array}$ & $\begin{array}{r}28 \\
40 \\
34 \\
7 \\
2\end{array}$ \\
\hline $\begin{array}{l}1991 \\
1992\end{array}$ & $\begin{array}{l}1992 \\
1993\end{array}$ & $\begin{array}{l}921 \\
888\end{array}$ & $\begin{array}{l}26 \\
21\end{array}$ & $\begin{array}{l}925 \\
893\end{array}$ & $\begin{array}{l}26 \\
21\end{array}$ & $\begin{array}{l}931 \\
896\end{array}$ & $\begin{array}{l}26 \\
21\end{array}$ & $\begin{array}{l}934 \\
902\end{array}$ & $\begin{array}{l}26 \\
21\end{array}$ & $\begin{array}{l}945 \\
937\end{array}$ & $\begin{array}{l}26 \\
23\end{array}$ & $\begin{array}{r}974 \\
1045\end{array}$ & $\begin{array}{l}24 \\
31\end{array}$ & $\begin{array}{r}999 \\
1102\end{array}$ & $\begin{array}{l}22 \\
34\end{array}$ & $\begin{array}{l}1045 \\
1168\end{array}$ & $\begin{array}{l}23 \\
36\end{array}$ & $\begin{array}{l}1174 \\
1364\end{array}$ & $\begin{array}{l}22 \\
36\end{array}$ \\
\hline
\end{tabular}


HIGHEST MEAN DISCHARGE, IN CUBIC FEET PER SECOND, AND RANKING FOR THE FOLLOWING NUMBER OF CONSECUTIVE DAYS FOR PERIOD OCT TO SEP

WATER YFAR RANGF

19291928

19331933

19341934

19351935

19361936

19371937

381938

19391939

19401940

19411941

19421942

19451945

$1946 \quad 1946$

19471947

19481948

9491949

19501950

19511951

9521952

19531953

19541954

19551955

19561956

19571957

19581958

19591959

19601960

19611961

9621962

19631963

19641964

9651965

19661966

19671967

19681968

19691969

19701970

19711971

$1972 \quad 1972$

19731973

19741974

19751975

19761976

$1977 \quad 1977$

$1978 \quad 1978$

19791979

19801980

$1981 \quad 1981$

19821982

19831983

19841984

19851985

19861986

19871987

19881988

9891989

19901990

19911991

19921992

19931993
1
$4750 \quad 24$

453028

$2810 \quad 46$

111003

628016

$4060 \quad 34$

362039

3025

193057

$2940 \quad 44$

$7280 \quad 10$

333040

$9160 \quad 5$

382036

79806

118002

42019

$7440 \quad 9$

201056

$4400 \quad 30$

508021

$1300 \quad 62$

91863

$4090 \quad 33$

$2450 \quad 50$

$7970 \quad 8$

629015

$3750 \quad 38$

172059

206055

$16900 \quad 1$

585017

$5850 \quad 17$

382037
$6500 \quad 14$

$2710 \quad 47$

$7980 \quad 7$

$3240 \quad 41$

$4440 \quad 29$

653013

2830
2330

224053

$2240 \quad 54$

$4110 \quad 32$

244051

469026

$1720 \quad 60$

$3150 \quad 43$

390035

503022

575018

319042

$4940 \quad 23$

$7030 \quad 12$

$2530 \quad 49$

146061

$\begin{array}{lll}4660 & 27\end{array}$

$2570 \quad 48$

106004
3
$4740 \quad 24$

474024
453027

279746

$10580 \quad 3$

600716

$3863 \quad 35$

$3573 \quad 39$

$4133 \quad 31$

193057

289744

$\begin{array}{lll}7060 & 11 \\ 1733 & 58\end{array}$

$\begin{array}{ll}1733 & 58 \\ 3283 & 40\end{array}$

86605

$3767 \quad 37$

76538

7143012
533319

706710

72809

199756

$4400 \quad 29$

501720

$1287 \quad 62$

91063

401333

244050

613315

370338

170360

205355

$16070 \quad 1$

$5823 \quad 17$

380036

$6317 \quad 14$

$\begin{array}{lll}2690 & 47\end{array}$

322041

440030

641313

$\begin{array}{ll}2810 & 45 \\ 2317 & 51\end{array}$

216354

221753

$4063 \quad 32$

228352

452728

$1710 \quad 59$

310043

$3877 \quad 34$

497721

568018

$3147 \quad 42$

490023

686312

249349

145361

458326

254748

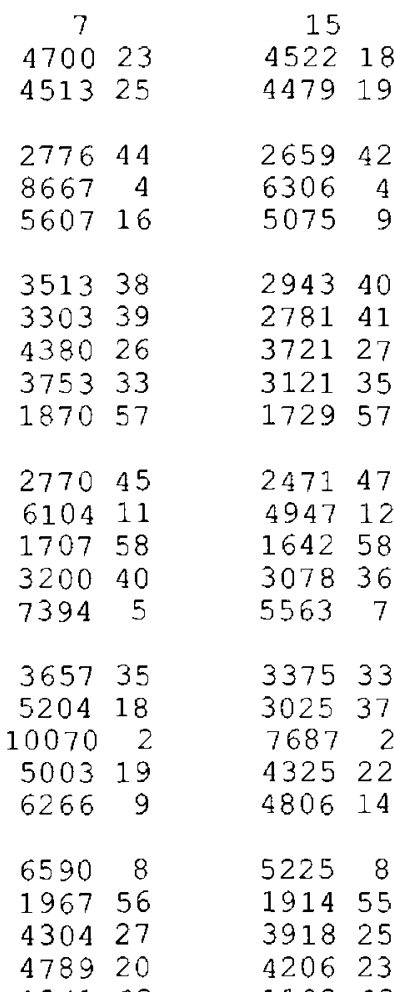

126162

89063

90863

362036

243348

61915

358437

166360

203054

13900

462924

$5626 \quad 14$

$\begin{array}{lll}3731 & 34\end{array}$

565313

$\begin{array}{rr}2630 & 47 \\ 6909 & 7\end{array}$

311441

422129

587712

272446
213753

202155

$2144 \quad 52$

382431

221351

414130

167759

292143

380132

475421

536317

298642

473622

621610

242750

143361

427328

4433
87213

61175

$4689=6$

$3445 \quad 32$

159759

196752

$\begin{array}{lr}9913 & 1 \\ 4064 & 24\end{array}$

498711

349330

470015

249346

299038

377726

485713

252345
191754

184356

194653

325134

211151

345831

156160

$2544 \quad 44$

356229

437721

$4673 \quad 17$

261743

443920

507110

227649

136961

370528

217350

\begin{tabular}{lr}
\multicolumn{2}{c}{30} \\
4169 & 11 \\
4214 & 10 \\
& \\
2270 & 44 \\
4460 & 6 \\
4421 & 7 \\
& \\
2441 & 40 \\
2478 & 38 \\
2961 & 33 \\
2470 & 39 \\
1705 & 55 \\
& \\
2139 & 47 \\
4415 & 8 \\
1557 & 58 \\
2864 & 34 \\
3955 & 13 \\
3152 & 27 \\
2513 & 37 \\
6433 & 1 \\
3470 & 23 \\
3387 & 24 \\
3936 & 14 \\
1761 & 52 \\
3179 & 26 \\
3547 & 22 \\
1113 & 62 \\
& \\
861 & 63 \\
2299 & 43 \\
2202 & 45 \\
4949 & 3 \\
3899 & 15 \\
3120 & 29 \\
1509 & 59 \\
1858 & 51 \\
6344 & 2 \\
3803 & 20 \\
& \\
3
\end{tabular}

$3781^{60}$

326611

183448

$3210 \quad 12$

288422

$2015 \quad 42$

223238

226336

199143

157453

197944

37985

14505

$2780 \quad 23$

292020

212041

$\begin{array}{lll}5161 & 1\end{array}$

268224

$3036 \quad 16$

153954

237232

292219

102962

$834 \quad 63$

186347

$2168 \quad 39$

$3956 \quad 3$

64033

313413

258626

141158

16605

42632

$4287 \quad 9$

310430

370521

$\begin{array}{rr}2170 & 46 \\ 4608 & 4\end{array}$ 
THIS PAGE INTENTIONALLY BLANK 
LOCATION.--Lat $29^{\circ} 48^{\prime}$, long $82^{\circ} 55^{\prime}$, in sec. 16 or 17, T.8 S., R. 14 E., on left bank at Rock Bluff Ferry, 4 '1/2 mi northwest of Bell and 10 miles downstream from Santa Fe River.

DRAINAGE AREA.--9,260 $\mathrm{mi}^{2}$, approximately

PERIOD OF RECORD,--June 1932 to December 1956 (discontinued).

GAGE.--Water-stage recorder. Datum of gage is $3.60 \mathrm{ft}$ above National Geodetic Vertical Datum of 1929 (levels by Corps of Engineers).

REVISIONS (water years).--WSP 822: 1928(M).

REMARKS.--Records good.
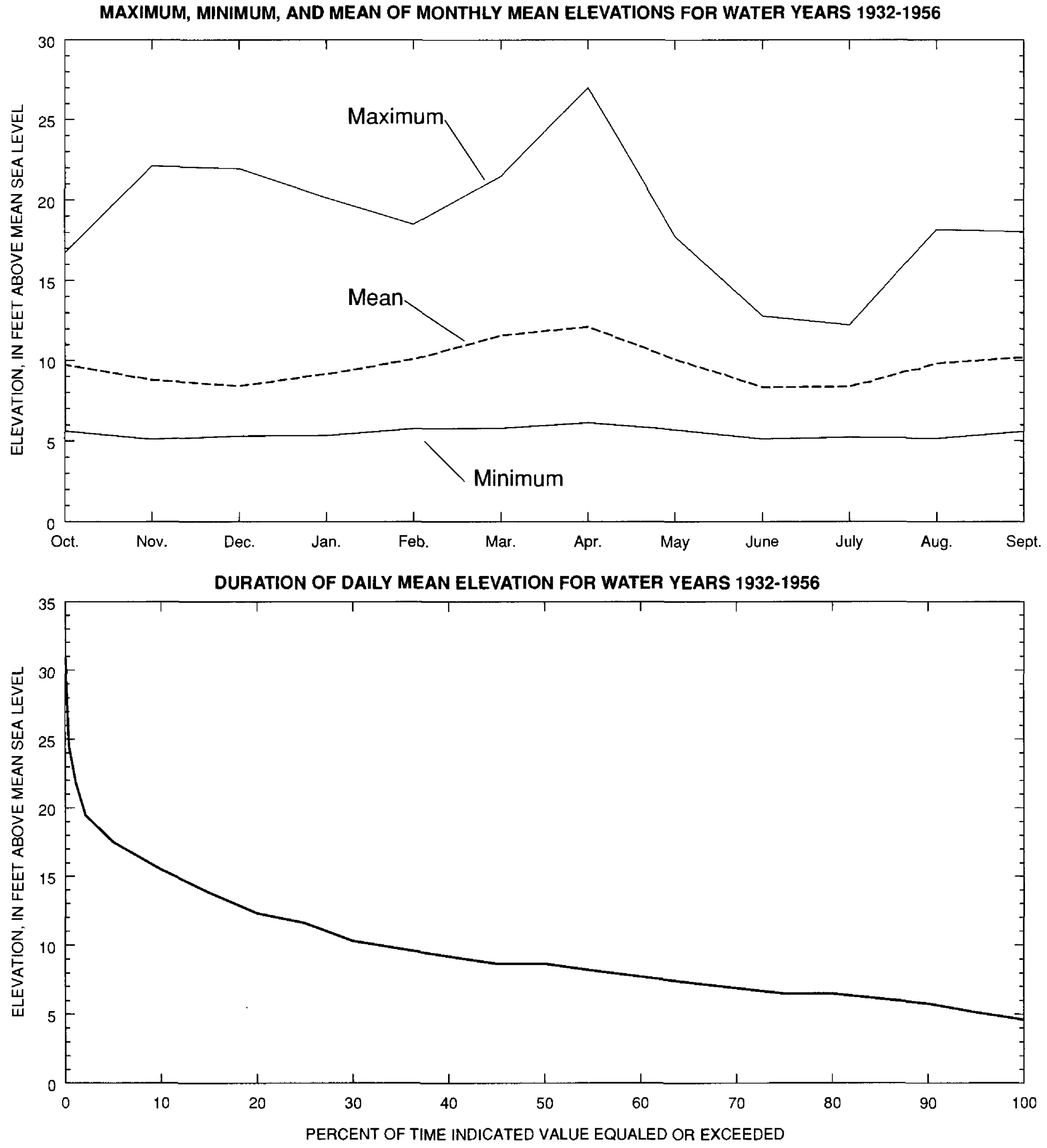
SUMMARY STATISTICS, IN CUBIC FEET PER SECOND UNLESS OTHERWISE INDICATED, FOR WATER YEARS $1932-1956$

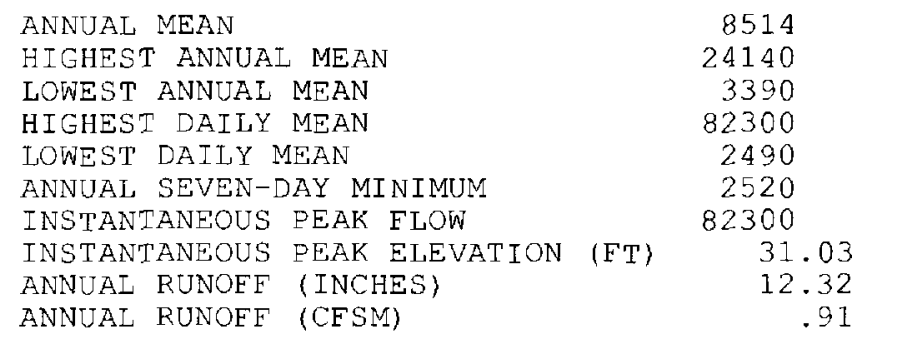

8514
24140
3390
82300
2490
2520
82300
31.03
12.32
.91

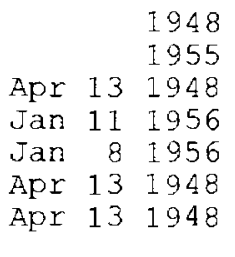

MAXIMUM, MINIMUM, AND MEAN OF MONTHLY MEAN DISCHARGES FOR WATER YEARS 1932-1956

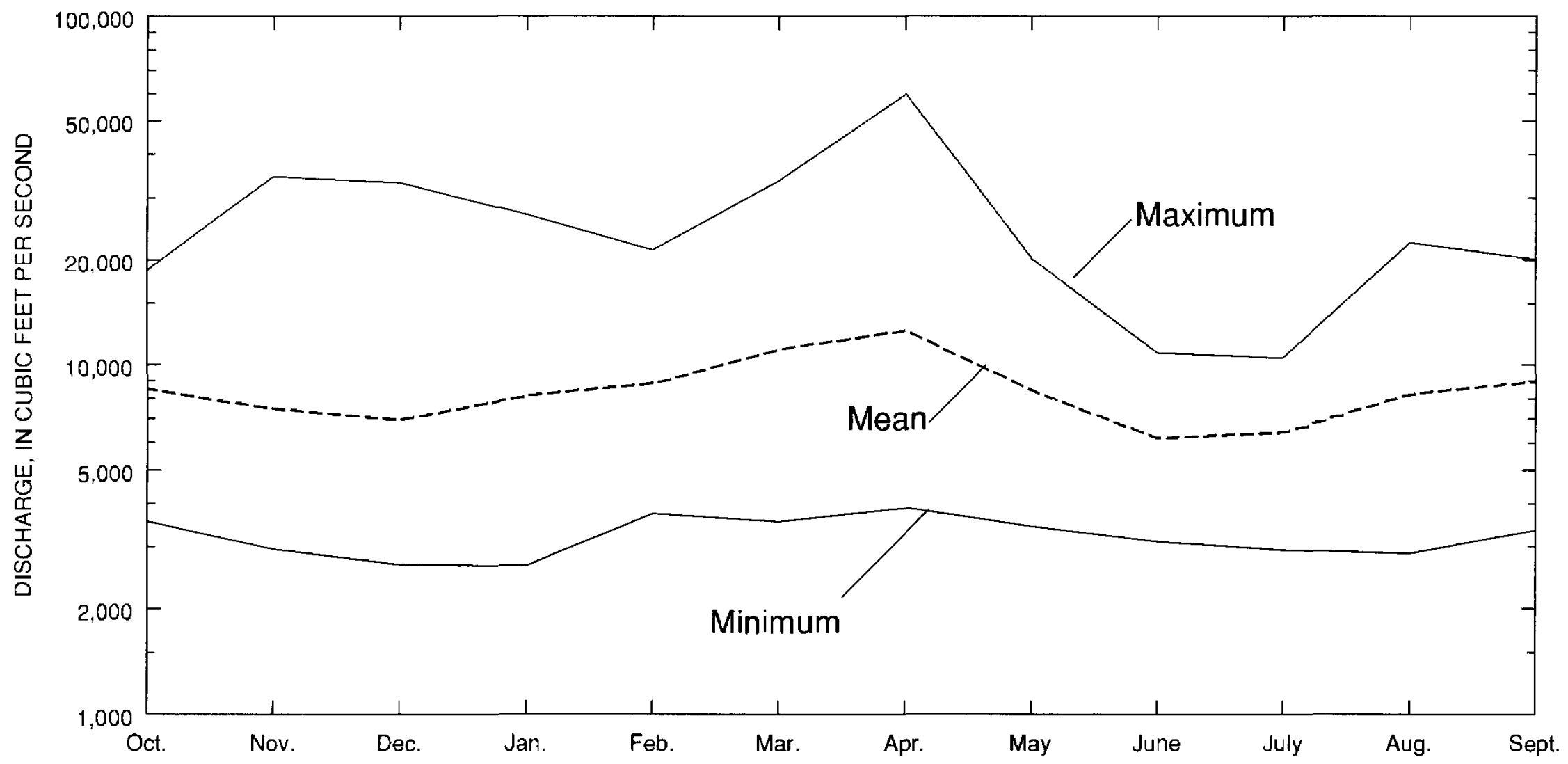

DURATION OF DAILY MEAN DISCHARGE FOR WATER YEARS 1932-1956

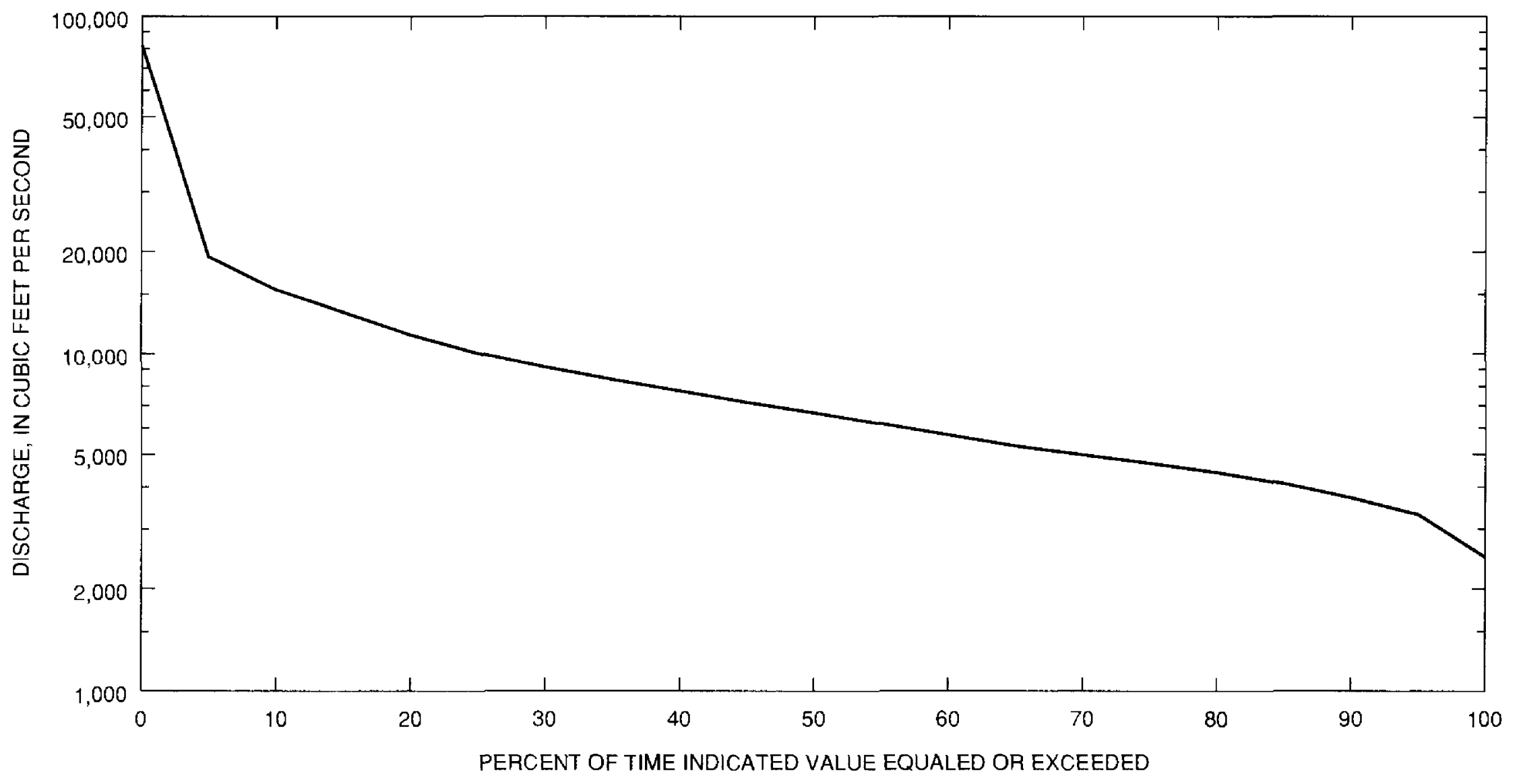


SUMMARY OF MONTHLY MEAN ELEVATION AND DISCHARGE STATISTICS FOR WATER YEARS 1932-1956

\begin{tabular}{lrrrrrr} 
& \multicolumn{3}{c}{ ELEVATIONS, } & \multicolumn{3}{c}{ DISCHARGE, } \\
& \multicolumn{2}{c}{ EEET ABOVE SEA LEVEL } & \multicolumn{2}{c}{ CUBIC EEET PER SECOND } \\
MONTH & MAXIMUM & MINIMUM & MEAN & MAXIMUM & MINIMUM & MEAN \\
OCTOBER & 16.68 & 5.62 & 9.74 & 18550 & 3550 & 8525.56 \\
NOVEMBER & 22.08 & 5.10 & 8.79 & 34280 & 2950 & 7478.88 \\
DECEMBER & 21.92 & 5.31 & 8.42 & 32940 & 2667 & 6942.00 \\
JANUARY & 20.11 & 5.34 & 9.17 & 26750 & 2648 & 8167.54 \\
FEBRUARY & 18.46 & 5.77 & 10.11 & 21170 & 3732 & 8839.25 \\
MARCH & 21.45 & 5.80 & 11.59 & 33390 & 3544 & 11035.08 \\
APRIL & 26.94 & 6.13 & 12.12 & 59430 & 3882 & 12554.83 \\
MAY & 17.69 & 5.70 & 10.09 & 20050 & 3437 & 8450.13 \\
JUNE & 12.76 & 5.12 & 8.34 & 10740 & 3106 & 6172.84 \\
JULY & 12.20 & 5.22 & 8.37 & 10400 & 2937 & 6426.20 \\
AUGUST & 18.13 & 5.15 & 9.84 & 22260 & 2870 & 8265.44 \\
SEPTEMBER & 18.00 & 5.59 & 10.21 & 19960 & 3348 & 8939.92
\end{tabular}

\section{DURATION OF DAILY MEAN VALUES FOR WATER YEARS 1932-1956}

PERCENT

OE TIME

EQTALD

DEC

JAN EEB

MAR

APR

MAY

JUNE

JULY

AUG

SEPT

$\begin{array}{rrrr}95.0 & 5.1 & 5.5 & 5.0 \\ 90.0 & 5.7 & 5.7 & 5.2 \\ 85.0 & 6.1 & 6.2 & 5.5 \\ 80.0 & 6.4 & 6.5 & 6.0 \\ 75.0 & 6.4 & 6.7 & 6.3 \\ 70.0 & 6.8 & 6.7 & 6.3 \\ 65.0 & 7.2 & 7.0 & 6.6 \\ 60.0 & 7.7 & 7.3 & 6.6 \\ 55.0 & 8.1 & 7.9 & 7.0 \\ 50.0 & 8.6 & 8.2 & 8.0 \\ 45.0 & 8.6 & 8.6 & 8.8 \\ 40.0 & 9.1 & 9.3 & 9.3 \\ 35.0 & 9.7 & 11.0 & 9.3 \\ 30.0 & 10.3 & 11.9 & 9.7 \\ 25.0 & 11.6 & 12.9 & 10.3 \\ 20.0 & 12.3 & 13.4 & 10.3 \\ 15.0 & 13.8 & 14.0 & 11.3 \\ 10.0 & 15.5 & 14.5 & 11.8 \\ 5.0 & 17.5 & 15.1 & 13.7\end{array}$

$\begin{array}{rr}4.8 & 4.8 \\ 5.0 & 5.3 \\ 5.3 & 5.5 \\ 5.8 & 5.8 \\ 5.8 & 6.1 \\ 6.1 & 6.1 \\ 6.4 & 6.4 \\ 6.4 & 6.7 \\ 6.7 & 7.4 \\ 7.4 & 7.4 \\ 7.8 & 7.8 \\ 8.2 & 8.2 \\ 8.6 & 9.0 \\ 8.6 & 9.5 \\ 9.0 & 11.0 \\ 10.0 & 12.7 \\ 10.5 & 14.0 \\ 11.0 & 15.4 \\ 15.5 & 17.9\end{array}$

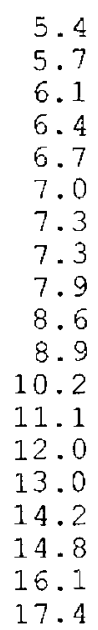

$\begin{array}{rr}5.9 & 5.8 \\ 6.8 & 6.1 \\ 7.1 & 6.4 \\ 7.1 & 7.1 \\ 7.4 & 7.5 \\ 7.8 & 8.3 \\ 8.5 & 8.8 \\ 9.4 & 9.2 \\ 9.8 & 9.7 \\ 10.3 & 10.3 \\ 11.3 & 12.0 \\ 11.9 & 12.7 \\ 13.0 & 14.1 \\ 13.6 & 14.9 \\ 14.3 & 15.7 \\ 15.0 & 15.7 \\ 15.7 & 16.5 \\ 17.2 & 17.4 \\ 19.8 & 20.4\end{array}$
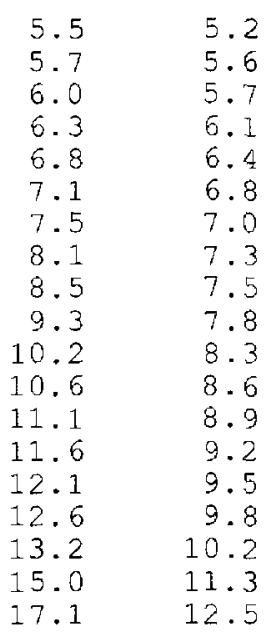

DISCHARGE IN CUBIC EEET PER SECOND

$\begin{array}{rrrr}95.0 & 3310.7 & 3526.3 & 3133.6 \\ 90.0 & 3711.0 & 3825.9 & 3301.7 \\ 85.0 & 4091.5 & 4197.2 & 3633.3 \\ 80.0 & 4411.7 & 4481.8 & 3986.2 \\ 75.0 & 4698.1 & 4702.2 & 4257.4 \\ 70.0 & 4982.1 & 4887.0 & 4572.9 \\ 65.0 & 5287.2 & 5213.7 & 4792.5 \\ 60.0 & 5700.6 & 5668.4 & 4980.0 \\ 55.0 & 6171.9 & 6144.8 & 5436.7 \\ 50.0 & 6664.2 & 6561.3 & 6374.5 \\ 45.0 & 7160.1 & 6962.0 & 7068.7 \\ 40.0 & 7733.2 & 8591.0 & 7580.9 \\ 35.0 & 8368.2 & 9784.7 & 7887.7 \\ 30.0 & 9101.1 & 11094.3 & 8199.2 \\ 25.0 & 10030.8 & 12092.0 & 8563.6 \\ 20.0 & 11352.3 & 13362.8 & 8988.0 \\ 15.0 & 13259.1 & 14173.8 & 9675.8 \\ 10.0 & 15439.1 & 15092.9 & 10381.2 \\ 5.0 & 19306.2 & 17208.3 & 11975.0\end{array}$

2961.
3128.9
3350.2
3610.0
3870.4
4131.
4378.0
4597.4
4807.8
5602.
5937.
6260.0
6647.5
7035.0
7525.
8225.2
8945.4
10281.8
15525.0

\begin{tabular}{|c|c|}
\hline & \\
\hline $\begin{array}{l}2989.1 \\
3384.0\end{array}$ & 3525.1 \\
\hline 3683.5 & 4237.0 \\
\hline 3942.9 & 4555.5 \\
\hline 4221.9 & 4874.3 \\
\hline 4485.2 & 5110.2 \\
\hline 4693.8 & 5359.7 \\
\hline 5084.7 & 5789.0 \\
\hline 53.1 & 6501.2 \\
\hline 574.3 & 6960.0 \\
\hline 5909.5 & 7482.0 \\
\hline 6498.0 & 8878.0 \\
\hline 7435.3 & 9868.9 \\
\hline 383.4 & 10666.9 \\
\hline 10563.1 & 11838.1 \\
\hline 5.5 & 13374.4 \\
\hline 3.6 & 14950.0 \\
\hline 0.0 & 16680.0 \\
\hline 770.5 & 20181.1 \\
\hline
\end{tabular}

3910.8
4801.8
5022.8
5286.3
5623.8
6310.6
7226.8
7618.0
8045.6
8521.2
9186.3
10541.8
11787.5
13102.5
14257.4
15209.2
16176.4
18142.9
25697.5

3773.0
4139.5
4657.3
5156.9
5943.3
6502.5
6919.5
7479.5
8100.0
8760.0
9879.1
11346.2
13007.9
14283.8
15688.2
16817.6
18958.3
21884.6
27150.0

3524.6
3769.8
4078.4
4618.9
4920.0
5206.7
5592.8
6107.7
6592.9
7197.0
7909.7
8591.8
9157.6
9793.1
10467.5
11360.0
12570.0
15060.0
18821.8

3205.2 3542.0 3827.5 4175.2 4397.4 4569.5

4806.5
4982.9 5405.0 5823.7

6185.0

6572.5 6958.1

7261.4
7538.9

7960.0

8335.0

9274.0

18821.8

10925.0

$\begin{array}{rrr}3278.9 & 3663.7 & 3446.0 \\ 4102.5 & 4146.6 & 3929.3 \\ 4344.0 & 4438.3 & 4375.4 \\ 4490.0 & 4714.1 & 4729.1 \\ 4617.6 & 5219.2 & 4975.9 \\ 4745.2 & 5714.1 & 5213.8 \\ 4922.8 & 6030.4 & 5507.7 \\ 5124.4 & 6573.8 & 5909.7 \\ 5372.0 & 6856.7 & 6355.3 \\ 5819.3 & 7148.7 & 6793.3 \\ 6205.3 & 7448.7 & 8022.2 \\ 6816.4 & 7938.0 & 9322.7 \\ 7169.5 & 8424.4 & 10304.5 \\ 7504.9 & 9005.8 & 11255.2 \\ 7889.4 & 9513.7 & 12213.8 \\ 8416.7 & 10223.6 & 13324.0 \\ 9123.3 & 11768.7 & 14936.4 \\ 9795.0 & 14725.0 & 16362.5 \\ 10641.7 & 18695.6 & 18878.9\end{array}$


LOWEST MEAN ELEVATION, IN FEET, AND RANKING FOR THE FOLLOWING NUMBER OF CONSECUTIVE DAYS

FOR PERIOD APR TO MAR

\section{WATER YEAR}

RANGE

$\begin{array}{ll}1933 & 1934 \\ 1936 & 1937\end{array}$

19371938

19431944

19451946

$1947 \quad 1948$

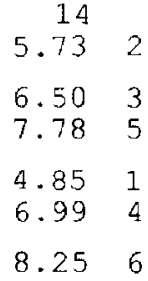

\begin{tabular}{ll}
\multicolumn{3}{c}{30} \\
5.76 & 2 \\
6.64 & 3 \\
7.93 & 5 \\
4.91 & 1 \\
7.11 & 4 \\
8.76 & 6
\end{tabular}

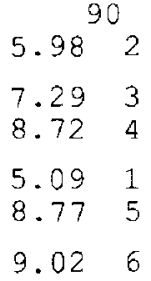

120
$6.15^{2}$

$7.48 \quad 3$

5.271

$9.24 \quad 5$

9.426
$6.63^{183}$

7.843

10.14

$\begin{array}{ll}5.68 & 1 \\ 12.3 & 6\end{array}$

11.25

HIGHEST MEAN ELEVATION, IN FEET, AND RANKING FOR THE FOLLOWING NUMBER OF CONSECUTIVE DAYS FOR PERIOD OCT TO SEP

\section{WATER YEAR}

RANGE
19331933

19341934

19361936

19371937

19381938

19421942

$1944 \quad 1944$

19561956

\begin{tabular}{|c|c|c|c|c|c|}
\hline 1 & & 3 & & 7 & \\
\hline 19.0 & 3 & 18.9 & 3 & 18.9 & 3 \\
\hline 14.9 & 7 & 14.7 & 7 & 14.3 & 7 \\
\hline 17.5 & 5 & 17.2 & 5 & 16.7 & 5 \\
\hline 19.1 & 2 & 19.1 & 2 & 19.0 & 2 \\
\hline 15. & 6 & 15.5 & 6 & 15.4 & 6 \\
\hline 20.9 & 1 & 20.8 & 1 & 20.8 & 1 \\
\hline & 4 & 18.8 & 4 & & \\
\hline
\end{tabular}

$\begin{array}{rr}15 & \\ 18.7 & 3 \\ 13.0 & 7 \\ 15.5 & 5 \\ 18.8 & 2 \\ 15.1 & 6 \\ 20.6 & 1 \\ 18.4 & 4 \\ & \end{array}$

\begin{abstract}
$18.1^{30}$
\end{abstract}
$17.3^{60} 2$

\subsection{7}

15.15

14.35

$\begin{array}{ll}18.1 & 2 \\ 14.2 & 6\end{array}$

$\begin{array}{ll}16.6 & 4 \\ 12.4 & 6\end{array}$

20.21

10.78

9.348

$\begin{array}{ll}19.0 & 1 \\ 17.3 & 3\end{array}$

$7.90 \quad 8$

\begin{tabular}{llll}
\multicolumn{2}{c}{90} & \multicolumn{2}{c}{120} \\
17.1 & 2 & 16.0 & 2 \\
10.1 & 7 & 9.50 & 7 \\
13.9 & 5 & 13.0 & 5 \\
15.6 & 4 & 14.6 & 3 \\
11.4 & 6 & 10.9 & 6 \\
17.9 & 1 & 17.3 & 1 \\
15.6 & 3 & 14.2 & 4 \\
7.55 & 8 & 7.42 & 8
\end{tabular}

183

8.327

$11.4 \quad 5$

$\begin{array}{ll}12.9 & 4 \\ 10.1 & 6\end{array}$

15.001

14.22

7.118

LOWEST MEAN DISCHARGE, IN CUBIC FEET PER SECOND, AND RANKING FOR THE FOLLOWING NUMBER OF

WATER YEAR

\begin{tabular}{|c|c|c|c|}
\hline & ANGE & 1 & \\
\hline 1933 & 1934 & 3530 & 8 \\
\hline 1934 & 1935 & 3380 & 6 \\
\hline 1935 & 1936 & 3020 & 4 \\
\hline 1936 & 1937 & 4260 & 13 \\
\hline 1937 & 1938 & 5440 & 19 \\
\hline 1938 & 1939 & 3630 & 10 \\
\hline 1939 & 1940 & 4090 & 12 \\
\hline 1940 & 1941 & 3270 & 5 \\
\hline 1941 & 1942 & 3510 & 7 \\
\hline 1942 & 1943 & 4410 & 14 \\
\hline 1943 & 1944 & 2790 & 2 \\
\hline 1944 & 1945 & 5900 & 21 \\
\hline 1945 & 1946 & 4680 & 16 \\
\hline 1946 & 1947 & 5850 & 20 \\
\hline 1947 & 1948 & 5950 & 22 \\
\hline 1948 & 1949 & 8110 & 23 \\
\hline 1949 & 1950 & 4850 & 17 \\
\hline & 195 & 4530 & 15 \\
\hline 951 & 195 & 3730 & 11 \\
\hline 152 & 195 & 3560 & 9 \\
\hline & 1954 & 4960 & 18 \\
\hline & 1955 & 2900 & 3 \\
\hline & 195 & 2490 & \\
\hline
\end{tabular}
CONSECUTIVE DAYS FOR PERIOD APR TO MAR

\begin{tabular}{rrrrrrrrrr}
3 & & 7 & & 14 & 30 & \multicolumn{2}{c}{60} \\
3603 & 9 & 3677 & 9 & 3694 & 9 & 3723 & 8 & 3791 & 7 \\
3380 & 6 & 3426 & 6 & 3452 & 6 & 3522 & 6 & 3580 & 6 \\
3020 & 4 & 3060 & 4 & 3095 & 4 & 3146 & 4 & 3298 & 4 \\
& & & & & & & & & \\
4260 & 13 & 4273 & 13 & 4346 & 13 & 4479 & 13 & 5031 & 15 \\
5440 & 19 & 5503 & 19 & 5636 & 19 & 5799 & 19 & 6370 & 20 \\
3660 & 10 & 3707 & 10 & 3759 & 10 & 3846 & 10 & 4047 & 10 \\
4120 & 12 & 4167 & 12 & 4206 & 12 & 4249 & 12 & 4410 & 12 \\
3270 & 5 & 3316 & 5 & 3333 & 5 & 3415 & 5 & 3539 & 5 \\
& & & & & & & & & \\
3537 & 7 & 3606 & 7 & 3656 & 7 & 3741 & 9 & 4072 & 11 \\
4443 & 14 & 4496 & 14 & 4510 & 14 & 4563 & 14 & 4670 & 13 \\
2843 & 2 & 2916 & 2 & 2996 & 2 & 3038 & 2 & 3087 & 2 \\
5967 & 21 & 6059 & 21 & 6365 & 22 & 7035 & 22 & 7775 & 22 \\
4680 & 16 & 4731 & 16 & 4764 & 16 & 4871 & 16 & 5774 & 18 \\
& & & & & & & & & \\
5883 & 20 & 5893 & 20 & 5932 & 20 & 6043 & 20 & 6299 & 19 \\
6077 & 22 & 6126 & 22 & 6179 & 21 & 6670 & 21 & 6806 & 21 \\
8110 & 23 & 8173 & 23 & 8267 & 23 & 8532 & 23 & 9746 & 23 \\
4887 & 17 & 4913 & 17 & 4968 & 17 & 5015 & 17 & 5199 & 16 \\
4530 & 15 & 4530 & 15 & 4561 & 15 & 4710 & 15 & 4823 & 14 \\
& & & & & & & & & \\
3763 & 11 & 3801 & 11 & 3823 & 11 & 3893 & 11 & 4040 & 9 \\
3573 & 8 & 3617 & 8 & 3659 & 8 & 3722 & 7 & 3840 & 8 \\
4967 & 18 & 4986 & 18 & 5050 & 18 & 5203 & 18 & 5633 & 17 \\
2957 & 3 & 3024 & 3 & 3085 & 3 & 3115 & 3 & 3146 & 3 \\
2497 & 1 & 2521 & 1 & 2552 & 1 & 2585 & 1 & 2645 & 1
\end{tabular}

HIGHEST MEAN DISCHARGE, IN CUBIC FEET PER SECOND, AND RANKING FOR THE FOLLOWING NUMBER OF

\begin{tabular}{rr}
\multicolumn{2}{c}{90} \\
3887 & 7 \\
3663 & 5 \\
3453 & 4 \\
& \\
5136 & 14 \\
6672 & 20 \\
4201 & 9 \\
4582 & 12 \\
3695 & 6 \\
& \\
4514 & 11 \\
4813 & 13 \\
3161 & 2 \\
8656 & 22 \\
6557 & 19 \\
& \\
6532 & 18 \\
6924 & 21 \\
10170 & 23 \\
5475 & 16 \\
5209 & 15 \\
& \\
4310 & 10 \\
3969 & 8 \\
6183 & 17 \\
3182 & 3 \\
2724 & 1
\end{tabular}

$\begin{array}{rr} & 120 \\ 4026 & 7 \\ 3801 & 5 \\ 3683 & 4 \\ & \\ 5328 & 14 \\ 6896 & 17 \\ 4431 & 9 \\ 4806 & 11 \\ 3882 & 6 \\ & \\ 5041 & 13 \\ 5025 & 12 \\ 3313 & 3 \\ 8888 & 22 \\ 7219 & 20 \\ & \\ 6914 & 18 \\ 7321 & 21 \\ 10730 & 23 \\ 5826 & 16 \\ 5432 & 15 \\ 4468 & 10 \\ 4143 & 8 \\ 6983 & 19 \\ 3252 & 2 \\ 2848 & 1\end{array}$

\begin{tabular}{rr}
\multicolumn{3}{r}{183} \\
4477 & 7 \\
4154 & 4 \\
6383 & 15 \\
& \\
5704 & 12 \\
8407 & 17 \\
4913 & 8 \\
6141 & 13 \\
4260 & 6 \\
& \\
5222 & 10 \\
5441 & 11 \\
3678 & 3 \\
9412 & 20 \\
0900 & 23 \\
& \\
8926 & 19 \\
9667 & 21 \\
0590 & 22 \\
7122 & 16 \\
6150 & 14 \\
& \\
5122 & 9 \\
4244 & 5 \\
8594 & 18 \\
3418 & 2 \\
3134 & 1
\end{tabular}
CONSECUTIVE DAYS FOR PERIOD OCT TO SEP

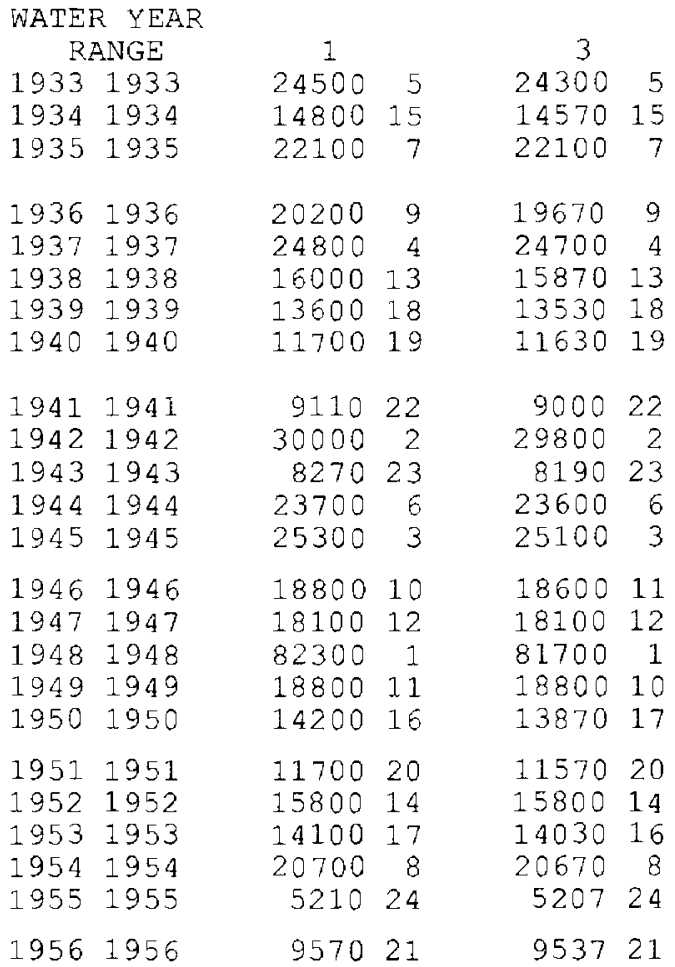

\begin{tabular}{rrrrr}
7 & & \multicolumn{2}{r}{15} \\
24030 & 5 & 23450 & 5 \\
13870 & 16 & & 12160 & 18 \\
21970 & 7 & 21570 & 7 \\
& & & \\
18410 & 11 & & 16070 & 12 \\
24540 & 4 & 23760 & 4 \\
15700 & 14 & 15140 & 14 \\
13300 & 17 & 12570 & 16 \\
11500 & 19 & & 11160 & 19 \\
& & & \\
8770 & 22 & & 8157 & 22 \\
29660 & 2 & & 29160 & 2 \\
8133 & 23 & & 7888 & 23 \\
23340 & 6 & 22430 & 6 \\
24960 & 3 & 24280 & 3 \\
18540 & 10 & 18390 & 9 \\
17900 & 12 & 17350 & 11 \\
79310 & 1 & 71990 & 1 \\
18660 & 9 & 18150 & 10 \\
13300 & 18 & 12470 & 17 \\
11310 & 20 & 10680 & 20 \\
15800 & 13 & 15770 & 13 \\
13900 & 15 & 13490 & 15 \\
20530 & 8 & 20010 & 8 \\
5141 & 24 & 4822 & 24 \\
9391 & 21 & 8705 & 21
\end{tabular}

\begin{tabular}{rr}
\multicolumn{2}{c}{30} \\
21970 & 4 \\
10210 & 18 \\
17760 & 9 \\
& \\
15130 & 13 \\
21940 & 5 \\
13890 & 14 \\
11130 & 16 \\
10160 & 19 \\
& \\
7207 & 23 \\
27870 & 2 \\
7847 & 21 \\
21210 & 6 \\
22820 & 3 \\
18100 & 8 \\
16800 & 11 \\
60040 & 1 \\
16860 & 10 \\
11120 & 17 \\
9871 & 20 \\
15450 & 12 \\
12680 & 15 \\
18220 & 7 \\
3980 & 24 \\
7272 & 22
\end{tabular}

\begin{tabular}{rr}
\multicolumn{2}{c}{60} \\
19850 & 4 \\
8678 & 19 \\
11850 & 13 \\
& \\
13960 & 12 \\
18600 & 6 \\
11330 & 14 \\
10000 & 16 \\
8968 & 18 \\
& \\
6452 & 22 \\
24380 & 2 \\
6949 & 21 \\
19460 & 5 \\
21440 & 3 \\
16720 & 7 \\
15710 & 8 \\
48210 & 1 \\
14780 & 10 \\
9515 & 17 \\
8386 & 20 \\
14430 & 11 \\
10420 & 15 \\
14790 & 9 \\
3712 & 24 \\
5764 & 23
\end{tabular}

\begin{tabular}{rr}
\multicolumn{2}{c}{90} \\
19370 & 3 \\
8230 & 19 \\
9241 & 16 \\
13430 & 10 \\
16740 & 5 \\
10100 & 13 \\
9476 & 14 \\
8310 & 18 \\
& \\
6091 & 22 \\
21830 & 2 \\
6567 & 21 \\
16540 & 6 \\
17400 & 4 \\
14670 & 8 \\
13760 & 9 \\
38790 & 1 \\
14770 & 7 \\
8523 & 17 \\
7286 & 20 \\
12850 & 12 \\
9321 & 15 \\
12900 & 11 \\
3792 & 24 \\
5402 & 23
\end{tabular}

$\begin{array}{rr} & 120 \\ 17480 & 3 \\ 7570 & 18 \\ 7721 & 17 \\ & \\ 12170 & 12 \\ 15050 & 4 \\ 9401 & 13 \\ 8921 & 14 \\ 7515 & 19 \\ 5542 & 22 \\ 20380 & 2 \\ 6283 & 21 \\ 14410 & 5 \\ 14340 & 6 \\ 13650 & 9 \\ 12310 & 11 \\ 35200 & 1 \\ 14180 & 7 \\ 7825 & 16 \\ 6781 & 20 \\ 12620 & 10 \\ 8610 & 15 \\ 13920 & 8 \\ 3680 & 24 \\ 5259 & 23\end{array}$


LOCATION.--Lat $29^{\circ} 35^{\prime} 22^{\prime \prime}$, long $82^{\circ} 56^{\prime} 12^{\prime \prime}$, in NW $1 / 4$ sec.29, T.10 S., R.14 E., Levy County, Hydrologic Unit 03110205 , on left bank about $400 \mathrm{ft}$ downstream from Fort Fannin Bridge on U.S. Highway 19, 2.0 mi southwest of Wilcox and 33 mi upstream from mouth.

DRAINAGE AREA.--9,640 $\mathrm{mi}^{2}$, approximately, includes part of watershed in Okefenokee Swamp which is indeterminate.

PERIOD OF RECORD.--October 1930 to September 1931, October 1941 to 1993 . Monthly discharge only for some periods, published in WSP 1304.

REVISED RECORDS.--WSP 1905: WDR FL-75-1: Drainage area.

GAGE.--Water-stage recorder. Datum of gage is $0.53 \mathrm{ft}$ below National Geodetic Vertical Datum of 1929. Prior to July 4,1931 , nonrecording gage at site $400 \mathrm{ft}$ upstream at present datum. July 4 to Sept. 30, 1931, and Mar. 26 to May 14, 1942, water-stage recorder, and May 15, 1942 to Jan. 24, 1951, nonrecording gage at present site and datum. Since Feb. 1, 1951, auxiliary water-stage recorder about 9.0 mi downstream from base gage. Datum of auxiliary gage is $2.99 \mathrm{ft}$ below National Geodetic Vertical Datum of 1929.

REMARKS.--Records fair. Flow generally affected by tide when discharge is less than $17,500 \mathrm{ft}^{3} / \mathrm{s}$.
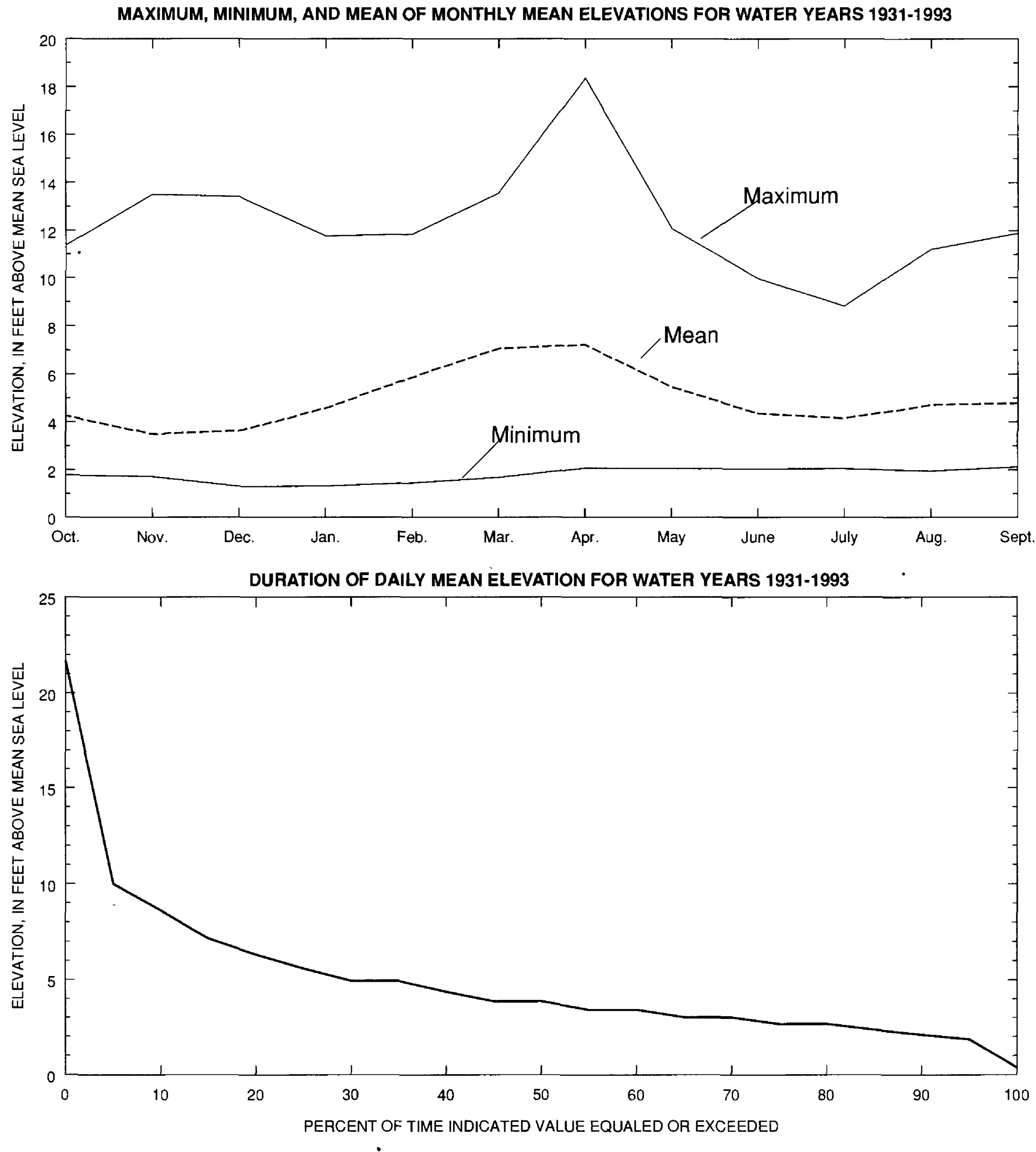

SUMMARY STATISTICS, IN CUBIC FEET PER SECOND UNLESS OTHERWISE INDICATED,
FOR WATER YEARS 1931 - 1993

\begin{tabular}{|c|c|c|c|}
\hline AL MEAN & 10540 & & \\
\hline EST ANNUAL MEAN & 24560 & & 1948 \\
\hline LOWEST ANNUAL MEAN & 4291 & & 1955 \\
\hline HIGHEST DATLY MEAN & 84700 & Apr 14 & 1948 \\
\hline LOWEST DAILY MEAN & 2950 & Oct 25 & 1981 \\
\hline NNUAL SEVEN-DAY MINIMUM & 3350 & Oct 21 & 1981 \\
\hline INSTANTANEOUS PEAK FLOW & 84700 & Apr 14 & 1948 \\
\hline INSTANTANEOUS PEAK ELEVATION & (FT) 21.79 & Apr 14 & 1948 \\
\hline INSTANTANEOUS LOW FLOW & 2960 & oct 25 & 1981 \\
\hline NNUAL RUNOFF (INCHES/CFSM & & & \\
\hline
\end{tabular}

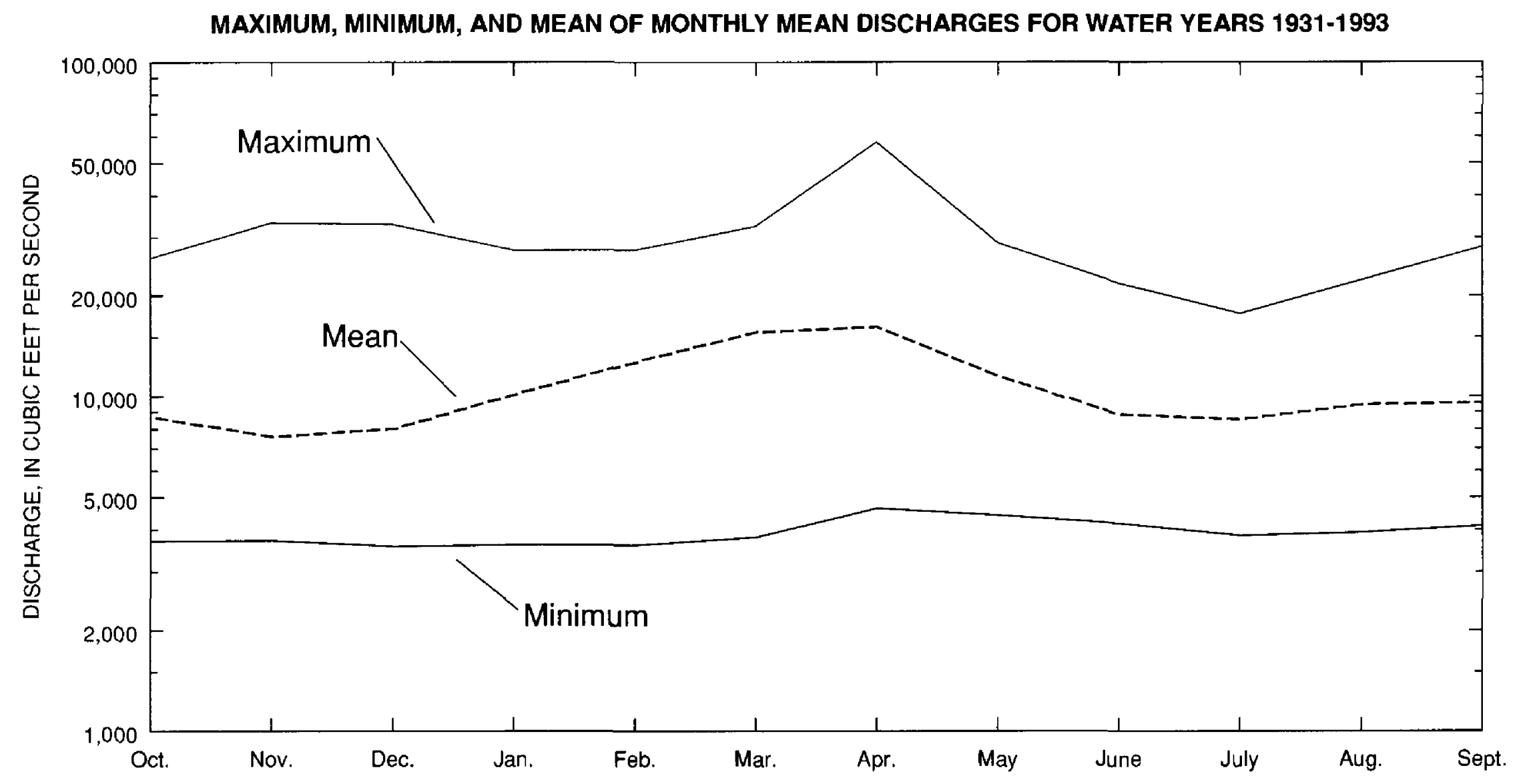

DURATION OF DAILY MEAN DISCHARGE FOR WATER YEARS 1931-1993

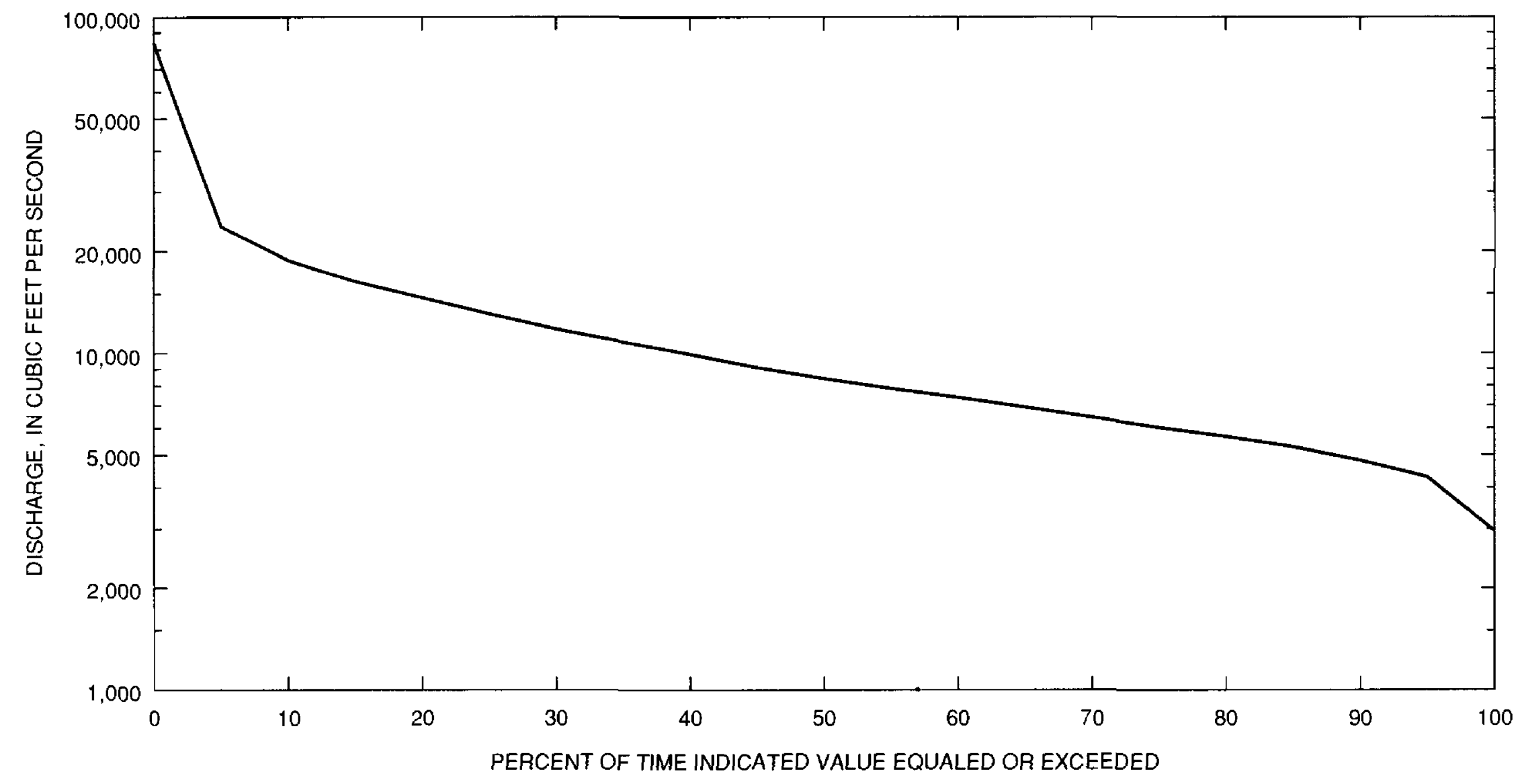


SUMMARY OF MONTHLY MEAN ELEVATION AND DISCHARGE STATISTICS FOR WATER YEARS 1931-1993

\begin{tabular}{lrrrrrr} 
& \multicolumn{3}{c}{ ELEVATIONS, } & \multicolumn{3}{c}{ DISCHARGE, } \\
& \multicolumn{2}{c}{ FEET ABOVE SEA LEVEL } & & \\
\multicolumn{1}{c}{ MONTH } & MAXIMUM & MINIMUM & MEAN & MAXIMUM & MINTMUM & MEAN \\
OCTOBER & 11.35 & 1.79 & 4.25 & 25810 & 3703 & 8710 \\
NOVEMBER & 13.48 & 1.72 & 3.50 & 33030 & 3718 & 7587 \\
DECEMBER & 13.39 & 1.31 & 3.64 & 32630 & 3575 & 8017 \\
JANUARY & 11.75 & 1.32 & 4.57 & 27320 & 3610 & 10120 \\
FEBRUARY & 11.82 & 1.46 & 5.89 & 27390 & 3602 & 12700 \\
MARCH & 13.53 & 1.69 & 7.06 & 32210 & 3796 & 15500 \\
APRTL & 18.32 & 2.07 & 7.23 & 57260 & 4631 & 16090 \\
MAY & 12.06 & 2.05 & 5.47 & 28690 & 4422 & 11510 \\
JUNE & 9.95 & 2.04 & 4.36 & 21690 & 4174 & 8838 \\
JULY & 8.80 & 2.05 & 4.15 & 17550 & 3844 & 8539 \\
AUGUST & 11.19 & 1.96 & 4.72 & 22190 & 3925 & 9462 \\
SEPTEMBER & 11.86 & 2.12 & 4.78 & 27910 & 4104 & 9568
\end{tabular}

DURATION OF DAILY MEAN VALUES FOR WATER YEARS 1931-1993

PERCENT

EQUALED OR

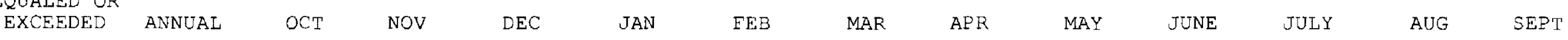

ELEVATION IN FEET ABOVE MEAN SEA LEVEL

$\begin{array}{rrrr}95.0 & 1.8 & 1.8 & 1.6 \\ 90.0 & 2.0 & 2.1 & 1.9 \\ 85.0 & 2.3 & 2.3 & 2.1 \\ 80.0 & 2.6 & 2.5 & 2.3 \\ 75.0 & 2.6 & 2.5 & 2.3 \\ 70.0 & 3.0 & 2.7 & 2.5 \\ 65.0 & 3.0 & 2.9 & 2.5 \\ 60.0 & 3.4 & 3.1 & 2.7 \\ 55.0 & 3.4 & 3.1 & 2.7 \\ 50.0 & 3.8 & 3.4 & 2.9 \\ 45.0 & 3.8 & 3.4 & 2.9 \\ 40.0 & 4.3 & 3.7 & 3.2 \\ 35.0 & 4.9 & 4.3 & 3.2 \\ 30.0 & 4.9 & 4.7 & 3.5 \\ 25.0 & 5.5 & 5.1 & 3.9 \\ 20.0 & 6.3 & 5.5 & 3.9 \\ 15.0 & 7.1 & 6.0 & 4.2 \\ 10.0 & 8.6 & 7.0 & 4.6 \\ 5.0 & 10.0 & 7.6 & 6.0\end{array}$

$\begin{array}{ll}1.4 & 1.4 \\ 1.5 & 1.8 \\ 1.9 & 2.0 \\ 2.1 & 2.2 \\ 2.1 & 2.5 \\ 2.3 & 2.8 \\ 2.5 & 2.8 \\ 2.5 & 3.1 \\ 2.8 & 3.4 \\ 2.8 & 3.4 \\ 3.0 & 3.8 \\ 3.0 & 4.3 \\ 3.4 & 4.8 \\ 3.4 & 5.3 \\ 3.7 & 5.9 \\ 4.1 & 6.6 \\ 5.0 & 7.4 \\ 6.0 & 7.4 \\ 8.1 & 9.8\end{array}$

$\begin{array}{rr}1.7 & 2.1 \\ 2.2 & 2.5 \\ 2.5 & 2.9 \\ 2.7 & 3.5 \\ 3.1 & 3.8 \\ 3.9 & 4.5 \\ 4.3 & 4.9 \\ 4.3 & 5.4 \\ 4.8 & 6.4 \\ 4.8 & 6.4 \\ 5.4 & 7.0 \\ 6.1 & 7.6 \\ 6.8 & 8.3 \\ 6.8 & 8.3 \\ 7.6 & 9.0 \\ 8.6 & 9.0 \\ 9.1 & 10.8 \\ 9.8 & 12.3 \\ 10.9 & 13.4\end{array}$

$$
\begin{array}{r}
2.3 \\
2.7 \\
3.2 \\
3.8 \\
4.1 \\
4.5 \\
4.9 \\
5.3 \\
5.8 \\
6.3 \\
6.8 \\
7.4 \\
7.4 \\
8.7 \\
9.5 \\
9.5 \\
11.2 \\
11.2 \\
14.5
\end{array}
$$

2.1
2.4
2.6
2.8
3.1
3.3
3.6
3.8
4.1
4.5
5.2
5.2
5.6
6.1
7.0
7.6
8.2
8.8
10.3

2.1
2.3
2.4
2.4
2.6
2.8
3.1
3.1
3.3
3.6
3.9
4.2
4.5
4.9
5.2
5.7
6.1
7.1
8.3

DISCHARGE IN CUBIC FEET PER SECOND

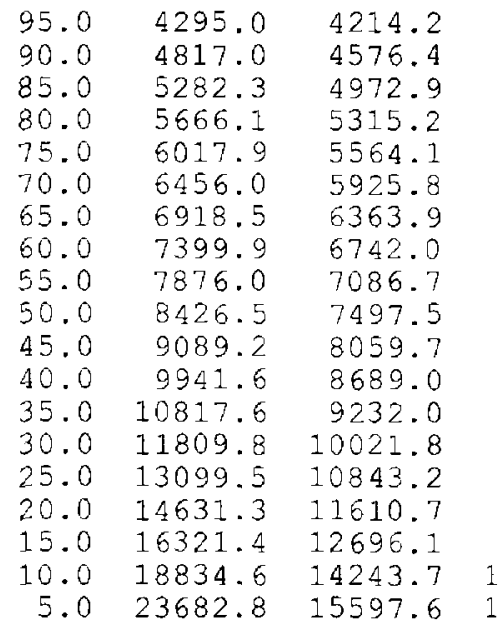

$\begin{array}{rr}4053.5 & 3981.6 \\ 4296.7 & 4309.4 \\ 4561.7 & 4623.2 \\ 4929.8 & 5045.5 \\ 5192.5 & 5314.9 \\ 5459.7 & 5517.7 \\ 5734.7 & 5937.8 \\ 6034.6 & 5241.5 \\ 6439.8 & 6511.6 \\ 6817.2 & 6747.7 \\ 7161.4 & 6994.8 \\ 7490.6 & 7258.5 \\ 7853.6 & 7567.5 \\ 8223.7 & 8180.8 \\ 8561.3 & 8522.6 \\ 9011.0 & 9362.8 \\ 9703.0 & 10429.1 \\ 10560.6 & 12757.2 \\ 12228.3 & 15345.0\end{array}$

4115.7

4701.6
5302.7

$4631.8 \quad 5393.6$

$5500.1 \quad 6194.6 \quad 5402.8$

5682.0

6118.2

6194.6
7248.0

6550.1

5979.3

8210.3

7178.3

4815.1

6409.3

$7174.5 \quad 8813.9 \quad 8405.3$

$9671.5 \quad 10618.6 \quad 10690.3$

$\begin{array}{rrrr}6736.6 & 9671.5 & 10618.6 & 10690.9 \\ 7075.8 & 10510.4 & 11556.9 & 11551.3\end{array}$

7499.5

$9004 \cdot 3$

$\begin{array}{lll}12210.3 & 14836.5 & 14663.0\end{array}$

10696.7

$13264.6 \quad 15822.5 \quad 15776.0$

11609.7
12902.8

$16060.3 \quad 18328.4 \quad 18179.5$

14703.3

$\begin{array}{llll}17118.9 & 20506.4 & 19877.6 & 14425.0 \\ 18141.6 & 22602.0 & 22314.3 & 15856.2\end{array}$

10802.0

$\begin{array}{lllll}1.24952 .3 & 25571.4 & 17613.7 & 13418.7\end{array}$

$\begin{array}{lll}21320.2 & 28754.1 & 28536.4\end{array}$

20013.8

22006.2

$24084.0 \quad 32063.8 \quad 35984.6$

$24565.5 \quad 17515.4$

$\begin{array}{rrrr}4375.3 & 4336.1 & 4256.0 & 4342.3 \\ 4850.0 & 4832.8 & 4668.8 & 4790.9 \\ 5250.1 & 5120.8 & 5085.8 & 5098.1 \\ 5542.2 & 5407.2 & 5553.6 & 5553.4 \\ 5781.8 & 5574.2 & 5831.2 & 5923.1 \\ 6031.2 & 5725.8 & 6359.9 & 6330.3 \\ 6342.3 & 6062.1 & 5996.0 & 6814.5 \\ 6618.6 & 6484.0 & 7449.4 & 7306.8 \\ 5957.3 & 7132.3 & 7735.4 & 7679.3 \\ 7542.2 & 7567.5 & 8075.4 & 8135.5 \\ 7971.4 & 7925.0 & 8480.6 & 8721.8 \\ 8646.0 & 8293.2 & 8972.5 & 9369.3 \\ 9274.7 & 8843.4 & 9789.4 & 10293.1 \\ 9979.8 & 9881.5 & 10729.4 & 11124.2 \\ 10802.0 & 10747.2 & 12237.0 & 11756.7 \\ 11926.0 & 11779.6 & 13629.8 & 12926.7 \\ 13418.7 & 13345.8 & 14733.6 & 14177.8 \\ 14723.7 & 14811.9 & 16193.3 & 16000.0 \\ 17515.4 & 16206.9 & 19250.7 & 19270.0\end{array}$

2.0

2.5 
WATER YEAR RANGE 19511952 19521953 19531954 19541955

19561957

19571958

19581959

19601961

19611962 19621963

19641965

19651966

19661967

19671968

19681969

19691970

19701971

19711972

19721973

19731974

$1974 \quad 1975$

$1976 \quad 1977$

19771978

19781979

19791980

19811982

19821983

19831984

19841985

19851986

$1987 \quad 1988$

19881989

19901991

19911992

19921993 $\begin{array}{cc}1 & \\ 2.04 & 21 \\ 1.43 & 8\end{array}$

2.2927
$.71 \quad 3$

$\begin{array}{ll}.37 \quad 1 \\ 1.76 & 16\end{array}$

$1.76 \quad 16$

1.5410

2.5630

$\begin{array}{rr}1.61 & 13\end{array}$

$\begin{array}{rr}1.24 & 6 \\ 4.4136\end{array}$

3.1533

2. 9232

1.5511

2.1725

2. 6131

$2.48 \quad 29$

$\begin{array}{rrr}1.33 & 7 \\ 2 & 08 & 24\end{array}$

1.9618

2.0522

$2.25 \quad 26$

$1.58 \quad 12$
$1.02 \quad 5$

2.0623

.632

1.68
2.4128

1.9619

$1.90 \quad 17$

$1.72 \quad 15$

1.529

$1.98 \quad 20$

$\begin{array}{ll}3.20 & 34 \\ 3.96 & 35\end{array}$

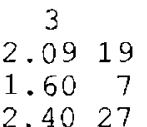

$\begin{array}{rr}1.60 & 7 \\ 2.40 \quad 27\end{array}$

.992

.561

1.7211

2.6829

$1.73 \quad 12$

1.426

3.2333

3.0432

1.689

$1.16 \quad 4$

$2.76 \quad 30$

$2.76 \quad 31$

$\begin{array}{ll}1.78 & 13 \\ 2.18 & 20\end{array}$

2.1821

.332

2. 3726

1.668

$\begin{array}{lr}1.27 & 5 \\ 2.27 & 23\end{array}$

1.013

$\begin{array}{ll}1.78 & 14 \\ 2.58 & 28\end{array}$

2.0818

$1.96 \quad 17$

1.8716

$1.71 \quad 10$

2.3024

$\begin{array}{ll}3.34 & 34 \\ 4.00 & 35\end{array}$
2.1416

$\begin{array}{rr}2.14 & 16 \\ 1.82 & 7\end{array}$

$\begin{array}{rr}2.52 & 25 \\ 1.23 & 2\end{array}$

1.051

$2.21 \quad 17$

$\begin{array}{rr}1.86 & 8 \\ 2.85 & 31\end{array}$

1. 919

$1.50 \quad 5$

4.4936
3.4233

3.1132

1.9811

2.4123

2.8329

2.8430

2.2218
2.40

2.2619

2.5226

2.5024

$\begin{array}{rr}2.01 & 12 \\ 1.59 & 6\end{array}$

2.3921

1.363

2.0213

2. 6628

2.2720
2.0715

1.9110

2.0514

2.6327

$3.48 \quad 34$
4.10

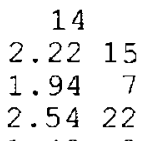

$\begin{array}{rr}2.54 & 22 \\ 1.42 & 2\end{array}$

1.231

$2.43 \quad 19$

$\begin{array}{rr}2.06 & 9 \\ 3.00 \quad 31\end{array}$

2.0810

1.826

4.5736

3.1832

$2.17 \quad 13$

$1.58 \quad 3$

2.5221

$2.97 \quad 30$

2.4218
2.60
23

$2.40 \quad 17$

2.6125

2.6726

$\begin{array}{rr}2.12 & 11 \\ 1.79 & 5\end{array}$

2.6024

1.634

$2.26 \quad 16$

2.8828

2. 4320

$2.04 \quad 8$

2.1412

2.8027

3.6534
$4.29 \quad 35$

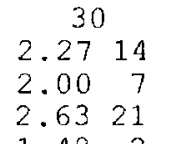

$\begin{array}{rr}2.63 & 21 \\ 1.48 & \end{array}$

$1.36 \quad 1$

2. 6220

2.2311

3.1331

2.168

1.906

4.8336

$3.34 \quad 32$

$2.23 \quad 12$

1.643

$2.70 \quad 23$

2.9828

$3.08 \quad 30$

2.4816

2.64
$2.48 \quad 17$

2.7224

2.8827

2.199

$\begin{array}{ll}1.87 & 5\end{array}$

1.724

$\begin{array}{rr}1.72 & 4 \\ 2.49 & 18\end{array}$

2.49
2.98
29

2.5119

$2.27 \quad 15$

2.2513

2.2110

2.8726

3.69
4.34

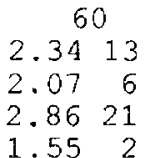

$1.38 \quad 1$

2.6920

2.4414

3.3331

$2.24 \quad 8$
1.98

5.6136

$\begin{array}{ll}5.61 & 36 \\ 3.85 & 34\end{array}$

3.5732

$\begin{array}{rrr}2.33 & 12 \\ 1.78 & 3\end{array}$

1.78
2.98

3.13 27

3.2930

$\begin{array}{ll}2.63 & 18 \\ 2.86 & 22\end{array}$

$\begin{array}{ll}2.86 & 22 \\ 2.57 & 17\end{array}$

2.8823

3.1929

2.249

2.137

1.854

2.5016

3.1628

2.6319

2.4715

2. 2710

2.97 24

$\begin{array}{rr}3.83 & 33 \\ 4.51 & 35\end{array}$
90

2.4312
2.09

$\begin{array}{lr}2.09 & 6 \\ 3.11 & 23\end{array}$

1.612

1.441

3.5329

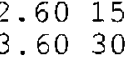

2.338

2.065

6.8036

4.0933

2.3810

1.833

2. 9921

3. $35 \quad 27$

3. 6431

2.7318

2.9720

2.8219

3.1324

3.2526

2. $48 \quad 13$

$\begin{array}{ll}2.24 & 7 \\ 3.1325\end{array}$

$1.84 \quad 4$

2. 5316

$\begin{array}{lll}3.36 & 28 \\ 2.69 & 17\end{array}$

2.69
17

2.5214

2. 369

3.0622

$4.08 \quad 32$
$4.48 \quad 35$
120

$2.51 \quad 12$

$\begin{array}{rr}2.15 & 5 \\ 3.48 & 26\end{array}$

$\begin{array}{rr}3.48 & 26 \\ 1.65 & 2\end{array}$

1.501

3.8429

$2.70 \quad 15$

3.9230

2.4410

4.5234

$4.45 \quad 33$

2.418

3.1221

3.5428

4.1431

2.88 18

3.0519

3.3925

3. 3824

3.3322

2.6014

$\begin{array}{ll}2.37 & 7 \\ 3.37 & 23\end{array}$

1.934

2. $86 \quad 17$

3.5027

2.7216

$2.57 \quad 13$

2.429

3.0920

$\begin{array}{ll}4.44 & 32 \\ 4.68 & 35\end{array}$

183

$\begin{array}{rr}2.73 & 9 \\ 2.25 & 5\end{array}$

4.1928

1.732

$1.70 \quad 1$

3.9125

3.1815 

FOR PERIOD OCT TO SEP (Continued)

\begin{tabular}{|c|c|c|c|c|c|c|c|c|c|}
\hline RANGE & 1 & 3 & 7 & 14 & 30 & 60 & 90 & 120 & 183 \\
\hline $1976 \quad 1976$ & 8.5321 & $8.51 \quad 21$ & $8.44 \quad 21$ & 8.1321 & $7.25 \quad 22$ & $6.49 \quad 22$ & $5.63 \quad 22$ & 5.0525 & 4.6425 \\
\hline $1977 \quad 1977$ & 10.615 & $10.5 \quad 14$ & $10.5 \quad 14$ & $10.5 \quad 14$ & 10.212 & 9.72 & 8.96 & 8.86 & $7.37 \quad 10$ \\
\hline $1978 \quad 1978$ & $9.58 \quad 17$ & $9.56 \quad 17$ & $9.51 \quad 17$ & 9.3017 & $8.82 \quad 16$ & $8.19 \quad 15$ & $7.53 \quad 16$ & 7.1715 & $6.19 \quad 15$ \\
\hline $1979 \quad 1979$ & 7.9623 & $7.92 \quad 23$ & 7.8623 & 7.5924 & 6.9325 & 5.9624 & 5.5124 & $5.30 \quad 22$ & 4.7323 \\
\hline 19801980 & 11.011 & $11.0 \quad 11$ & $11.0 \quad 11$ & 10.811 & 10.3 & $9.45 \quad 10$ & $8.31 \quad 13$ & $7.47 \quad 14$ & $6.28 \quad 14$ \\
\hline 19821982 & $5.82 \quad 32$ & 5.7932 & 5.6932 & $5.48 \quad 32$ & $5.26 \quad 32$ & 4.8531 & 4.8928 & 4.6626 & $4.25 \quad 26$ \\
\hline 19831983 & 12.57 & 12.5 & 12.57 & 12.57 & 12.27 & 1.95 & 10.95 & 9.86 & 8.27 \\
\hline $1984 \quad 1984$ & $16.5 \quad 2$ & 16.5 & 16.4 & 16.1 & 15.1 & 13.9 & 12.5 & 11.4 & 9.72 \\
\hline 19851985 & $7.30 \quad 27$ & $7.28 \quad 27$ & $7.20 \quad 27$ & $6.88 \quad 27$ & $6.38 \quad 27$ & 5.1429 & $4.30 \quad 32$ & 3.7932 & 3.3633 \\
\hline 19861986 & 15.1 & $25.0 \quad 4$ & 14.94 & 14.34 & 12.95 & 20.97 & 9.75 & 8.938 & 7.42 \\
\hline $1988 \quad 1988$ & 10.614 & $10.5 \quad 15$ & $10.4 \quad 15$ & 10.115 & $9.60 \quad 15$ & $8.15 \quad 17$ & $7.15 \quad 18$ & $6.25 \quad 19$ & $5.08 \quad 21$ \\
\hline 19911991 & 16.03 & 15.9 & 15.83 & $15.3 \quad 3$ & 14.23 & 13.13 & 12.1 & 11.32 & 10.3 \\
\hline 19921992 & $9.30 \quad 20$ & $9.25 \quad 20$ & 9.1720 & 9.0220 & $8.66 \quad 17$ & 8.1916 & $7.68 \quad 15$ & 7.0116 & 6.1516 \\
\hline 19931993 & 11.012 & 10.912 & 10.912 & $10.6 \quad 13$ & 10.014 & 9.51 & 9.52 & 8.94 & 7.75 \\
\hline
\end{tabular}

LOWEST MEAN DISCHARGE, IN CUBIC FEET PER SECOND, AND RANKING FOR THE FOLLOWING NUMBER OF CONSECUTIVE DAYS FOR PERIOD APR TO MAR

WATER YEAR

\begin{tabular}{rrrr} 
RANGE & \multicolumn{1}{c}{1} \\
1942 & 1943 & 5400 & 35 \\
1943 & 1944 & 4200 & 11 \\
1944 & 1945 & 7200 & 48 \\
1945 & 1946 & 5400 & 36 \\
1946 & 1947 & 6600 & 45 \\
1947 & 1948 & 7000 & 47 \\
1948 & 1949 & 9900 & 50 \\
1949 & 1950 & 6100 & 43 \\
1950 & 1951 & 5100 & 28 \\
& & & \\
1951 & 1952 & 4520 & 16 \\
1952 & 1953 & 4030 & 9 \\
1953 & 1954 & 5360 & 33 \\
1954 & 1955 & 3690 & 6 \\
1955 & 1956 & 3340 & 3 \\
1956 & 1957 & 3270 & 2 \\
1957 & 1958 & 4680 & 18 \\
1958 & 1959 & 4950 & 25 \\
1959 & 1960 & 8000 & 49 \\
1960 & 1961 & 6780 & 46 \\
1961 & 1962 & 4810 & 23 \\
1962 & 1963 & 4170 & 10 \\
1963 & 1964 & 3780 & 7 \\
1964 & 1965 & 10100 & 51 \\
1965 & 1966 & 6210 & 44 \\
1966 & 1967 & 5970 & 42 \\
1967 & 1968 & 4640 & 17 \\
1968 & 1969 & 3480 & 4 \\
1969 & 1970 & 4940 & 24 \\
1970 & 1971 & 5170 & 30 \\
1971 & 1972 & 5080 & 27 \\
1972 & 1973 & 4470 & 14 \\
1973 & 1974 & 5390 & 34 \\
1974 & 1975 & 5330 & 32 \\
1975 & 1976 & 5510 & 39 \\
1976 & 1977 & 5490 & 37 \\
1976 & &
\end{tabular}

$\begin{array}{cr}3 & \\ 5400 & 28 \\ 4200 & 9 \\ 7200 & 48 \\ 5400 & 29 \\ 6600 & 45 \\ 6600 & 45 \\ 7000 & 47 \\ 9900 & 50 \\ 6100 & 41 \\ 5230 & 25 \\ 4590 & 12 \\ 4590 & 12 \\ 4167 & 8 \\ 5417 & 31 \\ 3803 & 4 \\ 3433 & 3 \\ & \\ 3367 & 2 \\ 4780 & 17 \\ 5087 & 24 \\ 8067 & 49 \\ 6877 & 46 \\ & \\ 4960 & 22 \\ 4323 & 11 \\ 3870 & 6 \\ 10130 & 51 \\ 6313 & 44 \\ & \\ 6273 & 43 \\ 4827 & 18 \\ 3827 & 5 \\ 5030 & 23 \\ 5510 & 32 \\ & \\ 5317 & 26 \\ 4683 & 14 \\ 5600 & 35 \\ 6003 & 40 \\ 5943 & 39 \\ & \\ 5567 & 33 \\ & \end{array}$

$\begin{array}{rr}7 & \\ 5400 & 26 \\ 4200 & 8 \\ 7200 & 48 \\ 5400 & 27 \\ & \\ 6600 & 43 \\ 7000 & 47 \\ 9900 & 50 \\ 6100 & 39 \\ 5379 & 25 \\ 4627 & 12 \\ 4329 & 9 \\ 5440 & 28 \\ 3941 & 5 \\ 3484 & 2 \\ & \\ 3501 & 3 \\ 5029 & 19 \\ 5183 & 24 \\ 8089 & 49 \\ 6991 & 46 \\ & \\ 5110 & 23 \\ 4403 & 11 \\ 3933 & 4 \\ 10190 & 51 \\ 6423 & 42 \\ 6783 & 45 \\ 4943 & 17 \\ 4000 & 7 \\ 5060 & 21 \\ 5804 & 35 \\ 5573 & 30 \\ 4926 & 16 \\ 5784 & 34 \\ 6127 & 40 \\ 6284 & 41 \\ 5833 & 36 \\ 58 & \end{array}$

14
540025

42008

737148
5429

662943

662943
711447

992950

610037

544827

466412

43909

551428

$4024 \quad 5$

35292

$3564 \quad 3$

523622

524423

815249

705146

525124

452511

40064

1034051

649442

696344

518520

40816

515019

590633

572430

$5118 \quad 17$

590934

616539

636841

614438

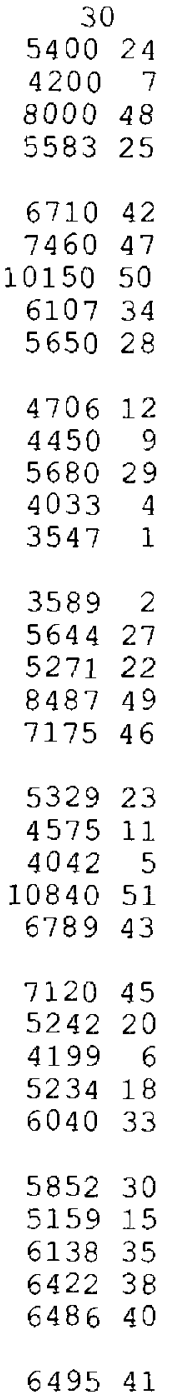

90

42535

973349

718638

710436

766943

$11830 \quad 50$

647629

$5884 \quad 24$

$\begin{array}{rr}5022 & 12 \\ 4693 & 9\end{array}$

678632

$4094 \quad 4$

$3617 \quad 1$

$3717 \quad 2$

$7578 \quad 40$

556819

927948

803845

544715

471610

$4286 \quad 6$

477051

839646

877347

560520

606826

683433

$7306 \quad 39$

545216

671931

$7038 \quad 35$

761241

775744
59420

43425

$9846 \quad 49$

766737

$7462 \quad 34$

809441

1245050

678529

611923

$5158 \quad 12$

$4825 \quad 9$

758736

41244

36821

$3760 \quad 2$

524243

580821

$9574 \quad 48$

872445

558215

500610

$4478 \quad 8$

1623051

920746

950547

575018

$371 \quad 6$
6375

631524

715132

826244

578720

689530

745733

816642

794340
183

637318 4587
$10430 \quad 46$

1157049

$9368 \quad 40$ 1028045

1232050

809332

$7021 \quad 23$

577013

48758

$9037 \quad 39$

$\begin{array}{ll}421.9 & 4 \\ 3937 & 1\end{array}$

$4042 \quad 3$

834133

669121

999043
1011044

634617

528210

52709

$\begin{array}{ll}19020 & 51 \\ 1058047\end{array}$

1147048

584914

46347

$\begin{array}{ll}7366 & 27 \\ 8368 & 34\end{array}$

860135

660320

788930

866836

894438

997942 

CONSECUTIVE DAYS FOR PERIOD APR TO MAR (Continued)

WATER YEAR

$\begin{array}{ll}\text { RANGE } \\ \text { RANGE } \\ 1977 & 1978 \\ 1978 & 1979 \\ 1979 & 1980 \\ 1980 & 1981 \\ & \\ 1981 & 1982 \\ 1982 & 1983 \\ 1983 & 1984 \\ 1984 & 1985 \\ 1985 & 1986 \\ 1986 & 1987 \\ 1987 & 1988 \\ 1988 & 1989 \\ 1989 & 1990 \\ 1990 & 1991 \\ 1991 & 1992 \\ 1992 & 1993\end{array}$

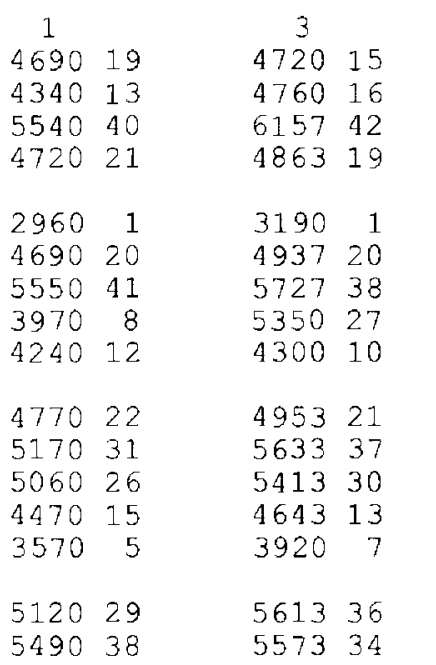

$\begin{array}{rr}7 & \\ 4799 & 13 \\ 4857 & 14 \\ 6621 & 44 \\ 4977 & 18 \\ 3349 & 1 \\ 5057 & 20 \\ 5911 & 37 \\ 5753 & 33 \\ 4336 & 10 \\ 5109 & 22 \\ 6047 & 38 \\ 5476 & 29 \\ 4857 & 15 \\ 3996 & 6 \\ 5743 & 31 \\ 5749 & 32\end{array}$

$\begin{array}{lr}30 \\ 5089 & 14 \\ 5076 & 13 \\ 7091 & 44 \\ 5175 & 16 \\ & \\ 3678 & 3 \\ 5241 & 19 \\ 6215 & 36 \\ 6007 & 32 \\ 4469 & 10 \\ 5258 & 21 \\ 6448 & 39 \\ 5594 & 26 \\ 5215 & 17 \\ 4248 & 8 \\ 6246 & 37 \\ 5954 & 31\end{array}$

\begin{tabular}{lr}
\multicolumn{3}{c}{90} \\
5500 & 17 \\
5415 & 14 \\
7628 & 42 \\
5392 & 13 \\
& \\
3903 & 3 \\
5864 & 23 \\
7157 & 37 \\
6263 & 27 \\
4811 & 11 \\
5630 & 21 \\
6592 & 30 \\
5985 & 25 \\
5559 & 18 \\
4325 & 8 \\
7020 & 34 \\
6419 & 28
\end{tabular}

120

555414

563316

792639

551413

$3890 \quad 3$

635025

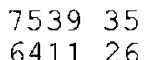

$\begin{array}{ll}6411 & 26 \\ 5101 & 11\end{array}$

$5773 \quad 19$

678228

665027

$\begin{array}{rr}5668 & 17 \\ 4409 & 7\end{array}$

766738

696231
183

555611

658319

802431

39732

742128

882137

669622
708424

$5908 \quad 15$

718926

715025

612316

45195

976141

$7788 \quad 29$

HIGHEST MEAN DISCHARGE, IN CUBIC FEET PER SECOND, AND RANKING FOR THE FOLLOWING NUMBER OF CONSECUTIVE DAYS FOR PERIOD OCT TO SEP

WATER YEAR

RANGE

19311931

19431943

19441944

19451945

19461946

19471947

19481948

19491949

19501950

19511951

19521952

19541954

19551955

19561956

19571957

19581958

19601960

19611961

19621962

19641964

19651965

19661966

19671967

19681968

19701970

$1971 \quad 1971$

19721972

19731973
19741974

19751975

19761976

19771977

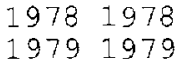

19801980

19811981

19821982

19831983

19841984

19851985

19861986

19871987

19881988

19891989

19901990

19911991 19921992

19931993

\begin{tabular}{|c|c|c|c|c|}
\hline $\begin{array}{r}1 \\
14500 \\
29200 \\
8000 \\
22300 \\
24300\end{array}$ & $\begin{array}{l}44 \\
12 \\
51 \\
23 \\
21\end{array}$ & $\begin{array}{r}3 \\
14500 \\
29200 \\
8000 \\
22200 \\
24300\end{array}$ & $\begin{array}{l}43 \\
12 \\
51 \\
23 \\
21\end{array}$ & $\begin{array}{r}7 \\
14460 \\
29030 \\
8000 \\
21960 \\
24210\end{array}$ \\
\hline $\begin{array}{l}18600 \\
17900 \\
84700 \\
20100 \\
15700\end{array}$ & $\begin{array}{r}31 \\
32 \\
1 \\
27 \\
38\end{array}$ & $\begin{array}{l}18400 \\
17770 \\
83830 \\
20100 \\
15300\end{array}$ & $\begin{array}{r}31 \\
32 \\
7 \\
27 \\
39\end{array}$ & $\begin{array}{l}18340 \\
17570 \\
79640 \\
19900 \\
14610\end{array}$ \\
\hline $\begin{array}{r}12400 \\
16500 \\
13800 \\
20000 \\
5890\end{array}$ & $\begin{array}{l}46 \\
36 \\
45 \\
29 \\
53\end{array}$ & $\begin{array}{r}12400 \\
16430 \\
13700 \\
20000 \\
5797\end{array}$ & $\begin{array}{l}46 \\
36 \\
45 \\
29 \\
53\end{array}$ & $\begin{array}{r}12170 \\
16230 \\
13510 \\
19890 \\
5617\end{array}$ \\
\hline $\begin{array}{l}10300 \\
15100 \\
25800 \\
40600 \\
28600\end{array}$ & $\begin{array}{r}49 \\
40 \\
17 \\
5 \\
13\end{array}$ & $\begin{array}{l}10230 \\
15100 \\
25730 \\
40530 \\
28500\end{array}$ & $\begin{array}{r}49 \\
40 \\
18 \\
5 \\
13\end{array}$ & $\begin{array}{r}9960 \\
14940 \\
25540 \\
40190 \\
28130\end{array}$ \\
\hline $\begin{array}{l}21300 \\
17100 \\
14700 \\
36700 \\
32900\end{array}$ & $\begin{array}{r}25 \\
35 \\
42 \\
7 \\
9\end{array}$ & $\begin{array}{l}21230 \\
17070 \\
14600 \\
36600 \\
32200\end{array}$ & $\begin{array}{r}25 \\
34 \\
41 \\
7 \\
10\end{array}$ & $\begin{array}{l}20960 \\
16930 \\
14510 \\
36130 \\
32090\end{array}$ \\
\hline $\begin{array}{r}33000 \\
20200 \\
7570 \\
11700 \\
27100\end{array}$ & $\begin{array}{r}8 \\
26 \\
52 \\
47 \\
15\end{array}$ & $\begin{array}{r}33000 \\
20130 \\
7107 \\
11470 \\
27100\end{array}$ & $\begin{array}{r}8 \\
26 \\
52 \\
48 \\
15\end{array}$ & $\begin{array}{r}32810 \\
20030 \\
6534 \\
11340 \\
27040\end{array}$ \\
\hline $\begin{array}{l}15500 \\
25800 \\
54900 \\
17300 \\
27400\end{array}$ & $\begin{array}{r}39 \\
18 \\
2 \\
33 \\
14\end{array}$ & $\begin{array}{l}15430 \\
25800 \\
54700 \\
17030 \\
27330\end{array}$ & $\begin{array}{r}38 \\
17 \\
2 \\
35 \\
14\end{array}$ & $\begin{array}{l}15370 \\
25710 \\
54010 \\
16810 \\
27130\end{array}$ \\
\hline $\begin{array}{l}20100 \\
25300 \\
22800 \\
18700 \\
26600\end{array}$ & $\begin{array}{l}28 \\
19 \\
22 \\
30 \\
16\end{array}$ & $\begin{array}{l}20100 \\
25270 \\
22730 \\
18600 \\
26600\end{array}$ & $\begin{array}{l}28 \\
19 \\
22 \\
30 \\
16\end{array}$ & $\begin{array}{l}19930 \\
25210 \\
22610 \\
18460 \\
26510\end{array}$ \\
\hline $\begin{array}{l}10100 \\
14800 \\
31100 \\
48300 \\
16500\end{array}$ & $\begin{array}{r}50 \\
41 \\
11 \\
3 \\
37\end{array}$ & $\begin{array}{l}10010 \\
14530 \\
31100 \\
48170 \\
16430\end{array}$ & $\begin{array}{r}50 \\
42 \\
11 \\
3 \\
37\end{array}$ & $\begin{array}{r}9886 \\
14390 \\
31100 \\
47730 \\
16230\end{array}$ \\
\hline $\begin{array}{l}41300 \\
32400 \\
25300 \\
11600 \\
14600\end{array}$ & $\begin{array}{r}4 \\
10 \\
20 \\
48 \\
43\end{array}$ & $\begin{array}{l}41130 \\
32330 \\
25100 \\
11530 \\
14470\end{array}$ & $\begin{array}{r}4 \\
9 \\
20 \\
47 \\
44\end{array}$ & $\begin{array}{l}40300 \\
32290 \\
24790 \\
11100 \\
14000\end{array}$ \\
\hline $\begin{array}{l}1300 \\
1500\end{array}$ & $\begin{array}{r}6 \\
34 \\
24\end{array}$ & $\begin{array}{l}38600 \\
17200 \\
21430\end{array}$ & $\begin{array}{r}6 \\
33 \\
24\end{array}$ & $\begin{array}{l}38030 \\
17030 \\
21270\end{array}$ \\
\hline
\end{tabular}

15

1395041

2840012

2116023

2366021

1824030

1706032

$68990 \quad 1$

1932028

1367042

1163046

1613036

1324045
1949027

522953

920050

1451039

2482019

$38740 \quad 4$

2017025

$16510 \quad 34$

1443040

$31530 \quad 10$

$32170 \quad 8$

321700
6540
6452

644752
1123047

2663014

$14790 \quad 38$

2539017

$51200 \quad 2$
$16200 \quad 35$

2632015

1910029

2503018

2208022

$17810 \quad 31$

2591016

$9374 \quad 49$

1343044

3093011

460503

$15850 \quad 37$

$37770 \quad 5$

32050

2419020

1.048048

1351043

$36090 \quad 6$

1667033
$20620 \quad 24$

$\begin{array}{rr} & 30 \\ 12840 & 43 \\ 27510 & 12 \\ 8000 & 50 \\ 20390 & 23 \\ 22330 & 21 \\ 17930 & 28 \\ 16560 & 31 \\ 57570 & 1 \\ 17920 & 29 \\ 12270 & 45 \\ 10510 & 46 \\ 15760 & 34 \\ 12860 & 42 \\ 18010 & 26 \\ 4826 & 53 \\ 7736 & 51 \\ 13230 & 40 \\ 22760 & 20 \\ 35360 & 4 \\ 24190 & 16 \\ 18150 & 25 \\ 15210 & 35 \\ 14230 & 36 \\ 27910 & 11 \\ 30850 & 8 \\ 30360 & 9 \\ 17970 & 27 \\ 6212 & 52 \\ 10140 & 47 \\ 24430 & 14 \\ 13810 & 38 \\ 24170 & 17 \\ 43860 & 2 \\ 13360 & 39 \\ 24120 & 18 \\ 16820 & 30 \\ 24310 & 15 \\ 20870 & 22 \\ 15960 & 32 \\ 24630 & 13 \\ 8872 & 49 \\ 12880 & 41 \\ 30260 & 10 \\ 41660 & 3 \\ 14180 & 37 \\ 32990 & 5 \\ 30900 & 7 \\ 22830 & 19 \\ 9187 & 48 \\ 12660 & 44 \\ 31970 & 6 \\ 15840 & 33 \\ 19180 & 24 \\ & 24 \\ 17\end{array}$

60

1218037
2432011

2432011

1877023

2056018

1677026

1559028

$45830 \quad 1$

1607027

$10570 \quad 45$

899446

$14800 \quad 33$

1085044

1085044
1524029
458853

624951

1108041

1986019

$22120 \quad 14$

1509030

$12340 \quad 36$
1302035

2408012

$28980 \quad 6$

2605010

$17030 \quad 25$
$6145 \quad 52$

614552
861248

1984020

1208038

2166016

339403

2075017

1506031

2312013

1933022

$13940 \quad 34$

2203015

875847

$11850 \quad 39$

291405

$\begin{array}{rr}36890 & 2 \\ 11010 & 42\end{array}$

$\begin{array}{ll}27120 & 9 \\ 29710 & 4\end{array}$

$19440 \quad 21$

806649
1173040

$28300 \quad 8$

$\begin{array}{lll}28300 & 8 \\ 14860 & 32\end{array}$

1788024

\begin{tabular}{rr}
\multicolumn{1}{c}{90} \\
11840 & 36 \\
22080 & 12 \\
7452 & 50 \\
16220 & 24 \\
16790 & 23 \\
14980 & 27 \\
13970 & 28 \\
38130 & 1 \\
15990 & 25 \\
9584 & 44 \\
8082 & 46 \\
13480 & 32 \\
9904 & 43 \\
13670 & 31 \\
4587 & 53 \\
5887 & 52 \\
10300 & 42
\end{tabular}

1016042

1841019

2004015

1394029

1079039

1163037

2248011

260206

2302010

1520026

606051

799148

1842018

1077040

2011014

296003

1051041

$12960-33$

1296033
2136013

2136013
$17920 \quad 20$

1267034

1886017

801947

$11960 \quad 35$

26610
32220

$\begin{array}{rr}32220 & 2 \\ 9014 \quad 45\end{array}$

240309

284304

1719022

772249
$11160 \quad 38$

254407

$13910 \quad 30$

$\begin{array}{ll}13910 & 30 \\ 17890 & 21\end{array}$
120

1136036
$20640 \quad 12$
708950

708950

$14270 \quad 25$

1407026

1403027

$12520 \quad 32$

$34680 \quad 1$

$15350 \quad 22$

887844

754247

1320029

921643

1449024

452653

$5725 \quad 52$

962542

$\begin{array}{lll}17160 & 17\end{array}$

1787016

$13120 \quad 30$

965741

1042037

2134010

$24960 \quad 5$

2049013

1339028

593651
759346

1702019

1038038

$18370 \quad 15$

267903

999240

$18730 \quad 14$

1161034

2111011

1695020

1232033

1705018

739449
1148035

1148035
$23960 \quad 6$

$\begin{array}{rr}29120 \quad 2 \\ 7879 & 45\end{array}$

$21880 \quad 9$

$21880 \quad 9$
$26000 \quad 4$

1511023

745248
1028039

$22960 \quad 8$

$\begin{array}{lll}22960 & 8 \\ 12650 & 31\end{array}$

$\begin{array}{ll}12650 & 31 \\ 16630 & 21\end{array}$

183

1040035
1694013

677550

1423021

1204029

1348024

1095032

$34210 \quad 1$

1427020
805044

$7490 \quad 45$ 


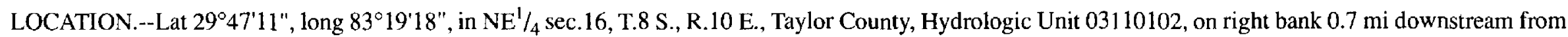
Atlantic Coast Line Railroad bridge, $0.7 \mathrm{mi}$ south of Clara, $13 \mathrm{mi}$ upstream from mouth, and $16 \mathrm{mi}$ northwest of Cross City.

DRAINAGE AREA.--350 $\mathrm{mi}^{2}$, approximately. See REMARKS.

PERIOD OF RECORD.--February 1950 to 1993.

REVISED RECORDS.--WSP 1234: 1950. WSP 1724: Drainage area.

GAGE.--Water-stage recorder. Datum of gage is $7.84 \mathrm{ft}$ above National Geodetic Vertical Datum of 1929.

REMARKS.--Records fair. Below about $500 \mathrm{ft}^{3} / \mathrm{s}$, all flow enters sinkhole $0.5 \mathrm{mi}$ downstream from gage. Above about $4,000 \mathrm{ft}^{3} / \mathrm{s}$, discharge measurements are made along U.S. Highways 19,98, and Alternate 27, measurements include all flow from about 3 mi northwest to 5 mi southwest of main channel, drainage area is increased by about $30 \mathrm{mi}^{2}$.

MAXIMUM, MINIMUM, AND MEAN OF MONTHLY MEAN ELEVATIONS FOR WATER YEARS 1950-1993
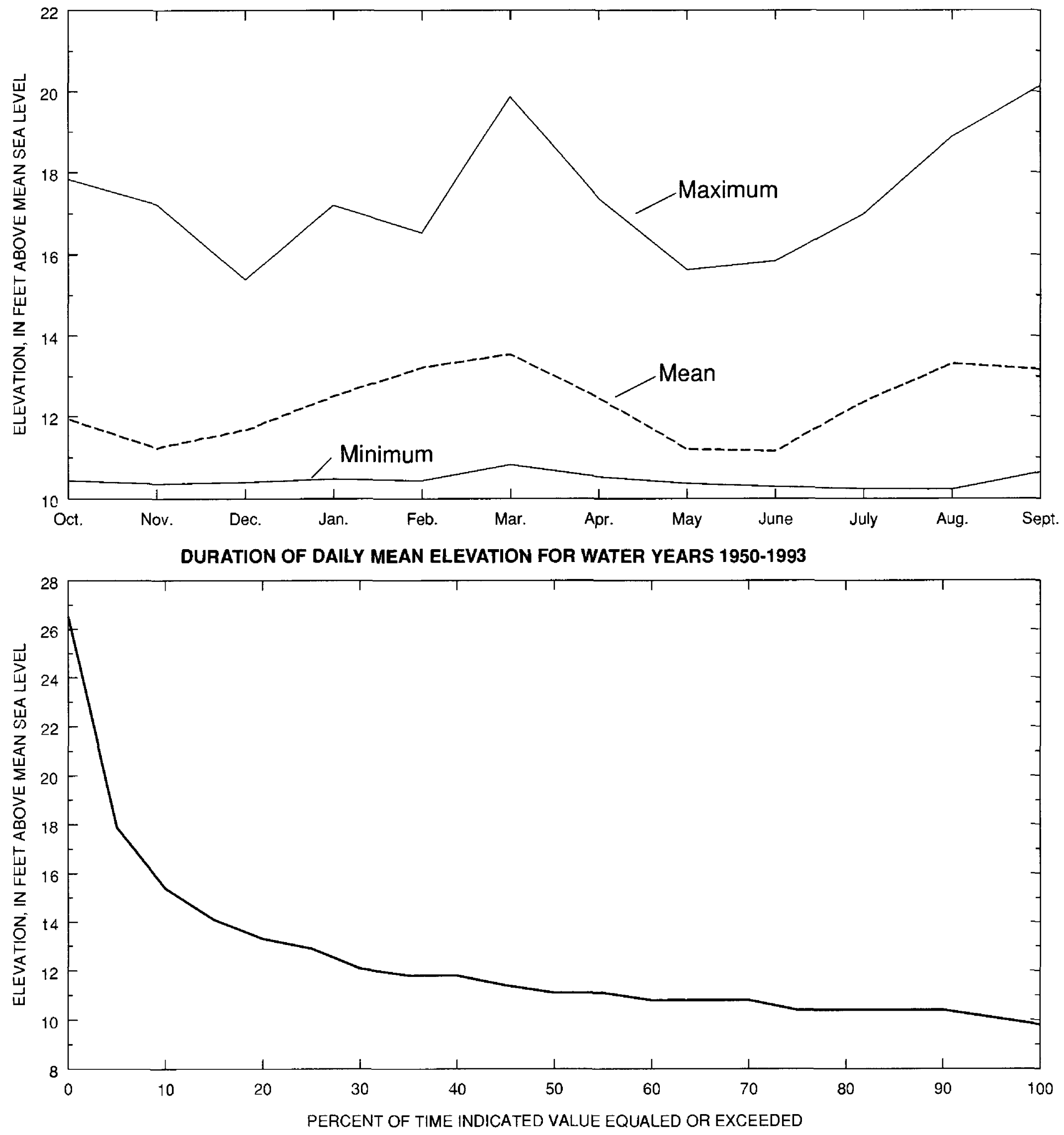


\section{SUMMARY STATISTICS IN CUBIC FEET PER SECOND}

FOR WATER YEARS $1950 \cdot 1993$

ANNUAL MEAN

HIGHEST ANNUAL MEAN

LOWEST ANNUAL MEAN

HIGHEST DATLY MEAN

IONEST DATIY MEAN

ANNUAL SEVEN-DAY MINIMUM

TNSTANTANEOUS PEAK FLOW

TNS'MANMANEOUS PEAK ETEVATTON

INSTANTANEOUS LOW FLOW

ANNUAL RUNOEE (INCHES/CESM)

$\begin{array}{ccrr}322 & & & \\ 901 & & & 1964 \\ 35.4 & & & 1956 \\ 16400 & \text { Sep } 14 & 1964 \\ 2.6 & \text { Jul } & 3 & 1981 \\ 2.8 & \text { Jul } & 2 & 1981 \\ 17600 & \text { Sep } 13 & 1964 \\ 26.74 & \text { Sep } 13 & 1964 \\ 2.5 & \text { Jul } 18 & 1981 \\ 12.50 / 0.92 & & \end{array}$

MAXIMUM, MINIMUM, AND MEAN OF MONTHLY MEAN DISCHARGES FOR WATER YEARS 1950-1993

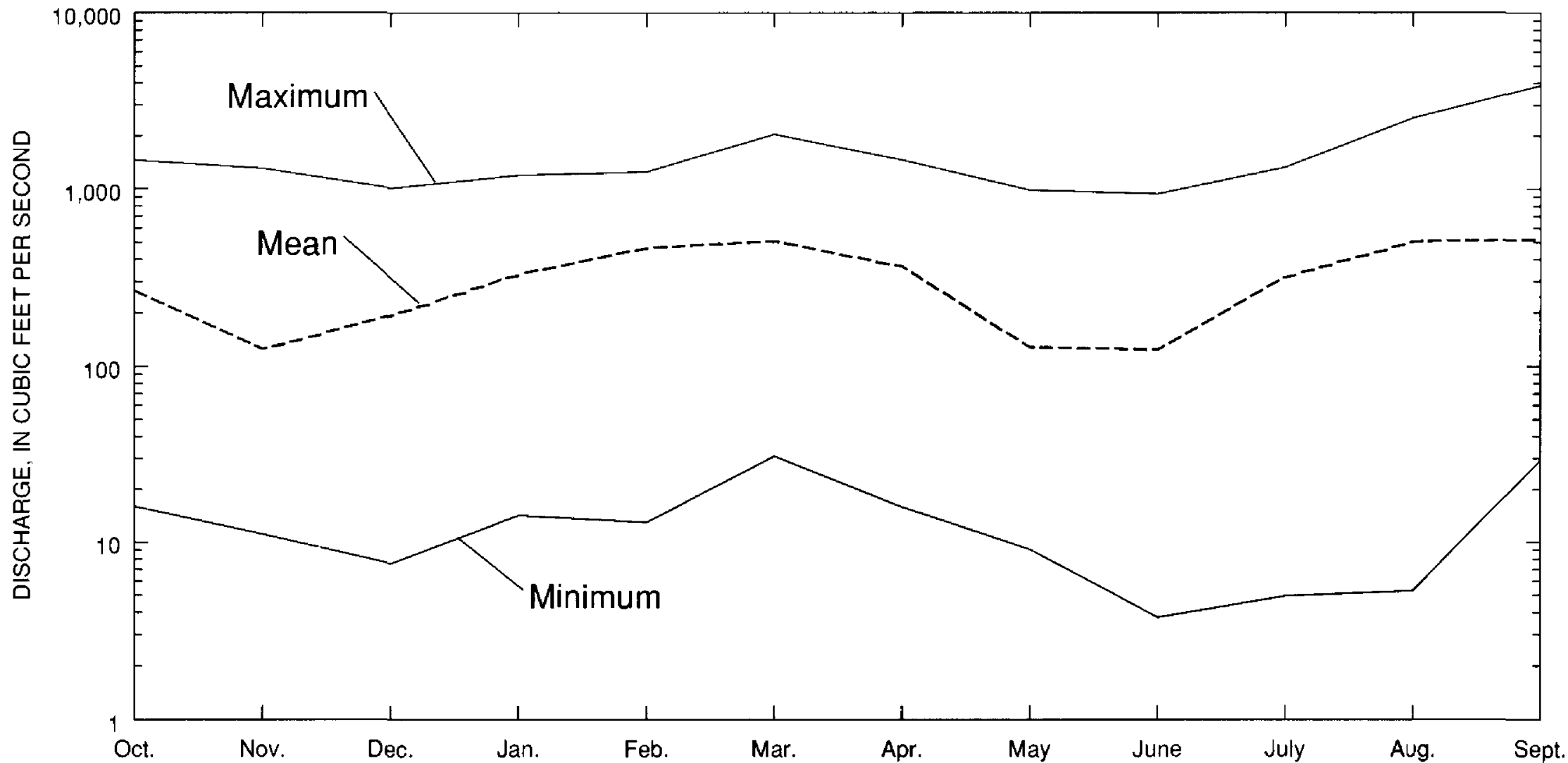

DURATION OF DAILY MEAN DISCHARGE FOR WATER YEARS 1950-1993

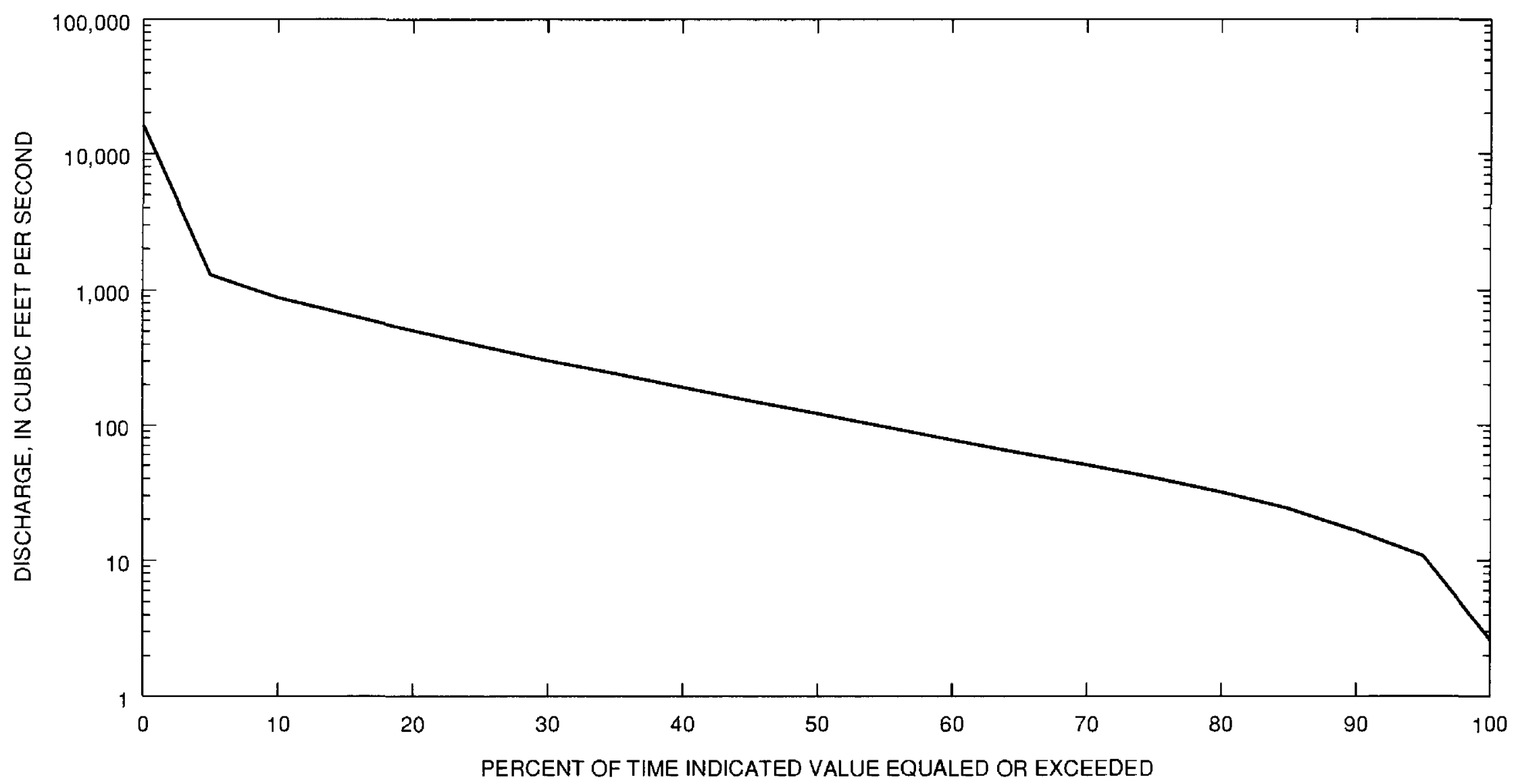


STEINHATCHEE RIVER BASIN

02324000 STEINHATCHEE RIVER NEAR CROSS CITY, FL--Continued

SUMMARY OF MONTHLY MEAN ELEVATION AND DISCARGE STATISTICS FOR WATER YEARS 1950-1993

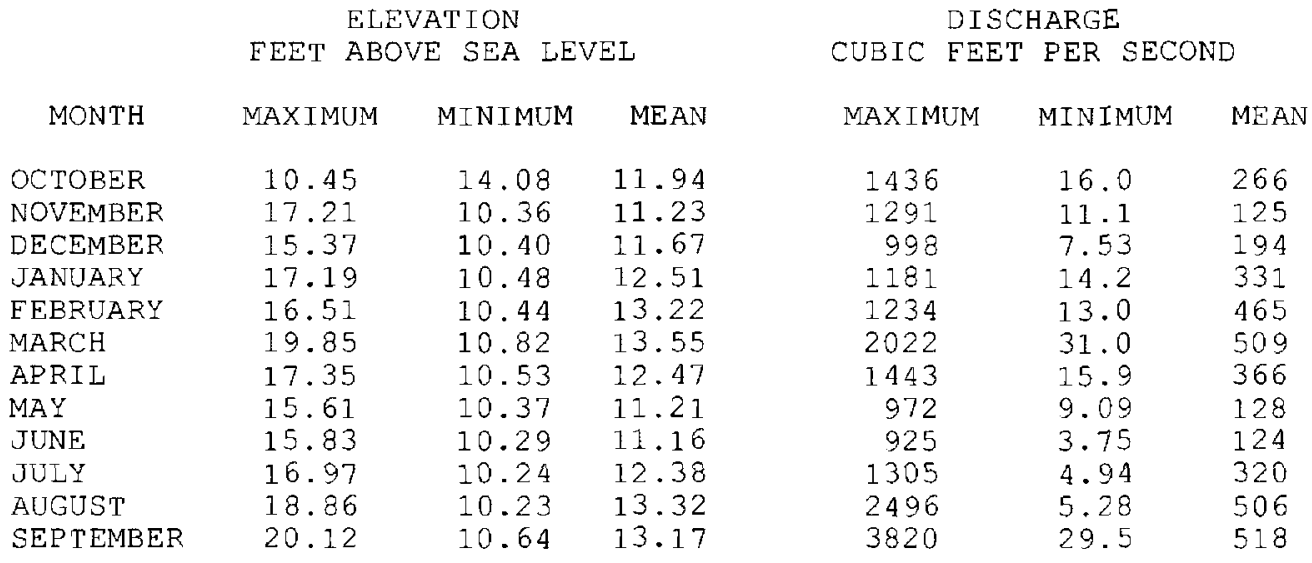

\section{DURATION OF DAILY MEAN VALUES FOR WATER YEARS 1950-1993}

PERCENT

OF TIME

XCEEDED ANNUAL OCT

DEC

JAN

$\mathrm{E} \Xi \mathrm{B}$

MAR

$\mathrm{APR}$

MAY

JUNE

JULY

AUG

SEPT

$\begin{array}{rlll}95.0 & 10.1 & 10.2 & 10.3 \\ 90.0 & 10.4 & 10.4 & 10.3 \\ 85.0 & 10.4 & 10.4 & 10.3 \\ 80.0 & 10.4 & 10.4 & 10.3 \\ 75.0 & 10.4 & 10.4 & 10.3 \\ 70.0 & 10.8 & 10.4 & 10.6 \\ 65.0 & 10.8 & 10.7 & 10.6 \\ 60.0 & 10.8 & 10.7 & 10.6 \\ 55.0 & 11.1 & 10.7 & 10.6 \\ 50.0 & 11.1 & 11.0 & 10.6 \\ 45.0 & 11.4 & 11.0 & 10.6 \\ 40.0 & 11.8 & 11.3 & 10.9 \\ 35.0 & 11.8 & 11.5 & 10.9 \\ 30.0 & 12.1 & 11.5 & 10.9 \\ 25.0 & 12.9 & 12.2 & 11.1 \\ 20.0 & 13.3 & 12.5 & 11.1 \\ 15.0 & 14.1 & 13.1 & 11.4 \\ 10.0 & 15.4 & 14.2 & 12.0 \\ 5.0 & 17.9 & 17.4 & 13.2\end{array}$

$95.0 \quad 10.8$

$90.0 \quad 16.5$

$85.0 \quad 23.9$

$80.0 \quad 31.8$

$\begin{array}{ll}75.0 & 40.5 \\ 70.0 & 50.6\end{array}$

$65.0 \quad 61.0$

$60.0 \quad 77.0$

$55.0 \quad 97.4$

$50.0 \quad 122.1$

$45.0 \quad 152.0$

$\begin{array}{ll}40.0 & 191.0 \\ 35.0 & 241.4\end{array}$

$30.0 \quad 302.3$

$25.0 \quad 389.3$

$20.0 \quad 502.7$

$15.0 \quad 660.0$

$\begin{array}{rr}5.0 & 1294.6\end{array}$

12.2
16.4
20.5
25.8
32.2
39.4
47.7
57.8
71.5
88.5
109.7
134.0
162.4
204.0
257.8
338.0
464.0
675.1
1185.2

11.1
13.1
14.9
20.7
27.6
31.4
34.9
38.3
43.9
50.7
58.3
65.9
74.6
85.5
99.5
123.8
157.5
215.4
419.2

13.3
20.8
24.0
28.0
33.2
39.0
46.0
55.1
65.4
77.8
94.2
115.9
143.
213.
266.
335.
405.0
531.
760.

$\begin{array}{ll}10.4 & 10 . \\ 10.4 & 10 . \\ 10.4 & 10.6 \\ 10.6 & 10 . \\ 10.6 & 10 . \\ 10.6 & 10 . \\ 10.6 & 11 . \\ 10.8 & 11 . \\ 10.8 & 11 . \\ 10.8 & 11 . \\ 11.0 & 11 . \\ 11.0 & 12 . \\ 11.2 & 12 . \\ 11.7 & 12 . \\ 12.1 & 13 . \\ 12.4 & 13 . \\ 12.9 & 14 . \\ 13.7 & 15 . \\ 14.8 & 16 .\end{array}$

$\begin{array}{ll}10.4 & 10.6 \\ 10.4 & 10.9 \\ 10.6 & 10.9 \\ 10.6 & 11.1 \\ 10.8 & 11.3 \\ 10.8 & 11.6 \\ 11.3 & 11.8 \\ 11.3 & 11.8 \\ 11.5 & 12.1 \\ 11.8 & 12.4 \\ 11.8 & 12.6 \\ 12.0 & 12.9 \\ 12.3 & 13.2 \\ 12.8 & 13.8 \\ 13.3 & 14.4 \\ 13.6 & 15.0 \\ 14.5 & 15.7 \\ 15.7 & 16.4 \\ 16.7 & 17.5\end{array}$

10.5
10.7
10.7
11.0
11.3
11.3
11.6
11.8
12.1
12.4
13.1
13.4
13.7
14.1
14.8
15.9
16.7
17.5
19.3

10.4
10.4
10.6
10.6
10.6
10.6
10.9
10.9
11.2
11.4
11.4
11.7
12.3
12.6
13.2
13.5
14.5
15.9
17.9

10.3
10.3
10.3
10.3
10.3
10.3
10.3
10.5
10.5
10.5
10.5
10.8
10.8
11.0
11.3
11.6
11.9
12.1
13.0

DISCARGE IN CUBIC EEET PER SECOND

10.2
10.2
10.2
10.2
10.2
10.5
10.5
10.5
10.5
10.5
10.7
10.7
11.0
11.3
11.3
11.5
12.1
13.3

10.

10.4
10.4

10.4

10.4

10.7

10.7

11.0

11.3

1.5

11.9

12.5

13.1

13.8
14.9

16.1

17.8

10.4
10.7

10.7

10.7

11.3

11.6

11.9

12.3
12.3

12.6

13.3

14.1

14.9

15.3
16.2

18.0

19.6

10.4

10.4

10.8

10.8

11.1

11.4

11.8
12.1

12.1

12.5

13.3

15.4

17.4

\section{$\begin{array}{llll}19.8 & 43.0 & 35.7 & 19.2\end{array}$}

$\begin{array}{rrrrr}13.3 & 19.8 & 43.0 & 35.7 & 19.2 \\ 20.8 & 31.6 & 61.5 & 48.2 & 28.4 \\ 24.0 & 45.7 & 83.0 & 60.7 & 33.7 \\ 28.0 & 57.7 & 103.9 & 78.2 & 41.4 \\ 33.2 & 73.4 & 128.3 & 105.4 & 50.2 \\ 39.0 & 89.8 & 158.8 & 128.9 & 59.9 \\ 46.0 & 120.3 & 195.7 & 154.0 & 72.8 \\ 55.1 & 143.9 & 240.6 & 189.7 & 90.7 \\ 65.4 & 169.1 & 277.6 & 242.8 & 114.9 \\ 77.8 & 197.3 & 323.0 & 307.0 & 140.5 \\ 94.2 & 231.5 & 382.1 & 374.9 & 175.0 \\ 15.9 & 274.5 & 439.9 & 444.4 & 221.8 \\ 43.3 & 326.5 & 512.7 & 521.7 & 289.7 \\ 13.0 & 391.0 & 612.8 & 602.7 & 373.3 \\ 266.7 & 468.3 & 710.5 & 717.1 & 463.3 \\ 335.0 & 553.4 & 825.0 & 869.7 & 573.3 \\ 05.0 & 669.2 & 962.2 & 1042.2 & 743.7 \\ 31.3 & 843.5 & 1108.6 & 1250.7 & 1044.8 \\ 760.4 & 1081.9 & 1329.6 & 1652.3 & 1485.0\end{array}$

8.6
10.9
12.8
14.5
16.5
19.0
22.7
27.4
33.7
43.4
53.4
65.7
80.5
97.8
122.3
158.2
214.0
291.4
459.4

5.9
7.2
8.5
10.7
13.3
16.1
19.6
24.3
30.5
37.6
44.9
52.9
64.5
91.6
122.1
155.6
208.2
295.3
535.9

6.6
15.0
21.0
28.5
37.4
49.3
59.8
72.7
95.3
121.0
158.6
193.3
234.5
296.0
394.0
530.0
711.5
904.0
267.5

13.8
34.9
46.3
57.6
74.2
100.8
140.2
183.1
223.5
269.2
316.1
384.9
481.1
599.4
726.2
864.8
1048.4
1303.5
1688.4

24.1
35.7
50.2
67.0
86.8
06.4
28.0
54.3
86.1
223.5
265.1
312.9
378.0
486.6
587.6
716.3
882.8
186.1
1667.9 

FOR PERIOD APR TO MAR

WATER YEAR

RANGE

19511952

19521953

19531954

19541955

19551956

19561957
1957

19591960

19601961

19611962

19621963

19631964

1.9641965

19681969

19691970

19701971

19711972

19751976

$1976 \quad 1977$

19771978

19781979

1981980

19851986

19871988

19891990

19911992

19921993

\begin{tabular}{|c|c|c|c|c|c|}
\hline 1 & & 3 & & 7 & \\
\hline 10.3 & 6 & 10.51 & 18 & 10.5 & 18 \\
\hline 10.4 & 13 & 10.41 & 10 & 10.4 & 10 \\
\hline 10.5 & 23 & 10.52 & 21 & 10.5 & 21 \\
\hline 10.3 & 4 & 10.3 & 4 & 10.3 & 4 \\
\hline 10.3 & 3 & 10.3 & 3 & 10.3 & 3 \\
\hline 10.4 & 16 & 10.41 & 15 & 10.4 & 15 \\
\hline 10.72 & 28 & 10.72 & 28 & 10.8 & 28 \\
\hline $10.7 ?$ & 27 & 10.72 & 27 & 10.8 & 27 \\
\hline 10.4 & 19 & 10.51 & 19 & 10.5 & 19 \\
\hline 10.3 & 8 & 10.3 & 6 & 10.3 & 6 \\
\hline 10.4 & 18 & 10.41 & 17 & 10.4 & 17 \\
\hline 10.4 & 17 & 10.41 & 16 & 10.4 & 16 \\
\hline 10.52 & 24 & 10.52 & 24 & 10.5 & 24 \\
\hline 10.3 & 9 & 10.4 & 8 & 10.4 & 7 \\
\hline 10.62 & 25 & 10.62 & 25 & 10.6 & 25 \\
\hline 10.52 & 22 & 10.53 & 23 & 10.5 & 23 \\
\hline 10.4$]$ & 14 & 10.41 & 11 & 10.4 & 13 \\
\hline 10.62 & 26 & 10.62 & 26 & 10.6 & 26 \\
\hline 10.5 & 20 & 10.52 & 20 & 10.5 & 20 \\
\hline 10.2 & 1 & 10.2 & 1 & 10.2 & 2 \\
\hline 10.3 & 7 & 10.3 & 7 & 10.4 & 8 \\
\hline 20.5 & 21 & 10.52 & 22 & 10.5 & 22 \\
\hline 10.41 & 11 & 10.41 & 12 & 10.4 & 11 \\
\hline 10.3 & 5 & 10.3 & 5 & 10.3 & 5 \\
\hline 10.4 & 15 & 10.41 & 13 & 10.4 & 12 \\
\hline 10.41 & 12 & 10.41 & 14 & 10.4 & 14 \\
\hline 10.4 & 10 & 10.4 & 9 & 10.4 & 9 \\
\hline 10.2 & 2 & 10.2 & 2 & 10.2 & 1 \\
\hline
\end{tabular}

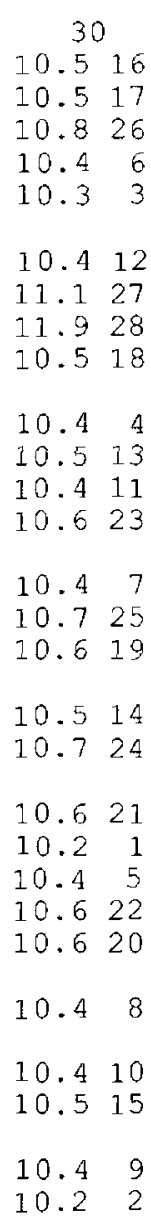

60

10.51
10.61

11.527

$\begin{array}{rr}11.5 & 27 \\ 10.4 & 6\end{array}$

$\begin{array}{ll}10.4 & 6 \\ 10.4 & 3\end{array}$

10.510

11.325

12.128

10.820

10.49

$10.5 \quad 15$

10.511

11.326

10.48

11.224

10.619

$10.5 \quad 13$

10.821

10.822

10.3

10.44

10.823

10.47

10.512

$\begin{array}{ll}10.4 & 5 \\ 10.3 & 2\end{array}$

\begin{tabular}{rr}
\multicolumn{1}{c}{90} \\
10.6 & 12 \\
10.6 & 17 \\
12.1 & 25 \\
10.5 & 6 \\
10.4 & 3 \\
10.5 & 7 \\
12.3 & 27
\end{tabular}

$\begin{array}{lll}12.3 & 28 & 12.828 \\ 11.2 & 23 & 11.724\end{array}$

10.58

$10.6 \quad 13$

$10.6 \quad 15$

$10.6 \quad 10$

$10.5 \quad 5$

11.424

10.67

10.815

$10.6 \quad 16$

11.422

10.921

10.3

10.511

10.920

11.019

10.42

10.69

$10.5 \quad 5$

$\begin{array}{ll}10.5 & 10 \\ 10.6 & 14\end{array}$

10.44
10.920

10.814

12.526

$\begin{array}{rr}10.6 & 11 \\ 10.5 & 3\end{array}$

10.54

$12.6 \quad 27$

10.68

11.120

11.723

11.018

$10.6 \quad 12$

10.916

183

$\begin{array}{ll}11.4 & 15 \\ 11.3 & 13\end{array}$

13.727

10.98

10.72

$10.8 \quad 7$

3.526

12.923

10.73

11.416

12.924

$10.7 \quad 1$

$\begin{array}{ll}13.5 & 25 \\ 11.4 & 17\end{array}$

12.321

11.519

11.110

11.212

$11.3 \quad 14$

11.111

12.722

$10.8 \quad 5$

10.56

10.86

10.74

HIGHEST MEAN ELEVATION, IN FEET, AND RANKING FOR THE FOLLOWING NUMBER OF CONSECUTIVE DAYS FOR PERIOD OCT TO SEP

WATER YEAR RANGE $\begin{array}{ll}1951 & 1951 \\ 1952 & 1952 \\ 1953 & 1953 \\ 1954 & 1954 \\ 1955 & 1955 \\ 1956 & 1956 \\ 1957 & 1957 \\ 1958 & 1958 \\ 1960 & 1960 \\ 1961 & 1961 \\ 1962 & 1962 \\ 1963 & 1963 \\ 1964 & 1964 \\ 1965 & 1965 \\ 1968 & 1968 \\ 1969 & 1969 \\ 1970 & 1970 \\ 1971 & 1971 \\ 1972 & 1972 \\ 1975 & 1975 \\ 1976 & 1976 \\ 1977 & 1977 \\ 1978 & 1978 \\ 1979 & 1979 \\ 1980 & 1980 \\ 1985 & 1985 \\ 1985 & \\ 1991 & 1991 \\ 1992 & 1992 \\ 1993 & 1993\end{array}$ \begin{tabular}{crrrr}
1 & & \multicolumn{3}{r}{3} \\
16.7 & 22 & & 16.5 & 22 \\
23.1 & 8 & & 23.0 & 8 \\
23.2 & 7 & & 23.0 & 7 \\
23.2 & 6 & & 23.1 & 6 \\
15.1 & 25 & & 15.0 & 25
\end{tabular} $12.929 \quad 12.729$ 22.511 $\begin{array}{rr}22.5 & 11 \\ 23.7 & 4\end{array}$ 22.313

22.610 16.323 17.421 26.5 18.419

16.124 $21.4 \quad 15$ 24.62

20.017 $\begin{array}{ll}22.0 & 14 \\ 17.6 & 20\end{array}$ 13.128 20.116 22.412 19.618 23.65 24.03

23.09 $\begin{array}{ll}13.9 & 27 \\ 14.7 & 26\end{array}$ $22.3 \quad 11$ $\begin{array}{ll}23.5 & 5 \\ 22.2 & 13\end{array}$ $22.5 \quad 10$ $15.6 \quad 24$

17.321 26.31 18.019

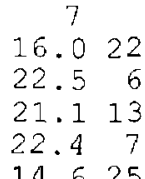

14.625

12.329

21.512

23.24

$21.8 \quad 10$

22.19

15.323

16.921

25.51

17.418

15.623

21.315

14.524

$20.8 \quad 15$

24.12

$19.7 \quad 17$

21.814
$17.5 \quad 20$

13.128

19.916

22.312

18.918

19.117

$\begin{array}{ll}21.0 & 14 \\ 17.2 & 19\end{array}$

13.028

19.316

21.711

$\begin{array}{rr}17.0 & 20 \\ 23.2 & 5\end{array}$

23.93

23.63

22.48

22.99

13.827
14.6
15

14.823

$\begin{array}{rr}20.4 & 8 \\ 19.6 & 10\end{array}$

19.411

3.725

$11.7 \quad 29$

19.013

21.3
$19.6 \quad 9$

21.25

14.822

16.618

24.01

15.920

13.326

$18.9 \quad 14$

17.916

$\begin{array}{ll}18.6 & 15 \\ 16.4 & 19\end{array}$

12.728

16.917

19.312

$\begin{array}{rr}15.9 & 21 \\ 21.2 & 6\end{array}$

22.73

20.77

$\begin{array}{ll}13.0 & 27 \\ 14.2 & 24\end{array}$

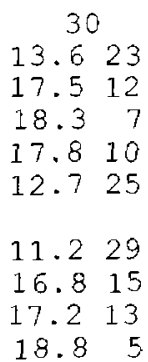

18.08

13.524

16.117

$\begin{array}{rr}21.1 & 1 \\ 14.7 & 20\end{array}$

12.128

$17.1 \quad 14$

17.511

$\begin{array}{ll}16.1 & 16 \\ 15.7 & 18\end{array}$

$12.2 \quad 27$

14.819

18.46

14.221

20.82

20.24

12.326
60 12.724
15.314

16.48

15.710

11.926

$11.0 \quad 29$

$15.5 \quad 12$

15.711

17.06

14.518

12.823

14.616

20.4

13.520

11.627

17.03

15.513

14.915

12.025

14.617

17.42

13.122

17.04

17.05

$\begin{array}{ll}11.4 & 28 \\ 13.5 & 21\end{array}$

12.90

14.315

15.57

14.812

11.029

14.614

14.910

13.319

12.123

13.618

19.11

13.121

$11.4 \quad 27$

15.65

16.23

14.016

$\begin{array}{rr}15.0 & 8 \\ 13.9 & 17\end{array}$

$11.9 \quad 25$

14.613

16.32

$\begin{array}{ll}12.9 & 22 \\ 14.8 & 11\end{array}$ 
LOWEST MEAN DISCHARGE, IN CUBIC FEET PER SECOND, AND RANKING FOR THE FOLLOWING NUMBER OF CONSECUTIVE DAYS FOR PERIOD APR TO MAR

\begin{tabular}{|c|c|c|c|c|c|c|c|c|c|c|c|c|c|c|c|c|c|c|}
\hline $\begin{array}{l}\text { WATE } \\
\text { RP } \\
1950\end{array}$ & $\begin{array}{l}\text { ANGE } \\
1951\end{array}$ & $\begin{array}{c}1 \\
3.60\end{array}$ & 3 & $\begin{array}{c}3 \\
3.63\end{array}$ & 3 & $\begin{array}{r}7 \\
3.84\end{array}$ & 3 & 5.23 & 4 & $\begin{array}{l}30 \\
11.31\end{array}$ & & $17.1^{60}=$ & $\begin{array}{l}0 \\
15\end{array}$ & $22.2^{9}$ & $\begin{array}{l}0 \\
13\end{array}$ & $76.5^{12}$ & $\begin{array}{l}20 \\
25\end{array}$ & $\begin{array}{r}183 \\
125 \quad 19\end{array}$ \\
\hline $\begin{array}{l}1951 \\
1952 \\
1953 \\
1954 \\
1955\end{array}$ & $\begin{array}{l}1952 \\
1953 \\
1954 \\
1955 \\
1956\end{array}$ & $\begin{array}{l}6.80 \\
11.0 \\
8.30 \\
9.80 \\
3.70\end{array}$ & $\begin{array}{r}11 \\
25 \\
13 \\
19 \\
4\end{array}$ & $\begin{array}{l}7.57 \\
11.0 \\
8.53 \\
9.80 \\
3.83\end{array}$ & $\begin{array}{r}11 \\
25 \\
13 \\
19 \\
5\end{array}$ & $\begin{array}{l}8.41 \\
11.6 \\
9.47 \\
10.3 \\
4.04\end{array}$ & $\begin{array}{l}11 \\
25 \\
14 \\
17 \\
4\end{array}$ & $\begin{array}{l}9.00 \\
12.6 \\
13.4 \\
11.7 \\
4.29\end{array}$ & $\begin{array}{r}10 \\
24 \\
25 \\
19 \\
3\end{array}$ & $\begin{array}{l}9.72 \\
19.7 \\
42.3 \\
12.9 \\
4.98\end{array}$ & $\begin{array}{r}8 \\
24 \\
39 \\
17 \\
3\end{array}$ & $\begin{array}{r}17.5 \\
26.3 \\
131 \\
15.6 \\
6.62\end{array}$ & $\begin{array}{r}17 \\
24 \\
42 \\
12 \\
3\end{array}$ & $\begin{array}{r}23.4 \\
33.5 \\
234 \\
22.2 \\
7.97\end{array}$ & $\begin{array}{r}9 \\
23 \\
40 \\
14 \\
2\end{array}$ & $\begin{array}{r}62.7 \\
47.9 \\
288 \\
38.6 \\
10.2\end{array}$ & $\begin{array}{r}23 \\
20 \\
41 \\
16 \\
2\end{array}$ & $\begin{array}{rr}126 & 20 \\
121 & 18 \\
533 & 43 \\
62.0 & 10 \\
23.2 & 1\end{array}$ \\
\hline $\begin{array}{l}1956 \\
1957 \\
1958 \\
1959 \\
1960\end{array}$ & $\begin{array}{l}1957 \\
1958 \\
1959 \\
1960 \\
1961\end{array}$ & $\begin{array}{l}5.90 \\
44.0 \\
21.0 \\
43.0 \\
14.0\end{array}$ & $\begin{array}{r}8 \\
43 \\
36 \\
42 \\
30\end{array}$ & $\begin{array}{l}6.30 \\
45.0 \\
22.7 \\
45.0 \\
15.7\end{array}$ & $\begin{array}{l}10 \\
42 \\
36 \\
43 \\
31\end{array}$ & $\begin{array}{l}6.60 \\
51.0 \\
29.1 \\
55.1 \\
18.0\end{array}$ & $\begin{array}{l}10 \\
42 \\
38 \\
43 \\
32\end{array}$ & $\begin{array}{l}7.61 \\
56.5 \\
35.0 \\
66.3 \\
23.1\end{array}$ & $\begin{array}{r}9 \\
42 \\
39 \\
43 \\
31\end{array}$ & $\begin{array}{r}11.3 \\
87.4 \\
50.9 \\
200 \\
26.6\end{array}$ & $\begin{array}{l}11 \\
42 \\
41 \\
43 \\
28\end{array}$ & $\begin{array}{r}14.3 \\
102 \\
75.3 \\
227 \\
52.1\end{array}$ & $\begin{array}{l}9 \\
39 \\
36 \\
43 \\
32\end{array}$ & $\begin{array}{r}16.8 \\
257 \\
84.7 \\
255 \\
110\end{array}$ & $\begin{array}{r}6 \\
42 \\
32 \\
41 \\
35\end{array}$ & $\begin{array}{r}20.5 \\
311 \\
82.1 \\
336 \\
195\end{array}$ & $\begin{array}{r}5 \\
42 \\
27 \\
43 \\
38\end{array}$ & $\begin{array}{rr}47.6 & 6 \\
474 & 39 \\
167 & 27 \\
524 & 42 \\
404 & 38\end{array}$ \\
\hline $\begin{array}{l}1961 \\
1962 \\
1963 \\
1964 \\
1965\end{array}$ & $\begin{array}{l}1962 \\
1963 \\
1964 \\
1965 \\
1966\end{array}$ & $\begin{array}{l}10.0 \\
5.90 \\
10.0 \\
17.0 \\
10.0\end{array}$ & $\begin{array}{r}20 \\
9 \\
21 \\
34 \\
22\end{array}$ & $\begin{array}{l}10.0 \\
5.90 \\
10.0 \\
18.0 \\
10.0\end{array}$ & $\begin{array}{r}20 \\
8 \\
21 \\
34 \\
22\end{array}$ & $\begin{array}{l}10.0 \\
6.24 \\
10.3 \\
20.9 \\
10.1\end{array}$ & $\begin{array}{r}15 \\
7 \\
18 \\
34 \\
16\end{array}$ & $\begin{array}{l}10.9 \\
6.81 \\
11.1 \\
30.6 \\
10.9\end{array}$ & $\begin{array}{r}13 \\
7 \\
16 \\
36 \\
14\end{array}$ & $\begin{array}{l}11.9 \\
8.06 \\
12.6 \\
35.5 \\
22.4\end{array}$ & $\begin{array}{r}13 \\
6 \\
15 \\
35 \\
27\end{array}$ & $\begin{array}{r}17.3 \\
13.7 \\
17.0 \\
125 \\
84.0\end{array}$ & $\begin{array}{r}16 \\
8 \\
14 \\
41 \\
37\end{array}$ & $\begin{array}{r}22.6 \\
16.8 \\
31.8 \\
286 \\
111\end{array}$ & $\begin{array}{r}15 \\
7 \\
21 \\
43 \\
36\end{array}$ & $\begin{array}{r}32.4 \\
23.6 \\
99.5 \\
279 \\
165\end{array}$ & $\begin{array}{r}11 \\
7 \\
30 \\
40 \\
36\end{array}$ & $\begin{array}{rr}42.4 & 3 \\
131 & 21 \\
149 & 24 \\
375 & 37 \\
245 & 30\end{array}$ \\
\hline $\begin{array}{l}1966 \\
1967 \\
1968 \\
1969 \\
1970\end{array}$ & $\begin{array}{l}1967 \\
1968 \\
1969 \\
1970 \\
1971\end{array}$ & $\begin{array}{l}39.0 \\
10.0 \\
4.70 \\
21.0 \\
15.0\end{array}$ & $\begin{array}{r}41 \\
23 \\
6 \\
37 \\
32\end{array}$ & $\begin{array}{l}39.3 \\
10.7 \\
5.00 \\
23.0 \\
17.0\end{array}$ & $\begin{array}{r}41 \\
24 \\
6 \\
37 \\
33\end{array}$ & $\begin{array}{l}40.6 \\
10.9 \\
5.04 \\
25.9 \\
20.0\end{array}$ & $\begin{array}{r}41 \\
23 \\
5 \\
36 \\
33\end{array}$ & $\begin{array}{l}42.4 \\
11.0 \\
5.60 \\
28.4 \\
27.1\end{array}$ & $\begin{array}{r}41 \\
15 \\
5 \\
34 \\
32\end{array}$ & $\begin{array}{l}50.8 \\
11.5 \\
5.90 \\
37.6 \\
32.2\end{array}$ & $\begin{array}{r}40 \\
12 \\
4 \\
37 \\
32\end{array}$ & $\begin{array}{r}86.3 \\
13.1 \\
7.99 \\
109 \\
42.2\end{array}$ & $\begin{array}{r}38 \\
7 \\
5 \\
40 \\
29\end{array}$ & $\begin{array}{r}153 \\
21.6 \\
12.4 \\
138 \\
52.2\end{array}$ & $\begin{array}{r}39 \\
11 \\
5 \\
38 \\
26\end{array}$ & $\begin{array}{r}199 \\
48.7 \\
21.8 \\
184 \\
94.9\end{array}$ & $\begin{array}{r}39 \\
21 \\
6 \\
37 \\
29\end{array}$ & $\begin{array}{rr}373 & 36 \\
83.6 & 13 \\
33.6 & 2 \\
484 & 40 \\
148 & 23\end{array}$ \\
\hline $\begin{array}{l}1971 \\
1972 \\
1973 \\
1974 \\
1975\end{array}$ & $\begin{array}{l}1972 \\
1973 \\
1974 \\
1975 \\
1976\end{array}$ & $\begin{array}{l}8.20 \\
8.80 \\
8.80 \\
24.0 \\
31.0\end{array}$ & $\begin{array}{l}12 \\
14 \\
15 \\
38 \\
39\end{array}$ & $\begin{array}{l}8.47 \\
9.10 \\
8.80 \\
24.3 \\
31.3\end{array}$ & $\begin{array}{l}12 \\
15 \\
14 \\
38 \\
39\end{array}$ & $\begin{array}{l}9.16 \\
11.3 \\
8.96 \\
27.0 \\
32.9\end{array}$ & $\begin{array}{l}13 \\
24 \\
12 \\
37 \\
40\end{array}$ & $\begin{array}{l}10.8 \\
13.7 \\
9.44 \\
28.7 \\
35.8\end{array}$ & $\begin{array}{l}12 \\
27 \\
11 \\
35 \\
40\end{array}$ & $\begin{array}{l}14.0 \\
21.0 \\
10.7 \\
30.6 \\
38.9\end{array}$ & $\begin{array}{r}19 \\
26 \\
9 \\
30 \\
38\end{array}$ & $\begin{array}{l}19.0 \\
30.6 \\
14.9 \\
39.7 \\
50.8\end{array}$ & $\begin{array}{l}18 \\
26 \\
11 \\
28 \\
31\end{array}$ & $\begin{array}{l}32.9 \\
66.0 \\
28.8 \\
66.1 \\
104\end{array}$ & $\begin{array}{l}22 \\
29 \\
19 \\
30 \\
34\end{array}$ & $\begin{array}{r}57.2 \\
77.7 \\
37.1 \\
118 \\
141\end{array}$ & $\begin{array}{l}22 \\
26 \\
14 \\
31 \\
34\end{array}$ & $\begin{array}{rr}303 & 35 \\
97.6 & 16 \\
54.8 & 7 \\
295 & 34 \\
165 & 26\end{array}$ \\
\hline $\begin{array}{l}1976 \\
1977 \\
1978 \\
1979 \\
1980\end{array}$ & $\begin{array}{l}1977 \\
1978 \\
1979 \\
1980 \\
1981\end{array}$ & $\begin{array}{l}16.0 \\
6.20 \\
11.0 \\
19.0 \\
13.0\end{array}$ & $\begin{array}{l}33 \\
10 \\
26 \\
35 \\
29\end{array}$ & $\begin{array}{l}16.7 \\
6.20 \\
11.0 \\
21.0 \\
13.7\end{array}$ & $\begin{array}{r}32 \\
9 \\
26 \\
35 \\
29\end{array}$ & $\begin{array}{l}17.6 \\
6.37 \\
11.9 \\
24.0 \\
14.3\end{array}$ & $\begin{array}{r}31 \\
8 \\
28 \\
35 \\
29\end{array}$ & $\begin{array}{l}20.0 \\
6.43 \\
12.3 \\
27.5 \\
17.8\end{array}$ & $\begin{array}{r}30 \\
6 \\
23 \\
33 \\
28\end{array}$ & $\begin{array}{l}27.8 \\
6.51 \\
12.6 \\
32.0 \\
33.1\end{array}$ & $\begin{array}{r}29 \\
5 \\
16 \\
31 \\
33\end{array}$ & $\begin{array}{l}54.1 \\
7.12 \\
15.8 \\
37.4 \\
57.3\end{array}$ & $\begin{array}{r}33 \\
4 \\
13 \\
27 \\
34\end{array}$ & $\begin{array}{l}71.9 \\
8.78 \\
27.6 \\
53.9 \\
64.8\end{array}$ & $\begin{array}{r}31 \\
3 \\
18 \\
27 \\
28\end{array}$ & $\begin{array}{r}88.1 \\
13.0 \\
42.1 \\
121 \\
64.1\end{array}$ & $\begin{array}{r}28 \\
3 \\
17 \\
32 \\
24\end{array}$ & 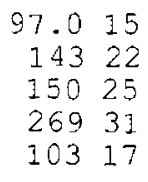 \\
\hline $\begin{array}{l}1981 \\
1982 \\
1983 \\
1984 \\
1985\end{array}$ & $\begin{array}{l}1982 \\
1983 \\
1984 \\
1985 \\
1986\end{array}$ & $\begin{array}{l}2.60 \\
31.0 \\
3.20 \\
11.0 \\
5.60\end{array}$ & $\begin{array}{r}1 \\
40 \\
2 \\
27 \\
7\end{array}$ & $\begin{array}{l}2.73 \\
31.3 \\
3.53 \\
11.0 \\
5.80\end{array}$ & $\begin{array}{r}1 \\
40 \\
2 \\
27 \\
7\end{array}$ & $\begin{array}{l}2.80 \\
31.7 \\
6.54 \\
11.6 \\
6.09\end{array}$ & $\begin{array}{r}1 \\
39 \\
9 \\
26 \\
6\end{array}$ & $\begin{array}{l}2.90 \\
33.0 \\
30.6 \\
13.4 \\
6.86\end{array}$ & $\begin{array}{r}1 \\
38 \\
37 \\
26 \\
8\end{array}$ & $\begin{array}{l}2.97 \\
37.1 \\
33.3 \\
15.7 \\
8.44\end{array}$ & $\begin{array}{r}1 \\
36 \\
34 \\
23 \\
7\end{array}$ & $\begin{array}{l}4.48 \\
49.9 \\
69.2 \\
20.4 \\
12.0\end{array}$ & $\begin{array}{r}1 \\
30 \\
35 \\
21 \\
6\end{array}$ & $\begin{array}{r}9.49 \\
90.2 \\
122 \\
25.6 \\
21.6\end{array}$ & $\begin{array}{r}4 \\
33 \\
37 \\
16 \\
12\end{array}$ & $\begin{array}{r}19.7 \\
152 \\
134 \\
28.0 \\
32.4\end{array}$ & $\begin{array}{r}4 \\
35 \\
33 \\
9 \\
12\end{array}$ & $\begin{array}{rr}42.7 & 4 \\
285 & 33 \\
179 & 28 \\
43.6 & 5 \\
507 & 41\end{array}$ \\
\hline $\begin{array}{l}1986 \\
1987 \\
1988 \\
1989 \\
1990\end{array}$ & $\begin{array}{l}1987 \\
1988 \\
1989 \\
1990 \\
1991\end{array}$ & $\begin{array}{l}14.0 \\
10.0 \\
9.40 \\
9.40 \\
9.00\end{array}$ & $\begin{array}{l}31 \\
24 \\
17 \\
18 \\
16\end{array}$ & $\begin{array}{l}14.7 \\
10.0 \\
9.43 \\
9.57 \\
9.50\end{array}$ & $\begin{array}{l}30 \\
23 \\
16 \\
18 \\
17\end{array}$ & $\begin{array}{l}15.7 \\
10.6 \\
10.5 \\
10.5 \\
10.5\end{array}$ & $\begin{array}{l}30 \\
22 \\
19 \\
20 \\
21\end{array}$ & $\begin{array}{l}18.7 \\
11.4 \\
11.5 \\
11.9 \\
11.8\end{array}$ & $\begin{array}{l}29 \\
17 \\
18 \\
21 \\
20\end{array}$ & $\begin{array}{l}20.6 \\
12.5 \\
14.4 \\
14.2 \\
14.1\end{array}$ & $\begin{array}{l}25 \\
14 \\
22 \\
21 \\
20\end{array}$ & $\begin{array}{l}28.8 \\
20.3 \\
23.7 \\
19.1 \\
22.4\end{array}$ & $\begin{array}{l}25 \\
20 \\
23 \\
19 \\
22\end{array}$ & $\begin{array}{l}38.5 \\
21.5 \\
29.2 \\
26.0 \\
35.7\end{array}$ & $\begin{array}{l}25 \\
10 \\
20 \\
17 \\
24\end{array}$ & $\begin{array}{l}47.7 \\
25.5 \\
36.9 \\
44.5 \\
38.1\end{array}$ & $\begin{array}{r}19 \\
8 \\
13 \\
18 \\
15\end{array}$ & $\begin{array}{rr}274 & 32 \\
57.6 & 8 \\
180 & 29 \\
86.7 & 14 \\
70.0 & 12\end{array}$ \\
\hline $\begin{array}{l}1991 \\
1992\end{array}$ & $\begin{array}{l}1992 \\
1993\end{array}$ & $\begin{array}{l}11.0 \\
3.70\end{array}$ & $\begin{array}{r}28 \\
5\end{array}$ & $\begin{array}{l}11.0 \\
3.70\end{array}$ & $\begin{array}{r}28 \\
4\end{array}$ & $\begin{array}{l}11.6 \\
3.79\end{array}$ & $\begin{array}{r}27 \\
2\end{array}$ & $\begin{array}{l}11.9 \\
4.09\end{array}$ & $\begin{array}{r}22 \\
2\end{array}$ & $\begin{array}{l}13.7 \\
4.38\end{array}$ & $\begin{array}{r}18 \\
2\end{array}$ & $\begin{array}{l}14.4 \\
5.68\end{array}$ & $\begin{array}{r}10 \\
2\end{array}$ & $\begin{array}{l}17.6 \\
6.34\end{array}$ & $\begin{array}{l}8 \\
1\end{array}$ & $\begin{array}{l}30.4 \\
9.25\end{array}$ & $\begin{array}{r}10 \\
1\end{array}$ & $\begin{array}{rr}62.4 & 11 \\
60.2 & 9\end{array}$ \\
\hline
\end{tabular}




\section{HIGHEST MEAN DISCHARGE, IN CUBIC FEET PER SECOND, AND RANKING FOR THE FOLLOWING NUMBER OF} CONSECUTIVE DAYS FOR PERIOD OCT TO SEP

WATER YEAR

RANGE

$1951 \quad 1951$

19521952

19531953

19541954

19551955

19561956

19571957

19581958

19591959

19601960

19611961

19621962

19631963

19641964

19651965

19661966

$1968 \quad 1968$

19691969

19701970

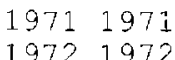

19731973

$1974 \quad 1974$

19751975

19761976

19771977

$1978 \quad 1978$

19801980

$1981 \quad 1981$

19821982

19831983

19841984

19851985

19861986

19871987

$1988 \quad 1988$

19891989

19901990

$1991 \quad 1991$

19921992

19931993
93236

356010

$3580 \quad 9$

37008

68439

33743

277016

42906

41907

267018

292014

93435

$1100 \quad 34$

64001

$1310 \quad 32$

$2240 \quad 21$

88437

201024

$1600 \quad 30$

$2320 \quad 20$

$3130 \quad 12$

$1530 \quad 31$

$1180 \quad 33$

$458 \quad 42$

$1610 \quad 29$

$2690 \quad 17$

$1650 \quad 28$

$4590 \quad 5$

50141

$2800 \quad 15$

191025

242019

300013

209023

$5010 \quad 4$

171027

183026

353011

58940

73138
3

$894 \quad 35$

341010

$3450 \quad 9$

35478

66739

31743

266316

41406

256018

280714

80636

$1090 \quad 34$

$15070 \quad 1$

124332

218721

2

7873

$\begin{array}{rr}1953 & 24 \\ 6717 \quad 2\end{array}$

$1550 \quad 29$

222320

306712

150330

116033

45742

$\begin{array}{lll}1570 & 28\end{array}$

$2613 \quad 17$

14873

44335

49741

$2717 \quad 15$

$\begin{array}{lll}1860 & 25\end{array}$

36719

52833

281313

203723

48734

164727

175026

330311

56740
724
15

82935

29338

224617

29039

60039

$258 \quad 43$

221018

37077

3743
226316

248913

$768 \quad 36$

$1032 \quad 34$

11160

112732

202920

163925

64138

179923

57172

144728

193721

275711

$1.410 \quad 30$

108933

$429 \quad 42$

$1450 \quad 27$

$2360 \quad 14$

113231

38675

$470 \leq 1$

259312

173424

213719

45563

235615

$1850 \quad 22$

43114

142929

154326

287010

50640
$673 \quad 37$
30

$630 \quad 37$

206212

159120

186613

47438

16443

153921

25917

165817

206311

$673 \quad 35$

97231

$\begin{array}{rr}6954 & 1 \\ 87434\end{array}$

167816

119127

42539

$1430 \quad 24$

$4340 \quad 2$

$1240 \quad 26$

143323

20859

125925

96832

$367 \quad 42$

106729

165818

$935 \quad 33$

27136

38941

208310

145622

$\begin{array}{lll}1743 & 14\end{array}$

$1715 \quad 15$

159819

31814

102630

109328

22598

41640

64136
60

31237

85120

102515

80423

20140

68.243

96.643

107924

14799

$\begin{array}{rr}1930 & 6 \\ 145410\end{array}$

$1377 \quad 14$

47136

88927

3858

66631

$1296 \quad 17$

78.129

$\begin{array}{lll}251 & 42\end{array}$

108823

28542

$1178 \quad 20$

92326

134915

104825

85928

28341

$748 \quad 30$

139713

$642 \quad 33$

$1714 \quad 7$

33138

16128

121119

127218

24963

$1167 \quad 21$

139812

19835

65132

61534

$2098 \quad 4$

28740

57935

82122

79124

12256

107710

73326

$348 \quad 36$

65828

30341

$473 \quad 32$

10939

56930

16741

107711

82621

74325

85719

89318

62829

$248 \quad 38$

$700 \quad 27$

12167

43733

96017

$240 \quad 39$

10988

$1058 \quad 13$

$1027 \quad 14$

14753 
LOCATION.--Lat $30^{\circ} 05^{\prime} 53^{\prime \prime}$, long $83^{\circ} 28^{\prime} 19^{\prime \prime}$, in NE $1 / 4$ sec.36, T.4 S., R.8 E., Taylor County, Hydrologic Unit 03110102, near left bank at downstream side of bridge on U.S. Highway $27,1.8 \mathrm{mi}$ upstream from small tributary, 4 mi northeast of Foley, and 32 mi upstream from mouth.

DRAINAGE AREA.--60 $\mathrm{mi}^{2}$ approximately.

PERIOD OF RECORD.--February to August 1955 (discharge measurements only); September 1955 to 1993.

REVISED RECORDS.--WSP 1905: Drainage area.

GAGE.--Water-stage recorder. Datum of gage is $53.59 \mathrm{ft}$ above National Geodetic Vertical Datum of 1929 (Florida Department of Transportation bench mark).

REMARKS.--Records fair.

MAXIMUM, MINIMUM, AND MEAN OF MONTHLY MEAN ELEVATIONS FOR WATER YEARS 1956-1993
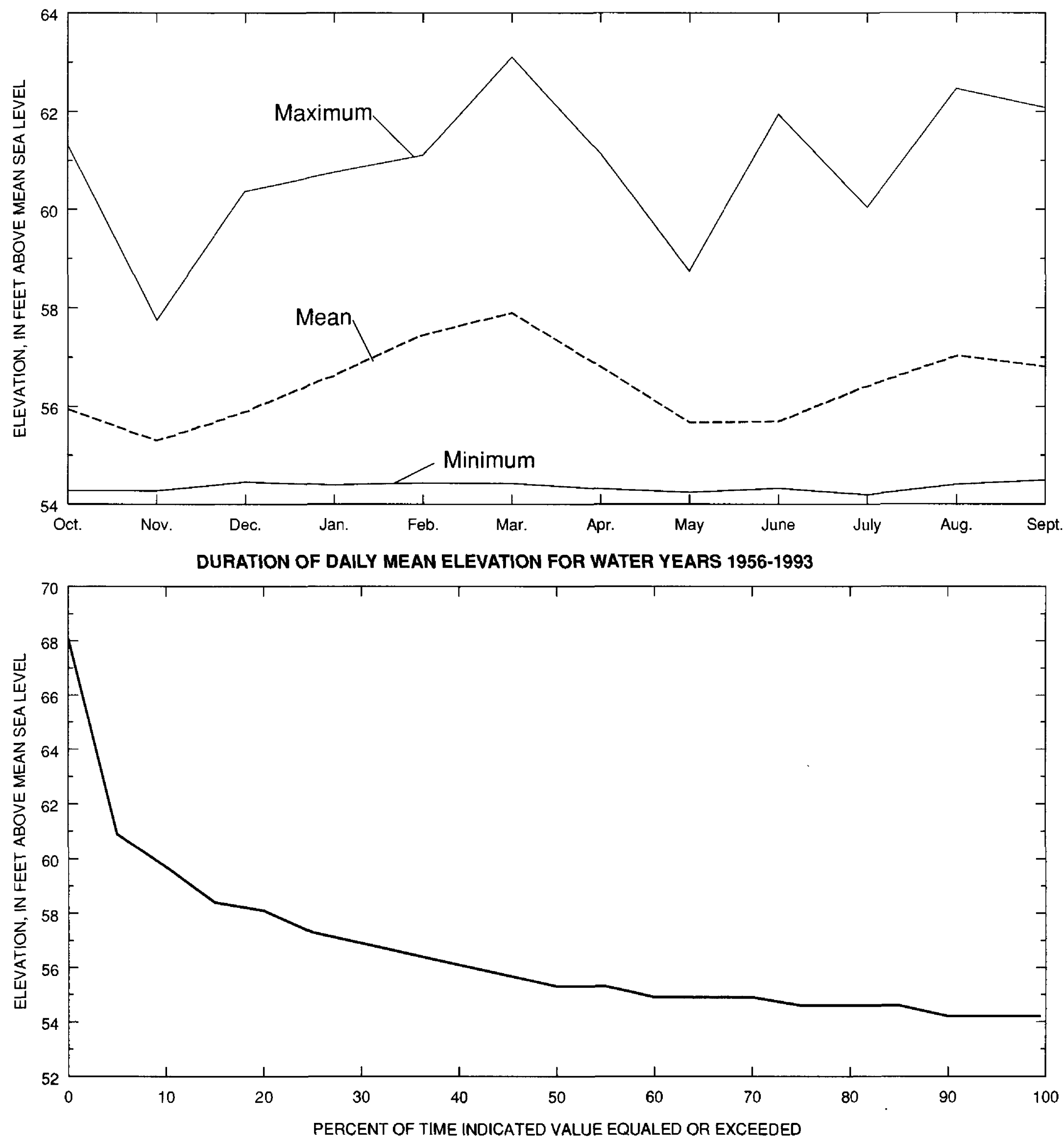
FENHOLLOWAY RIVER BASIN

02324400 FENHOLLOWAY RIVER NEAR FOLEY, FL--Continued

SUMMARY STATISTICS IN CUBIC FEET PER SECOND FOR WATER YEARS 1956 - 1993

ANNUAL MEAN

HTGHEST ANNUAL MF.AN

LOWEST ANNUAL MEAN

HIGHEST DAILY MEAN

LOWEST DAILY MEAN

ANNUAL SEVEN-DAY MINIMUM

INSTANTANEOUS PEAK ELOW

53.1

154

4.42

2710

$\begin{array}{lll} & & 1964 \\ & & 1968 \\ \text { Sep } & 12 & 1964 \\ \text { Aug } & 25 & 1993 \\ \text { Aug } 20 & 1993 \\ \text { Sep } & 12 & 1964 \\ \text { Sep } & 12 & 1964 \\ \text { Aug } & 25 & 1993 \\ 89 & & \end{array}$

INSTANTANEOUS LOW FLOW

ANNUAL RUNOEF (INCHES/CFSM)

$3210^{.41 \quad \text { Aug } 20} 1993$

(FT) $68.80 \quad$ Sep 121964

.32 Aug 251993

MAXIMUM, MINIMUM, AND MEAN OF MONTHLY MEAN DISCHARGES FOR WATER YEARS 1956-1993
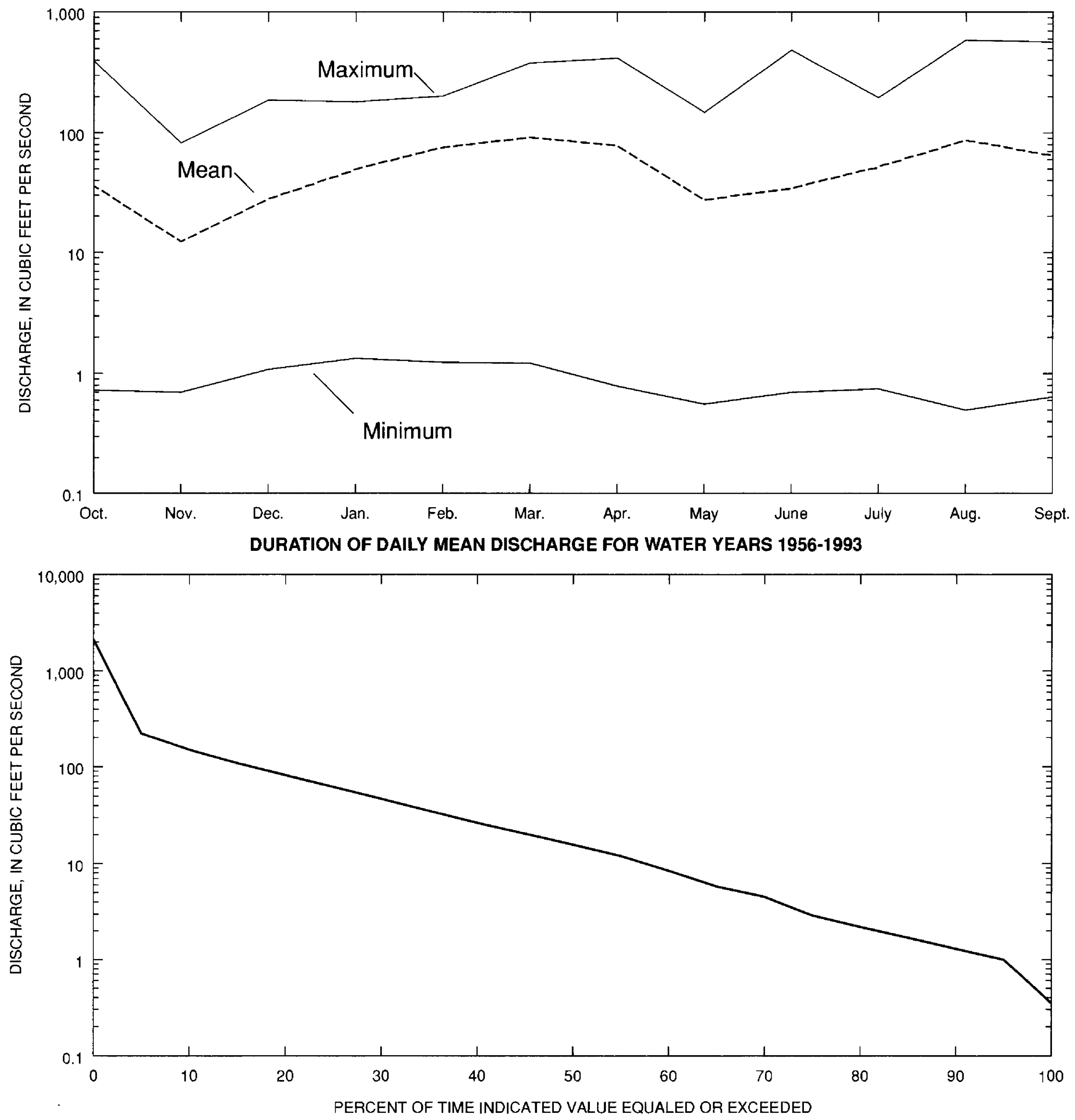
FENHOLLOWAY RIVER BASIN

02324400 FENHOLLOWAY RIVER NEAR FOLEY, FL--Continued

SUMMARY OF MONTHLY MEAN ELEVATION AND DISCHARGE STATISTICS FOR WATER YEARS 1956-1993

\begin{tabular}{|c|c|c|c|c|c|c|}
\hline & & EVATION & & & SCHARGE & \\
\hline & FEET & OVE SEA & VEI & CUBIC & ET FER S & OND \\
\hline MONTH & MAXIMUM & MINIMUM & MEAN & MAX IMUM & MINIMUM & MEAN \\
\hline OCTOBER & 61.29 & 54.27 & 55.94 & 389 & 73 & 36.0 \\
\hline NOVEMBER & 57.73 & 54.27 & 55.30 & 81.5 & .70 & 12.3 \\
\hline DECEMBER & 60.36 & 54.45 & 55.90 & 185 & 1.08 & 27.9 \\
\hline JANUARY & 60.75 & 54.39 & 56.64 & 179 & 1.34 & 49.9 \\
\hline FEBRUARY & 61.10 & 54.43 & 57.46 & 200 & 1.24 & 75.7 \\
\hline MARCH & 63.09 & 54.42 & 57.90 & 377 & 1.22 & 91.8 \\
\hline APRIL & 61.12 & 54.31 & 56.81 & 413 & .79 & 78.2 \\
\hline MAY & 58.72 & 54.24 & 55.67 & 147 & .56 & 27.5 \\
\hline JUNE & 61.93 & 54.32 & 55.69 & 478 & .70 & 34.6 \\
\hline JUIY & 60.02 & 54.19 & 56.42 & 194 & .75 & 52.3 \\
\hline AUGUST & 62.46 & 54.40 & 57.04 & 580 & .50 & 87.2 \\
\hline SEPTEMBER & 62.07 & 54.49 & 56.81 & 560 & .64 & 64.7 \\
\hline
\end{tabular}

DURATION OF DAILY MEAN VALUES FOR WATER YEARS 1956-1993

PERCENT

OF TIME

EQUALED OR

EXCEEDED ANNUAL

NOV

DEC JAN FEB MAR APR MAY

JUNE

JULY

AUG

SEPT

ELEVATION IN FEET ABOVE MEAN SEA LEVEL

$\begin{array}{rll}95.0 & 54.2 & 54.2 \\ 90.0 & 54.2 & 54.2 \\ 85.0 & 54.6 & 54.2 \\ 80.0 & 54.6 & 54.5 \\ 75.0 & 54.6 & 54.5 \\ 70.0 & 54.9 & 54.5 \\ 65.0 & 54.9 & 54.5 \\ 60.0 & 54.9 & 54.9 \\ 55.0 & 55.3 & 54.9 \\ 50.0 & 55.3 & 54.9 \\ 45.0 & 55.7 & 55.2 \\ 40.0 & 56.1 & 55.6 \\ 35.0 & 56.5 & 55.6 \\ 30.0 & 56.9 & 55.9 \\ 25.0 & 57.3 & 56.3 \\ 20.0 & 58.1 & 57.0 \\ 15.0 & 58.4 & 57.8 \\ 10.0 & 59.7 & 58.5 \\ 5.0 & 60.9 & 60.0\end{array}$

$\begin{array}{llll}54.4 & 54.4 & 54.3 & 54.4 \\ 54.4 & 54.4 & 54.6 & 54.7 \\ 54.4 & 54.6 & 54.6 & 55.0 \\ 54.4 & 54.6 & 55.1 & 55.3 \\ 54.6 & 54.6 & 55.1 & 55.6 \\ 54.6 & 54.6 & 55.3 & 55.9 \\ 54.6 & 54.8 & 55.3 & 56.2 \\ 54.7 & 54.8 & 55.6 & 56.8 \\ 54.7 & 55.1 & 55.6 & 57.1 \\ 54.7 & 55.1 & 55.8 & 57.1 \\ 54.9 & 55.1 & 55.8 & 57.4 \\ 54.9 & 55.3 & 56.3 & 57.7 \\ 55.1 & 55.5 & 56.6 & 57.7 \\ 55.1 & 55.5 & 57.3 & 58.0 \\ 55.3 & 56.0 & 57.9 & 58.3 \\ 55.7 & 57.0 & 58.4 & 58.9 \\ 55.9 & 57.7 & 58.9 & 59.6 \\ 55.5 & 59.0 & 59.7 & 59.9 \\ 57.8 & 60.0 & 60.6 & 60.9\end{array}$

DISCHARGE IN CUBIC FEET PER SECOND

$\begin{array}{rrr}95.0 & 1.0 & 0.9 \\ 90.0 & 1.3 & 1.1 \\ 85.0 & 1.7 & 1.4 \\ 80.0 & 2.2 & 1.4 \\ 75.0 & 2.9 & 1.8 \\ 70.0 & 4.5 & 2.3 \\ 65.0 & 5.7 & 2.9 \\ 60.0 & 8.4 & 3.7 \\ 55.0 & 12.0 & 5.5 \\ 50.0 & 15.7 & 7.5 \\ 45.0 & 20.5 & 9.7 \\ 40.0 & 26.5 & 12.7 \\ 35.0 & 35.1 & 16.0 \\ 30.0 & 47.0 & 20.8 \\ 25.0 & 61.8 & 28.1 \\ 20.0 & 82.0 & 38.7 \\ 15.0 & 109.0 & 57.0 \\ 10.0 & 150.0 & 87.0 \\ 5.0 & 220.8 & 146.4\end{array}$

$\begin{array}{rr}0.8 & 1.3 \\ 1.2 & 1.3 \\ 1.4 & 1.6 \\ 1.6 & 1.9 \\ 1.6 & 2.3 \\ 1.6 & 2.8 \\ 2.3 & 2.8 \\ 2.8 & 3.3 \\ 2.8 & 4.0 \\ 3.3 & 5.6 \\ 4.0 & 7.3 \\ 4.8 & 10.4 \\ 6.0 & 14.3 \\ 7.2 & 18.1 \\ 8.9 & 24.8 \\ 13.6 & 37.4 \\ 20.2 & 58.3 \\ 29.3 & 90.2 \\ 55.6 & 151.4\end{array}$

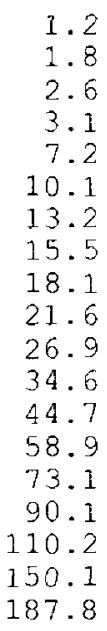

1.5
2.7
5.1
10.3
16.1
23.0
31.4
39.7
48.0
55.7
63.1
73.6
84.1
94.8
110.6
133.5
154.9
179.4
221.0
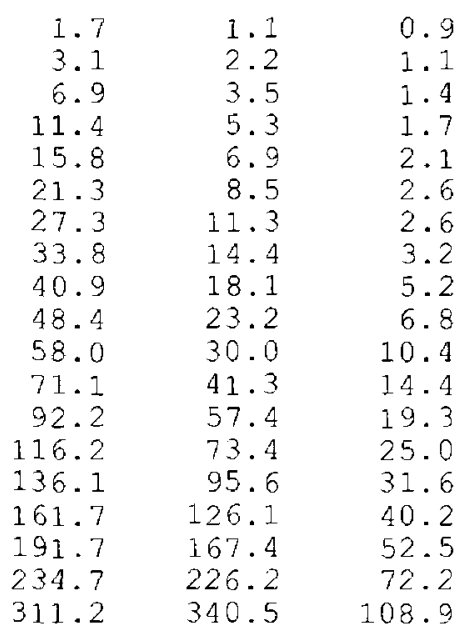
0.6
0.8
0.8
1.
1.7
2.2
2.8
4.7
5.8
6.7
9.5
13.5
18.2
23.3
30.7
43.4
70.6
166.3

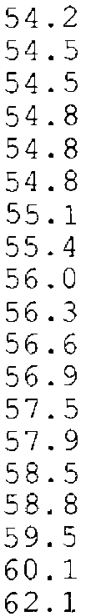

54.3

54.3

54.7

54.7

55.1

55.1

55.4

55.8

55.8

56.2

57.4

58.2

58.2
59.0

60.2

62.3 
FENHOLLOWAY RIVER BASIN

02324400 FENHOLLOWAY RIVER NEAR FOLEY, FL--Continued

LOWEST MEAN ELEVATION, IN FEET, AND RANKING FOR THE FOLLOWING NUMBER OF CONSECUTIVE DAYS FOR PERIOD APR TO MAR

WATER YEAR
RANGE

$\begin{array}{lr}1 & \\ 55.2 & 16 \\ 54.7 & 15 \\ 54.4 & 5 \\ 54.4 & 7 \\ 54.4 & 8 \\ 54.7 & 14 \\ & \\ 54.4 & 5 \\ 54.4 & 3 \\ 54.5 & 9 \\ 54.2 & 1 \\ 54.4 & 4 \\ 54.5 & 10 \\ 54.5 & 11 \\ & \\ 54.5 & 12 \\ 54.6 & 13 \\ 54.3 & 2\end{array}$

$\begin{array}{cr}3 \\ \\ 55.2 & 16 \\ 54.7 & 15 \\ & \\ 54.4 & 6 \\ 54.4 & 7 \\ 54.4 & 8 \\ 54.7 & 14\end{array}$

\begin{tabular}{cr}
7 \\
\multicolumn{3}{c}{7} \\
55.2 & 16 \\
54.7 & 14 \\
54.4 & 5 \\
54.4 & 6 \\
54.4 & 7 \\
54.7 & 15
\end{tabular}

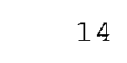

$54.4 \quad 5$

$1974 \quad 1975$

19751976

19761977

19771978

19781979

19791980

19801981

19861987

19871988

54.32

$\begin{array}{ll}54.4 & 8 \\ 54.4 & 3\end{array}$

54.59

54.21

54.44

$\begin{array}{ll}54.5 & 10 \\ 54.5 & 11\end{array}$

$54.5 \quad 12$

$54.6 \quad 13$

54.59

54.21

$54.4 \quad 4$

$\begin{array}{ll}54.5 & 10 \\ 54.5 & 11\end{array}$

54.612

54.613

$\begin{array}{rr}54.6 & 13 \\ 54.3 & 2\end{array}$ $\begin{array}{rr}55.3 & 16 \\ 54.7 & 14 \\ & \\ 54.4 & 5 \\ 54.5 & 7 \\ 54.4 & 6 \\ 54.9 & 15 \\ & \\ & \\ 54.5 & 8 \\ 54.4 & 3 \\ & \\ 54.6 & 10 \\ 54.2 & 1 \\ 54.4 & 4 \\ 54.6 & 13 \\ 54.6 & 9 \\ & \\ 54.6 & 11 \\ 54.6 & 12 \\ 54.3 & 2\end{array}$

\begin{tabular}{rrrrrrr}
\multicolumn{1}{c}{30} & \multicolumn{2}{c}{60} & \multicolumn{3}{c}{90} \\
55.4 & 16 & 56.2 & 15 & 57.5 & 16 \\
54.7 & 11 & 54.9 & 12 & 54.9 & 8 \\
& & & & & \\
54.4 & 5 & 54.4 & 2 & 54.5 & 2 \\
54.5 & 7 & 54.5 & 5 & 54.5 & 3 \\
54.5 & 6 & 54.5 & 6 & 54.5 & 5 \\
55.2 & 15 & 55.9 & 15 & 56.6 & 15 \\
& & & & & \\
54.5 & 8 & 54.6 & 8 & 55.0 & 10 \\
54.4 & 3 & 54.5 & 3 & 54.5 & 6 \\
& & & & & \\
54.8 & 13 & 55.2 & 14 & 55.6 & 14 \\
54.2 & 1 & 54.2 & 1 & 54.3 & 1 \\
54.4 & 4 & 54.6 & 7 & 54.6 & 7 \\
54.8 & 14 & 55.0 & 13 & 55.3 & 12 \\
54.7 & 10 & 54.9 & 11 & 55.5 & 13 \\
& & & & & & \\
54.7 & 12 & 54.8 & 10 & 55.1 & 11 \\
54.6 & 9 & 54.8 & 9 & 54.9 & 9 \\
54.4 & 2 & 54.5 & 4 & 54.5 & 4
\end{tabular}

90

120

183

$57.416 \quad 58.416$ 55.110

54.52

54.64

$54.6 \quad 5$

\section{$55.6 \quad 12$}

54.87

55.613

54.31

54.86

55.511

56.414

55.19

54.53
55.510

54.62

55.37

54.84

56.313

5.25

55.811

54.31

55.48

$\begin{array}{ll}56.1 & 12 \\ 56.5 & 14\end{array}$

55.49

$\begin{array}{ll}55.2 & 5 \\ 54.7 & 3\end{array}$

HIGHEST MEAN ELEVATION, IN FEET, AND RANKING FOR THE FOLLOWING NUMBER OF CONSECUTIVE DAYS FOR PERIOD OCT TO SEP

\begin{tabular}{|c|c|c|c|c|c|c|c|c|c|c|c|c|c|c|c|c|c|c|c|}
\hline \multicolumn{2}{|c|}{$\begin{array}{c}\text { WATER YEAR } \\
\text { RANGE }\end{array}$} & \multicolumn{2}{|l|}{1} & \multicolumn{2}{|l|}{3} & \multicolumn{2}{|l|}{7} & \multicolumn{2}{|l|}{15} & \multicolumn{2}{|c|}{30} & \multicolumn{2}{|c|}{60} & \multicolumn{2}{|c|}{90} & \multicolumn{2}{|c|}{120} & \multicolumn{2}{|c|}{183} \\
\hline $\begin{array}{l}1957 \\
1958\end{array}$ & $\begin{array}{l}1957 \\
1958\end{array}$ & $\begin{array}{l}67.6 \\
66.9\end{array}$ & $\begin{array}{l}2 \\
3\end{array}$ & $\begin{array}{l}67.5 \\
66.9\end{array}$ & $\begin{array}{l}2 \\
3\end{array}$ & $\begin{array}{l}67.0 \\
66.6\end{array}$ & $\begin{array}{l}2 \\
3\end{array}$ & $\begin{array}{l}65.3 \\
64.8\end{array}$ & $\begin{array}{l}2 \\
3\end{array}$ & $\begin{array}{l}62.5 \\
61.5\end{array}$ & $\begin{array}{l}2 \\
5\end{array}$ & $\begin{array}{l}59.8 \\
60.9\end{array}$ & $\begin{array}{l}7 \\
3\end{array}$ & $\begin{array}{l}59.0 \\
59.7\end{array}$ & $\begin{array}{l}5 \\
4\end{array}$ & $\begin{array}{l}59.6 \\
59.2\end{array}$ & $\begin{array}{l}3 \\
5\end{array}$ & $\begin{array}{l}58.4 \\
58.8\end{array}$ & $\begin{array}{l}5 \\
3\end{array}$ \\
\hline $\begin{array}{l}1961 \\
1962 \\
1963 \\
1964\end{array}$ & $\begin{array}{l}1961 \\
1962 \\
1963 \\
1964\end{array}$ & $\begin{array}{l}65.2 \\
59.2 \\
61.9 \\
68.5\end{array}$ & $\begin{array}{r}6 \\
17 \\
10 \\
1\end{array}$ & $\begin{array}{l}65.1 \\
59.1 \\
61.4 \\
68.1\end{array}$ & $\begin{array}{r}6 \\
17 \\
10 \\
1\end{array}$ & $\begin{array}{l}64.1 \\
58.9 \\
60.6 \\
67.3\end{array}$ & $\begin{array}{r}6 \\
17 \\
11 \\
1\end{array}$ & $\begin{array}{l}63.0 \\
58.4 \\
60.2 \\
65.5\end{array}$ & $\begin{array}{r}4 \\
17 \\
9 \\
1\end{array}$ & $\begin{array}{l}60.8 \\
57.5 \\
60.2 \\
63.1\end{array}$ & $\begin{array}{r}8 \\
16 \\
9 \\
1\end{array}$ & $\begin{array}{l}58.2 \\
56.2 \\
59.7 \\
62.6\end{array}$ & $\begin{array}{r}12 \\
17 \\
8 \\
1\end{array}$ & $\begin{array}{l}57.1 \\
55.6 \\
58.6 \\
51.5\end{array}$ & $\begin{array}{r}14 \\
18 \\
8 \\
1\end{array}$ & $\begin{array}{ll}57.0 & 1 \\
55.4 & 1 \\
57.9 & \\
60.0\end{array}$ & $\begin{array}{r}14 \\
18 \\
8 \\
2\end{array}$ & $\begin{array}{l}57.0 \\
55.3 \\
57.1 \\
59.1\end{array}$ & $\begin{array}{r}10 \\
17 \\
8 \\
2\end{array}$ \\
\hline $\begin{array}{l}1972 \\
1975\end{array}$ & $\begin{array}{l}1972 \\
1975\end{array}$ & $\begin{array}{l}62.7 \\
60.0\end{array}$ & $\begin{array}{r}9 \\
15\end{array}$ & $\begin{array}{l}62.2 \\
59.9\end{array}$ & $\begin{array}{r}9 \\
15\end{array}$ & $\begin{array}{l}61.3 \\
59.7\end{array}$ & $\begin{array}{r}9 \\
13\end{array}$ & $\begin{array}{l}60.1 \\
59.3\end{array}$ & $\begin{array}{l}11 \\
14\end{array}$ & $\begin{array}{l}59.1 \\
58.8\end{array}$ & $\begin{array}{l}11 \\
12\end{array}$ & $\begin{array}{l}58.5 \\
58.2\end{array}$ & $\begin{array}{r}9 \\
13\end{array}$ & $\begin{array}{l}58.4 \\
57.6\end{array}$ & $\begin{array}{r}9 \\
12\end{array}$ & $\begin{array}{l}58.2 \\
57.4\end{array}$ & $\begin{array}{r}7 \\
11\end{array}$ & $\begin{array}{l}57.7 \\
56.6\end{array}$ & $\begin{array}{r}7 \\
14\end{array}$ \\
\hline $\begin{array}{l}1976 \\
1977 \\
1978 \\
1970 \\
1980\end{array}$ & $\begin{array}{l}1976 \\
1977 \\
1978 \\
1979 \\
1980\end{array}$ & $\begin{array}{l}59.7 \\
62.7 \\
61.0 \\
60.8 \\
65.4\end{array}$ & $\begin{array}{r}16 \\
8 \\
11 \\
12 \\
5\end{array}$ & $\begin{array}{l}59.6 \\
62.6 \\
60.9 \\
60.7 \\
65.2\end{array}$ & $\begin{array}{r}16 \\
8 \\
11 \\
12 \\
4\end{array}$ & $\begin{array}{l}59.3 \\
61.9 \\
60.6 \\
59.5 \\
64.6\end{array}$ & $\begin{array}{r}16 \\
8 \\
10 \\
15 \\
4\end{array}$ & $\begin{array}{l}58.6 \\
61.3 \\
60.1 \\
59.0 \\
62.9\end{array}$ & $\begin{array}{r}16 \\
8 \\
10 \\
15 \\
5\end{array}$ & $\begin{array}{l}57.3 \\
61.1 \\
59.2 \\
58.1 \\
61.5\end{array}$ & $\begin{array}{r}17 \\
7 \\
10 \\
15 \\
4\end{array}$ & $\begin{array}{l}56.5 \\
60.4 \\
58.4 \\
57.3 \\
60.2\end{array}$ & $\begin{array}{r}16 \\
4 \\
10 \\
15 \\
5\end{array}$ & $\begin{array}{l}56.3 \\
59.9 \\
57.9 \\
57.2 \\
58.9\end{array}$ & $\begin{array}{r}16 \\
3 \\
10 \\
13 \\
6\end{array}$ & $\begin{array}{l}56.21 \\
59.4 \\
57.6 \\
57.01 \\
58.3\end{array}$ & $\begin{array}{r}16 \\
4 \\
10 \\
13 \\
6\end{array}$ & $\begin{array}{l}56.0 \\
58.6 \\
57.0 \\
56.4 \\
58.0\end{array}$ & $\begin{array}{r}16 \\
4 \\
9 \\
15 \\
6\end{array}$ \\
\hline $\begin{array}{l}1981 \\
1985\end{array}$ & $\begin{array}{l}1981 \\
1985\end{array}$ & $\begin{array}{l}60.3 \\
65.5\end{array}$ & $\begin{array}{r}14 \\
4\end{array}$ & $\begin{array}{l}60.2 \\
65.2\end{array}$ & $\begin{array}{r}14 \\
5\end{array}$ & $\begin{array}{l}59.6 \\
64.4\end{array}$ & $\begin{array}{r}14 \\
5\end{array}$ & $\begin{array}{l}59.3 \\
62.8\end{array}$ & $\begin{array}{r}13 \\
6\end{array}$ & $\begin{array}{l}58.4 \\
61.2\end{array}$ & $\begin{array}{r}14 \\
6\end{array}$ & $\begin{array}{l}57.6 \\
60.1\end{array}$ & $\begin{array}{r}14 \\
6\end{array}$ & $\begin{array}{l}56.9 \\
58.8\end{array}$ & $\begin{array}{r}15 \\
7\end{array}$ & $\begin{array}{l}56.91 \\
57.8\end{array}$ & $\begin{array}{r}15 \\
9\end{array}$ & $\begin{array}{l}56.8 \\
56.7\end{array}$ & $\begin{array}{l}11 \\
13\end{array}$ \\
\hline $\begin{array}{l}1987 \\
1989\end{array}$ & $\begin{array}{l}1987 \\
1989\end{array}$ & $\begin{array}{l}64.5 \\
59.0\end{array}$ & $\begin{array}{r}7 \\
18\end{array}$ & $\begin{array}{l}64.1 \\
58.9\end{array}$ & $\begin{array}{r}7 \\
18\end{array}$ & $\begin{array}{l}63.3 \\
58.6\end{array}$ & $\begin{array}{r}7 \\
18\end{array}$ & $\begin{array}{l}62.6 \\
58.0\end{array}$ & $\begin{array}{r}7 \\
18\end{array}$ & $\begin{array}{l}61.9 \\
56.9\end{array}$ & $\begin{array}{r}3 \\
18\end{array}$ & $\begin{array}{l}61.4 \\
56.1\end{array}$ & $\begin{array}{r}2 \\
18\end{array}$ & $\begin{array}{l}61.2 \\
55.7\end{array}$ & $\begin{array}{r}2 \\
17\end{array}$ & $\begin{array}{l}60.9 \\
55.41\end{array}$ & $\begin{array}{r}1 \\
17\end{array}$ & $\begin{array}{l}59.5 \\
55.1\end{array}$ & $\begin{array}{r}1 \\
18\end{array}$ \\
\hline 1993 & 1993 & 60.5 & 13 & 60.3 & 13 & 60.0 & 12 & 59.4 & 12 & 58.8 & 13 & 58.2 & 11 & $57.8=$ & 11 & 57.31 & 12 & 56.8 & 12 \\
\hline
\end{tabular}




\section{FOR PERIOD APR TO MAR}

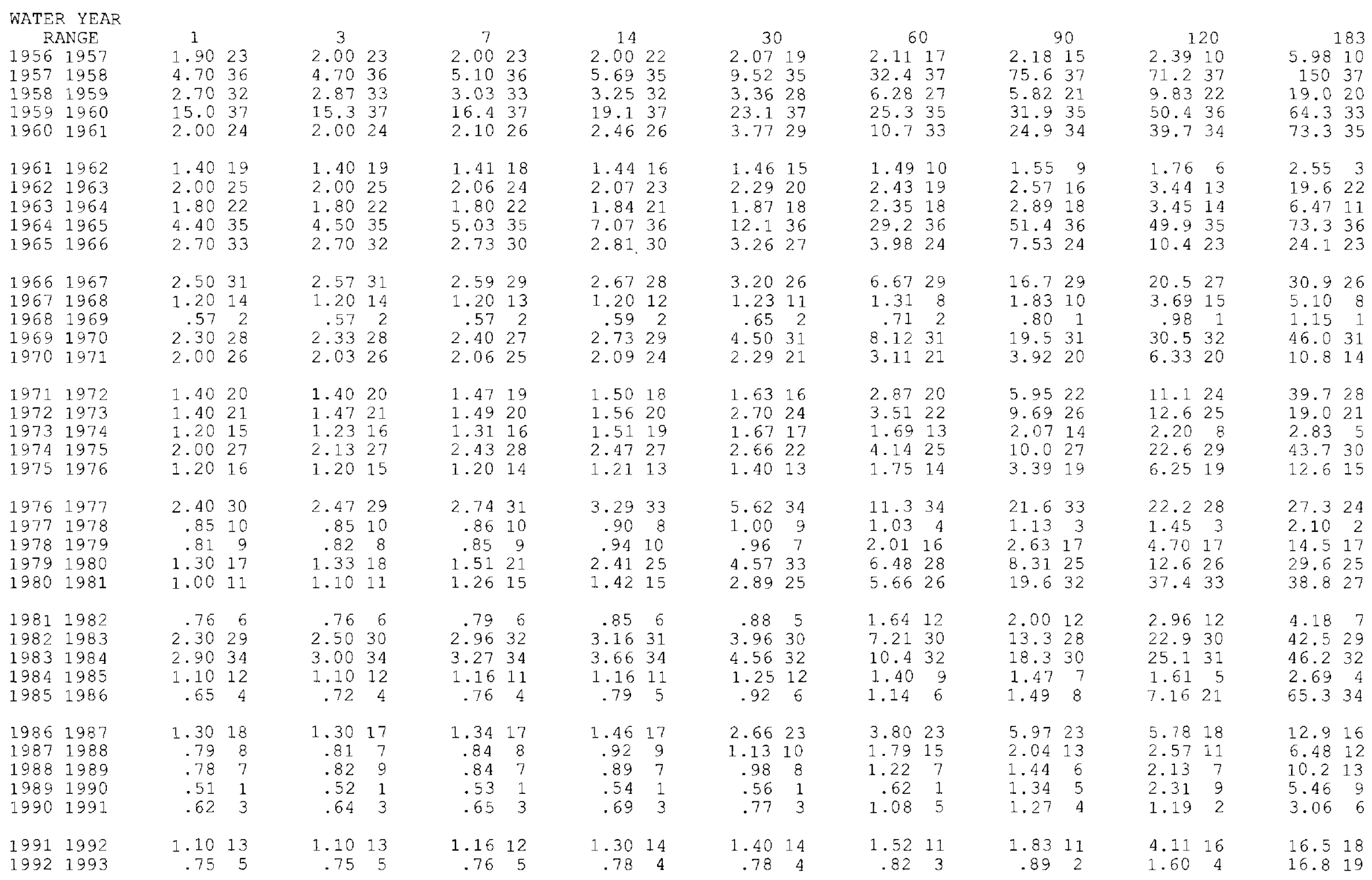



FOR PERIOD OCT TO SEP

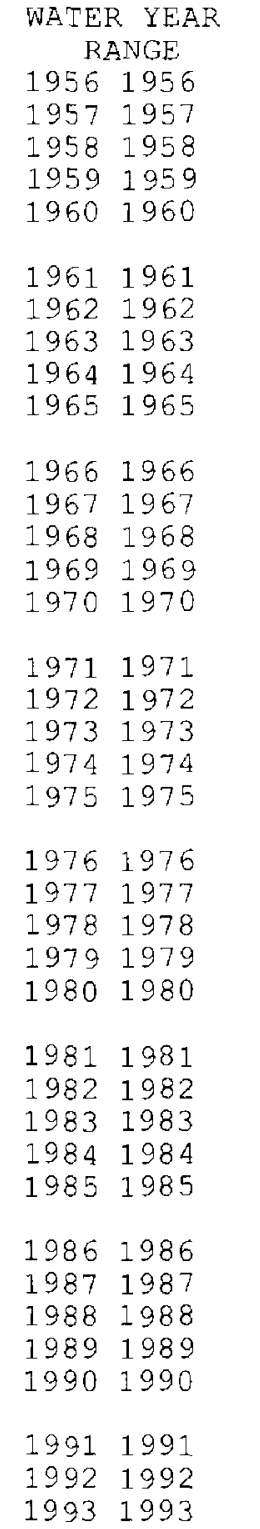
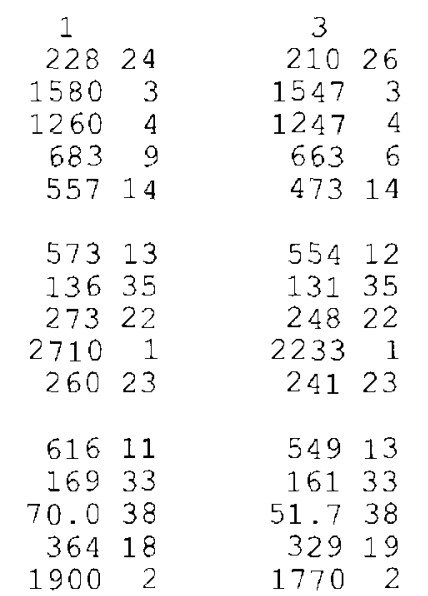

$554 \quad 12$

13135

24822

$\begin{array}{rr}2233 & 1\end{array}$

54913

16133

51.738

32919
1770

$224 \quad 26$

32321

8825

44116

17132

$151 \quad 34$

32620

$\begin{array}{ll}326 & 20 \\ 218 & 27\end{array}$

$198 \quad 29$

65510

17731

6848

48415

$\begin{array}{rr}615 & 12 \\ 697 & 7\end{array}$

35319

$394 \quad 17$

$228 \quad 25$

11036

$100 \quad 37$

7126

21228

18430
$208 \quad 27$

29621

8245

$\begin{array}{ll}412 & 16 \\ 169 & 31\end{array}$

$148 \quad 34$

31620

21225

19428

6199

16932

58410

43915

$\begin{array}{rr}556 & 11 \\ 635 & 8\end{array}$

$\begin{array}{ll}329 & 18 \\ 36 & 17\end{array}$

36117

22324

10636

98.037

6577

18729

17730
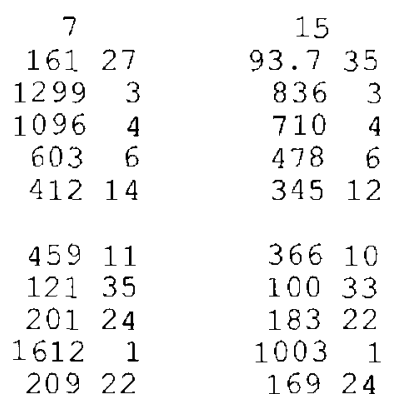

42513

$133 \quad 34$

$37.6 \quad 38$

272
1390

17926

24421

8055

33416

16028

$135 \quad 33$

$272 \quad 19$

19325

$\begin{array}{rr}137 & 32 \\ 517 & 8\end{array}$

$143 \quad 31$

45112

37915

48510

29218

$310 \quad 17$

$204 \quad 23$

93.036

86.737

$\begin{array}{ll}527 & 7\end{array}$

15929

$\begin{array}{rr}30 \\ 51.9 & 36 \\ 497 & 3 \\ 401 & 5 \\ 385 & 7 \\ 269 & 9\end{array}$

36610

10033

18322

10031

$314 \quad 14$

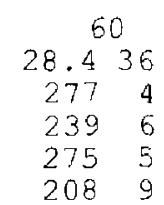

$241 \quad 12$

75.132

18120

$\begin{array}{rr}562 & 2 \\ 138 & 24\end{array}$

18819

98.634

23.338

22120

15526

17923

6365

25317

$\begin{array}{ll}137 & 27\end{array}$

$105 \quad 32$

$234 \quad 19$

16625

11430
$378 \quad 8$

13029

31315

$\begin{array}{ll}313 & 15 \\ 317 & 13\end{array}$

36311

370 9

$244 \quad 18$

27216

19321

72.736

$70.3 \quad 37$

4217

$\begin{array}{ll}112 & 31 \\ 132 & 28\end{array}$
62.934

15.638

16321

14323

13025

$414 \quad 4$

18818

11727

63.533

22216

12626

$\begin{array}{rr}77.1 & 31 \\ 275 & 8\end{array}$

93.029

$227 \quad 15$

23414

$258 \quad 10$

25611

19917

$237 \quad 13$

14622

$45.0 \quad 37$

52.035

3906

80.830

10228
12921

41.4
157
48

$157 \quad 18$
469

99.424

14719

51.431

9.9138

13120

10323

$\begin{array}{ll}103 & 23 \\ 109 & 22\end{array}$

2257

16817

93.526

$40.6 \quad 35$

18413

97.925

57.130

19211

64.829

18512

18512
20310

$173 \quad 15$

18214

16916

2118

88.627

25.237

$45.5 \quad 32$

2833

$44.6 \quad 33$
78.528

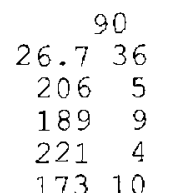

89.122

$28.5 \quad 35$

12117

$\begin{array}{rr}365 & 1 \\ 81.4 & 23\end{array}$

$107 \quad 19$

39.531

8.4538

$\begin{array}{rr}103 & 21 \\ 240 & 3\end{array}$

75.326

10620

2046

11818
76.9

35.433

16011

80.824

54.329

14014

48.630

$145 \quad 13$

1928

$137 \quad 15$

13116

15212

2007

61.128

18.237

$35.5 \quad 32$

$247 \quad 2$

35.134

64.627 \begin{tabular}{rrrr}
\multicolumn{2}{c}{120} & \multicolumn{2}{r}{183} \\
25.7 & 35 & 19.5 & 36 \\
228 & 2 & 160 & 4 \\
159 & 9 & 147 & 7 \\
202 & 4 & 171 & 3 \\
137 & 11 & 125 & 9
\end{tabular}

$\begin{array}{llll}79.3 & 23 & 70.721\end{array}$

$22.036 \quad 21.034$

$95.319 \quad 69.622$

$\begin{array}{rrrr}2.78 & 1 & 21.7 & 1 \\ 84.7 & 21 & 72.9 & 17\end{array}$

84.722

31.232

$7.08 \quad 38$

98.718

57.326

10016

1687

$92.6 \quad 20$

70.224

34.631

$\begin{array}{rl}1.40 & 10 \\ 9.2 & 25\end{array}$

48.029

11115

50.728

$\begin{array}{lll}115 & 14\end{array}$

1618

$\begin{array}{rr}132 & 12 \\ 99.0 & 17\end{array}$

$\begin{array}{lll}131 & 13\end{array}$

$190 \quad 5$

14.030

$\begin{array}{ll}14.2 & 37 \\ 31.1 & 33\end{array}$

$213 \quad 3$

$27.4 \quad 34$

50.827
72.718

$30.5 \quad 31$

5.2438

$\begin{array}{rr}71.9 & 19 \\ 178 & 2\end{array}$

40.327

80.216

12210

70.920
49.825

30.432

11012

53.124

$\begin{array}{rrr}37 & 3 & 28 \\ 11 & 6 & 11\end{array}$

46.826

95.115

1486

11013

65.323

$102 \quad 14$

1408

$31.7 \quad 30$

$9.72 \quad 37$

$21.8 \quad 33$

$158 \quad 5$

$19.8 \quad 35$

35.929 
LOCATION.--Lat $30^{\circ} 10^{\prime} 14^{\prime \prime}$, long $83^{\circ} 49^{\prime} 26^{\prime \prime}$, in NE $\frac{1}{4}$ sec.4, T.4 S., R.5 E., Taylor County, Hydrologic Unit 03110102 , on downstream side of concrete bridge, 3.0 mi downstream from Natural Well Branch, 14 mi upstream from mouth, and 14.7 mi northwest of Perry.

DRAINAGE AREA.--198 $\mathrm{mi}^{2}$.

PERIOD OF RECORD.--February 1950 to 1993.

REVISED RECORDS.--WSP 1905: Drainage area.

GAGE.--Water-stage recorder. Datum of gage is $14.35 \mathrm{ft}$ above National Geodetic Vertical Datum of 1929.

REMARKS.--Records good.

MAXIMUM, MINIMUM, AND MEAN OF MONTHLY MEAN ELEVATIONS FOR WATER YEARS 1950-1993
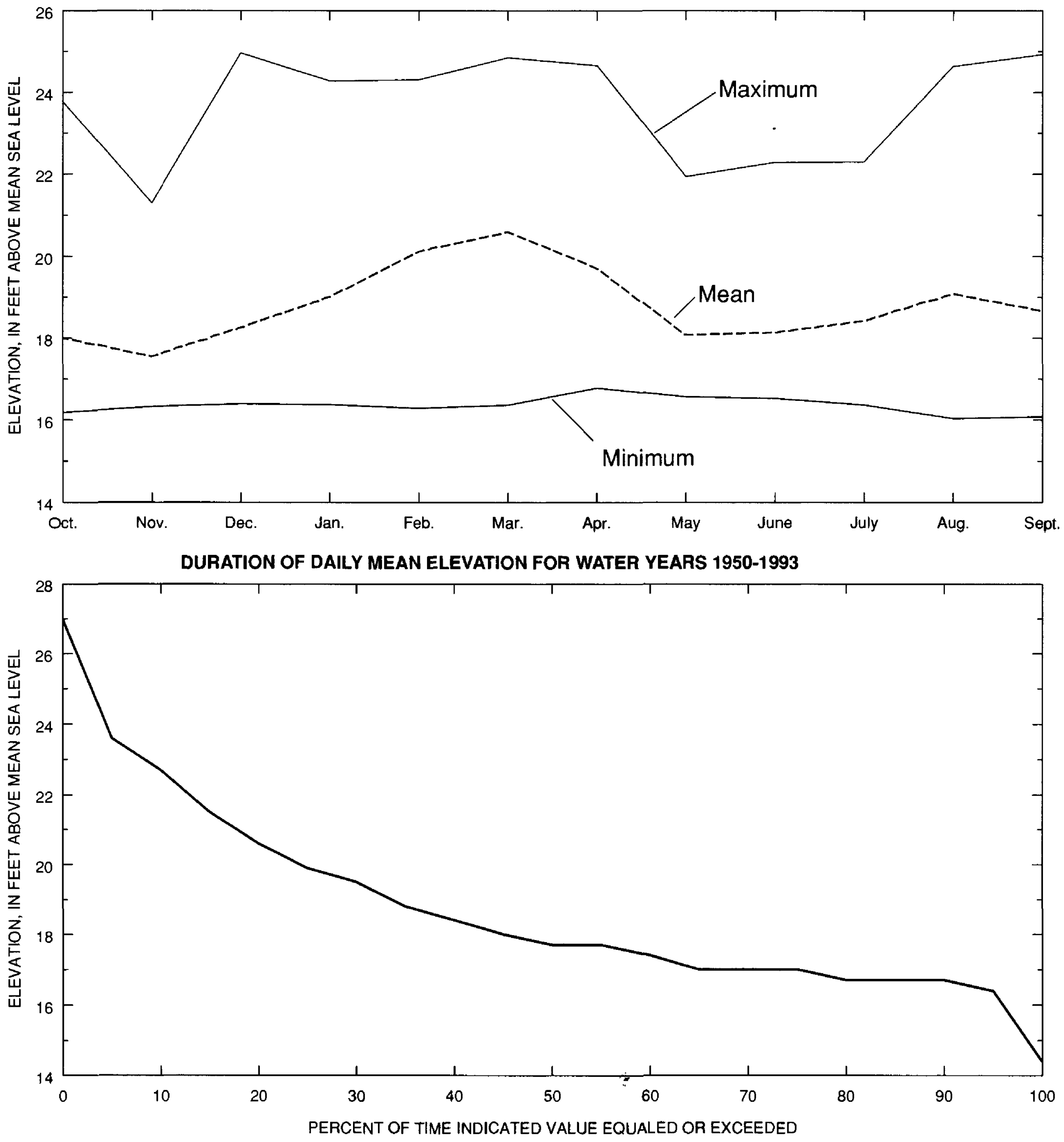
ECONFINA RIVER BASIN

02326000 ECONFINA RIVER NEAR PERRY, FL--Continued

SUMMARY STATISTICS IN CUBIC FEET PER SECOND FOR WATER YEARS 1950 - 1993

ANNUAL MEAN

HIGHEST ANNUAL MEAN

LOWEST ANNUAL MEAN

HIGHEST DAILY MEAN

LOWEST DAILY MEAN

ANNUAL SEVEN-DAY MINIMUM

INSTANTANEOUS PEAK FLOW

INSTANTANEOUS PEAK ELEVATION (FT)

INSTANTANEOUS LOW FLOW

ANNUAL RUNOFF (INCHES/CFSM)

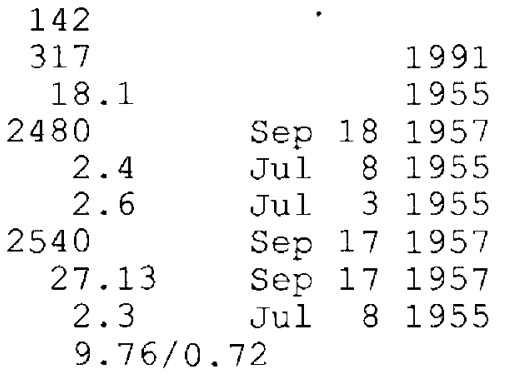

MAXIMUM, MINIMUM, AND MEAN OF MONTHLY MEAN DISCHARGES FOR WATER YEARS 1950-1993

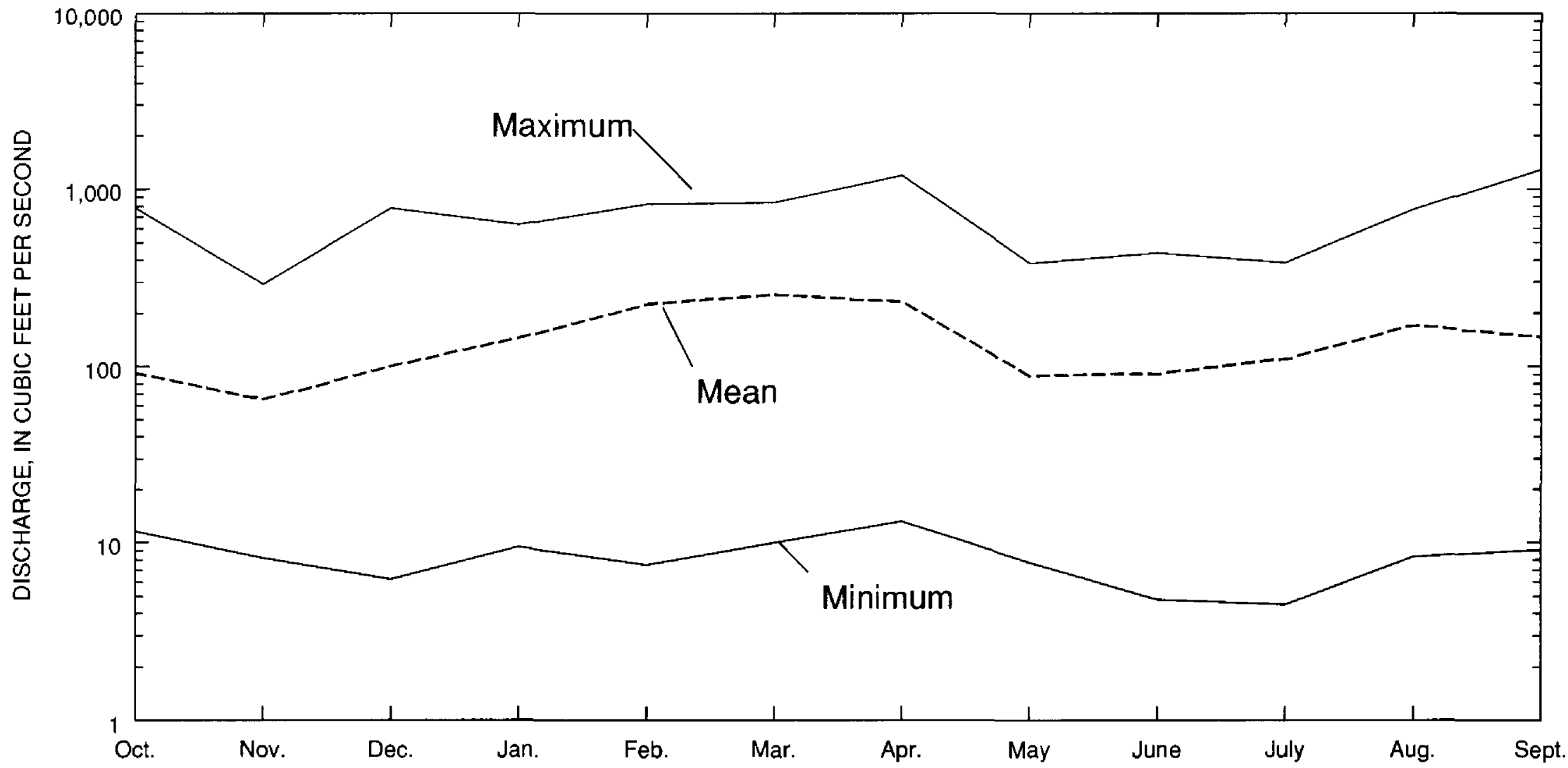

DURATION OF DAILY MEAN DISCHARGE FOR WATER YEARS 1950-1993

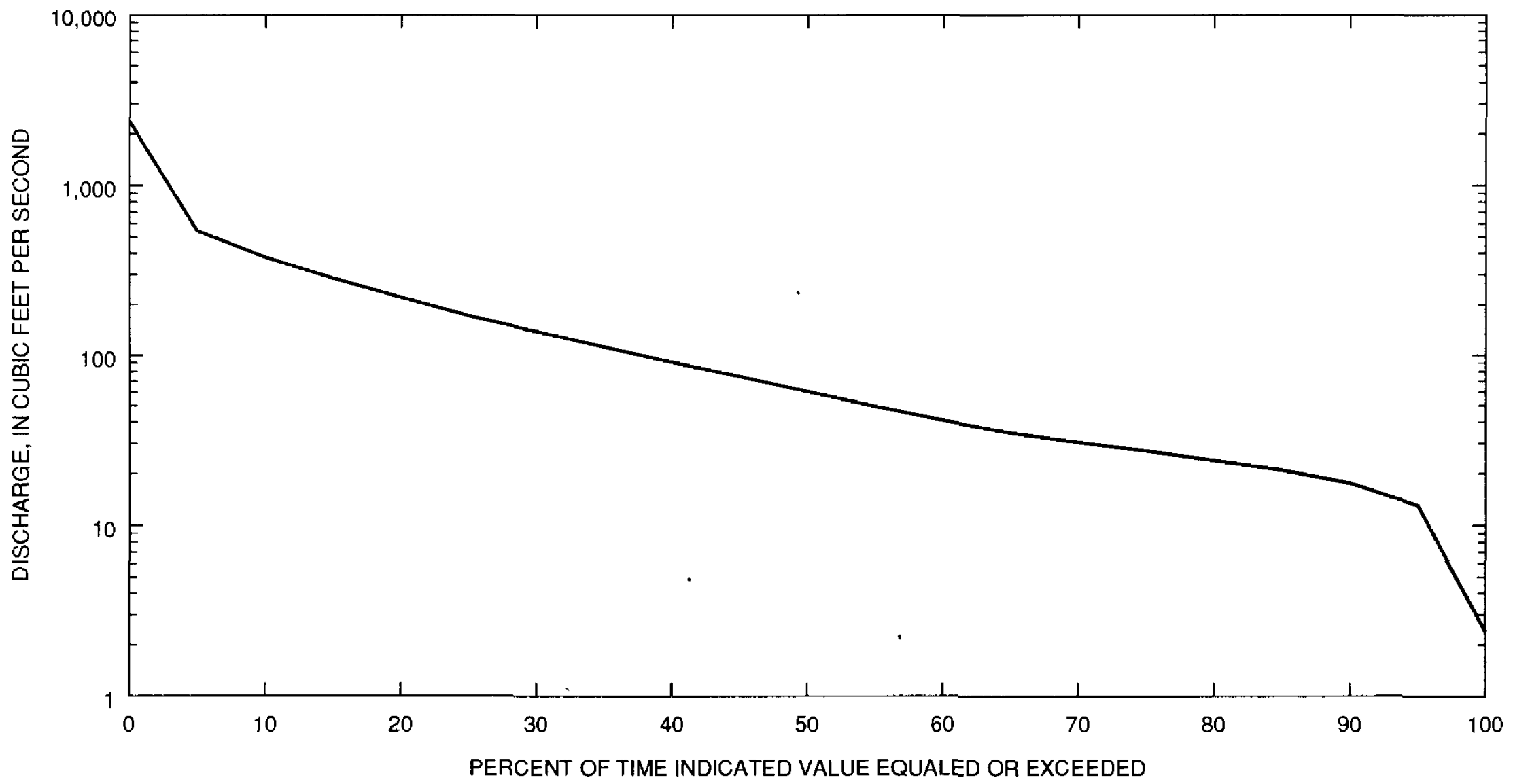


ECONFINA RIVER BASIN

02326000 ECONFINA RIVER NEAR PERRY, FL--Continued

\section{SUMMARY OF MONTHLY MEAN ELEVATION AND DISCHARGE STATISTICS} FOR WATER YEARS 1950-1993

ELEVATION
FEET ABOVE SEA LEVEL

\begin{tabular}{lrrr}
\multicolumn{1}{c}{ MONTH } & MAXIMUM & MINIMUM & MEAN \\
OCTOBER & 23.74 & 16.18 & 18.01 \\
NOVEMBER & 21.27 & 16.32 & 17.55 \\
DECEMBER & 24.94 & 16.39 & 18.26 \\
JANUARY & 24.26 & 16.37 & 19.01 \\
FEBRUARY & 24.29 & 16.28 & 20.12 \\
MARCH & 24.83 & 16.36 & 20.60 \\
APRIL & 24.64 & 16.78 & 19.72 \\
MAY & 21.92 & 16.56 & 18.08 \\
JUNE & 22.27 & 16.52 & 18.14 \\
JULY & 22.28 & 16.36 & 18.42 \\
AUGUST & 24.62 & 16.03 & 19.09 \\
SEPTEMBER & 24.91 & 16.08 & 18.66
\end{tabular}

DISCHARGE

CUBIC FEET PER SECOND

$\begin{array}{rcr}\text { MAXIMUM } & \text { MINIMUM } & \text { MEAN } \\ 772 & 11.6 & 91.6 \\ 288 & 8.18 & 65.2 \\ 771 & 6.22 & 100 \\ 624 & 9.47 & 145 \\ 813 & 7.50 & 225 \\ 828 & 9.97 & 255 \\ 1176 & 13.2 & 232 \\ 379 & 7.73 & 88.6 \\ 432 & 4.80 & 91.2 \\ 381 & 4.49 & 110 \\ 756 & 8.31 & 171 \\ 1266 & 9.12 & 147\end{array}$

\section{DURATION OF DAILY MEAN VALUES FOR WATER YEARS 1950-1993}

$\begin{array}{lllll}\text { EXCEEDED } & \text { ANNUAL } & \text { OCT } & \text { NOV } & \text { DEC } \\ & & & & \\ & & & & \\ & & & & \\ & & & & \\ 95.0 & 16.4 & 16.3 & 16.2 & 16.4 \\ 90.0 & 16.7 & 16.3 & 16.4 & 16.4 \\ 85.0 & 16.7 & 16.6 & 16.4 & 16.6 \\ 80.0 & 16.7 & 16.6 & 16.6 & 16.6 \\ 75.0 & 17.0 & 16.8 & 16.6 & 16.8 \\ 70.0 & 17.0 & 16.8 & 16.8 & 16.8 \\ 65.0 & 17.0 & 16.8 & 16.8 & 16.8 \\ 60.0 & 17.4 & 17.1 & 17.0 & 17.1 \\ 55.0 & 17.7 & 17.1 & 17.0 & 17.1 \\ 50.0 & 17.7 & 17.3 & 17.0 & 17.3 \\ 45.0 & 18.0 & 17.3 & 17.0 & 17.6 \\ 40.0 & 18.4 & 17.6 & 17.2 & 17.8 \\ 35.0 & 18.8 & 17.8 & 17.2 & 17.8 \\ 30.0 & 19.5 & 18.1 & 17.4 & 18.1 \\ 25.0 & 19.9 & 18.4 & 17.7 & 18.6 \\ 20.0 & 20.6 & 19.0 & 18.1 & 19.5 \\ 15.0 & 21.5 & 19.5 & 18.6 & 20.9 \\ 10.0 & 22.7 & 20.4 & 19.1 & 21.8 \\ 5.0 & 23.6 & 22.3 & 21.1 & 22.8\end{array}$

ELEVATION IN FEET ABOVE MEAN SEA LEVEL

$\begin{array}{ll}16.4 & 16.4 \\ 16.4 & 16.4 \\ 16.6 & 16.7 \\ 16.6 & 16.7 \\ 16.8 & 17.0 \\ 16.8 & 17.3 \\ 16.8 & 17.6 \\ 17.1 & 17.9 \\ 17.1 & 18.2 \\ 17.3 & 18.5 \\ 17.6 & 18.8 \\ 17.8 & 18.8 \\ 17.8 & 19.4 \\ 18.1 & 19.8 \\ 18.6 & 20.4 \\ 19.5 & 20.8 \\ 20.9 & 21.5 \\ 21.8 & 22.2 \\ 22.8 & 23.8\end{array}$

$$
\begin{aligned}
& 16.7 \\
& 16.9 \\
& 16.9 \\
& 17.9 \\
& 18.2 \\
& 18.4 \\
& 18.7 \\
& 19.0 \\
& 19.2 \\
& 19.8 \\
& 20.1 \\
& 20.4 \\
& 20.9 \\
& 21.2 \\
& 21.5 \\
& 22.2 \\
& 22.8 \\
& 23.5 \\
& 24.1
\end{aligned}
$$

$\begin{array}{ll}16.7 & 16.8 \\ 17.2 & 17.0 \\ 17.4 & 17.0 \\ 17.9 & 17.2 \\ 18.2 & 17.5 \\ 18.7 & 17.7 \\ 19.2 & 18.0 \\ 19.8 & 18.2 \\ 20.0 & 18.5 \\ 20.3 & 18.7 \\ 20.6 & 19.0 \\ 21.2 & 19.5 \\ 21.5 & 20.1 \\ 21.8 & 20.3 \\ 22.5 & 21.2 \\ 23.1 & 22.4 \\ 23.8 & 23.3 \\ 24.1 & 24.3 \\ 24.8 & 25.0\end{array}$

16.5
16.8
16.8
16.8
16.8
17.0
17.0
17.0
17.2
17.2
17.4
17.7
17.9
18.2
18.4
18.9
19.4
20.4
21.8
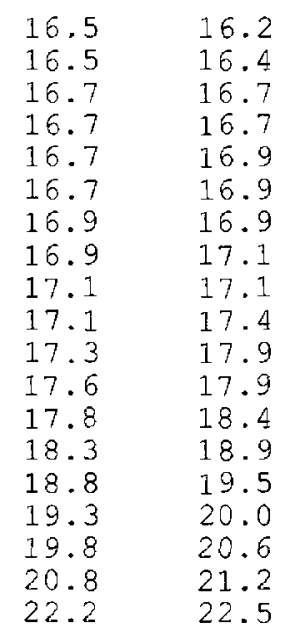

$$
\begin{aligned}
& 16.2 \\
& 16.7 \\
& 16.7 \\
& 16.7 \\
& 16.9 \\
& 17.1 \\
& 17.4 \\
& 17.9 \\
& 18.1 \\
& 18.4 \\
& 18.7 \\
& 18.9 \\
& 19.5 \\
& 19.7 \\
& 20.3 \\
& 20.9 \\
& 21.5 \\
& 23.1 \\
& 24.1
\end{aligned}
$$

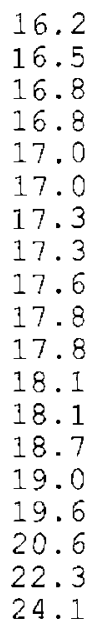

DISCHARGE IN CUBIC FEET PER SECOND

$\begin{array}{rrr}95.0 & 13.1 & 13.1 \\ 90.0 & 17.6 & 15.8 \\ 85.0 & 20.9 & 18.1 \\ 80.0 & 23.8 & 20.0 \\ 75.0 & 27.0 & 21.7 \\ 70.0 & 30.4 & 23.5 \\ 65.0 & 34.6 & 26.0 \\ 60.0 & 40.9 & 29.2 \\ 55.0 & 49.4 & 34.1 \\ 50.0 & 60.6 & 39.3 \\ 45.0 & 74.6 & 44.1 \\ 40.0 & 91.3 & 50.6 \\ 35.0 & 112.0 & 59.5 \\ 30.0 & 137.9 & 71.1 \\ 25.0 & 170.4 & 88.3 \\ 20.0 & 219.2 & 114.8 \\ 15.0 & 284.6 & 146.4 \\ 10.0 & 379.3 & 199.0 \\ 5.0 & 543.3 & 327.7\end{array}$

$\begin{array}{rrr}9.5 & 10.4 & 11.8 \\ 11.6 & 12.8 & 16.6 \\ 14.4 & 17.1 & 19.2 \\ 17.1 & 19.9 & 24.7 \\ 19.0 & 22.6 & 32.1 \\ 21.9 & 25.3 & 39.9 \\ 24.5 & 28.0 & 49.9 \\ 26.6 & 30.8 & 68.5 \\ 29.1 & 33.6 & 81.2 \\ 31.3 & 36.6 & 90.0 \\ 33.4 & 41.8 & 104.0 \\ 35.8 & 51.1 & 122.1 \\ 38.4 & 64.5 & 138.3 \\ 42.8 & 80.7 & 159.6 \\ 49.8 & 101.5 & 193.2 \\ 63.5 & 141.6 & 234.6 \\ 83.4 & 198.9 & 278.0 \\ 114.8 & 304.2 & 362.9 \\ 217.8 & 416.5 & 510.9\end{array}$

$\begin{array}{rr}18.0 & 19.1 \\ 20.5 & 27.2 \\ 27.1 & 34.3 \\ 32.5 & 46.1 \\ 56.7 & 62.3 \\ 83.3 & 82.1 \\ 105.5 & 103.5 \\ 122.8 & 133.4 \\ 141.8 & 162.6 \\ 158.6 & 189.2 \\ 187.6 & 213.0 \\ 218.5 & 246.4 \\ 248.5 & 285.6 \\ 285.2 & 321.7 \\ 328.4 & 362.0 \\ 374.7 & 414.8 \\ 429.0 & 494.1 \\ 495.1 & 587.6 \\ 606.1 & 735.5\end{array}$

20.8
26.3
30.2
35.8
42.9
50.6
59.5
69.7
82.8
99.3
120.9
146.5
179.0
218.6
284.7
389.4
512.5
643.4
833.3

15.1
21.5
23.3
25.0
26.6
28.3
30.5
32.9
36.0
40.8
47.0
55.4
67.8
83.3
98.6
118.1
152.5
225.4
338.7
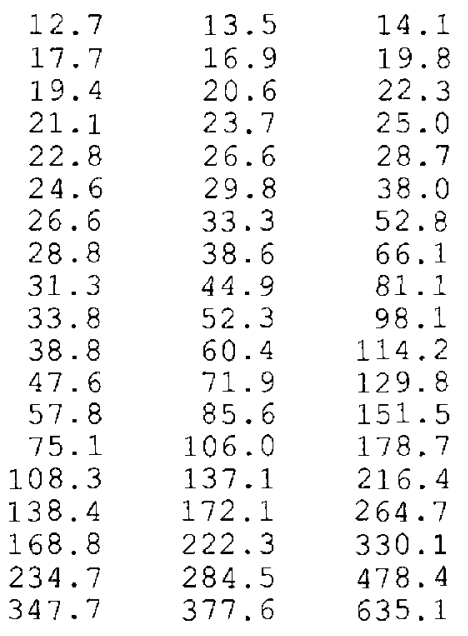

16.2
19.2
22.1
25.4
29.7
36.7
42.9
47.9
54.8
63.4
73.2
82.5
93.5
108.4
132.2
162.0
218.0
346.6
553.6 

FOR PERIOD APR TO MAR

WATER YEAR

\begin{tabular}{|c|c|c|c|c|}
\hline RANGE & 1 & 3 & 7 & \\
\hline $1951 \quad 1952$ & 16.813 & $16.8 \quad 12$ & 16.8 & 12 \\
\hline 19521953 & 16.815 & 16.914 & 16.9 & 15 \\
\hline 19531954 & 17.123 & 17.122 & 17.2 & 22 \\
\hline 19541955 & 16.4 & 16.4 & 16.4 & 6 \\
\hline $1955 \quad 1956$ & 16.35 & 16.3 & 16.3 & 4 \\
\hline $958 \quad 1959$ & 16.814 & 16.813 & 16.8 & 13 \\
\hline 19591960 & 17.525 & 17.625 & 17.7 & 25 \\
\hline 19611962 & 16.5 & 16.5 & 16.5 & r \\
\hline 19621963 & 16.4 & 16.4 & 16.4 & 5 \\
\hline $1963 \quad 1964$ & $16.7 \quad 11$ & 16.710 & 16.7 & 10 \\
\hline 9641965 & $17.5 \quad 24$ & $17.5 \quad 24$ & 17.6 & 24 \\
\hline 651966 & 17.122 & 17.121 & 17.1 & 21 \\
\hline $1975 \quad 1976$ & 14.4 & 17.223 & 17.2 & 23 \\
\hline 19761977 & 17.020 & 17.119 & 17.1 & 18 \\
\hline $78 \quad 1979$ & 16.916 & 16.915 & 16.9 & 14 \\
\hline 791980 & 17.018 & 17.017 & 17.0 & 17 \\
\hline 801981 & 16.917 & 16.916 & 17.0 & 16 \\
\hline 19811982 & 16.712 & 16.711 & 16.7 & 11 \\
\hline 321983 & 17.019 & $17.0 \quad 18$ & 17.1 & 19 \\
\hline 19831984 & 17.121 & 17.120 & 17.1 & 20 \\
\hline $1986 \quad 1987$ & 16.5 & 16.5 & 16.5 & 8 \\
\hline 71988 & 16.2 & 16.3 & 16.3 & 2 \\
\hline 31989 & 16.2 & 16.2 & 16.3 & 3 \\
\hline $90 \quad 1991$ & 15.9 & 15.9 & 15.9 & \\
\hline 115 & $16.6 \quad 10$ & 16.6 & 16.6 & 9 \\
\hline
\end{tabular}

\begin{tabular}{|c|c|c|c|}
\hline 14 & & 30 & \\
\hline 16.9 & 12 & 17.0 & 14 \\
\hline 16.9 & 15 & 17.0 & 1.5 \\
\hline 17.3 & 23 & 17.6 & 23 \\
\hline 16.5 & 6 & 16.5 & 6 \\
\hline 16.3 & 4 & 16.4 & 4 \\
\hline 16.91 & 13 & 16.9 & 12 \\
\hline 17.92 & 25 & 18.1 & 25 \\
\hline 16.5 & 7 & 16.5 & 7 \\
\hline 16.4 & 5 & 16.4 & 5 \\
\hline 16.7 & 10 & 16.8 & 11 \\
\hline 17.82 & 24 & 18.0 & 24 \\
\hline 17.22 & 20 & 17.2 & 19 \\
\hline 17.22 & 21 & 17.2 & 20 \\
\hline 17.11 & 18 & 17.4 & 22 \\
\hline 16.91 & 14 & 16.9 & 13 \\
\hline 17.01 & 17 & 17.2 & 16 \\
\hline 17.0 & 16 & 17.2 & 17 \\
\hline 16.71 & 11 & 16.8 & 10 \\
\hline 17.22 & 22 & 17.2 & 21 \\
\hline 17.11 & 19 & 17.2 & 18 \\
\hline 16.6 & 8 & 16.6 & 8 \\
\hline 16.3 & 2 & 16.3 & 2 \\
\hline 16.3 & 3 & 16.3 & 3 \\
\hline .9 & 1 & 15.9 & 1 \\
\hline 16.6 & 9 & 16.6 & 9 \\
\hline
\end{tabular}

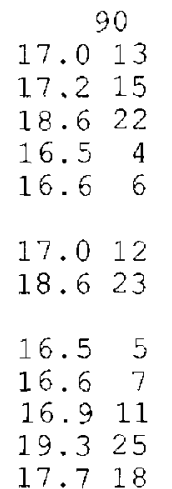

120

17.213

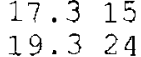

16.53

16.76

17.112

19.323

16.65

15.77

19.425

17.817

$17.7 \quad 16$

18.922

17.314

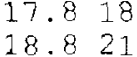

$17.0 \quad 10$

18.720

18.119

$\begin{array}{ll}15.8 & 8 \\ 16.5 & 2\end{array}$

16.64

16.11

17.09

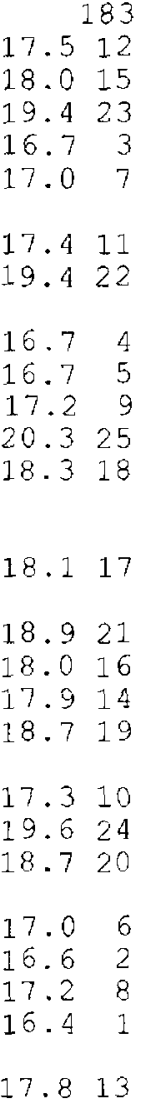

183

18.015

16.73

17.07

.41

6.7

17.75

0.325

8.117

8.921

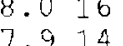

. 19

$\begin{array}{lll}9.6 & 24\end{array}$

18.720

6.6

6.41

17.813

HIGHEST MEAN ELEVATION, IN FEET, AND RANKING FOR THE FOLLOWING NUMBER OF CONSECUTIVE DAYS FOR PERIOD OCT TO SEP

WATER YEAR RANGE 1951195 19521952 19531953 19541954 19551955

19561956

19581958

19591959

19611961

19621962

19631963

19641964

19651965

19661966

19721972

19751975

19761976

$1978 \quad 1978$

9801980

19811981

19821982

19831983

19841984

19861986

19871987

19881988

19901990

19911991

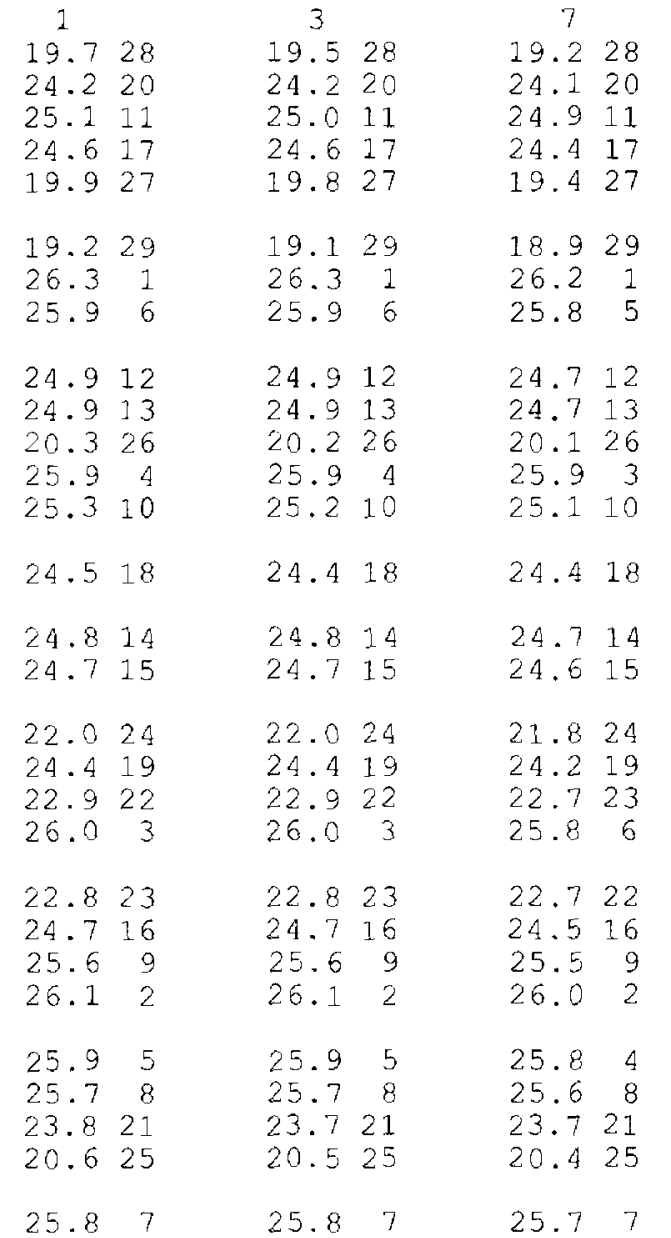

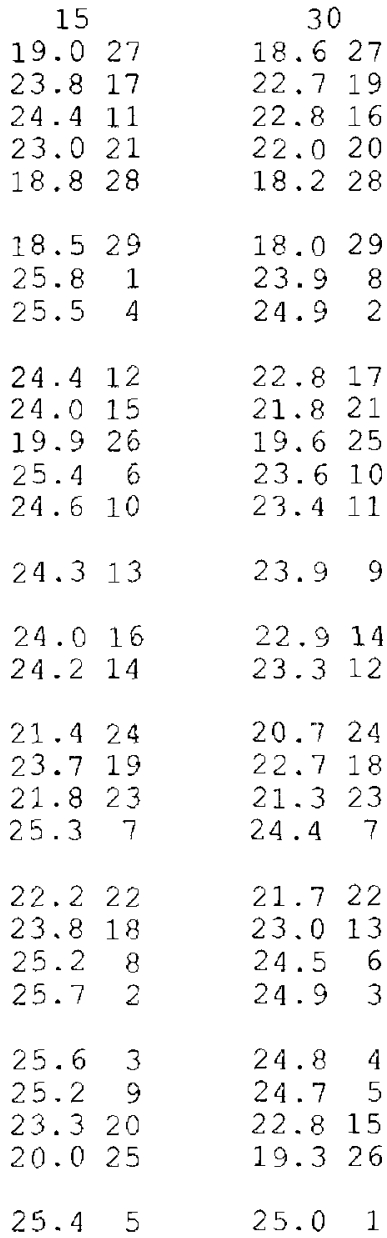

17.728

$\begin{array}{ll}23.2 & 8 \\ 23.3 & 6\end{array}$

20.322

19.724

19.026

23.37

21.715

$23.0 \quad 9$

$22.3=2$

22.311

20.223

$\begin{array}{ll}21.8 & 14 \\ 20.5 & 21\end{array}$

22.810

20.918

22.313

24.03

23.44

23.45

$24.4 \quad 1$

$21.0 \quad 17$

24.32
17. 90 17.927
20.717

21.016

20.219

17.329

17.728

22.26

120
17.727

$20.8 \quad 15$

20.716

20.219

$\begin{array}{rr}17.6 & 28 \\ 21.8 & 7\end{array}$

21.58

19.322

18.425

18.326

21.96

20.914

20.912

21.59

21.510

19.620

$\begin{array}{ll}20.5 & 17 \\ 20.5 & 18\end{array}$

20.913

19.521

21.011

23.32

22.94

22.45

24.11

$\begin{array}{ll}19.1 & 23 \\ 18.6 & 24\end{array}$

24.3

19.821
18.924

$23.6 \quad 3$
183
20.457

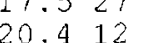
17.5
20.412
20.610 19.421

17.029

17.528 $21.5 \quad 5$ 20.88

19.520 17.925 20.97 20.511

20.116

20.69

20.313

19.322

19.817

19.718

19.519 $20.3 \quad 15$ $\begin{array}{ll}22.3 & 2 \\ 22.0 & 4\end{array}$

21.36

$22.3 \quad 3$

$\begin{array}{ll}18.2 & 23 \\ 18.1 & 24\end{array}$

$22.6 \quad 1$ 

CONSECUTIVE DAYS FOR PERIOD APR TO MAR

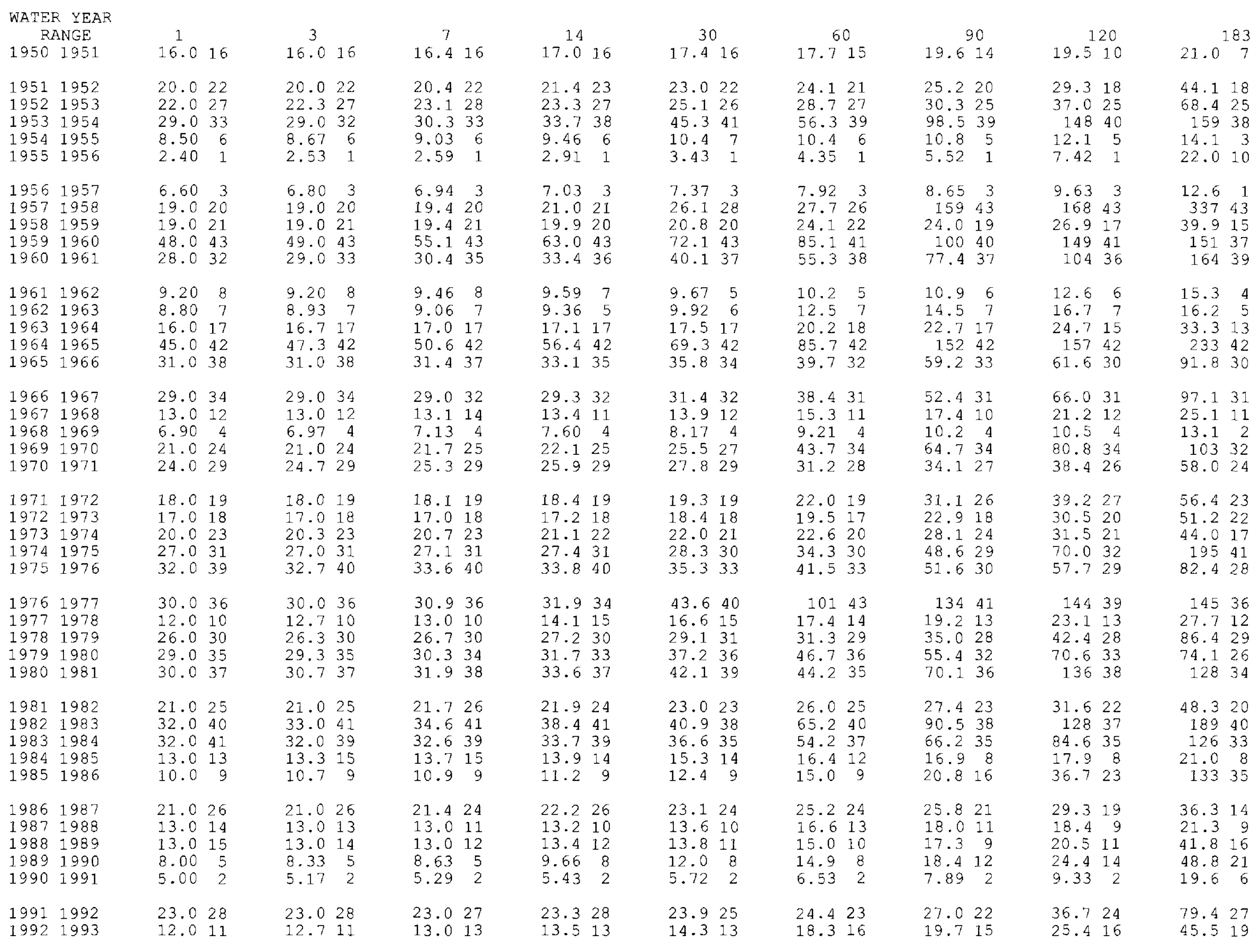


HIGHEST MEAN DISCHARGE, IN CUBIC FEET PER SECOND, AND RANKING FOR THE FOLLOWING NUMBER OF CONSECUTIVE DAYS FOR PERIOD OCT TO SEP

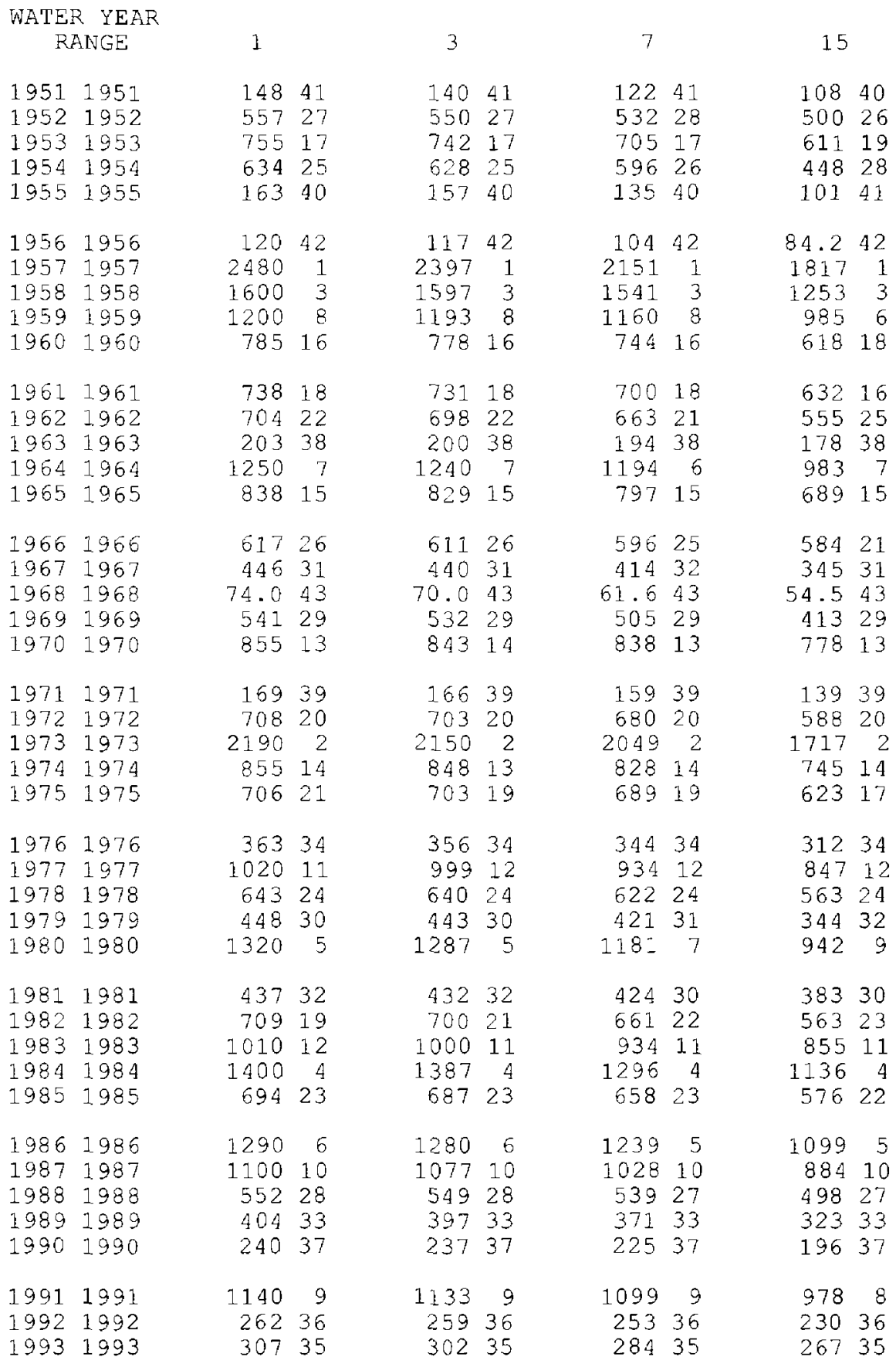

30

60

39326

43725

32729

74.841

$60.8 \quad 42$

$1266 \quad 1$

7937

$\begin{array}{lr}796 & 6 \\ 535 & 14\end{array}$

46120

$353 \quad 27$

15538

$\begin{array}{lll}669 & 12\end{array}$

$526 \quad 17$

52816

24134

43.543

31530

59513

$120 \quad 39$

$437 \quad 24$

$\begin{array}{rr}437 & 24 \\ 1176 \quad 2\end{array}$

53315

51318

$262 \quad 32$

7758

$448 \quad 22$

30631

67211

$340 \quad 28$

$468 \quad 19$

70210

8603

43923

$846 \quad 5$

7359

45221

24733

15537

8534

$210 \quad 36$

$236 \quad 35$
75.940 $282 \quad 24$ 27326

24129

51.541

49.242

8091

50512

$\begin{array}{rr}564 & 9\end{array}$

26227

$194 \quad 34$

12138

56410

35221

44115

17436

34.443

20331

41716

10139

37919

6895

53011

40917

22430

6904

$\begin{array}{lll}360 & 20\end{array}$

24628

49714

27525

40518

$618 \quad 6$

6127

32722

5928

7013

$300 \quad 23$

20332

13637

$710 \quad 2$

19035
20233
90

60.240

23525

29722

$\begin{array}{rr}211 & 28 \\ 36 & 42\end{array}$

48.241

6092

40012

41910

19231

13736

$\begin{array}{rr}105 & 38 \\ 439 & 9\end{array}$

29821

$336 \quad 19$

$138 \quad 35$

$33.7 \quad 43$

16332

35916

87.039

37814

$\begin{array}{ll}378 & 14 \\ 582 & 5\end{array}$

37215

34618

20329

5706

30220

24724

38213

21626

$347 \quad 17$

5854

5417

$248 \quad 23$

5038

6741

$\begin{array}{ll}216 & 27\end{array}$

15534

12637

6043

16233

19530
120

$51.5 \quad 40$

24823

25622

20825

28.243

46.841

5692

36011

$\begin{array}{ll}367 & 10 \\ 330 & 13\end{array}$

17429

10837

10837
85.238

3839

27720

$277 \quad 19$

11435

$31.8 \quad 42$

15132

31116

$78.3 \quad 39$

32914

4827

28718

33212

$188 \quad 27$

5036

25921

24324

31515

18428

29917

5274

5075

19326

4478

6261

$170 \quad 30$

12134

11236

$541 \quad 3$

$133 \quad 33$

16131
183

45.340

21322

25215

15628

22.043

41.441

3836

3309

$\begin{array}{ll}295 & 12 \\ 276 & 13\end{array}$

17526

77.437

65.038

$\begin{array}{ll}320 & 10 \\ 249 & 16\end{array}$

$215 \quad 21$

99.033

$8.4 \quad 42$

$\begin{array}{ll}129 & 30 \\ 247 & 18\end{array}$

63.339

26014

3627

$\begin{array}{ll}223 & 20 \\ 248 & 17\end{array}$

$\begin{array}{ll}170 & 27\end{array}$

$\begin{array}{ll}391 & 5 \\ 203 & 23\end{array}$

19024

29711

18425

24519

4273

4144

13329

$\begin{array}{ll}345 & 8\end{array}$

11932

86.836

87.435

$\begin{array}{rr}485 & 1\end{array}$

12131 


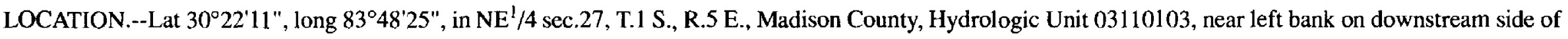
bridge on U.S. Highway 19, $0.6 \mathrm{mi}$ southeast of Lamont, and $34 \mathrm{mi}$ upstream from mouth.

DRAINAGE AREA.--747 $\mathrm{mi}^{2}$.

PERIOD OF RECORD.--February 1950 to September 1979; November 1983 to 1992 (gage heights and peak discharge only).

REVISED RECORDS.--WSP 1204, 1905: Drainage area. WSP 1504: 1953.

GAGE.--Water-stage recorder. Datum of gage is $42.90 \mathrm{ft}$ above National Geodetic Vertical Datum of 1929, unadjusted.

REMARKS.--Minor pumpage above and below station for irrigation during dry seasons. Since Aug. 27, 1963, low-head rock and concrete dam 0.6 mi downstream.

MAXIMUM, MINIMUM, AND MEAN OF MONTHLY MEAN ELEVATIONS FOR WATER YEARS 1950-1992
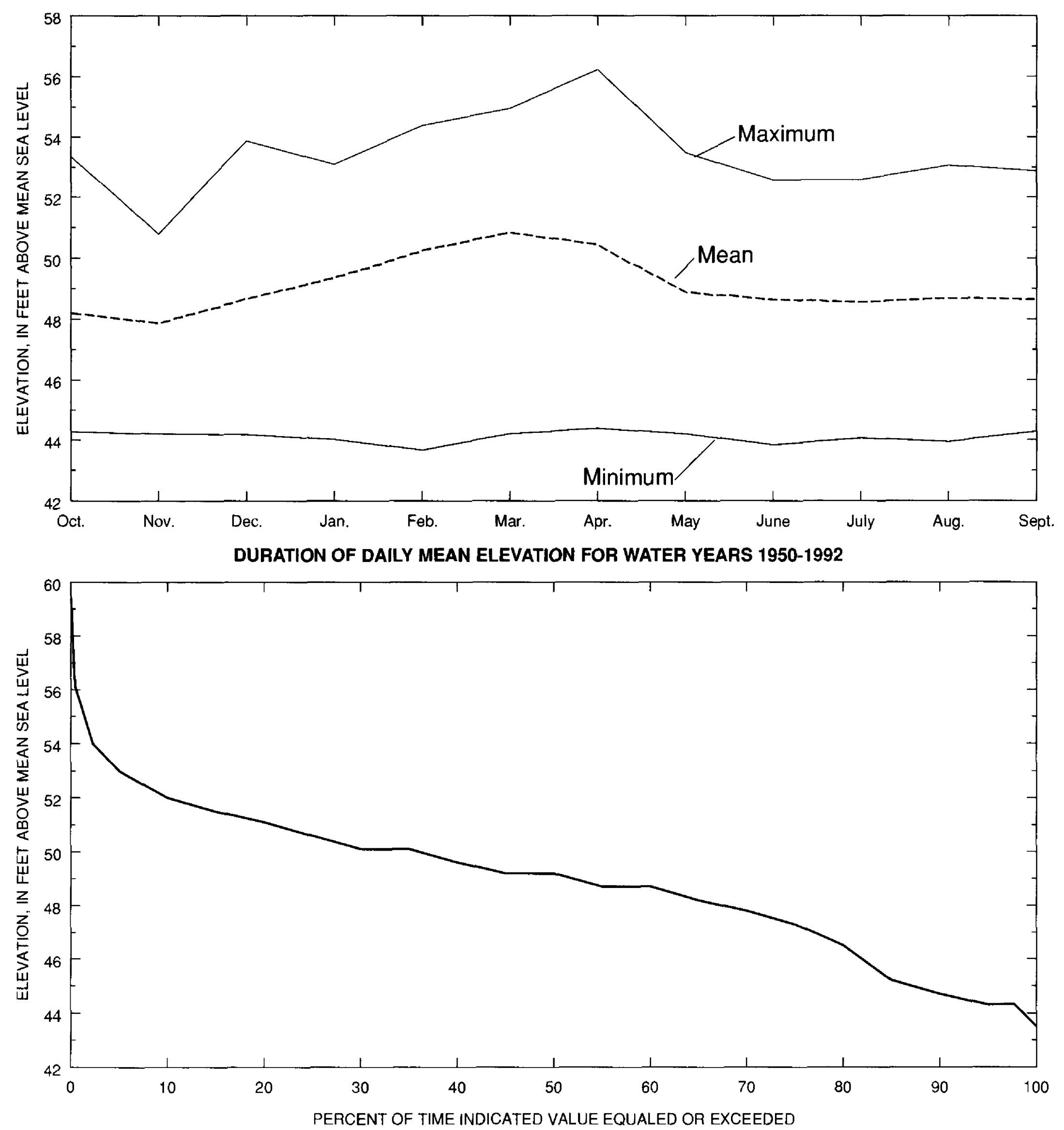
SUMMARY STATISTICS, IN CUBIC FEET PER SECOND UNLESS OTHERWISE INDICATED FOR WATER YEARS $1950-1992$

\begin{tabular}{|c|c|c|c|c|}
\hline NNUAL MEAN & 357 & & & \\
\hline HIGHEST ANNUAL MEAN & 1471 & & & 1965 \\
\hline LOWEST ANNUAL MEAN & 2.68 & & & 1955 \\
\hline HIGHEST DAILY MEAN & 11500 & Apr & 8 & 1973 \\
\hline LOWEST DAILY MEAN & .00 & Jun & 2 & 1955 \\
\hline ANNUAL SEVEN-DAY MINIMUM & .00 & Jun & 2 & 195 \\
\hline INSTANTANEOUS PEAK FLOW & 11500 & Apr & 8 & 197. \\
\hline INSTANTANEOUS PEAK ELEVATION (FT) & 59.47 & Apr & 8 & 197. \\
\hline INSTANTANEOUS LOW FLOW & .00 & Jun & 2 & 1955 \\
\hline ANNUAL RUNOFF (INCHES) & 6.50 & & & \\
\hline ANNUAL RUNOFF (CFSM) & 48 & & & \\
\hline
\end{tabular}

MAXIMUM, MINIMUM, AND MEAN OF MONTHLY MEAN DISCHARGES FOR WATER YEARS 1950-1992
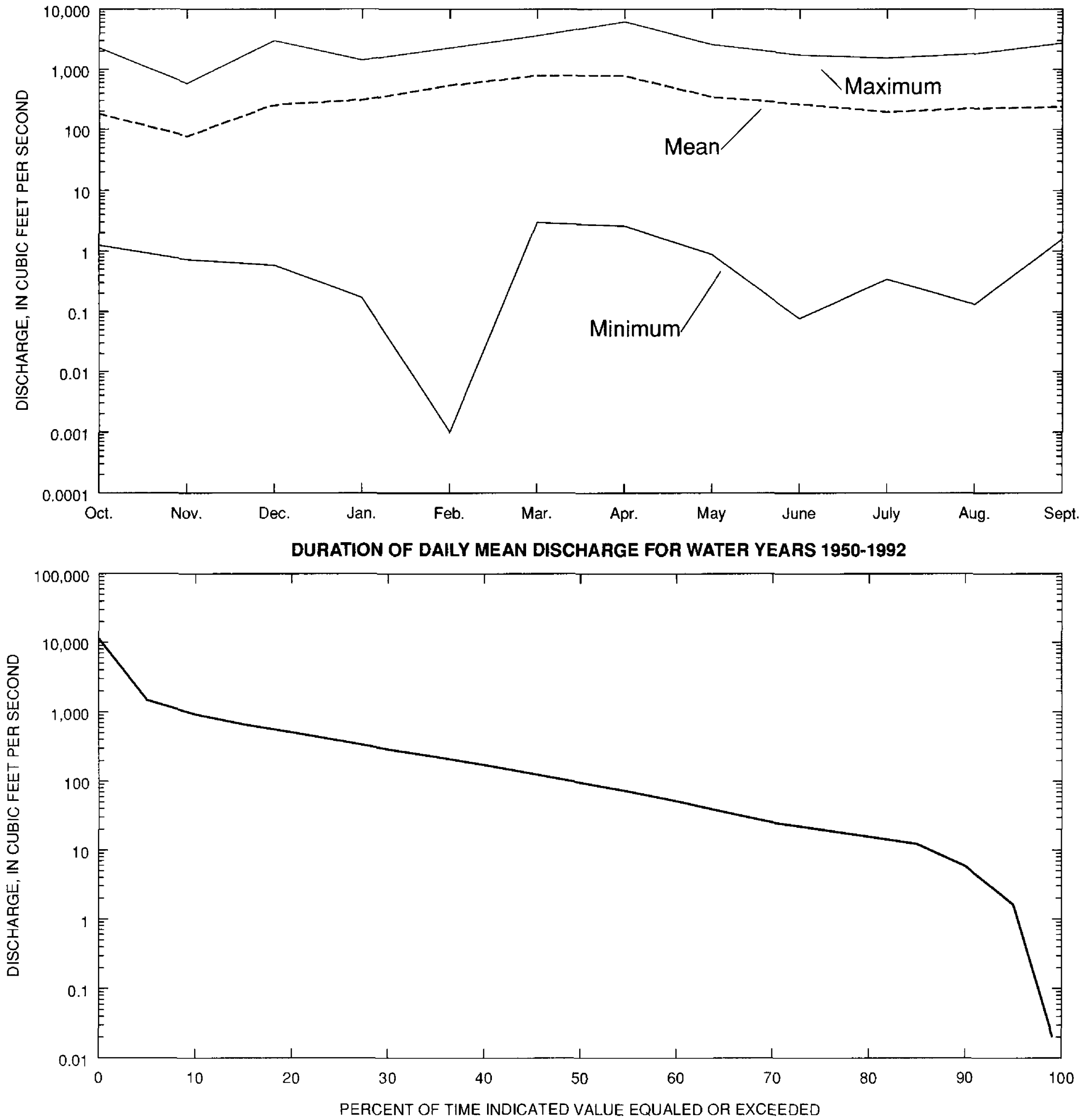
AUCILLA RIVER BASIN

02326500 AUCILLA RIVER AT LAMONT, FL--Continued

\section{SUMMARY OF MONTHLY MEAN ELEVATION AND DISCHARGE STATISTICS}

\section{FOR WATER YEARS 1950-1992}

\begin{tabular}{|c|c|c|c|c|c|c|}
\hline \multirow[b]{3}{*}{ MONTH } & \multicolumn{3}{|c|}{ ELEVATION } & \multicolumn{3}{|c|}{ DISCHARGE } \\
\hline & EEET AB & E SEA LE & $\mathrm{EL}$ & CUBIC FE & PER SEC & ND \\
\hline & MAXIMUM & MINIMUM & MEAN & MAXIMUM & MINIMUM & MEAN \\
\hline OCTOBER & 53.33 & 44.28 & 48.20 & 2220 & 1.25 & 179.85 \\
\hline NOVEMBER & 50.76 & 44.21 & 47.87 & 567.8 & .727 & 76.89 \\
\hline DECEMBER & 53.85 & 44.18 & 48.67 & 2923 & .584 & 258.71 \\
\hline JANUARY & 53.07 & 44.01 & 49.36 & 1402 & .171 & 306.91 \\
\hline FEBRJARY & 54.37 & 43.66 & 50.25 & 2222 & .000 & 544.91 \\
\hline MARCH & 54.93 & 44.21 & 50.83 & 3519 & 2.95 & 778.44 \\
\hline APRIL & 56.20 & 44.39 & 50.43 & 6000 & 2.58 & 783.52 \\
\hline MAY & 53.46 & 44.20 & 48.87 & 2524 & .887 & 344.73 \\
\hline JUNE & 52.54 & 43.83 & 48.64 & 1692 & .077 & 263.42 \\
\hline JULY & 52.55 & 44.06 & 48.56 & 1511 & .342 & 194.32 \\
\hline AUGUST & 53.04 & 43.94 & 48.69 & 1765 & .132 & 222.72 \\
\hline SEPTEMBER & 52.85 & 44.29 & 48.65 & 2674 & 1.57 & 237.03 \\
\hline
\end{tabular}

\section{DURATION OF DAILY MEAN VALUES FOR WATER YEARS 1950-1993}

PERCENT

EQUALED OR
EXCEEDED ANNUAL OCT
NOV DEC

ELEVATION IN FEET ABOVE MEAN SEA LEVEI

$\begin{array}{rlll}95.0 & 44.3 & 44.2 & 44.2 \\ 90.0 & 44.7 & 44.5 & 44.7 \\ 85.0 & 45.2 & 44.8 & 44.9 \\ 80.0 & 46.5 & 45.2 & 45.2 \\ 75.0 & 47.3 & 46.6 & 46.3 \\ 70.0 & 47.8 & 47.3 & 47.1 \\ 65.0 & 48.2 & 47.6 & 47.3 \\ 60.0 & 48.7 & 48.0 & 47.9 \\ 55.0 & 48.7 & 48.3 & 48.2 \\ 50.0 & 49.2 & 48.3 & 48.2 \\ 45.0 & 49.2 & 48.7 & 48.4 \\ 40.0 & 49.6 & 48.7 & 48.4 \\ 35.0 & 50.1 & 49.1 & 48.7 \\ 30.0 & 50.1 & 49.4 & 49.0 \\ 25.0 & 50.6 & 49.4 & 49.3 \\ 20.0 & 51.1 & 49.8 & 49.3 \\ 15.0 & 51.5 & 49.8 & 49.5 \\ 10.0 & 52.0 & 50.2 & 49.9 \\ 5.0 & 53.0 & 51.3 & 50.4\end{array}$

$\begin{array}{lll}44.4 & 44.2 & 44.5 \\ 44.8 & 44.9 & 44.9 \\ 45.1 & 45.5 & 45.8 \\ 45.1 & 46.9 & 47.5 \\ 46.7 & 47.9 & 48.3 \\ 47.4 & 48.3 & 48.7 \\ 47.7 & 48.6 & 49.9 \\ 48.1 & 48.9 & 49.9 \\ 48.4 & 49.3 & 50.3 \\ 48.8 & 49.7 & 50.7 \\ 48.8 & 50.0 & 51.1 \\ 49.1 & 50.0 & 51.1 \\ 49.5 & 50.4 & 51.5 \\ 49.5 & 50.4 & 51.5 \\ 49.8 & 50.8 & 51.9 \\ 50.2 & 51.5 & 52.4 \\ 51.3 & 51.9 & 52.8 \\ 52.4 & 52.7 & 52.8 \\ 53.5 & 53.0 & 53.2\end{array}$

44.4
46.3
47.1
48.3
49.2
50.0
50.4
50.8
50.8
51.3
51.7
51.7
52.1
52.1
52.5
53.0
53.0
53.5
54.4

$$
\begin{aligned}
& 44.6 \\
& 45.8 \\
& 47.1 \\
& 48.4 \\
& 48.8 \\
& 49.2 \\
& 49.7 \\
& 50.1 \\
& 50.1 \\
& 50.1 \\
& 50.6 \\
& 51.0 \\
& 51.0 \\
& 51.5 \\
& 51.9 \\
& 52.4 \\
& 52.9 \\
& 53.3 \\
& 54.3
\end{aligned}
$$

44.8
44.8
45.2
46.2
47.2
47.6
47.9
48.3
49.0
49.4
49.4
49.8
49.8
50.2
50.2
50.5
50.9
51.3
52.4

44.7
44.7
45.0
45.6
47.2
47.8
48.2
48.5
48.8
48.8
49.1
49.5
49.5
49.8
49.8
50.5
51.2
51.8
52.2

44.7
44.9
44.9
46.0
46.8
47.6
47.9
48.1
48.4
48.7
49.0
49.3
49.6
49.8
50.1
50.4
50.7
51.0
51.9

44.3
44.9
44.9
45.8
46.7
47.3
47.8
48.5
48.8
49.1
49.4
49.4
49.7
50.0
50.3
50.3
50.7
51.6
52.3

$44 \cdot 4$

DISCHARGE IN CUBIC FEET PER SECOND

$\begin{array}{rrr}95.0 & 1.6 & 0.8 \\ 90.0 & 5.8 & 1.9 \\ 85.0 & 12.3 & 4.0 \\ 80.0 & 15.7 & 5.4 \\ 75.0 & 19.9 & 10.5 \\ 70.0 & 25.3 & 13.8 \\ 65.0 & 35.7 & 16.2 \\ 60.0 & 51.2 & 18.7 \\ 55.0 & 71.1 & 21.7 \\ 50.0 & 95.9 & 24.7 \\ 45.0 & 128.5 & 30.0 \\ 40.0 & 172.7 & 37.0 \\ 35.0 & 226.2 & 52.7 \\ 30.0 & 289.4 & 90.8 \\ 25.0 & 385.0 & 138.8 \\ 20.0 & 507.2 & 193.4 \\ 15.0 & 661.5 & 292.7 \\ 10.0 & 916.9 & 498.5 \\ 5.0 & 1481.5 & 806.7\end{array}$

$$
\begin{array}{r}
2.3 \\
4.5 \\
8.4 \\
13.9 \\
15.9 \\
18.0 \\
20.4 \\
22.7 \\
27.7 \\
35.9 \\
44.5 \\
52.2 \\
56.5 \\
87.8 \\
119.8 \\
190.0 \\
335.9 \\
666.4 \\
1661.4
\end{array}
$$

4.1
12.1
19.5
66.4
108.5
166.0
256.2
309.6
380.0
436.0
486.6
544.8
603.7
678.2
757.1
856.9
989.3
1206.4
1728.7

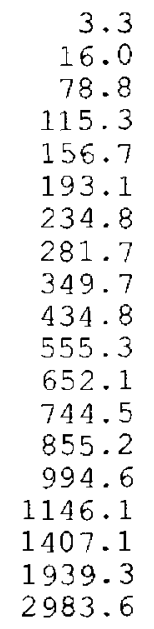

8.9
15.5
19.2
25.0
32.1
40.2
53.4
70.5
90.8
116.1
151.4
196.3
240.0
283.3
345.8
417.2
524.8
711.0
1195.7

3.8

8.1
10.6

14.1

17.0

20.4

$24 \cdot 2$

28.5
38.8

38.8
62.3

87.7

113.1

141.0

176.7

216.1

265.9

373.3

575.3
858.7

2.2
6.2
11.0
14.6
18.9
31.0
44.8
59.0
74.8
92.2
111.7
136.4
166.0
201.6
244.2
299.1
403.8
690.5
968.4

2.3 $4 \cdot 6$ 6.4
12.5 17.3 24.1 29.3 36.1 47.2 59.4 79.7
96.3 118.4 149.3 184.2 231.9 300.2 429.7 

FOR PERIOD APR TO MAR

WATER YEAR

RANGE

$\begin{array}{ll}1951 & 1952 \\ 1952 & 1953 \\ 1954 & 1955 \\ 1956 & 1957 \\ 1957 & 1958 \\ 1958 & 1959 \\ 1960 & 1961 \\ 1961 & 1962 \\ 1962 & 1963 \\ 1963 & 1964 \\ 1964 & 1965 \\ 1965 & 1966 \\ 1966 & 1967 \\ 1967 & 1968 \\ 1968 & 1969 \\ 1969 & 1970 \\ 1970 & 1971 \\ 1974 & 1975 \\ 1975 & 1976 \\ 1976 & 1977 \\ 1977 & 1978 \\ 1978 & 1979 \\ 1984 & 1985 \\ 1985 & 1986 \\ 1986 & 1987 \\ 1987 & 1988 \\ 1988 & 1989 \\ 1989 & 1990 \\ 1991 & 1992 \\ 1\end{array}$

$44.7 \quad 3$

$\begin{array}{ll}44.8 & 4 \\ 44.3 & 2\end{array}$

43.51

44.85

45.29

45.510

$45.0 \quad 8$

$44.9 \quad 7$

49.327

49.023

49.124

49.226

49.428

$48.8 \quad 22$

49.125

48.721

48.520

48.419

$\begin{array}{ll}48.1 & 18 \\ 47.6 & 14\end{array}$

47.816

47.815

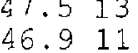

$47.4 \quad 12$
14

44.7

44.82
44.32

43.5

44.85

45.2
45.510

45.08

44.97

$44.9 \quad 6$

49.629

49.023

49.124

48.017

49.428

48.922

49.125

48.721

$48.5 \quad 20$

48.419

$48.1 \quad 18$

47.614

47.916

$47.8 \quad 15$

47.613

47.011

$47.4 \quad 12$

47.916

47.81
47.613

47.111

$47.4 \quad 12$
30

60

44.7
44.8

$\begin{array}{ll}44.8 & 4 \\ 44.32\end{array}$

$43.5 \quad 1$

45.29

$45.6 \quad 10$

45.08

44.97

44.95

$\begin{array}{rr}49.7 & 29 \\ 49.4 & 27\end{array}$

49.023

49.124

49.326

49.528

48.922

$49.2 \quad 25$

48.821

48.620

48.419

48.217

47.714

48.016

47.915

47.111

47.412

44.83

44.8
44.42

43.71

45.29

45.710

45.18

45.07

$44.9 \quad 5$

49.527

49.023

49.225

48.519

49.326

49.528

49.022

49.224

48.921

48.620

48.418

48.217
48.116

47.915

47.7
47.211

47.412
45.05

$\begin{array}{ll}44.9 & 3 \\ 44.4 & 2\end{array}$

43.81

45.28

$\begin{array}{lr}45.2 & 9 \\ 46.0 & 10\end{array}$

45.17

45.04

$50.6 \quad 29$

49.628

$\begin{array}{ll}49.1 & 23 \\ 49.5 & 26\end{array}$

48.820

49.425

49.527

49.121

49.324

49.122

$48.7 \quad 19$

48.418

$\begin{array}{ll}48.2 & 16 \\ 47.9 & 14\end{array}$

48.317

48.015

47.713

47.311

47.412
90

45.05

44.9
44.42

43.91

46.69

$\begin{array}{rr}45.3 & 8 \\ 46.6 \quad 10\end{array}$

45.27

45.16

51.329

49.928

49.322

49.727

49.120

49.624

49.321

49.626

49.323

$48.7 \quad 19$

48.518

48.216

48.317

48.014

47.713

47.411

47.512
120

183

45.26

45.14

44.52

$44.1 \quad 1$

47.910

46.99

45.76

$46.1 \quad 8$

51.629

50.628

49.923

49.924

49.320

50.125

$49.8 \quad 22$

50.326

50.327

48.816

48.818

48.512

48.316

$48.4 \quad 17$

48.014

48.013

48.817

48.211

$\begin{array}{ll}48.6 & 15 \\ 48.6 & 14\end{array}$

48.513

HIGHEST MEAN ELEVATION, IN FEET, AND RANKING FOR THE FOLLOWING NUMBER OF CONSECUTIVE DAYS FOR PERIOD OCT TO SEP

WATER. YEAR

RANGE

19511951

19521952

19541954

19561956 19571957

19581958

19611961

19621962

19631963

19641964

19651965

19661966

19671967

19681968

19691969

19701970

19711971

19731973

19751975

19761976 $1977 \quad 1977$

19781978

19791979

19821982

19851985

19861986

$1987 \quad 1987$

19881988

19891989

19911991

19921992
48.130

52.620

52.421

$46.3 \quad 31$

$\begin{array}{ll}5 / .8 & 2 \\ 56.7 & 5\end{array}$

51.924

$\begin{array}{rr}55.4 & 9 \\ 51.3 & 28\end{array}$

56.27

56.2

55.58

$\begin{array}{ll}53.1 & 17 \\ 50.5 & 29\end{array}$

51.626

53.316

51.527

59.4

54.611

54.612

54.014

$\begin{array}{ll}53.5 & 15 \\ 52.9 & 18\end{array}$

51.725
52.4
23

56.94

55.110

54.113

52.422

$\begin{array}{ll}5.7 & 7 \\ 52.7 & 19\end{array}$

$52.7 \quad 19$

15

48.030

52.620

52.421

46.231

57.53

51.924

$\begin{array}{ll}55.3 & 9 \\ 51.2 & 28\end{array}$

56.17

56.16

55.58

$\begin{array}{ll}53.1 & 17 \\ 50.5 & 29\end{array}$

51.526

53.316

51.527

59.4

54.611

54.612

54.014

53.415

52.918

$\begin{array}{lll}51.7 & 25 \\ 52.3 & 22\end{array}$ 

CONSECUTIVE DAYS FOR PERIOD APR TO MAR

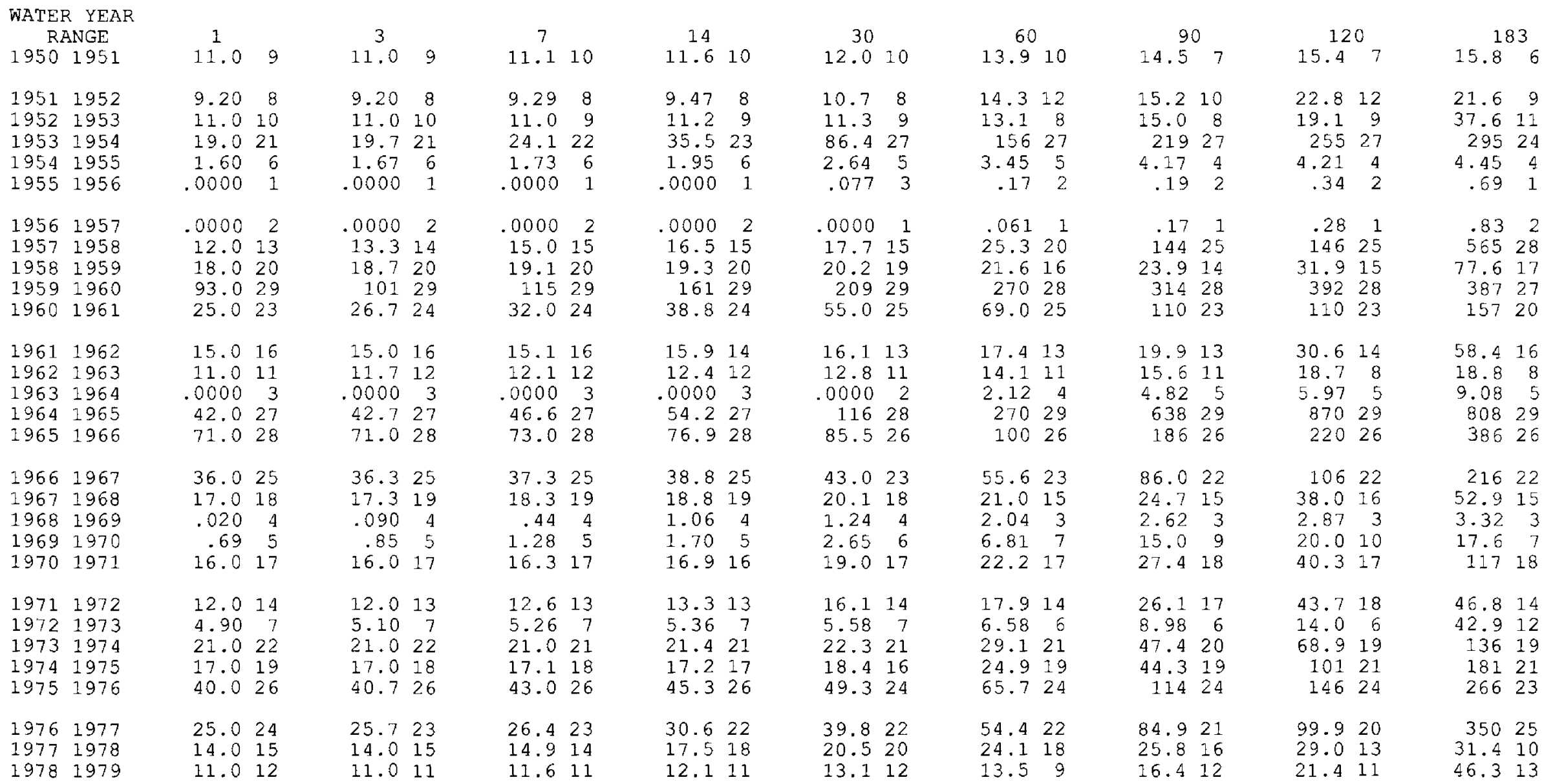
HIGHEST MEAN DISCHARGE, IN CUBIC FEET PER SECOND, AND RANKING FOR THE FOLLOWING NUMBER OF
CONSECUTIVE DAYS FOR PERIOD OCT TO SEP

WATER YEAR

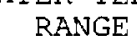

19511951

19521952

19531953

19541954

19551955

19561956

19571957

19581958

19591959

19601960

19611961

19621962

19631963

19641964

19651965

19661966

$1967 \quad 1967$

19681968

19691969

19701970

$1971 \quad 1971$

$1972 \quad 1972$

19731973

$1974 \quad 1974$

19761976

19771977

19781978

19791979

19821982

\begin{tabular}{|c|c|}
\hline 1 & 3 \\
\hline $\begin{array}{rl}216 & 27 \\
1040 & 20 \\
2050 & 13 \\
967 & 21 \\
13.0 & 30\end{array}$ & $\begin{array}{rr}211 & 27 \\
1033 & 20 \\
1993 & 13 \\
940 & 22 \\
11.3 & 30\end{array}$ \\
\hline $\begin{array}{rr}70.0 & 29 \\
6510 & 2 \\
5350 & 5 \\
4480 & 7 \\
3560 & 9\end{array}$ & $\begin{array}{l}67.0 \\
6277 \\
5250 \\
4437 \\
3513\end{array}$ \\
\hline $\begin{array}{rr}957 & 22 \\
4080 & 8 \\
764 & 23 \\
5820 & 4 \\
6000 & 3\end{array}$ & $\begin{array}{r}950 \\
3973 \\
759 \\
5717 \\
5923\end{array}$ \\
\hline $\begin{array}{rr}5120 & 6 \\
1990 & 14 \\
169 & 28 \\
268 & 26 \\
1620 & 17\end{array}$ & $\begin{array}{r}5070 \\
1967 \\
144 \\
261 \\
1593\end{array}$ \\
\hline $\begin{array}{rr}432 & 25 \\
1850 & 15 \\
11500 & 1 \\
1620 & 18 \\
3280 & 11\end{array}$ & $\begin{array}{r}415 \\
1817 \\
11370 \\
1573 \\
3200\end{array}$ \\
\hline $\begin{array}{ll}3300 & 10 \\
2370 & 12 \\
1780 & 16 \\
1440 & 19\end{array}$ & $\begin{array}{l}1757 \\
1420\end{array}$ \\
\hline
\end{tabular}

$627 \quad 24$

$\begin{array}{rr}7 & \\ 203 & 27 \\ 996 & 20 \\ 1799 & 14 \\ 830 & 22 \\ 9.31 & 30 \\ 58.9 & 29 \\ 5461 & 3 \\ 4743 & 6 \\ 4246 & 7 \\ 3393 & 9 \\ 927 & 21 \\ 3629 & 8 \\ 754 & 23 \\ 5314 & 4 \\ 5594 & 2 \\ 4901 & 5 \\ 1850 & 13 \\ 139 & 28 \\ 245 & 26 \\ 1486 & 17 \\ 405 & 25 \\ 1684 & 15 \\ 10990 & 1 \\ 1436 & 18 \\ 2934 & 10 \\ 2856 & 11 \\ 2233 & 12 \\ 1634 & 16 \\ 1311 & 19\end{array}$

62224

59524
15

$\begin{array}{lll}185 & 27\end{array}$

$\begin{array}{ll}1858 & 20\end{array}$

138315

$\begin{array}{lll}788 & 22\end{array}$

7.7730

54.129

$4322 \quad 3$

35857

3757
2982

86221

$2974 \quad 9$

71023
418

$4187 \quad 5$

$4595 \quad 2$

$\begin{array}{rr}4311 & 4 \\ 1570 & 13\end{array}$

12828

21126

135017

40225

136716

91731

238310

225711

$2168 \quad 12$

142814

$528 \quad 24$

30

14926

85919

95418

65022

7.2930

39.829

$2674 \quad 5$

$2278 \quad 7$

$\begin{array}{ll}3176 & 4 \\ 2141 & 8\end{array}$

65921

192010

$\begin{array}{lll}641 & 23 \\ 2637 & 6\end{array}$

$\begin{array}{ll}2631 & 6 \\ 3574 & 3\end{array}$

$3598 \quad 2$

$1084 \quad 15$

10428

14527

37325

109314

60001

167911

152612

19669

$\begin{array}{ll}1133 & 13 \\ 1080 & 16\end{array}$

$428 \quad 24$

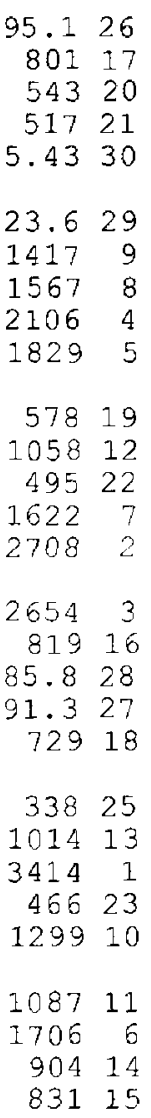

60
90

8.728
$642 \quad 16$

64216
49020

$403 \quad 22$

5.7430

22.929

99910

12748

1569
1452

52019

75313

38823

1666

2655

20013

62417
86.526

$\begin{array}{rl}86.5 & 26 \\ 74.0 & 27\end{array}$

$573 \quad 18$

28125

94111
2593

41021

12189

80912

13837

$\begin{array}{lll}743 & 14 \\ 657 & 15\end{array}$

35024
120

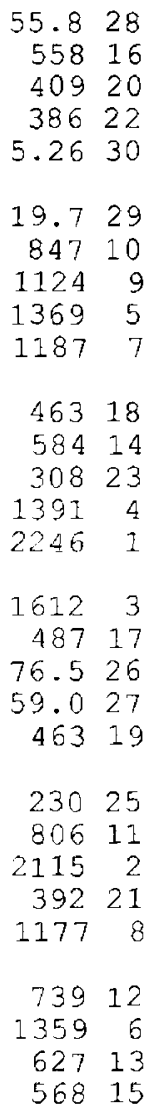

35924
183

$46.5 \quad 27$

$\begin{array}{lll}445 & 14\end{array}$

30921

4.4430

17.229

$\begin{array}{lll}56512 & 12\end{array}$

9457

9058

35619

$400 \quad 16$

$1230 \quad 3$

20601

1210
$355 \quad 20$

60.726

44.228

36118

16325

63911

$\begin{array}{ll}1590 & 2 \\ 297 & 22\end{array}$

8749

66710

$988 \quad 6$

46213
$413 \quad 15$

25123 
THIS PAGE INTENTIONALLY BLANK 


\section{AUCILLA RIVER BASIN}

02326512 AUCILLA RIVER NEAR SCANLON, FL

LOCATION.--Lat $30^{\circ} 13^{\prime} 52^{\prime \prime}$, long $83^{\circ} 55^{\prime} 08^{\prime \prime}$, in $\mathrm{SW}^{1} / 4$ sec.10, T.3 S., R.4 E., Taylor County, Hydrologic Unit 03110103 , on left bank, 3 mi west of Cabbage Grove, 6.9 mi north of Scanlon, 12 mi southwest of Lamont and 14 mi upstream from mouth.

DRAINAGE AREA.--805 $\mathrm{mi}^{2}$.

PERIOD OF RECORD.--March 1950 to November 1957, crest-stage partial-record station; May 1965, May 1967 (one discharge measurement made each water year); August 1976 to 1993.

REVISED RECORDS.--WRD FL-88-4: 1986, 1987.

GAGE.--Water-stage recorder. Datum of gage is $3.14 \mathrm{ft}$ above National Geodetic Vertical Datum of 1929, unadjusted. February 1950 to November 1957 , creststage gage at same site at present datum. August 30, 1976 to April 24, 1982, water-stage recorder at same site and datum. April 25, 1982 to October 24 , 1984, nonrecording gage.

REMARKS.--Records good.

EXTREMES OUTSIDE PERIOD OF RECORD.--Flood of April 9, 1973, reached a stage of about $23.5 \mathrm{ft}$, from floodmarks, (discharge not determined).

MAXIMUM, MINIMUM, AND MEAN OF MONTHLY MEAN ELEVATIONS FOR WATER YEARS 1976-1993

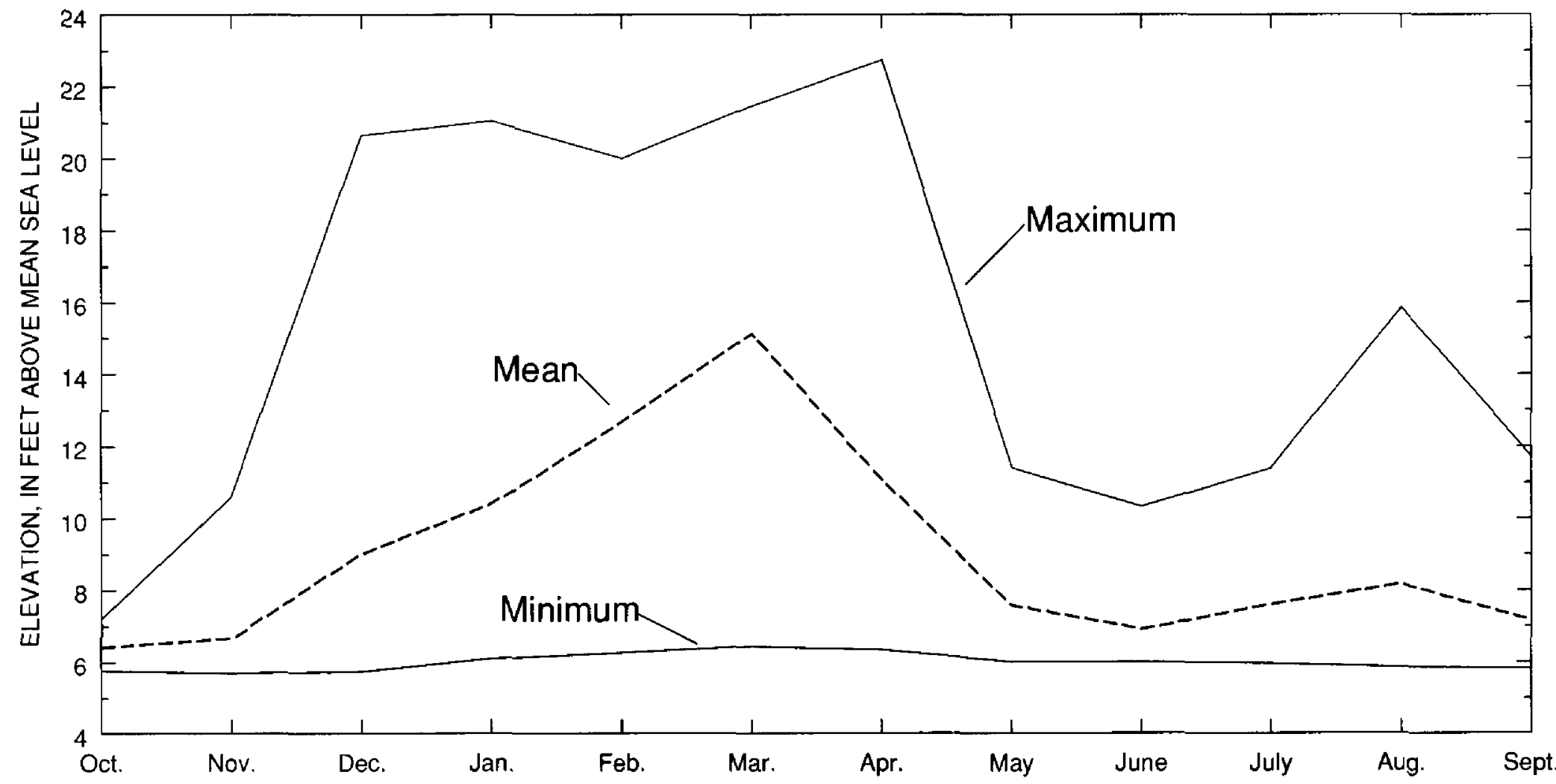

DURATION OF DAILY MEAN ELEVATION FOR WATER YEARS 1976-1993

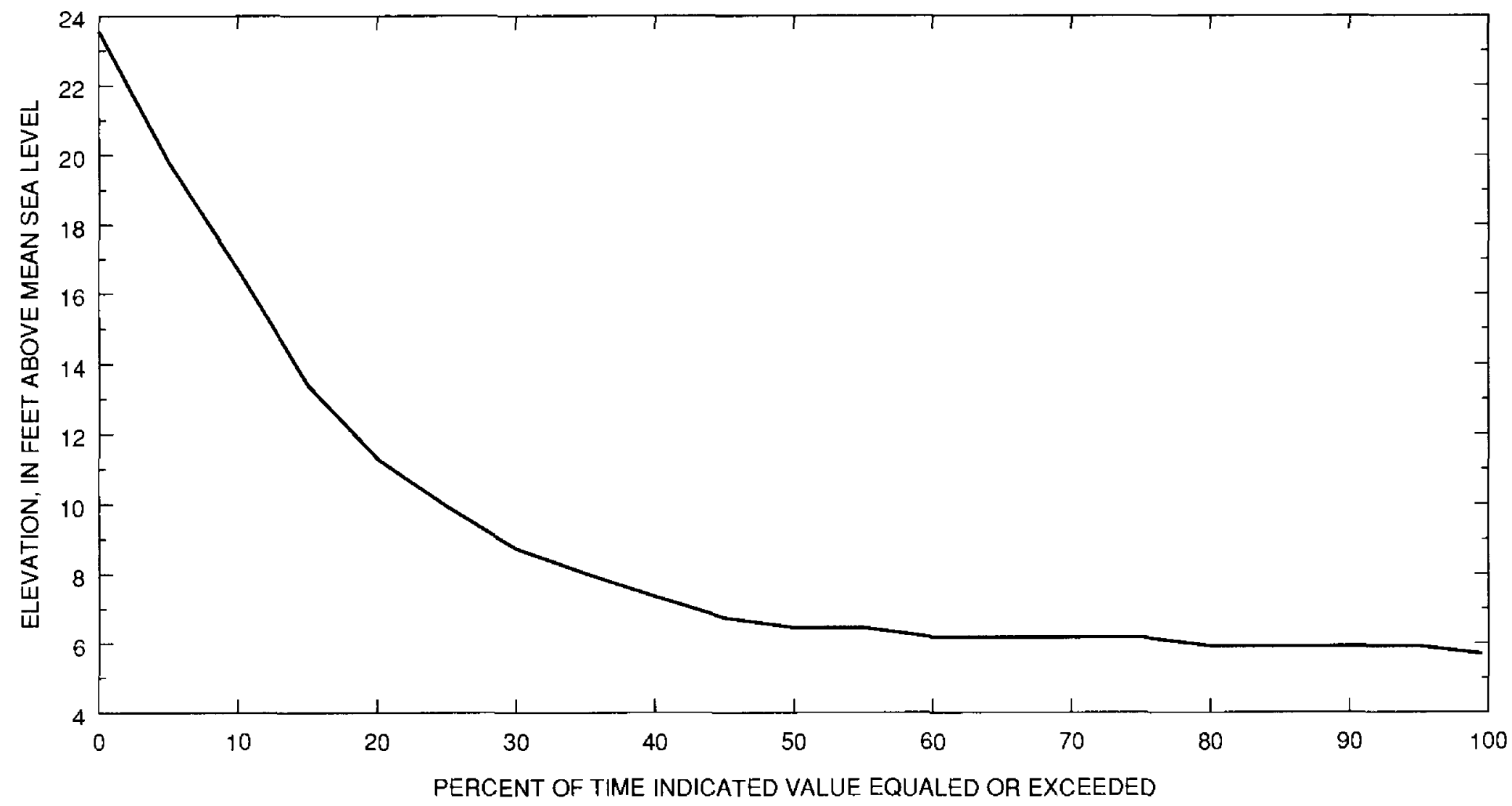


AUCILLA RIVER BASIN

02326512 AUCILLA RIVER NEAR SCANLON, FL--Continued

SUMMARY STATISTICS IN CUBIC FEET PER SECOND, UNLESS OTHERWISE INDICATED

FOR WATER YEARS 1976 - 1993

\begin{tabular}{|c|c|c|c|c|}
\hline ANNUAL MEAN & 549 & & & \\
\hline HIGHEST ANNUAL MEAN & 1200 & & & 1984 \\
\hline IOWEST ANNUAL MEAN & 160 & & & 1985 \\
\hline HIGHEST DAIIY MEAN & 7220 & Apr & 9 & 1984 \\
\hline LOWEST DAILY MEAN & 17 & Nov & 22 & 1990 \\
\hline ANNUAI SEVEN-DAY MINIMUM & 17 & Nov & 26 & 1990 \\
\hline INSTANTANEOUS PEAK FLOW & 7460 & Apr & 8 & 1984 \\
\hline INSTANTANEOUS PEAK ELEVATION (FT) & 23.61 & Apr & 8 & 1984 \\
\hline INSTANTANEOUS LOW FIOW & 16 & Nov & 28 & 1990 \\
\hline ANNUAL RUNOFF (INCHES/CESM) & & & & \\
\hline
\end{tabular}

MAXIMUM, MINIMUM, AND MEAN OF MONTHLY MEAN DISCHARGES FOR WATER YEARS 1976-1993

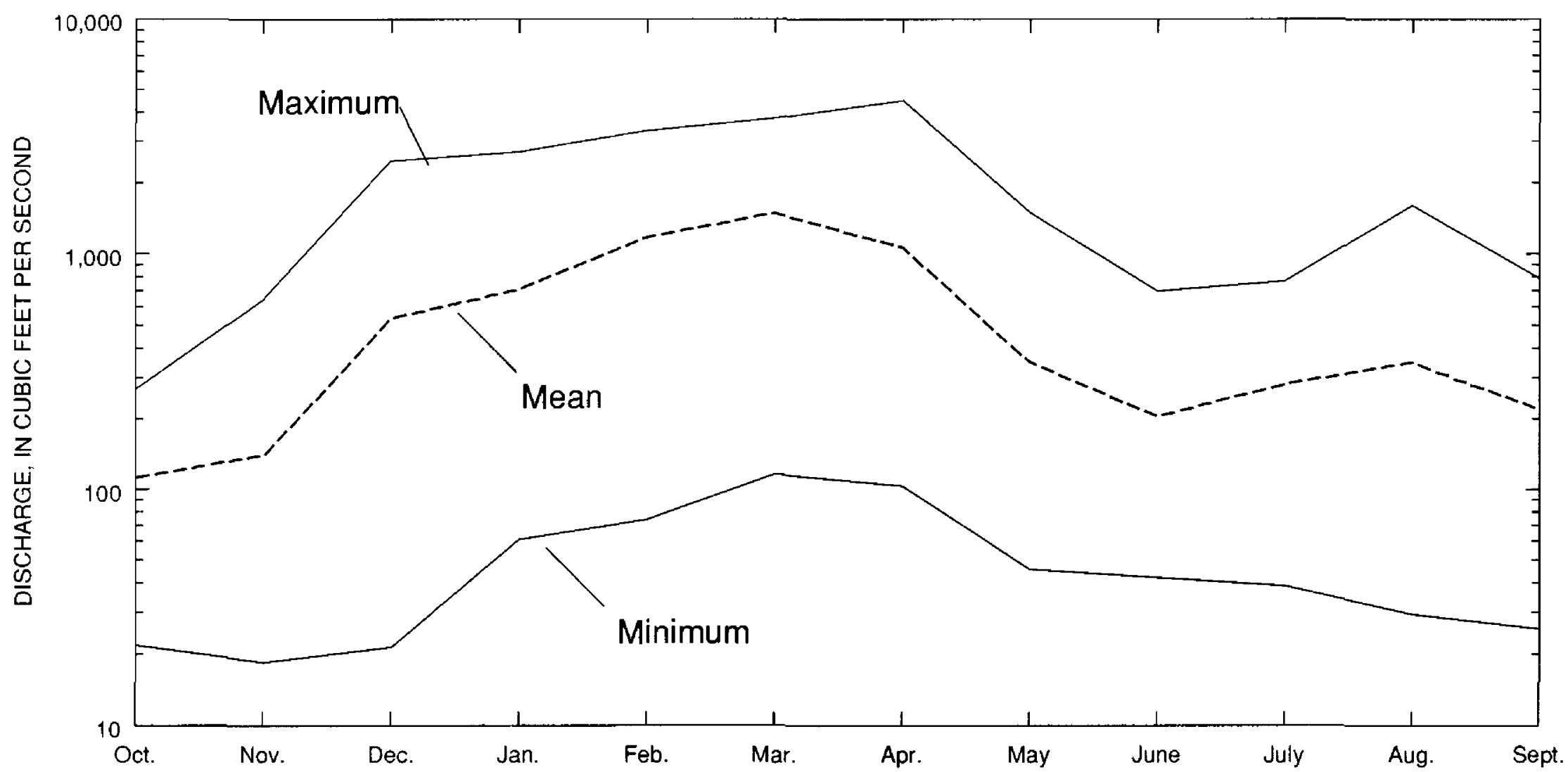

DURATION OF DAILY MEAN DISCHARGE FOR WATER YEARS 1976-1993

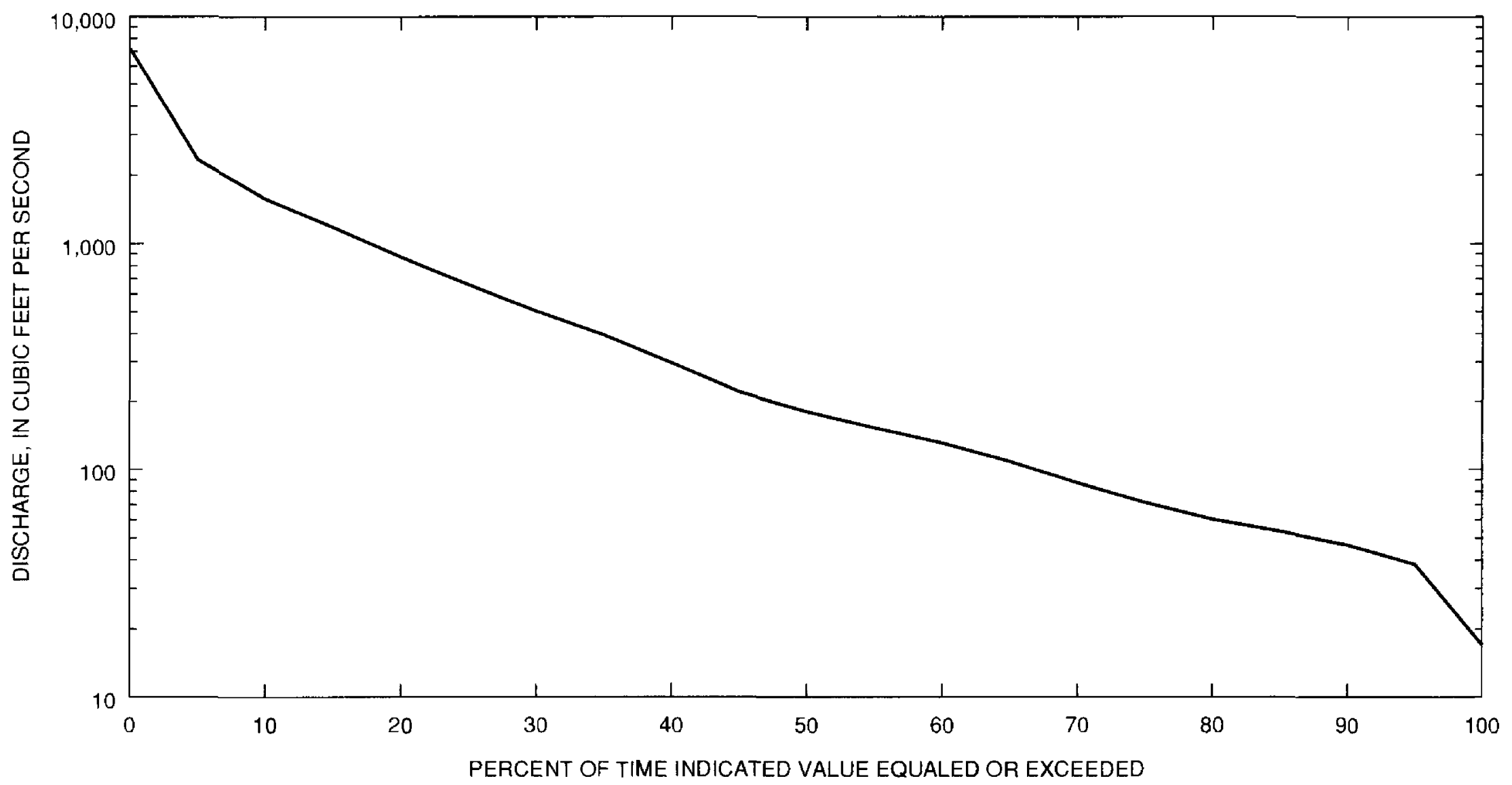


AUCILLA RIVER BASIN

02326512 AUCILLA RIVER NEAR SCANLON, FL--Continued

SUMMARY OF MONTHLY MEAN ELEVATION AND DISCHARGE STATISTICS FOR WATER YEARS 1976-1993

\begin{tabular}{lrrrrrr} 
& \multicolumn{3}{c}{ ELEVATION } & \multicolumn{3}{c}{ DISCHARGE } \\
& \multicolumn{2}{c}{ FEET ABOVE SEA LEVEL } & \multicolumn{2}{c}{ CUBIC EEET } & \\
\multicolumn{1}{c}{ MONTH } & MAXIMUM & MINIMUM & MEAN & MAXIMUM & MINIMUM & MEAN \\
& & & & & & \\
OCTOBER & 7.17 & 5.76 & 6.41 & 265 & 21.8 & 112 \\
NOVEMBER & 10.57 & 5.70 & 6.67 & 636 & 18.5 & 139 \\
DECEMBER & 20.62 & 5.75 & 9.02 & 2455 & 21.4 & 536 \\
JANUARY & 21.02 & 6.10 & 10.41 & 2680 & 60.8 & 707 \\
FEBRUARY & 19.97 & 6.26 & 12.66 & 3296 & 74.1 & 1175 \\
MARCH & 21.43 & 6.43 & 15.12 & 3737 & 116 & 1486 \\
APRIL & 22.70 & 6.34 & 11.10 & 4436 & 103 & 1063 \\
MAY & 11.39 & 6.01 & 7.57 & 1483 & 45.4 & 349 \\
JUNE & 10.29 & 6.01 & 6.92 & 689 & 42.0 & 205 \\
JULY & 11.36 & 5.96 & 7.61 & 760 & 38.8 & 281 \\
AUGUST & 15.81 & 5.86 & 8.17 & 1586 & 29.4 & 347 \\
SEPTEMBER & 11.69 & 5.82 & 7.19 & 780 & 25.6 & 219
\end{tabular}

$\begin{array}{rrr}95.0 & 5.9 & 5.7 \\ 90.0 & 5.9 & 5.9 \\ 85.0 & 5.9 & 6.0 \\ 80.0 & 5.9 & 6.0 \\ 75.0 & 6.1 & 6.0 \\ 70.0 & 6.1 & 6.1 \\ 65.0 & 6.1 & 6.1 \\ 60.0 & 6.1 & 6.1 \\ 55.0 & 6.4 & 6.1 \\ 50.0 & 6.4 & 6.1 \\ 45.0 & 6.7 & 6.2 \\ 40.0 & 7.3 & 6.3 \\ 35.0 & 8.0 & 6.3 \\ 30.0 & 8.7 & 6.4 \\ 25.0 & 9.9 & 6.5 \\ 20.0 & 11.3 & 6.6 \\ 15.0 & 13.4 & 6.8 \\ 10.0 & 16.7 & 7.1 \\ 5.0 & 19.8 & 7.9\end{array}$

5.7
5.8
5.8
5.8
6.0
6.0
6.0
6.0
6.0
6.0
6.0
6.0
6.2
6.2
6.5
6.7
6.9
8.2
10.2

$\begin{array}{rr}5.7 & 6.0 \\ 5.9 & 6.0 \\ 5.9 & 6.2 \\ 5.9 & 6.2 \\ 6.1 & 6.5 \\ 6.1 & 6.5 \\ 6.1 & 6.7 \\ 6.1 & 6.7 \\ 6.4 & 7.0 \\ 6.4 & 7.6 \\ 6.4 & 8.2 \\ 6.6 & 8.9 \\ 6.6 & 10.1 \\ 7.2 & 11.9 \\ 7.5 & 14.0 \\ 13.4 & 16.5 \\ 18.6 & 17.8 \\ 19.4 & 19.3 \\ 20.2 & 20.1\end{array}$

$$
\begin{array}{r}
6.1 \\
6.3 \\
6.3 \\
7.1 \\
8.7 \\
9.1 \\
9.5 \\
9.9 \\
10.3 \\
11.6 \\
12.1 \\
13.1 \\
14.2 \\
15.4 \\
16.7 \\
18.1 \\
18.9 \\
20.5 \\
22.2
\end{array}
$$

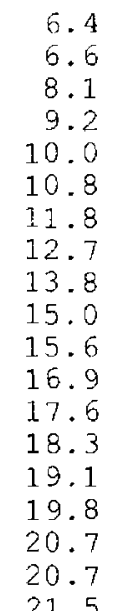

6.3
6.3
6.6
6.9
7.1
7.4
7.8
8.1
8.4
8.8
9.1
9.5
10.4
11.7
12.7
13.3
15.6
19.2
21.7

5.9
5.9
5.9
6.1
6.1
6.1
6.1
6.1
6.3
6.3
6.3
6.6
6.8
7.3
7.9
8.5
9.5
11.0
12.8

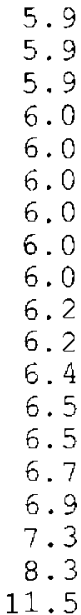

DISCHARGE IN CUBIC FEET PER SECOND

$\begin{array}{rrr}95.0 & 38.3 & 24.2 \\ 90.0 & 46.7 & 39.3 \\ 85.0 & 53.4 & 45.0 \\ 80.0 & 60.1 & 47.2 \\ 75.0 & 71.5 & 49.5 \\ 70.0 & 87.3 & 53.0 \\ 65.0 & 108.3 & 57.3 \\ 60.0 & 130.2 & 62.9 \\ 55.0 & 152.9 & 69.8 \\ 50.0 & 180.3 & 79.2 \\ 45.0 & 219.9 & 91.6 \\ 40.0 & 295.6 & 105.9 \\ 35.0 & 393.8 & 120.1 \\ 30.0 & 501.3 & 131.9 \\ 25.0 & 657.6 & 144.9 \\ 20.0 & 870.2 & 163.8 \\ 15.0 & 1181.5 & 185.4 \\ 10.0 & 1574.2 & 221.2 \\ 5.0 & 2341.3 & 334.9\end{array}$

$\begin{array}{rrrrrr}21.2 & 27.4 & 55.2 & 87.0 & 146.8 & 112.3 \\ 41.5 & 46.4 & 62.4 & 115.3 & 199.3 & 141.0 \\ 45.2 & 50.8 & 79.9 & 140.0 & 430.2 & 194.6 \\ 47.4 & 55.2 & 115.1 & 414.5 & 545.9 & 247.0 \\ 49.6 & 59.5 & 142.1 & 523.0 & 707.5 & 297.2 \\ 51.8 & 64.6 & 157.1 & 587.2 & 800.1 & 348.3 \\ 53.8 & 70.1 & 183.3 & 639.4 & 902.0 & 393.7 \\ 55.9 & 91.6 & 207.2 & 707.4 & 1038.9 & 430.1 \\ 57.9 & 113.5 & 246.4 & 794.4 & 1204.9 & 474.2 \\ 62.7 & 129.5 & 320.5 & 903.1 & 1329.7 & 522.9 \\ 71.6 & 148.0 & 385.6 & 1022.5 & 1436.1 & 573.3 \\ 80.8 & 166.0 & 544.1 & 1169.2 & 1533.7 & 655.3 \\ 89.0 & 182.8 & 664.2 & 1279.5 & 1658.5 & 782.5 \\ 99.0 & 257.3 & 804.0 & 1393.5 & 1799.5 & 971.3 \\ 131.6 & 312.4 & 1091.9 & 1510.7 & 2083.3 & 1194.0 \\ 162.6 & 1016.6 & 1451.4 & 1673.5 & 2355.9 & 1545.0 \\ 202.7 & 1659.2 & 1656.8 & 1884.2 & 2598.5 & 2325.0 \\ 336.1 & 2116.1 & 1982.7 & 2319.0 & 2918.6 & 3042.7 \\ 582.6 & 2533.7 & 2408.2 & 3580.0 & 3441.2 & 3871.9\end{array}$

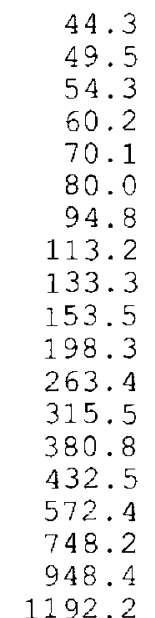

39.0
41.9
47.3
49.9
53.3
60.7
68.8
77.8
88.0
109.1
133.8
151.0
165.4
183.3
211.7
272.5
392.7
535.8
850.3

38.1
43.2
48.1
51.1
54.3
59.2
66.5
78.8
100.5
119.3
143.4
166.7
204.1
290.0
367.1
427.7
576.3
724.8
1058.0
FOR PERIOD APR TO MAR

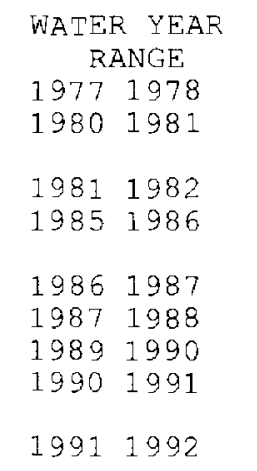

$\begin{array}{cccc}1 & & 3 & \\ 5.89 & 2 & 5.92 & 3 \\ 6.13 & 9 & 6.14 & 9 \\ 5.99 & 5 & 6.00 & 5 \\ 5.91 & 3 & 5.91 & 2 \\ 6.06 & 6 & 6.07 & 6 \\ 6.07 & 7 & 6.07 & 7 \\ 5.96 & 4 & 5.96 & 4 \\ 5.67 & 1 & 5.67 & 1 \\ 6.07 & 8 & 6.07 & 8\end{array}$

\begin{tabular}{llll}
14 & & \multicolumn{2}{c}{30} \\
5.98 & 3 & 6.03 & 3 \\
6.20 & 9 & 6.36 & 9 \\
& & & \\
6.03 & 5 & 6.05 & 5 \\
5.94 & 2 & 5.98 & 2 \\
& & & \\
6.12 & 8 & 6.15 & 8 \\
6.08 & 5 & 6.10 & 6 \\
5.99 & 4 & 6.04 & 4 \\
5.68 & 1 & 5.69 & 1 \\
& & &
\end{tabular}
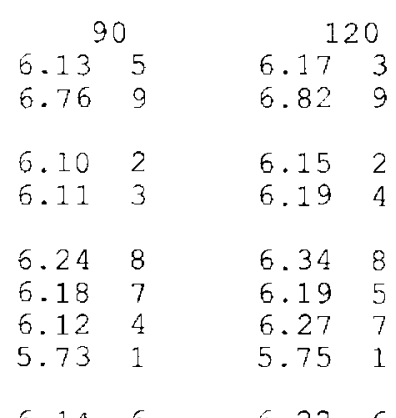

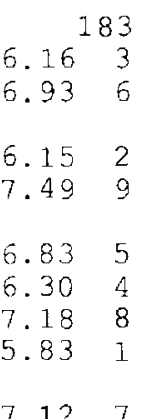


HIGHEST MEAN ELEVATION, IN FEET, AND RANKING FOR THE FOLLOWING NUMBER OF CONSECUTIVE DAYS FOR PERIOD OCT TO SEP

WATER YEAR

RANGE

RANGE
19771977
19781978

19781978

19811981

19821982

19861986

19871987

19891989

19901990

19911991

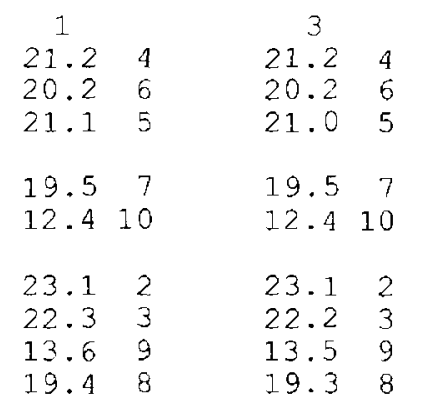

\begin{tabular}{|c|c|c|c|}
\hline 7 & & 15 & \\
\hline 21.2 & 4 & 21.1 & 4 \\
\hline 20.0 & 6 & 19.3 & 6 \\
\hline 20.8 & 5 & 20.3 & 5 \\
\hline 19.1 & 7 & 18.0 & 8 \\
\hline 2.11 & 0 & 11.3 & 10 \\
\hline 23.0 & 2 & 22.6 & 2 \\
\hline 22.2 & 3 & 21. & 3 \\
\hline 12. & 9 & 12.6 & 9 \\
\hline 19.0 & 8 & 18.0 & 7 \\
\hline
\end{tabular}

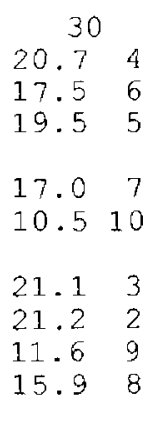

$20.0^{60} 3$

15.4
17.9

15.37

9.8910

$\begin{array}{ll}18.4 & 4 \\ 20.5 & 2\end{array}$

11.29

23.31

23.31

22.91

$21.8 \quad 1$

20.81

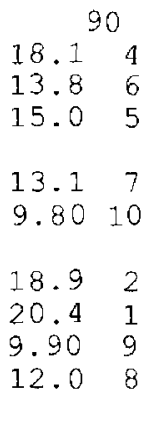

18.93

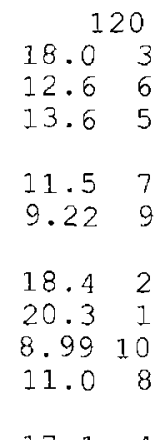

$17.1 \quad 4$
14.183

$\begin{array}{ll}14.8 & 3 \\ 10.7 & 6\end{array}$

12.05

9.957

15.42

17.21

$8.18 \quad 10$

9.408

14.54

LOWEST MEAN DISCHARGE, IN CUBIC FEET PER SECOND, AND RANKING FOR THE FOLLOWING NUMBER OF CONSECUTIVE DAYS FOR PERIOD APR TO MAR

WATER YEAR$$
\text { RANGE }
$$

19771978

19781979

19791980

19801981

19811982

19821983

19831984

19841985

19851986

19861987

19871988

19881989

19891990

19901991

19911992

19921993

\begin{tabular}{|c|c|c|c|}
\hline 1 & & 3 & \\
\hline 36.0 & 5 & 36.0 & 5 \\
\hline 42.0 & 6 & 42.0 & 6 \\
\hline 51.0 & 14 & 51.3 & 14 \\
\hline 55.0 & 15 & 56.3 & 15 \\
\hline 31.0 & 2 & 31.7 & 2 \\
\hline 50.0 & 12 & 50.0 & 12 \\
\hline 68.0 & 16 & 72.0 & 16 \\
\hline 46.0 & 8 & 46.3 & 8 \\
\hline 34.0 & 3 & 34.0 & 3 \\
\hline 50.0 & 13 & 51.0 & 13 \\
\hline 47.0 & 9 & 47.0 & 9 \\
\hline 45.0 & 7 & 45.7 & 7 \\
\hline 34.0 & 4 & 34.7 & 4 \\
\hline 17.0 & 1 & 17.0 & 1 \\
\hline 49.0 & 10 & 49.3 & 10 \\
\hline .0 & 11 & 49 & 11 \\
\hline
\end{tabular}

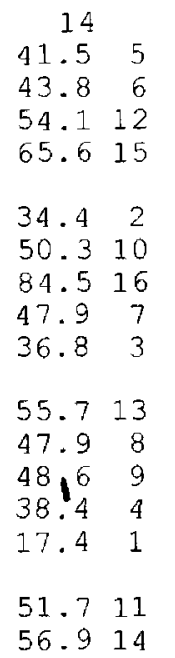

\begin{tabular}{rr}
\multicolumn{3}{c}{60} \\
56.7 & 9 \\
48.8 & 5 \\
86.0 & 13 \\
123 & 15 \\
& \\
40.0 & 2 \\
73.4 & 11 \\
144 & 16 \\
51.0 & 6 \\
47.3 & 3 \\
76.2 & 12 \\
58.2 & 10 \\
53.0 & 7 \\
47.9 & 4 \\
18.7 & 1 \\
& \\
55.1 & 8 \\
89.8 & 14
\end{tabular}

89.814

\begin{tabular}{rr}
\multicolumn{3}{c}{120} \\
65.7 & 6 \\
62.1 & 5 \\
144 & 14 \\
166 & 15 \\
& \\
49.6 & 2 \\
119 & 12 \\
220 & 16 \\
53.6 & 3 \\
77.5 & 8 \\
& \\
98.6 & 11 \\
61.7 & 4 \\
87.6 & 10 \\
81.1 & 9 \\
21.1 & 1 \\
& \\
72.3 & 7 \\
144 & 13
\end{tabular}

53.210

$\begin{array}{rr}30 & \\ 47.5 & 6 \\ 46.9 & 5 \\ 68.1 & 14 \\ 102 & 15 \\ 36.8 & 2 \\ 55.4 & 11 \\ 108 & 16 \\ 49.9 & 8 \\ 41.1 & 3 \\ 61.7 & 12 \\ 50.3 & 9 \\ 49.6 & 7 \\ 43.4 & 4 \\ 17.7 & 1 \\ 53.2 & 10 \\ 64.4 & 13\end{array}$

\begin{tabular}{rr}
\multicolumn{3}{c}{90} \\
57.6 & 6 \\
55.7 & 5 \\
133 & 14 \\
155 & 15 \\
& \\
41.8 & 2 \\
102 & 12 \\
169 & 16 \\
51.2 & 3 \\
61.7 & 9 \\
80.9 & 11 \\
60.3 & 8 \\
62.8 & 10 \\
54.5 & 4 \\
19.9 & 1 \\
58.6 & 7 \\
124 & 13
\end{tabular}

$144 \quad 13$
67. 183

96.06

$\begin{array}{rr}136 & 7 \\ 177 & 10\end{array}$

49.22

$221 \quad 14$

34216

$\begin{array}{rr}67.9 & 4 \\ 246 & 15\end{array}$

1669

85.45

1398

$\begin{array}{rr}219 & 13 \\ 27.8 & 1\end{array}$

21512

17911

HIGHEST MEAN DISCHARGE, IN CUBIC FEET PER SECOND, AND RANKING FOR THE FOLLOWING NUMBER OF CONSECUTIVE DAYS FOR PERIOD OCT TO SEP

WATER YEAR

$1977 \quad 1977$

19781978

19791979

19801980

19811981

19821982

19831983

19841984

19851985

19861986

19871987

19881988

19891989

19901990

19911991

19921992

19931993

\begin{tabular}{|c|c|c|c|}
\hline 1 & & 3 & \\
\hline 2760 & 6 & 2747 & 6 \\
\hline 2260 & 9 & 2227 & 9 \\
\hline 1570 & 12 & 1550 & 12 \\
\hline 2570 & 7 & 2553 & 7 \\
\hline 2000 & 10 & 1967 & 10 \\
\hline 854 & 17 & 853 & 17 \\
\hline 4750 & 4 & 4657 & 4 \\
\hline 7220 & 1 & 7097 & 1 \\
\hline 1230 & 15 & 1213 & 15 \\
\hline 5470 & 3 & 5403 & 3 \\
\hline 3610 & 5 & 3600 & 5 \\
\hline 2460 & 8 & 2437 & 8 \\
\hline 1080 & 16 & 1053 & 16 \\
\hline 1970 & 11 & 1953 & 11 \\
\hline 6200 & 2 & 6130 & 2 \\
\hline 1380 & 14 & 1350 & 14 \\
\hline 1550 & 13 & 1533 & 13 \\
\hline
\end{tabular}

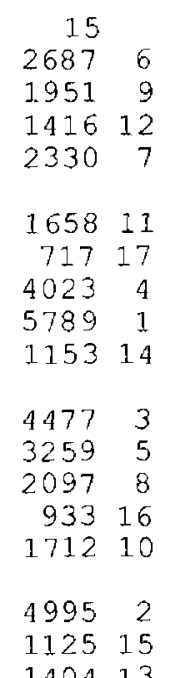

140413
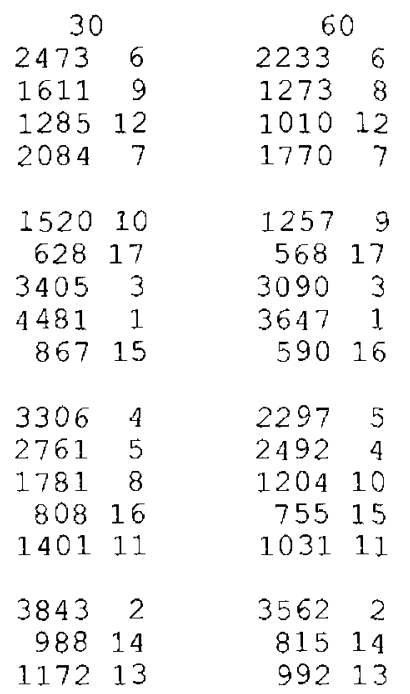

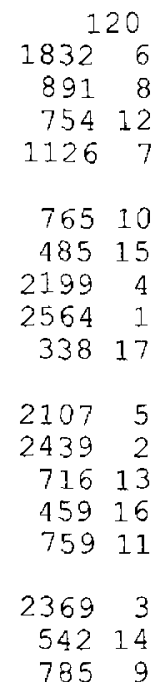

94510

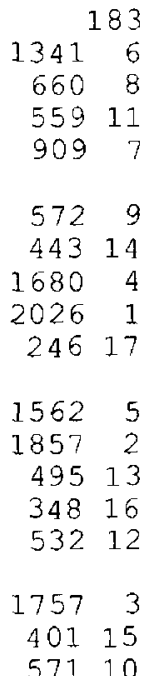

$\begin{array}{ll}401 & 15 \\ 571 & 10\end{array}$ 
THIS PAGE INTENTIONALLY BLANK 
STATISTICAL SUMMARIES OF HYDROLOGIC DATA

FOR LAKES 
LOCATION.--LAT $30^{\circ} 12^{\prime} 55^{\prime \prime}$, long $82^{\circ} 26^{\prime} 31^{\prime \prime}$, in SWl/4 sec.20, T.3 S., R. 19 E., Baker County, Hydrologic Unit 03070204, on south shore on private dock, $1.2 \mathrm{mi}$ northwest of Olustee and $11.3 \mathrm{mi}$ east of Lake City.

SURFACE AREA.--1,793 acres.

DRAINAGE AREA.--13.1 $\mathrm{mi}^{2}$.

PERIOD OF RECORD.--December 1974 to September 1993 (intermittent).

GAGE.- Nonrecording gage. Datum of gage is $150.40 \mathrm{ft}$ National Geodetic Vertical Datum of 1929. Gage readings have been reduced to elevations NGVD.

REMARKS.--Some natural diversion flow to the Suwannee River basin at times.

COOPERATION.--Gage readings were furnished by Suwannee River Water Management District.

EXTREMES FOR PERIOD OF RECORD.--Maximum observed elevation, I56.20 ft Feb. 25, 1988; minimum observed, 153.34 ft Jan. $3,1991$.

\begin{tabular}{|c|c|c|c|c|c|c|c|c|c|c|c|}
\hline & & & & Normal & $\begin{array}{l}\text { AN ELE } \\
\text { nthly me }\end{array}$ & $\begin{array}{l}\text { TON } \\
\text { s (All da) }\end{array}$ & & & & & \\
\hline oct & Nov & Dec & Jan & Feb & March & April & May & June & July & Aug & Sept \\
\hline 154.77 & 154.55 & 154.40 & 154.46 & 154.55 & 154.53 & 154.40 & 154.35 & 154.63 & 154.96 & 154.87 & 155.01 \\
\hline 154.81 & 154.66 & 154.88 & 155.32 & 155.45 & 155.23 & 154.76 & 154.30 & 154.12 & 153.93 & 153.85 & 154.17 \\
\hline 154.16 & 154.07 & 155.07 & 155.16 & 155.15 & 155.34 & 154.76 & 155.11 & 154.85 & 154.68 & 155.06 & 154.40 \\
\hline 154.00 & 153.78 & 153.80 & 154.14 & 154.59 & 154.40 & 154.62 & 154.54 & 154.40 & 154.20 & 154.24 & 155.68 \\
\hline 155.02 & 154.80 & 154.78 & 154.84 & 154.94 & 155.75 & 155.30 & 155.22 & 154.90 & 155.22 & 154.88 & 154.59 \\
\hline 154.58 & 154.48 & 154.42 & 154.20 & 154.99 & 154.91 & 154.72 & 154.20 & $\star$ & 154.36 & 154.46 & 154.96 \\
\hline 154.36 & $\star$ & 154.56 & 154.89 & 154.88 & $\star$ & 155.19 & 154.78 & 155.30 & 155.06 & 155.35 & 155.15 \\
\hline 155.28 & * & * & $\star$ & 155.18 & * & $\star$ & $\star$ & 154.28 & 155.34 & * & 155.90 \\
\hline 155.28 & * & 155.21 & 155.74 & 155.90 & 155.65 & 155.30 & 154.76 & 154.66 & 154.52 & 154.42 & 154.73 \\
\hline 154.46 & 154.40 & 155.10 & 155.82 & 156.00 & 156.01 & 155.28 & 154.95 & 154.78 & 154.82 & 155.76 & 155.50 \\
\hline 155.04 & 155.10 & 154.93 & 155.30 & 156.20 & 155.72 & 155.26 & 154.94 & 154.58 & 154.40 & 154.86 & 155.58 \\
\hline 155.16 & 155.20 & 155.06 & 154.98 & 154.82 & 154.90 & 154.60 & 154.68 & 154.68 & 154.70 & 154.64 & 155.04 \\
\hline 154.90 & 154.78 & 154.75 & 154.96 & 154.96 & 154.90 & 154.90 & 154.54 & 154.24 & 154.26 & 153.98 & 153.64 \\
\hline 153.70 & 153.55 & 153.36 & 153.34 & 154.00 & $\star$ & 155.00 & 155.70 & 155.45 & 155.43 & 155.82 & 155.68 \\
\hline 155.42 & * & 154.67 & 154.60 & $\star$ & 154.99 & 154.78 & * & 154.38 & 154.46 & 155.04 & 155.00 \\
\hline 155.22 & 155.10 & * & 155.48 & 155.74 & 155.66 & 155.20 & 154.60 & 154.40 & 154.66 & 154.20 & 154.18 \\
\hline
\end{tabular}

* Indicates a no-value month

Statistics on Normal monthly means (All days)

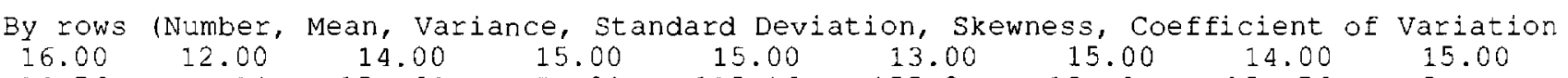

Numbe

12.00

15.00

15.00
154.88

0.44

0.29

0.29

0.54

0.44
0.66

155.16

55.16
0.37

0.61

13.00

$-36.26$

5.85

13.10

0.26

0.12

154.76

29.08

$-31.52$

0.00

5.85
0.00

13.10
0.00

$0.51 \quad 0.34$

$4.24-161.13$

0.17

25.52

154.64

0.16

0.40

$-10.46$

0.46
16.00
154.69
0.20
0.45

$-17.17$

0.00

$\begin{array}{rr}\text { Aug } & \text { Sept } \\ & \\ 15.00 & 16.00 \\ 154.76 & 154.95 \\ 0.36 & 0.43 \\ 0.60 & 0.66 \\ 2.97 & -11.62 \\ 0.00 & 0.00\end{array}$




\section{PALESTINE LAKE NEAR OLUSTEE, FL}

LOCATION.--Lat $30^{\circ} 07^{\prime} 46^{\prime \prime}$, long $82^{\circ} 24^{\prime} 34^{\prime \prime}$, in NW $1 / 4$ sec.22, T.4 S., R.19 E., Union County, Hydrologic Unit 03070204, on south side of private dock on northeastern shore, $6.2 \mathrm{mi}$ south of Olustee.

SURFACE AREA.--910 acres.

DRAINAGE AREA.--13.1 $\mathrm{mi}^{2}$

PERIOD OF RECORD.--August 1975 to September 1993 (intermittent).

GAGE.--Nonrecording gage. Datum of gage is $133.94 \mathrm{ft}$ National Geodetic Vertical Datum of 1929 (levels by Suwannee River Water Management District). Gage readings have been reduced to elevation NGVD.

REMARKS.--Formerly known as South Prong Pond near Lake Butler. Surface outlet through South Prong swamp to South Prong St. Marys River. Outflow into Olustee Creek will occur at elevations above $145 \mathrm{ft}$.

COOPERATION.--Gage readings are furnished by Suwannee River Water Management District.

EXTREMES FOR PERIOD OF RECORD.--Maximum observed elevation, 145.24 ft Oct.. 5, 1992; minimum observed, 141.14 ft Sept. $25,1989$.

MEAN ELEVATION

Normal monthly means (All days)

\begin{tabular}{|c|c|c|c|c|c|c|c|c|c|c|c|c|}
\hline Year & oct & Nov & Dec & Jan & Feb & March & April & May & June & Ju1y & Aug & Sept \\
\hline 1975 & * & * & * & * & * & * & * & * & * & * & 144.26 & 144.03 \\
\hline 1978 & * & * & $\star$ & $\star$ & $\star$ & 144.22 & 143.92 & 144.18 & 143.78 & 143.74 & 144.18 & 143.66 \\
\hline 1979 & * & 142.96 & * & 143.20 & 143.62 & 143.89 & 143.98 & 143.64 & 143.54 & 143.48 & * & 143.66 \\
\hline 1980 & 143.82 & $\star$ & 143.63 & 143.76 & 143.84 & 144.80 & 144.34 & 144.20 & 144.14 & 144.02 & 144.18 & 144.03 \\
\hline 1981 & 143.64 & 143.70 & 143.58 & 143.52 & 143.44 & 143.82 & 143.94 & 143.31 & 143.06 & 143.19 & $\star$ & 144.00 \\
\hline 1982 & 143.50 & 143.25 & 143.38 & 143.78 & 143.80 & 143.70 & 144.42 & 143.94 & 143.70 & 143.79 & 144.26 & 144.02 \\
\hline 1985 & 143.92 & 144.24 & 144.24 & 144.08 & 744.09 & 144.02 & 143.90 & 143.70 & 143.35 & 143.94 & 143.84 & 144.62 \\
\hline 1989 & $\star$ & $\star$ & $\star$ & $\star$ & $\star$ & $\star$ & t & $\star$ & $\pi$ & 142.92 & 142.92 & 142.12 \\
\hline 1990 & 143.12 & 142.90 & 142.76 & 142.93 & 142.96 & 142.94 & 142.96 & 142.61 & 142.64 & 142.62 & 142.40 & 142.20 \\
\hline 1991 & 141.99 & 141.70 & 141.59 & 141.76 & 142.49 & 143.25 & 143.92 & 144.15 & 144.01 & 144.10 & 144.26 & 143.99 \\
\hline 1992 & 143.88 & 143.68 & 243.36 & 143.26 & 143.44 & 143.70 & 143.84 & 143.61 & 143.62 & 143.61 & 143.79 & 144.23 \\
\hline 1993 & 144.58 & 143.95 & 143.79 & 143.84 & 144.34 & 144.23 & 144.04 & 143.46 & 143.11 & 143.36 & 143.32 & 142.98 \\
\hline
\end{tabular}

* Indicates a no-value month

Statistics on Normal monthly means (All days)

\begin{tabular}{|c|c|c|c|c|c|c|c|c|c|c|c|c|}
\hline & oct & Nov & Dec & $\operatorname{Jan}$ & Feb & March & April & May & June & July & Aug & Sept \\
\hline By & rows & (Number, Mean, & Variance, & Standard & Deviation, & - Skewness, & Coeff & it of & Variation & & & \\
\hline umber & 8.00 & 8.00 & 8.00 & 9.00 & 9.00 & 10.00 & 10.00 & 10.00 & 10.00 & 11.00 & 10.00 & 12.00 \\
\hline ean & 143.56 & 143.30 & 143.29 & 143.35 & 143.56 & 143.86 & 143.93 & 143.68 & 143.49 & 143.52 & 143.74 & 143.63 \\
\hline ar & 0.57 & 0.65 & 0.65 & 0.49 & 0.33 & 0.28 & 0.17 & 0.24 & 0.21 & 0.23 & 0.44 & 0.62 \\
\hline d & 0.75 & 0.80 & 0.80 & 0.70 & 0.57 & 0.53 & 0.41 & 0.49 & 0.46 & 0.48 & 0.66 & 0.79 \\
\hline$k \in w$ & 5.73 & -9.10 & 2.64 & 3.77 & -6.38 & -12.81 & -44.97 & 0.24 & 13.00 & -6.33 & -4.42 & 1.98 \\
\hline var & 0.01 & 0.01 & 0.01 & 0.00 & 0.00 & 0.00 & 0.00 & 0.00 & 0.00 & 0.00 & 0.00 & 0.01 \\
\hline
\end{tabular}




\section{SUWANNEE RIVER BASIN \\ 02313510 CHUNKY POND NEAR BRONSON, FL}

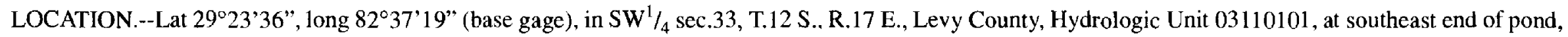
near center of outlet channel, $200 \mathrm{ft}$ upstream from culvert control and $3.7 \mathrm{mi}$ south of Bronson.

SURFACE AREA.--650 acres.

DRAINAGE AREA.--23 $\mathrm{mi}^{2}$, approximately.

PERIOD OF RECORD.--January 1967 to September 1993 (intermittent)..

GAGE.--Nonrecording gage. Datum of gage is National Geodetic Vertical Datum of 1929 (Florida Department of Transportation bench mark). Supplementary nonrecording gages about $600 \mathrm{ft}$ northeast; $0.5 \mathrm{mi}$ north; and $400 \mathrm{ft}$ west of north gage.

REMARKS.--Lake level outlet is culvert with lift gate in outlet canal. Outflow from lake is through a canal southward to a swampy area called Deerpen Pond and thence through Magee Branch to Waccasassa River.

COOPERATION.--Gage readings were furnished by Suwannee River Water Management District.

EXTREMES FOR PERIOD OF RECORD.--Maximum elevation observed, $56.00 \mathrm{ft}$ Sept. 7, 1967; lake dry at gage during November and December 1977, elevation not determined. Minimum observed since December 1977, $45.80 \mathrm{ft}$ Nov. 18, 1978.

MEAN ELEVATION

Normal monthly means (All days)

\begin{tabular}{|c|c|c|c|c|c|c|c|c|c|c|c|c|}
\hline Year & oct & Nov & Dec & Jan & Eeb & March & April & May & June & July & Aug & Sept \\
\hline 1967 & $\star$ & * & * & 55.39 & 54.85 & $\star$ & 54.70 & 54.22 & 53.82 & 53.99 & 55.15 & 55.87 \\
\hline 1968 & 55.57 & 55.36 & 55.24 & 55.13 & 54.81 & 54.54 & 53.86 & 53.12 & 52.91 & 53.06 & 53.57 & 53.71 \\
\hline 1969 & 53.49 & 53.46 & 53.21 & 53.03 & 52.72 & 52.99 & 52.93 & 52.59 & 52.34 & 52.37 & 52.58 & 52.51 \\
\hline 1970 & 52.64 & 52.59 & 52.93 & 53.62 & 54.66 & 54.66 & 55.30 & 54.90 & 54.95 & 55.01 & 55.57 & 55.51 \\
\hline 1971 & 55.17 & 54.92 & 54.71 & 54.81 & 54.91 & 54.92 & 54.73 & 54.17 & 53.54 & 53.23 & 54.11 & 54.78 \\
\hline 1972 & 54.87 & 54.88 & 54.85 & 54.78 & 54.93 & 54.65 & 54.75 & 54.53 & 54.31 & 53.99 & 54.00 & 54,20 \\
\hline 1973 & 53.54 & 53.25 & 53.57 & 53.36 & 53.62 & 53.75 & 54.31 & 53.79 & 53.56 & 53.34 & 53.46 & 53.36 \\
\hline 1974 & 53.03 & 52.24 & 51.85 & 51.87 & $\star$ & ^ & 50.89 & 50.53 & 50.68 & 51.29 & 52.05 & 52.84 \\
\hline 1975 & 52.62 & 52.28 & 52.20 & 52.24 & 52.06 & 51.62 & 51.36 & 50.58 & 49.95 & 50.35 & 50.26 & 50.59 \\
\hline 1976 & 51.31 & 51.42 & 51.18 & 51.40 & 51.26 & 50.69 & 49.98 & 49.79 & 49.42 & 49.50 & 49.37 & 49.24 \\
\hline 1977 & 48.85 & 48.50 & 48.51 & 48.90 & 49.11 & 49.03 & 48.44 & 47.20 & 47.42 & 46.79 & 46.34 & 46.25 \\
\hline 1978 & 46.32 & 45.85 & 47.26 & 47.28 & 48.39 & 49.88 & 51.42 & $\star$ & 50.53 & * & 54.12 & 54.66 \\
\hline 1979 & 54.37 & 54.00 & 53.50 & 54.00 & 54.20 & 54.10 & 53.78 & 53.98 & 53.72 & 52.95 & 53.23 & 53.75 \\
\hline 1980 & 53.93 & 53.72 & 54.00 & 54.03 & 54.31 & 54.28 & 54.38 & 54.56 & 54.02 & 54.45 & 54.95 & 54.56 \\
\hline 1981 & 54.02 & 53.66 & 53.09 & 52.48 & 52.41 & 51.82 & * & 50.68 & 49.98 & 49.61 & 49.56 & 49.53 \\
\hline 1982 & 48.96 & 48.73 & 48.03 & 48.02 & 47.59 & 47.30 & 49.81 & 49.95 & 50.29 & 50.97 & 52.53 & 54.20 \\
\hline 1983 & 54.26 & 54.05 & 53.89 & 53.83 & 54.44 & 54.52 & 54.36 & 54.35 & 54.32 & 54.39 & 54.43 & 54.67 \\
\hline 1984 & 54.61 & 54.60 & 54.58 & 54.80 & 54.59 & 54.61 & 54.66 & 54.20 & 54.20 & 54.78 & 54.91 & 54.56 \\
\hline 1985 & 54.32 & 54.12 & 53.90 & 53.69 & 53.58 & 52.84 & * & 52.28 & 51.57 & 52.49 & 52.47 & 53.71 \\
\hline 1986 & 53.76 & 53.83 & 53.82 & 53.98 & 53.93 & 53.98 & 53.84 & 53.21 & 52.36 & 52.10 & 52.86 & 54.36 \\
\hline 1987 & 53.90 & 53.75 & 54.20 & 54.04 & 54.38 & 54.56 & 55.02 & 54.98 & 54.36 & 54.04 & 54.50 & 54.36 \\
\hline 1988 & 53.90 & * & 53.36 & * & 53.76 & 54.00 & 53.74 & 53.34 & 53.64 & 53.20 & 53.34 & * \\
\hline 1089 & 54.25 & 54.40 & 54.40 & 54.09 & 53.85 & 52.27 & 52.10 & 52.00 & * & $\star$ & * & 52.30 \\
\hline
\end{tabular}

* Indicates a no-value montr

Statistics on Normal monthly means (All days)

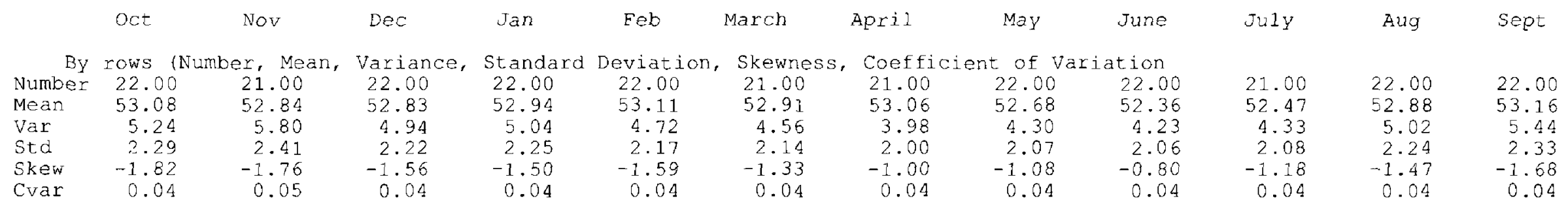




\section{SUWANNEE RIVER BASIN \\ 02319150 CHERRY LAKE NEAR CHERRY LAKE, FL}

LOCATION.--Lat 30 37'06”, long 83'25'20”, in NE $1 / 4$ sec.33, T.3 N., R.9 E., Madison County, Hydrologic Unit 03110203, on west shore on private dock, 2.7 mi northeast of the village of Cherry Lake and $10.2 \mathrm{mi}$ north of Madison.

SURFACE AREA.--483 acres.

DRAINGAGE AREA.--1.58 $\mathrm{mi}^{2}$.

PERIOD OF RECORD.--November 1974 to September 1993 (intermittent).

GAGE.--Nonrecording gage. Datum of gage is $148.13 \mathrm{ft}$ National Geodetic Vertical Datum of 1929. Gage readings have been reduced to elevations NGVD.

REMARKS.--Lake is landlocked.

COOPERATION.--Gage readings were fumished by Suwannee River Water Management District.

EXTREMES FOR PERIOD OF RECORD.--Maximum elevation observed, $153.97 \mathrm{ft}$ Apr. 15, 1975; minimum observed, $150.81 \mathrm{ft}$ Nov. $30,1990$.

EXTREMES OUTSIDE PERIOD OF RECORD.--Maximum elevation since April 1973, 154.41 ft from high-water mark by owner.

MEAN ELEVATION

Normal monthly means (All days)

$\begin{array}{ccccc}\text { Year } & \text { oct } & \text { Nov } & \text { Dec } & \text { Jan } \\ 1975 & \star & 152.54 & 152.60 & 153.15 \\ 1976 & 152.86 & 152.75 & 152.70 & 152.94 \\ 1977 & 153.12 & 153.32 & 153.45 & 153.27 \\ 1978 & 152.01 & 151.85 & 152.03 & 152.37 \\ 1979 & 152.09 & 151.93 & 152.23 & 152.43 \\ 1980 & 152.73 & 152.87 & \star & 153.04 \\ 1981 & 152.45 & 152.51 & 152.53 & 152.49 \\ 1982 & 151.91 & 151.79 & 151.89 & 152.33 \\ 1983 & 152.39 & 152.39 & 152.55 & 152.89 \\ 1984 & 152.59 & 152.81 & \star & 153.43 \\ 1985 & 152.27 & 152.23 & 152.25 & 152.39 \\ 1986 & 152.61 & 152.99 & 153.27 & 153.13 \\ 1987 & 151.81 & \star & 152.23 & 153.01 \\ 1988 & 151.59 & 151.87 & 151.75 & \star \\ 1990 & 151.98 & 151.85 & 152.31 & 152.57 \\ 1991 & 150.97 & 150.81 & 150.93 & 152.57 \\ 1992 & 152.19 & 151.95 & 152.03 & 152.71 \\ 1993 & 152.13 & 152.39 & 152.29 & 152.67\end{array}$

$\begin{array}{ccc}\text { Feb } & \text { March } & \text { April } \\ 153.49 & 153.45 & 153.70 \\ 153.07 & 153.15 & 153.15 \\ 153.06 & 153.06 & 152.72 \\ 152.69 & 152.94 & 152.65 \\ 152.99 & 152.99 & 152.87 \\ 153.03 & 153.13 & \star \\ 152.99 & 152.95 & 152.73 \\ 152.59 & 152.69 & 152.79 \\ 153.23 & 153.23 & 153.31 \\ 153.61 & 153.93 & 153.31 \\ 152.43 & 152.53 & 152.41 \\ 153.43 & 152.91 & 152.41 \\ 153.11 & 153.23 & 152.63 \\ 152.32 & 152.71 & 152.53 \\ 152.83 & 152.73 & 152.47 \\ 152.57 & 152.57 & 152.99 \\ 152.75 & 152.55 & 152.23 \\ \star & 152.89 & 152.59\end{array}$

May
153.53
153.39
152.46
152.64
152.77
152.97
152.37
152.53
153.09
152.89
152.21
152.17
152.41
152.23
152.05
152.59
152.13
152.27

$\begin{array}{cc}\text { June } & \text { JulY } \\ 153.30 & 153.22 \\ 153.31 & 152.98 \\ 152.14 & 151.90 \\ 152.56 & 152.80 \\ 152.53 & 152.89 \\ \star & 152.74 \\ \star & 152.14 \\ 152.83 & 153.29 \\ 153.19 & 152.83 \\ 152.82 & 153.21 \\ 152.07 & 152.35 \\ \star & 151.87 \\ 152.25 & 152.28 \\ \star & 151.98 \\ 151.85 & \star \\ \star & 152.90 \\ \star & \star \\ 152.33 & 152.21\end{array}$

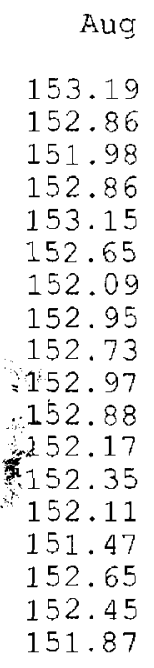

Sept

152.99 153.07

152.21

152.45

153.09

152.41

151.81

152.69

152.65

152.49

153.07

152.03

152.07

152.73

150.89

152.25

152.17

151.79

* Indicates a no-value month

\begin{tabular}{|c|c|c|c|c|c|c|c|c|c|c|c|c|}
\hline \multicolumn{13}{|c|}{ Statistics on Normal monthly means (All days) } \\
\hline & oct & Nov & Dec & $\operatorname{Jan}$ & $F e b$ & March & April & May & June & July & Aug & Sept \\
\hline By & rows & (Number, Mean, & Variance, & Standard & Deviat & n, skewr & is, coeff & cient of & Variation & & & \\
\hline Mean & 152.22 & 152.29 & 152.31 & 152.79 & 152.95 & 152.98 & $\begin{array}{r}17.00 \\
152.79\end{array}$ & 152.59 & $\begin{array}{r}12.00 \\
152.60\end{array}$ & $\begin{array}{r}16.00 \\
152.60\end{array}$ & $\begin{array}{r}18.00 \\
152.52\end{array}$ & 152.38 \\
\hline Var & 0.27 & 0.38 & 0.36 & 0.13 & 0.14 & 0.13 & 0.16 & 0.21 & 0.26 & 0.25 & 0.25 & 0.33 \\
\hline Std & 0.52 & 0.62 & 0.60 & 0.36 & 0.38 & 0.35 & 0.41 & 0.46 & 0.51 & 0.50 & 0.50 & 0.58 \\
\hline Skew & 3.32 & -17.28 & -8.58 & -22.92 & 31.88 & 42.65 & -9.56 & -33.64 & -11.15 & -0.65 & -30.20 & -16.41 \\
\hline Cvar & 0.00 & 0.00 & 0.00 & 0.00 & 0.00 & 0.00 & 0.00 & 0.00 & 0.00 & 0.00 & 0.00 & 0.00 \\
\hline
\end{tabular}


LOCATION.--Lat $30^{\circ} 36^{\prime} 34^{\prime \prime}$, long $83^{\circ} 12^{\prime} 10^{\prime \prime}$, in $\mathrm{NE}^{1} / 4^{\mathrm{sec}}$.3, T.2 N., R.11 E., Hamilton County, Hydrologic Unit 03110203, on northwest shore on private dock, $5.6 \mathrm{mi}$ west of Jennings, $8.8 \mathrm{mi}$ east of Pinetta.

SURFACE AREA.-- 198 acres.

DRAINAGE AREA.--3.0 $\mathrm{mi}^{2}$, includes $0.1 \mathrm{mi}^{2}$ estimated in Georgia.

PERIOD OF RECORD.--November 1974 to September 1993 (intermittent).

GAGE.--Nonrecording gage. Datum of gage is $99.36 \mathrm{ft}$ National Geodetic Vertical Datum of 1929 (levels by Suwannee River Water Management District). Gage readings have been reduced to elevations NGVD.

REMARKS.--Lake level control is lift gates at west end of lake by Lake Co-op (owners).

COOPERATION.--Gage readings were furnished by Suwannee River Water Management District.

EXTREMES FOR PERIOD OF RECORD.--Maximum observed elevation, $111.56 \mathrm{ft}$ Mar. 3, 1991, minimum observed $97.23 \mathrm{ft}$ Mar. $11,1985$.

MEAN ELEVATION

Normal monthly means (All days)

\begin{tabular}{|c|c|c|c|c|c|c|c|c|c|c|c|c|}
\hline Year & oct & Nov & Dec & Jan & Feb & March & April & May & June & July & Aug & Sept \\
\hline 1975 & * & 102.10 & 101.24 & 102.84 & 105.25 & 105.14 & 105.59 & 105.01 & 104.92 & * & * & 103.26 \\
\hline 1976 & * & 100.80 & $\star$ & 100.21 & 100.98 & 102.58 & $\star$ & 103.85 & $\star$ & * & 104.18 & * \\
\hline 1977 & * & 106.58 & 106.04 & 105.80 & 104.86 & $\star$ & * & 103.84 & 102.84 & * & 102.46 & * \\
\hline 1978 & 102.42 & * & * & $*$ & 103.30 & 105.24 & * & 104.18 & 103.42 & 104.38 & 106.08 & 102.67 \\
\hline 1979 & 102.04 & 100.84 & * & * & 101.96 & 103.99 & 103.58 & 103.68 & 103.54 & 103.44 & 102.78 & 102.67 \\
\hline 1980 & 100.58 & 100.63 & 100.56 & 101.62 & * & 101.72 & 103.90 & 103.38 & 102.72 & 102.72 & 102.75 & 102.98 \\
\hline 1981 & 102.62 & 102.30 & 101.86 & 101.12 & 100.79 & 102.94 & 105.02 & 103.44 & 101.74 & 102.61 & * & 102.96 \\
\hline 1982 & 102.18 & 101.94 & * & 100.36 & 102.56 & 102.91 & 104.08 & 104.18 & 102.72 & 102.65 & 104.76 & 103.37 \\
\hline 1983 & 102.72 & 99.86 & $\star$ & $\star$ & $\star$ & 103.30 & 105.82 & 105.72 & 105.14 & 105.98 & 104.68 & 103.27 \\
\hline 1984 & * & 101.70 & 102.96 & 105.72 & * & 105.88 & * & * & * & * & * & * \\
\hline 1985 & * & $\star$ & * & $\star$ & 97.75 & 97.95 & 99.82 & 100.25 & 100.26 & 100.99 & 102.33 & 102.68 \\
\hline 1986 & 102.36 & 102.49 & 103.53 & 104.75 & 106.27 & 105.67 & 104.30 & 102.74 & 102.60 & 102.34 & 102.40 & 102.21 \\
\hline 1987 & 105.63 & 105.82 & 107.02 & 109.67 & 110.01 & 110.04 & 109.80 & 108.28 & 106.78 & 106.63 & 106.39 & 106.38 \\
\hline 1988 & 105.29 & 105.38 & 105.65 & 105.64 & 106.87 & 108.84 & 107.82 & 106.92 & 104.91 & 102.11 & 100.36 & 106.35 \\
\hline 1989 & 108.08 & 107.15 & 106.35 & 105.08 & 103.04 & 101.35 & $\star$ & $\star$ & $\star$ & 99.37 & 99.37 & $\star$ \\
\hline 1990 & * & * & * & 101.10 & 104.31 & 107.26 & 107.54 & 105.68 & 103.08 & 99.40 & $\star$ & * \\
\hline 1991 & * & * & * & 107.27 & 109.81 & 110.95 & 109.51 & 108.99 & 108.33 & 108.77 & 109.69 & 108.61 \\
\hline 1992 & 107.99 & 107.37 & 106.50 & 106.62 & 108.41 & 108.78 & 108.40 & 107.59 & 106.97 & 107.29 & 107.41 & 107.78 \\
\hline 1993 & 107.94 & 107.34 & 106.98 & 108.78 & 109.00 & 108.81 & 108.14 & 106.81 & 106.23 & 105.99 & 105.86 & 105.64 \\
\hline
\end{tabular}

* Indicates a no-value month

Statistics on Normal monthly means (All days)

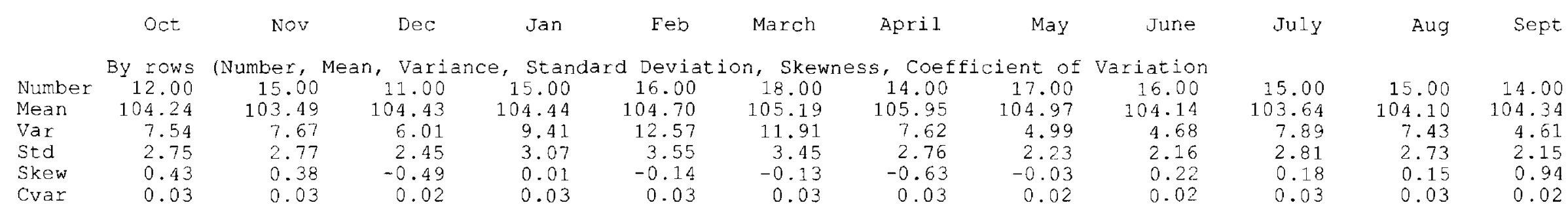


LOCATION.--Lat $30^{\circ} 02^{\prime} 27^{\prime \prime}$, long $83^{\circ} 07^{\prime} 11^{\prime \prime}$, in NE $1 / 4$ sec.21, T.5 S., R.12 E., Lafayette County, Hydrologic Unit 03110205 , on county pier, $235 \mathrm{ft}$ north of peninsula on west shore, $1.4 \mathrm{mi}$ southeast of Alton, 3.5 mi southeast of Mayo.

SURFACE AREA.--111 acres.

DRAINAGE AREA.--6.40 $\mathrm{mi}^{2}$.

PERIOD OF RECORD.--November 1974 to September 1993 (intermittent).

GAGE.--Nonrecording gage. Datum of gage $67.44 \mathrm{ft}$ National Geodetic Vertical Datum of 1929 (levels by Suwannee River Water Management District). Gage readings have been reduced to elevations NGVD.

REMARKS.--Lake is landlocked, Locally known as Koon Lake.

COOPERATION.--Gage readings were furnished by Suwannee River Water Management District.

EXTREMES FOR PERIOD OF RECORD.--Maximum elevation observed, 73.17 ft Aug. 8, 1980; minimum observed, 67.24 ft Oct. $16,1990$.

MEAN ELEVATION

Normal monthly means (All days)

\begin{tabular}{|c|c|c|c|c|c|c|c|c|c|c|c|c|}
\hline Year & oct & Nov & Dec & $\operatorname{Jan}$ & Feb & March & April & May & June & JuIy & Aug & Sept \\
\hline 1975 & $\star$ & 71.05 & 70.96 & * & 72.06 & 72.09 & 72.00 & 71.64 & 71.36 & * & 72.39 & 72.63 \\
\hline 1976 & 72.35 & 72.12 & 71.78 & 71.94 & 72.08 & 71.82 & $\star$ & $\star$ & $\star$ & 71.06 & $\star$ & 71.02 \\
\hline 1977 & $\star$ & 70.64 & $\star$ & 71.66 & $\star$ & $72.0 \leq$ & $\star$ & 71.04 & 70.49 & * & 70.79 & $\star$ \\
\hline 1978 & * & $\star$ & * & $\star$ & 72.19 & $\star$ & 71.76 & 72.20 & 71.94 & 72.43 & $\star$ & 71.91 \\
\hline 1979 & $\star$ & 70.86 & 70.61 & 70.66 & 71.04 & * & * & 72.04 & 71.35 & 70.89 & 70.98 & 72.69 \\
\hline 1980 & * & * & 72.12 & $\star$ & $\star$ & 72.74 & 72.46 & 72.14 & 71.98 & 72.00 & 73.17 & 72.80 \\
\hline 1981 & 71.74 & 71.86 & 71.86 & 71.54 & 71.32 & 72.00 & 71.66 & 70.99 & 70.42 & 70.13 & $\star$ & 70.23 \\
\hline 1982 & 69.57 & 69.23 & $\star$ & 69.72 & 69.78 & 69.79 & 71.09 & 71.18 & 70.64 & 71.38 & 72.32 & 72.00 \\
\hline 1985 & * & 70.78 & 70.36 & 70.14 & 70.00 & 69.69 & $\star$ & 69.99 & 68.15 & 67.91 & $\star$ & * \\
\hline 1986 & 69.62 & 70.62 & * & * & * & * & * & * & * & * & 70.52 & 70.32 \\
\hline 1987 & 69.70 & * & 70.84 & 71.89 & 72.44 & $\star$ & 71.94 & 71.42 & 71.02 & $\star$ & 71.28 & 71.50 \\
\hline 1988 & 70.42 & 70.99 & 69.96 & 69.92 & 70.82 & 71.18 & 70.86 & 70.39 & * & 69.19 & 69.39 & 70.54 \\
\hline 1989 & 71.22 & $\star$ & 70.06 & 69.62 & 69.16 & 68.76 & 68.38 & 67.94 & 67.80 & 68.02 & 68.53 & 68.88 \\
\hline 1990 & 69.24 & 68.88 & 68.94 & 69.00 & 69.04 & 68.92 & 68.56 & 67.99 & 67.73 & 68.22 & 67.53 & $\star$ \\
\hline 1991 & 67.24 & $\star$ & $\star$ & $\star$ & $\star$ & 69.74 & 70.13 & 70.66 & $\star$ & 70.71 & 71.72 & 71.62 \\
\hline 1992 & 70.80 & 70.46 & 70.22 & 69.88 & 70.04 & 70.02 & 69.40 & 68.82 & 68.40 & 67.68 & 69.08 & 69.28 \\
\hline 1993 & 69.92 & 69.80 & 69.50 & 70.08 & 70.32 & 71.70 & 70.52 & 70.10 & 69.32 & 69.00 & 68.30 & 67.90 \\
\hline
\end{tabular}

* Indicates a no-vaiue month

Statistics on Normal monthly means (All days)

\begin{tabular}{|c|c|c|c|c|c|c|c|c|c|c|c|c|}
\hline & oct & Nov & Dec & $\operatorname{Jan}$ & Feb & March & April & May & June & JuIy & Aug & Sept \\
\hline & By rows & (Number, & Mean, Var & e, sta & d Devi & ion, ske & ess, Coe & sient & Variation & & & \\
\hline Number & 11.00 & 12.00 & 12.00 & 12.00 & 13.00 & 13.00 & 12.00 & 15.00 & 13.00 & 13.00 & 13.00 & 14.00 \\
\hline Mean & 70.17 & 70.61 & 70.60 & 70.50 & 70.79 & 70.81 & 70.73 & 70.57 & 70.05 & 69.89 & 70.46 & 70.88 \\
\hline Var & 1.92 & 0.90 & 0.94 & 1.01 & 1.37 & 1.84 & 1.87 & 1.94 & 2.47 & 2.72 & 3.10 & 2.08 \\
\hline std & 1.39 & 0.95 & 0.97 & 1.01 & 1.17 & 1.36 & 1.37 & 1.39 & 1.57 & 1.65 & 1.76 & 1.44 \\
\hline Skew & -0.47 & -0.64 & -0.22 & 0.37 & -0.02 & -0.14 & -0.64 & -0.93 & -0.41 & 0.10 & -0.22 & -0.75 \\
\hline Cvar & 0.02 & 0.01 & 0.01 & 0.01 & 0.02 & 0.02 & 0.02 & 0.02 & 0.02 & 0.02 & 0.02 & \\
\hline
\end{tabular}


LOCATION.--Lat. 29 $59^{\prime} 18^{\prime \prime}$, long $83^{\circ} 02^{\prime} 53^{\prime \prime}$, in SW'/4 sec.5, T.6 S., R.13 E., Lafayette County, Hydrologic Unit 03110205, on north shore of southwest lobe of Iake 1.7 mi southwest of U.S. 27, and 7.5 mi west of Branford.

SURFACE AREA.--88 acres.

DRAINAGE AREA.--6.5 $\mathrm{mi}^{2}$ approximately.

PERIOD OF RECORD.--September 1977 to September 1993 (intermittent).

GAGE.--Nonrecording gage. Datum of gage is $48.12 \mathrm{ft}$ National Geodetic Vertical Datum of 1929. Gage readings have been reduced to elevations NGVD.

REMARKS.--Drainage divides are poorly defined and lake interconnects with Adams Lake and adjacent areas during high stage.

COOPERATION.--Gage readings were furnished by Suwannee River Water Management District.

EXTREMES FOR PERIOD OF RECORD.--Maximum elevation observed, $58.12 \mathrm{ft}$ July 27, 1980; minimum observed, $48.20 \mathrm{ft}$ May $15,1989$.

MEAN ELEVATION

Normal monthly means (All days)

\begin{tabular}{|c|c|c|c|c|c|c|c|c|c|c|c|c|}
\hline Year & Oct & Nov & Dec & $\operatorname{Jan}$ & Feb & March & April & May & June & July & Aug & Sept \\
\hline 1977 & * & * & * & * & * & * & * & $\star$ & * & * & $\star$ & 53.67 \\
\hline 1979 & $\star$ & $\star$ & $\star$ & * & * & * & 56.10 & 56.12 & 54.90 & 54.55 & 55.33 & 56.69 \\
\hline 1980 & 56.54 & 55.89 & 56.14 & 56.08 & 56.38 & 56.94 & 56.78 & 56.45 & 55.99 & 56.83 & 56.82 & 56.20 \\
\hline 1981 & 55.36 & 56.01 & 55.63 & 54.82 & 55.11 & 56.21 & 55.40 & 54.26 & 53.17 & 53.42 & 54.27 & 54.56 \\
\hline 1982 & 53.45 & 53.01 & 52.94 & 54.40 & 55.68 & 56.15 & 56.81 & 55.78 & 54.87 & 56.27 & 56.69 & 56.42 \\
\hline 1985 & * & 54.99 & 54.22 & 53.72 & 53.49 & $\star$ & 52.75 & 52.50 & 50.45 & 48.46 & 50.88 & 56.50 \\
\hline 1986 & 56.30 & 56.80 & 56.36 & 56.76 & 56.68 & 56.50 & 55.84 & 54.92 & 54.49 & 54.45 & 54.11 & 54.41 \\
\hline 1987 & 53.68 & 53.40 & 54.45 & 56.65 & 56.85 & 56.84 & 56.50 & 55.87 & 55.03 & 54.74 & 54.15 & 53.50 \\
\hline 1988 & 52.70 & $\star$ & 51.83 & * & 52.36 & 54.88 & 54.57 & 53.45 & 52.22 & 51.67 & 52.03 & 56.20 \\
\hline 1989 & 56.33 & 55.80 & 55.32 & 54.52 & 53.72 & 53.10 & 52.33 & 49.61 & 48.30 & 48.33 & 48.43 & 52.62 \\
\hline 1990 & 52.88 & 51.74 & * & 50.37 & 50.29 & 51.29 & 50.49 & 49.51 & 48.94 & * & * & $\star$ \\
\hline 1991 & * & * & * & * & 48.64 & 55.15 & 55.36 & 55.46 & 55.51 & 55.07 & 56.11 & 55.05 \\
\hline 1992 & 54.47 & 53.94 & 53.37 & 52.94 & 52.85 & 52.59 & 51.97 & 50.82 & 50.33 & 49.57 & 50.31 & 51.08 \\
\hline 1993 & 52.54 & 52.35 & 52.07 & 52.74 & 54.51 & 55.39 & 54.85 & 53.27 & 52.83 & 51.97 & 51.08 & 50.66 \\
\hline
\end{tabular}

* Indicates a no-value month

Statistics on Normal monthly means (All days)

\begin{tabular}{|c|c|c|c|c|c|c|c|c|c|c|c|c|}
\hline & oct & Nov & Dec & $\operatorname{Jan}$ & Feb & March & April & May & June & July & Aug & Sept \\
\hline By & rows (1 & (Number, Mean, & Variance, & Standard & Deviation, & Skewness, & Coeff & $t$ of & Variation & & & \\
\hline Number & 10.00 & 10.00 & 10.00 & 10.00 & 12.00 & 11.00 & 13.00 & 13.00 & 13.00 & 12.00 & 12.00 & 13.00 \\
\hline Mean & 54.42 & 54.39 & 54.23 & 54.30 & 53.88 & 55.00 & 54.60 & 53.69 & 52.85 & 52.94 & 53.35 & 54.43 \\
\hline Var & 2.55 & 3.03 & 2.69 & 3.90 & 6.54 & 3.56 & 4.23 & 5.97 & 6.77 & 8.58 & 7.57 & 4.18 \\
\hline Std & 1.60 & 1.74 & 1.64 & 1.98 & 2.56 & 1.89 & 2.06 & 2.44 & 2.60 & 2.93 & 2.75 & 2.05 \\
\hline Skew & 0.21 & -0.19 & -0.23 & -0.60 & -0.80 & -0.99 & -0.78 & -0.71 & -0.57 & -0.49 & -0.35 & -0.67 \\
\hline Cvar & 0.03 & 0.03 & 0.03 & 0.04 & 0.05 & 0.03 & 0.04 & 0.05 & 0.05 & 0.06 & 0.05 & 0.04 \\
\hline
\end{tabular}


LOCATION.--Lat $30^{\circ} 13^{\prime} 16^{\prime \prime}$, long $82^{\circ} 50^{\prime} 14^{\prime \prime}$, in $\mathrm{SW}^{\prime} / 4$ sec.17, T.3 S., R.15 E., Suwannee County, Hydrologic Unit 03110205 , on west shore on private dock, $1.0 \mathrm{mi}$ southwest of Wellborn.

SURFACE AREA.--69 acres.

DRAINAGE AREA.--2.89 $\mathrm{mi}^{2}$.

PERIOD OF RECORD.--November 1974 to September 1993 (intermittent).

GAGE.--Nonrecording gage. Datum of gage is $143.80 \mathrm{ft}$ National Geodetic Vertical Datum of 1929. Gage readings have been reduced to elevations NGVD.

REMARKS.--Lake is landlocked.

COOPERATION.--Gage readings were furnished by Suwannee River Water Management District.

EXTREMES FOR PERIOD OF RECORD.--Maximum elevation observed, $151.90 \mathrm{ft}$ Oct. 14, 1986; minimum observed, $147.06 \mathrm{ft}$ Nov 19 , 1987.

MEAN ELEVATION

Normal monthly means (All days)

\begin{tabular}{|c|c|c|c|c|c|c|c|c|c|c|c|c|}
\hline Year & oct & Nov & Dec & $\operatorname{Jan}$ & Eeb & March & April & May & June & July & Aug & Sept \\
\hline 1975 & $\star$ & 147.63 & $\star$ & 夫 & $\star$ & 147.95 & 147.92 & $\star$ & * & 148.61 & * & 148.38 \\
\hline 1976 & 148.22 & 148.10 & 148.01 & 148.10 & 148.12 & 148.14 & 147.98 & 148.31 & 147.92 & 147.95 & $\star$ & 148.16 \\
\hline 1977 & $\star$ & $\star$ & $\star$ & 148.26 & 148.68 & 148.26 & $\star$ & 147.54 & 147.29 & $*$ & 147.90 & $\star$ \\
\hline 1978 & 147.68 & * & * & $\star$ & 148.32 & 148.16 & 147.84 & 148.02 & 147.68 & 147.98 & 148.10 & 147.66 \\
\hline 1979 & 147.68 & $\star$ & 147.57 & 148.25 & 148.24 & $\star$ & 149.00 & 147.80 & 147.80 & 147.90 & 147.92 & $\star$ \\
\hline 1980 & 147.72 & 148.03 & 148.34 & 148.39 & 148.52 & 148.46 & 148.32 & 148.31 & 147.78 & 149.00 & 147.94 & 147.60 \\
\hline 1981 & 147.98 & 148.10 & 147.90 & 147.98 & 148.34 & 147.96 & 147.64 & 147.26 & 147.38 & 147.60 & 148.60 & 147.68 \\
\hline 1982 & 147.56 & 147.88 & 148.16 & 148.15 & 148.36 & 148.20 & 147.88 & 151.00 & 148.22 & 148.64 & 148.28 & 148.02 \\
\hline 1983 & 147.72 & 147.78 & 147.90 & 148.38 & 148.16 & 148.36 & 148.12 & 147.80 & 148.15 & 147.70 & $=47.60$ & 148.00 \\
\hline 1984 & 147.66 & 148.10 & 148.36 & 148.26 & 147.94 & 147.90 & 147.96 & 147.66 & 148.06 & 148.16 & 148.40 & $\star$ \\
\hline 1985 & * & * & $\star$ & $\star$ & * & 148.00 & 147.90 & 147.80 & 148.01 & 148.06 & 148.07 & 148.80 \\
\hline 1986 & 147.95 & $\star$ & 148.10 & 148.15 & 148.10 & 148.12 & * & 147.70 & 147.60 & 147.90 & 147.80 & 147.80 \\
\hline 1987 & 150.87 & 148.50 & 148.70 & * & 148.55 & 148.12 & * & * & * & 147.80 & 148.04 & $\star$ \\
\hline 1988 & 147.34 & 147.57 & $\star$ & 148.69 & 149.22 & * & 148.24 & 148.11 & * & 147.66 & 147.78 & 148.19 \\
\hline 1989 & 148.05 & 147.94 & 夫 & $\star$ & $\star$ & * & 148.46 & 148.52 & 148.56 & $\star \star$ & 147.90 & * \\
\hline 1990 & 147.76 & 147.68 & 147.99 & 148.82 & 149.00 & * & 148.38 & 148.37 & 148.26 & 149.10 & 149.02 & 148.95 \\
\hline 1991 & 149.13 & 149.08 & 148.86 & 149.14 & 148.96 & 149.45 & 149.31 & 148.56 & 149.24 & 148.55 & 148.72 & 148.50 \\
\hline 1992 & $\star$ & 1.48 .87 & 148.92 & 148.81 & 148.74 & * & 148.70 & 148.40 & 148.55 & 148.91 & * & 148.63 \\
\hline 1993 & * & 149.00 & * & 148.18 & 149.49 & 149.75 & 149.54 & 149.08 & 148.93 & * & 149.52 & 149.56 \\
\hline
\end{tabular}

* Indicates a no-value month

Statistics on Normal monthly means (All days)

\begin{tabular}{|c|c|c|c|c|c|c|c|c|c|c|c|c|}
\hline & oct & Nov & Dec & Jan & Feb & March & April & May & June & Ju1y & Aug & Sept \\
\hline By & rows ( & (Number, M & $n$, Var & e, Sta & 1 Devial & n, ske & $s$, coe & ient of & Variation & & & \\
\hline Number & 14.00 & 14.00 & 12.00 & 14.00 & 16.00 & 14.00 & 16.00 & 17.00 & 16.00 & 16.00 & 16.00 & 14.00 \\
\hline Mean & 148.09 & 148.16 & 148.23 & 148.40 & 148.55 & 148.34 & 148.32 & 1.48 .25 & 148.09 & 148.22 & 148.22 & 148.28 \\
\hline $\operatorname{Var}$ & 0.83 & 0.27 & 0.18 & 0.12 & 0.20 & 0.32 & 0.33 & 0.72 & 0. & 0.27 & 0.29 & 0.34 \\
\hline std & 0.91 & 0.52 & 0.43 & 0.34 & 0.45 & 0.56 & 0.57 & 0.85 & 0 . & 0.52 & 0.54 & 0.58 \\
\hline Skew & -2.33 & -33.82 & 7.35 & 39.66 & -20.17 & 0.53 & -15.53 & -1.65 & -3 & -5 & -18.91 & -22.33 \\
\hline Cvar & 0.01 & 0.00 & 0.00 & 0.00 & 0.00 & 0.00 & 0.00 & 0.01 & 0.00 & 0.00 & 0.00 & 0.00 \\
\hline
\end{tabular}


LOCATION.--Lat $29^{\circ} 45^{\prime} 38^{\prime \prime}$, long $82^{\circ} 04^{\prime} 30^{\prime \prime}$, in NW'/4 sec.35, T.8 S., R.22 E., Bradford County, Hydrologic Unit 03110206, on north shore of lake, on west side of private dock, 3.1 mi southwest of Keystone Heights.

SURFACE AREA.-5,299 acres, open water only, includes that of Little Santa Fe Lake.

DRAINAGE. AREA.--20.9 $\mathrm{mi}^{2}$, includes that of Little Santa Fe Lake.

PERIOD OF RECORD.--July 1957 to September 1993 (intermittent).

REVISED RECORDS.--WSP 2105: Drainage area, surface area.

GAGE.--Nonrecording gage. Datum of gage is $132.22 \mathrm{ft}$ National Geodetic Vertical Datum of 1929. Gage readings have been reduced to elevations NGVD.

REMARKS. - Lake is the headwaters of the Santa Fe River and has continuous outflow.

COOPERATION.--Gage readings were furnished by Suwannee River Water Management District.

EXTREMES FOR PERIOD OF RECORD.--Maximum elevation, $142.72 \mathrm{ft}$ Aug. 25, 1978 from highwater mark; minimum observed, $137.76 \mathrm{ft}$ Dec. 31 , 1990.

EXTREMES OUTSIDE PERIOD OF RECORD.--Maximum elevation since 1946, $143.9 \mathrm{ft}$, furnished by Department of Transportation, from information by local residents.

MEAN ELEVATION

Normal monthly means (All days)

\begin{tabular}{|c|c|c|c|c|c|c|c|c|c|c|c|c|}
\hline Year & oct & Nov & Dec & Jan & Feb & March & April & May & June & July & Aug & Sept \\
\hline 1957 & * & * & * & $\star$ & $\star$ & 夫 & * & $\star$ & « & 138.54 & 139.06 & 139.07 \\
\hline 1958 & 139.31 & 139.03 & 138.93 & 138.93 & 139.00 & 139.68 & 140.25 & 140.35 & 140.13 & 140.29 & 140.40 & 140.42 \\
\hline 1959 & 140.16 & 140.32 & 140.43 & 140.94 & 141.15 & 142.00 & 142.27 & 141.78 & 141.93 & 141.69 & 141.45 & 141.78 \\
\hline 1960 & 141.65 & 141.10 & 140.68 & 140.54 & 140.49 & 141.07 & 141.37 & 140.88 & 140.55 & 141.27 & 141.86 & 141.81 \\
\hline 1961 & 142.05 & 141.58 & 141.02 & 140.83 & 140.99 & 141.01 & 140.75 & 140.52 & 140.39 & 140.85 & 141.72 & 141.90 \\
\hline 1962 & 141.18 & 140.99 & 140.71 & 140.62 & 140.52 & * & 140.42 & * & 139.70 & * & 139.99 & 140.13 \\
\hline 1963 & * & 139.69 & * & 139.60 & * & 140.14 & 139.77 & 139.76 & 139.38 & 139.94 & $\star$ & 140.36 \\
\hline 1964 & 140.34 & * & 140.17 & 141.03 & * & 141.85 & * & * & 140.47 & * & 140.61 & 142.21 \\
\hline 1965 & 141.70 & 140.83 & $\star$ & 140.99 & * & 141.36 & 140.92 & $\star$ & 140.14 & * & 140.84 & 140.79 \\
\hline 1966 & 140.73 & 140.41 & 140.59 & 140.56 & 140.80 & 141.29 & 140.83 & 140.81 & 140.93 & 140.77 & 141.08 & 140.97 \\
\hline 1967 & 141.21 & 140.72 & 140.52 & 140.62 & 140.88 & 140.91 & 140.52 & 140.33 & 140.35 & 140.75 & 141.09 & 140.88 \\
\hline 1968 & 140.46 & 140.11 & 140.06 & 140.10 & 139.94 & 139.81 & 139.64 & 139.35 & 139.42 & 139.52 & 139.59 & 140.46 \\
\hline 1.969 & 140.38 & 140.31 & 140.15 & 140.07 & 140.14 & 140.40 & 140.54 & 140.25 & 139.99 & 139.86 & 140.08 & 140.31 \\
\hline 1970 & 140.82 & 140.86 & 140.91 & 141.35 & 142.17 & 141.86 & 142.08 & 141.23 & 140.96 & 140.93 & 140.88 & 140.89 \\
\hline 1971 & 140.58 & 140.29 & 140.06 & 140.21 & 140.34 & 140.39 & 140.31 & 140.18 & 139.88 & 140.04 & 140.34 & 140.63 \\
\hline 3972 & 140.44 & 140.41 & 140.57 & 140.72 & $\star$ & 140.72 & 141.02 & 141.01 & 141.72 & 141.65 & 141.18 & 141.62 \\
\hline 1973 & 141.01 & 140.90 & 141.00 & 141.10 & 141.22 & 141.20 & 141.65 & 141.22 & 141.17 & 141.17 & 141.18 & 141.20 \\
\hline 1974 & 140.92 & 140.51 & 140.56 & 140.63 & 140.51 & 140.34 & 140.26 & 140.11 & 140.32 & 140.77 & 141.02 & 141.36 \\
\hline 1975 & 140.90 & 140.40 & 140.44 & 140.62 & 140.82 & 140.85 & 340.66 & 140.42 & 140.27 & 140.35 & 140.50 & 140.53 \\
\hline 1976 & 140.73 & 140.48 & 140.34 & 140.38 & 140.52 & 140.19 & 139.75 & 139.91 & 140.09 & 140.34 & 140.13 & 140.06 \\
\hline 1977 & 139.76 & 139.50 & 139.66 & 139.84 & 140.13 & 140.65 & 140.15 & 139.71 & 139.66 & 139.42 & 139.25 & 139.54 \\
\hline 1978 & 139.12 & 138.82 & 138.95 & 139.29 & 139.92 & 140.89 & 140.32 & 140.22 & 140.42 & 140.70 & 142.72 & 141.70 \\
\hline 1979 & 141.02 & 140.41 & 140.39 & 140.63 & 141.04 & 141.04 & 140.98 & 141.01 & 140.73 & 140.35 & 140.30 & 140.47 \\
\hline 1980 & 140.44 & 140.35 & 140.61 & 140.92 & 141.19 & 141.16 & 141.13 & 140.85 & 140.57 & 140.41 & 140.67 & 140.41 \\
\hline 1981 & 139.80 & 139.95 & 139.77 & 139.55 & 139.57 & 140.15 & 140.05 & 139.50 & 139.08 & 139.12 & 139.58 & 139.80 \\
\hline 1982 & 139.63 & 139.60 & 139.51 & 139.86 & 140.29 & 140.61 & 140.99 & 140.87 & 140.88 & 140.94 & 140.72 & 140.58 \\
\hline 1983 & 140.58 & 140.55 & 140.42 & 140.38 & 140.69 & 141.32 & 141.63 & * & * & 141.24 & 141.06 & 140.99 \\
\hline 1984 & 140.96 & 140.92 & 241.04 & 141.49 & 141.34 & 141.62 & * & * & * & * & 141.12 & 140.97 \\
\hline 1985 & 140.69 & 140.54 & 140.44 & 140.10 & 140.86 & 139.57 & * & 139.71 & 139.54 & 139.58 & 140.15 & 141.18 \\
\hline 1986 & 141.02 & 140.81 & 140.73 & 140.62 & 140.75 & 141.38 & 341.08 & 140.74 & 140.32 & 140.27 & 140.49 & 140.46 \\
\hline 1987 & * & 139.87 & 140.15 & 140.42 & 141.01 & 141.55 & 141.80 & 141.27 & 140.94 & 140.61 & 140.51 & 140.57 \\
\hline 1988 & 139.89 & 139.91 & 139.78 & 139.88 & 140.72 & 140.94 & 141.64 & 140.72 & 140.32 & 140.04 & 139.88 & 141.06 \\
\hline 1989 & 140.92 & 140.72 & 140.63 & 140.52 & 140.29 & 140.25 & 140.04 & 139.80 & 139.60 & $\star$ & 139.48 & 139.41 \\
\hline 1990 & 139.35 & 139.05 & 138.97 & 139.05 & 139.20 & 139.31 & 139.13 & 139.78 & 138.72 & 138.82 & 138.72 & 138.37 \\
\hline 1991 & 138.17 & 137.97 & 137.85 & 137.84 & 138.01 & 138.39 & 138.54 & 138.88 & 139.25 & 139.64 & 140.52 & 140.54 \\
\hline 1992 & 140.23 & 139.91 & 139.67 & 139.57 & 139.87 & 140.16 & 140.03 & 139.69 & 139.89 & 139.86 & 140.25 & 140.61 \\
\hline 1993 & 141.12 & 140.88 & 140.65 & 140.74 & 140.73 & 141.13 & 141.11 & 140.55 & 140.25 & 140.10 & 139.81 & 139.69 \\
\hline
\end{tabular}

* Indicates a no-value month

Statistics on Normal monthly means (All days)

\begin{tabular}{|c|c|c|c|c|c|c|c|c|c|c|c|c|}
\hline & oct & Nov & Dec & Jan & Feb & March & April & May & June & July & Aug & Sept \\
\hline & By rows & (Number, & Mean, Var & e, sta & d Devi & $\mathrm{DH}, \mathrm{SKe}$ & $85, c$ & cient o & Variation & & & \\
\hline Number & 34.00 & 35.00 & 34.00 & 36.00 & 32.00 & 35.00 & 33.00 & 31.00 & 34. & 32.00 & 36.00 & 37.00 \\
\hline Gean & 140.51 & 140.25 & 140.19 & 140.29 & 140.47 & 140.72 & 140.66 & 140.37 & 140.23 & 140.31 & 140.51 & 140.64 \\
\hline Var & 0.65 & 0.55 & 0.52 & 0.57 & 0.64 & 0.64 & 0.72 & 0.45 & 0.50 & 0.61 & 0.69 & 0.70 \\
\hline Std & 0.81 & 0.75 & 0.72 & 0.75 & 0.80 & 0.80 & 0.85 & 0.57 & 0.71 & 0.78 & 0.83 & 0.84 \\
\hline Skew & -2.19 & -5.85 & -5.94 & -1.88 & -9.47 & -6.08 & -7.24 & -4.88 & 7.76 & -10.03 & 0.36 & -10.18 \\
\hline Cvar & 0.01 & 0.01 & 0.01 & 0.01 & 0.01 & 0.01 & 0.01 & 0.00 & 0.01 & 0.01 & 0.01 & 0.01 \\
\hline
\end{tabular}




\section{SUWANNEE RIVER BASIN \\ 02320630 LAKE ALTHO AT WALDO, FL}

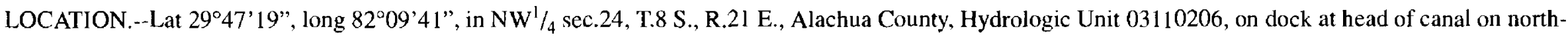
west shore of lake, $0.4 \mathrm{mi}(0.6 \mathrm{~km})$ east of Waldo, and $5.3 \mathrm{mi}$ southwest of Hampton.

SURFACE AREA. -555 acres.

DRAINAGE AREA.--3.39 $\mathrm{mi}^{2}$.

PERIOD OF RECORD.--March 1976 to September 1993 (intermittent).

REVISED RECORDS.--WDR FL-78-4; 1976-77.

GAGE.--Nonrecording gage. Datum of gage is $122.62 \mathrm{ft}$, National Geodetic Vertical Datum of 1929; gage readings have been reduced to elevations NGVD. REMARKS. - Lake interconnects with Santa Fe Lake (02320600) through Santa Fe canal on southeast end of lake.

COOPERATION.--Gage readings were furnished by Suwannee River Water Management District.

EXTREMES FOR PERIOD OF RECORD.--Maximum elevation observed, 143.38 ft Feb. 15, 1993; minimum observed, 136.86 ft Dec. 3, 1990.

MEAN ELEVATION

Normal monthly means (All days)

\begin{tabular}{|c|c|c|c|c|c|c|c|c|c|c|c|c|}
\hline Year & Oct & Nov & Dec & $\operatorname{Jan}$ & $\mathrm{F} e \mathrm{~b}$ & March & April & May & June & July & Aug & Sept \\
\hline 1976 & * & * & * & * & $\star$ & 140.20 & 140.07 & 140.03 & 140.27 & 140.34 & 140.23 & 140.18 \\
\hline 1977 & 139.88 & 139.54 & 139.75 & 140.26 & 140.39 & 140.48 & 140.17 & 139.74 & 139.50 & 139.47 & 139.34 & 139.52 \\
\hline 1978 & 139.34 & 138.90 & 139.01 & 139.56 & 140.10 & 140.53 & 140.42 & 140.33 & 140.38 & 140.46 & 140.98 & 140.99 \\
\hline 1979 & 140.21 & 140.01 & 140.25 & 140.78 & 141.02 & 140.78 & 140.92 & 140.78 & 138.62 & 140.46 & 140.57 & 140.64 \\
\hline 1980 & 140.54 & 140.44 & 140.74 & 141.06 & 141.01 & 141.05 & 141.10 & 140.85 & 139.80 & 140.74 & 140.67 & 140.22 \\
\hline 1981 & 140.10 & 140.00 & 139.73 & 139.70 & 140.26 & 140.04 & 139.66 & 139.30 & 139.80 & 139.30 & 140.52 & 139.63 \\
\hline 1982 & 139.50 & 139.50 & 139.70 & 140.20 & 140.36 & 140.52 & 140.36 & 140.50 & 140.68 & 140.20 & 140.26 & $\star$ \\
\hline 1983 & 140.46 & 140.40 & 140.40 & $\star$ & 140.84 & 140.98 & 140.38 & 140.44 & 140.80 & 140.20 & 140.26 & * \\
\hline 1984 & 140.67 & 140.74 & 140.98 & 140.98 & 141.06 & 140.50 & 140.58 & 140.69 & 140.67 & 140.73 & 140.65 & 140.66 \\
\hline 1985 & 140.44 & 140.40 & 140.38 & 140.33 & $\star$ & 140.26 & 139.91 & 139.84 & 139.71 & 139.94 & 140.55 & $\star$ \\
\hline 1986 & * & * & * & * & 140.96 & $\star$ & * & * & * & * & $\star$ & * \\
\hline 1987 & $\star$ & $\star$ & * & $\star$ & $\star$ & $\star$ & * & * & $\star$ & * & $\star$ & 140.74 \\
\hline 1988 & 140.14 & $\star$ & 140.44 & 140.72 & 141.22 & 141.14 & 140.94 & 140.74 & 140.34 & 140.24 & 140.52 & 140.96 \\
\hline 1989 & $\star$ & 140.76 & $\star$ & 140.69 & $\star$ & 140.49 & 140.22 & 139.93 & 140.04 & 140.07 & 139.95 & 139.83 \\
\hline 1990 & 139.60 & 139.44 & 139.40 & 139.47 & 139.53 & 139.61 & 139.54 & 138.61 & 138.98 & 139.30 & 139.00 & 138.72 \\
\hline 1991 & 138.50 & 138.01 & 1.36 .86 & 137.97 & 138.46 & 139.00 & 139.13 & 139.34 & 139.68 & 139.60 & 140.11 & 140.41 \\
\hline 19 & * & * & * & * & 139.89 & 139.95 & 139.86 & 139.61 & 139.82 & 139.85 & 139.95 & 140.27 \\
\hline 1007 & 140.50 & 140.28 & 140.23 & 140.29 & 141.09 & 140.45 & 140.51 & 140.31 & 140.32 & 139.95 & 139.60 & 139.60 \\
\hline
\end{tabular}

* Indicates a no-value month

Statistics on Normal monthly means (All days)

\begin{tabular}{|c|c|c|c|c|c|c|c|c|c|c|c|c|}
\hline & Oct & Nov & Dec & Jan & Feb & March & April & May & June & July & Aug & Sept \\
\hline By & rows & (Number, Mean, & Variance, & - Standard & Deviation, & Skewness &, Coeff & ent of & Variation & & & \\
\hline Jumber & 13.00 & 13.00 & 13.00 & 13.00 & 14.00 & 16.00 & 16.00 & 16.00 & 16.00 & 16.00 & 16.00 & 14.00 \\
\hline Mean & 139.99 & 139.88 & 139.84 & 140.15 & 140.44 & 140.37 & 140.24 & 140.06 & 139.96 & 140.05 & 140.20 & 140.17 \\
\hline $\operatorname{Var}$ & 0.39 & 0.63 & $1 . \pm 0$ & 0.71 & 0.60 & 0.31 & 0.30 & 0.42 & 0.38 & 0.22 & 0.29 & 0.42 \\
\hline sta & 0.63 & 0.79 & 1.05 & 0.84 & 0.77 & 0.56 & 0.55 & 0.65 & 0.62 & 0.47 & 0.54 & 0.65 \\
\hline Skew & -9.17 & -1.28 & -1.53 & -4.84 & 4.70 & -6.53 & $-18 \cdot 14$ & -4.26 & -10.29 & -13.54 & -6.69 & -4.50 \\
\hline Cvar & 0.00 & 0.01 & 0.01 & 0.01 & 0.01 & 0.00 & 0.00 & 0.00 & 0.00 & 0.00 & 0.00 & 0.00 \\
\hline
\end{tabular}


LOCATION.--Lat $29^{\circ} 55^{\prime} 16^{\prime \prime}$, long $82^{\circ} 10^{\prime} 01^{\prime \prime}$, in NE $1 / 4$ sec.2, T.7 S., R.21 E., Bradford County, Hydrologic Unit 03110206, on west shore at canal, 2.5 mi east of Sampson City, and $3.8 \mathrm{mi}$ southwest of Starke.

SURFACE AREA.--355 acres.

DRAINAGE AREA.-- $\mathrm{mi}^{2}$.

PERIOD OF RECORD.--February 1989 to September 1993.

GAGE.--Nonrecording gage. Datum of gage is National Geodetic Vertical Datum of 1929.

REMARKS.--Outflow from lake is through Sampson Lake to Santa Fe River.

COOPERATION.--Gage readings were furnished by Suwannee River Water Management District.

EXTREMES FOR PERIOD OF RECORD.--Maximum elevation observed, 135.64 ft April.1991; minimum observed, $129.79 \mathrm{ft}$ September and October 1990.

\begin{tabular}{|c|c|c|c|c|c|c|c|c|c|c|c|c|}
\hline \multirow[b]{2}{*}{ Year } & \multicolumn{12}{|c|}{$\begin{array}{l}\text { MEAN ELEVATION } \\
\text { Normal monthly means (All days) }\end{array}$} \\
\hline & oct & Nov & Dec & $\operatorname{Jan}$ & Feb & March & April & May & June & July & Aug & Sept \\
\hline $\begin{array}{l}1989 \\
1990 \\
1991 \\
1992 \\
1993\end{array}$ & $\begin{array}{c}* \\
131.07 \\
129.91 \\
131.19 \\
133.60\end{array}$ & $\begin{array}{c}\star \\
130.87 \\
129.94 \\
130.68 \\
131.28\end{array}$ & $\begin{array}{c}\star \star \\
130.82 \\
129.97 \\
130.53 \\
130.86\end{array}$ & $\begin{array}{c}{ }^{\star} \\
131.02 \\
130.38 \\
130.54 \\
130.83\end{array}$ & $\begin{array}{l}130.64 \\
131.08 \\
130.88 \\
131.01 \\
131.04\end{array}$ & $\begin{array}{l}130.75 \\
131.05 \\
132.06 \\
131.27 \\
131.58\end{array}$ & $\begin{array}{l}130.54 \\
130.80 \\
133.42 \\
131.07 \\
131.24\end{array}$ & $\begin{array}{l}130.33 \\
130.44 \\
132.34 \\
130.99 \\
130.50\end{array}$ & $\begin{array}{l}130.43 \\
130.52 \\
132.17 \\
130.89 \\
130.41\end{array}$ & $\begin{array}{l}130.59 \\
130.36 \\
131.81 \\
130.89 \\
130.74\end{array}$ & $\begin{array}{l}130.60 \\
130.10 \\
131.89 \\
131.16 \\
130.42\end{array}$ & $\begin{array}{l}131.20 \\
129.92 \\
131.11 \\
132.21 \\
130.34\end{array}$ \\
\hline
\end{tabular}

* Indicates a no-value month

Statistics on Normal monthly means (All days)

\begin{tabular}{|c|c|c|c|c|c|c|c|c|c|c|c|c|}
\hline & Oct & Nov & Dec & $\operatorname{Jan}$ & Feb & March & April & May & June & Ju1y & Aug & Sept \\
\hline $\begin{array}{r}\text { By } \\
\text { Number }\end{array}$ & $\begin{array}{c}\text { rows } 1 \\
4.00\end{array}$ & $\begin{array}{c}\text { (Number, Mean, } \\
4.00\end{array}$ & $\begin{array}{c}\text { Variance, } \\
4.00\end{array}$ & $\begin{array}{l}\text { standard } \\
4.00\end{array}$ & $\begin{array}{c}\text { Deviation, } \\
5.00\end{array}$ & $\begin{array}{c}\text { Skewness, } \\
5.00\end{array}$ & $\begin{array}{c}\text { Coeffi } \\
5.00\end{array}$ & $\begin{array}{l}\text { nt of v } \\
5.00\end{array}$ & $\begin{array}{r}\text { Variation } \\
5.00\end{array}$ & 5.00 & 5.00 & 5.00 \\
\hline Mean & 131.44 & 130.69 & 130.54 & 130.69 & 130.93 & 131.34 & 131.41 & 130.92 & 130.88 & 130.88 & 130.83 & 130.96 \\
\hline Var & 2.41 & 0.32 & 0.17 & 0.09 & 0.03 & 0.26 & 1.33 & 0.70 & 0.57 & 0.31 & 0.50 & 0.78 \\
\hline std & 1.55 & 0.57 & 0.41 & 0.30 & 0.18 & 0.51 & 1.15 & 0.84 & 0.75 & 0.56 & 0.71 & 0.88 \\
\hline Skew & 1.33 & -5.94 & 49.22 & -41.40 & -61.17 & 6.02 & 3.04 & 1.77 & -5.39 & -5.31 & -8.71 & 3.22 \\
\hline Cvar & 0.01 & 0.00 & 0.00 & 0.00 & 0.00 & 0.00 & 0.01 & 0.01 & 0.01 & 0.00 & 0.01 & 0.01 \\
\hline
\end{tabular}




\section{SUWANNEE RIVER BASIN \\ 02320750 LAKE SAMPSON NEAR STARKE, FL}

LOCATION.--Lat $29^{\circ} 56^{\prime} 11^{\prime \prime}$, long $82^{\circ} 10^{\prime} 15^{\prime \prime}$, in NE ${ }^{1} / 4$ sec.35, T.6 S., R.21 E., Bradford County, Hydrologic Unit 03110206, on northeast shore at end of canal, $2.7 \mathrm{mi}$ northeast of Sampson City, and $3.7 \mathrm{mi}$ southwest of Starke.

SURFACE AREA.--2,071 acres.

DRAINAGE AREA.--59.3 $\mathrm{mi}^{2}$.

PERIOD OF RECORD.--July 1957 to September 1993 (intermittent).

REVISED RECORDS.--WDR FL-74-1: Drainage area.

GAGE.--Nonrecording gage. Datum of gage is $127.49 \mathrm{ft}$ National Geodetic Vertical Datum of 1929 . July 1957 to September 1967 , at site 2.2 mi southwest at datum $1.23 \mathrm{ft}$ lower. Gage readings have been reduced to elevations NGVD.

REMARKS.--Outflow from lake is through Sampson River to Santa Fe River. Lake level control is concrete spillway culverts and lift gates 0.5 mi downstream from lake outlet. Prior to 1973, outflow through 12-inch drainage well on north side of lake to ground water.

COOPERATION.--Gage readings were furnished by Suwannee River Water Management District.

EXTREMES FOR PERIOD OF RECORD.--Maximum elevation observed, 136.15 ft Mar. 23, 1959; minimum observed, 129.66 ft Oct. 8, 1990.

MEAN ELEVATION

Normal monthly means (All days)

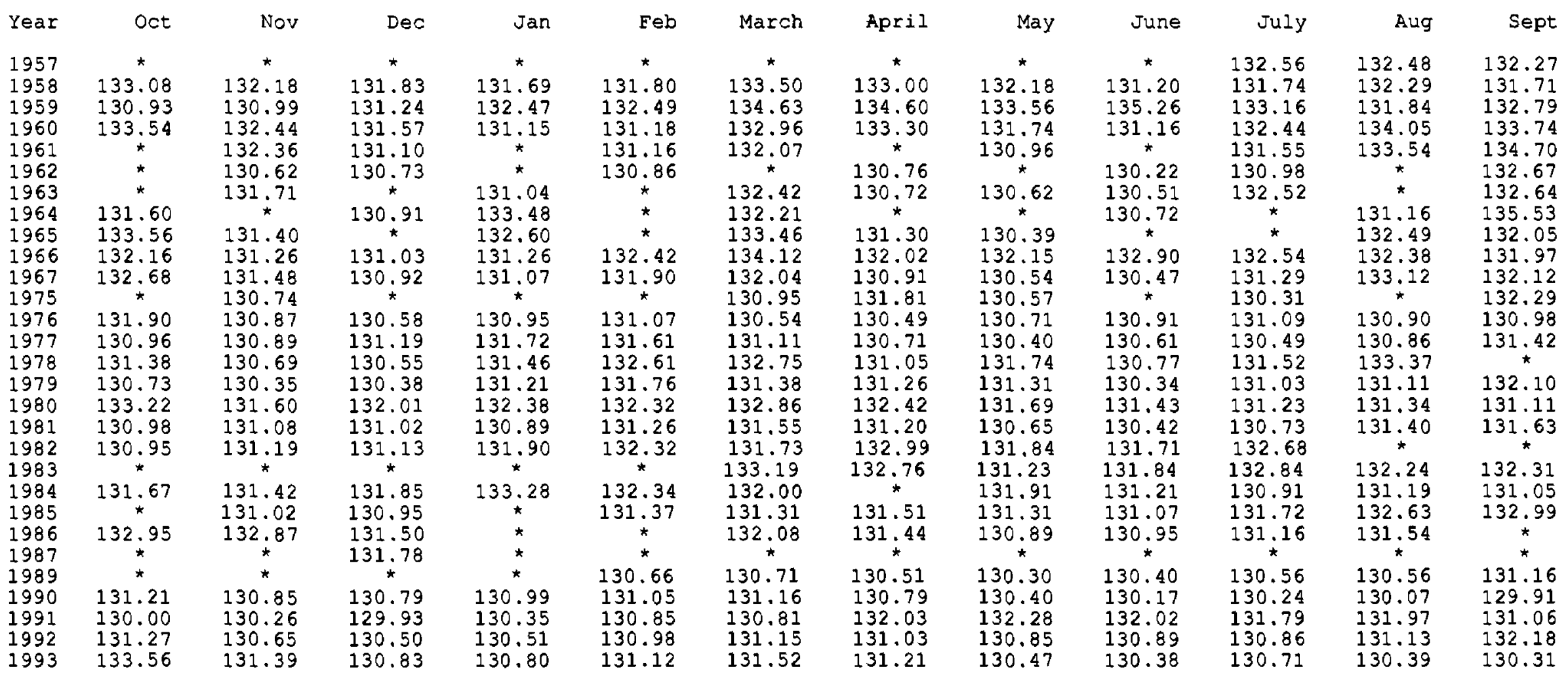

Statistics on Normal monthly means (All days)
By rows (Number, Mean, Variance, Standard Deviation, Skewness, Coefficient of Variation)

Number

Mean

Var

std

Skew

Cvar
131.92

1.21

1.10

0.90

0.01
24.00

31.26
0.47

0.68

$-5.13$

-5.13
0.01
23.00

131.06
0.30

0.54

$-23.84$

0.00
20.00
131.56

131.56
0.78

0.88

$-3.58$

0.01
21.00

131.58
0.42

0.42
0.64

0.64
-4.49

0.00
26.00

132.08
1.20

1.20
1.10
-3.06

$-3.06$

0.01
24.00

131.66
1.10

1.10
1.05

0.49

0.01
131.23

31.23
0.67

0.82

-4.61
0.01
131.15

31.15
1.21

1.10

2.12

0.01
July

26.00

131.49
0.75

0.87

$-6.32$

0.01

$\begin{array}{rr}\text { Aug } & \text { Sept } \\ & \\ 24.00 & 25.00 \\ 131.84 & 132.11 \\ 1.11 & 1.59 \\ 1.05 & 1.26 \\ -1.27 & -1.42 \\ 0.01 & 0.01\end{array}$




\section{SUWANNEE RIVER BASIN \\ 02321300 LAKE BUTLER AT LAKE BUTLER, FL}

LOCATION.--Lat 30'01'38', long 82 $20^{\prime} 18^{\prime \prime}$ ', in SE ${ }^{1} / 4$ sec.30, T.5 S., R.20 E., Union County, Hydrologic Unit 03110206, on south shore of 1ake at public park in Lake Butler.

SURFACE AREA.--437 acres.

DRAINAGE AREA.--3.94 $\mathrm{mi}^{2}$.

PERIOD OF RECORD.--July 1957 to September 1993 (intermittent).

REVISED RECORDS.--WDR FL-75-1: Drainage area.

GAGE.--Nonrecording gage. Datum of gage is $127.35 \mathrm{ft}$ National Geodetic Vertical Datum of 1929. July 1957 to September 1967 at same site at datum $3.41 \mathrm{ft}$ lower. Gage readings have been reduced to elevations NGVD.

REMARKS.--Outflow from lake is through a run to Butler Creek, thence to New River.

COOPERATION.--Gage readings were furnished by Suwannee River Water Management District.

EXTREMES FOR PERIOD OF RECORD.--Maximum elevation, $134.02 \mathrm{ft}$ about Sept. 13, 1964, from high-water mark; minimum observed, $128.71 \mathrm{ft}$ Dec. 30 , 1990.

MEAN ELEVATION

Normal monthly means (All days)

\begin{tabular}{|c|c|c|c|c|c|c|c|}
\hline Year & oct & Nov & Dec & $\operatorname{Jan}$ & Feb & March & April \\
\hline 1957 & $\star$ & * & * & $\star$ & * & * & $\star$ \\
\hline 1958 & 130.53 & 130.29 & 130.38 & 130.44 & 130.61 & 131.28 & 131.30 \\
\hline 1959 & 130.74 & 130.85 & 130.90 & 131.56 & 131.34 & 132.14 & 131.68 \\
\hline 1960 & 131.22 & 130.80 & 130.52 & 130.48 & 130.67 & 131.48 & 131.30 \\
\hline 1961 & 131.42 & 130.82 & 130.45 & 130.44 & 130.69 & 130.75 & 130.75 \\
\hline 1962 & 130.59 & 130.27 & 130.18 & * & 130.18 & $\star$ & 130.12 \\
\hline 1963 & $\star$ & 128.93 & $\star$ & 129.04 & * & 129.92 & 129.46 \\
\hline 1964 & 131.34 & $\star$ & 130.96 & 131.82 & * & 131.66 & $\star$ \\
\hline 1965 & 132.30 & 131.50 & $\star$ & 132.22 & * & 131.98 & 131.29 \\
\hline 1966 & 131.94 & 131.71 & 131.47 & 131.69 & 131.98 & 132.28 & 131.74 \\
\hline 1967 & 131.64 & 131.39 & 130.99 & 131.09 & 131.49 & 131.28 & 130.81 \\
\hline 1975 & $\star$ & 130.60 & 130.62 & 130.79 & 130.99 & 130.87 & 130.89 \\
\hline 1976 & 131.33 & 130.91 & 130.72 & 130.85 & 130.91 & 130.70 & 130.48 \\
\hline 1977 & 130.67 & 130.51 & 130.58 & 131.09 & 131.24 & 130.95 & 130.46 \\
\hline 1978 & 129.85 & 129.87 & 130.11 & 130.76 & 131.68 & 131.81 & 130.98 \\
\hline 1979 & 129.95 & 129.73 & 129.59 & 130.35 & 130.50 & * & 130.35 \\
\hline 1980 & 130.83 & 130.55 & 130.53 & 130.85 & 130.85 & 129.85 & 131.55 \\
\hline 1981 & 130.24 & 130.15 & 130.03 & 129.89 & 130.81 & 130.57 & 130.15 \\
\hline 1982 & 128.77 & 128.75 & 128.85 & 129.15 & 129.05 & 129.20 & 130.00 \\
\hline 1983 & 130.73 & 130.35 & 130.15 & 130.40 & 131.00 & 132.10 & 131.40 \\
\hline 1984 & * & 130.95 & 131.85 & 131.65 & 131.45 & 131.55 & 131.45 \\
\hline 1985 & * & 130.30 & 130.40 & * & 130.29 & 130.15 & 130.15 \\
\hline 1986 & 130.90 & 130.85 & 130.45 & 130.75 & 131.45 & 130.95 & 130.05 \\
\hline 1987 & 130.15 & 129.85 & 130.15 & 131.45 & 132.25 & 131.30 & 130.85 \\
\hline 1988 & 130.01 & 130.16 & 129.96 & 130.61 & 131.81 & 131.41 & 130.51 \\
\hline 1989 & 131.11 & 130.41 & 130.21 & 130.01 & 129.86 & 129.81 & * \\
\hline 1990 & 130.21 & 130.11 & * & 130.01 & 130.21 & * & 129.96 \\
\hline 1991 & 129.01 & 128.76 & 128.71 & 129.41 & 129.61 & 131.01 & 132.41 \\
\hline 1992 & 130.71 & 131.71 & 129.91 & 130.00 & 130.11 & 130.21 & 130.61 \\
\hline 1993 & 132.74 & 131.81 & 130.51 & 131.02 & 131.21 & 131.21 & 130.78 \\
\hline
\end{tabular}

July
130.86
130.82
131.29
131.18
130.27
$\star$
130.56
$\star$
$\star$
131.84
130.61
130.75
130.29
129.61
130.67
129.95
131.01
129.30
130.75
130.35
130.25
130.06
129.40
131.35
129.65
129.36
$\star$
131.86
130.61
131.21

Sept 130.58 131.00 131.09
131.44 131.44
130.99 129.26 131.50 133.61 132.09 131.53 130.49 130.49
131.78 130.75 130.10

131.43 130.55 130.24 130.80 130.17 132.43 130.15 132.30 130.56 129.01

131.51 129.71

* Indicates a no-value month

Statistics on Normal monthly means (All days)

Oct Nov Dec March April Man

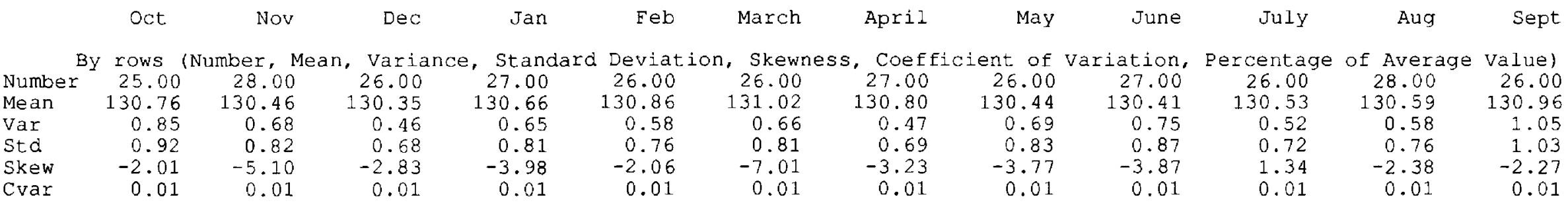


LOCATION.--Lat $29^{\circ} 42^{\prime} 31^{\prime \prime}$, long $82^{\circ} 43^{\prime} 59^{\prime \prime}$, in $\mathrm{NW}^{1} / 4$ sec.17, T.9 S., R.16 E., Gilchrist County, Hydrologic Unit 03110206 , on north shore of 1 ake near end of private dock, $8.2 \mathrm{mi}$ northeast of Trenton.

SURFACE AREA. -183 acres.

DRAINAGE AREA.--15.7 $\mathrm{mi}^{2}$.

PERIOD OF RECORD.--July 1972 to September 1993 (intermittent).

REVISED RECORD.--WDR FL-80-4: 1978 (M).

GAGE.--Nonrecording gage. Datum of gage is National Geodetic Vertical Datum of 1929. Prior to June 14, 1977, at site $60 \mathrm{ft}$ northeast at present datum.

REMARKS.--Concrete control at lake outlet at elevation $73.4 \mathrm{ft}$. There is some pumpage from lake for irrigation purposes.

COOPERATION.--Gage readings were furnished by Suwannee River Water Management District.

EXTREMES FOR PERIOD OF RECORD.--Maximum elevation observed, 75.98 ft Aug. 18, 1978; minimum observed, $55.10 \mathrm{ft}$ Feb. $6,1982$.

MEAN ELEVATION

Normal monthly means (All days)

\begin{tabular}{|c|c|c|c|c|c|c|c|c|c|c|c|c|}
\hline Year & oct & Nov & Dec & $\operatorname{Jan}$ & Feb & March & April & May & June & July & Aug & Sept \\
\hline 1972 & * & * & * & * & $\star$ & * & * & * & * & 72.35 & 72.47 & 74.28 \\
\hline 1973 & 73.48 & 72.90 & 73.26 & 73.55 & 74.11 & 74.23 & 74.35 & 73.59 & 73.11 & 72.60 & 72.13 & 71.31 \\
\hline 1974 & 70.23 & 69.43 & 68.82 & 68.14 & 67.42 & 65.74 & 65.83 & 65.09 & 64.62 & 64.17 & 64.02 & 64.52 \\
\hline 1975 & 64.84 & 64.09 & 63.98 & 64.37 & 65.00 & 65.44 & 65.42 & 65.01 & 54.24 & 64.36 & 65.31 & 66.07 \\
\hline 1975 & 68.21 & 68.48 & 67.64 & 67.60 & 67.20 & 66.38 & 65.61 & 65.20 & 55.19 & 66.77 & 68.08 & 70.29 \\
\hline 1977 & 71.47 & 70.68 & 70.24 & 70.89 & 71.75 & 71.88 & $\star$ & $\star$ & 58.23 & 67.54 & 67.06 & 66.72 \\
\hline 1978 & 65.78 & 64.87 & 64.56 & 64.49 & 64.87 & 66.05 & 66.18 & 65.66 & 67.72 & 68.52 & 75.98 & $\star$ \\
\hline 1979 & 73.11 & 71.97 & 70.98 & 70.80 & 70.80 & 70.24 & 69.75 & 70.84 & 70.31 & 70.13 & 72.90 & 74.46 \\
\hline 1980 & 74.32 & 73.79 & 73.50 & 73.45 & 73.90 & 74.16 & 74.34 & 73.93 & 73.02 & 72.67 & 72.89 & 72.37 \\
\hline 1981 & 71.61 & 71.04 & 70.29 & 69.61 & 69.53 & 69.97 & 69.68 & 68.41 & 58.06 & 66.75 & * & 66.22 \\
\hline 1982 & $\star$ & 64.98 & 59.49 & $\star$ & 55.55 & 59.99 & 66.21 & 72.73 & 72.92 & 73.90 & 74.79 & 74.84 \\
\hline 1984 & $\star$ & * & $\star$ & * & 71.22 & * & $\star$ & * & $\star$ & $\star$ & * & $\star$ \\
\hline 1985 & $\star$ & 70.18 & 69.04 & * & 71.22 & 70.95 & 70.01 & 69.34 & 68.75 & 68.40 & 68.47 & 74.09 \\
\hline 1986 & 73.76 & 73.62 & 73.34 & 73.31 & 73.83 & 73.76 & 73.33 & 72.57 & 71.80 & 71.01 & 71.66 & 73.27 \\
\hline 1987 & 73.30 & 72.79 & 72.84 & 72.99 & 73.49 & 74.02 & 73.96 & 73.32 & 72.87 & 72.58 & 72.00 & 71.28 \\
\hline 1988 & $\star$ & $\star$ & $\star$ & 68.98 & 70.12 & 73.88 & $\star$ & 72.76 & 71.63 & 71.08 & 71.18 & 73.70 \\
\hline 1989 & 73.23 & 72.68 & 69.90 & 71.63 & 70.93 & 70.10 & 69.23 & 68.73 & 67.73 & 67.86 & 67.76 & 69.78 \\
\hline 1990 & 69.88 & 68.94 & 68.73 & 68.38 & 68.08 & 67.73 & 67.63 & $\star$ & $\star$ & $\star$ & $\star$ & $\star$ \\
\hline 1991 & $\star$ & $\star$ & $\star$ & $\star$ & $\star$ & 68.67 & 72.23 & 73.53 & 72.70 & 72.28 & 73.36 & 72.64 \\
\hline 1992 & 73.13 & 71.18 & 70.43 & 69.70 & 69.86 & 69.83 & 69.63 & 68.78 & * & $\star$ & * & 69.60 \\
\hline 10 & $\star$ & 72.71 & 72.58 & 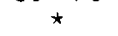 & 72.98 & 73.18 & 73.03 & 71.84 & 70.58 & 夫 & 69.83 & * \\
\hline
\end{tabular}

* Indicates a no-value month

Statistics on Normal monthly means (All days)

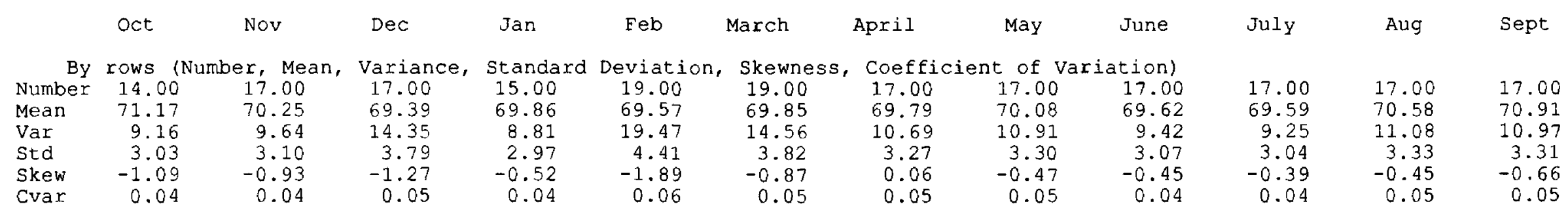


LOCATION.--Lat 3009'25", long 82³8'28", in NE $\frac{1}{4}$ sec.5, T.4 S., R.17 E., Columbia County, Hydrologic Unit 031 10206, on west shore of southern portion of lake, $2.0 \mathrm{mi}$ south of Lake City.

SURFACE AREA.--348 acres.

DRAINAGE AREA, $--15.4 \mathrm{mi}^{2}$.

PERIOD OF RECORD.--September 1965 to March, 1990 (intermittent).

GAGE.--Nonrecording gage. Datum of gage is $87.18 \mathrm{ft}$ National Geodetic Vertical Datum of 1929. Gage readings have been reduced to elevations NGVD. Prior to Oct. 1, 1974, nonrecording gage at site 2.0 mi northeast at same datum.

REMARKS.--Lake has several contributing creeks but no surface outlet; below about $95 \mathrm{ft}$ elevation, lake is separated into several small ponds.

COOPERATION.--Gage readings were fumished by Suwannee River Water Management District.

EXTREMES FOR PERIOD OF RECORD.--Maximum elevation observed, $99.98 \mathrm{ft}$ Mar. 1, 1966; minimum observed, $90.23 \mathrm{ft}$ Apr. $29,1968$.

MEAN ELEVATION

Normal monthly means (All days)

\begin{tabular}{|c|c|c|c|c|c|c|c|c|c|c|c|c|}
\hline Year & oct & Nov & Dec & Jan & $F \in b$ & March & April & May & June & July & Aug & Sept \\
\hline $\begin{array}{l}1965 \\
1966 \\
1967 \\
1968 \\
1969 \\
1970 \\
1971 \\
1972 \\
1973 \\
1974 \\
1975 \\
1976 \\
1977 \\
1978 \\
1979 \\
1980 \\
1981 \\
1982 \\
1983 \\
1984 \\
1985 \\
1986 \\
1987 \\
1988 \\
1989 \\
1990\end{array}$ & $\begin{array}{c}* \\
99.19 \\
99.05 \\
91.19 \\
92.42 \\
98.17 \\
97.25 \\
95.18 \\
95.29 \\
97.29 \\
97.22 \\
97.21 \\
93.47 \\
94.70 \\
\star \\
\star \\
\star \\
94.55 \\
\star \\
97.80 \\
96.40 \\
\star \\
94.81 \\
96.84 \\
\star \\
\star\end{array}$ & $\begin{array}{c}\star \\
98.38 \\
98.42 \\
91.14 \\
\star \\
97.32 \\
96.49 \\
\star \\
94.77 \\
95.64 \\
95.89 \\
95.83 \\
93.40 \\
94.69 \\
94.67 \\
94.37 \\
\star \\
94.65 \\
\star \\
\star \\
\star \\
\star \\
94.16 \\
95.95 \\
\star \\
\star\end{array}$ & $\begin{array}{c}\star \\
98.34 \\
97.74 \\
91.29 \\
91.55 \\
\star \\
\star \\
94.92 \\
94.83 \\
95.37 \\
94.95 \\
94.57 \\
93.68 \\
96.41 \\
94.38 \\
\star \\
96.29 \\
\star \\
96.04 \\
98.16 \\
\star \\
\star \\
94.30 \\
\star \\
\star \\
\star\end{array}$ & $\begin{array}{c}{ }^{\star} \\
98.72 \\
97.88 \\
93.22 \\
91.61 \\
98.69 \\
95.12 \\
96.86 \\
94.97 \\
94.76 \\
94.97 \\
94.37 \\
94.37 \\
97.60 \\
94.58 \\
94.39 \\
95.09 \\
94.90 \\
\star \\
\star \\
94.69 \\
98.08 \\
95.49 \\
94.51 \\
94.85 \\
\star\end{array}$ & $\begin{array}{c}{ }^{\star} \\
99.34 \\
98.40 \\
\star \\
\star \\
{ }^{\star} \\
94.77 \\
\star \\
96.92 \\
94.85 \\
95.09 \\
94.20 \\
96.01 \\
98.79 \\
94.92 \\
94.61 \\
\star \\
\star \\
\star \\
\star \\
94.31 \\
\star \\
\star \\
97.28 \\
95.11 \\
\star\end{array}$ & $\begin{array}{c}{ }^{\star} \\
99.56 \\
98.24 \\
91.08 \\
92.62 \\
98.38 \\
93.97 \\
97.65 \\
97.18 \\
94.53 \\
94.96 \\
94.09 \\
96.87 \\
98.87 \\
94.54 \\
98.43 \\
95.76 \\
94.53 \\
97.82 \\
98.64 \\
94.12 \\
\star \\
98.09 \\
98.06 \\
94.78 \\
96.17\end{array}$ & $\begin{array}{c}{ }^{*} \\
98.84 \\
97.26 \\
90.56 \\
94.23 \\
* \\
93.26 \\
97.30 \\
98.69 \\
\star \\
95.00 \\
93.91 \\
95.79 \\
98.00 \\
95.49 \\
95.20 \\
95.21 \\
\star \\
\star \\
98.56 \\
93.92 \\
97.88 \\
98.36 \\
\star \\
\star \\
\star\end{array}$ & $\begin{array}{c}\star \\
98.78 \\
96.26 \\
\star \\
\star \\
97.76 \\
93.65 \\
\star \\
97.69 \\
94.68 \\
96.18 \\
93.93 \\
94.67 \\
98.68 \\
95.45 \\
97.75 \\
93.96 \\
\star \\
\star \\
\star \\
93.76 \\
97.28 \\
97.16 \\
95.76 \\
\star \\
\star\end{array}$ & $\begin{array}{c}{ }^{*} \\
99.41 \\
95.15 \\
91.44 \\
94.65 \\
97.16 \\
\star \\
96.50 \\
97.86 \\
95.52 \\
96.38 \\
94.08 \\
94.03 \\
97.79 \\
94.52 \\
97.19 \\
\star \\
\star \\
\star \\
97.59 \\
93.67 \\
\star \\
95.60 \\
95.20 \\
\star \\
\star\end{array}$ & $\begin{array}{c}{ }^{\star} \\
99.40 \\
94.92 \\
93.65 \\
95.30 \\
\star \\
93.61 \\
\star \\
98.27 \\
95.62 \\
96.21 \\
94.05 \\
93.75 \\
97.18 \\
94.30 \\
98.49 \\
94.18 \\
\star \\
\star \\
\star \\
94.20 \\
95.18 \\
96.46 \\
\star \\
\star \\
\star\end{array}$ & $\begin{array}{c}{ }^{*} \\
99.59 \\
93.90 \\
\star \\
96.72 \\
98.72 \\
94.86 \\
96.18 \\
\star \\
96.36 \\
96.96 \\
93.79 \\
94.01 \\
97.86 \\
95.41 \\
97.76 \\
\star \\
\star \\
\star \\
\star \\
94.94 \\
95.50 \\
96.89 \\
\star \\
\star \\
\star\end{array}$ & $\begin{array}{c}98.93 \\
98.99 \\
93.42 \\
93.20 \\
98.05 \\
97.80 \\
95.97 \\
96.78 \\
98.13 \\
98.17 \\
97.64 \\
93.74 \\
94.79 \\
97.49 \\
95.08 \\
97.16 \\
\star \\
\star \\
97.80 \\
\star \\
\star \\
\star \\
98.24 \\
\star \\
94.97 \\
\star\end{array}$ \\
\hline
\end{tabular}

* Indicates a no-value month

Statistics on Normal monthly means (All days)

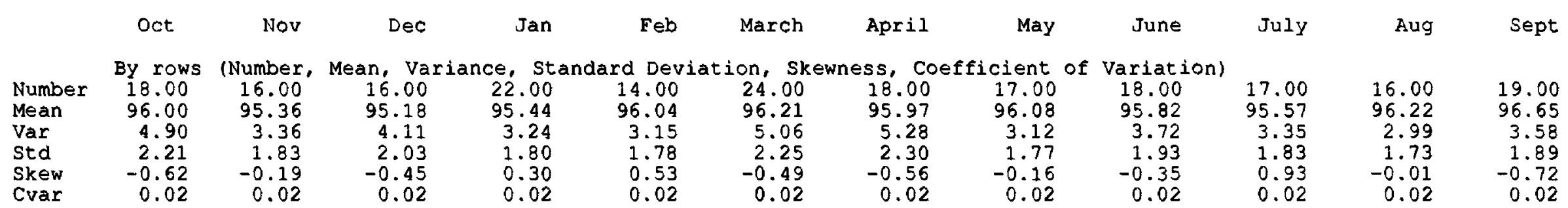


LOCATION.--Lat $29^{\circ} 45^{\prime} 08^{\prime \prime}$, long $83^{\circ} 02^{\prime} 18^{\prime \prime}$, in SE $1 / 4$ sec. 29, T.8 S., R.13 E., Dixie County, Hydrologic Unit 03110205 , on south shore of lake $10 \mathrm{ft}$ north of private dock, $9.8 \mathrm{mi}$ northeast of Cross City and $10.2 \mathrm{mi}$ northwest of Old Town.

SURFACE AREA.--153 acres $\left(0.24 \mathrm{mi}^{2}\right)$.

DRAINAGE AREA.--1.61 $\mathrm{mi}^{2}$.

PERIOD OF RECORD.--November 1974 to September 1993 (intermittent).

GAGE.--Nonrecording gage. Datum of gage is $46.72 \mathrm{ft}$ National Geodetic Vertical Datum of 1929 (levels by Suwannee River Water Management District.) Gage readings have been reduced to elevations NGVD.

REMARKS.--Lake is Iandlocked.

COOPERATION.--Gage readings were furnished by Suwannee River Water Management District.

EXTREMES FOR PERIOD OF RECORD.--Maximum elevation observed, 53.82 ft Oct. 2, 1988, Aug. 28, 1991; minimum observed, $47.86 \mathrm{ft}$ Aug. $16,1977$.

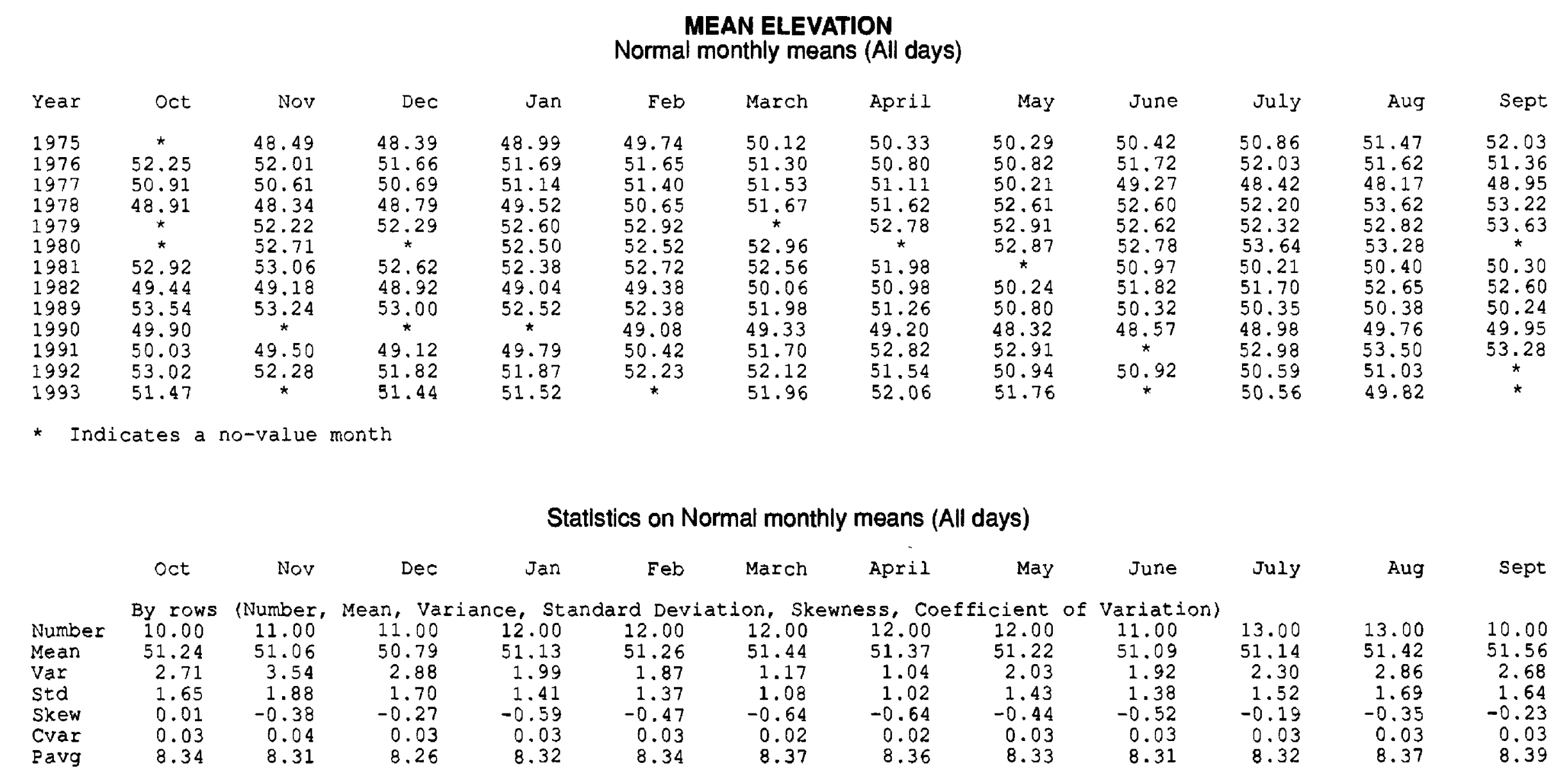


LOCATION.--Lat $30^{\circ} 23^{\prime} 03^{\prime \prime}$, long $83^{\circ} 32^{\prime} 20^{\prime \prime}$, in SE ${ }^{1} / 4$ sec.20, T.1 S., R.8 E., Madison County, Hydrologic Unit 03110102 , on west shore of the northwest portion of the lake $8.3 \mathrm{mi}$ southeast of Greenville, $9.5 \mathrm{mi}$ southwest of Madison.

SURFACE AREA.--453 acres.

DRAINAGE AREA.- $7.13 \mathrm{mi}^{2}$.

PERIOD OF RECORD.--November 1974 to June 1984 (intermittent)).

GAGE.--Nonrecording gage. Datum of gage is $99.61 \mathrm{ft}$ National Geodetic Vertical Datum of 1929. Gage readings have been reduced to elevations NGVD. REMARKS.--Headwaters Sampala Swamp, San Pedro Bay and Econfina River, outlet at south end of lake.

COOPERATION.--Gage readings were furnished by Suwannee River Water Management District.

EXTREMES FOR PERIOD OF RECORD.--Maximum elevation observed, $104.81 \mathrm{ft}$ March. 30, 1984; minimum observed, $102.69 \mathrm{ft}$ Aug. $4,1977$.

\begin{tabular}{|c|c|c|c|c|c|c|c|c|c|c|c|c|}
\hline \multirow[b]{2}{*}{ Year } & \multicolumn{12}{|c|}{$\begin{array}{l}\text { MEAN ELEVATION } \\
\text { Normal monthly means (All days) }\end{array}$} \\
\hline & Oct & Nov & Dec & Jan & Feb & March & April & May & June & July & Aug & Sept \\
\hline 1975 & * & 103.44 & 103.57 & 103.87 & 104.08 & 104.02 & 104.04 & 103.74 & 103.55 & 103.48 & 103.48 & 103,46 \\
\hline 1976 & 103.40 & 103.22 & 103.22 & 103.36 & 103.56 & 103.79 & 103.71 & 103.65 & 103.93 & 103.77 & 103.92 & 103.88 \\
\hline 1977 & 103.83 & 103.91 & 104.31 & 104.35 & 104.24 & 104.29 & 103.95 & 103.52 & 103.04 & 102.78 & 103.05 & 103.17 \\
\hline 1978 & 103.06 & 102.97 & 103.33 & 103.79 & 103.95 & 104.05 & 103.78 & 103.82 & 103.57 & 103.54 & 103.79 & 103.52 \\
\hline 1979 & 103.15 & 103.11 & 103.31 & 103.75 & 104.15 & 103.79 & 103.77 & 103.79 & 103.55 & 103.87 & * & 103.73 \\
\hline 1980 & 103.49 & 103.45 & 103.63 & 103.81 & 103.83 & 104.11 & $\star$ & 103.94 & 103.81 & 104.17 & 104.17 & 104.22 \\
\hline 1981 & 103.67 & 103.87 & 103.91 & 103.79 & 103.80 & 104.05 & 104.02 & 103.57 & 103.44 & 103.40 & $\star$ & 104.09 \\
\hline 1982 & 103.57 & 103.58 & $\star$ & 104.15 & 104.14 & 104.03 & 104.19 & 103.85 & 103.88 & 104.19 & 104.11 & 103.83 \\
\hline 1983 & 103.95 & 103.82 & $\star$ & 104.16 & * & 104.35 & 104.39 & 104.39 & 104.37 & 104.55 & 104.35 & 104.31 \\
\hline 1984 & $\star$ & 104.41 & 104.39 & 104.55 & * & 104.67 & 太 & 104.25 & 104.04 & $\star$ & * & $\star$ \\
\hline
\end{tabular}

* Indicates a no-value month

Statistics on Normal monthly means (All days)

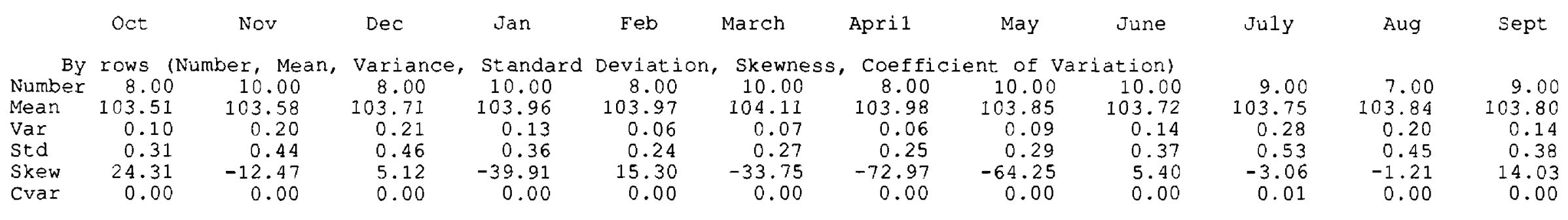




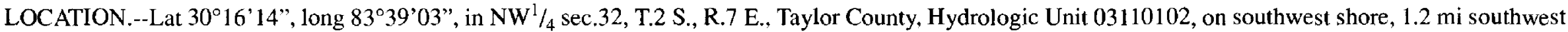
of Econfina River, $1.7 \mathrm{mi}$ southwest of Shady Grove, 2.1 mi northwest of Lake Bird Community, and 13.4 mi northwest of Perry.

SURFACE AREA.--44.5 acres.

DRAINAGE AREA,--0.34 $\mathrm{mi}^{2}$.

PERIOD OF RECORD.--November 1974 to September 1993 (intermittent).

GAGE.--Nonrecording gage. Datum of gage is $73.19 \mathrm{ft}$ National Geodetic Vertical Datum of 1929 (levels by Suwannee River Water Management District). Gage readings have been reduced to elevations NGVD.

REMARKS.--Lake will interconnect with Nose Lake at high stages, and at extreme high stage overflow into Econfina River Basin.

COOPERATION.--Gage readings were furnished by Suwannee River Water Management District.

EXTREMES FOR PERIOD OF RECORD.--Maximum elevation observed, $77.58 \mathrm{ft}$ Oct. 2, 1991; minimum observed, $73.90 \mathrm{ft}$ June $18,1993$.

MEAN ELEVATION

Normal monthly means (All days)

\begin{tabular}{|c|c|c|c|c|c|c|c|c|c|c|c|c|}
\hline Year & oct & Nov & Dec & $\operatorname{Jan}$ & Feb & March & April & May & June & July & Aug & Sept \\
\hline 1975 & $\star$ & 76.47 & * & 77.26 & 77.28 & 77.32 & 77.27 & 76.96 & 76.61 & 76.63 & * & 77.09 \\
\hline 1976 & * & 76.81 & 76.85 & $\star$ & 77.09 & 77.07 & 76.89 & 77.17 & 77.19 & 77.19 & * & * \\
\hline 1977 & * & $\star$ & 77.43 & 77.35 & 77.29 & $\star$ & 76.99 & $\star$ & $\star$ & $\star$ & 75.57 & $\star$ \\
\hline 1978 & * & * & $\star$ & * & $\star$ & $\star$ & $\star$ & * & 76.81 & * & * & $\star$ \\
\hline 197.9 & * & 76.39 & 76.51 & 76.78 & 77.06 & 77.15 & 77.23 & 77.10 & 77.24 & 77.17 & 76.97 & 77.01 \\
\hline 1980 & 76.85 & 76.66 & 76.79 & 76.74 & 76.90 & 77.43 & 77.00 & 76.59 & 76.61 & 76.59 & 77.25 & 77.41 \\
\hline 1981 & 76.99 & 77.10 & 77.07 & 76.92 & 76.89 & 77.08 & 77.02 & 76.44 & 75.99 & 75.85 & * & 76.26 \\
\hline 1982 & 76.19 & 76.38 & * & 77.11 & 77.19 & 77.05 & 77.27 & 76.89 & 76.69 & 77.03 & 77.21 & 77.11 \\
\hline 1983 & 76.89 & 76.51 & * & 76.83 & $\star$ & 77.17 & 77.25 & 77.23 & 77.19 & 77.15 & 77.05 & 76.78 \\
\hline 1984 & * & 76.83 & 77.13 & 77.27 & $\star$ & 77.30 & * & $\star$ & * & * & * & * \\
\hline 1985 & * & $\star$ & $\star$ & $\star$ & 75.79 & 75.61 & 75.29 & 75.13 & 74.54 & 75.09 & 75.53 & 76.09 \\
\hline 1986 & 75.59 & 75.85 & 75.90 & 76.78 & 77.29 & 77.11 & 76.79 & $\star$ & * & 75.63 & $\star$ & 75.99 \\
\hline 1987 & * & $\star$ & 76.47 & 77.25 & 77.53 & 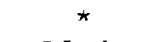 & 77.39 & 76.39 & 76.25 & 76.09 & 76.01 & 75.72 \\
\hline 1988 & 75.34 & 75.21 & 75.03 & 75.09 & 75.53 & 76.41 & 76.26 & 75.89 & 75.43 & 75.07 & * & * \\
\hline 1989 & * & 76.56 & 76.38 & 76.18 & 76.06 & 76.19 & 75.94 & 75.88 & 76.78 & 77.30 & 77.06 & 76.72 \\
\hline 1990 & 76.24 & 75.98 & 76.48 & * & 76.99 & 76.94 & 76.71 & 76.51 & 76.66 & 77.28 & 76.70 & 76.22 \\
\hline 1991 & 75.97 & 75.64 & 75.46 & 75.86 & 77.26 & 77.38 & 77.18 & 77.31 & 77.24 & 77.27 & * & 77.51 \\
\hline 1992 & 77.58 & 76.56 & 76.36 & 76.54 & 77.16 & 76.76 & 76.54 & 76.16 & $\star$ & 75.66 & 76.26 & 76.56 \\
\hline 1993 & * & 76.10 & 75.74 & 76.24 & 76.24 & 76.46 & 76.32 & 75.78 & 73.90 & 75.16 & 74.50 & 74.50 \\
\hline
\end{tabular}

* Indicates a no-value month

Statistics on Normal monthly means (All days)

oct

Nov

Dec

Jan

Feb

March

April

May

June

July

Aug

Sept

By rows (Number, Mean, Variance, Standard Deviation, Skewness, Coefficient of Variation)

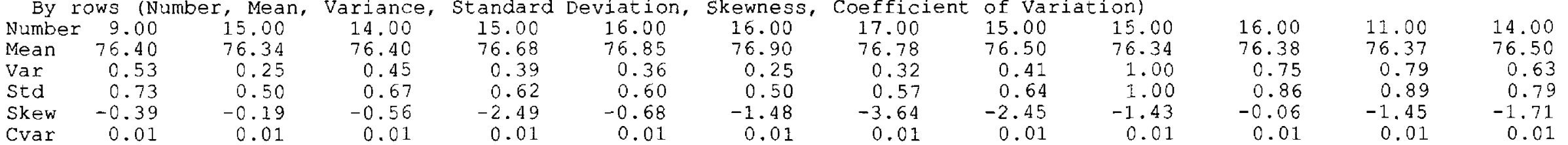

